\title{
On Trade-offs and Communal Breeding The Behavioural Ecology of Agta Foragers
}

\author{
Abigail Emma Page
}

A dissertation submitted in partial fulfilment of the

requirements for the degree of

Doctor of Philosophy

of

\section{University College London}

Department of Anthropology

University College London

March 2016 
I, Abigail E. Page confirm that the work presented in this thesis is my own. Where information has been derived from other sources, I confirm that this has been indicated in the thesis. 


\section{Abstract}

Time is finite and no organism can avoid the allocation dilemma that this necessarily entails. A quintessential trade-off is that between parental investment and reproduction, otherwise known as the quality-quantity trade-off. However, humans may be exceptional among apes given our high quantity production of high quality offspring. This success has been argued only to be possible by breeding communally. In this thesis I explore questions surrounding trade-offs, communal breeding and their fitness consequences in a small-scale foraging society, the Agta. The first analysis examines the composition of Agta childcare using an innovative form of data collection to maximise sample sizes, previously a major limitation in hunter-gatherer research. The Agta, like many small-scale societies are prolific communal breeders. However, contra previous conclusions, juveniles and non-kin appeared to provide more allocare than grandmothers. Interactions with non-kin were associated with significant decreases in maternal workload, while interactions with siblings and grandmothers were not. The next analysis explores why both kin and non-kin behave cooperatively, finding support for kin selection among close kin and reciprocity for distant kin and non-kin allocare. Communal breeding appears to be an important mechanism to ensure enough childcare was received in the absence of other strategies to counter shortfalls in household energy budgets. The next analysis asks, what are the fitness consequences of maternal social networks and allocare? Mothers' network centrality positively correlated with non-kin allocare as well as reproductive success, revealing the adaptive value of communal breeding. The final analysis investigates the health and fitness consequences of increased sedentism and cultivation from a life history perspective. Sedentary, cultivating mothers experienced higher fertility, disease load and child mortality, but nonetheless had more offspring reach maturity. These results highlight the optimising nature of hunter-gatherer cooperation and life history strategies. 


\section{Acknowledgements}

I am, of course, completely indebted to my supervisor Andrea Migliano for seeing my potential from the start and working tirelessly with me to reach it. Also, for putting emoticons in feedback, which always made my day $:-$. She has provided invaluable advice, insight and guidance as well as being a great friend. Ruth Mace, as my secondary supervisor, has also provided me with excellent advice and critique, which helped built my work into what it is here.

None of this would have been possible without the Agta, particularly all the children I got to know so well. I thank them unreservedly for having the patience to let me follow them around, measure them, and prick their fingers. Their understanding and acceptance is impressive. Particularly I would like to thank all the Agta mothers and girls for their care and acceptance and to Marinel and Jollibee for just being awesome. Of course, I was completely reliant on the advice, organisation and translation of our team of research assistants; Ata Christie, Gurly and Amay Crumpez and Imay Crumpez. Their Filipino spirit always made life fun and dynamic, as did the karaoke parties. I am very thankful to the local government in Palanan for allowing us to work with the Agta, and in particular to the Rural Health Unit and Palanan Station Hospital for working with me to organise the testing and treatment for over 400 Agta. It was hard, I didn't think it would be possible, but it was and thanks to the team I feel that we accomplished something to help, however tiny. I was not alone in the field, and thanks to the hard work of Mark Dyble, Sylvain Viguier and Daniel Smith we were able to collect such a wealth of data on all aspects of life. I particularly thank Mark for all the debates that livened up the evenings. All this research was made possible by funding from the Leverhulme Trust as part of the HunterGatherer Resilience Project led by Andrea Migliano, as well as Ruth Mace, Mark Thomas and Jerome Lewis.

The rest of the hunter-gatherer team - Deniz Salali, Nik Chaudhary, James Thompson, Janis Strods and Lucio Vinicius - have offered invaluable guidance on the structure of data collection, research questions, as well as the final product. Many people have offered critique and feedback on the different chapters and my thanks goes out to Matt Thomas, 
Sarah Myers, Heidi Colleran, Emily Emmott as well as all members of the Human Evolutionary Ecology group at UCL and members of the Evolutionary Demography Group at LSHTM for letting me gate-crash and present my work in its infancy.

Ultimately, I am unbelievably thankful to Wallace Hobbes (AKA Sylvain Viguier) who is literally the cleverest person I know, and without whom I would not have made it this far. His maths and coding lessons and patient understanding have opened up a new world for me. Special thanks also to Helen Cassidy who is always loving and makes me want to be better. My family have remained patient and caring even when I disappear to stare at a computer; they are the best and I am proud of them. 


\section{Contents}

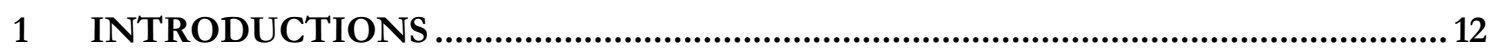

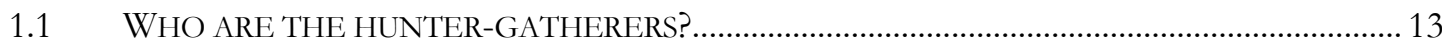

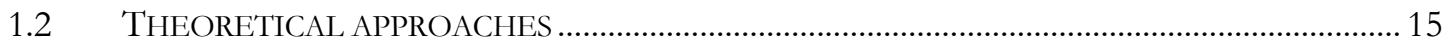

1.3 HUMAN LIFE HISTORY TRAITS AND COOPERATIVE BREEDING........................................... 19

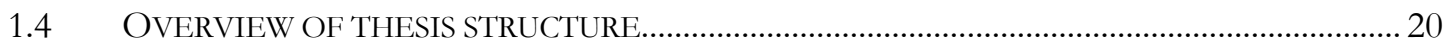

2 RISK AND THE EVOLUTION OF COOPERATIVE CHILDCARE .......................22

2.1 COOPERATIVE BREEDING: ITS DEFINITIONS AND QUESTIONS............................................ 22

2.2 WHY BREED COMMUNALLY? THEORETICAL PERSPECTIVES.......................................................

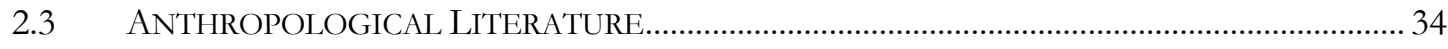

2.4 FLEXIBILITY, VARIABILITY AND RISK AVOIDANCE ............................................................ 43

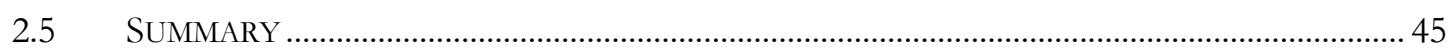

3 METHODOLOGICAL APPROACH AND DATA COLLECTION ...........................4

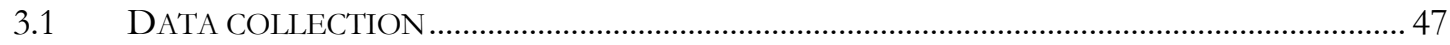

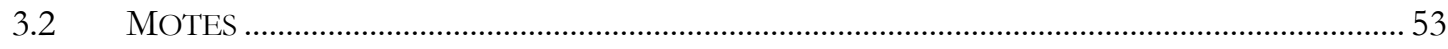

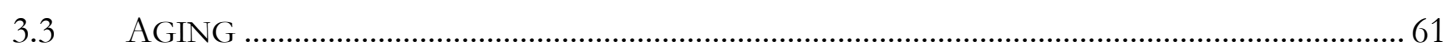

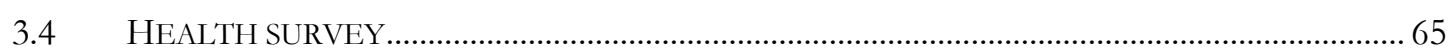

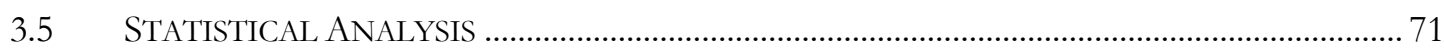

4 THE AGTA: RESILIENT FORAGERS UNDER TRANSITION? ............................78

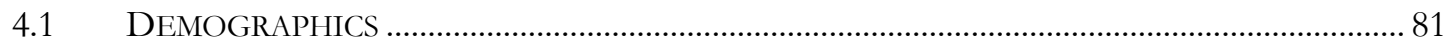

4.2 KINSHIP AND RESIDENCE PATTERNS ............................................................................ 86

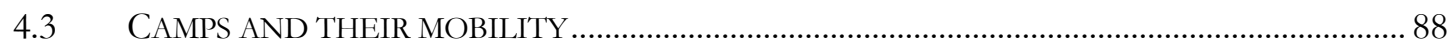

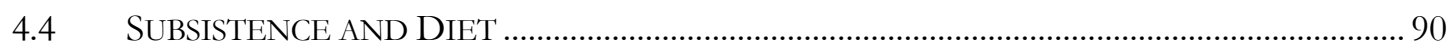

4.5 WEALTH, CAMP PERMANENCE, FOOD STORAGE AND SECURITY ………………………..... 97

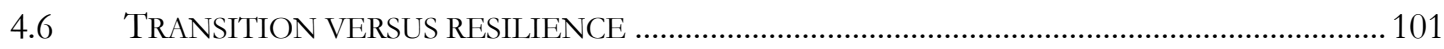

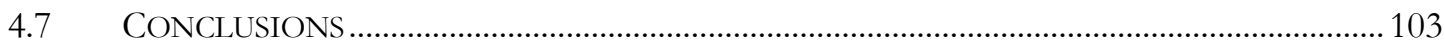

5 THE BEHAVIOURAL ECOLOGY OF COMMUNAL BREEDING: WHO CARES

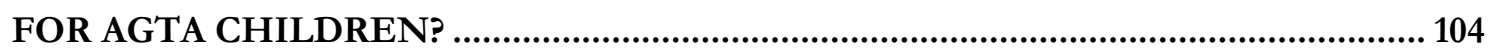

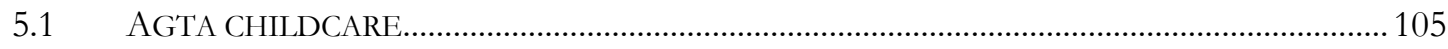

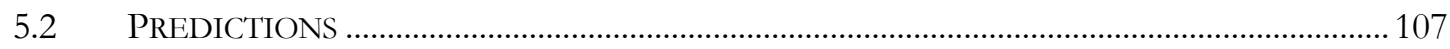

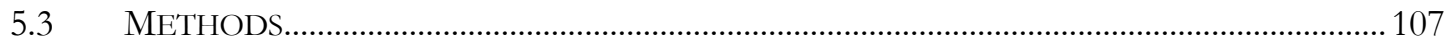

5.4 RESULTS: WHO CARES AND HOW MUCH? ..................................................................... 109 
5.5 RESULTS: SUBSTITUTIVE CARE...................................................................................114

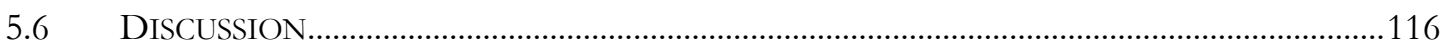

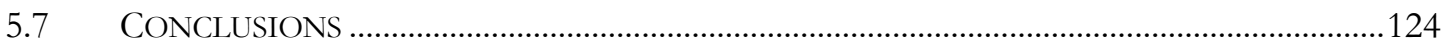

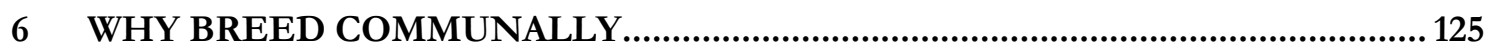

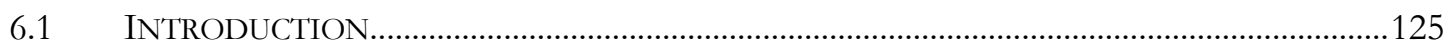

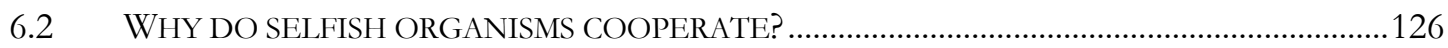

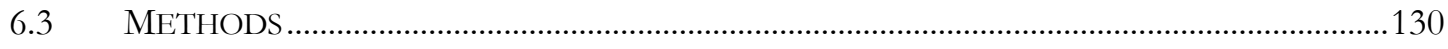

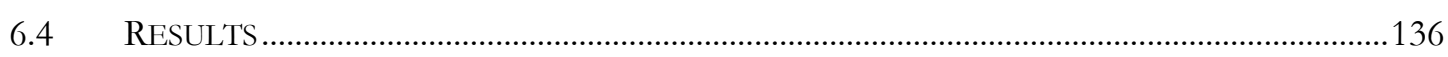

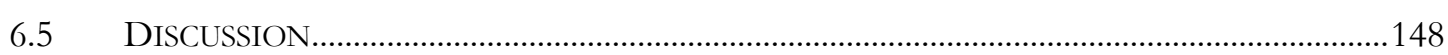

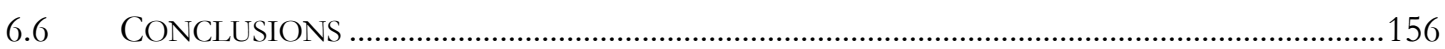

7 BETWIXT AND BETWEEN: SOCIAL NETWORK EFFECTS ON ALLOCARE

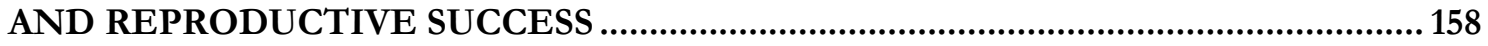

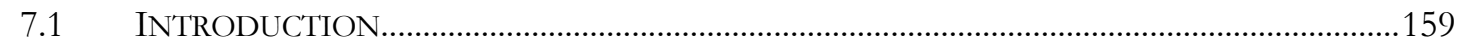

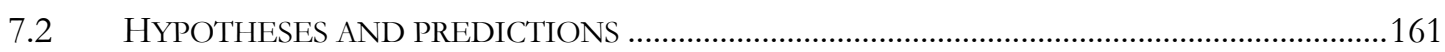

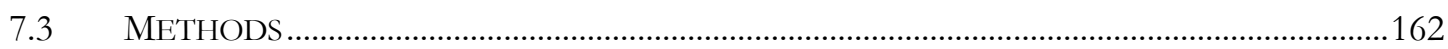

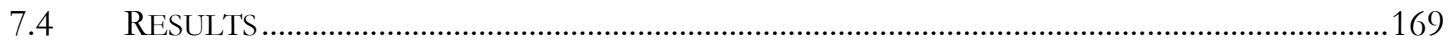

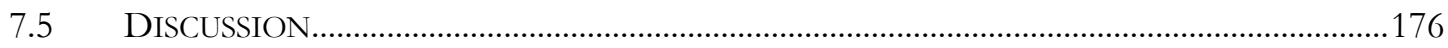

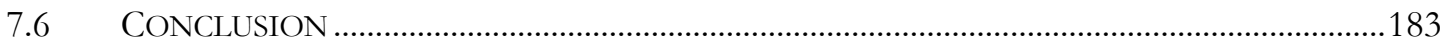

8 THE COST OF CULTIVATION AND SEDENTISM ............................................ 184

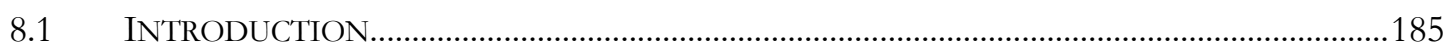

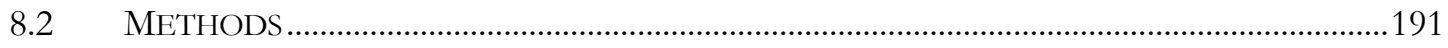

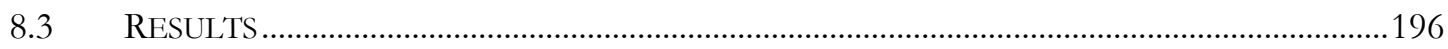

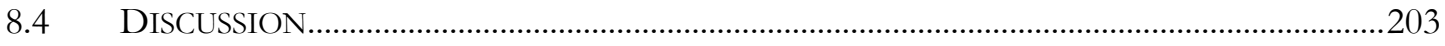

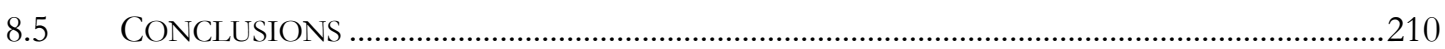

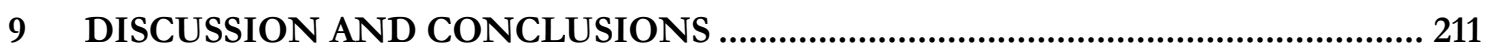

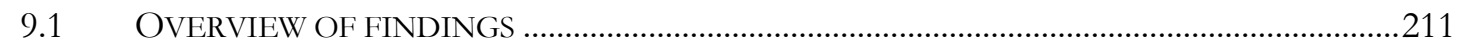

9.2 IMPLICATIONS FOR COOPERATION, RISK AND HUNTER-GATHERERS ...............................213

9.3 HUNTER-GATHERERS AND HUMAN EVOLUTION ………………………………………......216

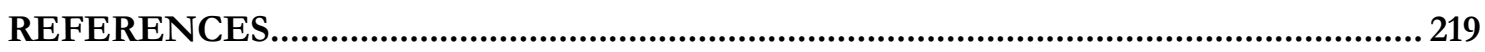

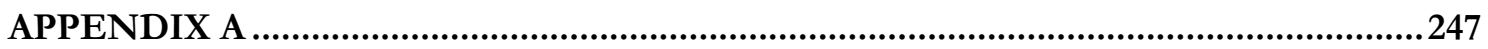

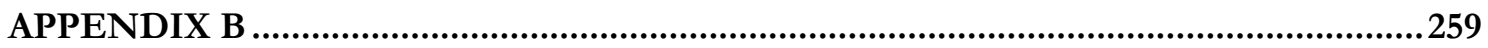

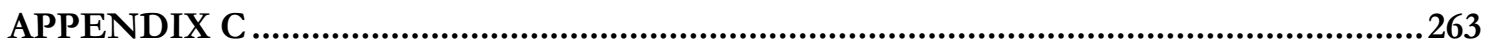

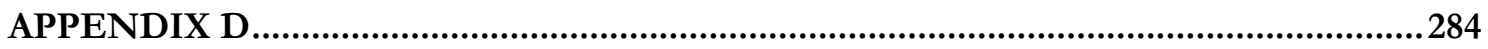

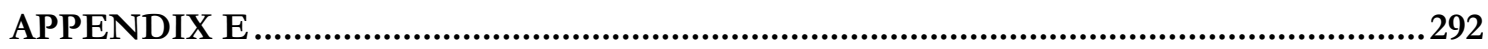




\section{List of Figures}

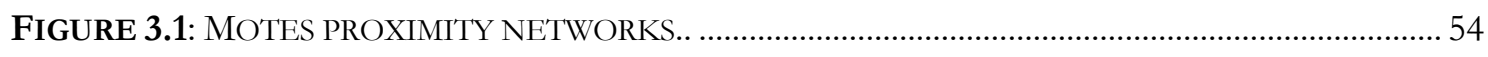

FIGURE 3.2: AGTA CAMP LAYOUTS AND MOTES THRESHOLDS......................................................... 56

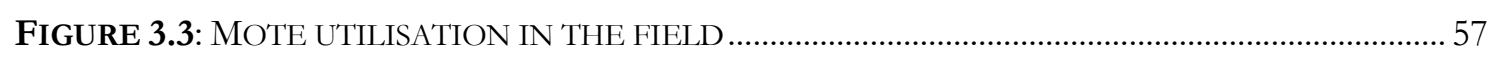

FIGURE 3.4: PROPORTION OF TIME TODDLERS SPENT WITH DIFFERENT KIN CATEGORIES FOR

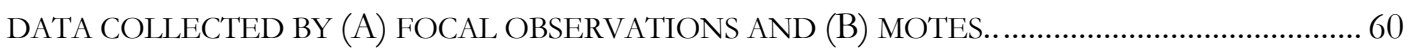

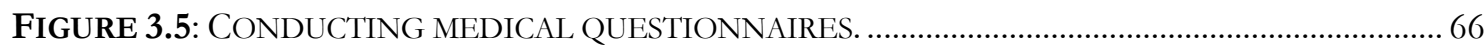

FIGURE 3.6: COLLECTION OF BLOOD SAMPLES FOR WBC DIFFERENTIAL ANALYSIS AND HAEMOGLOBIN COUNT.

FIGURE 3.7: RELATIONSHIP BETWEEN AGE AND A) FERTILITY, B) MATERNAL CHILD MORTALITY

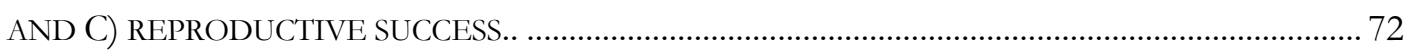

FIGURE 4.1: MAP OF THE SIERRA MADRE, ISABELA... ............................................................... 79

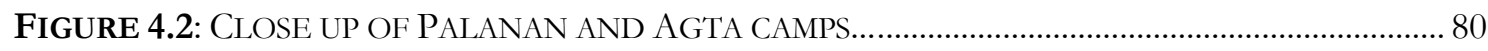

FIGURE 4.3: POPULATION PYRAMID FOR 914 AGTA CENSURED DURING 2013-2014....................... 82

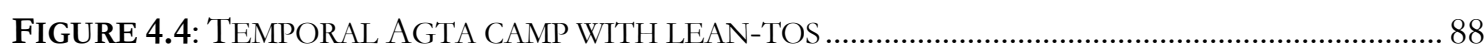

FIGURE 4.5: PERMANENT AGTA CAMP WITH A WOODEN HOUSE........................................................... 89

FigurE 4.6: TWO CAMPS (A-B) DJAGO AND (C-D) MATARAGAY BETWEEN TWO VISITS. .............. 90

FIGURE 4.7: SUMMARY DATA ON (A) THE PROPORTION OF TIME INDIVIDUALS SPENT IN ALL ACTIVITIES AND (B) PROPORTION OF TIME INDIVIDUALS SPENT IN DIFFERENT TYPES OF

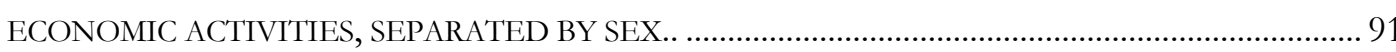

FIGURE 4.8: (A) PROPORTION OF EACH FOOD GROUP CONSUMED DEPENDING ON WHETHER A HOUSEHOLD LIVED ON LOCATION AND (B) THE PROPORTION FOODS WHICH WERE ACQUIRED VIA FORAGING (HUNTING, FISHING OR GATHERING) COMPARED TO CULTIVATION OR TRADE, DEPENDING ON DEGREE OF SETTLEMENT. 93

FIGURE 4.9: (A) THE PROPORTION OF TIME INDIVIDUALS SPENT IN DIFFERENT ACTIVITIES AND (B) PROPORTION OF TIME INDIVIDUALS SPENT IN DIFFERENT TYPES OF ECONOMIC ACTIVITIES, SEPARATED BY LOCATION. 95

FIGURE 4.10: (A) THE PROPORTION OF TIME INDIVIDUALS SPENT IN DIFFERENT ACTIVITIES AND (B) PROPORTION OF TIME INDIVIDUALS SPENT IN DIFFERENT TYPES OF ECONOMIC ACTIVITIES, SEPARATED BY DEGREE OF MOBILITY ............................................................... 97

FIGURE 4.11: CORRELATION PLOT FOR SIX KEY ‘TRANSITION’ VARIABLES....................................... 99 
FIGURE 4.12: GROUP MEANS FOR KEY TRAITS ASSOCIATED WITH IMMEDIATE RETURN FORAGERS SEPARATE BY (A) MOBILITY AND (B) LOCATION...................................................................100

FIGURE 5.1: MEAN PROPORTION OF INTERACTIONS WITH DIFFERENT KIN...................................110

FIGURE 5.2: PROPORTION OF A CHILD'S CLOSE-RANGE INTERACTIONS WITH CLOSE KIN AND UNRELATED INDIVIDUALS..

FIGURE 5.3: THE RELATIONSHIP BETWEEN NUMBER OF INTERACTIONS,

FIGURE 6.1: VISUALISATION OF THE CREATION OF THE CONTINGENCY VARIABLE. 131

FIGURE 6.2: RELATIONSHIP BETWEEN MATERNAL AGE AND HOUSEHOLD CARE NEED. 136

FIGURE 6.3: MODEL PREDICTED NUMBER OF CONTACTS BASED ON INTERACTIONS BETWEEN KIN TYPE AND A) HOUSEHOLD NEED AND B) GIVER COST.

FIGURE 6.4: MODEL INTERACTION PLOT SEPARATED BY KIN TYPE FOR RECIPROCATED ALLOCARE 142

FIGURE 6.5: THE RELATIONSHIP BETWEEN HOUSEHOLD BELONGINGS AND PROPORTION OF TIME SPENT WITH NON-KIN (A), OTHER KIN (B) AND GRANDPARENTS (C).

FIGURE 7.1: EXAMPLE NETWORK DEMONSTRATING LOW AND HIGH BETWEENNESS CENTRALITY 164

FIGURE 7.2: EXAMPLE NETWORK DEMONSTRATING LOW AND HIGH EIGENVECTOR CENTRALITY (EC). 165

FiguRE 7.3: THE RELATIONSHIP BETWEEN BETWEENNESS, DEGREE AND AGE-SPECIFIC RESIDUALS FOR FERTILITY, MORTALITY AND REPRODUCTIVE SUCCESS.

FIGURE 7.4: THE RELATIONSHIP BETWEEN NUMBER OF YOUNGER AND OLDER SIBLINGS AND

(A) BMI Z-SCORES AND (B) HAEMOGLOBIN LEVELS. 174

FIGURE 7.5: MATERNAL AND CHILD OUTCOMES ACCORDING TO MATERNAL CENTRALITY AND AMOUNT OF CARE RECEIVED FROM DIFFERENT CATEGORIES, 175

FIGURE 8.1: LYMPHOCYTOSIS BY OUT-OF-CAMP MOBILITY. 199

FIGURE 8.2: MEAN EOSINOPHIL CONCENTRATION BY CAMP HOUSING TYPE. 200

FIGURE 8.3: RELATIONSHIP BETWEEN MEASURES OF TRANSITION AND RESIDUALS OF AGESPECIFIC FERTILITY, MORTALITY AND REPRODUCTIVE SUCCESS. 


\section{List of Tables}

TABLE 1.1: LIFE HISTORY VARIABLES FOR HUMAN FORAGERS AND NON-HUMAN GREAT APES.. 19

TABLE 2.1: CROSS-CULTURAL REPORTS OF PROPORTION OF DIRECT CHILDCARE RECEIVED BY

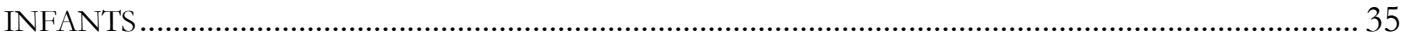

TABLE 3.1: LIST OF HOUSEHOLD OBJECTS AND THEIR WEIGHTING. ............................................... 52

TABLE 3.2: PROPORTIONS OF TIME TODDLERS SPENT WITH ANY GIVEN KIN CATEGORY FOR MOTES AND FOCAL OBSERVATIONS.

TABLE 3.3: RESULTS FROM POLYNOMIAL REGRESSION (WITH POISSON DISTRIBUTION) BETWEEN AGE AND FERTILITY. 73

TABLE 4.1: MAJOR CAUSES OF MORTALITY FOR 427 CASES REPORTED DURING FAMILY HISTORIES. 83

TABLE 4.2: MAJOR CAUSES OF CHILDHOOD MORTALITY FOR 202 CASES BROKEN DOWN BY AGE.84

TABLE 4.3: SEX RATIO OF MORTALITY RATES BY CAUSE OF DEATH. 85

TABLE 4.4: FORAGING RETURN RATES SEPARATED BY LOCATION.. 94

TABLE 5.1: PROPORTION OF ALL POSSIBLE INTERACTIONS KIN SPENT WITH CHILDREN UNDER 12 YEARS.. 109

TABLE 5.2: PROPORTION OF CHILDREN UNDER 12 YEARS WITH DIFFERENT TYPES OF GRANDPARENTS PRESENT IN CAMP. 111

TABLE 5.3: MLM RESULTS ON THE EFFECT OF INTERACTIONS WITH DIFFERENT KIN TYPES ON PARENTAL-OFFSPRING INTERACTIONS. 115

TABLE 6.1: EVOLUTIONARY MODELS EXPLAINING COOPERATION AND ALLOCAREE. ................. 128

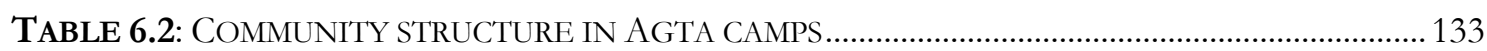

TABLE 6.3: DESCRIPTIVE STATISTICS FOR BINARY VARIABLES AT THE DYADIC LEVEL..................134

TABLE 6.4: DESCRIPTIVE STATISTICS FOR CONTINUOUS VARIABLES 135

TABLE 6.5: RESULTS FROM MULTILEVEL MODELS EXAMINING DIFFERENT PREDICTORS FOR THE

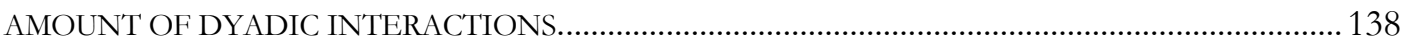

TABLE 6.6: PREDICTORS OF ALLOCARE SEPARATED BY KIN TYPE................................................... 140

TABLE 6.7: RESULTS FROM INTERACTION ANALYSIS WITH KIN TYPES..............................................143

TABLE 6.8: MODEL SELECTION DETAILS FOR EACH OF THE SIX ANALYSES SEPARATED BY KIN TYPE. 145

TABLE 6.9: MLM RESULTS FOR PREDICTORS OF CARE FROM DIFFERENT KIN TYPES.....................146

TABLE 6.10: SUMMARY OF THE MAIN RESULTS FROM THE ‘WHY CARE’ ANALYSIS. ...........................148

TABLE 7.1: DESCRIPTIVE STATISTICS FOR MOTHERS AND THEIR CHILDREN..................................... 168 
TABLE 7.2: MULTILEVEL MODELS OF MATERNAL NETWORK CHARACTERISTICS, FERTILITY AND REPRODUCTIVE SUCCESS (SURVIVORSHIP TO AGE 16). 169

TABLE 7.3: NON-AGE CONTROLLED FERTILITY AND REPRODUCTIVE RATES FOR WOMEN AGED 25 YEARS AND OVER ACCORDING TO BETWEENNESS AND DEGREE CENTRALITY. 170

TABLE 7.4: MULTILEVEL MODELS EXAMINING THE RELATIONSHIP BETWEEN THE PROPORTIONS OF INTERACTIONS BETWEEN NON-KIN, NON-HOUSEHOLD AND KIN PROXIMITY AND MATERNAL CENTRALITY. 171

TABLE 7.5: MULTILEVEL MODELS EXAMINING THE RELATIONSHIP BETWEEN NON-KIN ALLOCARE, FEMALE FERTILITY, AND REPRODUCTIVE SUCCESS..

TABLE 7.6: RESULTS SUMMARY. 177

TABLE 8.1: WHITE BLOOD CELL TYPES, MEDICALLY ABNORMAL THRESHOLDS AND HEALTH IMPLICATIONS 192

TABLE 8.2: DESCRIPTIVE STATISTICS FOR CATEGORICAL INDEPENDENT VARIABLES. 193

TABLE 8.3: DESCRIPTIVE STATISTICS FOR THE CONTINUOUS DEPENDENT AND INDEPENDENT VARIABLES. 194

TABLE 8.4: SELECTION PROCESS FOR CAMP SEDENTARIZATION VARIABLES . 195

TABLE 8.5: FERTILITY, MORTALITY AND SURVIVORSHIP TO AGE 16 FIGURES AVERAGED BY MOBILITY AND CAMP TYPE

TABLE 8.7: WHITE BLOOD CELL TYPES, MEDICALLY ABNORMAL THRESHOLDS, FREQUENCY, PREDICTORS AND HEALTH IMPLICATIONS. 


\title{
Chapter One
}

\section{Introductions: Human Behavioural Ecology and Hunter-Gatherers}

\author{
"Feign illness, feign fatigue, go to the limit of all lies and feign frigidity. But no more \\ children, biccoughed to conception. Four were enough. But how she loved them when she beld \\ them first, heavy-lidded, helpless, the head out of all proportion... Given security she'd bear \\ a dozen, but not as things were. No paupers for the parish". Daphne Du Maurier, \\ Mary Anne: 70
}

Awareness of reproductive trade-offs is not limited to the realms of academia or the evolutionary study of human behaviour. Each mother faces the decisions that define the study of life history, such as how many children to have, when to have the next child, how much to invest in which child and when to start and when to stop reproducing. Like all organisms our time, and hence our energy, is finite (Charnov 1993). Thus, we all face an allocation dilemma; too many children and we will not be able to provide for them all. Life history theory is a theoretical approach within behavioural ecology which takes for granted that reproductive behaviours and decision-making, such as expressed by the fictional character Mary-Anne above, are fitness optimising (Hill \& Hurtado 1996). This by no means requires an conscious break down of calorific energy expenditure versus proportion of genetic representation in future generations - this laborious task is left to human behavioural ecologists - but rather, natural selection favours behaviours which increase fitness regardless of whether they are couched in cultural or social emic explanations (Davies et al. 2012).

Humans, like several gregarious mammals do not raise their children in isolation but are commonly defined as cooperative breeders (Hrdy 2005). Cooperative breeding is, in its simplest form, when non-mothers provide childcare and provisioning to offspring, a theory predominately developed to explain the extreme cooperation in co-nesting birds 
(Koenig \& Dickinson 2004), or reproductive suppression in mammals such as naked mole rats (Heterocephalus glaber, (Solomon \& French 1997)) and eusocial insects (Wilson 2008). When mothers have access to additional assistance in childrearing, trade-offs are altered; earlier weaning and a quicker resumption of ovulation is possible when someone else can take an infant off your hands. That human someone has often been argued to be grandmothers, and consequentially, our exceptional survival post-reproduction has been associated with grandmaternal importance in the successful production of multiple offspring (Hawkes et al. 1997; Hrdy 2005).

This thesis investigates cooperative breeding, life history and their adaptive values. This research is firmly grounded in behavioural ecology and seeks the ultimate explanation of behaviour in its given ecological context (Nettle et al. 2012). Hypotheses are developed following key life history predictions, focusing on parental investment and inclusive fitness explanations of cooperation. In conducting this research with the Agta - a Philippine foraging group - I aim to further the understanding of life history, reproduction and cooperation in a natural fertility population which spends the majority of subsistence activities foraging. However, significant variability exists within the Agta, as with any population, allowing the exploration of the 'reactive norms' of behaviour in different contexts. Foragers face a unique situation among humans (but not animals) in which they survive without wealth, leading many to argue that the origins of cooperation and sociality lie in the importance of relational wealth buffering inherent risks and shortfalls in foraging niches (Winterhalder 1986). I aim to explore the variability in wealth, cooperation and reproduction among the Agta to shed light on the adaptive role of human social networks.

\subsection{Who are the hunter-gatherers?}

The definition of hunter-gatherers is fraught with difficulties and contention. Their very name implies that the distinction is an economic one. Thus, hunter-gatherers are those without domesticated plants and livestock (Kelly 2013). However, this excludes the majority of ethnographically known foraging populations since most are involved in some form of market exchange and a diversification of subsistence (Hitchcock \& Biesele 2000). Given that hunter-gatherers could not be defined as 'Pleistocene economies' in 1968 during the first symposium on foraging societies since "such a definition would effectively eliminate most, if not all, of the peoples reported..." (Lee \& DeVore 1968: 4), this is certainly not possible today. Attempts to define hunter-gatherer groups in this way is 
reflective of nostalgia for Jean-Jacques Rousseau's concept of the Noble Savage and of Sahlins' 'original affluent society' (Sahlins 1968). This harps back to early thought in anthropology of hunter-gatherers as primitive, uncultured fossils who had yet to evolve 'civilisation' (Kelly 2013). Yet, such topological definitions are unnecessary, and predictably hide more variability that exists between the remaining hunter-gatherers today. Here, I am in complete agreement with Kelly (2013) who argues that the term hunter-gatherer has no explanatory weight; however there are aspects of hunter-gatherer lifeways which the Agta share with other foraging groups that poses specific constraints, pressures and contextual influence, which (I expect to) condition human behaviour. The exact percentage of time spent in foraging is meaningless ${ }^{1}$ as a taxonomic division between 'foragers' and 'nonforagers'. However, foraging modes of subsistence poses a unique set of behavioural challenges which individuals are expected to meet. Consequentially, it is the variability among hunter-gatherers that can inform our understanding of behaviour.

A division is commonly draw between 'complex' or 'delayed-return' hunter-gatherers and 'primitive' or 'immediate-return foraging ${ }^{2}$ societies. The former labels are problematically value ridden. As a result I will use Woodburn's (1982) 'return' terminology here. Delayed return foragers are those epitomised by North West American hunter-gatherers, who were more sedentarised, practiced mixed methods of food production, produced surplus requiring storage, leading to the accumulation of wealth, social status and hierarchy (Kelly 2013). In contrast, immediate-return foragers, as the name suggests, produce and consume food on a daily basis; there is no food storage and individuals move freely throughout the environment as there is little concept of property, territoriality and belongings are rare. Thus, the distinction between these groups is based on degree of egalitarianism associated with key features of mobility and wealth (Woodburn 1982). The Agta fall into the latter, allowing me to draw parallels between other egalitarian groups such as the Aka, BaYaka, Hadza, !Kung and Batak (to name a few). Over the next section I will introduce the theoretical framework underpinning this research and discuss humans' key life history traits.

\footnotetext{
${ }^{1}$ For instance, what is exactly the 'right' amount of foraging? More than $50 \%, 75 \%$ or $95 \%$ of subsistence activities? Who sets this threshold and what is the model for comparison?

2 I use both terms 'hunter-gatherers' and 'foragers' interchangeably throughout this thesis for linguistic variance only.
} 


\subsection{Theoretical approaches}

\subsubsection{Evolutionary perspectives}

Evolution is simply change over time, often driven by natural selection which itself is the product of three observable aspects of traits: (i) heritability; (ii) variability; and (iii) differential reproductive outcomes (Darwin 1859). There is no sense of progression here. Instead, adaptation by natural selection will simply favour the propagation of traits which maximises individuals reproductive success or lifetime fitness, i.e. the number of genetic copies present in future generations (Gross 2005; West et al. 2011). It is through behaviour that most animals interact with the environment. Consequently, behavioural strategies have a large influence on fitness, and thus, a target of evolution (Kappeler et al. 2013). For instance, A South African brown fur seal (Arctocephalus pusillus) who, rather than swim in a large mobbing group, goes for a solitary dip on a regular basis would soon find herself face-to-teeth with a great white (Carcharodon carcharias). In other words, antipredatory behaviours are a key survival strategy. The evolutionary analysis of behaviour falls under the discipline of behavioural ecology which seeks to understand how and why behaviours are optimal in fitness terms given their ecological context (Davies et al. 2012; Winterhalder $\&$ Smith 2000). At the heart of this is the assumption that individuals tend to be optimising due to the evolution of reactive norms which increase lifetime reproductive success (Grafen 2002; Nettle et al. 2013). A reaction norm is simply the evolved ability to respond adaptively to differential circumstances. This means that there is no need for behaviour to be rigorously under the control of genes (Sear 2015) but it is the ability to respond which is under selective pressure. This reactive norm is expected to result in a fitness payoff. However, this is tempted by a series of phylogenetic, developmental, morphological and genetic constraints (Kappeler et al. 2013; Hill \& Hurtado 1996). Therefore, the underlying assumption in behavioural ecology predictions and hypotheses is that "phenotypes should be approximately optimal (i.e. fitness maximizing)" (Hill \& Hurtado 1996: 13).

\subsubsection{Human behavioural ecology}

Human behavioural ecology is an offshoot of behaviour ecology specifically dealing with humans. In complete agreement with Nettle's et al. (2013) recent review of the field, there is no fundamental difference in approach between the study of human or other animal behaviour; humans are expected to have evolved behavioural strategies which increase their genetic representation in future generations, given ecological context and constraints. 
This starting point generates hypothesis for further analysis to explore both ultimate and proximate explanations about the evolution of behaviour. As originally laid out by Tinbergen (1963), ultimate explanations are those which ask the evolutionary why question, which can be answered in terms of phylogeny - which marine bird species have evolved cooperative breeding? - or functionality - do mothers increase their reproductive success by breeding cooperatively? Proximate questions are those that ask bow, and include ontological investigations - how do children learn to cooperate? - and mechanistic questions - what maintains an individual's cooperative behaviour? The boundaries between whether an explanation is casual or mechanistic can become blurred, especially in the study of cooperation (Barrett \& Stulp 2013). Here, I focus on ultimate explanations for behaviour as I seek to directly quantify the fitness consequences of reproductive trade-offs and cooperative breeding, as well as understanding the roles of kin selection and reciprocity in cooperation. However, the measurement of fitness is difficult given the long life of human subjects (Orr 2009). Therefore, as a proxy for lifetime fitness, similar to many human behavioural ecologists, I use age-controlled fertility and survivorship to maturity rates to explore the fitness consequences of life history trade-offs among the Agta (Sear 2015).

\subsubsection{Life history theory}

Life history schedules are the outcome of individuals' allocation of time and energy to optimise inclusive fitness, given extrinsic mortality rates (Charnov 1993). The production of offspring requires energy, the harvesting of which takes time. Time is also required to grow and develop, the longer time spent in which, the greater the amount of energy can be extracted from the environment (Hill \& Kaplan 1999). Organisms face a trade-off between time spent in reproduction (shortened by time spent in growth) and adult reproductive rate (increased due to growth and somatic investment), or otherwise put, a trade-off between current and future reproduction (Stearns 1992; Kaplan et al. 2000a). The solution to this dilemma is represented in a number of predictable life history traits across species, such as: size at birth, age at maturity, size at maturity and length of life (Stearns 1992).

The longer an organism survives for, the more energy can be devoted to growth and the accumulation of resources and skills. This is somatic effort and it is crucial for fitness as height, body mass index (BMI) and resources are strongly positively correlated with reproductive success (Gibson \& Mace 2003; Allal et al. 2004; Lawson \& Mace 2011; Kaplan 
et al. 2000a). However, the future is not certain, and the amount of time invested in somatic effort is dictated by extrinsic mortality risks. Hence, in an environment in which mortality is higher, we would expect less investment in growth and life history schedules to be speeded up (Charnov 1993). Yet too early reproduction can result in diminishing returns as intrinsic mortality increases (Charnov \& Berrigan 1993; Stearns 1992). The distinction between intrinsic and extrinsic mortality is thus crucial in life history theory, the former being influenced by the allocation decisions of organisms and the latter being uninfluenced by trade-offs (Stearns 1992). However, this distinction can be difficult to make (Promislow 1991) as many forms of mortality could be arguably be influenced by investment at some points but not at others; a child of better nutritional condition may fight influenza, however nutritional condition may do little in the face of a highly virulent zoonotic pathogen. Ultimately then age at maturity and cessation of growth is dependent on the costs and benefits of maturation of different ages and sizes. If individuals are facing high extrinsic mortality risks it follows that early maturing individuals will be more likely to survive and thus reproduce, maximising their reproductive success (Stearns 1992). However, this simultaneously reduces time spent in growth and development. As a result extrinsic risks in the environment can be considered to result in either 'fast' (develop fast, reproduce lots, die young) or 'slow' (develop slowly, invest in offspring, live long) life history traits as organisms attempt to optimise allocations given constraints (Nettle 2010; Promislow \& Harvey 1990).

Fast life history strategies are not only represented by features like earlier maturation and smaller body size (Migliano et al. 2007), but also by the trade-off between the number and quality of offspring, otherwise referred to as the quantity-quality trade off. The quantityquality premise states that the optimal level of fertility is that which balances the number of offspring produced against their survival and future reproductive success (Borgerhoff Mulder 2000; Lack 1954). A parent cannot invest excessively in multiple offspring, and thus must choose between fewer high quality offspring or multiple low quality offspring (Hill \& Hurtado 1996). While some evidence is supportive of natural fertility populations acting 'optimally' in this regard (Borgerhoff Mulder 2000; Strassmann \& Gillespie 2002; Lawson et al. 2012), the evidence is inconclusive as data from the Ache and !Kung reveal that women would have achieved higher fitness by exhibiting higher fertility (Pennington \& Harpending 1988; Hill \& Hurtado 1996). 
One cause may be unmeasured heterogeneity among women or in their environments (Lawson \& Mace 2011). For instance, a mother's allocation of effort is dependent on the degree of risk in the environment. In high mortality environments mothers are expected to invest in quantity rather than quality. Quinlan (2007) demonstrated that maternal care shows a quadratic relationship with pathogen load. Maternal investment occurs for as long as an offspring's survival is responsive to care. Once the mother starts to receive diminishing returns from this investment due to unalterable environmental assaults, her care drops dramatically. At this time, a mother increases her fitness by investing elsewhere. This demonstrates the blur between intrinsic and extrinsic risk, revealing the usefulness of thinking of risk as a continuum. Desai (1995) compared child outcomes with sibship size, finding that in resource poor contexts sibling quantity had little influence of child quality. This indicates that parental ability to influence child outcomes plays an important role in life history strategies, potentially obscuring quantity-quality trade-offs if left unmeasured (Lawson \& Mace 2011). Unlike the Dogon and Kipsigis (agropastoralists) for whom quality-quantity trade-offs have been demonstrated (Strassmann \& Gillespie 2002; Borgerhoff Mulder 2000) variance in reproductive success of the Ache is more dependent on fertility than it is mortality (Hill \& Hurtado 1996; Strassmann \& Gillespie 2002). Thus, in environments where there is little variance in mortality (i.e. childhood and juvenile mortality occurs regardless of parental interventions) fitness is maximised by fertility, suggesting that the point of diminishing returns for higher fertility may be at a substantially higher threshold than in lower mortality environments, in which mortality has a larger relative influence of reproductive success.

The quality-quantity trade-off is part of the subset of life history theory that falls under 'parental investment'. Trivers (1972: 139) defines parental investment as "any investment by the parent in an individual offspring that increases the offspring's chance of surviving (and hence reproductive success) at the cost of the parent's ability to invest in other offspring”. A mother's reproductive success is not only the result of the production, survival and future reproductive value of one offspring, but a combination of all her current offspring's survival and expected reproductive value plus all her future offspring's survival and expected reproductive success (Gross 2005). However, this equation is further influenced by the fact that not all children are born equal. Due to differential mortality and skewed sex ratios, the reproductive value of different offspring, often dependent on age and sex, varies. As a result, in some scenarios males have significantly higher reproductive value than their sisters, and thus are favoured in terms of parental investment (Mace 1996). 
Ultimately then, the art of reproduction is one of the optimal allocation of time following expectations of reproductive value given estimated extrinsic mortality risks. So how in general do humans solve this allocation problem?

\subsection{Human life history traits and cooperative breeding}

Humans have been noted for their combination of 'fast' and 'slow' life history traits defined by long lives, late maturation and prolonged growth as well as early weaning, shorter interbirth intervals and high fertility (Charnov \& Berrigan 1993; Robson \& Wood 2008). Compared to other great apes we manage quite a feat by producing and simultaneously raising, on average, six to eight highly dependent offspring (Table 1.1, (Campbell and Wood, 1988)). This extreme dependency of infants stems from our energetically expensive brains (Aiello \& Wheeler 1995) as gestation and lactation both significantly increase the energetic demands on the mother by $25 \%$ and $39 \%$, respectively (Aiello \& Key 2002)). Furthermore, given our prolonged juvenile period and arguably minimal productive ability, offspring remain dependent on parents for many years as they, like many animals with slow life histories, invest in growth, development and skills (Kaplan et al. 2003). This is possible because of the doubling of adult life expectancy between humans and chimpanzees (Hill et al. 2001; Kaplan et al. 2000a), setting humans on a slow life history path.

Table 1.1: Life history variables for human foragers and non-human great apes.

\begin{tabular}{lcccc}
\hline & Human foragers & Orangutans & Gorillas & Chimpanzees \\
\hline Mean age at first reproduction & 19.7 & 15.6 & 10 & 14.3 \\
Mean age at last reproduction & 39 & 38 & - & 27.7 \\
Mean inter-birth interval (IBI) & 3.72 & 8.1 & 4.4 & 5.5 \\
Age at weaning & 2.8 & 7 & 4.1 & 4.5 \\
Expected age of death at 15 & 54.1 & 39 & - & 29.7 \\
Total fertility rate (TFR) & 6.1 & - & 3 & 2 \\
\hline Human forager data is the average of Ache, Hadza, Hiwi, and !Kung data reported in (Marlowe \\
2010; Howell 1979; Hill \& Hurtado 1996; Kaplan et al. 2000a). The chimpanzee (Pan sp.) data is the \\
average for Bossou, Gombe, Kibale, Mahale, and Tai reported also reported in (Kaplan et al. 2000), \\
except for age at weaning which is taken from (Alvarez 2000). Data for Orangutans (Pongo sp.) is \\
reported in (Alvarez 2000) for age at weaning and IBI, and in (Wich et al. 2004) for age at first and \\
last reproduction and life span. Age at weaning for gorillas (Gorilla spp.) is the mean reported for G. \\
beringei (3.6 years (Fletcher 2001)) and G. gorilla (4.6 years (Nowell \& Fletcher 2007)), TFR as \\
reported by (Kaplan et al. 2000a) and IBI and age at weaning as reported by (Alvarez 2000).
\end{tabular}


However, unlike other species with late maturation and extended life span, humans wean infants at a younger age resulting in shorter interbirth intervals and high fertility. Across mammals infants are weaned when they reach a third of the mother's body weight or at the eruption of the first permanent molar (Charnov \& Berrigan 1993). For humans this suggests we should wean our offspring at around 6 years of age, closer to the Orangutan (Pongo spp.) pattern. However, human populations average at around half this at 2.8 years (Lee et al. 1991; Alvarez 2000), significantly earlier than all other apes. Consequently humans have combined strategies and invest in a high number of high quality offspring, made possible, according to the cooperative breeding hypothesis, by allomaternal assistance (Hrdy 2011; Kennedy 2005).

Allomothering $^{-3}$ refers to care from any individual other than the mother (Tecot et al. 2012) and is assumed critical for the successful rearing of multiple dependents as they provide the additional investment required by mothers (Snopkowski \& Sear 2013; Hrdy 2005). As a result, allocare can be considered to lift some of the energetic constraints off a mother allowing her to invest in both quality and quantity. For instance, mothers with assistance have additional energy to invest in subsistence labour, childcare or their own somatic condition and fertility. Thus, in the ultimate sense, cooperative breeding is expected to be associated with increases in either fertility or child survivorship and quality (dependent on environmental risks). Such evidence is apparent among callitrichids in which allocarer presence increased the amount of time mothers spent feeding (Mitani \& Watts 1997) and their foraging efficiency (Tardif 1997) as well as pup weight gain and survivorship in Kalahari meerkats (Suricata suricatta (Clutton-Brock et al. 2001)). Not wishing to cherry pick examples, some studies do fail to demonstrate fitness consequences of cooperative breeding, however overall among both birds (Dickinson \& Hatchwell 2004) and mammals there appears a consensus that allocare is associated with reductions in parental investment and load lighting, leading to increases in recipient fitness (Solomon \& French 1997; Russell 2004), a trend matched in the human literature (Sear \& Mace 2008).

\subsection{Overview of thesis structure}

This thesis explores and brings together these different aspects of human life history cooperative breeding, reproductive trade-offs and parental investment - in a population of

\footnotetext{
3 Alloparent on the other hand refers to care from any individual who is not the parent, the distinction being whether male or bi-parental care is of theoretical interest.
} 
hunter-gatherers living in the Philippine rainforest. My intention is to offer unique insights to the complexities around cooperative behaviour, parental investment and fitness outcomes in a foraging group which continues to be largely reliant on mobility and foraging modes of subsistence. However, the Agta, like many hunter-gatherers today, also exhibit significant variability in terms of degree of foraging, cultivation, sedentism and wealth accumulation. Rather than sweep this variability under the carpet, this thesis seeks to explore the relationship variability has with different behavioural strategies and demographic consequences.

After an introduction to my data collection, methodology and the Agta themselves I examine several interrelated questions across four analysis chapters. The literature review found in chapter two and analyses in chapters five and six focuses on the explanation of the occurrence of cooperative breeding from an inclusive fitness standpoint. I ask firstly, who provides allocare among the Agta (chapter five), and secondly what causes apparently selfish individuals to provide costly allocare (chapter six). Chapter seven also deals with cooperative breeding, but this time asks, what is its adaptive value? In this analysis I explore the fitness consequences of allocare and maternal social networks. The analysis in chapter eight deals with quality-quantity trade-offs and explores how mothers maximise their reproductive success. Here, I ask what is the adaptive function of behavioural change towards increased settlement and cultivation, and what hypothesises can be put forward to understand the paradox of Neolithic health deterioration alongside population growth. Finally, chapter nine brings together these analysis and comments on the overarching themes of this work in the context of the wider literature. I focus on the implications of these research findings for perspectives of human cooperation, life history as well as the role of hunter-gatherers studies in human behavioural ecology. 


\section{Chapter Two \\ Risk and the Evolution of Cooperative Childcare}

\subsection{Cooperative breeding: its definitions and questions}

Cooperative breeding occurs in $9 \%$ of bird species (Riehl 2013), compared to only 3\% of mammals (Hrdy 2009). Yet, within the mammalian class cooperative breeding is widely distributed and has independently evolved in rodents, Herpestidae (monogooses), Canidae (dogs) and primates (Lukas \& Clutton-Brock 2012). While cooperative breeding is absent from all non-human great apes (Hrdy 2009) it occurs extensively among humans (Ivey 2000; Hewlett 1991; Konner 2005) and the benefits of kin presence has been recorded in temporal and spatial cross-sections of the world's populations (Sear \& Mace 2008).

Given cooperative breeding's wide phylogenetic dispersal, one clear direction of research is exploring why it evolved repeatedly and independently. As a 'cooperative' act, cooperative breeding denotes the evolution of a childcare behaviour because it is beneficial to other individuals, not simply the individual helping (West et al. 2011; West et al. 2007). Cooperative acts often appear costly as the energy invested into another individual's offspring cannot be re-invested elsewhere (Tardif 1997). Thus, it is puzzling why individuals behave 'altruistically'. Strictly speaking, altruism is any action which results in an ultimate fitness cost to the altruist but benefits others, thus is the true 'selfless' act (Foster et al. 2006). Such altruism appears contradictory to the selfish gene paradigm in which each gene strives to increase its frequency in future generations (Dawkins 1989), thus evolutionary biologists and anthropologists attempt to reconcile apparent altruism with evolutionary theory. This has resulted in confusion over the term altruism; research often describes behaviour as altruistic if it is costly in the short-term and ignores possible benefits, such as indirect (kin altruism) or direct but delayed (reciprocal altruism) benefits (West et al. 2007). As stated by West and colleagues (2007), this results in altruism becoming synonymous with cooperation. While this appears like simple semantics, they 
require different ultimate explanations; cooperation entails the quantification of fitness returns. Here, altruism will be used only in its strictest form as I seek to explore what benefits individuals receive from assisting other individual's offspring.

As Solomon \& French (1997: 2) put it, there is a "dizzying array of labels in the literature" for cooperative breeding which are often used interchangeably, lacking any clear definition. In the strictest sense cooperative breeding implies a monopolisation of breeding by a dominant female who is aided by non-breeding allomothers. This is also referred to as singular (or pair-nesting in birds (Riehl 2013)). This system is found in callitrichids (Bales et al. 2000) and meerkats (Clutton-Brock et al. 2001). Communal breeding (or plural breeding and cooperative polygamy) refers to systems in which multiple females reproduce, often pooling their resources and young (Lukas \& Clutton-Brock 2012). Communal breeding does not preclude the presence of non-breeding helpers, only the absence of a breeding monopoly (Riehl 2013). Communal breeding is rarer in mammals, found in some nocturnal strepsirrhines, black-and-white ruffed lemurs (Varecia variegata) and humans in the order primate (Baden et al. 2013). In the human literature this distinction is unclear. The term cooperative breeding is used loosely to refer to when someone other than the mother assists in the rearing of offspring (Russell 2004; Jennions \& Macdonald 1994), following the general definition of cooperation. Others argue that humans demonstrate elements of both communal and cooperative breeding as pre-reproductive and postreproductive life-stages can be considered to be 'reproductive suppression' (Lukas \& Clutton-Brock 2012; Hawkes \& O'Connell 1998). Therefore, to a degree there is an unwillingness to place humans into either category, hence the uncritical use of terminology.

However, the difference between these two systems is profound, as sacrificing reproduction entails a large cost. As reproduction is not delayed in communal breeders, individuals can gain direct and immediate benefits from breeding in a group (Lewis \& Pusey 1997). The most important feature of communal systems is plurality in breeding; even if some helpers are non-reproductive it is unclear if such a trait arose specifically to facilitate cooperative breeding (Riehl 2013). Furthermore, cooperative breeding systems are defined by strict dominance-subordinate relationships where dominant mothers firmly and often violently suppress subordinates reproduction (Hrdy 2009). Yet immediate-return foraging groups are broadly egalitarian, as is common in other communal breeding species (Baden et al. 2013). Hence, the motivations and structures surrounding these two systems are expected to differ significantly. Consequentially, here I define humans as communal 
breeders, with an awareness that this may be facilitated by extensive juvenile and postreproductive periods. This bears little weight on the review of existing literature on human allocare, given that few researchers had these definitions in mind. However, the distinction increases in importance for inter-species comparisons and theoretical perspectives; the evolution of cooperative breeding has been mainly explained with indirect fitness benefits in constrained environments (Emlen 1982), however, if multiple individuals can breed simultaneously one ultimate explanation of communal breeding could lie in reciprocity. Thus, throughout this thesis I will refer to humans as communal or plural breeders, using cooperative breeding to refer to singular breeders and as a catch-all phrase when discussing the 'cooperative breeding literature' on all species.

The anthropological approach to communal breeding has focused on topics such as the evolution of pair-bonding, male provisioning and the post-menopausal lifespan (Bogin 1997). An assumption in early research was that the evolution of pair bonding guaranteed males provisioning of women and children, who would otherwise be unable to procure enough food for survival (Darwin 1871; Lovejoy 1981; Kaplan et al. 2003). However, Hawkes et al. (1997) argued that post-reproductive Hadza females devoted significant amounts of energy to grandchildren. Energy from gathered foods can easily overtake that of hunted foods due to high failure rates of hunts (Hawkes 2000; Blurton Jones et al. 2000). Thus, Hawkes and colleagues have argued that humans' post-menopausal lifespan evolved due to the fitness benefits conferred on grandchildren when grandmothers were present (Hawkes \& Coxworth 2013). Problematically, however, data from South American foraging groups reveals that grandmothers are of little importance as males supply $97 \%$ of calories to their young (Kaplan et al. 2003). Even through females commonly contribute a higher proportion of items (Kelly 2013), the calorific value of hunted goods is often greater (Gurven \& Kaplan 2006; Hill 1993).

Little consensus has been reached on this topic and the debate remains active today, however Marlowe (2003) cautions about swinging between extremes of the spectrum. Both fathers and grandmothers have important roles in different contexts; among the Hadza adult females brought more calories back to camp when they did not have infants, if they did, males' production was higher. Due to a particular focus on these two extremes, anthropological research has primarily sought to understand how care from one particular kin type could increase child quality or survival (Sear et al. 2000; Meehan et al. 2014; Sear \& Mace 2008; Sear et al. 2002; Hawkes et al. 1997), maternal fertility (Sear et al. 2003; Crognier 
et al. 2001; Bereczkei 1998; Kramer et al. 2009; Tymicki 2004) or how allocarers reduce the maternal energetic burden (Meehan et al. 2013a). As a result the role of unrelated individuals and why they would provide care has been under-examined (Apicella et al. 2012). This is not, however, the case within the large food sharing literature in which reciprocity has been demonstrated to play an important role in who shares with whom (Gurven, 2006, 2004a; Jaeggi and Gurven, 2013; Kasper and Borgerhoff Mulder, 2015). Food provisioning is a major form of cooperation in humans (Hill \& Hurtado 2009), as is common in other communally breeding species (Brown 2014; Caraco \& Brown 1986). I take extensively from this literature to inform my analysis of childcare and I will now review the major theories explaining why individuals cooperate.

\subsection{Why breed communally? Theoretical perspectives}

\subsubsection{The primacy of kin (selection)}

Cooperative breeding theory was developed in birds with singular breeding systems. In avian species allomothers are usually previous years clutches which remained philopatric due to resource competition hindering independent breeding (Emlen 1995). As a result, much cooperative breeding theory and research has centred on species which primarily nested with closely related individuals (Solomon \& Hayes 2009; Hatchwell 2007). Therefore, indirect fitness stemming from kin selection (Hamilton 1964) was considered a sufficient explanation for allocare. Hamilton's theory states a costly strategy may be selected for, if it benefits individuals carrying the same genes (measured by coefficient of relatedness, $r$ ), depending on the degree of the costs to the helper $(C)$ weighted by expected benefits $(B)$, or otherwise put: $r B>C$ (Hamilton 1964). Thus, an individual would be expected to help a relative if the benefit to their indirect fitness outweighs the costs of the initial action, a benefit determined by the number of shared genes. Extensive evidence has been produced supporting the role of relatedness in a number of cooperative species including eusocial insects (Foster et al. 2006), birds (Koenig \& Dickinson 2004) and mammals (Solomon \& French 1997), including small and large scale human societies (Nolin 2010; Koster \& Leckie 2014; Alvard 2009; Nowak 2006).

Whilst this is a pervasive explanation for cooperative behaviour, the explanatory power of this theory has been overstated (Clutton-Brock 2002). Firstly, many cooperative breeding groups are not as closely related as first thought. For instance, callitrichids originally 
assumed to be monogamous, now appear to live in polyandrous and polygynous groups (Tardif 1997). This fact alongside the frequent inter-group migration means that relatedness is significantly lower than originally assumed and males often provide care for non-related offspring (Clutton-Brock 2002)). Furthermore, approximately 44\% of bird species nest in groups containing unrelated helpers who are engaged in cooperative breeding (Riehl 2013). The trend of living in family groups is much more common in singular rather than plural breeders. As such, kin selection may have less explanatory importance in communal breeding species. For instance, hunter-gatherers have been demonstrated to reside in camps which contain a significant number of non-kin (Dyble et al., 2015; Hill et al., 2011). Given that on average individuals reside with only 25\% consanguineal kin (Dyble et al. 2015), in an average camp of 30 individuals, only seven individuals would be consanguineously related to ego, significantly limiting within kin allocare options. For this reason alone it is essential to examine the role of non-kin allocare.

Even if a group was comprised of closely related individuals, individuals may be cooperating for different reasons. Individuals may assist one another due to shared space rather than shared genes (Clutton-Brock 2002). For instance, neighbours cooperate due to lower transaction costs, greater knowledge of the cost-benefit ratio of any given action and an increased likely of future interactions (Nolin 2010). This fact of co-residence may lead to an overstatement of the role of kin selection if one simply examines who provides care, rather than why. This is what has been termed as an association index in the anthropological literature (Koster \& Leckie 2014), a measure of the 'multi-faceted interdependences that characterise [households] relationships' (Koster \& Leckie 2014: 101) as households cooperate in multiple arenas, often entailing physical proximity. The association index is a measure of this proximity, as a proxy for the longer-term dependent relationships. Thus, as kin in small-scale societies characterise these relationships, the role of association is overlooked while the importance of kinship is overstated. For instance, Alvard (2009) explored the composition of Lamalera (whale hunters from Indonesia) cooperative hunting crews. While finding they were significantly more related than expected by chance, the composition of hunting crews was better predicted by lineage membership than simple relatedness. Without the inclusion of lineage membership in the model, the importance of relatedness was significantly overestimated. While, a number of studies have indicated the importance of the association index in food sharing (Nolin 2010; Gurven et al. 2000; Gurven 2004a; Allen-Arave et al. 2008; Koster \& Leckie 2014), it is 
important to note that co-residence is not random, but is frequently structured around close kin (Gurven \& Hill 2010; Nolin 2010). Consequentially, even if individuals cooperate due to shared space, if they preferentially share space with kin then kin selection continues to occur. As a result, it is difficult to separate the influence of these two forces. Nonetheless, these complexities reveal that kin selection is not the 'magic bullet' of cooperation; it is part of a complex web of other explanatory variables that structure human interaction.

Hence, to investigate if indirect fitness explanations are valid, one must examine how the degree allomothering varies according to relatedness (Griffin and West 2003). More related individuals are expected to provide more care. However, single species studies paint an inconsistent picture on this front; when related and unrelated infants were presented to captive pygmy marmosets (Cebuella pygmaea) there was no difference in caregiving response (Wamboldt et al. 1988). Similarly, Eberle and Kappeler (2006) find that while grey mouse lemurs (Microcebus murinus) have the ability to discriminate kin, they do not offer discriminatory care. However, these distinctions were made by male meerkats (Suricata suricatta) when feeding related young (Clutton-Brock et al. 2004) and male golden lion tamarins (Leontopithecus rosalia) who both preferentially carried more related offspring (Baker 1991). Griffin and West (2003) examined this question across 18 cooperatively breeding vertebrates (15 birds and three mammals), finding that relatedness significantly predicted allomothering and accounted for $10 \%$ of its variance. However, this effect was dependent on the fitness cost (i.e. less resources to reproduce) and fitness benefits (i.e. increased survivorship of related broods) of helping; when the benefits were low the motivation was removed and care reduced (Griffin and West 2003). This is a vital and frequently overlooked point: any test of kin selection must examine how cooperation varies according to the cost and benefit. For instance, Clutton-Brock et al. (2001a, 2000) found that while allocaring meerkats (Suricata suricatta) were related to offspring, the allocarers physical condition (indicating the cost of the care) predicted the amount of care provided significantly better than variance in relatedness (Griffin \& West 2002). As a result, kin selection predicts that related individuals should preferentially assist those in greater need of their help, particularly if costs are low and benefits are high (Allen-Arave et al. 2008).

Few empirical studies have explored this relationship between costs, benefits and relatedness. For instance, Kasper and Borgerhoff Mulder (2015) found that the best predictor of cooperation was having needy relatives to help. As need is high, this implies 
that the fitness benefits are also high. Thus, following predictions of kin selection, aid was given to relatives in need (i.e. Hamilton's B) dependent on degree of relatedness. This finding is reflected in Hooper et al. (2015) examination of food sharing according to cost and need (number of producers versus dependents in the receivers and givers household). Hooper and colleagues (2015) found that among the Tsimane, individuals with high production transferred more resources to households with high consumption, low production and high relatedness. Thus, this matches the clear predictions set out by kin selection.

Few studies of direct childcare rarely test kin selection in such a manner. For instance, as evidenced below, the majority of studies limit their perspective to comparing different kin types (i.e. fathers versus grandmothers) rather than comparing non-kin versus kin or a continuous variance in the coefficient of relatedness (Sear \& Mace 2008; Snopkowski \& Sear 2013; Sear \& Coall 2011a; Apicella \& Crittenden 2013). The food sharing literature is based on advanced multivariate analysis controlling for association index, reciprocity, costbenefit ratios, dyadic relationships, clustering and non-independence. In contrast, childcare analysis is often simplified into linear regressions examining whether care increases with relatedness (Crittenden \& Marlowe 2008; Ivey 2000).

\subsubsection{Paternity certainty}

When paternity is unknown significant differences in magnitude of care are predicted between matrilineal and patrilineal kin. A mother is always sure of her $50 \%$ contribution to her offspring, however due to internal gestation, a father can never have the same level of confidence of his 50\% share. This insecurity can affect a male's desire to invest in a child as he may be misdirecting his care (Kleiman \& Malcolm 1981). This same uncertainty influences matrilineal and patrilineal kin. Grandparents should be $25 \%$ related to their grandchild, however their confidence will change according to their lineage and sex: a maternal grandmother is $100 \%$ sure of her $25 \%$ stake in a grandchild, followed by maternal grandfather who has to consider the uncertainty in his relationship with his daughter and the paternal grandmother who has to consider the likelihood of her son's cuckoldry. Finally, the paternal grandfather is the most uncertain as he must consider the likelihood of his son's cuckoldry as well as his own. Thus, if investment is based on degrees of kinship, we should find this pattern in the amount of allocare offered, assuming paternity certainty is of a significant level. Consequently, the benefit received by the offspring and/or mother 
should be proportional to the degree of relatedness and paternity uncertainty (Danielsbacka et al. 2011).

\subsubsection{Reciprocal altruism (cooperation $\left.{ }^{4}\right)$}

Among the Agta "the return may be made at a later date but it will be expected" (Peterson 1978: 40)

Trivers (1971) developed the theory of reciprocal altruism to explain how a costly behaviour can be selected for when the recipient is distantly or completely unrelated to the giver. Here, cooperation can evolve if the cost of helping in the present is outweighed by the benefits the giver receives at some point in the future, lessened by the probability that this benefit may or may not occur. As a result, due to long-term interactions cooperation can evolve without relatedness. This was formalised into the Tit-For-Tat (TFT) model of cooperation by Axelrod and Hamilton (1981). In its strictest form TFT requires significant score-keeping and extremely discriminative players: as a result unlikely to occur in nature (Gurven 2004b; Gurven 2006). In reality, reciprocal exchanges are frequently unequal because the marginal value of a resource varies between the giver and receiver. For instance, the marginal value of an additional kilo of meat produced by the skilled hunter is significantly lower than for an inefficient juvenile (Hooper et al. 2015), thus it costs little to give and benefits a lot. However, in future this situation will reverse as the juvenile develops and the skilled hunter has a run of bad luck. Even if the skilled hunter never receives the exact same number of kilos in return, as long as the transaction meant both parties benefited more than had they not participated in the exchange, reciprocal altruism can be favoured (Gurven 2006). This reveals that the cost benefit ratio is as important in reciprocal altruism as it is in kin selection, thus cannot be used to distinguish between the models (Franzen 2004). What does firmly separates the models, however, is contingency, defined as the relationship between what A gives B with what B gives A (Gurven 2004b). Contingency does not predict kin selection as the benefits are indirect and do not require future reciprocity.

Reciprocal altruism theory is clear: help an individual if the cost of helping now is lower than the expected future returns. However, demonstrating contingent cooperation 'on the ground' is more difficult, particularly if it is not perfectly balanced (Allen-Arave et al. 2008; Gurven 2004a). Two major difficulties in quantifying reciprocal altruism include the appropriate time frame for examining contingency and which 'currency' this contingency is

\footnotetext{
${ }^{4}$ If the altruist receives long-term benefits for their costly activity this act is no long strictly altruistic but cooperative (Foster et al. 2006).
} 
in. Jaeggi and Gurven (2013) argue that helping is best predicted by long-term contingency, however, as many studies are conducted over several weeks or months they do not capture exchange which occurs over a generation or lifetime (Gurven 2004b). Thus, a lack of an association may be due to study duration. Furthermore, reciprocity may be present but in multiple currencies, leaving studies focusing only on food sharing or childcare unable to capture the explanatory power of reciprocity (Allen-Arave et al. 2008). Here the association index is of importance, given that long-term, more generalised contingency is more likely in individuals who frequently associate (Gurven 2006), however this is rarely explicitly examined. Finally, individuals may suffer an significantly unbalanced relationship if it functions to signal their generosity or 'sharing intent', permitting enlarged networks to fallback on in times of need (Allen-Arave et al. 2008; Gurven et al. 2000; Wiessner 2002; Patton 2005). For instance, Nolin, (2012) found that among the Lamelera, men holding leadership roles gave more than expected based on their household productivity while among the Ache sharing was predicted by relative generosity (i.e. total given divided by total produced (Gurven et al. 2000)). Quantifying all these dimensions in actual populations is difficult and never (understandably) conducted in its totality.

While these limitations of the examination of reciprocal altruism must be kept in mind, researchers have nonetheless found a significant role for reciprocal altruism in many populations including the Dolgan and Nganasan (Ziker \& Schnegg 2005), Ache and Hiwi (Gurven 2004a; Gurven et al. 2000; Allen-Arave et al. 2008), Lamelera (Nolin 2010) and among the Mpimbwe (Kasper and Borgerhoff Mulder 2015). In fact, in a recent metaanalysis of food sharing in human and non-human primates Jaeggi and Gurven (2013b) find that contingency accounted for $20 \%$ of the variance in food sharing among 32 primate species, more (but not significantly so) than relatedness. However, it is an error to suggest reciprocal altruism and kin selection are mutually exclusive; many of their predictions overlap and both processes can be acting simultaneously (Jaeggi and Gurven, 2013; Nolin, 2010). For instance, among the Ache Allen-Arave et al. (2008) find that nepotistic reciprocal altruism best describes food sharing; individuals are more likely to participate in long-term exchange partnerships with close kin who they trusted and have a high likelihood of continued interaction with. Furthermore, kin were not significantly more likely to give food if they had not received food from the household previously, thus it appeared kin selection had little role in maintaining nepotistic food transfers. Similar results have been found in the Lamalera (Nolin 2010) and Dolgan (however, kinship still independently predicted sharing here (Ziker \& Schnegg 2005)) indicating that kinship may 
be important in partner selection, but contingency is what maintains it (Nolin 2015). This is perhaps not surprising given that reciprocity will overtake kin selection whenever the probability of future interaction is higher than the dyadic coefficient of relatedness, a common situation in small-scale societies (Axelrod \& Hamilton 1981; Gurven et al. 2001; Allen-Arave et al. 2008).

\subsubsection{Loss and unpredictability}

Regardless of whether ego is closely related or not, what motivates individuals to cooperate and take a risk on whether their cooperation will be returned (Hames 2004)? Given that much of the cooperative literature has stemmed from food sharing among huntergatherers, a primary explanation of function arose from the inherent daily variance in food returns in a stochastic environment (Winterhalder 1986). Strictly speaking, risk refers to the probability of loss given the distribution of outcomes, while variance refers to stochastic or unpredictable outcomes (Winterhalder 1986; Winterhalder et al. 1999). Food sharing is seen as a form of risk-pooling to reduce the variance across individuals; individuals share today since they may not be successful hunting for a week, thus will later be dependent on their sharing partners, be that kin or unrelated individuals (Gurven 2004b; Gurven 2006; Nolin 2010).

Hunting success rates are surprisingly low in many foraging groups: only $23 \%$ and $27 \%$ of hunts were successful among the savannah !Kung and Hadza, respectively (Lee 1979; Hawkes 1991). These figures are increased among South Asian tropical forest foragers: $59 \%$ and $63 \%$ of hunts were success among the Batek and Palanan Agta, respectively (Endicott \& Endicott 2008; Griffin \& Griffin 2000). However, from our own data it is apparent that fishing success rate $(89 \%)$ is significantly higher than hunting $(65 \%$, Dyble n.d.). Winterhalder (1996) established that given small degrees of asynchrony (i.e. success rates) between foragers, sharing as a form of risk pooling mitigated the likelihood of shortfalls even among groups of only six to eight foragers. As a result, sharing has been demonstrated to lead to significant nutritional improvements among the Ache (Kaplan \& Hill 1985) and significantly lowers mortality (Jaeggi and Gurven, 2013). Unsurprising then, egalitarianism and sharing norms are often strongly enforced in foraging societies, and 'levelling' tactics commonly used to keep the best hunters arrogance in check (Wiessner 1996; Lee 1979; Woodburn 1982). 
Individual variance is also influenced by sickness and disability; among South American forager-horticulturalists, Ache adults were found to be sick on $6.5 \%$ of all days, the Yora $8 \%$ and $90 \%$ of Shiwiar were reported to have suffered a disability for 14 days or longer (Gurven et al. 2000; Sugiyama 2004; Sugiyama \& Chacon 2000). As a result, Sugiyama (2004) reports that $60 \%$ of the Shiwiar in his sample would have been unlikely to survive in the absence of food sharing. Thus, individuals survival also depends on medium term 'health insurance', by perhaps signalling generosity in periods of good health to ensure provisioning is received during periods of ill health when returns cannot be immediate (Sugiyama \& Chacon 2000; Gurven et al. 2000).

These theories have been specifically developed with economic transfers, such as food sharing, in mind. Thus, their applicability to childcare appears, at a glance, obscure. However, given individuals energy budgets must encompass food production, household tasks and childcare there is plenty of room for stochasticity in childcare. For instance, if a husband is unsuccessful hunting then the mother's forging efforts require increasing, significantly reducing the amount of time she is available for direct childcare. Or, if a child falls ill they require significant increases in high intensity care, which a mother can provide but then her foraging efforts and direct care to other offspring must decrease (Sugiyama \& Chacon 2000). Simply put, a deficit in one area due to unpredictability results in a deficit of childcare which can be met with allocare. Within the human communal breeding literature reciprocity is rarely (if ever) examined, however, environmental uncertainty has been theoretical linked to the evolution of cooperative breeding in birds and mammals.

Emlen (1982) modelled the evolution of allocare by unrelated individuals, finding that fitness payoffs were higher for helping if the environment was marginal, unpredictable and restrictive for reproduction. This model has been supported by Rubenstein and Lovette (2007) who conducted a phylogenetically controlled comparative analysis of 45 species of African starlings, finding that cooperative breeding was more prevalent in areas of high temporal variability. Furthermore, Jetz and Rubenstein (2011) found, among 9,310 nonmarine avian species, that temperature and rainfall variability were strong predictors of cooperative breeding. Cooperative breeding has also been associated with unpredictable habitats in naked mole rats (Heterocephalus glaber, (Faulkes et al. 1997)) and black-and-white ruffed lemurs (Varecia variegata, (Baden et al., (2013)), while environmental uncertainty has been forwarded as an explanation for allomaternal care in Malagasy strepsirrhines in general (Tecot et al. 2012). 
Human mothers do not only face short-term unpredictability but also face what Hill and Hurtado (2009) label as 'predictable life-history shortfalls'. Given humans' production of multiple highly dependent offspring, there are specific times during a mothers reproductive life course that she cannot provide all the specific care an infant, toddler or dependent child require (Kramer 2010). However, as parents age previously dependent children function as allocarers (Kramer 2011), until they cease reproduction and ultimately have more household provisioners than dependents. As a result, there are predictable times in a household's life course in which it will be in need of care and others when it can provide care. While this is not related to environmental stochasticity it is a risk which requires buffering: either by siblings once available or if not then distant or non-kin. Huntergatherer mothers face a particularly difficult predicament in which they must deal with a baseline childcare deficit, and then meet any further care deficits arising from illness, disability and foraging failure. Perhaps then, the phrase "it takes a village to raise a child" is not so surprising (Apicella \& Crittenden 2013).

In summary, there seems a significant amount of evidence that humans require assistance in childcare, which is likely structured by kin selection and reciprocal altruism. Yet, the role of reciprocal altruism has been comparatively ignored in the cooperative breeding literature outside of food sharing. This is a product of a poor conception of what is reciprocal altruism and how it can maintain cooperation. For instance, Clutton-Brock (2009) argued that delayed returns from reciprocity are not likely to be a driving force behind allomothering, due to a problem of free-riders over a long time period. This point holds true in singular breeding species in which allocare entails a significant fitness cost (i.e. the cessation of the allomothers own reproduction) and unpredictability of when/if the current allomothers will become the dominant reproducing female. However, plural breeding species hold no such cost, nor necessitate extended periods between reciprocity. Multiple females (and their non-dependent offspring) can aid each other in breeding, creating a crèche style of caretaking. Moreover, even dependent children produce a significant amount of resources which are transferred to older generations, thus as Kramer (2010) points out, resource transfers are often bidirectional. As pointed out by Ivey (2000) and Kramer (2010) the costs of allocare for juveniles may be relatively low, given their lack of economic productivity and fact they may often be provided by adults in terms of food, shelter and other resources. Young females may also gain direct benefits from allocaring; Lancaster (1971) posited that they learn and develop mothering skills, especially given the higher infant mortality rate experienced by nulliparous mothers (Baker \& Wood 1992). 


\subsection{Anthropological Literature}

There is a large array of anthropological literature on which type of kin provides the most care and what benefit this bestows on both mothers and their offspring. Research spans from small-scale societies in which data collection consists of intensive observation or large-scale correlational studies (in modern and historical populations) examining the benefits associated by kin presence or absence. Rarely are these approaches combined (for exception, see Gibson and Mace (2005)). It is important to note that much of the observational research is based on childcare received by a small number $(10-30$, on average) of infants (2.5 years or under); little is known about who cares for toddlers and dependent children and data may be easily skewed by small sample sizes. The larger scale studies are based on much bigger sample sizes, however run into a problem of a lack of data depth. Often analyses are conducted on whether or not a grandparent or father was 'present' in a village or residing nearby and the effect this has on child and maternal outcomes. This makes causation more difficult to trace. Nonetheless, a combination of both types of studies in the following review produces a meaningful view of communal breeding in humans.

\subsubsection{The essentiality of mothers}

Mothers are the main caretaker of infants: in Kramer's (2010) review on the topic mothers were found to, on average, provide $48.8 \%$ of direct care (nursing, feeding, carrying, holding and grooming) to younger children (mainly infants, but ranges to 0-4 years; Table 2.1) in small-scale societies. Overall, when examining the different subsistence types in Table 2.1 there is remarkable consistency of maternal care regardless of whether the population are hunter-gatherers (Aka, Efe, Agta, Alywara and Hadza) or horticulturalists (Ye'Kwana, Maya, rural Trinidad and Toba). Thus, mothers are consistently important caretakers of infants. Similarly, Sear and Mace's (2008) meta-analysis of 45 studies on 'who keeps children alive' (ranging from historical Japan to small scale pastoral villages in the Gambia) found that maternal death has a highly detrimental effect on infant survival. However, this effect is strongly dependent on child age; after weaning the effect on child mortality almost disappears. Thus, while few observational studies examine who provides care to children post-weaning, it appears likely that the maternal effect lessens as additional allocarers take over (Sear \& Mace 2008). Crittenden and Marlowe (2008) explore which camp mates carried Hadza children under the age of four $(n=56)$ and found that age negatively 
predicts the amount of time a child is held, while Meehan et al. (2014) found that among the Aka maternal care declines rapidly from late infancy into the second and third years of life.

Table 2.1: Cross-cultural proportions of direct childcare from different caretakers

\begin{tabular}{|c|c|c|c|c|c|c|c|c|}
\hline Group & Moth & Fath & Bro & Sis & Sib & $\overline{G M}$ & Other & Ref \\
\hline Ye'kwana $^{a}$ & $49.0 \%$ & $2.7 \%$ & $1.9 \%$ & $16.7 \%$ & - & $11.2 \%$ & $20.6 \%$ & Hames (1988) \\
\hline $\mathrm{Aka}^{\mathrm{b}}$ & $42.7 \%$ & $15.8 \%$ & - & - & - & - & $13.2 \%$ & Hewlett (1988)* \\
\hline Efe & $50 \%$ & $6.0 \%$ & $14.0 \%$ & $13.0 \%$ & - & $9.0 \%$ & $9.0 \%$ & Ivey (ND)* \\
\hline Agta & $51.7 \%$ & $4.4 \%$ & $1.1 \%$ & $10.2 \%$ & - & $7.6 \%$ & - & Goodman et al. (1985)* \\
\hline Maya & $46.1 \%$ & $1.6 \%$ & $4.6 \%$ & $31.6 \%$ & - & $1.2 \%$ & $\begin{array}{c}\text { OTH: } \\
11.2 \% \\
\text { NON: } \\
2.8 \%\end{array}$ & Kramer (2005) \\
\hline Alyawarac $^{c}$ & $53 \%$ & $<1 \%$ & - & - & $31.0 \%$ & - & $16.0 \%$ & Denham (1974)* \\
\hline Trinidad $^{\mathrm{d}}$ & $44.2 \%$ & $10.3 \%$ & - & - & $16.3 \%$ & - & $29.3 \%$ & Flinn (1992) \\
\hline Mardu $^{e}$ & $32.3 \%$ & $2.7 \%$ & - & - & $5.0 \%$ & $14.3 \%$ & $29.8 \%$ & Scelza (2009) \\
\hline Toba & $50 \%$ & - & $4.0 \%$ & $33.0 \%$ & - & $13.0 \%$ & - & Valeggia (2009) \\
\hline Hadza $^{c f}$ & $68.7 \%$ & $7.1 \%$ & - & $1.2 \%$ & - & $\begin{array}{c}\text { MGM: } \\
3.7 \% \text {, } \\
\text { PGM: } \\
1.2 \%\end{array}$ & $\begin{array}{c}\text { OTH: } \\
3.6 \% \\
\text { NON: } \\
12.4\end{array}$ & $\begin{array}{c}\text { Crittenden and Marlowe, } \\
\text { (2008)* }\end{array}$ \\
\hline Averages & $48.8 \%$ & $6.3 \%$ & $5.1 \%$ & $17.6 \%$ & $17.4 \%$ & $7.7 \%$ & $14.8 \%$ & \\
\hline
\end{tabular}

Notes: Direct childcare includes nursing, feeding, carrying, holding and grooming. a includes children 0-40 months. b values for mothers and fathers include children aged 1-18 month(s). Other may include siblings and grandmothers. ${ }^{\mathrm{c}}$ values reported for carrying children only. $\mathrm{d}$ includes children $0-4$ years old. ${ }^{e}$ includes children $0-3$ years old. ${ }^{\mathrm{f}}$ includes children $0-4$ years, includes carrying children only, sister is older sisters only. GM stands for grandmother. References followed by $\mathrm{a}^{*}$ are those conducted with immediate-return foragers most similar to the Agta. The other groups (Ye'Kwana, Maya, rural Trinidad and Toba) are small-scale horticulturalists and agriculturalists. The Mardu aboriginals were once mobile hunter-gatherers but have since experienced extensive settlement and acculturation. Adapted from Kramer (2010: 421).

\subsubsection{Is father care obligate?}

While previous work has focused on paternal support as key for maternal and child survival (Kaplan et al. 2000b), sole dependence on male childcare appears a risky strategy (Hrdy 2008) and in only in 47\% of studies $(n=15)$ did fathers have an effect on child survival (Sear \& Mace 2008). Nonetheless, while overall fathers provide a fraction of the care of mothers (averaging at 6.3\%), among the Aka, fathers interact significantly more (15.8\% (Hewlett 1988)). Fathers may offer lower investment care (such as proximity, 
watching or touching) rather than high investment care (carrying, grooming or feeding), thus are relatively ignored in child observational studies which focus on high investment care (frequently carrying). Furthermore, a father's importance may increase in later life as he plays a greater role in arranging marriages, teaching or playing with older children (Scelza 2009; Sear \& Mace 2008; Sear 2011). Consequentially, the consistent sampling of infants or children under the age of four years may systematically underestimate the role of fathers. Furthermore, paternal care may be dependent on the presence of other caregivers. Meehan (2005) recorded how, when an Aka family resided matrilocally, infants received 2.5 times more physical contact from allomothers. However, in a patrilocal camp where the number of allomothers was significantly reduced males increased their interactions by $20 \%$. Therefore, a lack or presence of allomothers and their effect, may depend on who else is investing in a child. Overall while paternal care appears variable across populations this does not demonstrate any consistent pattern according to subsistence.

\subsubsection{Helpful Grandmothers and child survival}

Grandmothers, as originally argued by Hawkes et al. (1997), are expected to be major allocarers of children. As they are post-reproductive their costs are low and inclusive fitness benefits high (i.e. $r=0.25$, assuming no paternity uncertainty). Thus, their care and presence are expected to have positive effects on child survival and/or maternal fertility. Sear and Mace (2008) found maternal grandmothers have the most consistent positive effect, correlating with child survival in $69 \%$ of studies $(n=46)$. Paternal grandmothers were positively associated with child survival in $53 \%$ of statistically valid studies $(n=17)$. Furthermore, Sear et al., (2002, 2000) found that maternal grandmother presence increased child height, weight and survival from the age of 18 months in rural Gambia. This is likely related to the importance of assistance during weaning, a period of significantly higher mortality (Cassidy 1980). In rural Ethiopia it appeared that maternal grandmothers had beneficial effects on child survival and height due to increased engagement in heavy domestic tasks (Gibson \& Mace 2005). Similarly, in Meehan's (2013) study Aka grandmothers were found to significantly decrease maternal energy expenditure by 150 kcal.

Observationally grandmothers appear to be of importance, often providing more care than fathers (Hames 1988; Ivey 2000; Goodman et al. 1985; Scelza 2009). Furthermore, grandmothers appear to be more likely to provide more demanding childcare tasks (Scelza 
2009). However, several studies show that grandparents have little involvement with their grandchildren (Crittenden \& Marlowe 2008; Kramer 2005), particularly among more immediate-return hunter-gatherers (Table 2.1). For instance, among the Hadza, Efe and Agta the average amount of direct care is $5.4 \%$, compared to horticultural populations with an average of $9.9 \%$. However, many hunter-gatherer studies are limited by their small sample sizes. Due to the labour intensive nature of observational studies, sample frequently contain around 10 - 15 infants. Only a few studies include more than 20 infants (Crittenden \& Marlowe 2008; Meehan et al. 2013a). As a result it is difficult to examine variability within the sample. For instance, in a large-scale Indonesian study $(n=16,250)$, grandmothers assistance was mediated by the needs of her dependents; grandmothers provided more financial help to poorer couples or conducted more household tasks for working mothers (Snopkowski \& Sear 2015). This suggests that grandmaternal care may be contextual according to need, prevailing conditions and what other types are carers are available. For instance, among 127 Aka children aged 0 to 10 years Meehan et al. (2014) found that grandmothers had a positive influence on child developmental and nutritional outcomes (weight-for-age, height-for-age and weight-for-height) during the $9-36$ month period. However, children in matrilocal camps did not suffer when maternal grandmothers were not present, while children in patrilocal camps did. Thus, it appears that the importance of grandmothers may lie with access to other allocarers, potentially distorting overall results.

The fact that sample sizes are small is indicative of another practical limitation on the scale of grandmothers' assistance. For instance, in Ivey's (2000) study the sample size consisted of 20 infants with four grandmothers, i.e. a significant number of infants did not have a grandmother around to provide allocare and those that did would have to share. Considering the likelihood of grandmothers death and co-residence, Hill and Hurtado (2009) established that only around 10\% of middle aged (defined as late 30's) Ache women co-resided with their mothers. Thus, similar to fathers it seems a risky strategy to rely on the presence of a grandmother. Furthermore, even once a grandmother is present she is likely to also invest in other offspring and grandchildren, particularly if some are in greater need than others. This indicates the possible negative side of kin; bigger kin networks can lead to competition among close relatives for resources in constrained environments (Borgerhoff Mulder 2007). 


\subsubsection{Not so helpful grandmothers and grandfathers?}

Sear (2008) found in a matrilineal Mali society that maternal grandmothers and aunts were associated with an increase in child mortality. This is the result of excessive competition for resources at the household level, as older individuals drain limited household resources (Strassmann \& Garrard 2011; Strassmann 2011). Furthermore, given earlier ages at first birth, grandmothers are often still reproducing when their first child reproduces, suggesting that they may wish to devote little energy outside of their own direct reproductive interests (Tymicki 2004). Furthermore, in a historical German population it appeared that while maternal grandmothers increased the odds of 5 -year survival for children by $23 \%$, paternal grandmothers decreased it by $19 \%$ (Beise \& Voland 2002). The relative impact of both matrilineal and patrilineal kin does appear dependent on context. Among the Pimbwe, a horticultural population from Tanzania, women with larger kin networks suffered if they had greater wealth due to excessive demands on their resources. As a result a child's nutritional status was dependent on wealth and it's interaction with the number of kin, revealing significant breeding competition rather than cooperation (Hadley 2004). Similar results have also be found among the Kipsigi, in which paternal kin are of greater importance in richer patrilineages while maternal kin buffer offspring from the negative influences of poorer patrilineages (Borgerhoff Mulder 2007).

The negative influence of paternal grandparents on child outcomes may equally be the product of increased investment in fertility rather than child quality. For instance, in the same 18th century German population as above, a household containing a paternal grandmother was associated with higher overall fertility alongside reduced child survival as the father's kin appeared to increase fertility, not survival (Beise 2005). Sear and Coall (2011b) review of kin effects on fertility established that paternal grandmothers and grandfathers were significantly more likely to affect a daughter-in-law's fertility rate, an effect replicated in rural Gambia (Sear et al. 2003) and Thailand (Snopkowski \& Sear 2013). The proximate mechanisms are unclear, but it may be related to kin influence on the age at first reproduction (Snopkowski \& Sear 2013) or kin priming of information which encourages higher fertility (Mathews \& Sear 2013). This emphasis on fertility is a possible cause of the negative association between paternal grandfather presence and child survival in 25\% of studies in Sear and Mace's (2008) meta-analysis, while maternal grandfathers had positive influences on child survival in $17 \%$ of studies. No negative associations were found for maternal grandfathers, however they are rarely associated with providing much care or had much positive influence on child outcomes (Sear et al. 2000; Kramer 2010). 
Consequently, maternal grandfathers appear to have a greater positive impact than paternal grandfathers while overall grandfathers seem to matter little. This pattern is demonstrated in the absence of grandfather interactions or allocare in observational studies (Meehan 2005; Meehan et al. 2013a; Ivey 2000; Crittenden \& Marlowe 2008).

\subsubsection{Siblings}

Siblings are 50\% related, the same amount they would be to any future offspring. Thus, they can be predicted to invest heavily in one another (Hamilton 1964). Furthermore, children do not suffer from opportunity costs due to helping as they are not yet reproductively active (Tucker \& Young 2005). As a result, during the juvenile years a child's best fitness strategy may be to invest in embodied capital and their younger siblings (Kramer 2011). This appears the case in the observational data as siblings provide, on average, $17.4 \%$ of direct childcare across nine populations. There is a trend towards siblings providing more childcare in foraging populations (16.7\% versus $14.5 \%)$, however the results are patchy, as studies do not consistently separate brothers from sisters or report care from siblings at all (Table 2.1). Thus the pattern is unclear, particularly since children can also be considered to be competitors for parental investment and household resources (Jones 1986; Bohler \& Bergstrom 1995; Alam 1995). As such, having a large number of siblings has often been associated with poorer child outcomes, given the relationship between household size and child survival (Lawson et al. 2012). Therefore, siblings are predicted to have a mixed effect on child and maternal outcomes, mediated by birth order and household resources (Kramer 2010).

Turke's (1988) seminal piece in Ifaluk found that having a first-born girl increased a woman's fertility by acting as 'helpers-at-the-nest' and reducing the woman's labour and childcare demands. This is hypothesised to occur in the 'ecological constraints' model (Emlen 1982) when offspring are not yet able to establish territory or gain enough resources for independent breeding. Rather than invest everything into growth juveniles invest some energy into indirect fitness via sibling care, particularly if in high mortality environments, their long-term survival is unknown (thus making excess investment in embodied capital a poor evolutionary strategy (Hagen \& Barrett 2009)). For instance, Hadza children (aged 3-17) while not net producers, collected a substantial proportion of daily nutritional requirements, thus made significant contributions to the family pot (Crittenden et al. 2013). This opens the door to bidirectional transfers and the pooling of 
resources. Kramer et al. (2009) argues that children contribute to the 'energy budgets' (created with provisioning, domestic, economic or childcare labour) of their siblings and mothers. For instance, without child production Maya parents (small-scale farming society from Mexico), would have to increase their workload by 2.5 fold (Lee \& Kramer 2002) while Venezuelan Pumé forgers would not be able to enter reproductive maturity at 15.5 years (Kramer et al. 2009). Thus, juvenile production should not be ignored (Kramer \& Boone 2002; Kramer 2011; Kramer 2005; Kramer 2010; Lee \& Kramer 2002).

These predictions are broadly found in the literature. Bereczkei and Dunbar (2002) found that in a Hungarian Gypsy population, mothers whose firstborn was a girl had a higher completed fertility compared to mothers with firstborn boys. While similar results have been found in the Aymara, the gender of the helper child is not always important (Crognier et al. 2002). However, in 89 !Kung women who had completed their fertility, Hames and Draper (2004) found no effect of having a first born girl on mothers fertility or survivorship. This they argue may be due to the dangerous nature of the Kalahari bush; mothers would often have to supervise the childcare by older siblings. Therefore, the role of siblings might be dependent on the ecology; in dangerous or difficult environments children may need increased time to learn the skills to be a competent caretaker and provisioner (Kramer 2005).

Other studies have demonstrated that sibling presence is correlated with negative fertility influences. For instance, in the Gambia while older sisters had no fertility effect, older brothers decreased the probability of giving birth. In this patrilineal society it may be that after giving birth to a girl, women slow down reproduction to avoid having another girl (Sear et al. 2003). Similar results are apparent in a historical Polish dataset where it appeared that the absence of siblings increased the risk of transition to next birth, as much as by $40 \%$ (Tymicki 2004). The influence of siblings may also be gender dependent; in the Kipsigis the greater number of brothers limited parental investment in sons, resulting in marriage delays. However, more sisters or brothers had no influence on female fertility while more sisters increased parental investment in sons (Borgerhoff Mulder 1998). This is the result of sex biases in parental investment resulting in son competition for limited resources only. Thus, siblings cannot be assumed to act as helpers-at-the-nest.

The evidence of the influence of siblings on child survival and nutritional status is similarly mixed. Overall, five of six studies examining the influence of siblings found positive effects, however the topic is less commonly investigated and comparisons are few (Sear \& 
Mace 2008). The research conducted by Sear and colleagues (2002) in the Gambia also found that that older sisters increased the survival of younger siblings. However, one study among Shuar hunter-horticulturalists found that number of sisters negatively correlated with BMI while brothers had a quadratic relationship with BMI; the optimal number of brothers was two (Hagen \& Barrett 2009). Hagen and Barrett (2009) suggest that girls receive preferential feeding to improve fecundity and marriage prospects leaving less food for young children, hence the negative effect of female sibs. Similarly, among the Tsimane, girls with older brothers and younger sisters had a lower nutritional quality regardless of household wealth (Magvanjav et al. 2012). Thus the role of siblings appears mediated by ecological context, household resources and biases in parental investment (Sear \& Mace 2008; Sear et al. 2003).

\subsubsection{Distant kin and unrelated individuals}

The literature is extremely limited on the influence of allocare from more distantly related kin and unrelated individuals on child survival and maternal fertility. However, humans live in societies, whether they are small-scale villages or large urban centres, which contain a high proportion of unrelated individuals. Extensive cooperation is still common without the necessity of relatedness, indicating the importance of wider social networks in human social evolution (Apicella \& Crittenden 2013). Furthermore, a brief review of the literature above reveals the importance of flexible caregiving, particularly in highly mobile societies like foragers. Given the high mortality rate and likelihood of being resident with any given kin type, sex or age, it would be a highly risky strategy to place all your eggs into one allocare basket (Hrdy 2005). Thus, throughout the life course women may have to rely on alternative sources of help dependent on conditions (Kramer 2010). As a result these networks are likely to encompass not only distant kin (aunts and uncles, cousins (of all types) and affines) but also individuals who are not related consanguineously. Given that this consists of around 75\% of hunter-gatherer camps (Dyble et al. 2015; Hill et al. 2011), focusing cooperative networks on only kin significantly reduces who can actually provide for children.

There is more outcome data for the presence of aunts and uncles. As they share $25 \%$ of genetic material with their nieces and nephews, exactly the same as grandparents, they could be expected to provide a significant proportion of care. However, unlike grandparents, aunts and uncles are reproductively active during a similar time period as the 
focal mother, and therefore have their own children to invest time and energy into. Consequently, their impact on child outcomes is dependent on the cost of their actions (i.e. less resources to invest into their own reproduction) and the overall benefit; the benefit of investing into a niece or nephew would need to be twice that of investing into one's own reproduction. Sear and Mace (2008) cite only six studies which have examined the topic, each of which produced opposing results. For instance, Hill and Hurtado (1996) without distinguishing between matrilineal and patrilineal kin, found no effect of aunts and uncles on Ache child survival. However, Ache women suffered lower fertility when they coresided with adult brothers, whose fertility benefited from adult sisters' presence. Thus, sisters supplemented male fertility, perhaps by increased caretaking. Similarly, among the historic Polish sample, female siblings significantly reduced their sisters fertility and total survivorship to age 16, indicating clear competitive relationships of maternal kin, particularly while they are reproductively active (Tymicki 2004). While negative effects of maternal and paternal aunts have been found on child survivorship in both Malawi and historical China (Sear 2008; Campbell \& Lee 2002), among Hungarian Gypsies number of adult siblings significantly increased maternal fertility (Bereczkei \& Dunbar 2002). Following kin competition hypothesis however, these relationships may be dependent on interactions local resource availability (Griffin et al. 2004). For instance, among the Pimbwe, the number of maternal aunts and uncles increased child relative weight, however this was contingent on household socioeconomic status. Richer households suffered from many requests on their resources, while poorer households could rely on these richer households to provide these resources due to kin ties (Hadley 2004). However, only a few studies have examined the influence of kin competition by resource availability (Borgerhoff Mulder 1998; Borgerhoff Mulder 2007; Mace 1996) limiting any firm conclusions.

There are two non-mutually exclusive possibilities for why distant kin may be greater competitors than non-kin. Firstly when considering costs of caring, the reproductive scheduling of some distant kin will match the focal mother's scheduling. For instance, siblings (and thus the child's aunts and uncles) are commonly reproducing at the same time as the mother, thus childcare from them is particularly costly. This can also be true of younger grandparents under the age of approximately 50 years. Likewise, older children within the aunt's household (cousins of the focal child) may be involved in caretaking of their siblings. Thus, families of the same generation, as is common with distant kin, can compete for allocare resources. Of course, this is not the case for all distant kin. Similarly, non-kin may equally be at their peak reproductive stage, however a difference is that they 
will be dependent on a different pool of resources. This is the second possibility of why distant kin maybe more competitive than close kin. Among the Agta we have found that households tend to cluster into groups with two or three other households between which the majority of food sharing occurs. These cluster households contained significantly more grandparents, uncles and aunts than expected by chance (Dyble et al. n.d.). As a result, distant kin are dependent on the same pool of energy and labour resources and increasing number of dependents will act as competitors for their food pot. In contrast, non-kin reside outside of the 'resource clusters' and are not in competition for resources.

In the few observational studies which have reported on the investment from aunts and uncles they seem to provide exceedingly little care; among the Hadza maternal aunts provided only $1.9 \%$ of direct care (carrying in this instance (Crittenden \& Marlowe 2008)), while among the Agta maternal aunts averaged around $0.9 \%$ of direct care, paternal aunts only $0.5 \%$ (Goodman et al. 1985). However, in comparison among the Hadza, non-kin frequently carry children (12.4\%) particularly female juveniles (aged 8-12 years), a pattern also noted in the Aka (Meehan 2005), Ache and Hiwi (Hill \& Hurtado 2009). Furthermore, Ivey (2000) reported that when parents lacked support, unrelated allocarers were recruited. As a result, it appears that while distant kin are competitive, non-kin have an important role in the hunter-gatherer contexts of ensuring children receive the support they require by providing flexible childcare. However, only one study I am aware of examines the influence of non-kin on child outcomes. Borgerhoff Mulder et al. (2011) finds among the horticultural Pimbwe that a mother's relational wealth, (her social support network) is the largest predictor of child survival. However, because kin had a negative effect on child survival, the most important components of a mother's social network are unrelated. This role for non-kin may be theoretically more important in foraging populations given the stochastic nature of not only the resource ecology but also demography (Kramer 2010).

\subsection{Flexibility, variability and risk avoidance}

Human allomothering systems are highly flexible and context dependent. Who exactly increases the survival or fertility of a mother and her children varies according to the demographics, residence patterns, levels of resource competition, mortality risks and type of subsistence. However, these trends within a single population have only been explored by one study. Valeggia (2009) examined how allomothering varied among two Tabo groups 
from the South American Gran Chaco and found that in the 'traditional' community mothers provided $50 \%$ of care to infants under 24 months, young unrelated girls provided $33 \%$, and grandmothers $13 \%$. Fathers provided only $2 \%$ of care. However, in the urbanised community mothers provided $77 \%$, fathers $7 \%$, sisters $4 \%$ and grandmothers $3 \%$ of care. While this study does not explicitly test what features of these communities specifically correlated with allocarer change, the differences are extreme enough to indicate an effect of urbanisation. If, as supposed throughout the cooperative breeding literature, cooperative breeding evolved in response to environmental hardship and uncertainty (Emlen 1982; Jetz \& Rubenstein 2011) then the influence of cultural and/or subsistence change on the structure of allocare may be significantly related to risk avoidance.

Above, I discussed communal breeding as a buffer from predictable and unpredictable shortfalls in the environment. However, there are multiple ways to buffer an individual from risk (i.e. probability of loss), which depends on the resources available and subsistence patterns. Aktipis et al. (2011) discusses four ways of dealing with risk. Firstly, risk reduction includes any action that lowers the probability of loss, such as moving to a resource-rich and predictable environment. A second form is risk avoidance, which occurs when individuals diversify subsistence by participating in different economic activities or reducing exposure to risk across space with mobility (Winterhalder et al. 1999). This tactic is common in east African pastoralists attempting to mitigate the influence of cattle disease or climatic fluctuations (Little et al. 2001) as well as in foragers. The third type is risk transfer which is any action which exchanges risk between individuals or groups (Fafchamps 1992) and defines informal insurance networks worldwide, particularly in developing nations (Caudell et al. 2015). Risk pooling is an example of this where individuals/groups face an immediate small loss but decrease the severity of larger, unpredictable losses in the future. As a result, it can be best described as a needs-based transfer (Hao et al. 2015; Aktipis et al. 2011), thus matching reciprocity exactly. In particular, for reciprocity to be effective individuals within the pooling network must be independent and the number of units exposed to the risk large (Cashdan 1985). As a result, risk pooling is a useful method of reducing risk exposure when individuals face shortfalls independently. This remains true of foragers in terms of variability production, sickness and lifetime childcare deficits; the mixed age demographic of hunter-gather groups means that there is always a significant number of non-reproductive individuals present. 
The fourth and final way of dealing with risk is risk retention, which involves accepting risk by being able to absorb losses. This includes increases in wealth and food storage, two forms of risk buffer often placed in contrast to reciprocity in hunter-gatherer studies (Winterhalder et al. 1999; Cashdan 1985). When individuals have increased wealth their need for pooling-networks are reduced as they are independently able to protect themselves from shortfalls. Furthermore, individuals with increased wealth may, given the need-cost dynamics of reciprocity, be obligated in sharing networks to provide a significant amount of support for individuals in need (Hadley 2004). Thus, ultimately risk-pooling becomes less viable than wealth accumulation and wealthier individuals attempt to remove themselves from such wide sharing networks (Fafchamps 1992). Furthermore, reciprocity often hinders wealth accumulation and shortage, given its association with widespread sharing and egalitarian social norms (Wiessner 1996). As a result, in foraging populations involvement in cultivation and/or wage labour is associated with increased sedentarisation (thus swapping one risk avoidance strategy for another). However, without mobility the limitations of wealth accumulation and food shortage are removed, reducing their dependence on risk pooling (Cashdan 1985).

There is sufficient theoretical work to suppose that subsistence and/or lifestyle change in small-scale foraging communities will result in significant changes to sharing networks. Certainly, among the Mpimbwe it appeared that wealthier individuals engaged less in reciprocal exchange, and rather engaged in the cash economy (Kasper \& Borgerhoff Mulder 2015). Similar results have been found in the Dobe !Kung (Yellen 1990), Machiguenga forager-horticulturalists from Peru (Henrich 1997) and in rural Tanzania (La Ferrara 2002). However, among the Tsimane Gurven et al. (2015) recently found that market integration was not a like-for-like substitute of traditional resource pooling, and individuals with more wealth gave a higher percentage of food to others. Why these differences in patterns occur is unclear, and warrants significant further investigation. Nonetheless, it appears that we may expect childcare behaviour to vary significantly according to factors such as wealth accumulation, food storage and involvement in diverse forms of subsistence.

\subsection{Summary}

Overall, it appears that human allomothering is defined by its flexible nature and context dependency (Sear \& Mace 2008). In exploring 'who cares' and what are the consequences 
of this allocare it appears there is no human universal allocarer, or singular 'ace-in-the-hole' (Hrdy 2005). Particularly, in foraging societies defined by fluctuating group membership, short life spans and limited resources, it appears that maintaining a wide, flexible childcare networks may be a less risky strategy than focusing on one single allocarer. Following this, of particular interest is the significant variability in allocarer providers within populations. Given the hypothesis that allocare acts as a buffer to risk, it is theoretically interesting to explore how then allocare changes with different ways of dealing with risk.

It is explicitly evident that direct childcare studies rarely test how cooperative breeding can be maintained among populations of selfish individuals. I am aware of two observational studies which approach this problem (Ivey 2000; Crittenden \& Marlowe 2008), finding that the amount of care given is significantly related to kinship. However, without exploring need, costs, association, among other influences it seems to be premature to come to the conclusion that "...nepotistic investment appears to be the primary motive for allomothering among the Hadza" (Crittenden 2009: 109). At the same time, the literature contains many examples of a significant proportion of care being offered by non-kin, a topic currently unexplored due to the focus on infants in observational studies. Furthermore, given that mother's successful recruitment of allocarers appears imperative for her children's survival, we know very little about how mothers ensure they have access to the allocarers they need.

As a result, my work on cooperative breeding (chapters 5 to 7) will focus on (i) robustly testing the predictive power of different cooperative hypotheses, specifically kin selection, reciprocal altruism, helpers-at-the-nest and grandmothering; (ii) examine the influence of risk, subsistence (i.e. increased cultivation), ecology and wealth on childcare; (iii) explore how mothers may manipulate their social networks to facilitate childcare; (iv) examine the consequences of allocare on child outcomes and/or maternal fertility; $(v)$ focus not only on infants, but also on all dependent children under the age of 12 years, using a significantly larger sample size; $(v i)$ and explicitly examine the role of non-kin and their importance in flexible caregiving. 


\section{Chapter Three}

\section{Methodological Approach and Data Collection}

Here I summarise all data collection and statistical analysis used throughout this research. Any methodological approach or statistical analysis applied in only one chapter are discussed in depth in their relevant chapters. This thesis is a product of one year's worth of data collection, spanning two field seasons. During this time the Hunter-Gatherer team collected a range of genealogical, anthropometric, behavioural and aging data. We also piloted an innovative data collection procedure to examine proximity networks and childcare with the application of motes (section 3.2). Using the latest in technological advances it was possible to collect a depth of data on contact networks which has not been possible in hunter-gatherers previously. Data collection was a joint effort of the team (Mark Dyble, Daniel Smith, Sylvain Viguier, Andrea Migliano and myself), allowing us to capture information on different aspects of life (detailed in section 3.1). The coding, design and development of the motes was Sylvain Viguier's arena while we both implemented them in the field. My data collection was focused on all the anthropometric and health related data detailed in section 3.4. Section 3.3 deals with how the team created a method for reliable aging; while data collection and protocols were developed collaboratively with the Hunter-Gatherer team, the subsequential analysis was developed by Yoan Diekmann, Pascale Gerbault and Mark Thomas and remains whole heartily their work. Full data collection protocols and forms can be found in appendix A while more technical details about the motes and the statistical method used in the aging process can be found in appendix B.

\subsection{Data collection}

We collected observational, interview, anthropometric and health data over two field seasons from April to June 2013 and February to October 2014. During this time we met 
914 Agta from a population of around 1000 residing in the Northern Sierra Madre Natural Park (NSMNP). Permits for all fieldwork were awarded by the Department of Environment and Natural Resources based in the NSMNP as well as the local government in Palanan. We also sought permissions to work with the Agta from several 'chiefs' prior to the start of data collection. In the first fieldtrip we (MD, DS and I) conducted a census, genealogical interviews and anthropometrics. Our major aim here was to meet a large number of Agta across a number of different camps. As a result, this data collection was primarily conducted over two to three days in each camp, depending on its size. We were based in the barangay (district) centre and would travel out to the camps by boat or foot depending on the location. Travel time was a short as a one-hour walk or as long as a sixhour boat ride. On this occasion we worked with two field assistants who would translate and assist in data collection. When we first arrived in a camp we would introduce ourselves and arrange a community meeting in which we would show posters and act out/explain what it was we were doing and why. During this time we would collect informed consents from each of the adults who would sign on behalf of their children. After this we would conduct a camp map and find out about everyone living in the camp and broadly who was related to whom. After all of this we would conduct the genealogical interviews as well as collect anthropometric data (described fully below).

In the second period of fieldwork the aim was the return to the camps in which we had originally conducted the census to collect more detailed information. On this occasion we (MD, DS, SV, AM and I) were primarily based in Palanan town where we would get supplies. From here we would travel out to the surrounding Agta camps in all directions for 10 - 14 days at a time (depending on the size of the camp and if the weather permitted leaving). Between February and July 2014 (the dry season) we revisited 13 camps throughout Palanan. We each conducted shared data collection (genealogies, interviews and camp scans) as well as our own focused data collection (specified below). After July we (SV and I) returned to all the camps once more to conduct shorter data collection focused on the medical aspects of this thesis. We collected anthropometrics for all children and adults, as well as blood samples and conducted the medical questionnaire. Therefore, we had visited the majority of camps three times over the two-year fieldwork period, noting births, deaths, individual movements as well as camp changes. I will now detail the exact protocols we followed, separated into observational data collection, interviews, reproductive histories, medical survey and the application of the motes. 


\subsubsection{Camp Scans}

In each camp we conducted daily camp scans to record activity patterns. These scans were based on spot observation techniques (Gibson \& Mace 2005; Borgerhoff Mulder \& Caro 1985). We categorized each individual's activity at the allocated time, and if they were out of camp their reported location and activity was recorded. We found out about their location from enquiry with family members who were in camp during the scan time. To produce an unbiased time sample the first scan was rotated daily (start times from 6:30 to 9:30 at 30 minute intervals and then three more scans were conducted every four hours from this starting point). We also recorded with whom individuals were with while conducting this activity. We recorded individuals as a 'group' if they were within three meters of one another (i.e. relaxing in a lean-to). The activity groups included childcare, foraging, cash labour, agriculture, household tasks, relaxing, being out-of-camp, playing and socialising. Therefore, we had four points during each day we knew the exact composition and activities of each member of the camp. From this data it is possible to extract the variables such as how long individuals spent foraging compared to hunting, fishing, cash labour and food production.

\subsubsection{Food Diaries}

The dietary data was collected at the household level at the end of each day. Our data collection for diet was primarily based around activities, rather than an in-depth dietary recall. We asked the mother and the father at the end of the day (between 17:00 - 18:00) what foods they had eaten that day. To create variables from this data we counted up the total amount of meals we had recorded for a household and established what proportion of these consisted of meat, vegetables, fruits, honey and rice. This data was primarily collected by Mark Dyble and Andrea Migliano.

\subsubsection{Interviews}

We conducted household interviews to quantify demographics, household wealth and food security and storage. For consistency, we conducted the questionnaire with the mother of the household.

\subsubsection{Reproductive histories and genealogies}


During both our fieldwork periods we collected full genealogies and reproductive histories from each mother. We recorded not only living children, but also miscarriages, stillbirths and infant and child mortality. To help establish ages (see aging methodology in section 3.3 below) we would ask roughly how old was the first child when the second was born (i.e. had he/she stopped breastfeeding, or started walking etc.). We would always specifically enquire whether a mother had experienced any miscarriages or stillbirths, as these would often go unreported. If there was a large interbirth interval between any two children we would enquire if there was a specific reason, which may prompt a mother to report a deceased child. If a child had died, we would enquire about the causes, roughly how old the child was and when it happened. Often mothers did not know 'when' or 'how old' but it was always possible to associate an individual's age and year of death with another event that we did know. For instance, we would ask, "who were you breastfeeding when child X died?" or "which of your children is the most similar in age to child X when he/she died?" Since we robustly aged all living children, with this information it is much easier to triangulate ages and date of death for deceased children, however for older individuals this becomes increasingly more difficult since life-stages become a lot larger. Often individuals were said just to be 'older than me' or 'older than my parents' at death. Therefore, our ages for childhood mortality (under 16 years old) are more precise than our ages for adult mortality.

We then went up the genealogy and enquired about ego's parents, siblings, and grandparents. For many, it was quite rare that they would know who their grandparents were, what area they had lived in or what they had died of. Similarly, people seemed to know family members who lived nearby very well, but families of any brothers or sisters who had moved to a more distant area were often unknown. We would ask about individuals' sibling's husbands or wives and their offspring if they were not co-resident in camp. As we often collected the same family tree from several individuals, we did find inconsistencies in the data, such as an additional child or a very different birth order. To produce the most accurate genealogy we took either the genealogy from the most knowledgeable individual (i.e. the mother over the aunt) or the genealogy that reduced other inconsistencies (i.e. avoiding impossibilities such as six month interbirth intervals).

Overall the genealogies we collected contained 2,953 living and dead Agta from Palanan and neighbouring municipalities of Maconacon, Divilican and San Mario. From this data it 
was possible to establish the consanguineous relatedness $(r)$ of each individual we met and calculate maternal fertility and childhood mortality rates.

\subsubsection{Food security}

To establish how secure or insecure individuals felt in terms of food supply we asked a series of food security questions adapted from the 2014 survey for US households (Coleman-Jensen et al. 2014). By removing questions related to grocery shopping, access to resources and money we were left with 10 questions. Each individual answer was scored 1 to 3 marks according to their answer. The most insecure answer was scored with 1 while the most secure answer was scored with 3 marks. We then totalled these marks up for each mother. To control for the fact that some individuals did not have children, the answers were weighted according to how many questions they answered. For instance, if a mother answered all questions she would have had a maximum score of 30 . For a wife without children questions 5, 6, 8 and 10 were not relevant. As a result, her maximum score was 18. Therefore, if the mother with children had a score of 15 her adjusted score was $0.5(15 / 30)$ while the wife without children with the same score would have an adjusted score of 0.83 $(15 / 18)$. At the same time, we inquired about how much rice $(\mathrm{kg})$ each family had in their household at time of interview as a measure of food storage. We did not weigh this rice directly, thus measurement may be unreliable according to interviewees skill at estimation.

\subsubsection{Household belongings}

Our interview also included a quantification of the numbers of belongings owned or 'material wealth'. To create an 'emic' based list, we first sought to establish the most important items from a sub-sample $(n=16)$ of households. We asked each household to name 10 of the most important belongings an Agta could own. Based on this we created a list of 14 household items that were mentioned the most frequently. This list was then shown to each household, asking whether they had these items and if they did, how many did they have. As some items were more important than others we weighted each item according to the number of times it appeared in the list. For instance, as most households owned cooking pots, a family without one would be considered quite 'poor' since these are an essential daily item. Thus, these items were weighted the highest. This system assumes that the 'most common' are the most valued, since it would be erroneous to compare cooking pots to spoons 1-to-1. However, it does undervalue rare, luxury items (such as radios or guns). The object, count and proportion can be found in Table 3.1. Overall, this 
method was thought to be more nuanced than taking the monetary value of items since this is unlikely to be directly reflective of the value the Agta place in the items.

Table 3.1: List of household objects and their weighting used in creation of household belonging variable.

\begin{tabular}{l|rrr}
\hline Item & \multicolumn{1}{c}{$\boldsymbol{n}$} & Proportion & Weight \\
\hline Goggles & 31 & 0.053 & 5 \\
Blanket & 37 & 0.063 & 6 \\
Hunting bow & 7 & 0.012 & 1 \\
Cups & 65 & 0.111 & 11 \\
Air gun & 5 & 0.009 & 1 \\
Kettle & 45 & 0.077 & 8 \\
Knife & 65 & 0.111 & 11 \\
Mat & 15 & 0.026 & 3 \\
Net & 12 & 0.020 & 2 \\
Plates & 93 & 0.158 & 16 \\
Cooking pot & 123 & 0.210 & 21 \\
Radio & 4 & 0.007 & 1 \\
Spear gun & 35 & 0.060 & 6 \\
Spoon & 50 & 0.085 & 9 \\
Total & 587 & 1.000 & \\
\hline
\end{tabular}

\subsubsection{Camp descriptions and mobility}

During the two years of fieldwork we visited each camp multiple times (at least twice, sometimes three times depending on whether it was abandoned or not), therefore we were able to create mobility variables. This was broken down to individual level mobility and out-ofcamp mobility. For camp-level mobility we conducted a camp survival analysis that quantifies the proportion of individuals leaving camps. If all individuals who had been present on our first visit remained so during later visits, the camp had a survival rating of 1 . If, however, camp composition completely changed the camp had a survival rating of 0 . Therefore, this measure quantifies out-of-camp mobility. Leaving was defined as any departure from camp which was longer than overnight. At the individual-level people were either allocated as mobile or settled depending on whether or not we had ever witnessed them to move (again for longer than one night) at least once during our fieldwork.

While these mobility variables capture peoples' movement, the degree of camp sedentarisation was also coded according to housing type. The housing variable is on a 
three-point scale, 0 being the most temporary and includes camps with lean-to shelters, which frequently change in either location or position. Camps allocated to 2 on the scale were fully settled camps in which the houses were permanent (wooden huts with metal roofs) and unable to move. Camps with a mixture of both of these features had a temporary measure of 1 . Finally, for a binary analysis the camps were simply separated into a category of settled or not, based on the presence of permanent housing, churches and infrastructure such as water pumps. Therefore, with these measures we have both a sense of the permanence of camps as well as individuals' mobility in and out of them.

\subsection{Motes}

Sylvain Viguier and I designed the motes as a new methodology of increasing the resolution of studies of hunter-gatherer social networks. In particular, they have been designed to capture proximity interactions which mimic traditional childcare observations. The coding and testing was conducted by Sylvain Viguier and further details of which can be found in appendix B.

Motes are low-powered and compact wireless sensing devices. Multiple devices communicate and store these communications without the need for infrastructure (such as WiFi or local hubs (Cattuto et al. 2010)) and create ad hoc wireless sensing networks (Panisson et al. 2012). The device we utilised was the UCMote Mini (with a TinyOS operating system) with some custom modifications. It comprises of a main processor, a wireless communication module, a memory storage unit and a battery which allows the devices to run for up to four weeks with one charge. Motes allow us to produce highresolution proximity networks for a larger sample than previously possible. Each device sends a message that contained it's unique ID, a time stamp and the signal strength at a programmed interval. This message is picked up and stored by any other mote within specified diameter around the emitting mote (Figure 3.1). At the end of the experiment these data are downloaded for analysis.

\subsubsection{Previous applications of ad hoc networks}

The growing interest in emerging dynamics of social networks in both humans and nonhuman animals has led to the development of a variety of technological advances to 'data 
mine real-world interactions' (Isella et al. 2011). For instance, mobile phones signals have been utilised to track and map mobility patterns of 100,000 individuals (González et al. 2008). Others have focused on examining the structural elements of social interactions using WiFi and Bluetooth networks created by mobile phones (O’Neill et al. 2006; Eagle \& Pentland 2006). In a developed, industrial world where individuals are rarely separated from their mobile phones and other essential electronics, the electronic signals they emit are valid proxies for social interactions (Cattuto et al. 2010). However, these have little application in traditional anthropological fieldsites without electricity, infrastructure, telecommunication systems or portable electronics.

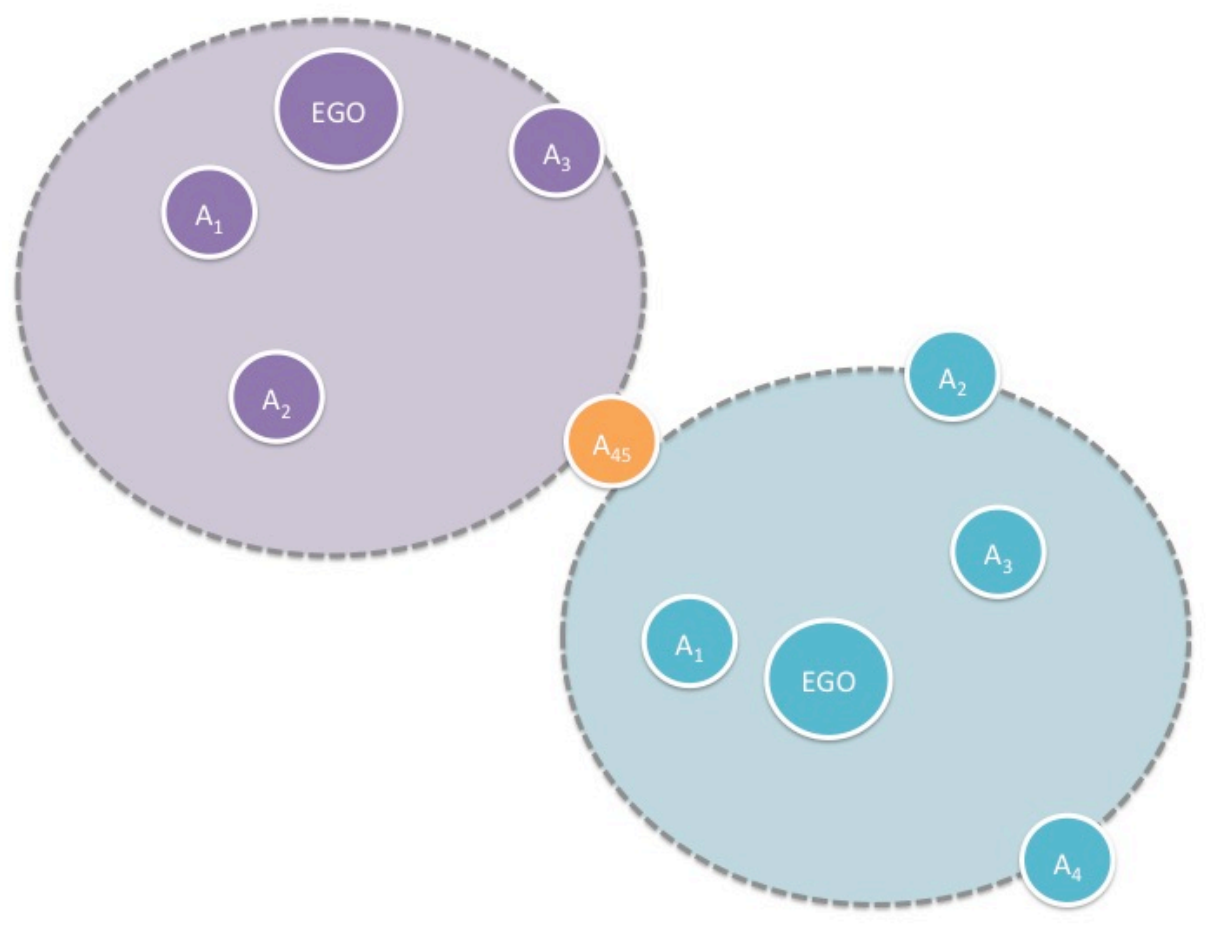

Figure 3.1: Motes proximity networks. Visualising the functionality of the motes. Each individual wears a mote, which transmits and receives messages every two minutes from other motes within approximately 3-metres. Messages beyond this threshold are disregarded and not stored. For instance, here all the blue nodes are within 3-metres of the blue Ego, and these interactions are stored. However, the purples nodes are beyond the threshold and these packets are disregarded. The singular orange node straddles both proximity networks and is stored by both Egos.

The Sociopatterns project have pioneered and developed small, inexpensive and unobtrusive active RFID (Radio Frequency Identification Devices) which sense face-to- 
face interactions up to one meter proximity (Cattuto et al. 2010; Isella et al. 2011; Barrat \& Cattuto n.d.). Passive RFID technologies include contactless payment systems and urban travel cards (such as the Oyster card used by Transport for London). However, these are read-only devices that means a unidirectional communication with a reader. Cattuto et al. (2010) altered these standard RFID tags so they were able to engage in bi-directional communications among themselves, in a peer-to-peer fashion, thus, forming an ad hoc network. Each tag has a 'transmit phase' in which packets are sent out on a radio channel. While the other tags are in a 'receiver phase' they listen to the same channel to pick up a packet sent within the programmed signal strength (i.e. distance). However, this technology was designed to function in modern contexts (Stehlé et al. 2011). This means they are dependent on a data collection infrastructure, comprised of fixed radio receivers connected to a computer via the Local Area Network (LAN). Furthermore, data collection is limited then to the zone covered by the RFID readers; if individuals leave this area their data is not collected. Consequentially, we required autonomous devices, which also contained a memory and battery for prolonged, independent functioning.

These conditions are met by motes, and to our knowledge have only been used once before by Kazandjieva and colleagues (2010) in exploring social contact networks and infectious disease spread in a US high school. They conducted their study over one day, which involved 792 motes sending broadcast messages to approximately 9-meter diameter every 20 seconds. This meant at the end of the 9-hour experiment they collected and downloaded 3 million records of contacts between participants. This study revealed that since each of these 7-byte interactions needed to fit into the $1 \mathrm{MB}$ flash memory, there is a significant trade-off on the duration, frequency and thresholds (i.e. two metres versus 15) applied in any study. This research also highlighted the importance of the 'human factor' since their motes experienced significant reboots (1500 over 500 devices) due to sudden shocks and loose batteries (i.e. banging motes against hard surfaces).

\subsubsection{Range and calibration}

The radio links were adjusted to allow a mote to record all other radio signals within a radius of approximately three metres. Three metres was utilised as not too include too many individuals in the camp who may not be necessarily interacting with ego. Since camps are small and open-plan, some individuals with huts around 10 metres away may have no meaningful interaction but continually stored as proximate by the motes (Figure 3.2). The 
second factor was that three meters is a common threshold used in child observation studies (Meehan 2009; Meehan et al. 2013b; Crittenden 2009) to denote when a carer may be providing low-effort investment (such as watching or 'proximity' babysitting) as well as more high-investment activities such as teaching, playing or talking. Therefore, we felt this was a suitable threshold that captures close proximity which is necessarily for important interactions, such as childcare, playing, hunting, foraging, cultural exchange (i.e. showing, learning and sharing) as well as disease transmission (Kazandjieva et al. 2010; Stehlé et al. 2011). Thus, 3-meter spatial networks are a proxy for meaningful behavioural interactions (Barrat \& Cattuto n.d.). Three-metres equates to a programmed power level of 5 within the embedded software, therefore, if signal strength is lower than 5 the packet is discarded. If it falls within this threshold the entry is stored in a buffer in the volatile memory, which once it is full it is written to the flash memory.

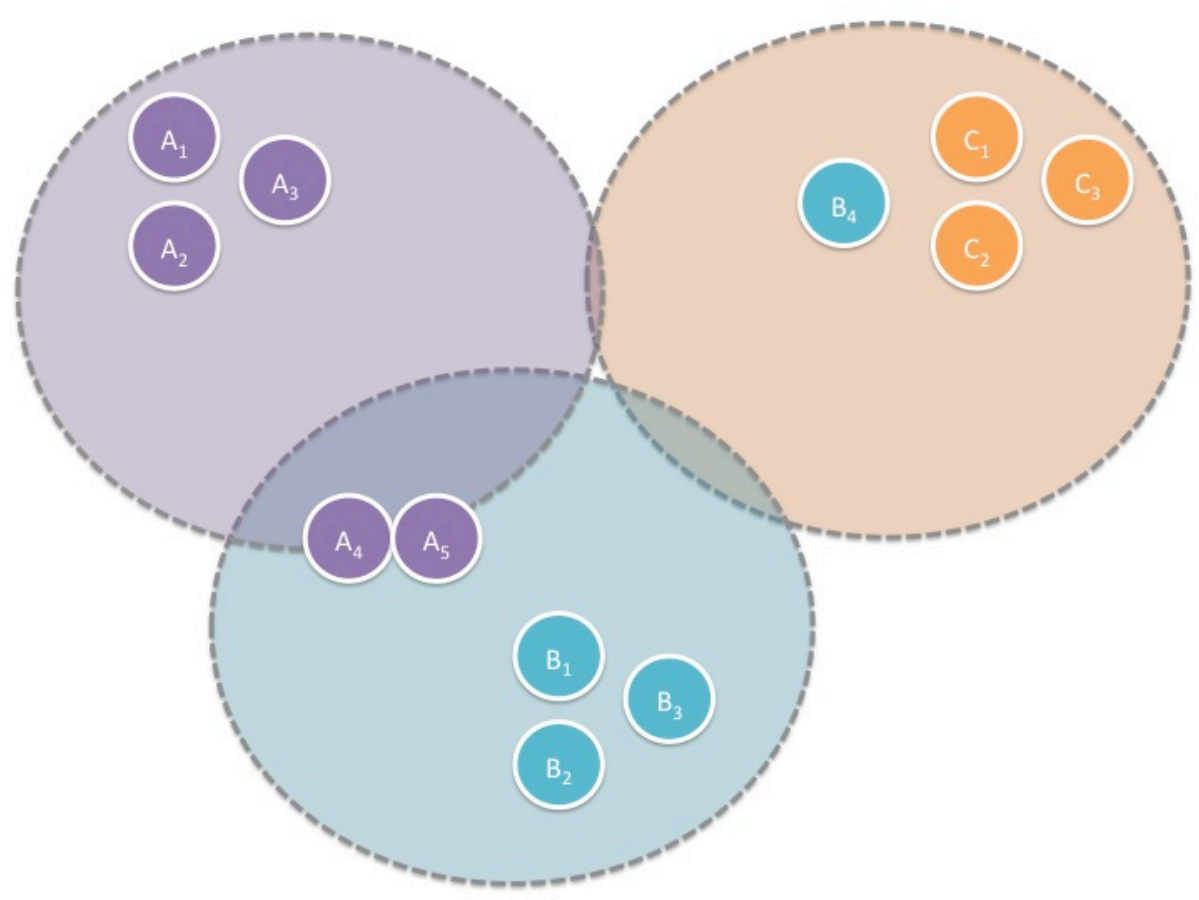

Figure 3.2: Agta camp layouts and motes thresholds. Here, when some members of household A come closer to household B their mote messages will be stored, however the reminder of household $\mathrm{A}$ and household $\mathrm{C}$ remain outside the signal range and will be discarded. 


\subsubsection{Motes utilisation in the field}

Once the motes were protected from water damage, they were sealed into wristbands, armbands or belts (depending on the size of the individual, Figure 3.3). Out of the total 13 camps we visited we conducted the motes experiment in six camps containing 200 individuals. The study was undertaken in one camp at a time. After explanation of methods and discussion of data anonymity, participants signed informed consent forms and were given a mote. Each mote was labelled with a unique number and identified with coloured string to ensure swaps did not occur. All individuals within a camp (from newborns to elderly individuals) wore the motes from a period ranging from five to seven days depending on the camp. A few individuals per camp decided they did not want to partake in the experiment, however this was rare as once a few people took part everyone would. If a mother or father did not wear a mote, their whole family was removed from the experiment in the data processing. This was so we did not bias the data used in the childcare analysis with apparently 'absent' parents.

A

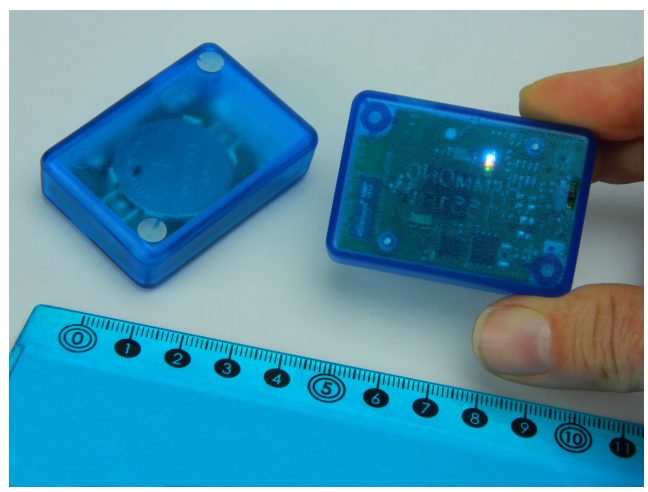

B

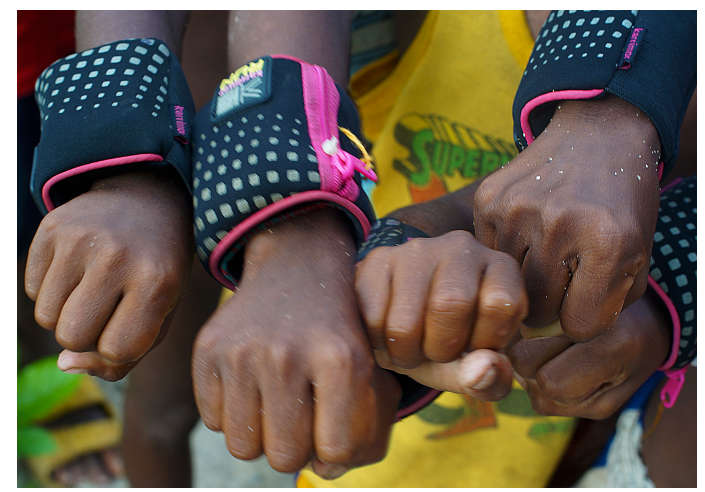

Figure 3.3: Mote utilisation in the field. (A) Motes switched on before packaging and (B) Agta children wearing their motes in armbands. Credit: Rodolph Schlaepfer and Sylvain Viguier

While the motes were worn throughout the night, data was only selected from between 5:00 and 20:00. This was to avoid long hours of simply recording who slept in the same lean-to. If individuals arrived at a camp during the experiment they were promptly given a mote, and entry time was recorded. Similarly, if an individual left a camp at any time before the end of the experiment, the time they returned the mote was recorded. A small compensation (usually a thermal bottle or cooking utensils) was given to each participant 
when the mote was returned at the end of the experiment. To ensure swaps did not occur individuals were regularly (twice daily) asked to check they were wearing the correct armband. All mote numbers were also checked when they were being handed back to ensure we always knew who had worn each mote. Any swaps were recorded during the experiment and adjusted in the final data processing.

\subsubsection{Data processing}

Data recovery from the motes produced a two-by-two matrix containing the frequency of interactions between each dyad that partook in the experiment. These raw frequency matrixes were then adjusted according to the length of the experiment; the raw number of interactions was divided by the number of hours the experiment was conducted for. Thus, the resultant dependent variable reflects the hourly average number of interactions between an allocarer and a child. I refer to this variable throughout the analysis as 'number' or 'frequency' of interactions. To bring my results into line with cooperative breeding literature in hunter-gatherers in general, I have also presented the descriptive statistics as proportion of interactions. These were produced by establishing the total number of hours an allocarer was present in camp (representing possible interactions), then dividing this by the number of actual interactions between an allocarer and child. Consequentially, this figure represents how long an allocarer spent with a child as a proportion of the allocarers total time while in camp. This result is referred to as 'proportion of interactions'.

\subsubsection{Comparison of motes data to observed proximity data}

The innovative usage of remote sensing technologies to create high-density proximity networks required justification. Therefore, to establish whether or not the motes were, in fact, recording proximity at approximately three meters we compared this data to observational data from five toddlers (aged between two to five years) produced using focal sampling techniques (Meehan 2005; Meehan et al. 2013b; Fouts et al. 2005; Hewlett et al. 2000). This comprised of a large part of my data collection where Sylvain Viguier and I observed 80 children (aged 0 to 6 years) for nine hours. This is, to my knowledge, the largest sample of focal follows among hunter-gatherer children. However, this has not been used in this thesis due to the amount of data processing required in a limited amount of time. Nonetheless, it will form the basis of future work on this topic. In this technique a 
focal child is observed for 12 hours over several days to ensure a range of activities are captured. This 12-hour period is broken into three 4-hour intervals $(6: 00-10: 00,10: 00-$ 14:00 and 14:00 - 18:00) during which, the researcher records who is interacting with a child and what type of interaction this is every 20 seconds (observe for 20 seconds, record for 10 seconds) within a three meter radius. These 4-hour intervals were conducted on non-consecutive days to reduce any sampling bias (i.e. the father was out of camp for those two days). Due to the intensive nature of the data collection, 15-minute breaks are essential every hour, thus in total each child was observed for 9 hours. This produces 1,080 observational points per child over three days, compared to an average of 3,150 mote points over one week.

Table 3.2: Proportion of time toddlers spent with any given kin category for motes and focal observations. GP refers to grandparents. Non-kin are all individuals related less than $r=0.125$, and other kin between 0.25 and 0.125 . Categories that include multiple individuals (such as GP, siblings, other and non-kin) are summed across category, thus toddlers spend $23 \%$ of time with all non-kin, however, on average they spend only $2 \%$ of their time with any given non-kin individual. These proportions are not out of 1 since children can often be with more than one individual.

\begin{tabular}{lrr}
\hline & Motes & Focals \\
\hline Mother & 0.34 & 0.37 \\
Father & 0.11 & 0.19 \\
GP & 0.06 & 0.02 \\
Siblings & 0.24 & 0.24 \\
Other kin & 0.07 & 0.08 \\
Non-kin & 0.23 & 0.24 \\
\hline
\end{tabular}

To compare these two types of data, means were produced for the proportion of time five toddlers spent with specific kin categories. These differences are minimal, and the distribution of observations with specific kin types is not significantly altered between the two methods. For instance, the motes recorded that toddlers spent on average $34 \pm 26 \%$ of time with mothers, $11 \pm 5 \%$ of time with fathers, $24 \pm 13 \%$ of time with siblings and 6 $\pm 6 \%, 7 \pm 7 \%$ and $23 \pm 13 \%$ for grandparents, other kin $(r \leq 0.25$ and $\geq 0.125)$ and nonkin $(r<0.125)$, respectively (Table 3.2). These same toddlers were observed to spend $37 \pm$ $26 \%$ of time within three-meters of their mothers, $19 \pm 19 \%$ with fathers, $24 \pm 19 \%$ with siblings and $2 \pm 1 \%, 7 \pm 8 \%$ and $24 \pm 20 \%$ of their time with grandparents, other kin and 
non-kin, respectively (Figure 3.4). Therefore, the two types of data collection produce remarkably consistent and similar pictures of proximity at three meters. Overall, the consistency between the observational and motes data leads us to conclude motes have a high reliability and represent proximity at approximately three meters. Therefore, my chapters on childcare (chapters 5 to 7 ) use mote interactions as a proxy for low intensity or proximity childcare.

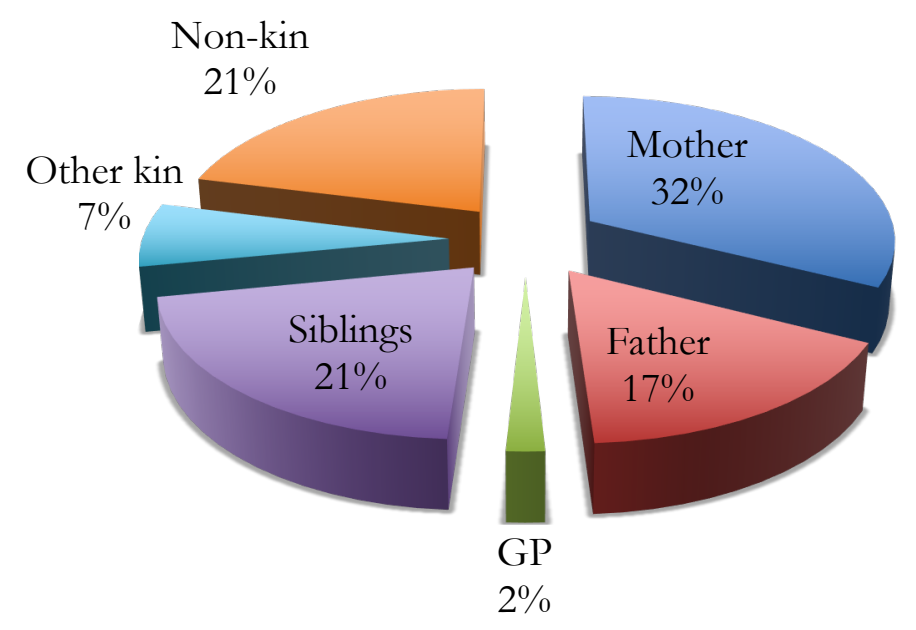

A

B

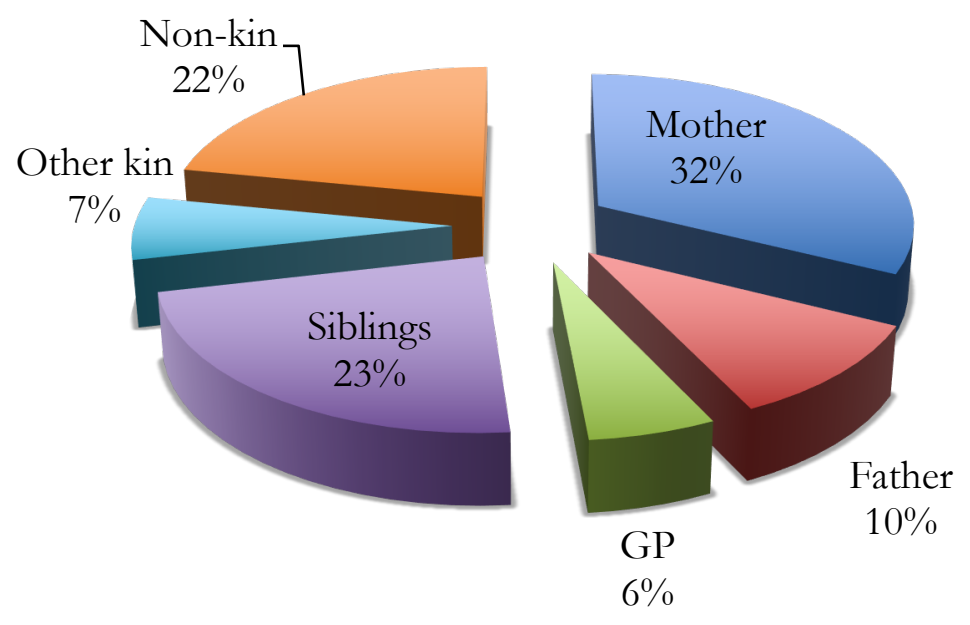

Figure 3.4: Proportion of time toddlers spent with different kin categories for data collected by (A) focal observations and (B) motes. GP represents grandparents. 


\subsection{Aging}

The aging protocols were developed and conducted by the whole Hunter-Gatherer team. The subsequential analysis method was developed and conducted by Yoan Diekmann, Pascale Gerbault and Mark Thomas and remains whole heartily their work. Further details of the Gibbs sampling Markov chain Monte Carlo (MCMC) analysis can be found in appendix B.

Many studies of hunter-gatherers struggle to accurate age their population, particularly if this is the first quantitative data collection with the group. Foragers do not have the same concepts of dates, years or time as their researchers, thus little idea about how old they are. When asked they will often suggest any reasonable (or unreasonable) figure, or simply state 'old' or 'young'. However, aging is essential for the exploration of life history events (age at first menarche, age at first and last birth, age at menopause and age at death) as well as accurate anthropometrics and growth projections for children and age-controlled fertility for women. Thus, it was essential that we were able to accurately age all individuals in the population.

Surprisingly, as a fundamental and underlying problem in much of the hunter-gatherer research, there has been limited discussion and exploration on this topic. Some researchers often make use of what Hill and Hurtado (1996: 112) refer to as "guesstimates", based on how old you personally consider an individual to look, to establish the ages of their sample (Chagnon 1974). However, this is problematic as we expect the physical appearance trajectories of hunter-gatherers to be significantly different. Babies are often born smaller and remain so, thus the age of children is frequently underestimated. On the flipside, due to harsh environmental conditions, adults frequently appear older to western researchers resulting in an overestimation of their ages. Other researchers, as reviewed by Hill and Hurtado (1996, chap. four), use: dental examinations based on teeth eruptions (however, the extreme range in when teeth erupt results in a large age bracket); relative age lists and clustering into age cohorts; and statistical techniques (Blurton Jones et al. 1992; Wood et al. 1985; Borgerhoff Mulder 1989). Early and Headland (1998) use an 'age calendar' to establish unknown ages, which entailed a calendar of dated local events (the arrival of a mine at one location, when anthropologists came or when particularly big typhoons made landfall) which Agta mothers were asked to relate the birth of their children to.

Two studies - Hill and Hurtado (1996) and Howell (1979) - have attempted to use robust methodologies to overcome this key limitation in forager research. Howell's (1979) 
demography of the Dobe !Kung contained an extensive aging procedure referred to as a 'steady-state model'. Based on a relative age list of all individuals (i.e. A is older than B and $\mathrm{E}$, but younger than $\mathrm{C}$ and $\mathrm{D}$, while $\mathrm{D}$ is older than $\mathrm{C}$ ) this method assumes a static population structure based on fertility and mortality trends. By comparing the !Kung demography to an appropriate stable population model, Howell was able to age individuals by taking the life-table which most overlapped this her own data. Thus, individuals are assigned ages due to the expected proportion of the population who should fall into each age group (i.e. $79 \%$ of infants surviving to their first birthday, $64 \%$ to their 15 and $10 \%$ to their $50^{\text {th }}$ birthday). Hill and Hurtado (1996) however, extensively critique this method on a number of points. Firstly, stable population models are unfeasible as populations are not stable due to epidemics, drought and famine as well as periods of population growth and expansion. Secondly, these population models are based on western population schedules, and they should not be considered to be universal. There is no reason to assume that mortality rates are proportional between different age brackets, as these models do. For instance, Migliano et al. (2007) suggest that pygmies suffer from high mortality rates, leading to reduce growth and faster life-histories. Thus, attempting to force foragers' unique demography and life history schedules into these models risks obscuring actual patterns.

Consequently, Hill and Hurtado (1996) demography of the Ache is based on a robust aging method which did not rely on static population models. The method follows a number of processes to arrive at more accurate ages. Firstly, the population was broken into age cohorts, in which individuals we asked to age-rank all others in their own cohort, as well as cohorts above and below them. Age-ranking was ego based, thus each individual was asked if alter was older or younger than them. A final relative age list was produced by using the ranking that minimised the number of contradictions, and the age cohorts were joined together to create a master list. To move from relative ages to absolute ones, Hill and Hurtado utilised dated events (similar to Early and Headland (1998)), photos of children at first contact, estimates of age differences between children to create an 'age chain' of birthdays and birth certificates as an anchor. The age chain method consisted of establishing which child A of known age now is of a similar age to ego when a child B (also of known age) was born. Thus ego's age is age of A plus the age of person B. By the end of this process the ages of 97 individuals out of 443 were either known or estimated. With these 97 individuals as anchors, the ages of the ranked individuals were established by 
mapped them on a fifth-order polynomial regression, using relative age as the independent variable and age of the known individuals as the dependent variable.

While this is certainly an elaborate and more robust method comparative to previous methodologies there remains significant limitations. For instance, the use of the age chain is problematic due to the uncertainty associated with an individual's age at the time of another person's birth (Diekmann et al. n.d.). Each of these small errors accumulate and amplify along the age chain. Hill and Hurtado (1996: 127) find a mismatch between actual and estimated ages to be $+0.52 \pm 1.2$ years, thus in the age chain method ages may have been overestimated by a half a year each 12.5 years (the average age of person $A$ in the age chain). As a result, the oldest individuals may be around two years older than estimated. This results in a deviation between +6.8 and -2.8 once the level of error is factored into the analysis. However, Hill and Hurtado (1996) only used point estimates that do not account for this uncertainty in age estimates.

We used a new statistical methodology, developed and conducted by Yoan Diekmann, Pascale Gerbault and Mark Thomas (paper in preparation) and data collection methodology (developed by the hunter-gatherer team), employing a Gibbs sampling Markov chain Monte Carlo (MCMC) algorithm that avoids these shortcomings. Unlike Hill and Hurtado's (1996) method, this approach takes the inherent uncertainty of age estimates into account, as well as age-rank structures among individuals and produces a distribution of possible ages as an output for each individual. This approach produces a more accurate age point estimate than regression-based approaches. The initial age range or distribution can be chosen to reflect the level of confidence in the a priori estimate. For example, if an individual's birthday is known with certainty (e.g. when a birth certificate is available) one can assume a point mass as the initial distribution, while wider a priori distributions may be chosen for age estimates solely based on physical appearance. Additionally, this method does not require age 'anchors' of known or estimated ages to produce age estimates for all individuals in a population. This is particularly useful in populations where absolute ages for all or most individuals are not known, as an age distribution will still be produced for each individual, and can be sampled in a Monte Carlo fashion for downstream analyses. 


\subsubsection{Methodology of aging}

In the field, for infants and younger children we established birth years and seasons as much as possible. When collecting reproductive histories, we noted birth order of all children and asked mothers to approximate the gap between these children. Thus, at the family level we knew the exact age of the youngest child, particularly if it had been born during our fieldwork, and then the order of the remaining children. Furthermore, during anthropometric data collection we took the teeth age of children under 13 years of age. This meant that we would have an age bracket to place each child in. We then placed all individuals into age groups (0-2 years; $2-5$ years; 5-10 years; $10-15$ years; $15-25$ years; $25-45$ years and $45+$ years) based on the above information sources. As we had photos of each of the individuals in each age group, we printed these out to conduct a pile sort. Each individual was asked to sort out their own age group according to age, starting with themselves. Therefore, they were first presented with their own picture. Next they were shown a picture of individual $\mathrm{A}$, and asked are they older or younger than you? Or do you not know them well to know their relative age? This process continued until all individuals had been allocated a relative rank. Then the individual would be asked to do this for the age group one older and one younger themselves. For the young age groups (0-2 and 2-5 years) mothers and teenagers were asked to conduct the aging, since younger children were not capable of relatively aging individuals. Thus, at the end of this process with had multiple relative age lists for each age category.

After we have the age ranks, each individual has given an age bracket based on the additional information we collected in the field (such as teeth or estimated ages). This bracket was smaller for children, or larger for older adults as there is little to anchor their age by (i.e. the difference between 70 and 80 is difficult to ascertain). A screening procedure was then developed to check the accuracy for each of the age ranks. Firstly, given the age brackets developed, are any individuals placed in the wrong order (i.e. if person A has an age 3-5 years, yet has been placed as older than person B who is between 6-9 years). Secondly, using known birth orders, checking whether siblings are placed in the wrong order in their age ranks. Any inconsistencies were then removed from the age rank. After this first trimming stage, each individual's age ranks were collated. For instance, if one individual had ranked their group as $\mathrm{ABC}$, and another had ranked the group as BCD, this would be merged into $\mathrm{ABCD}$. At this point MCMC algorithm is used to allocate ages, based on a probabilistic framework. The Gibbs sampler approach significantly reduces the difference be the known and estimate ages compared to the regression methods (0.94), 
compared to third-order polynomial regression (2.37) used by Blurton Jones et al. (1992), or the fifth-order polynomial regression (2.33) used by Hill and Hurtado (1996). Therefore the MCMC method is a significant and large improvement on all previous methods of aging in hunter-gatherer populations.

\subsection{Health survey}

As part of my research I conducted a health survey with 420 individuals in our sample. This entailed more traditional assessment of nutritional condition using anthropometrics, as well as recent medical history, faecal analysis for intestinal helminths and blood tests for iron-deficient anaemia and white blood cell composition to examine the types of pathogens the Agta face. To organise this data collection I worked in close collaboration with the Palanan field hospital and Rural Health Unit. I would, roughly each week go to one of the Agta camps with two field assistants and one trained health care assistant from the Rural Health Unit to collect the health data and conduct medical tests. I would then return to Palanan town with the samples and test results for diagnosis by the doctor and to collect all relevant medicines (primarily for helminth infections and iron deficiency) and get these to the relevant participants. Individuals with suspected TB or medical conditions which required antibiotics or further treatment (primarily respiratory conditions or gastrointestinal disorders) were taken to the hospital and made sure they received all medical treatment. At the end of the fieldwork I was also awarded funds to conduct a health and medical care awareness workshop with the Agta and medical providers.

\subsubsection{Anthropometrics}

The anthropometric measurements were conducted according to standards developed by FANTA (Cogill 2003) using a Harpenden anthropometer. Height was measured to the nearest $0.1 \mathrm{~mm}$. Ensuring the subject was standing upright, with a straight back without shoes. Weight was taken to the nearest $0.1 \mathrm{~kg}$ on bathroom scales, used on a hard, flat surface with subjects wearing light clothing. Since anthropometrics have wide interobserver reliability all anthropometrics used in this research (conducted during 2014) were done by one researcher (myself). 


\subsubsection{Medical Questionnaire}

I conducted a medical questionnaire to quantify symptom history over the past two weeks as well as any longer illness such as TB. These questions were developed with the assistance of medical doctors at the Palanan Station Hospital, with reference to WHO's and Medecins San Frontiere book, Clinical Guidelines: Diagnosis and Treatment Manual (Broek et al. 2013). The questionnaire was broken down into a few sections: gastro-intestinal disease, influenza and fevers, respiratory tract infections, intestinal parasite symptoms, skin conditions, malnutrition and physical status and finally questions relating to medical care, frequency of access and cost. The questions for each of these conditions specifically focused on key diagnostic symptoms, such as a long-term wet cough (TB), cyclical fevers (malaria) or an itchy anus (helminths). I would also enquire how severe or common the symptoms were, and whether the individual had sought any medical attention for them. The questionnaire was conducted with a qualified health care assistant (Ata Rosie, Figure 3.5). The completed questionnaire was then handed back to the doctor along with the blood test results for diagnosis.

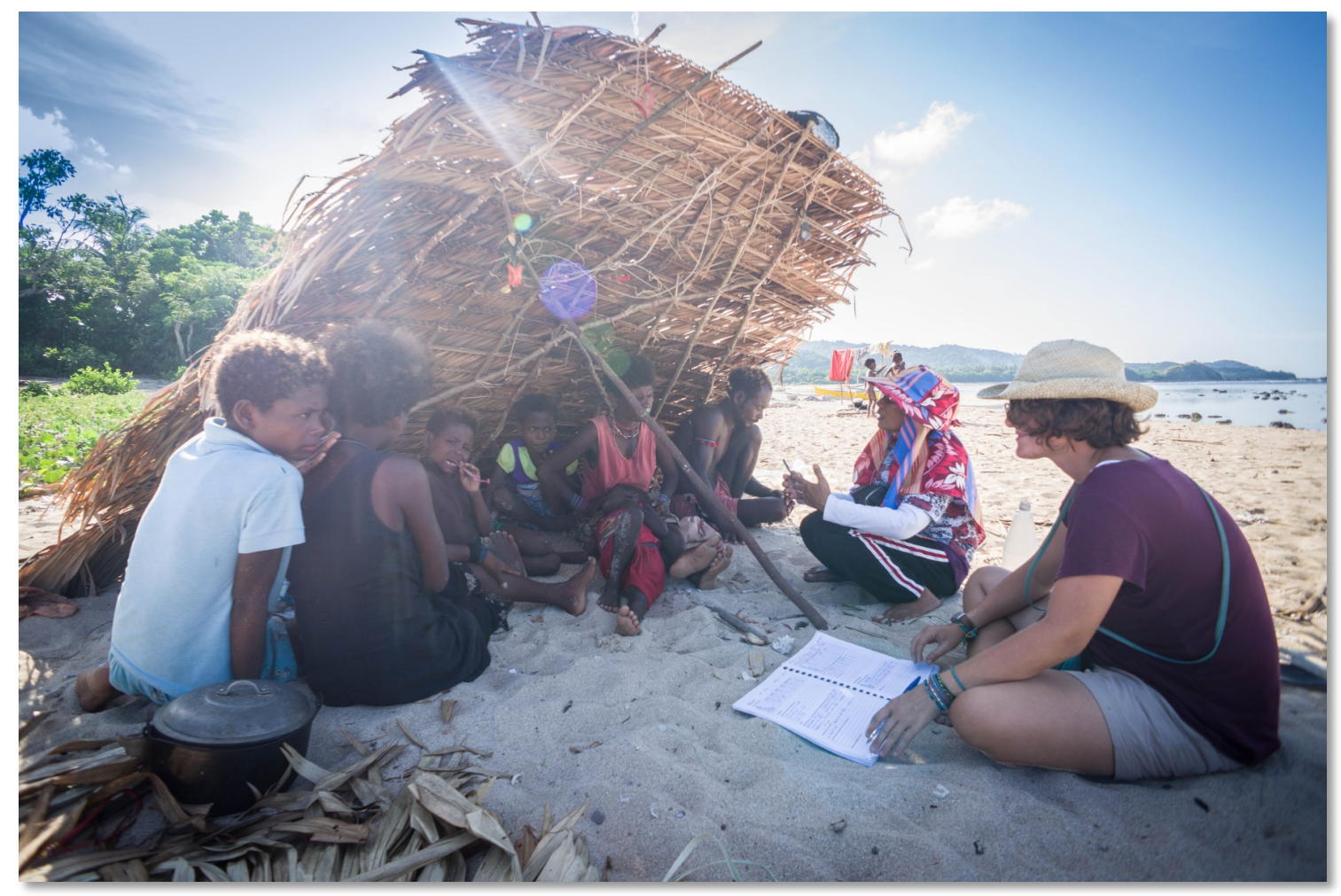

Figure 3.5: Conducting medical questionnaires. 


\subsubsection{Blood composition analysis}

Blood composition analysis was conducted as a proxy for examining the different types of disease pressures the Agta face. I looked at haemoglobin concentrations and white blood cell differentials to examine both nutritional condition and infectious diseases. The methodology and rationale behind this approach is discussed below.

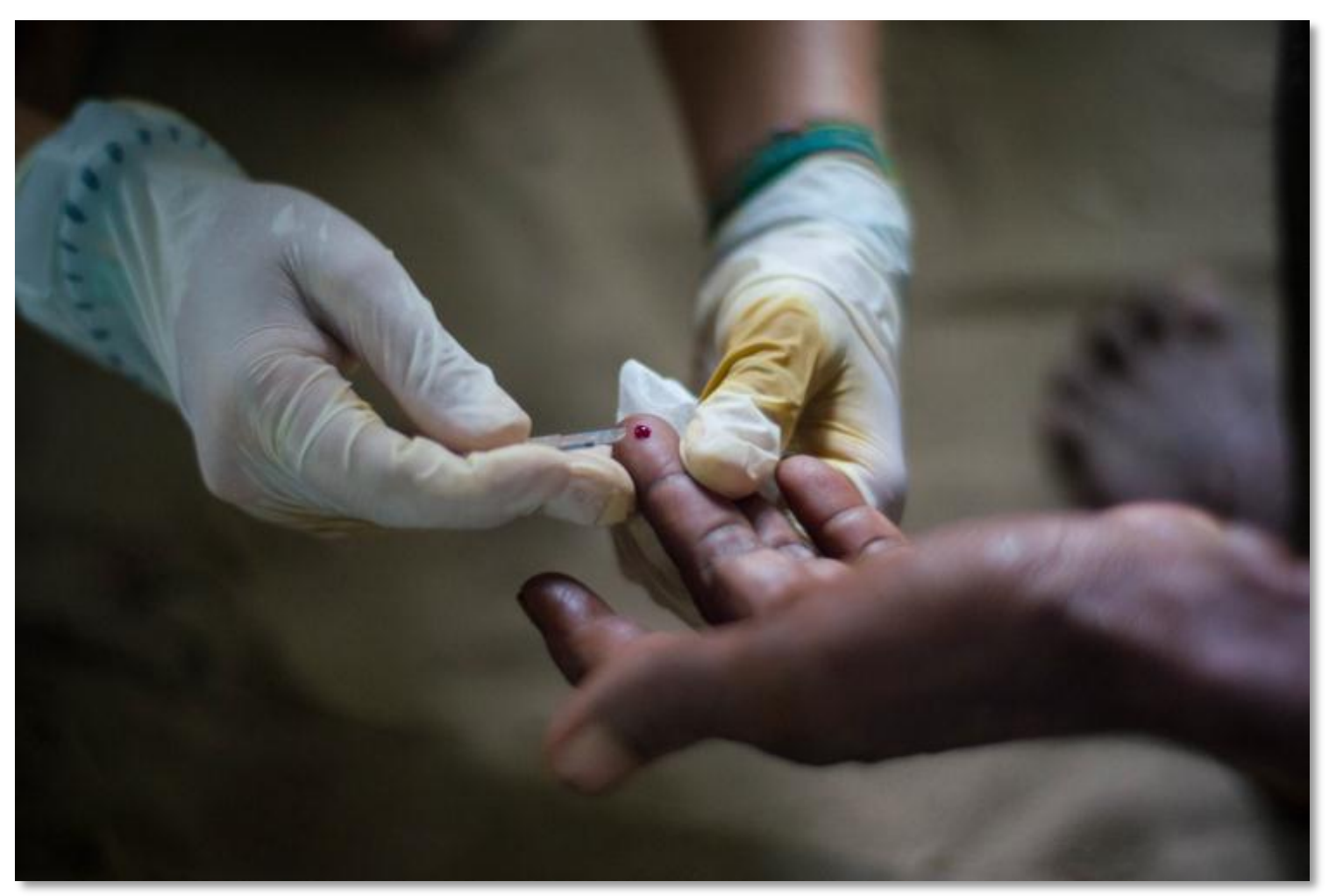

Figure 3.6: Collection of blood samples for WBC differential analysis and haemoglobin count.

\subsubsection{Blood Collection Protocol}

The standard protocol for blood collection is as follows. A blood sample, obtained by a Haemolance Normal Flow lancet, of approximately $10 \mu \mathrm{L}$ was drawn into the cavity of the specially designed microcuvette by capillary action. Following best procedure, blood was always taken from the end of the middle or ring finger on the right hand (Morris et al. 1999, Figure 3.6). The first two or three drops were wiped away. Clotted samples were discarded, and the sample was always taken within one minute prior to analysis. If multiple samples were required (due to a lost sample, clotting or lack of flow) a different finger was used each time, as skin puncture causes the body's defence system to increase the number of 
WBC close to the wound, affecting the overall measurement. If possible the microcuvettes for both the Hb 301 and WBC DIFF systems were filled from the same prick. If blood flow clotted before this was possible, a second prick was made on a different finger. The blood flow was never encouraged by squeezing due to the altering effect this has on the blood sample (Morris et al. 1999). The microcuvette was then placed into each analyzer. The WBC DIFF produces a full white blood cell differential within five minutes using staining and image analysis within the analyzer. The Hb 301 displays its results within 10 20 seconds by measuring the absorbance of the whole blood at an $\mathrm{Hb} / \mathrm{Hb} 02$ isobestic point.

\subsubsection{Haemoglobin}

Haemoglobin is an iron-based component of red blood cells, which binds oxygen, helping the red blood cells to transport oxygen from the lungs to the rest of the body (Mitaishvili 2010). There is a wide normal range of haemoglobin levels for men, women and children, which are subject to daily variation. However, haemoglobin values below $130 \mathrm{~g} / \mathrm{L}$ for males, $120 \mathrm{~g} / \mathrm{L}$ for females and $\leq 110 \mathrm{~g} / \mathrm{L}$ children aged are considered anaemic. Haemoglobin analysis was conducted on HemoCue ${ }^{\circledR} \mathrm{Hb} 301$ for 345 individuals older than three months. The Hb 301 system is calibrated against the hemiglobincyanide (HiCN) method, the international reference method for the determination of the haemoglobin concentration in blood. The measuring range of the $\mathrm{Hb} 301$ is $0-25.6 \mathrm{~g} / \mathrm{dl}$.

While comparative studies have found the $\mathrm{Hb} 301$ system to have remarkably accuracy and precision (HemoCue 2014a) Morris and colleagues (1999) discuss the problems of reliability in association with samples taken from the left or right hand (correlation of variation of $6.3 \%)$ and measurements taken on four consecutive days $(7 \%$ correlation of variation). There is significant indication that this within-subject variability arises from (i) not consistently using the same finger, (ii) incorrect handling of the finger, i.e. 'milking' leading to contamination with extracellular fluid (Morris et al. 1999), (iii) incorrect filling of microuvettes and (iv) the presence of air bubbles (Tatsumi et al. 2002; Muñoz et al. 2005). These factors result in significant alterations to the reliability of correlation coefficients between researchers (Neville 1987), and inconsistent results (Rippmann et al. 1997; Agarwal \& Heinz 1992). However, the reliability can be greatly reduced by reducing operator error with proper training, standardized procedures and consistency (Morris et al. 1999; Neville 1987; Agarwal \& Heinz 1992; Rippmann et al. 1997; Tatsumi et al. 2002; Briggs et al. 2012; Srivastava et al. 2014). 
As a portable, battery operated machine, which is easy to use the HemoCue system remains optimal particularly for fieldwork over large, remote areas without adequate laboratory facilities (Srivastava et al. 2014; Sari et al. 2001; Akhtar et al. 2008). Furthermore, the majority of studies have found the HemoCue system to have remarkable precision, accuracy and sensitivity in both adults and children (Sari et al. 2001; Akhtar et al. 2008; Srivastava et al. 2014; von Schenck et al. 1986; Rechner et al. 2002; Medina Lara 2005; Rosenblit et al. 1999; Hudson-Thomas et al. 1994; Teli et al. 2002; Kim et al. 2013; Morris et al. 1999; Gwetu et al. 2014; Hinds et al. 2007; Spielmann et al. 2012; Neufeld et al. 2002; Sanchis-Gomar et al. 2013). While studies with less supportive results do exist (Bhaskaram et al. 2003; Saxena \& Malik 2003; Neufeld et al. 2002; Neville 1987; Seguin et al. 2011), such as differences between capillary and venous samples (capillary samples generally being less accurate than venous ones (Shahshahani et al. 2013; Mills \& Meadows 1989; Srivastava et al. 2014)) it is argued that these differences are not clinically relevant if correct, standardized procedures are used (Schalk et al. 2007; Radtke et al. 2005; Tatsumi et al. 2002; Muñoz et al. 2005). Furthermore, the majority of recent systematic reviews on the reliability, accuracy and precision of the HemoCue $\mathrm{Hb}$ technique finding the majority of work to be in support of this method of assessment of anaemia, particular in rural resource poor settings in the developing world (Gwetu et al. 2014; Sanchis-Gomar et al. 2013; Srivastava et al. 2014; Akhtar et al. 2008).

\subsubsection{White blood cell composition}

White blood cell (WBC) analysis was conducted on HemoCue ${ }^{\circledR}$ WBC DIFF for in-vitro white blood cell composition in capillary blood. As a portable, battery operated system WBC DIFF provides immediate values for total white blood cell count and a differential count of the five main leukocytes (neutrophil, lymphocyte, monocyte, eosinophil and basophil) each of which have specific functions and morphologic appearance, making classification possible (Greer et al. 2013). There is large intra- and inter personal variation in WBC concentrations, therefore the majority of the analysis has been conducted with internationally accepted medically abnormal concentrations of each cell type. These are based on a normal range (two standard deviations from population norms) primarily from European and North American populations (Beck 2008; van Assendelft 2002; Greer et al. 2013).

Neutrophils are the major component of our WBC, and it is their activation and death at the sites of infections and wounds which causes pus (Mitaishvili 2010). Severe neutrophilia 
is suggestive of septicaemia or other serious microbial infections (Beck 2008). Raised neutrophil levels above $7.0 \times 10^{9} / \mathrm{L}$ for adults and $8.0 \times 10^{9} / \mathrm{L}$ for children under 12 indicate bacterial infections (Wahed \& Dasgupta 2015). Lymphocytosis is a lymphocyte concentration of more than $3 \times 10^{9} / \mathrm{L}$ for adults over the age of 12 . For children there is much greater normal variation, especially less than two years (normality range from $4-12$ x $\left.10^{9} / \mathrm{L}\right)$ while for children aged 2 - 12 the ranges steadily reduces into adulthood $(2-6$ years normal range $6-9 \times 10^{9} / \mathrm{L} ; 6-12$ years normal range of $1-5 \times 10^{9} / \mathrm{L}$ ). Such raised lymphocyte levels are indicative of viral infections, such as Rotoviruses and Calicivirses, which are major causes of gastroenteritis and diarrhoea common among the Agta (Wahed \& Dasgupta 2015). Eosinophils are an extremely valuable marker of the presence of parasites, particularly when eosinophilia is moderate (i.e. between 1.5 and $5 \times 10^{9} / \mathrm{L}$ ). Parasites cause an eosinophilic response when they invade the tissue of the body. Particularly helminths with a tissue migratory phase (such as hookworm and Ascaris spp. as they pass through the lungs) cause severe eosinophilia (above $5 \times 10^{9} / \mathrm{L}$ ). On the other hand helminths such as Trichuris trichura (whipworm) cause mild and occasional eosinophilia (depending on helminthic load) as it attaches to the intestinal mucosa and feeds on tissue secretions rather than blood (unlike the hookworm, (Beck 2008)).

While these are the most likely causes of neutrophilia, lymphocytosis and eosinophilia, it is not always possible to delimitate the effects so neatly. For instance, both neutrophilia and lymphocytosis can be caused by non-acute conditions such as toxoplasmosis, Addison disease and autoimmune disorders and gout (Beck 2008). Likewise eosinophils are a major effector cell in many types of allergic inflammation. However, increased counts of neutrophils, lymphocytes and eosinophils are here taken to, on average, indicate bacterial, viral and helminthic infections, respectively (Beck 2008).

All quality control studies of the WBC DIFF system find the results to be confidently repeatable with small standard deviations between tests and produce similar results to other methods (HemoCue 2014b). Overall automated methods, if properly calibrated and conducted are found to be more precise than manual procedures (Greer et al. 2013; Bentley et al. 1993; van Assendelft 2002). Traditionally the collection of biological samples has been very difficult from foraging groups due to their remote locations and the difficulties of storing biological samples in often hot conditions. The HemoCue systems successfully overcome these limitations by allowing the blood differential to be made immediately and then the sample discarded. 


\subsubsection{Faecal analysis}

To further examine parasite load among the Agta we collected stool samples. This was the more complex part of the data collection as the samples had a short storage time, particularly in tropical conditions. Therefore, I have only a limited sample $(n=30)$ from a few camps (two coastal producing 13 samples and three inland with 17 samples). The sample was comprised equally of $50 \%$ males and $50 \%$ females, however the majority of individuals were under 16 (median age $=9.98$ years). Due to difficulties in collection we only sampled individuals who had parasitic symptoms. As such $100 \%$ of samples were positive for one to three common species of nematodes (whipworm (Trichuris trichiura), hookworm (Ancylostoma duodenale or Necator americanus as it was not possible to distinguish between species ova) and roundworm (Ascaris lumbricoides)). These are the three "soiltransmitted helminths" and collectively infect millions worldwide (Bethony et al. 2006). These data cannot be used to examine if individuals were infected with helminths (given that the samples had only been taken from individuals with parasitic symptoms) but they can say something about parasite load by examining correlates of polyparasitic infection. Each stool sample was placed in a sterile and sealed collection pot and preserved with polyvinyl-alcohol and received by the local hospital laboratory for microscopic analysis within 24 - 36 hours. Collection was conducted by a fully trained healthcare assistant and microscopic analysis was conducted by the Palanan hospital laboratory technician.

\subsection{Statistical Analysis}

All chapter specific forms of analysis are discussed in-depth within the relevant chapter. However, since I use reproductive residuals, multilevel models and the same modelling procedure throughout this thesis this is discussed below.

\subsubsection{Fertility, mortality and reproductive success residuals}

To examine fertility and survivorship to age 16 the non-linear effect of age needed to be eliminated. To create age-specific fertility and survivorship to age $16 \mathrm{I}$ used the residuals from a polynomial regression between fertility (all live births), survivorship to age 16 (my proxy for reproductive success) and child mortality. All models were run with a Poisson distribution due to the discrete nature of the data. The polynomial regressions for reproductive success and fertility were fitted with three degrees while mortality has fitted to 
two degrees to compromise increasing complexity, and thus better accuracy, of the fitted model with the significance of the coefficients (residual models results shown in Table 3.3).
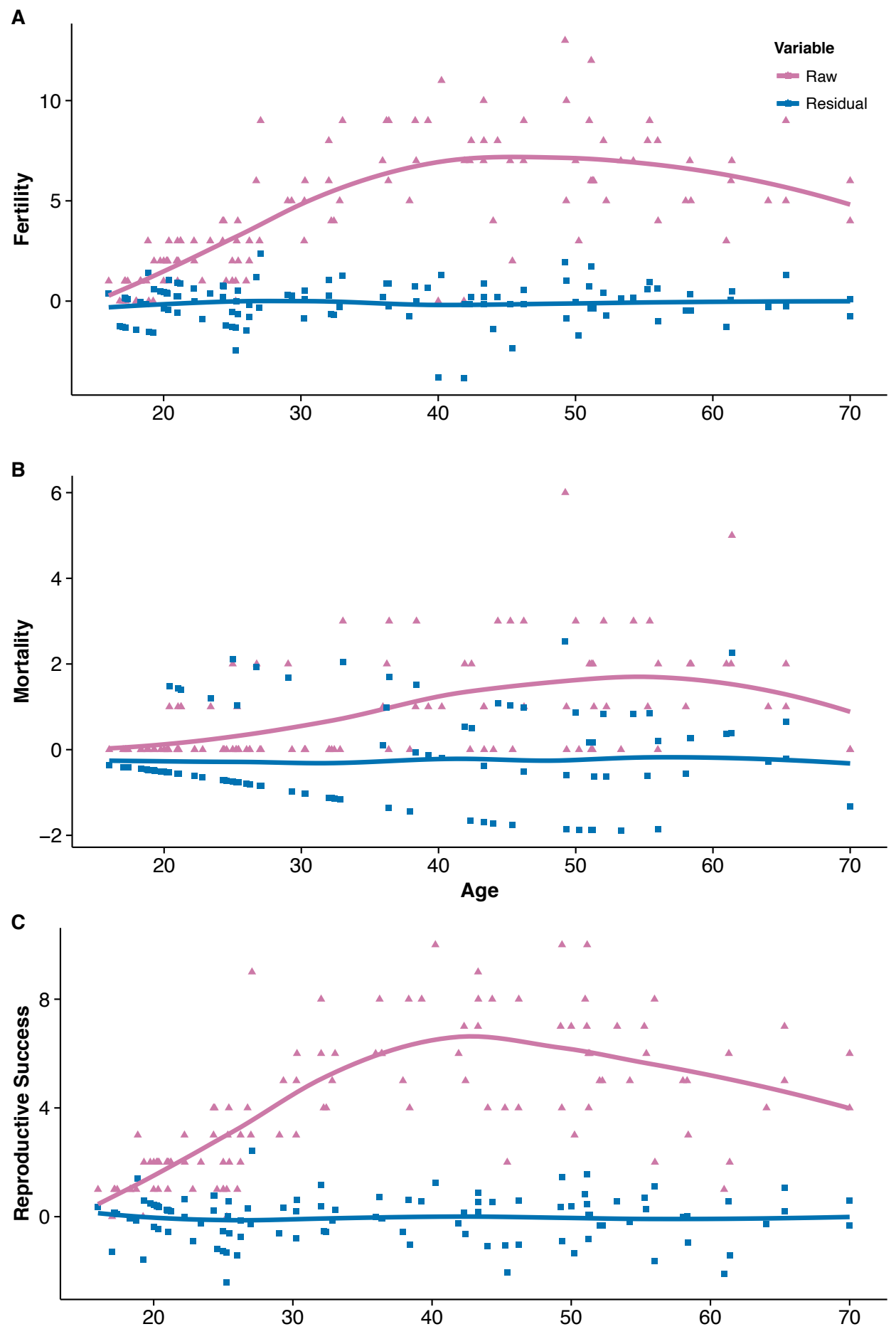

Figure 3.7: Relationship between age and A) fertility, B) maternal child mortality and C) reproductive success. The pink line represents the raw data and its relationship with age. The blue line is the age-specific residual age controlling for age using a polynomial regression with a Poisson distribution. 
By using each mother's residuals from this analysis I can examine how high or low their fertility is, given their age. Thus, an age-specific fertility residual of 0 represents the average fertility of the sample of that given age, negative values are below average fertility while positive residuals are above average. These residuals had no significant relationship with age and its quadratic term ( $p=1$ in all cases) after this transformation. Thus, these residuals are used in all final analysis and no longer present a significant relationship with age (Figure 3.7).

Table 3.3: Results from polynomial regression (with Poisson distribution) between age and fertility, $n=117$.

\begin{tabular}{crrl}
\hline Model & Degree & Coefficient & $\boldsymbol{p}$ \\
\hline \multirow{3}{*}{ Fertility } & 1 & 0.4370 & 0.0000 \\
& 2 & -0.0083 & 0.0001 \\
\multirow{3}{*}{ Mortality } & 3 & 0.0000 & 0.0025 \\
& 1 & 0.2537 & 0.0000 \\
\multirow{2}{*}{ RS } & 2 & -0.0024 & 0.0001 \\
& 1 & 0.4471 & 0.0000 \\
& 2 & -0.0087 & 0.0002 \\
\hline
\end{tabular}

\subsubsection{Kin categories}

Throughout this thesis I use three different kin categories: close kin, distant kin and nonkin. Close kin refers to all individuals who are related $r>0.25$, thus includes parents and siblings. Distant kin $(0.125 \leq r \leq 0.25)$ includes grandparents, half siblings, aunts and uncles and first cousins. Non-kin $(r<0.125)$ subsumes both distantly related individuals (first cousins once removed and double second, second, third and fourth cousins) as well as individuals completely unrelated. Distant cousins accounted for $29.6 \%$ of the non-kin sample (50\% of which share coefficients of $r<0.031)$. This division has been made since in family histories individuals could rarely trace genealogical relationships beyond grandparents and their immediate siblings and cousins. 


\subsubsection{Definition of children, adults, carers and dependents}

I have consistently established the cut-off between dependent children who require the receipt of some allocare at 11 years. While children are nowhere near net producers at this age, they no longer are fully dependent on adult care. From our own observations from the age of 12 juveniles are free to travel between camps and do not receive any particular 'caring' attention from adults. Such patterns are common among hunter-gatherers in which older children experience significant freedoms (Konner 2005; Kramer 2005; Kramer 2002; Peterson 1978). Furthermore, I draw a line between younger and older children within the under 12 category. Before the age of 6 children are fully dependent on other individuals, be that mothers, siblings or older children in playgroups. They are not capable of providing care to younger children. However, between the ages of $6-12$ years children can be defined as both dependents and carers. It is not uncommon for a six or seven year old child to carry a younger sibling around in a playgroup, which is often overseen by one to two teenagers. As a result, while younger children's childcare requires additional supervision it should not be ignored. Thus, when exploring cooperative breeding I consider all proximity care received by children aged under 12 years. These children can be cared for by a child over the age of 6 if they are younger than that child: i.e. a six year old can provide care for a three year old while a six year old cannot provide allocare to a nine year old.

\subsubsection{Multilevel models}

As the Agta are hierarchically nested (individuals within households residing in camps) logistic and linear multi-level models (MLM) were used to avoid problems of nonindependence. MLM distributes the response variance into each of the levels. These levels are the 'random-effects' entered into each model (Koster et al., 2015; LEMMA, 2015). All models were originally run with level 1 as the individual, level 2 as the household and level 3 as the camp. Levels that failed to improve the model were removed to produce the most parsimonious model.

Due to the levels in MLM, an additional concern is sample size at each cluster of the analysis; while one may have many level 1 observations (for instance, here in one analysis I have 2,045 observations), levels 2 and 3 are necessarily significantly reduced in a hierarchical structure (here reducing to six camps in analysis based on the motes data). 
Some have argued for setting a role of thumb of a minimum of ten observations at each level of the analysis (Maas \& Hox 2005). However, such specifications are difficult to adhere to, particularly in hunter-gatherer studies where population sizes are often small. Nonetheless, how problematic small sample sizes are depends on the research objective of the analysis and the parameter being tested (Snijders 2005; Bell et al. 2010). Here, I am primarily interested in the predictive value of fixed effects and regression coefficients which are distributed across multiple levels to account of the non-independence of observations rather than the variance distribution of the random effects per se. A small sample size (as small as two level-2 units) in one the levels can result in biased random effect variance estimates (Clarke \& Wheaton 2007; Bell et al. 2010). However, studies have consistently showed little or no bias to the estimates of fixed effects, which is of interest here (Clarke \& Wheaton 2007; Maas \& Hox 2005; Bell et al. 2010; Gelman 2006). In chapter 7 it is level one which is primarily of interest (i.e. maternal centrality) while in chapter 5 it is level one, two and three which is of interest (i.e. the dyad, the giver and the receiver), all of which have larger sample sizes (ranging from $n=39$ to $n=2,045$ ). Thus, it is these sample sizes which are of main importance; the other cluster sizes (i.e. $n=6$ in level 2, 3 or 4 depending on the analysis) are not as important for the power of such tests (Snijders 2005). Smaller sample sizes at higher levels may result in the computed variance of 0 , however given that non-MLM are simply models without a group-level variance (i.e. variance pre-set at 0 ) then having the ability to attribute some variance due to nonindependent clustering is preferable (LEMMA 2015). As a result, I retained the camp as a level in the multilevel models to capture the nested nature of the sample.

The relative explanatory value of random effects is established using variance partition coefficients (VPC); a VPC is calculated by dividing the variance of one level by the sum of all other variance components. 'Fixed-effects' are then entered into this base model (referred to as intercept-only), which includes all predictive variables of interest. To produce an estimation of variance reduction with the inclusion of fixed-effects (i.e. the predictors) I followed the procedure cited by (Koster et al., 2015; LEEMA, 2015). This procedure allows for an estimation of the percentage reduction in the unexplained variance at each level by the inclusion of a predictor variable. To establish this figure an interceptonly model is produced which contains only random-effects (i.e. the levels). The residual variance in this model is used as the baseline to examine how much residual is reduced once fixed-effects are included by removing the new residual figure from the intercept-only 
figure and dividing this by the original figure (see Koster et al., (2015) for a fuller discussion). This figure can then be compared to the variance explained by the control only model to compute how much of the variance the measure independently accounts for. As a result, VPC provide insight into the influence of fixed-effects has on the overall variance in the dependent variable (Koster et al. 2015). Variance explained figures are reported in the main results tables (labelled as VAR or variance reduction).

\subsubsection{AIC}

Only theoretically interesting variables are included in each set of models as well as controls known and/or suspected to confound relationships. As a result, no variables are systematically removed from the model (i.e. stepwise model fitting) to increase model precision. Rather information theoretical (IT) approach is relied on to establish the 'best fit' model. IT approaches favour models that have the most explanatory value without being overly complex (i.e. containing many parameters (Burnham \& Anderson 2004)). One measure of this is Akaike's Information Criterion (AIC), which estimates the distance of the model from the data weighted according to the complexity of the model. Models with too many parameters which do not significantly reduced the distance between the model and data are penalised with a higher AIC score (Burnham \& Anderson 2004). The AIC value in and of itself means very little, however it is useful for model selection as it allows for relative comparison between models to see which fall closest to the data. Burnham and Anderson (2004) state the common threshold that models with AIC scores 2.0 lower that others are considered the 'best-fit', a threshold I follow here. While I do not conduct any systematic model selection, I do present a 'best-fit' model to justify additional complexity with the inclusion of interactions as well as when comparing different sets of predictors.

\subsubsection{Software}

All statistical analysis was conducted on $\mathrm{R}$ version 3.1.2 (R Core Team, 2014) using the following packages:

- Multilevel modelling in Ime4 (Bates et al. 2013)

- Social network analysis in igraph (Csardi 2015) and CePa (Gu 2012) 
- Genealogical analysis and coefficient of relatedness in pedigree (Coster 2012) and kinship2 (Therneau et al. 2015).

- Data manipulation in plyr (Wickham 2011)

- Variance inflation factor in car (Fox and Weisberg 2011)

- Plotting in ggplot2 (Wickham 2009) and cowplot (Wilke \& Wickham 2015) 


\section{Chapter four}

\section{The Agta: Resilient Foragers Under Transition?}

The Agta are one of several Negrito populations residing in the Philippines. Negrito refers to a phenotypically similar group - dark skinned, short statured and curly haired - of hunter-gatherers in Southeast Asia, ranging from the western Andaman Islanders in India, to the Semang of Malaysia and ending at the north-eastern Agta of the Philippines (Omoto 1984; Endicott 2013). Other hunter-gatherer groups in the Philippines include the Batak from Palawan, the Aeta of western Luzon and the Ata from Negros. Some have rejected the term Negrito as derogatory as it means ‘little black person' (Minter 2010). However, it remains a useful terminology to refer to a genetically similar population dispersed throughout the region, separating populations like the Aeta and Agta from the other 'indigenous peoples' (IPs) of the Philippines who arrived in later population migrations (Scholes et al. 2011).

Archaeological and genetic data suggests that anatomically modern hunter-gatherers reached Southeast Asia around 50,000 years ago and resided in Luzon for the last 4540,000 years based on radiocarbon and luminescence dating (Bellwood 2010; Higham 2013). Investigations into the genetic diversity of the Aeta and Agta reveal long-term settlement of the region due to high levels of autosomal differentiation (Heyer et al. 2013). Interestingly, however, recent evidence reveals that there is no significant Y-chromosome differentiation between Negritos and non-Negrito Philippine populations (Delfin et al. 2011), supported by mtDNA and Y-chromosome genetic variation from the Batak revealing a lack of a common Negrito ancestor which excludes non-Negritos (Scholes et al. 2011). Finally, Philippine Negritos have been found to be more genetically similar to nonNegrito neighbours than one another (Migliano et al. 2013). Thus, while some of the 'Negrito' phenotypes, such as dark skin colour, are likely due to deep ancestry and survival of isolated populations, other phenotypes, such as short stature, may occur due to convergent evolution (Migliano et al. 2013; Scholes et al. 2011; Endicott 2013). 
From around 5-4,000 years ago systemic agriculture arose in the region as a later wave of migration of Austronesian-speaking farmers spread from Taiwan throughout the Philippines (Lipson et al. 2014). Linguistic evidence suggests heavy interaction at this point as the 'original' Agta languages were replaced by the Austronesian languages (Diamond 2014; Bellwood 2010; Reid 2013). Despite this significant interaction, hunter-gatherer groups remain in some of the Philippines most isolated regions, maintaining lifestyles that match many of the features of other immediate-return foragers.

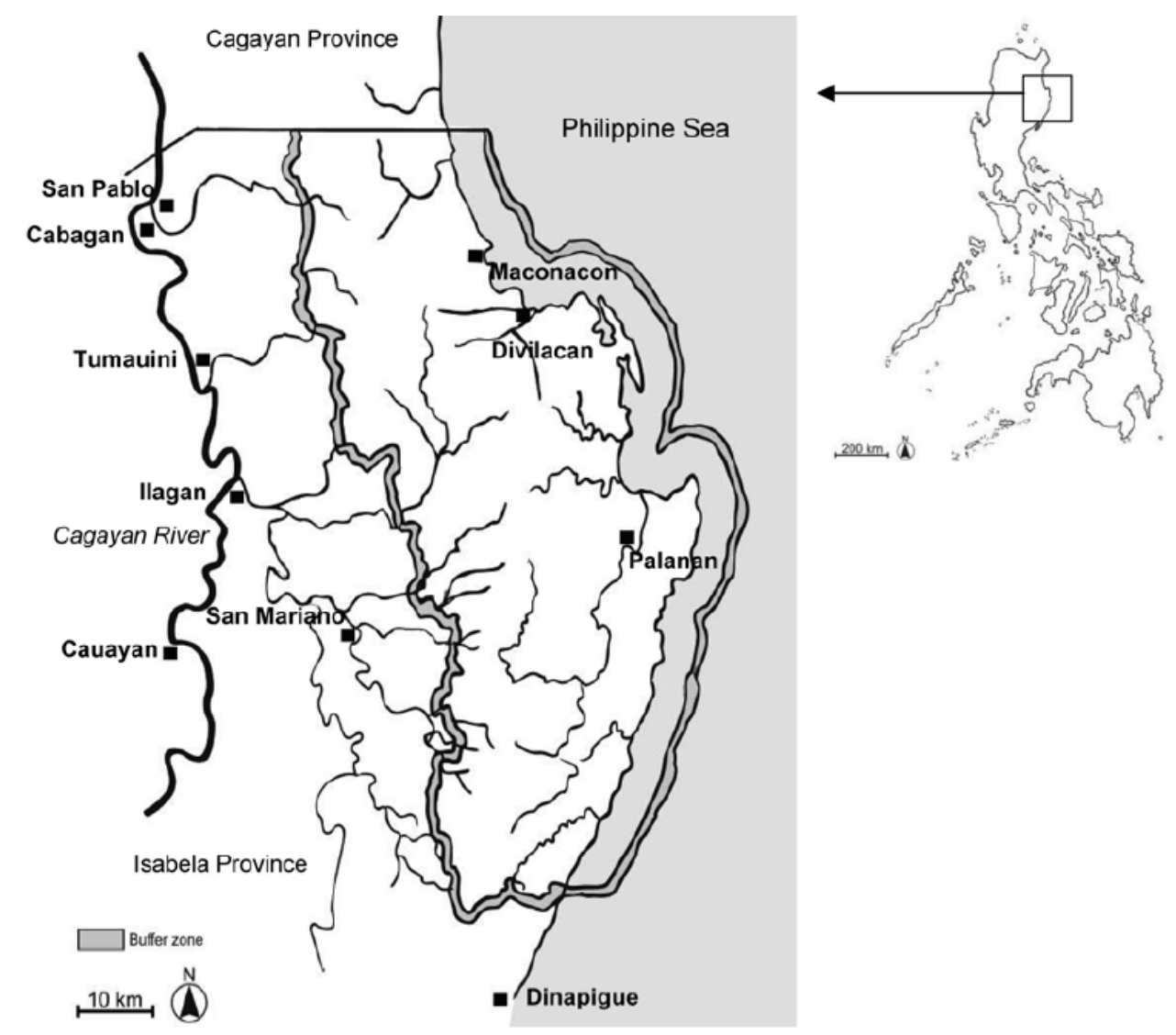

Figure 4.1: Map of the Sierra Madre, Isabela. Thick line represents the National Park boundaries (NSMNP). The Palanan Agta reside within its boundaries. From Minter (2010: 2).

There are around 1,800 Agta living within the Northern Sierra Madre Natural Park (NSMNP) found in Isabela Province, the second largest administrative district located in the north east of Luzon (Figure 4.1). The NSMNP is a the largest protected zone in the Philippines, covering around 360,000 hectares and includes mountainous tropical rainforest, river watersheds and the beaches, coral reefs and diverse marine eco-system of 
the Pacific Ocean (Minter 2010). This study focuses on a population of 1000 Agta living within Palanan. During 2013-2014 we met 914 of these Agta residing in a total of 20 mobile camps (Figure 4.2). As discussed by Rai (1990) the Agta throughout the region form distinct linguistic groups, broadly adhering to municipal lines. Thus, there is in fact limited interaction between the Agta living in San Mariano (Rai 1990), Cagayan (Griffin \& Griffin 1992), Maconacon (Griffin \& Griffin 1997) and Casiguran (Early \& Headland 1998; Headland et al. 1989; Headland 1989). From our own records, we find that marriage between these groups is rare. This perhaps is not surprising since many Agta do not know Tagalog, the national language of the Philippines, making communication difficult between linguistic groups.

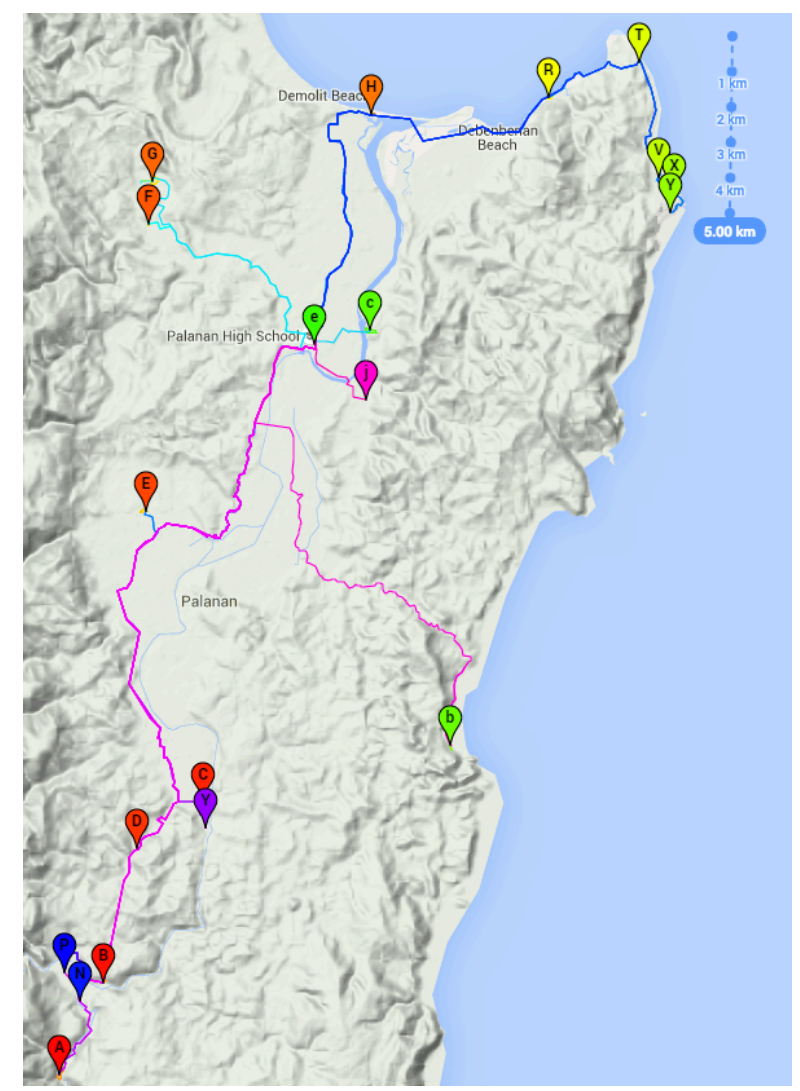

Figure 4.2: Close up of Palanan and Agta camps. Markers are each camp we visited during our fieldwork. The lines are the major routes taken from these camps to Palanan.

Some of the best-known literature on the Agta stems from groups living in Casiguran, to the south of Palanan and the NSMNP. Early and Headland (1998) and Headland (1989) demographic data reveals the Casiguran Agta to be at the extremes of known rates of 
infant mortality, homicide and life span, revealing a rapidly declining population $(1.2 \%$ per year between 1936 to 1976). In part, Headland argues that this is the result of external forces in the area with the rise of migrants, logging, mining and other extractive exploits (Headland 1984). These events were related to the construction of a road in 1977 to Dilasag, bringing an influx of violence, alcoholism, homicide and environmental and subsistence loss (caused by land grabbing, fishing with poisons and dynamite as well as land clearing (Early \& Headland 1998)). Comparatively, the Agta of Palanan avoided this turmoil and social upheaval. No road joins Palanan to 'the mainland', as locals refer to the rest of Luzon. The only access to the interior of the NSMNP remains an irregular boat service from Dilasag (depending on weather conditions) or an expensive light-air craft journey across the mountains (also dependent on the weather and pilot availability). Therefore, the area has remained undeveloped, and there remains a minimal presence of logging and mining in the area (Minter, Personal communication).

\subsection{Demographics}

There are 914 Agta living in Palanan at the time of this study in 2013-2014. The sex ratio appears significantly skewed at 117.6 (one-side proportion test $\mathrm{x}^{2}=5.8, p=0.02,95 \% \mathrm{CI}$ $[0.51,0.57])$. The population pyramid below (Figure 4.3) reveals that this skew is mainly caused by higher proportion of males in the under-five age category, that has a sex ratio of $141.3\left(n=222, \mathrm{x}^{2}=6.2, p=0.01,95 \%\right.$ CI $\left.[0.52,0.65]\right)$. Accordingly, if the under-fives are removed from the sample, males no longer out number females $\left(n=692, \mathrm{x}^{2}=1.8, p=0.2\right.$, $95 \%$ CI $[0.49,0.56])$. Any remaining skew is the product of more males surviving beyond 55 than females (ratio of 250). Thus, during the reproductive years $(16-55)$ the sex ratio is in fact 1:1. Similarly male skewed ratios from birth have been reported among the Ache (125, Hill and Hurtado, 1996) and the Hiwi at 117 (Hurtado \& Hill 1987) which may be influenced by male-specific mortality rates. If, as Fisher's (1930) observation states more males die before reaching maturity, the males who actually survive to reproduce experience significantly higher reproductive success given the lack of competition. As a result, there would exist selective pressure on producing more males than girls at birth. Thus, male biased sex ratios during infancy, as evident among the Agta, may be the result of a compensation for higher male mortality rates, a hypothesis supported by the absence of a biased sex ratio after the $1-5$ age group. 


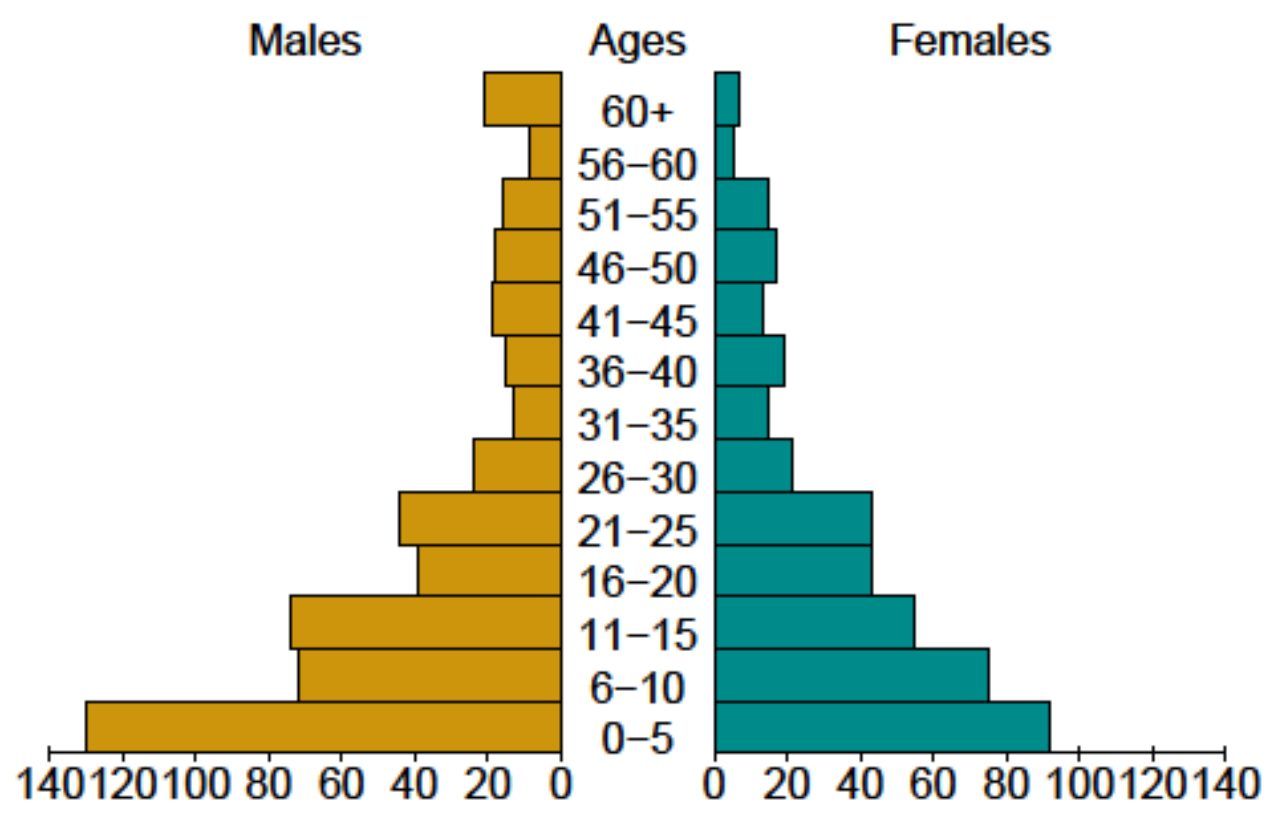

Figure 4.3: Population pyramid for 914 Agta censured during 2013-2014 separated by sex and 5 year age groups.

\subsubsection{Marriage and fertility}

Minter (2010) finds that marriage normally occurs at age 22 for men and 19 for women. Early and Headland (1998) argue this occurs soon after menarche at age 17, however our preliminary data suggests that $50 \%$ of Agta teens have reached menarche at age 13.8, indicative of a significantly earlier entry into reproduction. Nonetheless, the mean age at first birth is 20.4 among the Casiguran Agta, similar to our own figure at 20.2 years.

Previous estimates of Agta fertility has placed them at the higher end of the foraging fertility spectrum, for instance, Headland (1988) finds a total fertility rate (TFR) of 6.3 among the Agta from Casiguran and 5.9 from Agta of Palanan. Similarly Early and Headland (1998) report a TFR of 7 for the San Ifendo Pensiula Agta, while Goodman et al. (1985) find TFR of 6.5 among the Cagayan Agta to the North. This is high compared to estimates for the Batek (5.2), Batak (3.7), Efe (2.6), Mbuti (5.0) and !Kung (4.1-4.7) (Kelly, 2013 and references within). Accordingly, we find a period TFR of 7.7 based on births occurring during 2013-2014, one of the highest reported for hunter-gatherers (Kramer (2008) reports a TFR for the Pumé of 7.8 while Hill and Hurtado (1996) report a TFR of 8 for Ache still residing in the forest). Our sample contains 27 post-reproductive females (aged 50 or over), and their completed family sizes are significantly lower than the TFR at 6.13. This may be the consequence of under-reporting by older women, or perhaps 
indicates a more recent increase in fertility, driven perhaps by recent emphasis on reducing mobility; the TFR for women living in a settled camp was significantly higher than the TRF for women living in a mobile camp, 7.7 versus 6.6 , respectively.

\subsubsection{Life expectancy and Mortality}

Minter (2010) finds life expectancy from birth to be at 25 years, due in part to extremely high childhood mortality rates: 283 per 1000 live births based on reproductive histories with 159 households. Headland finds infant mortality rates of $34-37 \%$, and childhood mortality (under 15 years) at 49-50\% of all live births in Agta from Casiguran (Headland 1988; Early \& Headland 1998). Comparing these results with our data, it appears the infant and child mortality rates are lower. Out of 520 reported live births during reproductive histories with 124 women, $19 \%$ children were reported to die before age one, $13.9 \%$ between the ages one to five and 6\% between five to fifteen years. This means the Agta experience a childhood mortality rate of 388.5 per 1000 live births. Including later term miscarriages and stillbirths reveals that $17.9 \%$ of 592 conceptions do not result in a live birth, increasing the total mortality rate to 15 years to 520.3 per 1000 live births. Thus, whilst lower than previously reported the Agta can still be considered a high mortality population.

Table 4.1: Major causes of mortality for 427 cases reported during family histories. RTI stands for respiratory tract infection, GID for gastrointestinal disease, neonatal for all cases of infant mortality soon after birth for unknown reasons.

\begin{tabular}{lccc}
\hline Cause of death & $\boldsymbol{n}$ & $\mathbf{\%}$ & Cum \\
\hline Unknown & 71 & 0.17 & 0.17 \\
Violence & 10 & 0.02 & 0.19 \\
Malaria & 26 & 0.06 & 0.25 \\
Tuberculosis & 35 & 0.08 & 0.33 \\
Accident & 21 & 0.05 & 0.38 \\
Old Age & 23 & 0.05 & 0.44 \\
RTI & 36 & 0.08 & 0.52 \\
Childbirth & 51 & 0.12 & 0.64 \\
GID & 35 & 0.08 & 0.72 \\
Other disease & 56 & 0.13 & 0.85 \\
Measles & 13 & 0.03 & 0.88 \\
Neonatal & 31 & 0.07 & 0.96 \\
Alcoholism & 19 & 0.04 & 1.00 \\
\hline
\end{tabular}




\subsubsection{Causes of mortality}

The major causes of mortality among the Agta are similar to those reported in other foraging groups (Hill \& Hurtado 1996; Hill et al. 2007; Gurven et al. 2007; Howell 1979; Headland 1989; Early \& Headland 1998; Minter 2010; Froment 2014) and include several respiratory diseases such as tuberculosis (TB) and pneumonia, as well as gastrointestinal infections and malaria. In our full sample of 427 deaths reported during family histories from direct family members, infectious disease accounted for $86 \%$ of cases. A full breakdown of causes of mortality are reported in Table 4.1, and match reports from both Minter (2010) and Early and Headland (1998) in neighbouring Agta populations.

Table 4.2: Major causes of childhood mortality for 202 cases broken down by age. RTI stands for respiratory tract infection, GID for gastrointestinal disease, neonatal for all cases of infant mortality soon after birth for unknown reasons.

\begin{tabular}{l|cc|ccccc}
\hline \multicolumn{1}{c}{} & \multicolumn{2}{c}{$<$ 1 year old } & \multicolumn{2}{c}{$\mathbf{1 - 1 5}$ years } & \multicolumn{2}{c}{$<$ 15 years } \\
\cline { 2 - 7 } & $\boldsymbol{n}$ & $\mathbf{\%}$ & $\boldsymbol{n}$ & $\mathbf{\%}$ & $\boldsymbol{n}$ & $\mathbf{\%}$ \\
\hline Malaria & 9 & 0.09 & 15 & 0.15 & 24 & 0.12 \\
Hepatitis & 0 & 0.00 & 5 & 0.05 & 5 & 0.02 \\
RTI & 20 & 0.20 & 13 & 0.13 & 33 & 0.16 \\
GID & 8 & 0.08 & 16 & 0.16 & 24 & 0.12 \\
Other disease & 0 & 0.00 & 14 & 0.14 & 3 & 0.01 \\
Measles & 10 & 0.10 & 22 & 0.21 & 32 & 0.16 \\
Subi-subi & 17 & 0.17 & 3 & 0.03 & 20 & 0.10 \\
Accident & 4 & 0.04 & 15 & 0.15 & 19 & 0.09 \\
Infection & 6 & 0.06 & 0 & 0.00 & 6 & 0.03 \\
Neonatal/Unknown & 25 & 0.25 & 0 & 0.00 & 36 & 0.18 \\
\hline
\end{tabular}

The distribution of causes of death varies significantly according to the age of the individual. If we take the 202 childhood deaths, we see that accidents (15\%) become an increasingly important cause of mortality in children aged one to fifteen years while RTI $(20 \%)$ and nutritional conditions such as 'subi-subi' (17\%) are major causes of mortality in the under one's (Table 4.2). Subi-subi is caused by a thiamine deficiency, and otherwise known as beri-beri (Minter, 2010). Malnutrition has a role to play in many of these deaths. In a nutritional study among Casiguran Agta, De Souza (2006) found that 34\% of Agta 
adults were undernourished while $10 \%$ were extremely malnourished. This suggests that chronic under nutrition is a significant secondary factor in infectious disease mortality.

\subsubsection{Skewed mortality sex ratios}

One possible explanation for the extreme male-bias in the 1-5 year category above is the occurrence of sex-specific mortality rates in the under-fives. Looking at only infant (aged under one) mortality figures, there is no significant sex bias the mortality results, as females account for $54 \%$ of deaths. However, there is a significant bias in the 1-5 age category, with a mortality sex ratio of 170.6 (Table 4.3), substantially higher than the 1-5 sex ratio (141.3). This reduces slightly during the 5 - 15 ages to 118.8. In adulthood, mortality becomes skewed towards females with a ratio of 0.8 , likely due to the risks associated with childbirth, explaining why substantially fewer women survive to age 55 and beyond. A significantly higher proportion of male mortality is associated with accidents (mortality sex ratio 5), and virulent infectious diseases (malaria: 4, hepatitis: 2, RTI: 3 and measles: 4). Otherwise there seems to a significant nutritional element to female dominated causes of death (subi-subi: 0.33 and GID: 0.5). Therefore, these results indicate that accidents are a far greater cause of mortality for boys, likely due to their early adventures in the sea, river and trees. It also indicates they are either more likely to catch infectious diseases, are more susceptible to them or receive differential care from parents. However, the mortality skews are likely affected by the small sample sizes per cause of mortality in the 1-5 age group. Nonetheless, for all causes of mortality the sample is larger $(n=144)$, suggesting that males are far more likely to die during the ages 1-15 years, as such this may indicate an adaptive reason for such an extremely skewed sex ratio from birth.

Table 4.3: Sex ratio of mortality rates by cause of death, $n=144$.

\begin{tabular}{ccc|cc}
\hline Cause of death & $\mathbf{1 ~ - 5}$ years & $\boldsymbol{n}$ & $\mathbf{0 ~ - ~ 1 5 ~ y e a r s ~}$ & $\boldsymbol{n}$ \\
\hline Malaria & 4.0 & 5 & 1.43 & 17 \\
Hepatitis & 2.0 & 3 & 0.67 & 5 \\
RTI & 3.0 & 4 & 0.75 & 21 \\
GID & 0.5 & 6 & 1.17 & 13 \\
Other & 1.0 & 5 & 0.81 & 29 \\
Measles & 4.0 & 10 & 2.0 & 21 \\
Subi-subi & 0.33 & 4 & 0.7 & 17 \\
Accident & 5.0 & 6 & 2.67 & 11 \\
Unknown & 2.0 & 3 & 2.0 & 9 \\
Total & 1.71 & 46 & 1.15 & 144 \\
\hline
\end{tabular}


In our whole sample, other 'social' causes of death such as violence $(2.3 \%)$ and alcoholism (4.4\%) appear much less important than infectious disease or accidents. If we take the adult only sample $(n=108)$ we find 6 cases of death associated with alcoholism $(5.5 \%)$ and 8 cases associated with violence (7.4\%). Demographic data on 2,953 individuals included in the Agta genealogy (ranging from roughly 1920 to today) reveal that only 10 homicides have occurred over the last 90 years, producing an effective homicide rate of 3.39. This is significantly lower than the rates reported for both the San Ildefonso Agta (129 per 1000 or 11 homicide since 1950 (Early \& Headland 1998) and 326 in the Casiguran Agta (Headland 1989)). Similarly, Minter (2010) finds the influence of violence to be less manifest' among the Agta of the NSMNP. This is likely linked to the isolation of Palanan from the negative influences of logging and mining, and external population pressures (such as land grabbing). This rate puts the Palanan Agta to a violence level much closer to that found in the Batek (Endicott \& Endicott 2008) and Hadza (Marlowe 2010).

In his 1989 paper on 'population decline' Headland argues that the majority of Agta adults from Casiguran are alcoholics. We did not witness such extreme rates of drinking during our fieldwork in Palanan. Alcohol consumption was limited to a few households, never the majority. Nonetheless, the Agta attribute $4 \%$ of deaths to alcohol-related causes, not too far from the 6\% Headland found in Casiguran. This mismatch between the witnessed levels of alcohol consumption and reported causes of alcohol-related deaths could stem from either (i) the Agta citing alcohol as the cause of death when they do not know and/or felt an individual was of poor 'moral' character or (ii) as our mortality records data back 90 years, perhaps alcohol consumption was higher historically when Headland was conducting his fieldwork. However, the difference in social cohesion between the two populations at Casiguran and Palanan suggests that the Palanan Agta have never experienced such high rates of alcoholism, and I believe scenario (i) to be much more likely.

\subsection{Kinship and Residence Patterns}

Most authors portray the Agta as following a bilateral or cognatic descent system, in which ego gives equal weight to both lines of descent (i.e. matrilineal or patrilineal, Headland 1987; Rai 1990; Griffin 1996; Minter 2010)). Having a large but flexible kin base is important as it allows access to collectively held land (Minter 2010), a common feature in many immediate-return foraging societies (Kelly 2013). 
On average, households move once every 10 days, but this varies according to the camp. This figure was established from individuals' presence in daily camp scans; each day households were recorded as there or away. Some households moved regularly between nearby sites (a trend noted by Peterson 1978), while some never moved. When this data is transformed into a binary variable of either moved once or never moved we find that $27.5 \%$ out of 444 households were witnessed to move camp at least once. Rai (1990) finds among the Disabungan Agta that households moved once every 18 days in the late 1970's, while during the summer months Minter (2010) witnessed households to change camp as much as once every couple of days. Families cited a number of reasons for their move, often involving visiting missed relatives, seasonal fishing grounds, increasing social tensions and a dislike of the camp they were in. Often, individuals would make short distance moves because their previous location was dirty or smelly or families moved just because they liked to be mobile. The most mobile of individuals were teenagers, who would often go further afield visiting relatives without their immediate family. This trend is likely related to seeking out suitable marriage partners. Marriage is prohibited within individual's own specific area (often referring to three to five camps spanning a relatively short distance) as the Agta are exogenous and avoid marriage with close kin. Marriage partners are most commonly found in neighbouring areas that have a few, but significantly less distant kin links (Minter 2010).

Marriages are monogamous and they appear highly stable. Marriage more commonly end due to death than divorce. Headland (1987) discusses a period of brideservice occurring in Casiguran Agta that resulted in a period of matrilocal residence, after which the young family reverts to patrilocality. However, Peterson (1978) and Rai (1990) both argue that there is a matrilineal preference, leading to families joining the wife's family. This debate is a common one in anthropology, as the traditional view, that foragers are often patrilocal to best facilitate cooperative hunting, defence or warfare, appears hard to support given the cross-cultural evidence (Marlowe 2004). In part, the lack of clarity on this topic is due to hunter-gatherers ambilocal or bilocal residence pattern. As they are highly mobile and do not follow a unilineal descent system they do not need one set residence rule. Rather, they live with both the maternal and paternal kin depending on their needs (i.e. childcare early in life might simulate a matrilocal bias) and environmental conditions (i.e. good fishing where paternal kin might cause patrilocality). Therefore, it is more accurate to examine residence systems across a whole life course which demonstrates the majority of warm-climate 
foragers (68.8\%) are bilocal (Marlowe 2004). This trend is found in the Palanan Agta, who live with both matrilineal and patrilineal kin without particular bias (Dyble et al. 2015).

\subsection{Camps and their mobility}

The nature of camps varies according to location and degree of acculturalisation and cultivation. The Agta reside in broadly three types of camps. The most mobile of these are comprised of lean-tos, temporary shelters which are constructed in less than an hour, and frequently moved according to rain, wind and sun (Figure 4.4). These shelters are made of crossed poles and palm leaves for roofing, thus are commonly abandoned (Rai 1990; Peterson 1978). These type of camps do not have a church or water pump, but rather are based around a fresh water source. The composition of these camps changes frequently, both in terms of the number, position and location of structures and the individuals residing within the camp. New individuals arrive and leave every couple of days.

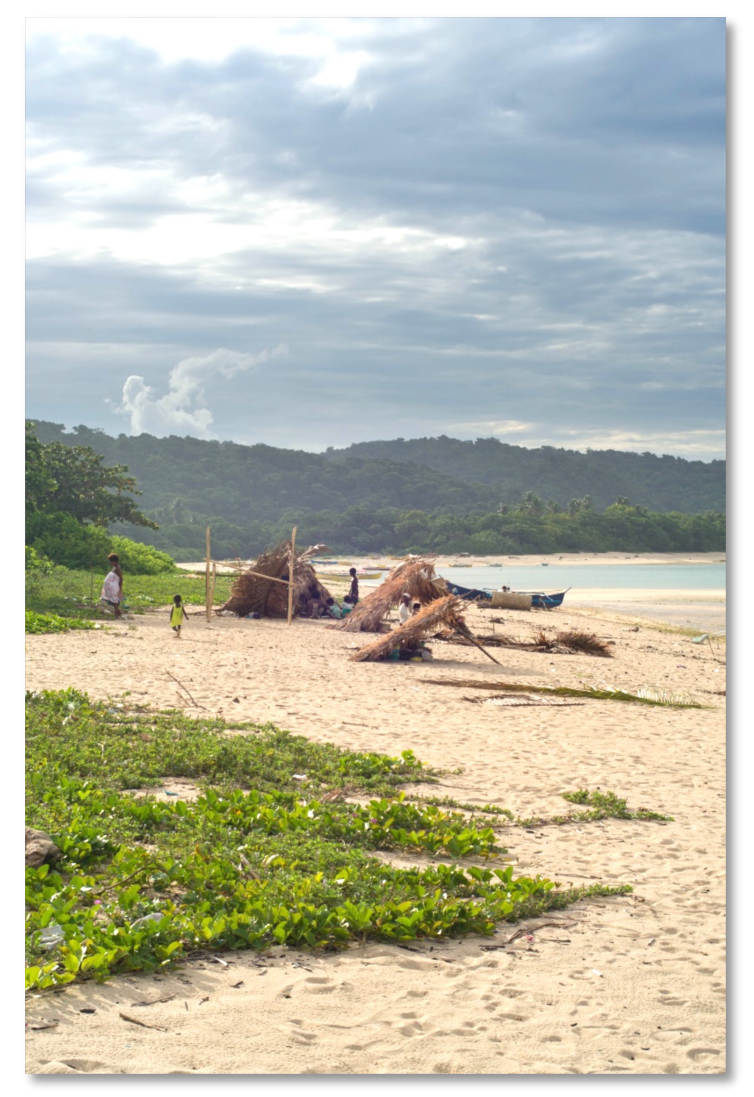

Figure 4.4: Temporal Agta camp with lean-tos 
A semi-permanent camp often contains a mixture of lean-tos and more permanent buildings, perhaps with a water pump. The more permanent type of Agta shelters are still built over a matter of days, consisting of natural materials. However, these are moved less often and commonly expanded or altered rather than abandoned. Finally, permanent camps have often been built around a church, garden or water pump and mainly consist of houses made by the Philippine government, which are made of wooden planks and iron roofs (Figure 4.5). These houses remain unaltered but individuals living in them may change.

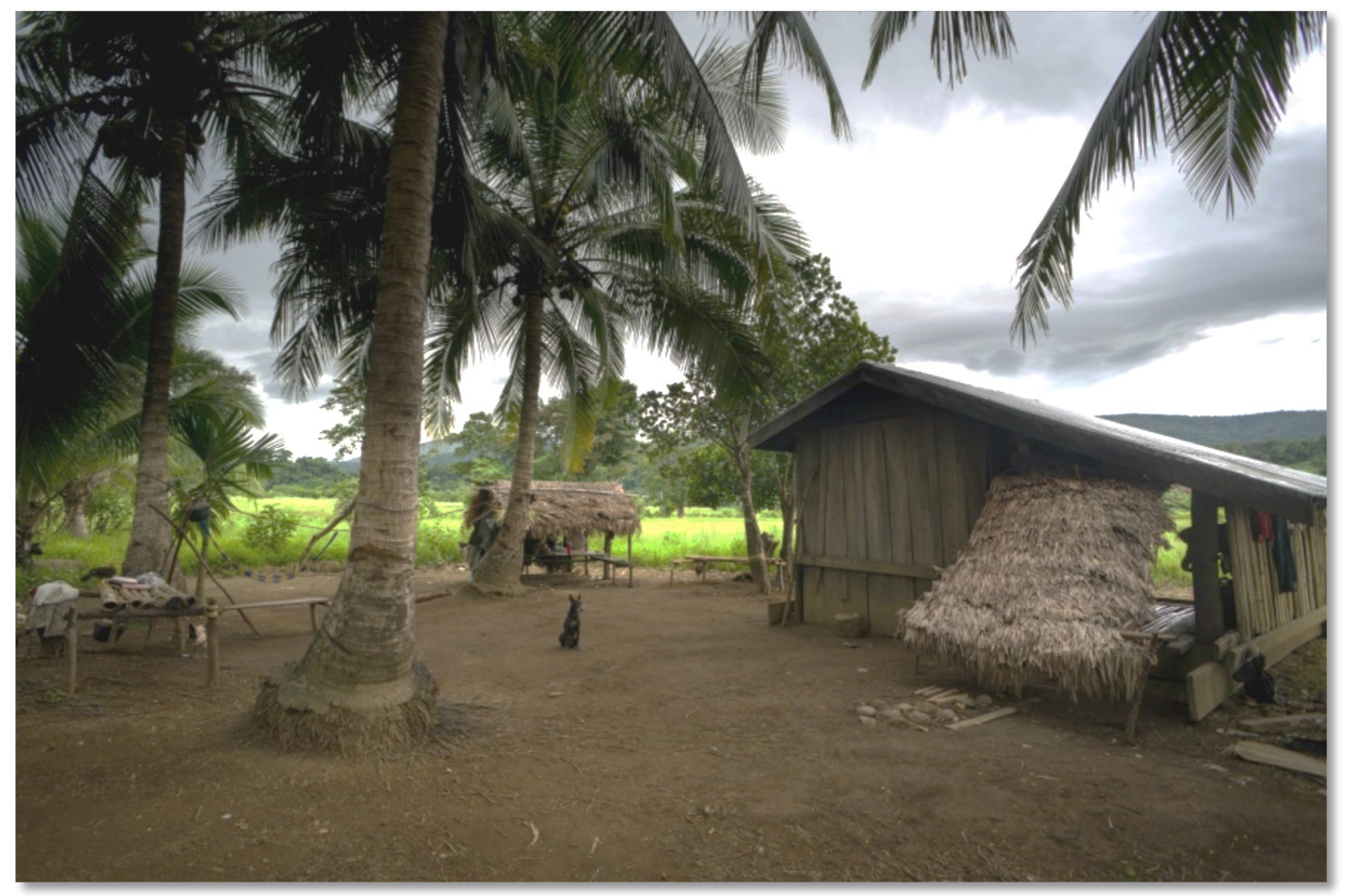

Figure 4.5: Permanent Agta camp with a wooden house.

During Minter's fieldwork in 2002-2005 only one of the camps in Palanan was heavily settled, now this trend has extended and at least four of 20 camps in our sample have churches, permanent housing and water pumps. Therefore, the average camp movements may have reduced in recent time (but without solid data this is difficult to establish) and more camps are becoming more permanent. Nonetheless, we find that $26.8 \%$ of Agta households were in mobile camps, comprised of temporal housing (lean-tos) without the presence of infrastructure such as toilets or water pumps, or the presence of a church. As 
we conducted our research collection over two years we were able to get a sense of the changing nature of camps, demonstrated in Figure 4.6. There is significant variability in the degree of mobility between camps, as some camps such as Djago, in the top of Figure 4.6, had $83 \%$ the same individuals living in the camp between two visits, while another camp had only $24 \%$. This figure ranged from $0 \%$ (when the camp was completely abandoned) to a maximum of $86 \%$ similarity. The mean figure was $0.59 \pm 0.23$.

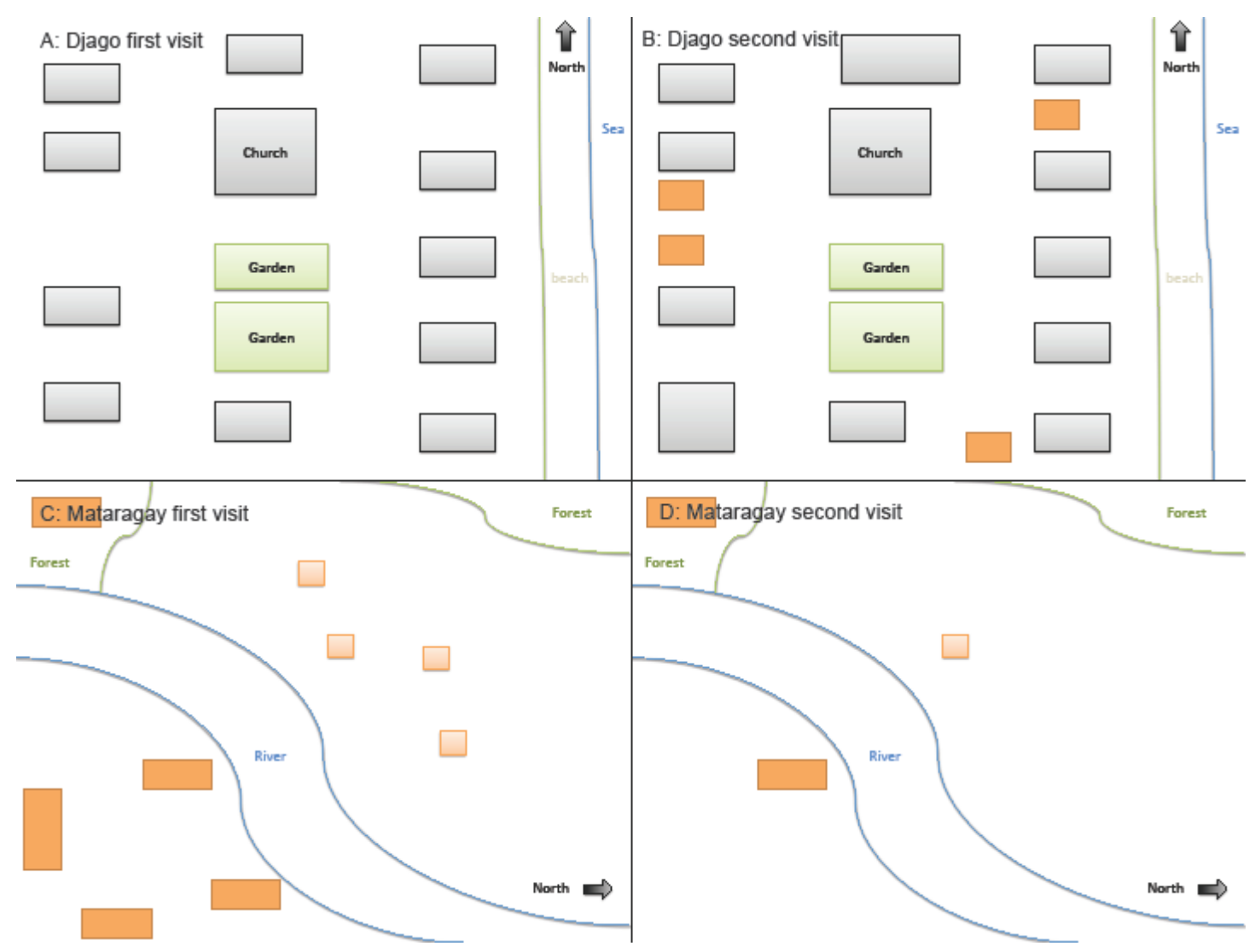

Figure 4.6: Two camps (A-B) Djago and (C-D) Mataragay between two visits (left first visit and right second). Black icons are wooden houses, dark orange are semipermanent Agta huts and pale orange are lean-tos. Any other key camp features are labelled on the maps.

\subsection{Subsistence and Diet}

The Agta are predominantly marine and river fisher-gatherers, as out of 2,168 work activities 53.6\% were spent fishing, 2.9\% hunting, and 20\% gathering. Non-foraging activities consist of cash labour (9.5\% of activities), cultivation (12\%) and trade $(2.1 \%)$. Thus, the Agta continue to rely heavily on foraging modes of subsistence $(76.5 \%)$ versus 
non-foraging activities (23.5\%). Nonetheless, this varies significantly according to gender, location (coastal or inland ecologies) and degree of sedentarisation.
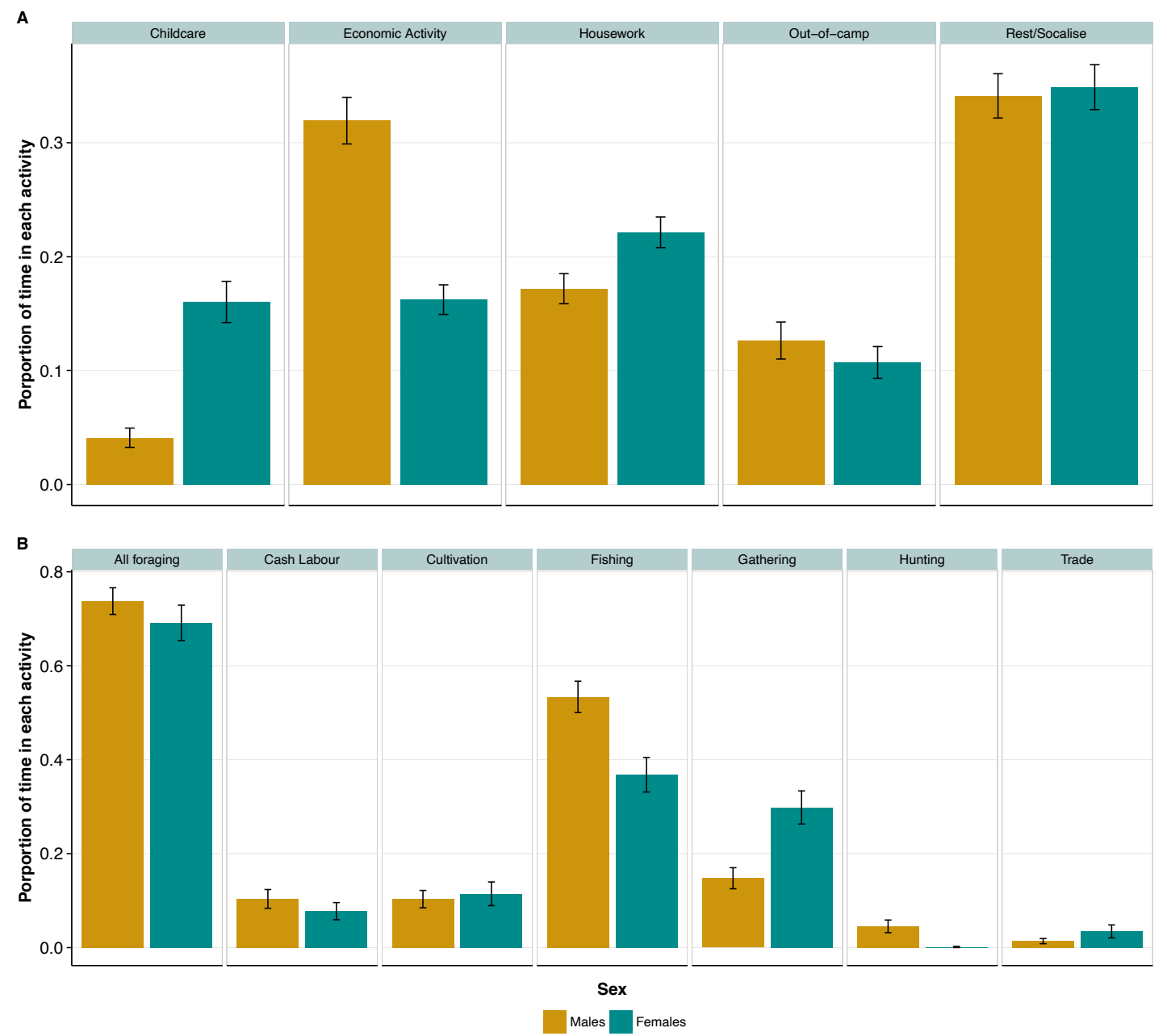

Figure 4.7: Summary data on (A) the proportion of time individuals spent in all activities and (B) proportion of time individuals spent in different types of economic activities, separated by sex. Here, and in the figures below, 'Economic activity' in plot A includes all activities broken down in plot B. 'All foraging' represents all fishing, gathering and hunting activities in opposition to all 'non-foraging' tasks which includes cash labour, cultivation and trade. In this case, this reveals that while males and females spent the same amount of economic activities foraging, males spend more time in fishing and foraging while women spend more time in gathering $(n=914)$. Error bars are standard errors of the mean.

\subsubsection{Division of labour}

The sexual division of labour among all human groups, including hunter-gatherers appears universal (Kelly 2013). Commonly cited reasons for this division include the 
incompatibility between hunting and childcare (Washburn \& Lancaster 1968; Lee 1968). Gathering is seen as more conducive to reproduction as children can be placed into a 'crèche', gathering can be put-on-hold when required and tubers and fruits are not scared away by the sound of children. This has led to the paradigm of 'man the hunter' and 'women the gatherer' in anthropological studies of foragers (Estiko \& Griffin 1981; Lee 1968). However, among the Agta of Cagayan, women have been historically known to hunt habitually, along with reports in the Mbuti, Tiwi and Ainu (Goodman et al. 1985; Estiko \& Griffin 1981; Estioko-Griffin \& Griffin 1985). Estioko-Griffin and Griffin (1985) report six women hunting on 16 days after 163 observation-days, and found no significant difference in fertility (or fertility measures such as age at menarche) between women who hunted or not. Thus, Estioko-Griffin and Griffin conclude that hunting is compatible with normal fertility patterns. Such extensive female hunting has not been reported since among the Agta.

In our own data, hunting is significantly less important, and many men were never witnessed to hunt. Out of 2,168 work activities only one woman was witness to go hunting once, producing an effective female hunting rate of 0.001 (Figure 4.7B). Nonetheless, women do spend a significant amount of time fishing (36.5\%), catching octopus, crabs and other shellfish. Often these trips would consist of family day trip to a good fishing spot, where they would fish with their husband and older children. Women would also go fish alone or with other women, particularly younger, unmarried teenagers. There is still a gender bias in gathering however, as while males spend more of their work activities in fishing they spend less time gathering. Examining Figure 4.7A also reveals a significant difference between men and women. Women conduct far less subsistence related work activities in general, however maintain similar proportion of their time 'resting' (which includes socialising, sleeping and resting) as males. This is because women spend significantly higher proportion of their time involved in childcare activities (including carrying, playing or feeding as well as keeping an eye on children) and housework. Thus, the Agta match trends seen in many other populations in relation to the gendered division of labour (Kelly 2013).

\subsubsection{Ecological variability in diet and activity}

On average only $19.6 \%$ of food is produced from cultivation while the reminding $80.4 \%$ is produced by foraging activities (fishing, hunting and gathering), matching the activity data. 
However, this figure varies significantly according to the degree of settlement; settled camps produce less food by foraging $(78.4 \% \pm 5.7 \%)$ compared to mobile camps $(90.4 \%$ $\pm 9.2 \%$, Figure 4.8). Furthermore, there is significant ecological variability in these trends, depending on whether groups live at the coast or inland.
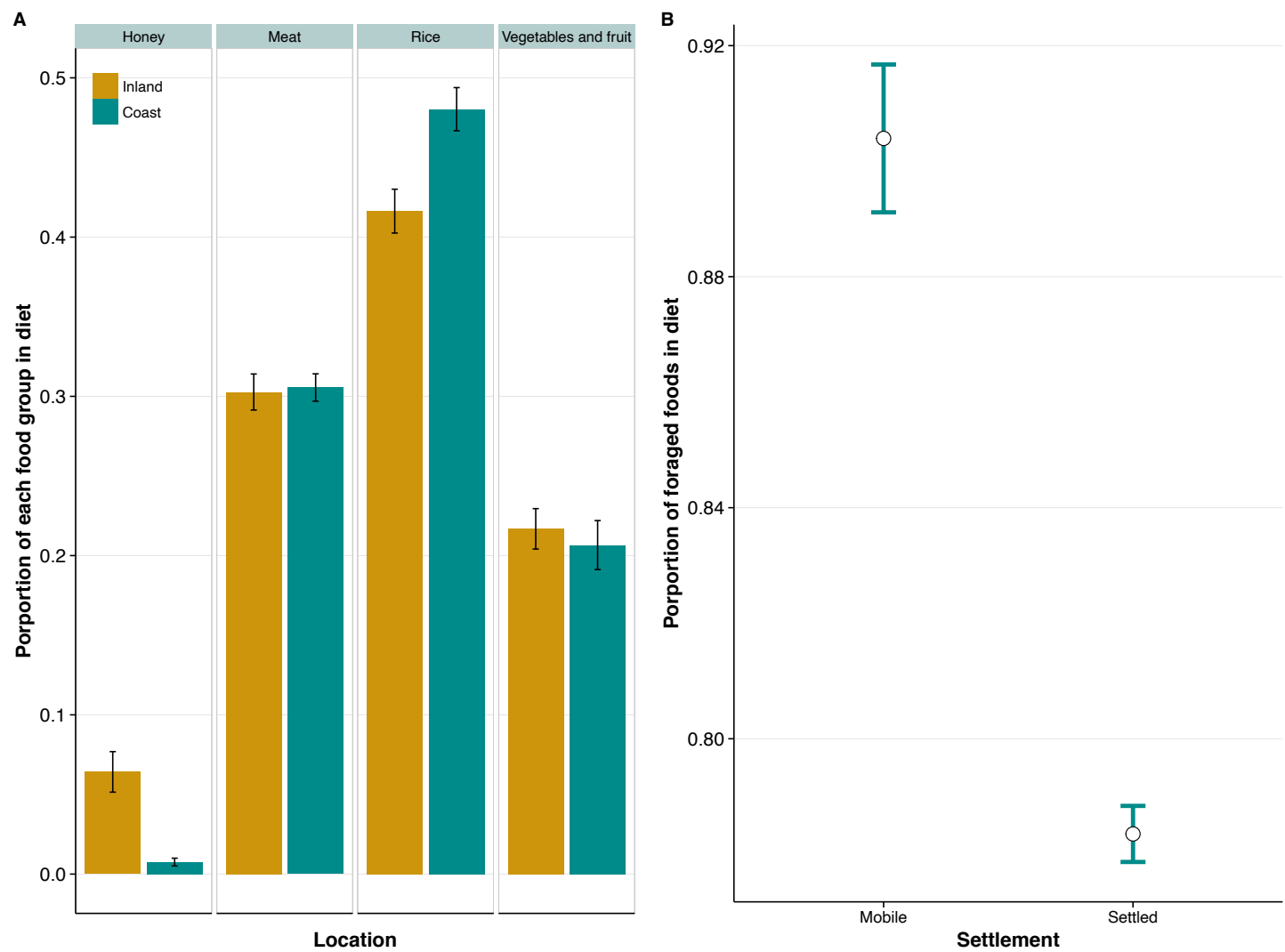

Figure 4.8: (A) proportion of each food group consumed depending on whether a household lived on location and (B) the proportion foods which were acquired via foraging (hunting, fishing or gathering) compared to cultivation or trade, depending on degree of settlement $(n=914)$. Error bars are standard errors of the mean.

\subsubsection{Coastal Agta}

The main task for males is spear fishing in the shallower coastal waters. Women also plant small swiddens in the treeline. Minter (2010) argues that the gathering of fruit or vegetable products does not seem a significant element in subsistence. Allen (1985) also noted the distinct lack of vegetables in the Agta diet. However, we see little variance in proportion of vegetables and fruit between inland and coastal Agta (Figure 4.8). What we do see is a significant increase in rice consumption in coastal groups. Since rice is often directly 
exchanged for fish and other marine resources, this increased rice consumption is likely reflective of a higher fishing return rate at the coast. Examining the breakdown of daily activities below by location (Figure 4.9A), it does appear that costal groups spend significantly longer resting, implying that the coast may be a more bountiful ecology. Figure 4.9B demonstrates that coastal groups spent more time fishing than cultivation, gathering and hunting suggesting they are focused on marine resources. These results are supported by foraging returns data (Table 4.4) as mean package size is $20.1 \%$ larger at the coast than inland, while fishing at the coast is significantly more effective as hourly returns are $62.2 \%$ higher. Thus, it is likely that at the coast foragers reach their daily needs faster, thus spend longer in other activities. Furthermore, it appears that coastal foraging trips were significantly less variable comparatively to inland trips; coefficient of variation at the coast represents only $49.5 \%$ and $62.3 \%$ of the inland variation in package size and the hourly calorie return rate, respectively. Thus, while the variability in foraging return rate is nonetheless high (the standard deviation is consistently larger than the mean), reflective of hunter-gatherer foraging niches in general (Kelly 2013), coastal environments appear relatively less variable.

Table 4.4: Foraging return rates separated by location. SD represents standard deviation, CV coefficient of variation. Data collected by Mark Dyble.

\begin{tabular}{cccccccc}
\hline Location & $\begin{array}{c}\text { Number } \\
\text { of trips }\end{array}$ & $\begin{array}{c}\text { Mean } \\
\text { package } \\
\text { size } \\
\text { (kcals) }\end{array}$ & $\begin{array}{c}\text { SD } \\
\text { package } \\
\text { size }\end{array}$ & $\begin{array}{c}\text { CV } \\
\text { package } \\
\text { size }\end{array}$ & $\begin{array}{c}\text { Mean } \\
\text { kcal } \\
\text { per } \\
\text { hour }\end{array}$ & $\begin{array}{c}\text { SD } \\
\text { kcal } \\
\text { per } \\
\text { hour }\end{array}$ & $\begin{array}{c}\text { CV } \\
\text { kcal } \\
\text { per } \\
\text { hour }\end{array}$ \\
\hline Costal Camp & 193 & 2322.7 & 3024.2 & 1203.47 & 704.7 & 901.4 & 58.56 \\
Inland camp & 99 & 1855.3 & 4874.5 & 1874.04 & 266.7 & 522.8 & 14.23 \\
\hline
\end{tabular}

\subsubsection{Watershed Agta}

The Agta living inland are more dependent on hunted resources. A good male hunter is highly valued as a husband and camp mate (Minter 2010: 112). Hunting has traditionally been with a bow and arrow but now also occurs with the more powerful homemade shotguns. However, shotguns are not always preferred due to their noise and the difficulty in reloading with speed compared to the silent and speedy bow and arrow. Hunted items include the long tailed macaque (Macaca fascicularis), Philippine warty pig (Sus philippinensis) 
and the Philippine brown deer (Cervus mariannus), as well as a number of bird and bat species that adults and children regularly shoot down as snacks.
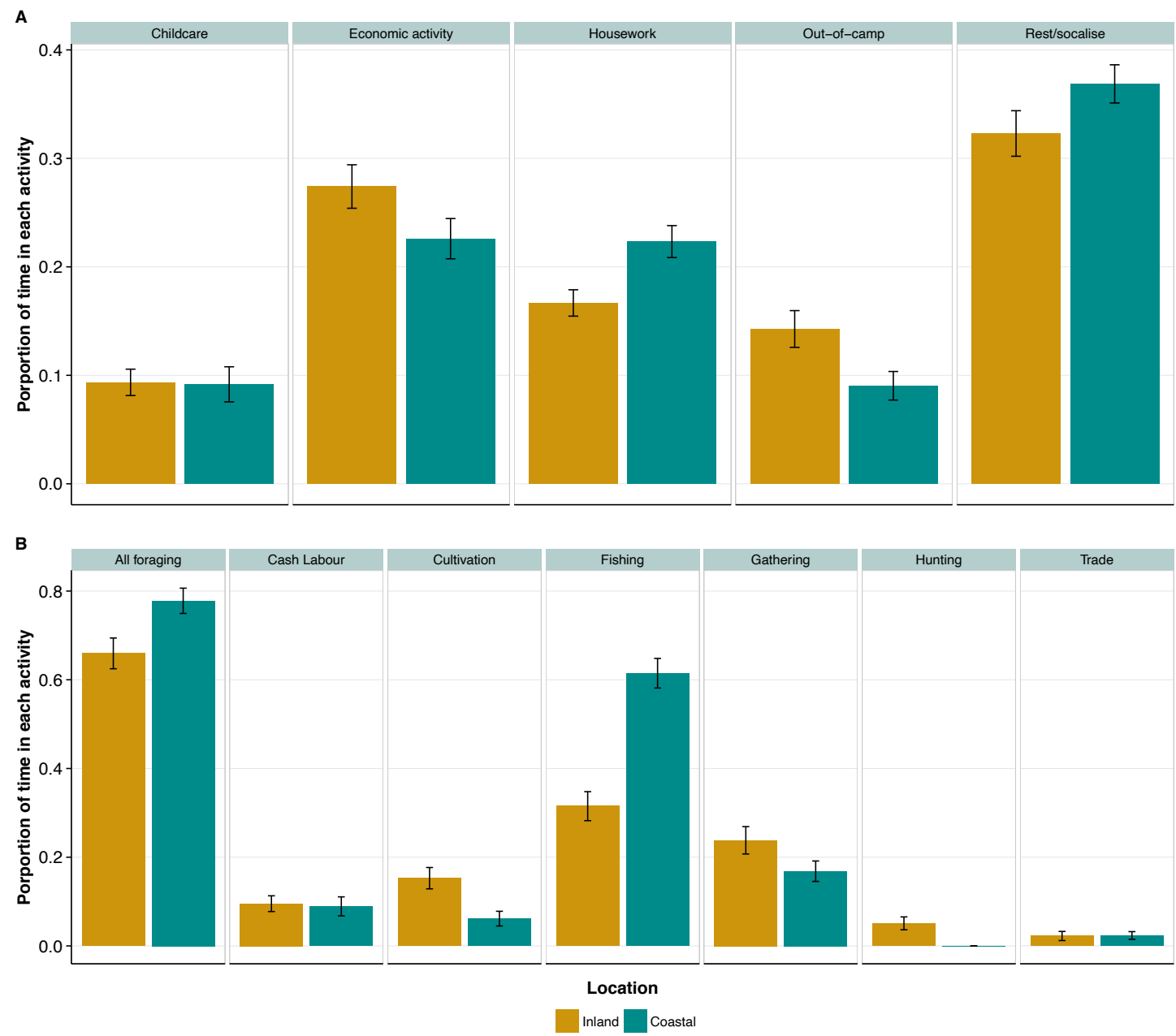

Figure 4.9: (A) the proportion of time individuals spent in different activities and (B) proportion of time individuals spent in different types of economic activities, separated by location $(n=914)$. Error bars are standard errors of the mean.

We witnessed no hunting in coastal groups while it consisted of $0.05 \pm 0.2$ of activities in inland groups. Thus, fishing is still a primary subsistence strategy $(0.32 \pm 0.4)$. Individuals living inland devote more time to gathering than their peers on the coast $(0.24 \pm 0.35$ versus $0.17 \pm 0.24$ ). Commonly gathered foods include fern shoots (pako; Deplazium esculentum) and the hearts and shoots of various palms and forest fruits. Inland groups also spend significantly more time in cultivation $(0.15 \pm 0.27$ versus $0.06 \pm 0.18)$. Minter (2010) finds similar trends, with inland groups tending to their own land a higher percentage of 
their time than coastal groups (0.08 versus 0.05$)$. This implies overall there is a significantly different subsistence strategy between these two groups, even when they are often as little as $10 \mathrm{~km}$ apart.

Minter (2010: 282) also argues that the coastal-dwelling Agta's diet are much richer in animal protein compared to the river groups; one coastal group she studied from Dimasalansan consumed animal protein at $79 \%$ of their meals $(n=90)$, compared to a river group in Diangu at $41 \%(n=102)$ and another watershed group in Disabungan who consumed animal products at $26 \%$ of meals $(n=51)$. We do not find this result in our data, however. It appears that coastal $(0.31 \pm 0.09)$ and inland Agta $(0.30 \pm 0.1)$ in Palanan consume remarkably similar proportions of animal protein in their diets.

\subsubsection{Variability by sedentarisation}

As argued by Minter (2010) the agricultural practices, and their economic importance, of the Agta has often been ignored. The literature portrays them as 'unsophisticated' or 'hobby' farmers as they gain few and irregular returns from their efforts (Estioko-Griffin \& Griffin 1981; Griffin 1985; Headland 1986; Rai 1982). However, in Minter's recent research she finds that both the domestication of animals and cultivation is becoming increasingly present, however varies greatly by location. Time spend in cultivating their own land (clearing, planting, tending and harvesting) ranged between $4-11 \%$ for males and $3-13 \%$ for females in three different environments (coastal, interior and watershed). From this work the crop yielded an average of $283 \mathrm{~kg}$ of rice per household, enough for 113 days given an average daily rice consumption of $2.5 \mathrm{~kg}$. Accordingly, we find that $13.1 \%$ of all activities were spent in cultivation. However, this figure not only varies by ecology but also degree of sedentarisation (Figure 4.10B). Mobile groups spend only 3.5\% of their work activities in cultivation and $0 \%$ of activities in cash labour and trade. Comparatively, settled groups spend $11.6 \%, 10 \%$ and $2.5 \%$ of their work activities in cultivation, cash labour and trade, respectively. As a result, while settled groups spent only $69.3 \% \pm 36.1 \%$ of time in 'foraging' activities (the combination of gathering, fishing and hunting), mobile groups spent a mean of $96.2 \% \pm 10.3 \%$. Interestingly, while settled groups appear to spend significantly less time in work activities overall $(0.24 \pm 0.2$ versus $0.33 \pm 0.29)$ this is not translated into greater rest time on average. The major increase is in time spent out-ofcamp for settled groups, which is often associated with going to the market to trade goods. Thus, while non-foraging activities may have higher returns (hence less time spent in food production itself), they do involve additional activities to exchange these items for foods or 
cash. As a result it seems that settled, mobile, inland and coastal Agta all follow different subsistence strategies.
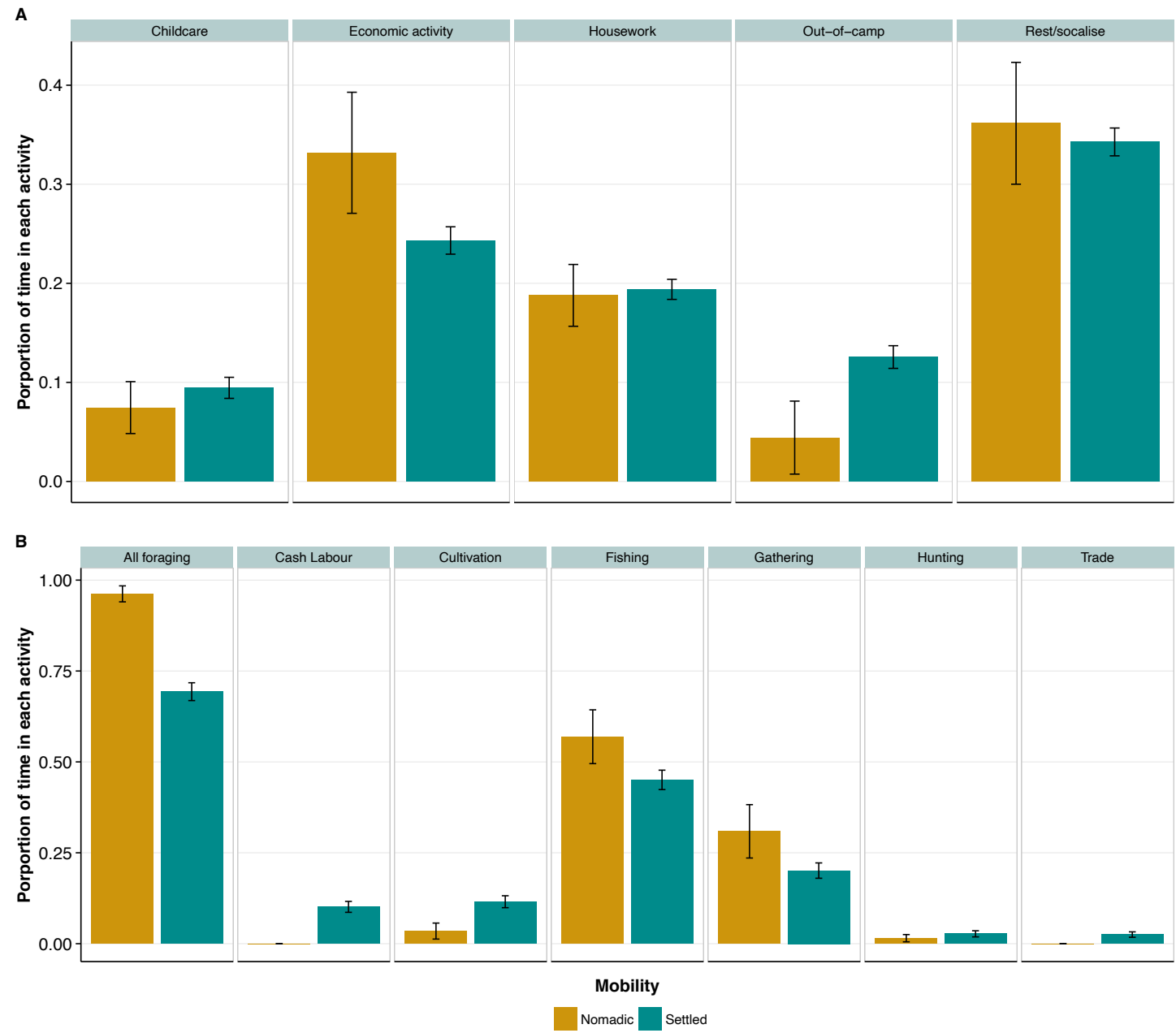

Figure 4.10: (A) the proportion of time individuals spent in different activities and (B) proportion of time individuals spent in different types of economic activities, separated by degree of mobility $(n=914)$. Mobility is denoted by whether they were witnessed to move camp or not at least once during the two-year field period. Error bars are standard errors of the mean.

\subsection{Wealth, camp permanence, food storage and security}

As previously discussed, immediate-return hunter-gatherers are often defined by a few highly connected traits, such as low levels of food storage and wealth, low camp permanence, and a high level of mobility. These features are all linked to the high mobility and fluidity of these groups as they extract what they require from the environment (Woodburn 1982; Smith et al. 2010). In particular many of these features have been 
associated with the ways of mitigating risk in the foraging niche (see section 2.4 on risk management). For instance, high mobility is a form of risk reduction as individuals spread their exposure to risk spatially. Depending on the degree of mobility, this strategy significantly limits other forms of dealing with risk. Wealth and food storage are forms of risk retention and only possible in more sedentarised communities. Additionally, as forms of risk retention are not possible, hunter-gatherers have been argued to focus more on risk transfer, including risk pooling (i.e. reciprocity). Extensive norms of sharing are often associated with egalitarianism, demand sharing and 'levelling' behaviours to ensure individual accumulation does not occur (Wiessner 1996; Gurven 2006; Cashdan 1985). Consequentially, risk pooling and risk retention are two methods of mitigating risk as individuals who accumulate wealth and/or food storage are in less need of risk pooling and often extract themselves from sharing networks (Fafchamps 1992). As a result specific traits are often highly associated with one another.

Examining the topic archaeologically, during the Neolithic revolution, traits commonly associated with increased cultivation and intensified agriculture include sedentarization, increases in wealth accumulation, camp permanence and camp size (Hayden 1995; Piperno \& Pearsall 1998). This is not to imply that this is a matter of progression or that sedentarization only occurs in the context of cultivation; settlement should occur when there is local abundance and the costs are lower for settling than moving to patches of lower productivity or unknown/unpredictable returns. Thus, cultivation is only one possible example of this scenario (Kelly, 2013). Historically, delayed returned huntergatherers were able to settle due to significant resource abundance, defendable resources, food storage and seasonality, such as North Western Native American populations. However, if cultivation increases an individual's resource return rate, reduces variability in returns and produces enough food from a single location to ensure survival, then it can result in decreased mobility. Sedentism can be either in the form of more permanent villages or reductions in individual movement between locations as the need to care for and defend cultivated land increases (Kelly 2013). Furthermore, cultivation results in food storage making mobility increasingly difficult. Thus, it is possible to consider these traits as different strategies to deal with variability and risk; if extensive food sharing and mobility are one form of insurance against risk then subsistence diversification, wealth accumulation and food storage are another. 


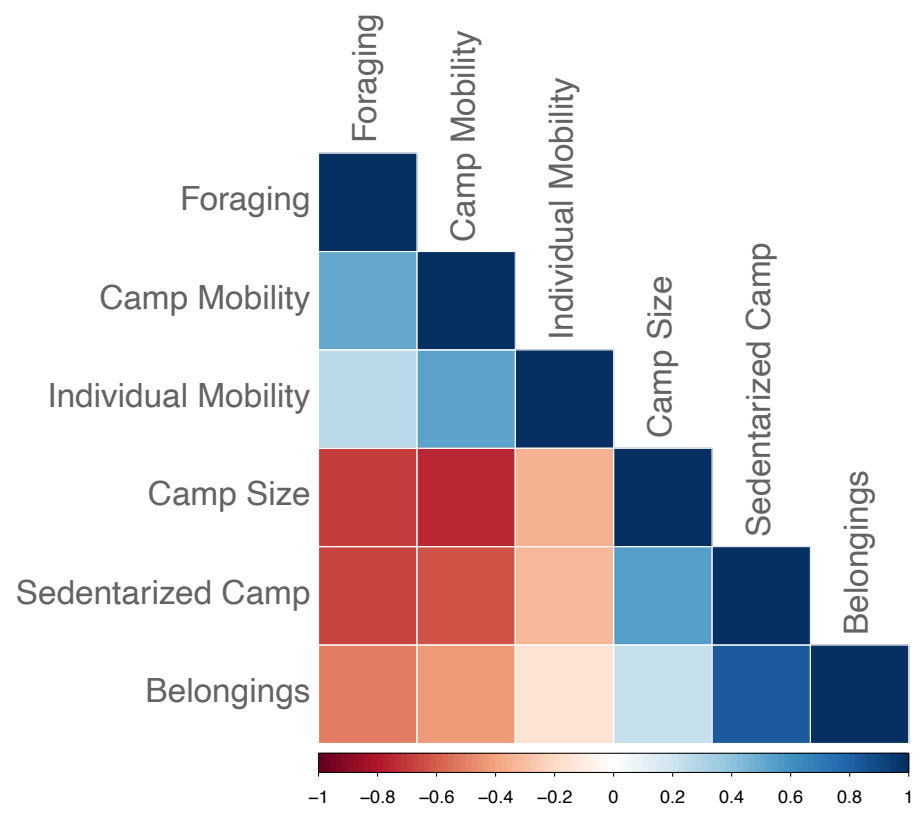

Figure 4.11: Correlation plot for six key 'transition' variables. Positive correlations are marked in blue shades, negative in red shades. The stronger the shade, the stronger the correlation, represented on the bar legend. All correlations are statistically significant at $p<0.05$. Variables are: sedentarised camp (number of permanent structures within a camp); belongings (an index quantifying household wealth); individual mobility (whether an individual was ever witnessed to move camp over a two-year period, $0=$ never moved); camp mobility (number of people leaving a camp); and foraging (proportion of food that individuals acquired from foraging activities versus food production and wage labour).

Figure 4.11 shows significant correlations between proportion of food produced from

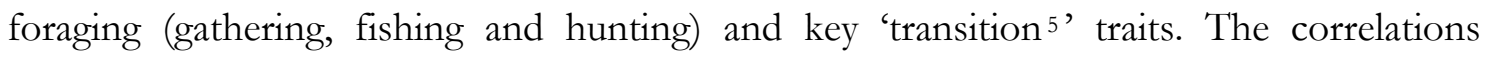
indicate that while extensive foraging, low camp size, high mobility and few household belongings characterize some Agta camps; others exhibit more food production, larger camp size, little mobility, and a greater number of household belongings. Furthermore, Figure 4.12A reveals that settled groups have significantly higher food storage, house permanence and household wealth. As argued by Kelly (2013), these traits should not create a binary system, rather there is extensive variance both between and within populations, which is demonstrated by the Agta in terms of significant variance of wealth, camp permanence and food storage depending on their ecology.

\footnotetext{
${ }^{5}$ I use the word 'transition' to refer to the process of change in mobility, subsistence, sharing and egalitarian ethos. Transition needs not be unidirectional, permanent or inevitable but dependent on ecological conditions following the principles of HBE.
} 

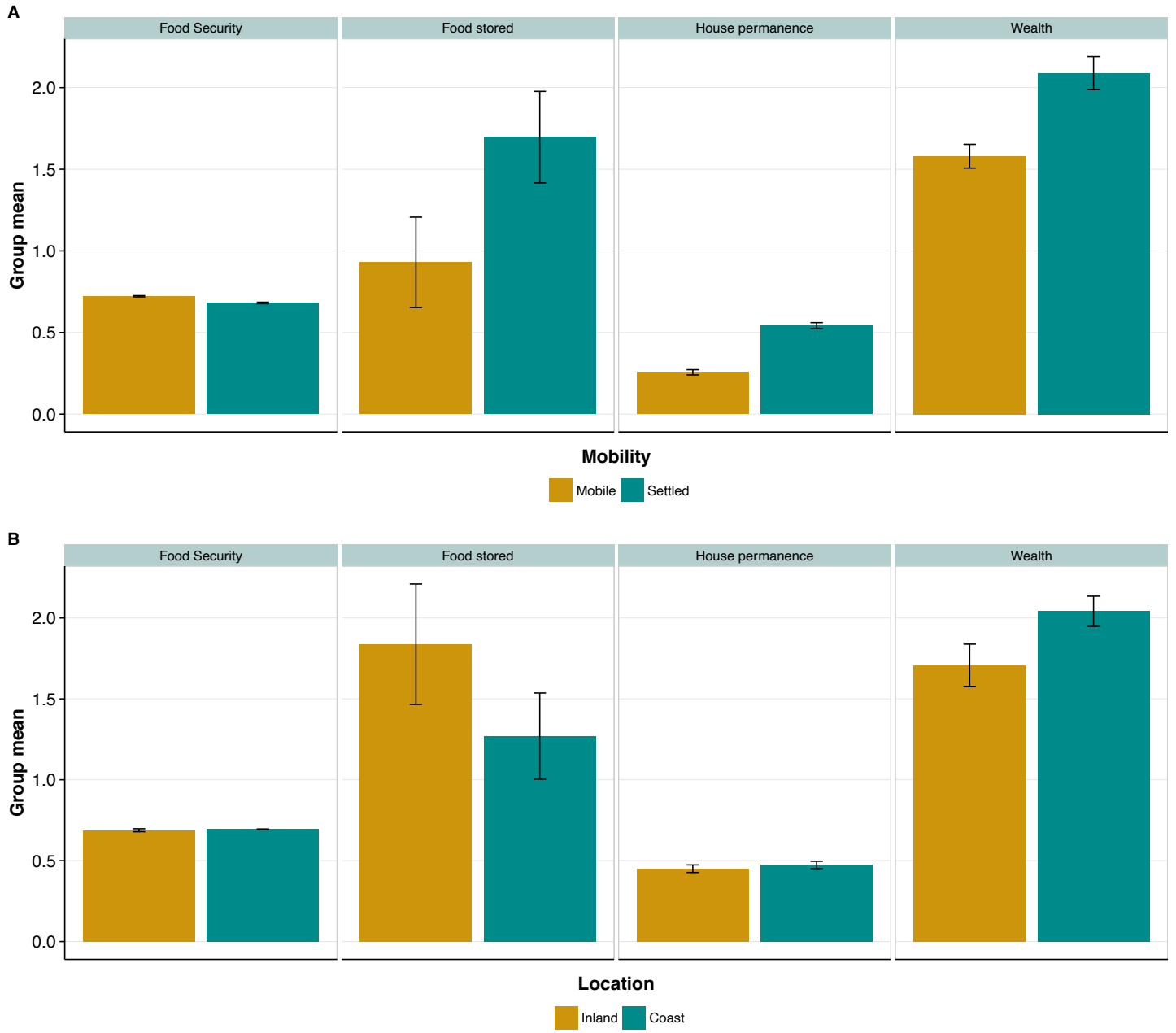

Figure 4.12: Group means for key traits associated with immediate return foragers separated by (A) mobility and (B) location. Error bars are standard errors of the mean $(n=914)$.

I have argued above that the coastal environment can be considered less risky given its higher return rate and more predictable resources given the lower coefficient of variation. As a result, we may expect individuals to report greater food insecurity inland. The fact that the levels on food insecurity are equal (Figure 4.12B) in both environments, indicates that individuals have successful buffers in place to mitigate shortfalls. For instance, above we saw that cultivation is greater inland than at the coast, as is food storage. Thus, perhaps inland groups are practicing risk reduction by subsistence diversification and storage. However, wealth is significantly higher at the coast, which is likely the reflection of increase trade at the coast; as marine returns are high (up to $7 \mathrm{~kg}$ in one trip), individuals frequently trade additional fish for cash and rice. 


\subsection{Transition versus resilience}

The ethnographic literature on the Agta is some of the most pessimistic, focusing on their extinction due to high mortality, increasing social ills (such as violence and alcoholism), encroachment and exploitation from 'outsiders' and deforestation (Minter 2010). Certainly, the Agta are not an example of an isolated, untouched tribe (but then if anthropology was to rely on this definition of foragers the field of hunter-gatherer studies would all but disappear). The assimilation of the Austronesian language family long before linguistic records indicates a history of long contact once farmers arrived between 5,000 and 3,500 years ago (Bellwood 2010). The archaeological record worldwide suggests that continuous and significant interactions between indigenous populations and farming migrants (Piperno \& Pearsall 1998; Bar-Yosef \& Meadow 1995; Price \& Gebauer 1995; Inomata et al. 2015). Thus, it is not necessarily the case that when foraging groups first come into contact with outsiders they are immediately replaced or wiped out. Nonetheless, in the rapidly developing, globalised world these pressures are significant and important to document to understand their consequences.

A high proportion of the ethnographic record on the Agta predicts their cultural loss and extinction. Rai's $(1990,1982)$ work focuses on the Agta's transition to a 'non-foraging way of life' and intensive dependence on non-traditional strategies of trade, cash labour and horticulture. Since this is argued to be in 'sharp contrast to their earlier stable system', it is portrayed as a progressive, linear move away from foraging subsistence. Rai (1990: 3) believes he is witnessing the last traditional activities as "time seems to be finally catching up with the Agta..." due to environmental degradation and socioeconomic subjugation. Similarly, Headland's (1986) research is focused on population decline, acculturation and loss of knowledge and group cohesion in the Casiguran Agta due to external forces, high mortality and the Agta's inability to defend their culture and acceptance of their subordination (Early \& Headland 1998). In fact, Griffin and Headland (1994: 73) go so far as to predict that the "ancient Agta culture will be extinct as well [by the year 2005]". Wrapped up in these grandiose statements is the assumption of a once pristine, static and ancient Agta culture being rapidly lost in the second half of the $20^{\text {th }}$ century. Such concepts of static culture are unsupported in anthropology, particularly human behavioural ecology, as individuals are understood to adapt to environmental (be in natural or social) change. Inflexible cultural systems are improbable, and there is no reason to suspect that once 'non-foraging' behaviours occur the pathway to farmers or landless labourers is inevitable, or irreversible (Oota et al. 2005). For instance, Griffin (1996, p. 15) later argued that one 
period of fieldwork can be deceptive as you accept all 'new' behaviour as cultural change, and ignore how cyclical it can be. This is precisely the perspective I take of Agta 'transition' and behaviour. Some individuals may be more settled than others, and as discussed above this leads to significant alterations to their subsistence strategies. However, by no means do I expect this to be a permanent change. Rather, I view these behaviours as adaptations to prevailing conditions.

Both Headland (1986) and Griffin and Griffin (2010) have argued that the Agta are failed farmers due to their subordination to non-Agta neighbours who seize or destroy their land. Thus, the Agta become marginalised, landless labourers rather than peasants (Early \& Headland 1998). However, I have already discussed the significant amount of cultivation the Agta are involved in, remaining remarkably consistent between Minter's 2002-2005 fieldwork and our own. The Agta of Palanan appear able to consistently engage in farming to a degree which brings in a significant amount of calories. Furthermore, rather than being completely subjugated by non-Agta farmers, there appears to be an element of mutualism in their relationships (Peterson 1978; Minter 2010). The notion of a 'grim future' of the Agta in part stems from the particular social history of the Agta living in Casiguran studied by Headland, as it is this population that collapsed once a road was built from Dilasag to Casiguran in 1977. A sudden influx of migrants, logging and extractive industries brought with it increased violence, homicide and alcoholism as well as increased mortality rates. Thus, the Agta became synonymous with population decline, social problems and cultural loss. However, this is only one population and focusing on it ignores the resilience and adaptability of the Agta groups throughout the Philippines who have remained a cohesive population in the face of external pressures.

In our own data, we have found a wide variety of foraging and non-foraging strategies. The Agta, 40 to 50 years after the original 'grim future' predictions were made are still a cohesive population involved in a significant about of 'traditional' foraging modes of subsistence. Thus, Minter's (2010) argument for the resilience and adaptation of the Agta is far more convincing. Resilience is the concept of how systems can withstand an external and internal insult and adapt to meet its challenges to ensure its survival. This concept then, falls completely in line with key concepts of human behavioural ecology, and underscores this thesis. We should not expect the Agta to have maintained a 'traditional' culture up until 40-50 years ago, rather the degree they are involved in cultivation, hunting, 
fishing and the nature of their interaction with non-Agta would had varied throughout time according to their needs (Minter 2010).

\subsection{Conclusions}

The Agta have many features in common with other immediate return foragers worldwide. However, they do seem exceptional for their high fertility. It seems also that the Agta of Palanan suffer slightly lower infant and childhood mortality rates than reported for surrounding groups. There appears a significant sex bias in both births and deaths, suggesting that more males are born to counter the particularly high male death rate during the ages $1-5$. While the Agta have had intensive interaction with farming populations for many generations, they have maintained some important components of the forager lifestyle such as mobility, flexible residence and bilateral descent systems, little material wealth and inequality and a significant proportion of time devoted to foraging. Nonetheless, these traits all significantly vary according to location and degree of sedentarisation. It is this variation and its consequences I seek to explore in the following analysis chapters. 


\section{Chapter five}

\section{The Behavioural Ecology of Communal Breeding: Who Cares for Agta Children?}

The review in chapter 2 revealed different types of kin have been demonstrated to play important roles in childcare. Primarily focus has been placed on both grandmothers and juveniles as 'low-cost' helpers, while fathers have primarily been highlighted as food providers rather than allomothers. Hunter-gatherer studies however, reveal that grandmothers do not always interact a significant amount with their grandchildren and the results are mixed in relation to the role of siblings, fathers and more distantly related individuals. A limiting factor in observational studies in the past has been their sample size, making it difficult to examine trends. Here I explore the composition of childcare among the Agta using three-meter proximity data from the motes as a proxy for low investment childcare. With this innovative technology I have captured allocare relationships between 200 individuals during a full week at two-minute intervals. I examine not only infants, but also allocare received by all dependent children under the age of 12. In particular, I focus on the roles of grandmothers, juveniles and unrelated individuals to explore the composition of communal breeding among the Agta. I find that grandmothers were rarely co-resident and shared few interactions with their grandchildren. Revealing the importance of kin selection dependent on costs, however, was the fact that siblings offer significantly more care than grandmothers, as did fathers. Nonetheless, non-kin can also be considered significant allocarers as it is only proximity from non-kin that decreased maternal care, suggestive of a reduction in workload. This suggests that communal breeding cannot be explained by kin selection alone, opening the path to explore why individuals breed communally in chapter 6 . These results have significant implications for our understanding of the role of grandmothering and the juvenile period, particularly in reference to human life history evolution. 


\subsection{Agta childcare}

As with most hunter-gatherer populations the Agta style of childcare has been argued to be indulgent, affectionate and intensive; infants and toddlers are permanently held, cuddled or played with (Minter 2010: 67). The majority of childcare among the Palanan Agta is high intensity, which includes breastfeeding, carrying, playing, feeding and providing medical care. Younger Agta children are frequently looked after by various member of their families as well as spending a significant amount of time in mixed-aged play groups which form a type of ad hoc crèche (Konner 2005) in which care was provided by unrelated children. Griffin and Griffin (1992) sum up the situation perfectly:

"The baby remains against the body of the mother nearly constantly in its first weeks, but it is also in contact with the father, siblings if any, and other kin that may drop in to visit, nap or play... Grandparents may take in toddlers and older children on a "drop-in" basis or in the case of the parents overnight departure for hunting and fishing. During the first 12 months an infant is usually carried in a sling at the mother's back, side, or front... Nursed on demand, it is returned to the back for sleeping after suckling... An infant under 1 year may be passed among several adults and youths being return to its mother if it becomes fussy" (Griffin \& Griffin 1992: 301).

Chapter 2 discussed the importance of communal breeding; without able helpers providing direct childcare and provisioning, foraging mothers would have to extend their economic and childcare activities to such a point that they could not sustain multiple dependent offspring at one time. Thus, we return to Lack's (1954) principle that the number of offspring is a product of both the mother's fertility and her ability to ensure dependents reach reproductive maturity. Ultimately then, it is of theoretical interest whether direct allocare significantly increases maternal reproductive success and how this occurs, a topic not yet explored among the Agta. Here my aim is to detail the nature of Agta childcare focusing on the importance of grandmothers, juveniles and non-kin. Reviewing the current evidence stemming from observational studies in small-scale societies reveals some inconsistencies, arguably due, in part, to small-sample sizes and short observational times.

Observational studies are extremely labour intensive, and require one researcher to follow a focal child for a number of hours, recording observations at a set time interval. The standard developed by Meehan et al. (2013a) involves the researcher observing one child for nine hours over three days in four hour periods making observations each 20 seconds. The amount of detail captured by such a method is impressive, and it is possible to observe 
the complex dynamics of childcare as well as maternal activities and household tasks. However, it reduces the sample size as it takes a long time to observe a significant number of children (for instance, it took eight months to observe 80 Agta children with two researchers observing children six to nine hours a day). Sample sizes are often in the range of 10 - 20 (Ivey 2000; Meehan et al. 2013b; Scelza 2009), making variability (such as by age or sex) difficult to explore. Several studies with larger sample sizes rely on hourly instantaneous camp scans to observe a particular activity (Crittenden \& Marlowe (2008) observed 68 children 13 times a day to quantify infant carrying) or self-reported childcare (Valeggia (2009) collected information of childcare behaviour from 200 adults), thus lack the same breadth and detail as the observational studies. Furthermore, given that older children are hard to follow or find, observational studies are often limited to infants and younger toddlers (approximately at 2.5 years), while Crittenden and Marlowe's (2008) focus on child carrying meant their sample contained children aged less than four years. As a result, the majority of what we know about allocare from small-scale societies is about who cares for infants, rather than the wider system of allocare for dependent children.

Overall, research of direct allocare is much more patchy than the investigation of indirect childcare (i.e. food provisioning), thus summaries are far from definitive. Nonetheless, bearing in mind the small sample sizes and focus on infants, it seems that grandmothers do not appear as important in hunter-gatherer societies compared to other small-scale societies, while care from fathers, siblings, extended family members and non-kin seems variable and understudied (Table 2.1 and references therein). As a result, this chapter has three interconnected aims: (i) to use high-resolution data for a larger sample of individuals than previously possible in observational studies in exploring (ii) who cares for Agta infants, toddlers and children aged less than 12 years and (iii) how this allocare substitutes maternal care, theoretically allowing her to invest additional energy into increases in child quality and/or quantity.

This chapter will proceed as follows: I will summarise the major predictions based on the review of the literature in chapter 2 and detail the specific methods used in this analysis. This will be followed by descriptive and inferential statistics about who looks after Agta children and how this care substitutes maternal and paternal care. The results will be summarised in a discussion of the relevance of this work to the existing literature, focusing on the role of grandmothers, juveniles and non-kin. Why individuals' provide allocare and the fitness consequences of this allocare is explored in chapters 6 and 7. 


\subsection{Predictions}

Kin selection predicts that individuals should provide allocare dependent on indirect fitness benefits weighted by degree of relatedness $(r B)$ and the direct fitness cost of the cooperative action (C, Hamilton (1964)). As a result, closely related family members, such as juvenile siblings, are expected to provide a high proportion of care given that their relatedness is high and their costs are lower as they are currently not reproductively active (Kramer 2005). Thus, I predict that juvenile siblings to spend a significant proportion of time interacting with dependent children.

Grandparents are predicted to provide a significant proportion of childcare to their grandchildren given their $25 \%$ genetic similarity and completion of their reproductive lifespans. Following the grandmothering hypothesis, I expect being a grandmother significantly increases the number of interactions with a dependent child (Hawkes et al. 1997). This is predicted to be meditated by paternity certainty; maternal grandmothers are completely sure of their $25 \%$ genetic share in a grandchild, while this confidence decreases for both maternal grandfathers and paternal grandmothers, being lowest for paternal grandfathers (Danielsbacka et al. 2011). Thus, maternal grandmothers are expected to provide the most contact interactions with grandchildren, followed by maternal grandfathers, paternal grandmothers and finally paternal grandfathers. I expect extended family members (referred to throughout this thesis as distant kin when $r \leq 0.25$, thus including grandparents, aunts and uncles and cousins) to offer significantly less care than closer kin. However, childcare may also be motivated by reciprocity, particularly from less related individuals as indirect benefits are easily overtaken by direct returns when $r$ is reduced (Axelrod \& Hamilton 1981). Thus, non-kin are also expected to provide allocare.

Overall, if care of any type is to be beneficial to mothers it must reduce their workload. As a result, I predict that a child's contact with close kin, distant kin and non-kin to substitute maternal care, allowing the mother to re-invest energy into fertility and/or child survival resulting in increased fitness for the mother and indirect fitness for the allocarer.

\subsection{Methods}

The level of the analysis was the child $(n=81)$ and the dependent variable was the proportion of interactions different types of caregivers shared with a child. Children were defined as all dependents under the age of 12. Caregivers were separated into close family 
(mother, father, brothers and sisters), grandparents (separated by lineage), distant kin (cousins, uncles and aunts; $0.125 \leq r \leq 0.25)$ and non-kin $(\mathrm{r}<0.125)$. I conducted a twosample Wilcoxon test to compare if the proportion of interactions were significantly different between types of kin (i.e. grandmothers versus sisters). The non-parametric version of the t-test was used due to the data's non-normal distribution (established by Shapiro-Wilk tests, $p<0.05)$. This analysis is followed by a multilevel model (MLM) which explored the relationship between care from allocarers (siblings, distant kin, grandparents and non-kin) on the number of interactions parents had with their children.

To bring my results into line with the cooperative breeding literature in hunter-gatherers in general I have presented the descriptive statistics as proportion of interactions. This variable was produced by establishing the total number of hours an allocarer was present in camp and dividing this by the number of actual interactions recorded between an allocarer and child. Consequentially, this figure represents how long an allocarer spent with a child as a proportion of the allocarers total time while in camp. This result is referred to as 'proportion of interactions'. To keep data manipulation to the minimal for the inferential analysis (substitutive care) I used the number of interactions two individuals shared on average per hour of the motes experiment (to control for differences in experiment time in different camps) as the dependent variable. I refer to this variable throughout the analysis as 'number' or 'frequency' of interactions. I use this latter variable for all future analysis.

Over six Agta camps (three being coastal camps, three inland) I conducted the motes experiment with 200 individuals $(107$ males). Of this sample, 81 were children under the age of 12 (49 males). The average age of the child sample was $5.2 \pm 3.3$ years (ranging from 0.2 - 11.5 years) while the average giver age was $25.6 \pm 3.2$ years. Children under 12 received on average 4,287 $\pm 1,687$ mote messages over the course of the experiment from 148 allocarers, representing close proximity interactions (at three metres). However, this varied widely according to camp size, duration of the experiment and child age; range 4488,128 messages. Children were almost always in the presence of several individuals and rarely left unattended while young or without friends and siblings when older. In total, I recorded 2,195 dyadic interactions, of which parents represented $6.8 \%$, siblings $6.3 \%$, grandparents $2.3 \%$, distant kin $19.8 \%$ and non-kin $64.6 \%$ of said dyads. The average number of interactions between a dyad per hour was $1.2 \pm 2.2$ but ranged between 0 to 20.3 recorded messages. On average, carers spent $4.5 \% \pm 8 \%$ of possible interactions with a dependent child, peaking at $67.9 \%$ for mothers with young infants. 


\subsection{Results: who cares and how much?}

Figure 5.1 and Table 5.1 reveal that mothers were the single most important caregivers as they spent $30.9 \%$ of all possible interactions with their offspring. This significantly increased to $51.9 \%$ for infants less than 2.5 years old. Mothers were in close interaction with toddlers between 2.5 to 5 years and children over 5 years for $32.8 \%$ and $19 \%$ of the time, respectively. Paternal interactions with infants, toddlers and children were roughly half that of mothers, but nevertheless, paternal care diminished as offspring aged. Comparatively, sibling care remained more consistent throughout childhood and brothers spent $34.7 \%$ more time than sisters interacting with siblings (Wilcoxon test; $\mathrm{W}=2417, p=$ $0.047)$.

Table 5.1: Proportion of all possible interactions kin spent with children under 12 years. PGF refers to paternal grandfather; PGM: paternal grandmother; MGF: maternal grandfather; and MGM: maternal grandmother. Non-kin are all individuals related $r<0.125$ (excluding any paternity uncertainty). Infants are aged under two years, toddlers from two to five years while children are aged 6 to 11 years inclusively.

\begin{tabular}{l|ccccccccccc}
\hline \multicolumn{10}{c}{} & \multicolumn{10}{c}{ Kin type } \\
\hline $\begin{array}{l}\text { Age } \\
\text { group }\end{array}$ & Mother & Father & Sis & Bro & PGF & PGM & MGM & MGF & $\begin{array}{c}\text { Other } \\
\text { kin }\end{array}$ & $\begin{array}{c}\text { Non- } \\
\text { kin }\end{array}$ \\
\hline All & 0.309 & 0.167 & 0.15 & 0.202 & 0.01 & 0.006 & 0.022 & 0.009 & 0.053 & 0.048 \\
Infants & 0.520 & 0.255 & 0.11 & 0.263 & 0.02 & 0.008 & 0.037 & 0.016 & 0.056 & 0.039 \\
Toddler & 0.328 & 0.157 & 0.16 & 0.210 & 0.01 & 0.001 & 0.032 & 0.007 & 0.042 & 0.040 \\
Child & 0.190 & 0.107 & 0.16 & 0.165 & 0.01 & 0.007 & 0.008 & 0.007 & 0.058 & 0.057 \\
\hline
\end{tabular}

It is surprising that, on average, brothers interacted with siblings more than sisters, given that female juveniles are frequently expected to provide more allocare. However, this average is significantly influenced by an absence of sisters, particularly in households with infants and toddlers. Here, the median number of interactions between a sister and an infant was 0 while between a sister and a toddler the median was 0.01 . This appears to be a product of the skewed sex ratio in the sample, therefore is reflective of which type of sibling is present rather than how much care is preformed when they are present. In fact, being a female juvenile compared to a male juvenile positively predicted the amount of interactions with a dependent child, indicating that, when available, females do provide more allocare $(B=0.6, p<0.001,95 \%$ CI $[0.3,1])$. 

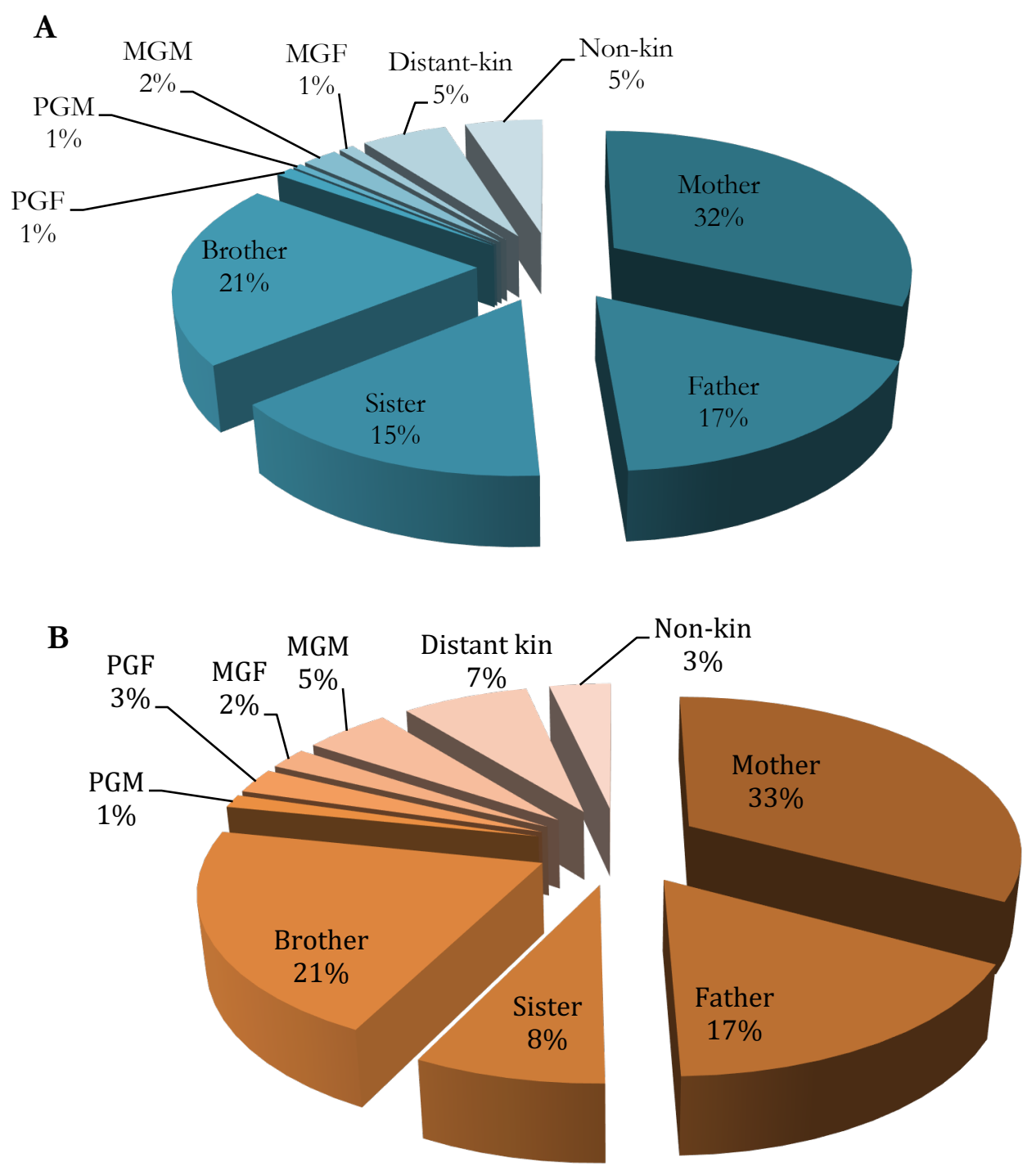

Figure 5.1: Pie chart of the mean proportion of interactions with different kin. A) Full sample $(n=81)$. Each of these proportions are significantly different from each other category at $p<0.05$, except between fathers and sisters (Wilcoxon tests, W $=3172, \mathrm{p}=0.45)$, fathers and brothers $(\mathrm{W}=2615, \mathrm{p}=0.21)$, sisters and nonkin $(\mathrm{W}=3442, p=0.08)$ and PGF versus MGF $(\mathrm{W}=3051, p=0.6) . \mathrm{B})$ Reduced sample $(n=34)$ adjusting for grandparental presence. Includes children with grandparents present.

Neither paternal nor maternal grandparents spent much time in close proximity with their grandchildren. Co-residence with any grandparent was not the norm as only $41.5 \%$ of children resided with at least one type of grandparent (Table 5.2). There is a slight (marginally significant in a two-way proportion test, $p=0.09$ ) matrilineal bias as $21 \%$ of children reside with a maternal grandmother, compared to $15 \%$ residing with paternal grandfathers. Overall, however, $72 \%$ of Agta children did not reside with either 
grandmother, while only $8 \%$ resided with two grandparents (which is either patrilineal or matrilineal, never combined). Thus, grandmothers are not a common resource among the Agta. Switching to the grandparental perspective $(n=27), 48.2 \%$ did not live with any grandchildren. Examining just grandmothers $(n=14)$ reveals that $50 \%$ live with at least one grandchild, while on average grandmothers co-resided with $48.9 \% \pm 0.36 \%$ of their living grandchildren.

Table 5.2: Proportion of children under 12 years with different types of grandparents present in camp at time of data collection $(n=81)$.

\begin{tabular}{c|rrrrcc}
\hline Number of children & MGM & MGF & PGM & PGF & Grandmothers & Grandparents \\
\hline 0 & 0.79 & 0.81 & 0.94 & 0.85 & 0.72 & 0.76 \\
1 & 0.21 & 0.19 & 0.06 & 0.15 & 0.28 & 0.16 \\
2 & - & - & - & - & 0 & 0.08 \\
\hline
\end{tabular}

Examining the reduced data set of 34 children living with either maternal or paternal grandparents we can explore the amount of care grandparents offer when they are present (Figure 5.1B). Re-running the Wilcoxon rank sum tests revealed that while most other kin categories continue to offer significantly more care than each type of grandparent even when that grandparent is present, the difference no longer reaches statistical significance for sisters (MGF, $p=0.8$; MGM, $p=0.5$; PGF, $p=0.8$; PGM, $p=0.08$ ). While there is no statistical difference in the amount of care offered between grandfathers, MGM offer $55 \%$ more care than PGM $(\mathrm{W}=686, p=0.005)$. Furthermore, MGM now are more important than the average non-kin individual, offering $28.3 \%$ more care than the average non-kin individual $(\mathrm{W}=372, p=0.06)$, however the relationship is of marginal significance.

\subsubsection{Kin versus non-kin}

The average distant kin and non-kin individuals spent $5 \%$ of their time with all offspring, increasing to $6 \%$ for children over 5 years of age. However, the importance of kin is influenced by the method of quantification of proximity across the sample. Here, proportion of interactions with non-kin represents the average over multiple non-kin. Averages are significantly impacted by extremes, particularly since the frequency 
distribution of ties among unrelated individuals demonstrates a right skew (Figure 5.2). As a rule, children exhibit a pattern of intense interactions with one or two individuals and weaker connections with a large number of other non-kin. This trend does not only occur among children (here 2 to 11 years), but peaks in teenagers (12 to 17 years, Figure 5.2). Children have between 1-2 individuals they interact with as much as close kin. This does not occur in infants who interact mainly with close kin. Thus, this pattern is reflective of children having one or two close non-kin allocarers who interact with them just as much as their mother, father and siblings.

A

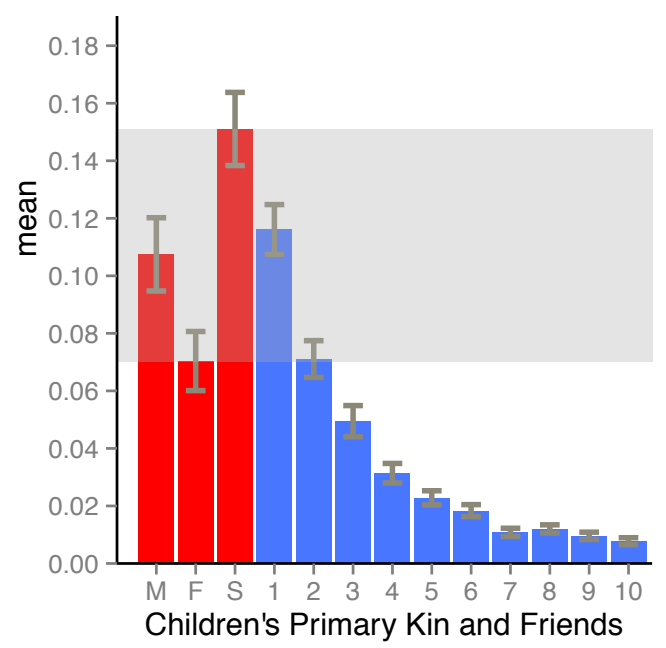

B

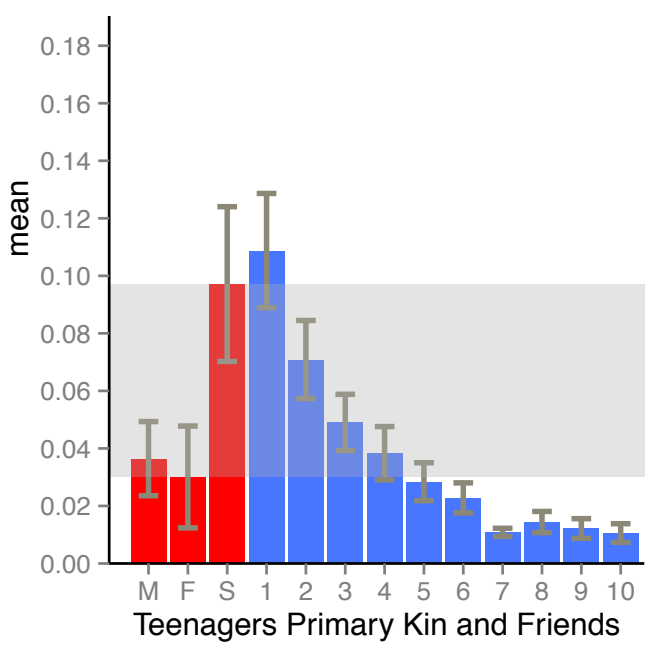

Figure 5.2: Proportion of a child's close-range interactions with close kin and unrelated individuals. (A) children (2-11 years) and (B) teenagers (12-17). Red bars: from left to right, proportion of interactions with mother, father and siblings. Blue bars: proportion of interactions with unrelated individuals ranked from left to right by frequency of interactions, up to the $10^{\text {th }}$ strongest relationship. Shaded area represents the range of frequency of interactions with close kin. Error bars represent $\operatorname{SEM}(n=81)$.

The effect this relationship has on the distribution of the data is portrayed in Figure 5.3. Figure 5.3A represents the proportion of interactions a child has with the average individual from each kin category, and is reflective of the pie chart in Figure 5.1. Here we see that mothers are the most important caregiver until age 9, when sisters, distant and non-kin provide more care. Figure 5.3B is based on the proportion of a child's time they spent with different kin types. Now that distant kin and non-kin are no longer averaged 
over many individuals we see that children spend a higher proportion of their own time with distant relatives and unrelated individuals. Thus, examining the interactions as a proportion of the child's time by kin group, I find that children spend on average $34 \pm$ $26 \%$ of their time with mothers, $11 \pm 5 \%$ of time with fathers, $24 \pm 13 \%$ of time with siblings and $6 \pm 6 \%, 7 \pm 7 \%$ and $23 \pm 13 \%$ for grandparents, other kin and non-kin, respectively (Figure 3.4). Both of these perspectives are important as, on the one hand they reveal the cost of childcare to mothers and how this cost diminishes with age, while on the other hand showing that children receive a significant amount of care from less related individuals, which increases with age.
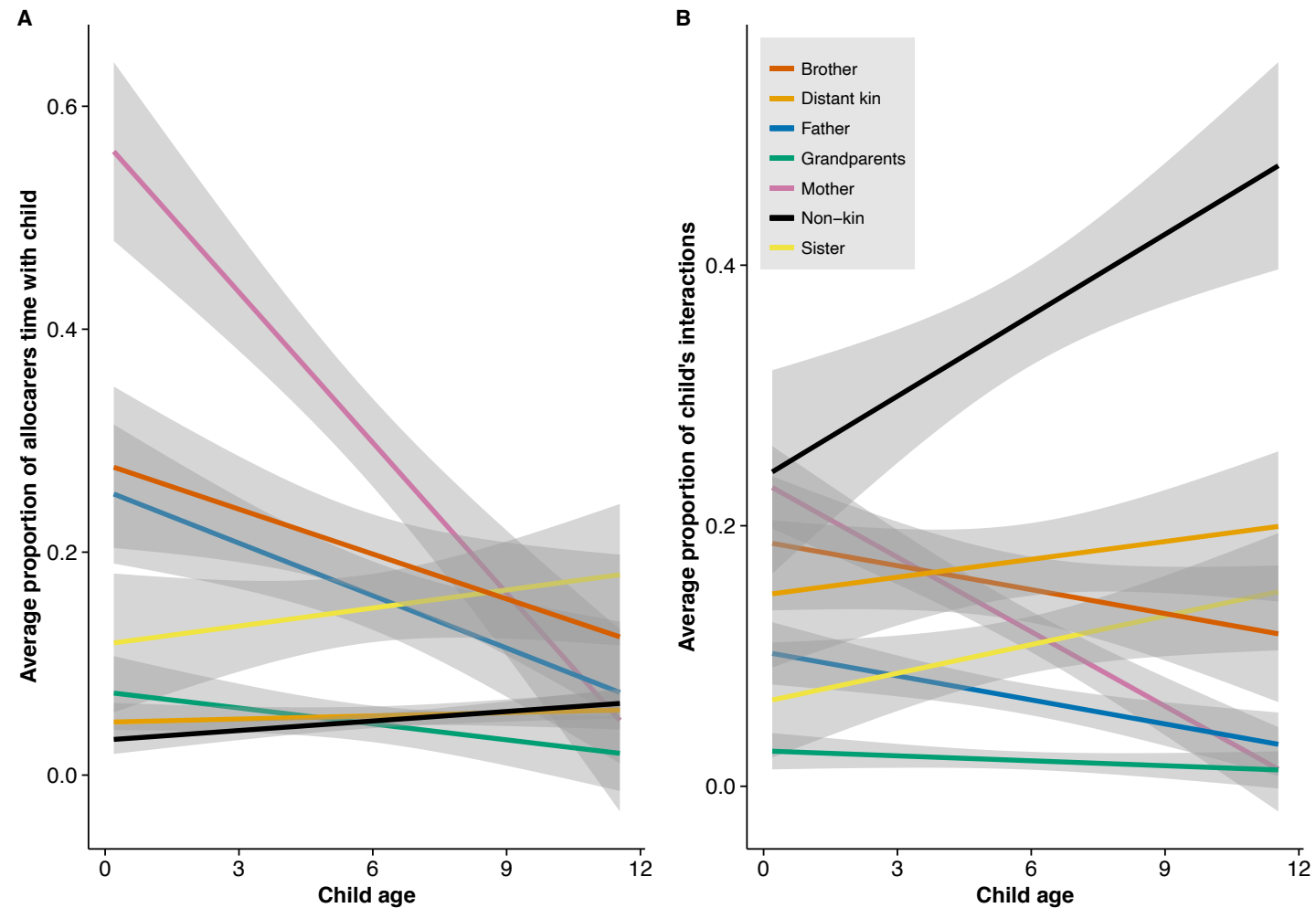

Figure 5.3: The relationship between number of interactions and age separated by whether the dependent variable is A) the average proportion of allocarers' time spent in childcare or B) the average proportion of care a child received from different types of allocarers $(n=81)$.

To conclude this section, it is evident that I did not confirm my original predictions. Grandparents are not significant allocare providers. However, following the expectations of paternity certainty maternal grandmothers did provide the most care amongst grandparents. Nevertheless, there is no significant difference between maternal 
grandfathers, paternal grandmothers and paternal grandfathers in the amount of interactions. Furthermore, paternal grandfathers' interactions with grandchildren are the second highest after maternal grandmothers, in opposition to the paternity certainty hypothesis, painting an inconsistent picture. Overall, non-kin seem more important than expected based on kin selection theory alone, while juveniles do appear to be frequently proximate to their dependent siblings, more so than fathers at later ages. Thus, by examining the composition of Agta childcare it appears that, after mothers, it is siblings who provide a significant amount of care while the role of non-kin should not be underestimated.

\subsection{Results: substitutive care}

I explored the relationship interactions from different individuals had with the amount of interactions a child had with their mother and father. For instance, is maternal care low when grandmaternal care is high? The aim was the capture whether care from parents was reactive to care from allocarers or vice versa (as causality cannot be distinguished). Fathers consistently provided significantly more care (blue line in Figure 5.3) than grandmothers. However, several grandparents did provide a significant amount of care (maximum proportion of interactions: $\mathrm{MGM}=0.34 ; \mathrm{MGF}=0.18$ ), particularly when fathers were absent or provided little direct care. This suggests that grandparental care may be of greater importance when parents were unavailable. Thus, I predicted that grandparental allocare would increase when paternal care is low. It is expected that for mothers to receive fitness benefits from allocare that this allocare is significantly associated with reductions in maternal workload, allowing her to reinvest the freed up time and/or energy into current or future reproduction. Consequently, I predicted that care from different caregivers would decrease maternal childcare.

MLMs were run with level 1 as the child, level 2 the household and level 3 the camp. Child age and sex were controlled for in each analysis. Five separate models were run containing one grandparent each to avoid overfitting the model with an excess number of predictors with diminishing sample size (for instance, only 6 children resided with a sister and maternal grandparents, none resided with all four grandparents). These models (Table 5.3) revealed that increased contacts from fathers $(B=0.35)$, siblings $(B=0.4)$ and PGM $(B=$ 1.1), had a positive effect on the number of maternal interactions. Furthermore, distant kin demonstrate a similar trend $(B=0.36)$, however the relationship is not significant $(B=$ 
0.09). MGM demonstrated no significant influence on maternal childcare. Thus, increased contact with close and distant kin did not reduce the amount of interactions a mother had with a child.

Table 5.3: MLM results on the effect of interactions with different kin types on parentaloffspring interactions.

\begin{tabular}{|c|c|c|c|c|c|c|}
\hline & & & Covari & es par & neters & \\
\hline Dependent variable & Model & Covariate & $\mathbf{B}$ & SE & $p$ & $n$ \\
\hline & & Age & -0.710 & 0.147 & $<0.0001$ & \\
\hline & & Sex & 0.162 & 0.912 & 0.86 & \\
\hline & & Father & 0.346 & 0.113 & 0.002 & \\
\hline & 1 & Siblings & 0.402 & 0.062 & $<0.0001$ & 01 \\
\hline Number of maternal & & Distant Kin & 0.361 & 0.214 & 0.09 & \\
\hline contacts & & Non-kin & -1.452 & 0.709 & 0.04 & \\
\hline & 2 & MGM & 0.697 & 0.577 & 0.227 & 20 \\
\hline & 3 & MGF & -0.211 & 1.134 & 0.85 & 18 \\
\hline & 4 & PGM & 1.052 & 0.198 & $<0.0001$ & 6 \\
\hline & 5 & PGF & -1.094 & 0.942 & 0.25 & 14 \\
\hline & & Age & -0.255 & 0.099 & 0.01 & \\
\hline & & Sex & 0.327 & 0.583 & 0.575 & \\
\hline & 1 & Mother & 0.157 & 0.064 & 0.01 & 81 \\
\hline & & Siblings & -0.070 & 0.054 & 0.19 & \\
\hline Number of paternal & & Distant Kin & 0.526 & 0.175 & 0.003 & \\
\hline contacts & & Non-kin & -1.376 & 0.549 & 0.012 & \\
\hline & 2 & MGM & -0.555 & 0.372 & 0.13 & 20 \\
\hline & 3 & MGF & -2.013 & 0.875 & 0.02 & 18 \\
\hline & 4 & PGM & 0.800 & 0.118 & $<0.0001$ & 6 \\
\hline & 5 & PGF & -0.206 & 0.723 & 0.77 & 14 \\
\hline
\end{tabular}

Note: Due to diminishing sample size five separate models were run: one containing the nuclear family, distant kin (excluding grandparents) and non-kin and the reminding four separating the different types of grandparents. The table shows the results for five independent models which are numbered and separated by shades of grey. Sample sizes vary according to the model and are reported separately for each model in the table. Dependent variable is the number of interactions reported with parents averaged across the length of the experiment. 
Care from non-kin is significantly associated with a reduction in maternal $(B=-1.5)$ and paternal childcare $(B=-1.6)$. In terms of paternal care, it is interesting to note (bearing in mind the extremely small sample sizes at this point, $n=6$ ) that while PGM appear to increase paternal interactions (i.e. fathers' mothers are more likely to be present at the same time as fathers themselves), maternal grandparents demonstrate the opposite relationship. Contact from both MGF $(B=-2.0)$ and MGM $(B=-0.6)$ reduces the amount of time fathers are in contact with their offspring (albeit insignificantly in the case of MGMs, $p=$ 0.1) suggesting that maternal grandparents offer more care when fathers are otherwise engaged or vice versa.

Consequentially, while it appears that paternal and maternal grandparental care are reactive to one another, suggestive of substitutive relationships, it is only proximity from non-kin which significantly reduces maternal contact with her offspring. However, the small numbers of grandparents removes the power from this analysis and my ability to speak about their relationship with parental care with any certainty.

\subsection{Discussion}

The picture of Agta childcare cannot be simply explained by 'kin selection', nor is it particularly defined by grandmothering. It is evident that non-kin do have a significant role in childcare among the Agta, and consist of around 23\% of children's interactions. Furthermore, all children interact with one or two unrelated individuals as much (if not more) than close kin. However, these dynamics are frequently overlooked due to a focus on distant kin (i.e. grandmothers). Overall, I find little evidence in support of grandmothering, while juveniles and fathers appeared to be more important allomothers. Supportive of previous findings (Sear \& Mace 2008) I find that maternal care decreases with age, however by exploring allocare with a larger age range of children reveals that grandparental and paternal care all also decrease with increasing age. On the other hand, siblings and non-kin become increasingly important, particularly after ages five to six. Therefore, the importance of allocarers is dependent on the age of the child as well as relatedness and the ability of the individual to provide allocare, revealing the advantages of larger sample sizes spanning different age groups. 


\subsubsection{Absent grandmothers}

The extensive human post-reproductive lifespan has remained an open evolutionary question: given that natural selection selects for traits that increase reproductive success, not being able to reproduce for 10, 15 or 20 years seems like a significant limitation on this (Williams 1957; Hawkes et al. 2003; O’Connell et al. 1999). It is not reproductive senescence which is particularly 'unique' (Whitehead 2015; Foote 2008; Hawkes \& Coxworth 2013; Brent et al. 2015), nor is its timing, but the fact that humans' have an extended postreproductive lifespan (Hawkes et al. 2003). While foragers, on average, survive for up to 2022 years (based on post-menopausal life expectancy from the !Kung, Hadza and Ache), less than $6 \%$ of chimpanzees are alive past the age of last birth (Kaplan et al. 2000; Hawkes et al. 1997; Hill \& Hurtado 1996; Howell 1979; Hill et al. 2001). As a result, Hawkes and colleagues (Hawkes et al. 1997; Hawkes et al. 2003; Hawkes \& Coxworth 2013) put forward the grandmothering hypothesis for post-menopausal longevity in humans. Mothers whose mothers assist in childcare increase fertility without deteriorating reproductive success as grandmaternal presence allows for shortened birth intervals without increasing childhood mortality (Hawkes et al. 1997). As a result long living post-menopausal grandmothers will increase their fitness relative to shorter-lived grandmothers due to indirect fitness gains (Hawkes et al. 2003), propagating the post-menopause longevity trait.

Hawkes and colleagues argued that grandmothering is part of a cluster of life history traits in humans that create our 'unique' fast and slow strategy, given that extended longevity drives delayed maturity (Charnov \& Berrigan 1993), resulting in the excessive dependency of offspring. It is the care and provisioning from grandmothers which allows us to have multiple dependent offspring as their allocare permits for earlier weaning and shortened IBI (Hawkes et al. 2003). As a result, a major quest in the anthropology of cooperative breeding has remained the quantification of the role of grandmothers and their influence on child survival and maternal fertility. As reviewed in chapter 2, in a range of small-scale and historical populations, maternal grandmothers play a significant role in improving child quality and maternal fertility (Sear \& Mace 2008; Sear et al. 2002; Snopkowski \& Sear 2015; Sear et al. 2003). Consequentially, many have come to see grandmaternal care as a defining feature of human life history (Hill \& Kaplan 1999).

The picture is not, however, so clear in foraging societies, particularly among the Agta. For instance, referring back to Table 2.1 , it is apparent among the more immediate-return hunter-gatherer groups (Agta, Aka, Efe and Hazda) the proportion of high-investment care 
received from grandmothers is low, averaging at 5.4\%. Similarly, in this study, grandmothers were only proximate to children for $1.6 \%$ of the time they were in camp, peaking at 3.7\% for maternal grandmothers. Simply put, all other kin and non-kin provided more care than grandparents. As suggested by Hill \& Hurtado (2009), this is in part influenced by the shorter life expectancy in hunter-gatherers matched with fluid camp composition, meaning that many children do not reside with any grandparent, maternal or otherwise. For instance, Migliano et al. (2007) demonstrated that among many pygmy populations residing in high mortality environments, post-15 life expectancy spans between 20 to 23.5 years. As a result only $13-31 \%$ of pygmy mothers survived to past last reproduction. In contrast, the Turkana (pastoralists) survive for 46.6 years after age 15 and 63\% survive past last reproduction. Consequentially, high mortality environments, such as those common in many foraging populations (Hill \& Hurtado 1996; Hill et al. 2007; Kramer \& Greaves 2007; Hill et al. 2001; Gurven et al. 2007; Stock \& Migliano 2009; Early \& Headland 1998), are not conducive to the presence of grandmothers. This suggests that the role of grandmothers may lie in lower mortality environments where they can be relied on as more predictable sources of allocare (Kaplan et al. 2000).

Furthermore, the high mortality of foraging populations like the Agta poses another problem for the role of grandmothers. Given that age at maturity is governed, in part, by expected age at death (why risk waiting to reproduce when the life expectancy at birth is as low as 20-25 years (Charnov \& Berrigan 1993; Promislow \& Harvey 1990)) populations residing in higher mortality environments tend to reach reproductive maturation earlier. For instance, Turkana women start reproducing four years later than the Aeta (Migliano et al. 2007), a trend apparent in developed nations (Nettle 2010; Nettle 2011) and throughout the Primate order (Walker et al. 2006). Consequentially the generational time is shortened and when grandmothers are present they often still have dependent offspring. This is certainly the case among the Agta: only $4.1 \%$ of the 148 allocarers could be defined as completely post-reproductive (i.e. grandmothers without dependent children aged under 12 years). However, their average age was 69.5 years and only one of the three grandmothers was physically able to provide care and/or food. All other grandparents still had dependent (i.e. under the age of 12) offspring, and thus perhaps unable to offer much assistance. The results detailed above are suggestive of this pattern, for both grandmothers and distant kin in general. 
Nonetheless, we also see that grandparents are reactive to paternal care; increases in the number of interactions with maternal grandfathers and grandmothers correlated with reductions in paternal contacts (albeit only of marginal statistical significance for grandmothers). The causality is, of course, unclear here as fathers may do less when grandparents do more, or vice versa. However, these result follow those of Meehan et al. (2014) who found that maternal grandmothers presence only influenced child nutritional outcomes in patrilocal camps where less allocarers were present. Thus, the importance of grandmothers may lie in substituting paternal care, as unlike in other studies the Agta fathers provided consistently more allocare than grandmothers (Scelza 2009; Ivey 2000). Similar to the Aka (where fathers provide $15.8 \%$ of care (Hewlett 1988)) then, Agta fathers are important caregivers as they spend $16.7 \%$ of their time with their offspring. An important distinction here is the difference in measurement; I have recorded proximity only, thus many fathers may spend their time 'caring' by simply being present and conducting other tasks. In many other studies investigating high quality care, the father's role may have been overlooked if they commonly provided lower investment care. Being within three meters of a child can be considered an important form of childcare in terms of watching over them and being able to intervene when required. It does not seem that fathers actively increase their proximity care as children grew, as this drops down to $10 \%$ after the age of 5 years. Thus, studies focusing on younger children may not necessarily underestimate the father's role, yet studies focusing just on tasks like carrying, feeding or grooming may do just that. It is only possible to conclude that fathers are less important than mothers in direct childcare, however their importance likely stems also from family provisioning. Combining both direct and indirect childcare is a clear avenue of future research, allowing for greater insight into the evolution of cooperative breeding and pair bonding in humans (Kaplan et al. 2000; Washburn \& Lancaster 1968; Kaplan et al. 2003).

\subsubsection{Helpers-in-the-lean-to}

Agta siblings appear to be major providers of allocare, however, compared to grandmothers, care from juveniles has received little attention for their role in cooperative breeding (Kramer 2011). Theoretically sibling care should be significant: they are 50\% related thus suffer no fitness costs relative to care they could provide to future offspring (assuming paternity certainty here); as juveniles are in a pre-reproductive period they do not suffer a reproductive opportunity cost in caring; and they can develop skills required to 
be a successful parent later in life (Lancaster 1971). Consequentially, juveniles can be considered both dependents and carers. However, this is frequently overlooked as siblings are expected to be competitors for parental resources and large sib sets are associated with decreases in survival and poorer outcomes (Lawson et al. 2012). A lot of early work focused on the optimality of human reproduction, based on Lack's (1954) original observation that fitness is maximised by ensuring offspring survival, extended by Triver's (1972) theory that parental investment will be biased to offspring who can maximise reproductive success. While the evidence for a quantity-quality trade-off optimising human reproduction is mixed (Strassmann \& Gillespie 2002; Hill \& Hurtado 1996; Blurton Jones 1987; Pennington \& Harpending 1988; Borgerhoff Mulder 2000), there is substantial support for the presence of older siblings having a negative influence on maternal fertility (Sear et al. 2003; Tymicki 2004; Borgerhoff Mulder 1998) and child nutritional condition (Hagen \& Barrett 2009; Magvanjav et al. 2012). Significant sex and wealth effects mediate many of these relationships as siblings often compete more for resources which are limited and/or with siblings of the same sex (Borgerhoff Mulder 1998). As a result, juveniles have more often than not been considered competitors rather than co-operators. However, as stated by Kramer (2011: 533), there is a significant difference between raising a brood of offspring simultaneously and raising a newborn into a household with existing offspring.

Evidence from the Agta clearly supports this hypothesis, as does the majority of observational studies in small-scale societies; of the six studies which document the amount of care received from both grandmothers and siblings in Table 2.1, 66.7\% report that sibling care as higher than grandmaternal care. The exception being the Mardu, where siblings carried children only $5 \%$ of the time compared to grandmothers who carried them for $14.3 \%$ and the Hadza where siblings carried children for $1.2 \%$ of observations. However, Crittenden et al. (2013) argue that children's role in the Hadza is focused on food production rather than direct childcare (Crittenden et al. 2013; Crittenden \& Zes 2015). Siblings, on average, are reported to engage in direct childcare $17.4 \%$ of the time across the ten studies reported in Table 2.1, firmly in line with $15 \%$ and $20.2 \%$ reported here for sisters and brothers, respectively. This care, alongside other subsistence activities (such as foraging, tending animals, cultivation, food processing, firewood and water collection) enables mothers to re-invest freed-up energy and time into other activities (Kramer 2011). In short, if children were unable to provide this assistance (i.e. be completely dependent) parents would be unable to increase their workload to sufficiently subsidise all their offspring and ensure their survival (Kramer 2005; Lee \& Kramer 2002). As a result, 
Kramer (2011) argues that humans are able to maintain their fast/slow life history traits because juveniles provide allocare (direct or indirect) which allowed mothers to start reproducing earlier (Kramer 2008) and shortening birth intervals without significant increases in mortality.

Focusing on juvenile help may shed more light on the hypothesis of the evolution of the highly dependent juvenile period due to the embodied capital requirements in skilled foraging niches and increased cognitive development (Kaplan et al. 2000; Kaplan 1994; Walker et al. 2006). Lancaster and Kaplan (2009) posited that the extended juvenile period evolved as a result of humans' intensive extraction of high quality foods using advanced techniques. Human's foraging return rates and brain sizes are significantly higher than chimpanzees, and following Kaplan and colleagues' (2003) argument, this results in a 1.4fold longer juvenile stage devoted to skills and intellectual development. Thus, the juvenile period is selected for as it ensures the investment in embodied capital (i.e. growth, soma, immune function as well as skills and knowledge) which allows for a higher reproductive rate once maturity is reached which maximises reproductive success (Kaplan et al. 2000; Pagel \& Harvey 2002). Delayed maturation is only possible in environments of reduced mortality; compared to chimpanzees hunter-gatherers today experience double the life expectancy at birth and adulthood (Hill et al. 2001). In Kaplan and colleagues' (2000) model of human life history evolution they find that if, unlike Charnov's (1993) model, actors can influence their mortality rates (i.e. they are not completely exogenously determined) by investing in embodied capital then energy is diverted to mortality reduction by extending the juvenile period.

However, across the Primate order, more complex foraging skills (denoted by diet) are not consistently associated with prolonged juvenile periods, particularly in New World monkeys, while diet has no influence on relative brain size (Walker et al. 2006). Consequentially the link between foraging niche, cognition and prolonged development is not clear-cut, and other social factors may have an important role alongside skill development (Pagel \& Harvey 2002; Dunbar 2003). An alternative and comparatively unexplored hypothesis is that juvenile periods may also be selected for due to the social role of juveniles. The under-exploration of this question results from a systematic neglect of children's contributions due to a focus on 'net' production (i.e. the age at which an individual can sustain themselves) rather than production, which is not insignificant, albeit not self-sustaining (Kaplan 1994; Lee \& Kramer 2002; Cain 1977). Few hunter-gatherer 
studies examine the food production of children, even though it appears to be substantial (Crittenden \& Zes 2015; Crittenden et al. 2013), while children's heavy involvement in domestic tasks are equally ignored (Kramer 2011; Meehan et al. 2013a). If juveniles play an important role in ensuring maternal reproductive success, and vis-à-vis, their own indirect fitness, delayed maturation would also be selected for if this combination of investment into current indirect fitness (allocare) and delayed reproduction (via embodied capital) maximised genetic contributions to future generations. Including indirect fitness returns into life history models would greatly benefit understanding when it pays to cease growing and providing allocare given environmental mortality rates. This would be an intriguing avenue of future research, given the current limiting dichotomy between pair bonding and grandmothering in attempting to explain human longevity, allocare and juvenile periods.

Nonetheless, there is a snag in this argument: increased contact with siblings did not significantly reduce the amount of time a mother spent with that child, and in fact increases it. This pattern is also true for paternal and grandmaternal care, thus these allocarers do not replace maternal care, and without significant reduction of the maternal energy burden it is unclear how allocare would be associated with improvements to child condition, survival and/or maternal fertility. Certainly this study is limited to direct childcare only and does not explore maternal activities and domestic tasks. Furthermore, due to the coarse nature of the data (i.e. only proximity), the quality of the level of care is hidden. For instance, Scelza (2009) suggests that older siblings conduct more demanding, high quality childcare tasks which significantly reduce the maternal energy burden. Thus, while mothers are still present (here within three meters), the simultaneous presence of older siblings to groom, feed, play with and hold children means that the mother can relax more or conduct higher investment activities in another child. Anecdotally, the occurrence of playgroups with one or two mothers watching from a distance was not uncommon, suggestive of this pattern. However, a quantitative examination is critical to further explore the role of juveniles as allocarers.

Nonetheless, proximity care from non-kin did significantly decrease the number of interactions mothers had with their children, indicating that there is a direct substitutive effect. This reveals that non-kin may have significance in hunter-gatherer systems; given the stochastic variation (mortality, mobility, sickness and variability in food production) in who is actually available to provide allocare, reliance on any one particular carer, such as an older sister, grandmother or father would be a risky strategy (Kramer 2010). As a result, 
one possible way of mitigating losses is ensuring very distantly or unrelated individuals are able to provide care.

\subsubsection{Kin selection and non-kin allocarers}

The data clearly supports the hypothesis that close kin provide the most allocare. This is in keeping with Crittenden \& Marlowe's (2008) finding that among the Hadza, more closely related individuals held a child for longer, as well as Ivey's (2000) work with the Efe showing that relatedness consistently predicted care from adult males, juveniles, reproductively and post-reproductively aged women. However, here I have also demonstrated that non-kin provided a significant proportion of care received by a child $(23 \%)$, and while the majority of non-kin interact infrequently, a few provide a level of contact comparable to close kin. Thus, similar to the results among the Hazda (Crittenden \& Marlowe 2008) and Efe (Ivey 2000), non-kin are providing a significant amount of care, indicating their importance. This begs the question: why do unrelated Agta allocarers provide care? I suggest that reciprocal altruism is a potential solution to shortfalls in household energy budgets as allocare consists of a form of risk pooling. This hypothesis is tested in the next chapter (6, "Why breed Communally"). Furthermore, as argued in section 2.2.1, kin selection cannot be tested by simply demonstrating that specific kin types provide more allocare than less related individuals, as this may well be equally influenced by reciprocity, association or spatial distance. To explore the ultimate 'why' behind communal breeding the confounding effects of relatedness, reciprocity, costs, benefits and association must be separated in multivariate analysis. This analysis is conducted in chapter 6.

One evident reason for previous results emphasising the importance of grandparents and deemphasising the importance of siblings and non-kin is the problem of small-sample sizes and a focus on infants. While Agta grandparents never offer a large amount of care, it is most certainly increased during infancy where maternal grandmothers spend $3.7 \%$ of their time proximate to an infant. By age six onwards this decreases to $0.8 \%$. Therefore, arguably the importance of grandmothers lies in earlier years. These results are mimicked in Meehan et al. (2014) study of the influence of presence of different kin on weight-for-age, heightfor-age and weight-for-height z-scores among 127 Aka children aged less than 10 years. Here grandmothers were associated with higher z-scores (for weight-for-age, height-forage, and weight-for-height) during the 9 to 36 month period, with diminishing effect sizes 
over time. Similar results are apparent in a large cross-section of studies on grandmaternal effects on child outcomes (Sear \& Mace 2008).

The role of siblings and non-kin has not been explored with the same level of detail of grandparents, thus there is little to compare these results to. However, it follows that as children age and parental care decreases, it is likely that these interactions are taken up by other types of individuals. This trend is demonstrated by Figure 5.2, which reveals that children spend more time with siblings than parents, and share intensive relationships with one or two non-kin individuals. While this trend increases further into teenage years, it is not present in infants aged less than two years who do not interact with non-kin as much as they do with primary kin. Therefore, by exploring allocare across a large sample containing a range of ages, it is possible to see the importance of non-kin and siblings.

Finally, the larger sample size also produces more confidence in our results, as they are less likely to be biased by a few individuals who happen to provide significantly more care. Nonetheless, even here there are simply not enough grandparents to explore these relationships in-depth with any confidence, further indicating the problem of relying on grandparents for allocare.

\subsection{Conclusions}

Here I have explored allocare through close proximity interactions and thanks to the application of the motes, the larger sample size of 200 individuals sampled over a week greatly increases the statistical power of this analysis. These data should go hand-in-hand with more time-consuming but finer grained observational data to aid better interpretation of the results, setting a direction for future research. Nonetheless, being able to examine allocare across a larger age range of children has revealed some interesting trends. Contra to my original predictions Agta childcare is defined by a lack of grandmothers and the importance of juveniles and non-kin. This undermines the grandmothering hypothesis and indicates more research should be focused on a comparable 'juvenile hypothesis' as well exploring non-kin allocare. The question of why individuals provide costly allocare has yet gone unanswered and I turn to this question in the next chapter. 


\section{Chapter Six Why breed communally}

The review in chapter 2 revealed that the literature is awash with examples of indirect and direct benefits which occur from cooperation, yet this paradigm is rarely (if ever) applied with the same robusticity to allocare. Therefore, here I examine the role of both kin selection and reciprocity in understanding why individuals breed communally. As in chapter 5 I use highresolution proximity data from 200 individuals, including 81 dependent children to explore the ultimate reasons behind allocare, in particular exploring the influence of costs, benefits and reciprocity as well as relatedness. Allocare appears driven by kin selection; however, this is primarily limited to related juveniles. Grandmothers provide little care and are unreactive to household childcare needs, as are distant kin in general. These relationships may be defined more by competition for resources than cooperation. Reciprocity does not predict allocare in the full sample; however, reciprocity is important for more distantly and unrelated individuals, suggesting their care is provisional on short-term returns. These results support the hypothesis that allocare is a form of risk mitigation, as does the finding that degree of cultivation and material wealth influences the nature of allocare, shifting the perspective from wider, unrelated networks to the nuclear family. This chapter demonstrates the importance of hypothesis testing, revealing that communal breeding is much more complex than kin care and can be considered an important behavioural strategy when facing risky environments with many dependent offspring without material wealth.

\subsection{Introduction}

Given that the successful reproduction of offspring who survive to maturity is a key component of an individual's lifetime fitness, getting additional childcare from allocarers surely would result in a fitness payoff (assuming the childcare standards were high enough of course). Thus, why mothers allow other individuals to provide allocare for their children is not such an evolutionary puzzle. However, the same cannot be said for understanding 
why the allocarers provide costly childcare, particularly when they are distantly related or unrelated. The majority of research on communal breeding in humans has indicated that allocarers care due to indirect fitness benefits (Crittenden \& Marlowe 2008; Mace \& Sear 2005; Sear \& Mace 2008; Meehan et al. 2014; Kramer 2010). However, as revealed in the literature review contained in chapter 2 , previous studies have rarely separated the effects of relatedness from reciprocity, shared proximity as well as the confounding effects of costs and benefits. The majority of studies that explore these questions do reveal that more related individuals provide more allocare (Ivey 2000; Crittenden \& Marlowe 2008) or that more closely related individual have a positive influence on child outcomes (Sear \& Mace 2008). However, they cannot reveal that this trend occurs due to kin selection or explain why non-kin provide costly allocare. As a result, here I intend to explore why do allocarers care by further extending the results established in chapter 5 . This requires reconciling the apparently costly behaviour (allocare) with inclusive fitness theory, in which individuals should be optimising behavioural strategies to ensure increased genetic representation in future generations. This question will be separated into two interconnected parts in this chapter. Firstly, I will explore what benefits do the givers of allocare receive from interacting with dependent children. This can be broadly separated into indirect fitness (kin selection) and direct benefits (reciprocity). Secondly, I further explore the role of cooperative breeding as a form of risk reduction by examining how it varies according to ecology and foraging returns as well as subsistence diversification and wealth accumulation.

\subsection{Why do selfish organisms cooperate?}

Exploring why individuals cooperate from an evolutionary perspective has led to the development of a series of models, some specific to cooperative breeding, others more general. Following evolutionary logic they attempt to explain human behaviour from the perspective of individualistic gains as true altruism is not an evolutionary stable strategy (Foster et al. 2006; Rand \& Nowak 2013). While these theories have been discussed in depth in chapter 2, they are now recapped below and their main predictions are summarised in Table 6.1. It is important to note that these are not mutually exclusive hypotheses and are all expected to be in operation to some degree. For instance, an extended family member may be more likely to receive help because they share, on average, $25 \%$ of their genes with the recipient. However, given that they do not share $75 \%$ of their genes, cooperation may be dependent on reciprocation, which is not predicted by kin 
selection. Thus, their behaviour is predicted by both reciprocal altruism and kin selection. Furthermore, grandmothering and helpers-in-the-nest are sub-predictions within kin selection that consider differences in reproductive stages and the influence this has on the cost benefit ratio in kin selection.

\subsubsection{Kin selection}

Kin selection predicts that individuals should provide allocare dependent on indirect fitness benefits weighted by degree of relatedness $(r B)$ and the direct fitness cost of the cooperative action (C, Hamilton (1964)). As a result, care should diminish with increasing cost and decreasing relatedness, but increase the greater the benefit of the recipient. Care driven by kin selection is not expected to be influenced by reciprocity (Gurven 2006). As in chapter 5, I predict that juveniles or 'helpers-at-the-nest' will provide a significant proportion of care given that their relatedness is high and cost is low. However, following the lack of importance of grandmothers in chapter 5 I predict that being a grandmother will not significantly predict the number of interactions with a child. Nonetheless, I expect that juveniles and grandmothers to offer more care when the need of the recipient is high enough, as the benefits would then outweigh the costs.

Overall, given that kin can afford, in fitness terms, to invest a significant amount in their relatives' offspring, they can be considered to be reliable caretakers, particularly due to their likely co-residence. This would result in tightly clustered childcare networks around the nuclear family.

\subsubsection{Reciprocity}

Childcare motivated by reciprocity should be dependent on the needs of the recipient household and the cost of the action to the giver (Gurven 2004b). However, unlike kin selection, reciprocity is defined by contingency (i.e. cooperation which is dependent on expectations of future cooperation based on prior sharing patterns (Allen-Arave et al. 2008)). Reciprocity does not need to be evenly balanced, but the giver should expect that the long-term benefits of giving outweigh the immediate cost of giving (Gurven 2006). Contingency should become an increasing important predictor of cooperation as $r$ diminishes as direct benefits soon outstripped indirect ones (Allen-Arave et al. 2008). Thus, 
I predict that distantly related kin (referred to throughout this chapter as distant kin when $r$ $\leq 0.25$, thus including grandparents, aunts and uncles and cousins) and unrelated individuals' allocare will depend on contingency, the need of the receipt household and giver cost.

Table 6.1: Evolutionary models explaining cooperation and allocare. None of these models are mutually exclusive.

\begin{tabular}{|c|c|c|c|c|c|}
\hline & \multicolumn{5}{|c|}{ Indicators } \\
\hline Theory & $r$ & Contingency & Benefit & Cost & Clustering \\
\hline $\begin{array}{l}\text { Kin selection: care } \\
\text { pooling (Hamilton } \\
\text { 1964) }\end{array}$ & $\begin{array}{c}\text { Increases } \\
\text { care }\end{array}$ & $\begin{array}{l}\text { Not present; } \\
\text { needs based } \\
\text { imbalance }\end{array}$ & $\begin{array}{l}\text { Essential: to } \\
\text { the offspring } \\
\text { and/or } \\
\text { mother } \\
\text { (indirect } \\
\text { fitness). }\end{array}$ & $\begin{array}{l}\text { Dependent } \\
\text { on relatedness } \\
\text { and benefit }\end{array}$ & $\begin{array}{l}\text { Cooperative } \\
\text { networks } \\
\text { clustered } \\
\text { around kin }\end{array}$ \\
\hline $\begin{array}{c}\text { Reciprocal } \\
\text { altruism: risk } \\
\text { pooling } \\
\text { (Trivers 1971; } \\
\text { Aktipis et al. 2001; } \\
\text { Gurven 2006; } \\
\text { Cashdan 1985) }\end{array}$ & - & $\begin{array}{l}\text { Essential but } \\
\text { may be } \\
\text { delayed and } \\
\text { unbalanced }\end{array}$ & $\begin{array}{l}\text { Essential: to } \\
\text { the offspring } \\
\text { and/or } \\
\text { mother }\end{array}$ & $\begin{array}{l}\text { Proportional } \\
\text { to expected } \\
\text { returns }\end{array}$ & $\begin{array}{l}\text { Dispersed } \\
\text { networks } \\
\text { ensuring wide } \\
\text { risk transfer }\end{array}$ \\
\hline $\begin{array}{c}\text { Helpers-at-the- } \\
\text { nest } \\
\text { (Lancaster 1971; } \\
\text { Lee \& Kramer } \\
\text { 2002) }\end{array}$ & $\begin{array}{l}\text { Siblings } \\
\text { provide care } \\
\text { due to } \\
\text { indirect } \\
\text { fitness }\end{array}$ & Not present & $\begin{array}{l}\text { Essential: to } \\
\text { the offspring } \\
\text { and/or } \\
\text { mother } \\
\text { (indirect } \\
\text { fitness). }\end{array}$ & $\begin{array}{l}\text { Low: } \\
\text { juveniles are } \\
\text { not yet } \\
\text { reproductively } \\
\text { active }\end{array}$ & $\begin{array}{l}\text { Cooperative } \\
\text { networks } \\
\text { clustered } \\
\text { around } \\
\text { household }\end{array}$ \\
\hline $\begin{array}{l}\text { Grandmothering } \\
\text { (Hawkes et al. 1997; } \\
\text { Sear \& Mace 2008; } \\
\text { Strassmann \& } \\
\text { Garrard 2011) }\end{array}$ & $\begin{array}{l}\text { Increased } \\
\text { care from } \\
\text { MGM due to } \\
\text { paternity } \\
\text { certainty }\end{array}$ & Not present & $\begin{array}{l}\text { Essential: to } \\
\text { the offspring } \\
\text { and/or } \\
\text { mother } \\
\text { (indirect } \\
\text { fitness). }\end{array}$ & $\begin{array}{l}\text { Low: care } \\
\text { from post- } \\
\text { reproductive } \\
\text { females }\end{array}$ & $\begin{array}{l}\text { Cooperative } \\
\text { networks } \\
\text { clustered } \\
\text { around the } \\
\text { distant kin }\end{array}$ \\
\hline
\end{tabular}

Note: throughout this thesis I separate close kin (primary family including parents and siblings) from distant kin (grandparents, aunts and uncles etc. at $r \geq 0.125)$ and non-kin $(r<0.125)$. These divisions are consistent. Throughout this analysis I do not focus on the role of the father, thus I am examining alloparenting rather than allomothering.

\subsubsection{Risk-pooling}

Communal breeding is arguably a form of risk-pooling to mitigate the losses occurring from either short-term variance (i.e. foraging failure) or long-term need given mothers' life history schedules (referred to as predictable life history shortfalls (Hill \& Hurtado 2009)). I divide these two types of risk pooling into variance pooling and care pooling, respectively. 
Variance pooling ensures that individuals can face short-term shocks without suffering larger losses as they pay an immediate smaller cost by cooperating now (Aktipis et al. 2011). Foraging failure or periods of sickness will influence direct childcare as individuals' energy budgets encompass food production, household tasks and childcare. Therefore, a shortage in one area such as food production can result in a deficit in childcare, which can be met by allocare. For instance, household A provides childcare to household B because household $\mathrm{B}$ is experiencing a bad run of luck in food production, thus spends more time foraging. Household A will expect to be helped by household B at some point in the near future when the scenario is reversed. Variance pooling is expected to occur between distantly or unrelated individuals as the household has 'maxed-out' its energy budget: networks are expected to be wide given the necessity for exposure units to be independent (Cashdan 1985) to deal with variance and demographic variability in mobile camps. Variance pooling is thus expected to occur with distant kin and non-kin, however, distant kin should be less sensitive to the costs of providing allocare due to shared reproductive interests mitigating some imbalances in cooperation.

Care pooling occurs when a mother is chronically unable to provide care for her offspring due to a high number of dependents. Given the long-term nature of the exchange mothers are not readily able to reciprocate care, thus, siblings and extended family members are likely to provide care given their higher likelihood of future interactions, increased trust and degree of relatedness compensating for any allocare unreturned (Allen-Arave et al. 2008). Thus, I expect extended family to offer significantly less care than closer kin, and this care may be motivated by reciprocity as well as kin altruism as relatedness is low and indirect benefits are easily overtaken by direct returns (Axelrod \& Hamilton 1981). Care from distant kin is expected to be more sensitive than close kin to the immediate fitness costs associated with cooperation, as $r$ is significantly lower. Likewise, distant kin should provide care to the households in the most critical of needs since $B$ has to be significant enough to adjust for the lower level of $r$. Thus, I predict that distant kin will provide more care the 'needier' a household is without immediate reciprocation. Non-kin on the other hand will be more determined by variance pooling which is dependent on short-term exchanges.

A second set of predictions centres on the effects of different types of risks and strategies to mitigate these risks. There are significant differences in variance of food production between coastal and inland groups (section 4.4.2); individuals relying on marine resources 
experience significantly larger package sizes, higher mean calorific return per hour and face lower levels of variance (Table 4.4). As a result, I predict that childcare networks at the coast will be more focused around the household than the extended family and unrelated individuals, as there is less need for wide childcare networks. Furthermore, markers of transition to more settled, cultivating and/or wage labour forms of subsistence are expected to alter the means by which individuals buffer risks. I predict that households involved in more foraging activities will have children who are cared for more by distant and unrelated individuals as it is difficult to conduct many foraging tasks in the presence of children (after weaning). Furthermore, as individuals spend more time working in cultivation and wage labour settlement increases, as does wealth accumulation (section 4.5). As a result, risks may be buffered by risk retention (i.e. storage of food and wealth) and wealthier households will extract themselves from wider networks to avoid demands on their wealth (Fafchamps 1992). Consequently, I expect that households with greater wealth will rely less on childcare from wider cooperative networks (such as the extended family and non-kin) and focus more on the nuclear household.

\subsection{Methods}

\subsubsection{Dependent variables}

The dependent variable for the statistical analysis is the number of interactions two individuals shared on average per hour of the motes experiment. I refer to this variable throughout the analysis as 'number' or 'frequency' of interactions. The first analysis 'why allocare' is conducted on the dyadic relationships between each child and allocarer. The second analysis 'variability in allocare' is conducted at the child level have been summed into one outcome variable per kin type (mother, father, siblings, grandparents, distant kin and nonkin).

\subsubsection{Independent variables}

Measures of transition have been described at length in the methodological section 3.1, these are used in the second analysis examining the influence of subsistence change, mobility, wealth and food storage on the amount of allocare different individuals provide. The binary variables of grandmothers and juveniles were created to test both the helpersat-the-nest and grandmothering hypotheses. If an allocarer was pre-reproductive (aged 15 
or under) they were coded as 1 , all other individuals were coded as 0 . All maternal and paternal grandmothers were coded as 1 . Descriptive statistics for all dependent and independent variables are presented in Table 6.3 and Table 6.4.

\subsubsection{Reciprocity}

To test the influence of reciprocity, a contingency variable was created for each dyad (Nolin 2010). For each giver's household (i), the care that dependent children received from all other carers from the current recipient child's (j) household was summed and transformed into an independent variable. In Figure 6.1 this value is 25 (i.e. the sum of the dyadic interactions between the carer, father and mother in $j$ ). This value then is the independent variable entered into the analysis to predict the value of interactions between any dyad between the two households (in this instance, interactions between the mother from $i$ and the dependent child from $j$ ). Thus, the reciprocity value reflects the number of interactions the giver's (i) household received from all givers in the receiving household $(j)$.

\section{Receiver household (j) Giver household (i)}

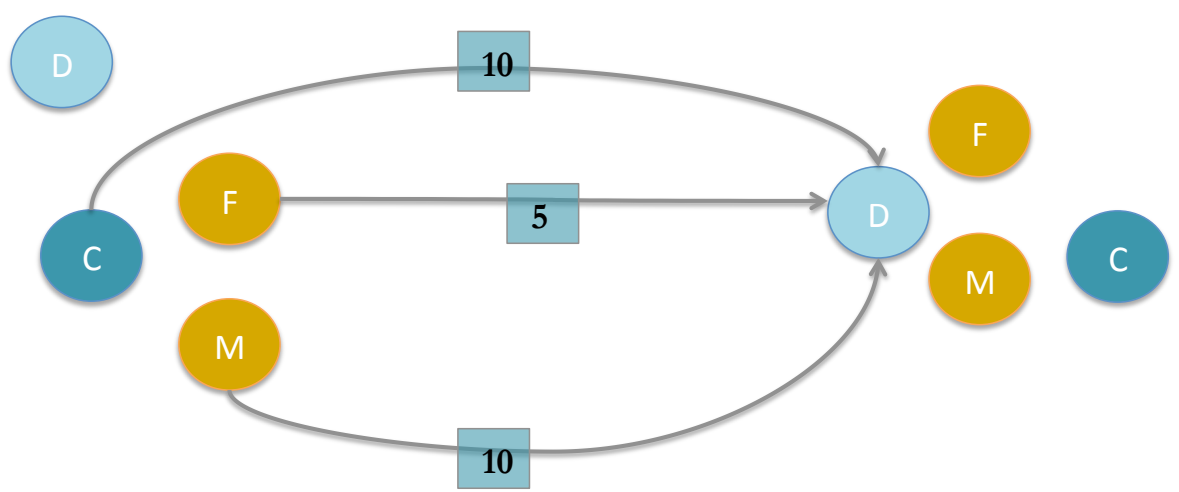

Interactions between household $j$ to household $i=25$

Figure 6.1: Visualisation of the creation of the contingency variable. D represents dependent child, $\mathrm{C}$ carer, and $\mathrm{M}$ and $\mathrm{F}$ mother and father respectively Blue boxes represent the number of interactions between each individual in household $j$ and to all dependent children in household $i$. 


\subsubsection{Giver cost and receiver household need}

Variables for giver cost and household care need were computed from the data. The cost $(c)$ of offering proximity care is computed based on the number of dependents $(d)$ and allocarers $(a)$ in the giver household and ego's importance as a carer in their own household (defined below) $(e)$, included to gage opportunity costs of helping (Winterhalder 1996). This is weighted according to whether an infant was present in the household (infant present $=2$, no infant $=1$, infant defined as children aged under two years) $(i)$ as allocare from households with infants are present is expected to be costlier. Children can be allocarers when they were aged 6 or older. Thus, $c$ is a product of:

$$
c=\left(\left(\frac{\mathrm{d}}{\mathrm{a}}\right) * \mathrm{e}\right) * i
$$

Ego's importance is based the distribution of care evident in the descriptive data (see section 5.4 above). As mothers provide the most care they were weighted as 0.5 ; fathers and siblings provide a significant amount of care but approximately half that of the mother, thus are weighted at 0.25 . Thus, for a mother $(M c)$ from a household with four non-infant children and two allocarers the equation is:

$$
M c=((4 / 2) * 0.5) * 1=4
$$

It is important to note the circularity with this variable; older siblings are considered to be one of the household carers, thus care is given at a 'cost', even if they are providing care for offspring within their own household. As a result, siblings residing in the same household as the focal child were removed from the estimation of giver cost by removing one point of the giver cost estimation. Receiver household care need (D) is the product of the number of dependent children divided by the number of carers in a household multiplied by 2 if one dependent was an infant. Thus, the equation is:

$$
D=\left(\frac{d}{a}\right) * i
$$

\subsubsection{Community detection}

To attempt to understand association effects (Koster \& Leckie 2014) a parental cluster variable was created. Here if the child's parents and allocarer belonged to the same proximity cluster they were coded as 1 , otherwise 0 . These clusters were created using a community structure algorithm based on modularity optimisation (Brandes et al. 2007) to attribute structure to a network; individuals who share more social ties and few ties with 
individuals from other groups form a structure (Newman 2004). Different combination of clusters are given modularity scores; the higher the modularity, the more optimal the partition and the less individuals in different clusters share ties (Newman 2010). Specifically, modularity is defined as the fraction of ties that fall within communities minus the expected value of the same quantity if ties are assigned at random (see Newman (2004) for equations). A value of 0 occurs when no more within-community ties occurred than is expected at random, and the maximum possible value is 1 . Values above 0.3 are reflective of effective graph partitions. Here, I used an optimal community structure algorithm that iterates through all possible graph partitions and returns the partition that maximized modularity (Csardi 2015; Brandes et al. 2007). This algorithm first divides a network into two random, but equal groups, then considers if the inclusion of each node in the network would improve the modularity or not. Nodes that improve modularity (or do not significantly decrease it) are moved and this process is repeated until all nodes have been moved once. After this, the partition with the highest modularity is selected as the starting 'state'. This process is repeated until no combination of nodes improves the modularity (Newman 2010).

Table 6.2: Community structure in Agta camps

\begin{tabular}{ccccc}
\hline Camp & $\boldsymbol{n}$ & Clusters & Average size & Modularity \\
\hline Djibbut & 22 & 3 & 7.33 & 0.425 \\
Cemento & 8 & 3 & 2.67 & 0.306 \\
Dinipan & 20 & 4 & 5 & 0.483 \\
Djago & 24 & 4 & 6 & 0.412 \\
Dipaguiden & 17 & 3 & 5.67 & 0.215 \\
Didikeg & 23 & 5 & 4.6 & 0.528 \\
\hline
\end{tabular}

To remove circularity clustering was only computed for the adult network (12 years or over). This resulted in camps sized between 8 and 24, in which the optimal number of clusters ranged between 3 and 5 (Table 6.2). The average size of these clusters was $5.2 \pm$ 1.4, which contained two to three separate households. Overall, the modularity scores for 
each of the camps partition was robust, averaging at $0.4 \pm 0.1$. Only one camp demonstrated a modularity score below 0.3 (Dipaguiden, 0.22) indicating its less clustered nature. From this analysis, each individual's cluster was recorded and children under the age of 12 were given the same cluster membership of their parents. If a carer and a child were in the same cluster they were recorded as 1 . As a result, the cluster variable represents the predictive value of the interactions between a child and their parents 'associates' or 'neighbours'. Due to the fact that individuals were frequently clustered with close and distant kin the model may have suffered from collinearity when both the clustering variable and coefficient of relatedness were included. Consequentially, I checked the variance inflation factor (VIF) in full models and confirmed that VIFs did not raise above 2.5 and clustering and relatedness both had VIFs of 1.04, far below acceptable limits (Zuur et al. 2010).

Table 6.3: Descriptive statistics for binary variables at the dyadic level $(n=2,195)$

\begin{tabular}{|c|c|c|c|}
\hline Variable & Type & $n$ & $\%$ \\
\hline \multirow[t]{2}{*}{ Giver sex } & Male & 1006 & 49.2 \\
\hline & Female & 1039 & 50.8 \\
\hline \multirow[t]{2}{*}{ Child sex } & Male & 1172 & 57.3 \\
\hline & Female & 873 & 42.7 \\
\hline \multirow[t]{2}{*}{ Pre-reproductive Juveniles } & False & 1259 & 61.6 \\
\hline & True & 786 & 38.4 \\
\hline \multirow[t]{2}{*}{ Grandmothering } & False & 2020 & 98.8 \\
\hline & True & 25 & 1.2 \\
\hline \multirow[t]{2}{*}{ Mobility } & Mobile & 886 & 43.8 \\
\hline & Settled & 1136 & 56.2 \\
\hline
\end{tabular}

\subsubsection{Statistical analysis: why allocare}

I ran multilevel models to explore the effects of coefficient of relatedness, giver cost, household need and reciprocity on the number of interactions a carer had with a child. In each analysis I controlled for location, child age and child sex. In contrast to the "who cares' analysis in chapter 5 , here interactions between parents and children were removed 
from the dataset, thus all remaining interactions reflect alloparents. The unit of analysis in the MLM was the dyadic relationship (level 1, $n=2,195$ ) between a child and the giver (i.e. any individual interacting with them over the age of 6 years). As dependent children are all those under the age of 12 years there is overlap between the child and giver categories. To avoid this circularity children could only be 'cared' for by individuals who were older then themselves. For instance, a child of five years could be 'cared' for by an individual aged 8 years, a situation not uncommon from our observations and within the childcare literature in hunter-gatherers (Kramer 2010; Konner 2005). However, a child of 10 years could not be 'cared' for by the same 8 year old. These thresholds were felt to capture the crossover of juveniles as both dependents and carers (Kramer et al. 2009).

Table 6.4: Descriptive statistics for continuous variables $(n=200)$

\begin{tabular}{ccccc}
\hline Variable & Minimum & Mean & Maximum & SD \\
\hline Dyadic relatedness & 0 & 0.086 & 0.500 & 0.137 \\
Giver cost & 0 & 0.458 & 2.500 & 0.458 \\
Receiver need & 0.5 & 2.228 & 5 & 1.200 \\
Reciprocity & 0 & 5.239 & 53.4 & 9.481 \\
Food storage & 0 & 1.527 & 14 & 3.407 \\
\hline Household wealth & 0.18 & 1.734 & 3.24 & 0.869 \\
\hline Maternal foraging & 0 & 0.601 & 1 & 0.342 \\
\hline
\end{tabular}

Working with dyadic data results in the pseudoreplication as each child and giver appears in the dataset multiple times. Consequentially, they are not independent data points and violate the basic assumptions in ordinary least squares regressions. However, the use of MLM overcomes this issue as it allows for clustering within the data (for full discussion see (Pollet et al. 2015)). The models in this analysis consider the fact that both the giver (level 2) and the child (level 3) are not unique entries, as well as the fact they are hierarchically clustered into camps (level 4). These models seek to test the competing hypothesis for why individuals perform costly care (kin selection, reciprocal altruism, grandmothering and helpers-at-the-nest). Thus I explore the relationships between coefficient of relatedness, 
household need, giver cost, reciprocity, association and reproductive stage on the number of interactions between each dyad.

\subsection{Results}

\subsubsection{Do Agta mothers require allocare?}

In short, yes. By examining the distribution of the household need index by maternal age (Figure 6.2) it is evident that swiftly after commencement of reproduction mothers have more dependents than carers. This peaks between the ages of 25 to 30 when mothers have several young offspring. For instance, it was not uncommon to witness young mothers with a newborn infant, a young toddler and an older child around five years. This childcare deficit does not start to significantly drop-off until between the ages of 35-40 years, when mothers have several older children to provide childcare. As a result, Agta mothers face up to 15 years of their reproductive lifespan with a shortage of care, indicating the importance of allocare from not only siblings (when available), but also from individuals outside the nuclear family. As a result, it is expected that distant kin may provide more care the more in childcare 'need' a household is in when mother's do not have older siblings available to provide allocare.

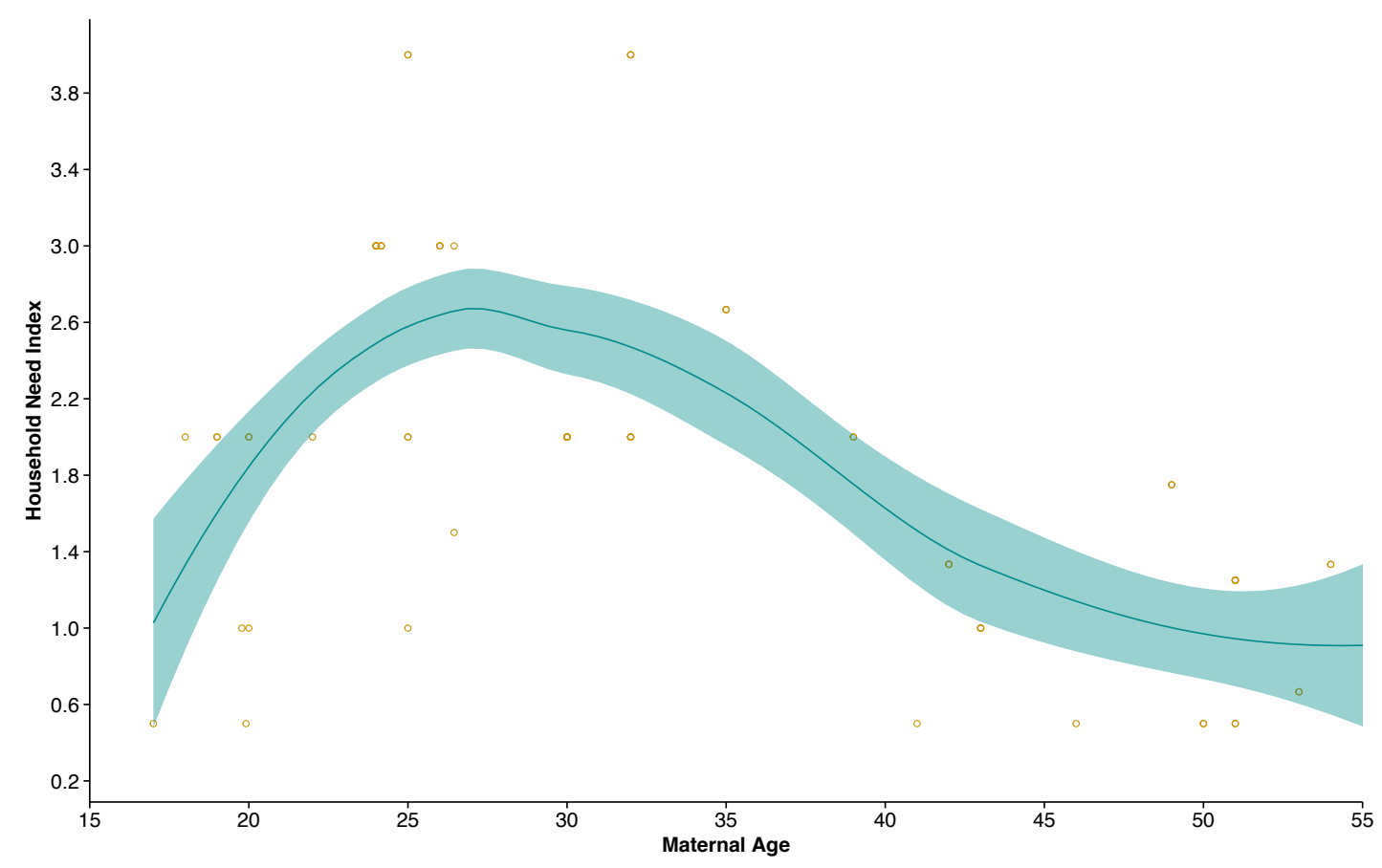

Figure 6.2: Relationship between maternal age and household care need. One outliner (need $=5$ ) has been removed here to allow an improved view of the majority of the data. Shaded area represents 95\% confidence interval of the smoothed regression between the household need index and age (with loess smoothed fit curve), $n=39$. 


\subsubsection{Why allocare?}

All models are presented in Table 6.5, the first panel being the intercept-only model which contains only random effects (i.e. the levels) with additional covariates being included in each following model (Koster et al. 2015). The VPCs (variance partition coefficients, see methods section 3.5.2) in the intercept-only model reveal that $12.9 \%, 7.6 \%$ and $7.5 \%$ of the variance in proximity interaction was attributed to the giver, receiver and camp factors. The remaining $72 \%$ of variance is attributed to unique dyadic factors. Giver cost $\left(B_{4}=\right.$ $2.5)$, relatedness $\left(B_{5}=6.2\right)$ and clustering $\left(B_{6}=2\right)$, all predict the number of interactions between an allocarer and child. While related individuals or those who associated frequently with a child's parents are in contact with a child significantly more often, individuals whose proximity care comes at a higher cost (measured by the cost index noted above) are in contact significantly less. Giver cost, relatedness and clustering reduce the unique dyadic variance by $16.6 \%, 5.4 \%$ and $12.1 \%$, respectively. Consequently, coresidence and cost seem to explain more variance than relatedness by itself.

Contra predictions, reciprocity did not significantly predict the amount of dyadic contacts; its inclusion in the model increased the AIC (from 7987.9 to 7989.9 ) indicating its lack of predictive power. Furthermore, household need held a negative relationship with the number of dyadic interactions $\left(B_{8}=-0.2, p=0.03,95 \%\right.$ CI $\left.[-0.3,-0.01]\right)$. However, the inclusion of interactions demonstrates that more care is offered to the recipient household the higher the household need if the giver is related. The inclusion of interactions results in a reduction of the dyadic variance by $1.3 \%$ from the previous model. However, relatedness no longer independently predicts the number of dyadic contacts $(p=0.3)$, implying that the care offered by kin is contingent on recipient need. Furthermore, the interaction also revealed that allocarers less closely related to a child provide significantly less care when that child is from a needy household $\left(B_{8}=-0.2\right)$.

Being a grandmother $\left(B_{9}=0.6\right)$ did not significantly predict dyadic proximity. However, juveniles were in proximity to dependent children significantly more, dependent on relatedness (final panel, 'full model' in Table 6.5). The juvenile covariate had a significant positive interaction with relatedness, demonstrating that related juveniles provided more care to children. This effect is large and very significant $(B=1.6, p<0.0001)$. As a result, unrelated juveniles did not offer significantly more care in the full sample $\left(B_{10}=0.2, p=\right.$ 0.2). Thus, this indicates that a significant proportion of the relationship between kinship and proximity is driven by juvenile allocare. It is worthwhile noting that in the early model, 
kinship had a large effect on the amount of care offered to children $\left(B_{5}=6.2\right)$, however the inclusion of the measures clustering $\left(B_{3}=3.5\right)$, household need $\left(B_{3}=2.7\right)$ and juveniles $(B$ $\left.{ }_{3}=-0.5\right)$ resulted in a large reduction in effect and significance. Finally, grandmothers appeared, contra predictions, to provide significantly less care when the household was in need $(B=-0.8)$ compared to when they were not in need $\left(B_{9}=2.3\right)$. This reveals that grandmaternal care is not reactive to long-term care deficits of their offspring (as measured by household need).

Table 6.5: Results from multilevel models examining different predictors for the amount of dyadic interactions. Table continues below.

\begin{tabular}{|c|c|c|c|c|c|c|c|c|}
\hline \multirow[b]{2}{*}{ Parameter } & \multicolumn{2}{|c|}{ Intercept-model } & \multicolumn{2}{|c|}{ Giver cost } & \multicolumn{2}{|c|}{ Relatedness } & \multicolumn{2}{|c|}{ Cluster } \\
\hline & $B$ & CI & $B$ & CI & $B$ & CI & $B$ & CI \\
\hline$B_{0}$ Intercept & $1.5^{* *}$ & $0.8,2.1$ & $2.3^{* *}$ & $1.5,3.1$ & $1.09 * *$ & $0.3,1.9$ & 0.73 & $-0.3,1.7$ \\
\hline$B_{1}$ Child age & - & - & 0.02 & $-0.04,0.1$ & 0.01 & $-0.04,0.1$ & 0.01 & $-0.04,0.1$ \\
\hline$B_{2}$ Female child & - & - & -0.11 & $-0.5,0.2$ & -0.11 & $-0.4,0.2$ & -0.11 & $-0.4,0.2$ \\
\hline$B_{3}$ Costal & & & -0.05 & $-1,1$ & -0.14 & $-1.2,1$ & 0.12 & $-1.2,1.5$ \\
\hline$B_{4}$ Giver cost & - & - & $-2.5^{* * *}$ & $-2.8,-2.1$ & $-0.52^{* *}$ & $-0.8,-0.2$ & $-0.48^{* *}$ & $-0.8,-0.2$ \\
\hline$B_{5}$ Relatedness & - & - & - & - & $6.2^{* * *}$ & $5.4,6.9$ & $3.5^{* * *}$ & $2.7,4.2$ \\
\hline$B_{6}$ Cluster & - & - & - & - & - & - & $1.97^{* * *}$ & $1.8,2.2$ \\
\hline Giver variance & & $(12.9 \%)$ & 1.53 & $29.5 \%)$ & 0.65 & $15.6 \%)$ & 0.667 & $(17.2 \%)$ \\
\hline Receiver variance & & $(7.6 \%)$ & 0.42 & $(8.2 \%)$ & 0.41 & $(10 \%)$ & 0.403 & $(10.4 \%)$ \\
\hline Camp variance & & $3(7.5 \%)$ & 0.17 & $(3.3 \%)$ & 0.24 & $(5.9 \%)$ & 0.409 & $(10.5 \%)$ \\
\hline Dyadic variance & & $6(72 \%)$ & 3.05 & $(59 \%)$ & 2.849 & $(68.6 \%)$ & 2.405 & $(61.9 \%)$ \\
\hline Model AIC & & 755.7 & & 20.8 & & 98.8 & & 87.9 \\
\hline
\end{tabular}

Model betas are reported alongside 95\% confidence intervals. Significant p-values at $p=0.05$ are represented

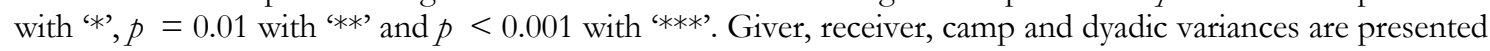
alongside VPC in brackets and represent the importance of each of these levels as sources of variation in interactions. 


\begin{tabular}{|c|c|c|c|c|c|c|c|c|}
\hline \multirow[b]{2}{*}{ Parameter } & \multicolumn{2}{|c|}{ Reciprocity } & \multicolumn{2}{|c|}{$\begin{array}{c}\text { Household } \\
\text { need }\end{array}$} & \multicolumn{2}{|c|}{$\begin{array}{l}\text { Reproductive } \\
\text { stage }\end{array}$} & \multicolumn{2}{|c|}{ Full model } \\
\hline & $B$ & $\mathrm{CI}$ & $B$ & $\mathrm{CI}$ & $B$ & $\mathrm{CI}$ & $B$ & $\mathrm{CI}$ \\
\hline$B_{0}$ Intercept & 0.74 & $-0.25,1.8$ & $1.2^{* *}$ & $0.2,2.3$ & $1.09 *$ & $0,2.2$ & $1.15^{*}$ & $0,2.3$ \\
\hline$B_{1}$ Child age & 0.01 & $-0.04,0.1$ & 0.00 & $-0,0.1$ & 0.00 & $-0.1,0.1$ & 0.00 & $-0.1,0.1$ \\
\hline$B_{2}$ Female child & -0.11 & $-0.4,0.2$ & -0.15 & $-1,0.2$ & -0.14 & $-1,0.2$ & -0.16 & $-1,0.2$ \\
\hline$B_{3}$ Costal & 0.13 & $-1.2,1.5$ & 0.14 & $-1.2,1.5$ & 0.14 & $-1.2,1.5$ & 0.17 & $-1.3,1.7$ \\
\hline$B_{4}$ Giver cost & $-0.5^{* *}$ & $-0.8,-0.2$ & $-0.6^{* * *}$ & $-1,-0.3$ & $-0.49 * *$ & $-0.8,-0.2$ & $-0.34 * *$ & $-0.7,-0.03$ \\
\hline$B_{5}$ Relatedness & $3.5^{* * *}$ & $2.7,4.2$ & 0.59 & $-0.6,1.8$ & 0.65 & $-0.6,1.8$ & -0.48 & $-1.7,0.8$ \\
\hline$B_{6}$ Cluster & $2^{* * *}$ & $1.8,2.2$ & $2^{* * *}$ & $1.8,2.2$ & $2.0^{* * *}$ & $1.2,2.0$ & $2^{* * *}$ & $1.8,2.2$ \\
\hline$B_{7}$ Reciprocity & 0.00 & $-0,0$ & 0.00 & $-0,0$ & -0.01 & $-0,0$ & -0.01 & $-0,0$ \\
\hline$B_{8}$ Household need & - & - & -0.20 & $-0.6,1.8$ & $-0.2^{* *}$ & $-0.4,-0.1$ & $-0.19 *$ & $-0.4,-0.1$ \\
\hline Relatedness*need & - & - & $1.4^{* * *}$ & $0.9,1.8$ & $1.3^{* * *}$ & $0.9,1.8$ & $1.3^{* * *}$ & $0.8,1.7$ \\
\hline$B_{9}$ Grandmother & - & - & - & - & 0.59 & $-0,0$ & $2.3 * *$ & $0.6,4.0$ \\
\hline$B_{10}$ Juvenile & - & - & - & - & $0.3^{* * *}$ & $0,0.7$ & 0.24 & $-0.9,0.6$ \\
\hline Grandmother*need & - & - & - & - & - & - & $-0.77^{*}$ & $-1.4,-0.1$ \\
\hline Juvenile*r & - & - & - & - & - & - & $1.6^{* * *}$ & $0.5,2.8$ \\
\hline Giver variance & \multicolumn{2}{|c|}{$0.665(17 \%)$} & \multicolumn{2}{|c|}{$0.669(17.5 \%)$} & \multicolumn{2}{|c|}{$0.637(16.5 \%)$} & \multicolumn{2}{|c|}{$0.63(16.1 \%)$} \\
\hline Receiver variance & \multicolumn{2}{|c|}{$0.403(10.3 \%)$} & \multicolumn{2}{|c|}{$0.402(10.5 \%)$} & \multicolumn{2}{|c|}{$0.401(10.4 \%)$} & \multicolumn{2}{|c|}{$0.42(10.6 \%)$} \\
\hline Camp variance & \multicolumn{2}{|c|}{$0.43(11 \%)$} & \multicolumn{2}{|c|}{$0.405(10.6 \%)$} & \multicolumn{2}{|c|}{$0.465(12 \%)$} & \multicolumn{2}{|c|}{$0.51(13.2 \%)$} \\
\hline Dyadic variance & \multicolumn{2}{|c|}{$2.41(61.6 \%)$} & \multicolumn{2}{|c|}{$2.36(61.5 \%)$} & \multicolumn{2}{|c|}{$2.36(61.1 \%)$} & \multicolumn{2}{|c|}{$2.34(60.1 \%)$} \\
\hline Model AIC & \multicolumn{2}{|c|}{7989.80} & \multicolumn{2}{|c|}{7958.40} & \multicolumn{2}{|c|}{7955.10} & \multicolumn{2}{|c|}{7947.30} \\
\hline
\end{tabular}

Once all fixed-effects are entered into the final model, the resultant dyadic variance is 2.3 , representing an overall decrease of $36 \%$, mainly produced by relatedness and parental clusters, with a positive interaction between receiving household's childcare need and the relationship between pre-reproductive females and relatedness. The camp and receiver variances were otherwise unchanged, while giver variance decreased by $4.1 \%$ in the final model. Overall, it seems that lower costs and higher benefits of caring outweighed relatedness, particularly given the dependency of relatedness on reproductive stage (i.e. being a juvenile) and household need. Reciprocity appears to offer little explanatory power in the full sample, thus it is unclear why less related or unrelated individuals provided proximity care. However, I hypothesised that this was due to different types of kin having different motivations. Thus, I made the following predictions: (i) care from close kin will be better predicted by kin selection, weighted by costs and benefits; (ii) distant kin and non-kin provide more care according to reciprocity, weighted by costs and benefits, 
however (iii) distant kin will offer costlier care than non-kin given that they can also receive indirect benefits if care is never returned.

\subsubsection{Analysis by kin group}

To explore how the predictive power of household care need, giver cost and dyadic reciprocity vary according to kin type the dataset was separated into the three kin categories: close kin $(n=136)$, distant kin $(n=485)$ and non-kin $(n=1,424)$. Given that parents have been removed from this analysis, close kin includes only full siblings. These siblings range from age 6.2 to 27 , with a mean age of $12.8 \pm 4.8$ years. Thus, the majority $(73.5 \%)$ are juveniles while the rest are young adults. As a result, their giver cost measures are consistently lower. Distant kin includes grandparents, aunts and uncles and first cousins. Non-kin subsumes both distantly related individuals (first cousins once removed and double second, second, third and fourth cousins) as well as individuals completely unrelated. All multilevel models control for age, sex and location, retained the dyad at level one, giver at level two, child at level three and camp at level four. Full results are presented in Table 6.6.

Figure 6.3 reveals, as above, that close kin had significantly more interactions with children than distant kin and non-kin, however the slope of these relationships was dependent on the giver cost and household need. There was a significant interaction between household need and kin type; care from distantly and unrelated individuals decreased with recipient need, while care from close kin increased (Table 6.6).

Table 6.6: Predictors of allocare separated by kin type. Multilevel model with dyadic relationship at level 1, giver at level 2, child at level 3 and camp at level 4 . Sample sizes vary according to model.

\begin{tabular}{l|ccc|ccc|ccc}
\hline & \multicolumn{3}{c|}{ Close kin $(\boldsymbol{n}=\mathbf{1 3 6})$} & \multicolumn{3}{c|}{ Distant Kin $(\boldsymbol{n}=\mathbf{4 8 5})$} & \multicolumn{3}{c}{ Non-kin $(\boldsymbol{n}=1,424)$} \\
\cline { 2 - 10 } Parameter & $\mathbf{B}$ & $\boldsymbol{p}$ & $\mathbf{C I}$ & $\mathbf{B}$ & $\boldsymbol{p}$ & $\mathbf{C I}$ & $\mathbf{B}$ & $\boldsymbol{p}$ & CI \\
\hline Intercept & 0.072 & 0.973 & $-4.2,4.5$ & 4.287 & $<0.001$ & $2.6,5.8$ & 1.075 & $<0.001$ & $0.6,1.5$ \\
Child age & 0.253 & 0.085 & $-0.1,0.6$ & -0.149 & 0.011 & $-0.3,-0.03$ & 0.036 & 0.038 & $0,0.07$ \\
Child female & -0.144 & 0.846 & $-1.6,1.4$ & 0.066 & 0.862 & $-0.7,0.8$ & -0.118 & 0.297 & $-0.3,0.1$ \\
Coastal & -0.388 & 0.817 & $-4.2,3.5$ & -0.579 & 0.380 & $-2,1.4$ & -0.216 & 0.215 & $-0.6,0.1$ \\
Cost & -2.372 & 0.049 & $-4.8,0$ & -1.436 & 0.004 & $-2.4,-0.4$ & -0.402 & 0.017 & $-0.8,-0.1$ \\
Need & 1.380 & 0.002 & $0.5,2.3$ & -0.429 & 0.007 & $-0.8,-0.1$ & -0.122 & 0.018 & $-0.2,-0.02$ \\
Reciprocity & -0.024 & 0.585 & $-0.1,0.1$ & 0.048 & 0.031 & $-0.01,0.1$ & 0.025 & 0.002 & $0.01,0.04$ \\
\hline
\end{tabular}


Proximity care offered by close kin, distant kin and non-kin decreases the more care was costly to the giver, however the effect was much stronger for distant kin and the weakest for close kin. As evident by Figure 6.3, at cost 1.5 non-kin are predicted to offer more care than distant kin. In contrast, close kin are comparatively unreactive to increasing cost of care, as the relationship only just reaches statistical significance $(p=0.049)$.
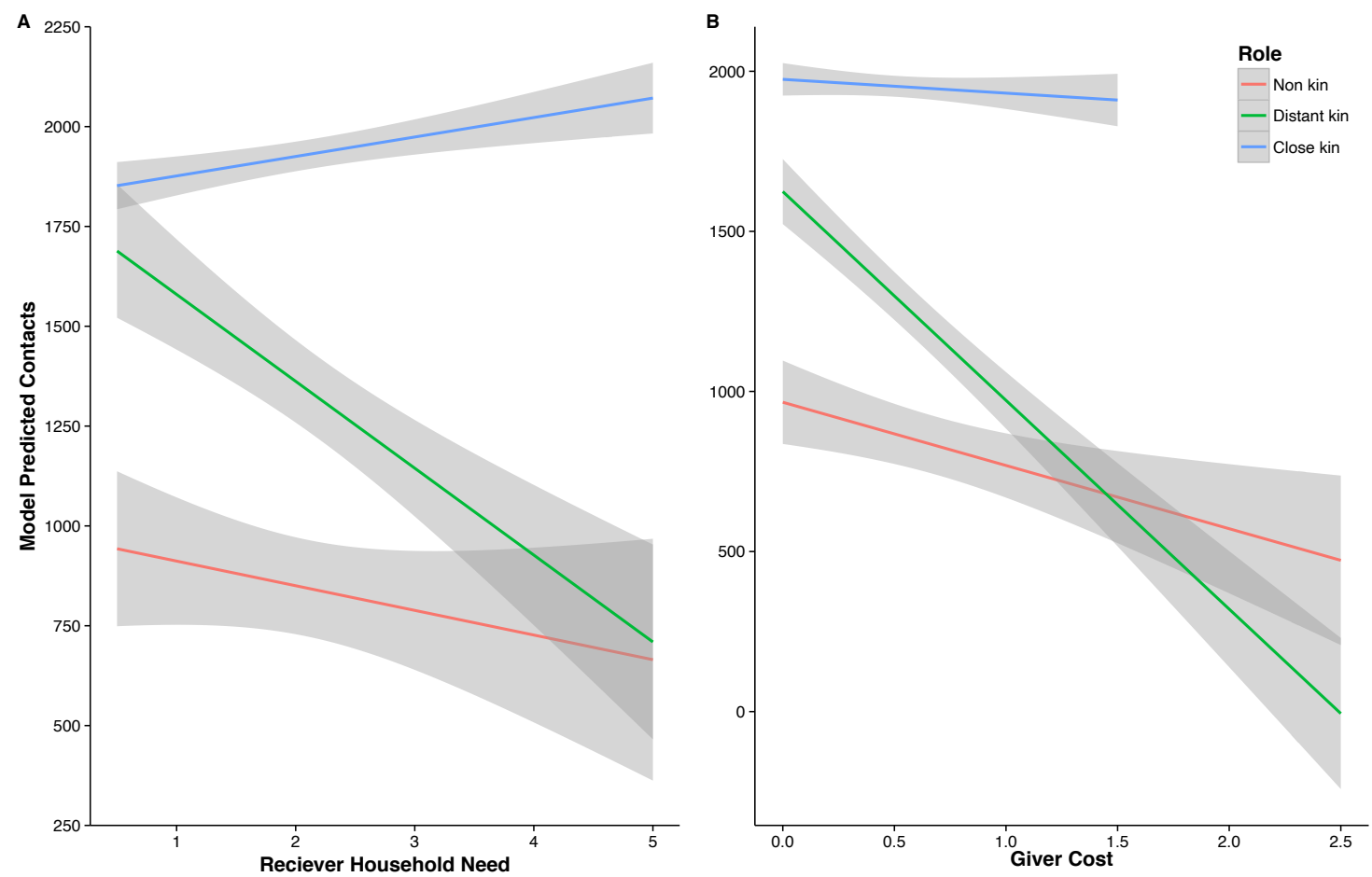

Figure 6.3: Model predicted number of contacts based on interactions between kin type and A) household need and B) giver cost. Siblings in plot B have a lower range in cost because their care is not as 'costly' as they are providing care within the household. Siblings outside the household are relatively young and have a lower giver cost as only just starting reproduction.

Therefore, distant kin as compared to non-kin, appeared more sensitive to household need, however the relationship is opposite to the predicted relationship. Rather than interacting significantly more when recipient need is high, suggestive of high benefits of care, at the highest need (4-5) distant kin care falls to the same level of non-kin care. Similarly, contra predictions I find that distant kin interactions are more sensitive to giver cost than non-kin interactions. Therefore, the predictions stemming from kin selection in relation to distant kin care are not met. However, close kin interactions appear to meet the predictions of kin 
selection exactly, as they offer the most care particularly at increasing household need, and are less reactive to the costs of the interaction.

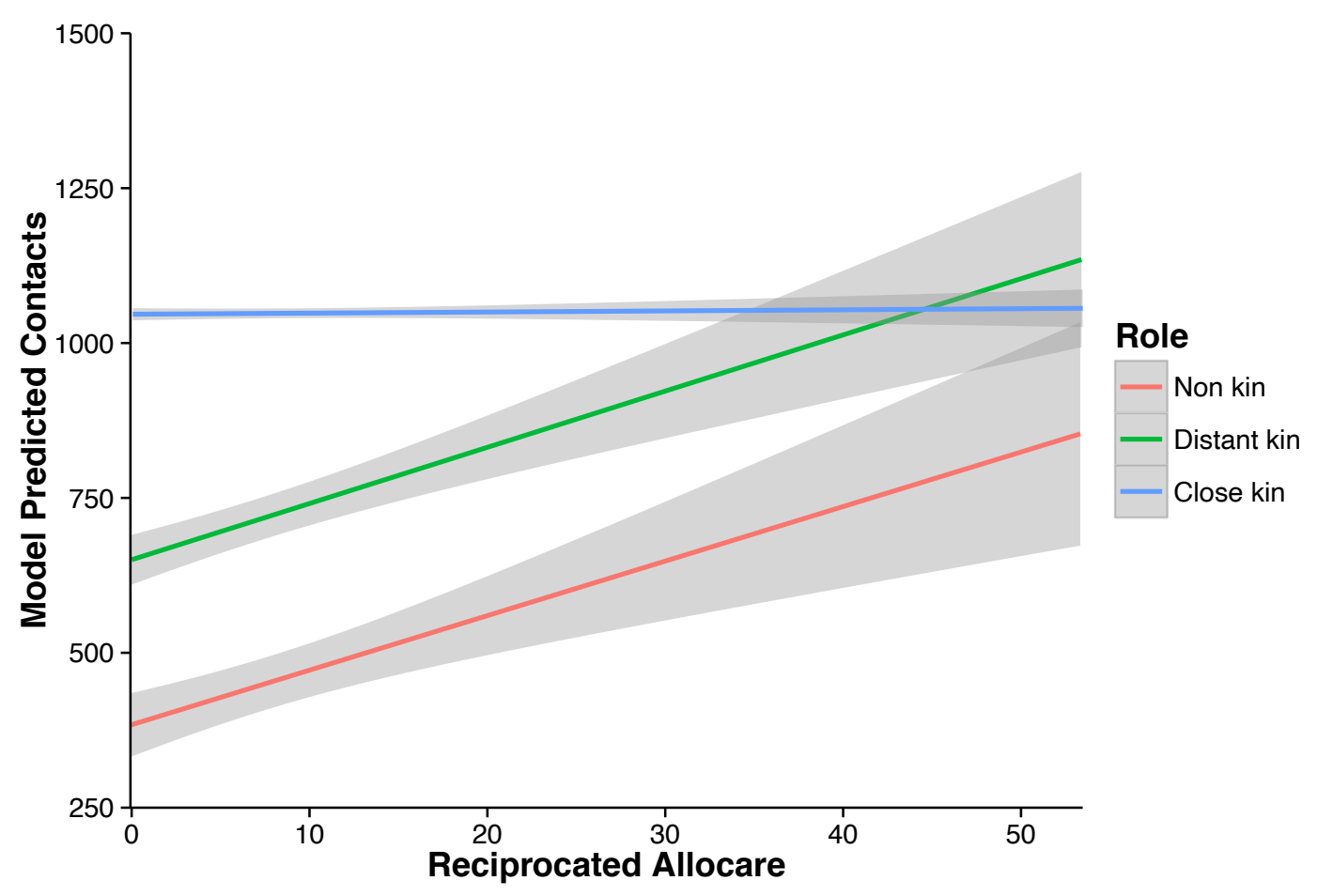

Figure 6.4: Model interaction plot separated by kin type for reciprocated allocare.

Proximity care by non-kin and distant kin increases the more this care is reciprocated (Figure 6.4), however care from close kin significantly decreases the less care is reciprocated between the households. This follows, given that close kin assist households more in 'need' of allocare, thus these are the households least able to reciprocate care, given their high numbers of dependents compared to carers.

Overall I find support for the predictions that care from close kin (here restricted to siblings as parents have been removed from the analysis) was best predicted by kin selection while distant kin and non-kin allocare was better predicted by reciprocity (Table 6.7). Again siblings' importance is reinforced as they interacted the most with dependent children, and were particularly reactive for long-term need. However, neither distant kin nor non-kin were reactive to long-term needs of the household, which makes the benefit of cooperation unclear. This may be because I have not captured the dynamics of shortterm need, which they may be more reactive to (i.e. daily variability in childcare). 
Nonetheless, it appears that non-kin may be more reliable allocarers at high cost than distant kin. This begs the question of what benefits do non-kin gain from interacting with dependent children particularly at these high costs and how is this different from distant kin.

One likely factor is that the role of unrelated juveniles in providing childcare in the form of playgroups. Observationally mixed-age and mixed-sex playgroups were common and included children aged from 2.5 to late teens $(13-15$ years). Often a few older children would watch over many of the younger children. While siblings would be present in this group, many of the interactions were with unrelated children. Thus, childcare is likely low cost for these children as they are providing care for multiple individuals simultaneously: some who are related, some who are not. Suggestive of this trend is the finding that in the non-kin dataset $(n=1,424)$ being a juvenile aged less than 16 significantly increased the number of interactions with a non-kin child compared to individuals of reproductively active age group (aged 16 to 50 years, $B=0.4, p=0.006,95 \%$ CI [0.1, 0.7]). However, no such trend was apparent in the distant kin data set $(n=485)$ as juveniles did not provide significantly more care than reproductively active individuals $(B=0.5, p=0.3,95 \%$ CI $[-2$, 1.2]). Thus, while non-kin care is defined by low-cost, juvenile allocare, distant kin care is not, and if many distant kin (i.e. aunts, uncles and grandparents) are reproductive active adults then their allocare would be highly influenced by their heavy cost of allocare due to reproductive stage.

Table 6.7: Results from interaction analysis with kin types. Minus symbols represent when the amount of interactions decreases with a predictor while plus signs are present when proximity care increases. Thus, in reading the second row "household need" this reveals that contacts from close kin increase with household need but decrease with household need from distant kin and non-kin.

\begin{tabular}{l|c|c|c}
\hline \multirow{2}{*}{ Predictor } & Close kin & Distant Kin & Non-kin \\
\hline Contact & High & Medium & Low \\
Household Need & + & - & - \\
Giver Cost & - & - & - \\
Reciprocity & - & + & + \\
\hline Supportive of ... & Kin selection & Reciprocity & Reciprocity \\
Allocarers are... & Siblings & All ages & Juveniles \\
\hline
\end{tabular}




\subsubsection{Variability in allocare: influence of ecology and 'transition'}

Here, I explored the relationship between subsistence change and ecology on the proportion of interactions with different kin type. The total sample was reduced to 66 children due to missing wealth data. Separate models were run for each of the different dependent variables (interactions with mother, father, siblings, grandparents, distant kin and non-kin). All models initially included all four 'transition' predictor variables (settlement, household foraging, belongings, food storage), however the maximal model containing all predictors suffered from multicollinearity (variance inflation factors above 2.5 (Zuur et al. 2010)). As a result, following AIC 'best fit' procedures (Burnham \& Anderson 2004), food storage and mobility were removed from the models (in all cases except the grandparental care model, in which mobility was retained as it produced the model with the lowest AIC score). The relationship between foraging and childcare appeared mediated by location, thus all models were ran with an interaction between foraging and location. If this interaction was non-significant it was removed to produce the most parsimonious model. Details and AIC values of the different models are shown in (Table 6.8). Due to the focus on the mother throughout this thesis I have chosen to use maternal proportion of work activities spent foraging as the household measure. However, in the case of examining what predicts paternal care it was appropriate to use paternal time spent in foraging, thus paternal foraging is used in the paternal care analysis only. The key predictions in this section are: (i) children at the coast receive care from a smaller network of individuals focused on the nuclear family due to relative bounty and a reduction of variance in foraging returns; and (ii) children from wealthier households with increased involvement in wage labour and cultivation will receive care from a smaller network of individuals focused on the nuclear family as risk-pooling is replaced by risk retention.

Final model results are detailed in Table 6.9 and demonstrate three trends. Firstly, as predicted, the proportion of time spent in foraging is associated with a reduction in interactions within the nuclear family (mother, father and siblings). However, there was no significant positive relationship between foraging and distant kin or foraging and non-kin. Nonetheless, it appears that more household belongings are associated with decreases in care from extra-familial sources, including grandparents, distant kin and non-kin (Figure 6.5). Furthermore, if we simply take all close kin $(r=0.5)$ we find that household wealth is significantly associated with more interactions with close kin $(B=11.2, p<0.001,95 \% \mathrm{CI}$ $[3.9,18.2])$. Thus it appears, that household wealth results in a more nuclear family focused network while increased foraging decreases it. 
Table 6.8: Model selection details for each of the six analyses separated by kin type. Lowest AIC represent the best fit model and were used in the final analysis. Each maximal model consisted of all four 'transition' variables (food storage, mobility, household belongings and proportion of time spent foraging) as well as child age, sex and location (coastal or inland). Interactions were run with measures of transition of location in the first instance, all insignificant interactions were removed in final models. Z-scores greater than 1.96 are statistically significant $(n=66)$.

\begin{tabular}{|c|c|c|c|c|}
\hline Dependent variable & Model & DF & AIC & Removed variables \\
\hline \multirow{3}{*}{ Maternal care } & Maximal model & 12 & -17.276 & \multirow{3}{*}{ Food storage and mobility } \\
\hline & Best fit model & 10 & -20.516 & \\
\hline & Non-interaction & 9 & -14.197 & \\
\hline \multirow{3}{*}{ Paternal care } & Maximal model & 12 & -64.886 & \multirow{3}{*}{ Food storage and mobility } \\
\hline & Best fit model & 10 & -67.610 & \\
\hline & Non-interaction & 9 & -63.583 & \\
\hline \multirow{3}{*}{ Sibling care } & Maximal model & 12 & 7.831 & \multirow{3}{*}{ Food storage and mobility } \\
\hline & Best fit model & 10 & 4.033 & \\
\hline & Non-interaction & 9 & 9.847 & \\
\hline \multirow{3}{*}{ Grandparental care } & Maximal model & 11 & -154.850 & \multirow{3}{*}{ Food storage } \\
\hline & Best fit model & 10 & -156.400 & \\
\hline & \multicolumn{3}{|c|}{ Interaction non-significant $(z=-1.100)$} & \\
\hline \multirow{3}{*}{ Other kin care } & Maximal model & 11 & -239.302 & \multirow{3}{*}{ Food storage and mobility } \\
\hline & Best fit model & 9 & -241.053 & \\
\hline & \multicolumn{3}{|c|}{ Interaction non-significant $(\mathrm{z}=-1.070)$} & \\
\hline \multirow{3}{*}{ Non-kin care } & Maximal model & 11 & -258.948 & \multirow{3}{*}{ Food storage and mobility } \\
\hline & Best fit model & 9 & -259.583 & \\
\hline & Interaction non-s & ificant & $(z=0.112)$ & \\
\hline
\end{tabular}

The relationship between foraging and contact care from the nuclear family was dependent on location; children at the coast whose households partook in more foraging interacted more with their mothers, siblings and fathers. Such divergent results were unexpected; however, this result may be influenced by the relative bounty at the coast compared to 
inland. Food production data comparing four inland and four coastal camps demonstrates the higher efficiency of marine fishing (Table 4.4). Consequently, individuals at the coast spent significantly less time in food production and more time socialising, resting and playing (Figure 4.7). Primary kin may then, have more time to spend with offspring if they spent a higher proportion of activities fishing. Anecdotally the nature of fishing trips was different depending on whether they occurred in the intertidal zone or in a river. River fishing was primarily conducted by males and unmarried or post-reproductive females; it was rare for younger children to be taken along. However, fishing on the reef allows a range of activities for older males (spearfishing) and women and children (octopus, shrimp and shellfish catching as well as collecting many other types of mollusc). As a result, fishing trips were often family affairs conducted over the course of a day as the family created a temporary shelter by the fishing spot. Thus, increased marine fishing need not necessitate a reduction in family care, as does hunting, gathering or river fishing.

Table 6.9: MLM results for predictors of care (age, location, subsistence and wealth) from different kin types. All covariates entered into the models are reported. Model betas are presented alongside $95 \%$ confidence intervals. Variables significant at $p<0.05$ are highlighted in bold, $n=66$. Child sex is controlled for in this analysis. GP stands for grandparents.

\begin{tabular}{|c|c|c|c|c|c|}
\hline & \multicolumn{5}{|c|}{ Predictive variables } \\
\hline Kin type & Child age & Coastal & Foraging & $\begin{array}{c}\text { Coast* } \\
\text { Foraging }\end{array}$ & Household belongings \\
\hline Mother & $-0.05[-0.1,-0.03]$ & $0.05[-0.1,0.2]$ & $-1.1[-1.8,-0.4]$ & $1.1[0.5,1.8]$ & $-0.07[-0.2,0.0]$ \\
\hline Father & $-0.01[-0.02,-0.0]$ & $-0.02[-0.5,0.01]$ & $-0.4[-0.7,-0.1]$ & $0.4[0.1,0.7]$ & $-0.03[-0.1,0.1]$ \\
\hline Sibling & $0.01[-0.03,0.01]$ & $0.2[-0.6,0.1]$ & $-1.5[-2.8,-0.4]$ & $1.8[0.7,3.1]$ & $-0.1[-0.2,0.1]$ \\
\hline GP & $-0.004[-0.01,0.0]$ & $0.01[0.01,0.2]$ & $0.1[-0.00,0.14]$ & - & $-0.04[-0.1,-0.00]$ \\
\hline Distant kin & $0.002[-0.00,0.0]$ & $0.06[0.03,0.1]$ & $0.02[-0.01,0.05]$ & - & $-0.02[-0.04,-0.01]$ \\
\hline Non-kin & $0.002[0.0,0.01]$ & $0.02[-0.02,0.1]$ & $-0.02[-0.1,0.01]$ & - & $-0.02[-0.04,-0.01]$ \\
\hline
\end{tabular}

Child age and location have an effect on care from some kin types. Matching the results from chapter 5 (Table 5.1), it is evident that mothers and fathers provided statistically more care the younger the child was; however, with increasing child age non-kin became more important (Figure 5.3). Residing at the coast was independently associated with increases in 
care from grandparents and distant kin, however this did not reach statistical significance for non-kin $(p=0.2)$. Thus, there appears to be a trend of wider childcare networks at the coast (Figure 6.5, plots B and C) contra predictions. A possible explanation is that given the $25.2 \%$ larger packet sizes at the coast (mean size in kcal 2322.7 versus 1855.3) compared to inland, resources appear less constrained implying that the presence of greater numbers of distant kin may be sustainable at the coast (i.e. less competitive and demanding relationships), thus allowing for the aggregation of larger extended families.

A

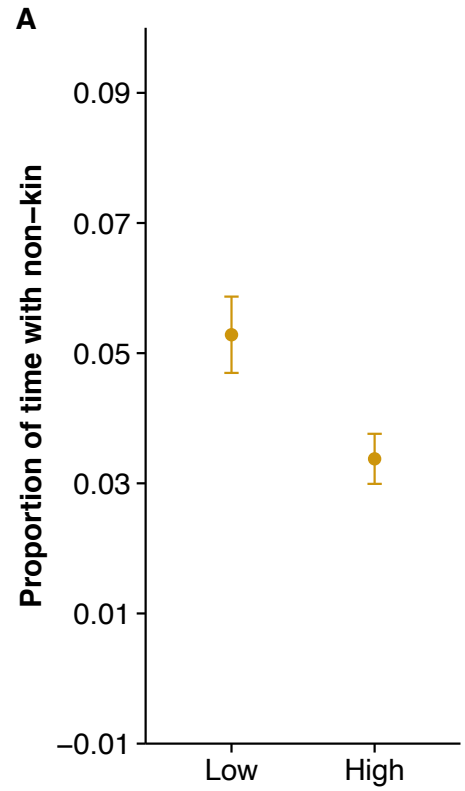

B

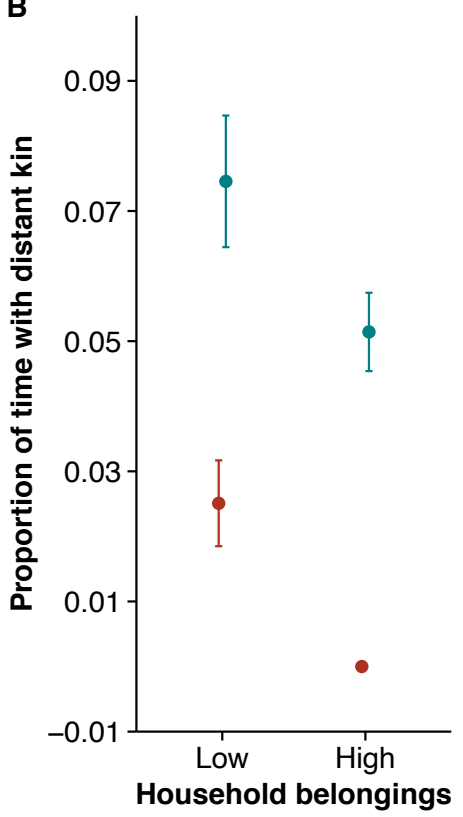

C

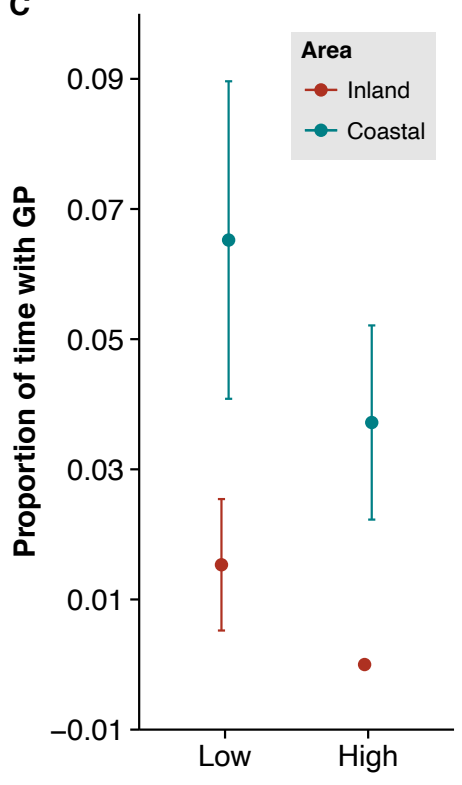

Figure 6.5: The relationship between household belongings and proportion of time spent with non-kin (A), other kin (B) and grandparents (C). Proportion of time spent with distant kin and grandparents is significantly affected by location; coastal children spent more time with individuals distantly related to them $(0.125 \leq r \leq$ 0.25). High household belongings are those above the mean (1.7), and those with low household belongings are at or below the mean. Plot A only has one set of data points as there is no significant interaction between non-kin interactions and location $(n=66)$.

To summarise this section, the evidence is supportive of prediction (ii) that wider cooperative networks become replaced by increasing wealth and alterations in subsistence. However, the evidence does not support the prediction (i) that coastal groups will have smaller cooperative networks. 


\subsection{Discussion}

\subsubsection{Kin selection, reciprocity and the role of the unrelated allocarer}

The data clearly supports the hypothesis that close kin provide care due to inclusive fitness benefits. Interactions increases with the coefficient of relatedness; close kin offer more care when the recipient household is in need; allocare by close kin is uninfluenced by contingency. Thus, this fills each expectation stemming from Hamilton's (1964) equation $r B>C$, and it can be deduced that siblings provide care primarily for inclusive fitness gains. However here, it is helpers-at-the-nest or siblings who provide the majority of allocare, not grandmothers (Table 6.10).

Table 6.10: Summary of the main results from the 'why care' analysis. If the prediction was met the indictor is marked with a $\boldsymbol{V}$, otherwise with a $\boldsymbol{X}$. If the indicator was not explored, it is marked with a question mark.

\begin{tabular}{|c|c|c|c|c|c|}
\hline \multicolumn{6}{|c|}{ Indicators } \\
\hline Theory & $\mathbf{R}$ & Contingency & Benefit & Cost & Clustering \\
\hline \multirow[t]{2}{*}{$\begin{array}{l}\text { Kin selection: } \\
\text { care pooling }\end{array}$} & $\begin{array}{l}\text { Closer kin } \\
\text { offer } \\
\text { more care }\end{array}$ & $\begin{array}{l}\text { Not present } \\
\text { among close kin; } \\
\text { care given } \\
\text { according to } \\
\text { need }\end{array}$ & $\begin{array}{l}\text { Increases care } \\
\text { from close kin }\end{array}$ & Decreases care & $\begin{array}{c}\text { Close kin offer } \\
\text { the most care }\end{array}$ \\
\hline & $\checkmark$ & $\checkmark$ & $\sqrt{ }$ & $\checkmark$ & $\checkmark$ \\
\hline \multirow[t]{2}{*}{$\begin{array}{l}\text { Reciprocal } \\
\text { altruism: } \\
\text { variance } \\
\text { pooling }\end{array}$} & - & $\begin{array}{l}\text { Distant kin and } \\
\text { non-kin care } \\
\text { increases with } \\
\text { reciprocation }\end{array}$ & $\begin{array}{c}\text { Stochastic need } \\
\text { not measured }\end{array}$ & $\begin{array}{l}\text { Care decreases } \\
\text { with increasing } \\
\text { cost; non-kin less } \\
\text { reactive than } \\
\text { distant kin }\end{array}$ & $\begin{array}{l}\text { Individuals } \\
\text { outside the } \\
\text { nuclear family } \\
\text { participate in } \\
\text { variance } \\
\text { pooling }\end{array}$ \\
\hline & - & $\checkmark$ & $?$ & $\sqrt[V]{ } / X$ & $\checkmark$ \\
\hline \multirow[t]{2}{*}{$\begin{array}{l}\text { Helpers-at- } \\
\text { the-nest }\end{array}$} & $\begin{array}{l}\text { Related } \\
\text { juveniles } \\
\text { interact } \\
\text { more }\end{array}$ & $\begin{array}{l}\text { Sibling care was } \\
\text { not predicted by } \\
\text { contingency }\end{array}$ & $\begin{array}{l}\text { Increases care } \\
\text { from siblings }\end{array}$ & $\begin{array}{c}\text { Low cost } \\
\text { significantly } \\
\text { predicts care as } \\
\text { siblings interact } \\
\text { heavily }\end{array}$ & $\begin{array}{l}\text { Care from } \\
\text { within the } \\
\text { household }\end{array}$ \\
\hline & $\checkmark$ & $\checkmark$ & $\checkmark$ & $\sqrt{ }$ & $\checkmark$ \\
\hline \multirow[t]{2}{*}{ Grandmother } & $\begin{array}{l}\text { MGM } \\
\text { offer } \\
\text { more care } \\
\text { (chapter } \\
5 \text { ) }\end{array}$ & $\begin{array}{c}\text { Distant kin offer } \\
\text { more reciprocal } \\
\text { care }\end{array}$ & $\begin{array}{l}\text { Grandmothers } \\
\text { offer less care } \\
\text { when the } \\
\text { benefits are high }\end{array}$ & $\begin{array}{l}\text { Grandparents } \\
\text { offer little care } \\
\text { comparative to } \\
\text { other kin types } \\
\text { regardless of the } \\
\text { low cost }\end{array}$ & $\begin{array}{l}\text { Extended kin } \\
\text { do not offer } \\
\text { significant } \\
\text { amounts of } \\
\text { care (chapter } \\
\text { 5) }\end{array}$ \\
\hline & $\checkmark$ & $x$ & $x$ & $x$ & $x$ \\
\hline
\end{tabular}


Human mothers, due to their high reproductive rate, have multiple dependent offspring. Here, I have revealed that mothers face a major proportion of their lifespan with more offspring than they could arguably care for alone. This is what Hill \& Hurtado (2009) refer to as predictable life history shortfalls as each mother will face the deficit of care arising from the relatively rapid production of offspring. This is a long-term shortfall, thus reciprocity should not be foreseeable in the near future, and consequentially kin are expected to provide more allocare given indirect fitness gains from cooperation (AllenArave et al. 2008; Hooper 2015). Accordingly, I have demonstrated here that siblings' allocare meets the expectations of 'care-pooling': that they offer more care the higher the need of the household (i.e. the more dependents versus carers) resulting in an imbalance, as care is unidirectional. Thus, I argue that without this, mothers would not be able to maintain such a reproductive rate, suggesting the adaptive value of helpers-at-the-nest. These results further support the results and conclusions of chapter 5.

However, contra predictions we do not see the same pattern among distant kin, given that distant kin, and grandmothers in particular, appeared to reduce interactions with increasing household need. This may occur because grandmothers increase food production to cater for more dependent grandchildren, thus combining food provisioning with direct childcare is an essential next step to explore these questions further. Nonetheless, here it appears that direct childcare is not unbalanced according to need (as predicted by kin selection) and distant kin cannot be argued to be care pooling due to long-term life history shortfalls, driven by indirect fitness gains. Similar results have been found elsewhere, as the effects of kinship quickly evaporate as $r$ decreases (Koster \& Leckie 2014). This dynamic is not unexpected as more distantly related individuals receive a higher fitness return from following a tit-for-tat strategy rather than kin selection (Axelrod \& Hamilton 1981). Titfor-tat is expected when $B p>C$ ( $p=$ the probability of future interactions). Whenever the value of $p$ exceeds the dyadic coefficient of relatedness, cooperation should be governed by reciprocal altruism, not kin selection (Allen-Arave et al. 2008; Gurven et al. 2001). This appears the case among the Agta; while in the full sample reciprocity was not a significant predictor of allocare, it is among distantly and unrelated individuals. Therefore, among distant kin, as has been found in food sharing among the Ache (Allen-Arave et al. 2008), it seems that even if cooperative dyads are related, their sharing is motivated by reciprocal altruism rather than kin selection. Thus, it is foolhardy to argue that nepotistic mechanisms drive cooperative breeding without exploring costs, benefits and conducting multivariate analyses to weigh up different hypotheses (Barclay \& Reeve 2012). While this strategy does 
not reveal causality, it does effectively discriminate between causal hypotheses and allows for the controlling of confounders, such as association (Shenk \& Mattison 2011).

Apicella \& Crittenden (2013) lament the lack of data about childcare from unrelated individuals. Given that all societies maintain significant social ties with unrelated individuals, it seems a large oversight to ignore their role in communal breeding. In chapter 5 I demonstrated that non-kin provided a significant proportion of care received by a child, and while the majority of non-kin interact infrequently, a few provide a level of contact comparable to close kin. Thus, similar to the results among the Hazda (Crittenden \& Marlowe 2008) and Efe (Ivey 2000), non-kin are providing a significant amount of care, indicating their importance. Given that, as revealed by Figure 6.2, that even considering the role of older 'caring' siblings, mothers face periods of care shortage which can be amplified due to further unpredictable shortfalls in care, having additional allocarers fully outside the household can be considered to be important strategy of mitigating risk. This begs the question: why do unrelated Agta allocarers provide care? I suggest that reciprocal altruism is a potential solution for unpredictable shortfalls as a form of risk pooling.

Risk transfer is any action which distributes risks between different 'exposure units', in this case individuals and households. Units all accept immediate, but small losses (i.e. cooperation) when they can afford to do so and thus, mitigate the severity of future unpredictable and larger losses (Aktipis et al. 2011). As argued in section 2.2.3, this is another expression of reciprocal altruism in which actors suffer short-term costs for higher total returns at some point in the future. Cashdan (1985) argued that for risk transfer to be an effective method of buffering unpredictable shortfalls, the network of reciprocators must be large and the exposure units independent. Thus, it follows that here I find that contingency best predicts the amount of care received from distant kin and non-kin only. These individuals are outside of the immediate household, thus experiencing different patterns in foraging returns influenced by sickness and disability as well as hunting success. Furthermore, reciprocity in childcare appears dependent on costs as both distant kin and unrelated individuals offer significantly less care when they themselves experience a high cost in childcare, supporting the variance-pooling hypothesis. However, I have not measured stochastic need in this study; thus it is not possible to conclude that short-term reciprocity is dependent on short-term childcare needs. A complete quantification of this question requires collection of data on fluctuations in foraging success and sickness for 
each household simultaneously as data is collected on childcare. Nonetheless the current evidence is supportive of the predictors for variance pooling laid out in Table 6.10.

Arguably, then reciprocal cooperation in terms of communal breeding is an important behavioural strategy to ensure that children receive an adequate amount of childcare regardless of household shortfalls in childcare labour or food production. Similar to the original argument by Emlen (1982) then, I argue that communal breeding may result in increased fitness returns for the giver when they are unrelated or more distantly related due to unpredictable environments. There is supportive evidence of the role of environmental unpredictability in the evolution of cooperative breeding in birds (Rubenstein \& Lovette 2007; Jetz \& Rubenstein 2011) as well as naked mole rats (Faulkes et al. 1997) and Malagasy strepsirrhines (Tecot et al. 2012). Therefore, an individual provides costly care now while they can afford the labour costs to ensure that at some point in the future they will receive allocare when they require it more. If by receiving allocare mothers and the household can spend more time in food production, somatic maintenance and/or child quality ultimately reciprocal allocare will increase child survival and/or maternal fertility. It is these questions I investigate further in chapter 7.

\subsubsection{Less than cooperative distant kin and more cooperative non-kin?}

By-and-large the aforementioned results matched predictions laid out in Table 6.1, however several results did not follow expectations. As argued above, reciprocity is not possible in the short-term for life history shortfalls, thus kin are likely to bear the highest immediate cost in cooperating (Alvard 2009). As a result, distant kin are expected to be reactive to long-term household needs. This was not the case, and in fact grandmothers only offer significantly more care than non-grandmothers when their offspring have 'low' need scores, or very few dependent offspring compared to number of carers. The second finding is that at high cost when distant kin are predicted to offer more allocare than nonkin due to the increased likelihood of future relationships and possible indirect benefits, it is non-kin who interact more with dependent offspring. Therefore, why do distant kin not provide care according to indirect fitness gains? Furthermore, why do non-kin react less to high cost allocare and ultimately provide more care than distant kin at high cost? I propose that the explanations may lie in distant kin focusing on their own families who face equally high care shortages and are more likely to compete over resources and that non-kin allocarers are juveniles who crèche children, resulting in low cost care. 
Mothers have a high need index when they have more dependent children compared to the numbers of carers available in the household. This peaks at the age of 25-30 years when mothers can have a newborn, toddler and a child less than five years. However, if we take the average age of first birth of 19.7 years among our sample, then a young mother aged 26 (who on average had 2.8 offspring at this age) has a mother who is 45.7 years old and still has, on average, 2.8 dependent children under the age of 12 . Thus, even for later born children, a grandmother may not be fully available to provide care until later in life, when the hazards in the environment start to take their toll on older individuals. Therefore, at exactly the time when a mother is in need of allocare, co-residing grandmothers are unable to provide costly allocare without short-term reciprocation. Similarly, a mother likely has some co-residing siblings or siblings-in-law who are undergoing a similar reproductive stage, thus have high cost in providing allocare. Therefore, distant kin may be unreactive to needs due to simultaneously high childcare needs. Of course, non-kin also will be reproductively active and not all distant kin are reproductively active, however a key difference is that kin co-reside and may compete over limited resources.

Mathematically and experimentally increasing local competition due to limited dispersal of relatives significantly diminishes the predictive value of $r$ in $r B>C$ as the cost benefit ratio is altered (West et al. 2001; Griffin et al. 2004). Given that resources are always finite, and kin prefer to co-reside (Dyble et al. 2015), an individual's main competitors are kin who come with a spouse and, in all likelihood, dependent offspring. As argued above, food sharing occurs primarily among $3-4$ households which are significantly more related to one another than expected by chance, and in particular, contained significantly more grandparents, uncles and aunts (Dyble et al. n.d.). In contrast, non-kin reside outside of the 'resource clusters' and thus, are not in competition. Therefore, increasing the fitness of a siblings' offspring may expand the number of competitors. While this may increase an individual's indirect fitness gains it would decrease their direct fitness: at low levels of $r$, the fitness returns required to compensate for the fitness cost are significantly high (Hamilton 1964) and investing in direct fitness and providing allocare in a tit-for-tat manner may result in the highest fitness returns. Thus, distant kin are still cooperative, only they gain inclusive fitness gains vis-à-vis direct benefits of reciprocal cooperation rather than the indirect benefits associated with kin selection.

In Strassmann and Garrard's (2011) meta-analysis of 17 studies examining the effects of grandparents in patrilocal societies the data supported a local resource competition 
hypothesis. Given that children in patrilineal societies are in competition with paternal relatives - whose fitness interests are never fully in line with each child's fitness interests maternal grandparents have a positive influence, as they are non-resident and thus, noncompetitors. This is why, Strassmann and Garrad (2011) argue, maternal grandmothers and grandfathers consistently have a more positive effect on child survival than paternal grandparents. Similar results have been reported among the Kipsigi (Borgerhoff Mulder 2007) and Pimbwe (Hadley 2004). Specifically, among the Ache females during the forest period experienced a 10\% reduction in age-specific fertility for each additional co-residing adult sibling, thus Hill and Hurtado (1996) interpret these results in terms of kin competition for group resources. Therefore, when kin co-reside cooperation may be reduced when the benefits and/or relatedness is not sufficient to counteract the high cost of allocare and increases in resource competition. These factors will significantly reduce the predictive power of kin selection (Hamilton 1967). A limitation here is that I have grouped together all distant kin regardless of lineage, reproductive stage and age. All these factors are likely to have a different influence of the degree of competitive or cooperative nature of the relationship. Further examinations should explore amount of care offered according to resource competition, particularly focusing on food sharing clusters, reproductive stages and cooperation between different kin types.

Turning to the second question above - why do non-kin provide more care at higher cost than distant kin? - this is in part answered by the fact that if kin competition reduces an individual's fitness, providing allocare to increase their kin's fitness would only further reduce their lifetime fitness. Thus accordingly, I find that distant kin are highly reactive to giver cost, quickly reducing allocare when the price is high. However, why do non-kin appear relatively uninfluenced by the cost of allocare? Certainly the cost does reduce the number of dyadic interactions, however the slope is not dramatic and as a result they provide more allocare than distant kin. This, I argue, is the result of juvenile allocarers 'crèching' multiple children into playgroups, thus the addition of unrelated children costs the providers very little.

The ethnographic observation of mixed-age, mixed-sex playgroups in hunter-gatherer societies is significant (Konner 2005). Among the Agta, children from the age of two years have been reported to spend a significant amount of their time in playgroups, which is in agreement with my own observations (Peterson 1978). Often within these playgroups, children are involved in observational and experimental learning, particularly under the 
guidance of older siblings (Griffin \& Griffin 2010; Kelly 2013; Peterson 1978). The playgroups consist of unrelated children who are watched over by a couple of older children who have one or two siblings in the group. My point is one of diminishing costs: if an older child must provide care for a sibling, the increase in cost of also providing proximity care to other children may be small or even insignificant. Perhaps the care will be of lower quality (see chapter 7), but it would certainly allow a mother to conduct other activities (as seen in chapter 5 where non-kin care significantly reduced maternal childcare). Supporting this hypothesis is that fact that relationships with non-kin are significantly assorted by age. We have shown here and previously that children aged between 2 and 12 years spend more time interacting with non-kin of their own age group (i.e. 2 - 12 years) than older individuals (Migliano et al. 2016). Furthermore, here I demonstrated that this is not the case for distant kin, indicating why these two types of allocarers interact differently with increasing cost. One essential test would be to quantify observations of playgroups, understanding their structure and costs compared to the benefits received from participating mothers (i.e. those mothers who leave offspring in playgroups).

\subsubsection{Subsistence change, risk and how to mitigate it}

The finding that, as predicted, subsistence change and wealth accumulation influence who provides allocare is supportive of cooperation driven by reciprocal altruism and risk pooling. Time spend foraging is associated with decreases in the amount of time spent with the nuclear family while increases in household belongings decrease contact with more distant kin and increase care from close kin (mothers, fathers and siblings). Due to the strong relationship between the amount of time spent in either cultivation and/or trade (i.e. non-foraging activities) activities and the amount of household wealth (section 4.5), these traits are considered significantly interrelated. Thus, more acculturated, settled Agta camps focus increasingly on the nuclear family compared to wider networks based on reciprocal altruism and risk pooling. However, this relationship is influenced by an interaction with location: while the proportion of time spent foraging has a negative influence on the amount of time spent with nuclear families inland, the opposite occurs at the coast. This result may be the outcome of both the higher returns per hour from marine sources, resulting in adults spending more time in camp resting, socialising or conducting domestic tasks (Figure 4.10). This would increase the likelihood that members of the nuclear family are proximate to a child. 
Nonetheless, the fact that changes in subsistence and wealth accumulation are associated with altered caregiving dynamics meets expectations based on risk mitigation. Risk can be dealt with either by risk transfer (i.e. cooperative breeding), risk reduction (residing in a resource-abundant environment), risk avoidance (subsistence diversification and mobility) and risk retention (using wealth and/or food storage to directly absorb and buffer losses (Aktipis et al. 2011; Hao et al. 2015). Individuals may mitigate risks in multiple ways, however some strategies close the door to others (Cashdan 1985; Winterhalder et al. 1999; Fafchamps 1992). For instance, mobility becomes increasingly difficult with increasing food storage and wealth, and individuals with a lot of wealth may want to extract themselves from sharing networks (Hadley 2004; Fafchamps 1992). This trend was found among the Mpimbwe of Tanzania, where individuals more engaged in the cash economy could afford to avoid risk pooling and removed themselves from sharing networks (Kasper \& Borgerhoff Mulder 2015). In his review of the research on food storage, foraging, mobility and environment variability, Kelly (2013) considers mobility as essential for gathering social ties to ensure an insurance network is in place to help buffer from environment shortfalls without food shortage. Consequently, I would argue that rather than one single method of reducing risk, individuals have multiple options which function in different contexts. Movements towards cultivation and/or wage labour can result in the accumulation of household wealth and resources which reduce the need and ability to be mobile, while making other households dependent on wealthier households. Thus, wealthier households withdraw from insurance networks, resulting in more closed networks, as risks no longer require wide distribution but can be retained and absorbed within the household (Fafchamps 1992). A similar trend was found among two Tabo groups from the South American Gran Chaco in which more 'traditional' communities were defined by care from grandmothers and non-related juveniles, while increasing acculturation resulted in increasing amounts of childcare from mothers and fathers (Valeggia 2009).

This reveals that focusing on variance within populations and not seeking a singular description of childcare is key to understanding the selective pressures on the evolution of cooperative breeding. These results further support the hypothesis that communal breeding is related to unpredictable environments: if we, as I do here, assume that foraging modes of subsistence produce increased variability in food returns compared to mixedmodes of production then more extended networks of allocare dependent on reciprocity or variance pooling should be expected in foraging contexts, as we see here. Examining 
variability further within a population is an exciting avenue of future research that should, rather than assume that subsistence diversification reduces resource stochasticity, measure this directly and quantify the influence on behavioural strategies, particularly cooperation.

The results from the effect of ecology on childcare did not, however, meet predictions, as it appeared that coastal households relied more on care from grandmothers and distant kin. However, this trend was non-significant for non-kin painting a less consistent picture. The hypothesis that best explains these results is that residing in a resource-abundant environment (denoted by the larger package sizes at the coast) results in more cooperative, larger aggregations of extended families. Above I argued that in locally resource-poor areas, kin often become competitors due to limited dispersal (Griffin et al. 2004). However, if resource competition is limited and consistent in neighbouring areas then any competition between relatives is negligible and the importance of indirect fitness gains increases (West et al. 2001). Thus, in a resource-abundant area, as coastal regions often are (Kelly 2013), distant kin may cooperate more as the indirect fitness gains are higher without competition for resources. While this requires a significant amount of exploration, it does reveal interesting avenues of future research focused on how behaviour is affected by ecological variability.

\subsection{Conclusions}

This chapter set out to explore why individuals provided costly allocare, focusing on indirect fitness and the role of reciprocity in risk reduction. Overall, the predictions from kin selection are overwhelmingly met for close kin allocare. Thus, siblings gain direct inclusive fitness benefits from providing allocare. However, kin selection does not predict allocare from distant kin, which appears reactive to reciprocity rather than long-term childcare needs. Thus, I have argued that due to co-residence, lower levels of relatedness may result in a reduction in cooperation, particularly at higher costs, thus confounding the relationship between allocare and relatedness. Kin competition should be an equally important consideration in future research. Nonetheless, these results reveal that just because a dyad is related does not mean that allocare stems from nepotism; competing effects of reciprocity, costs and benefits must be separated for a more accurate portrayal of cooperation. Both distant kin and non-kin allocare are reactive to reciprocity suggesting that short-term returns are important. This follows the predictions of variance pooling which suggests that communal breeding is adaptive when individuals require buffering 
from unpredictability. In particular, I found that increasing wealth and subsistence diversification reduces wider allocare networks, which are dependent on reciprocity. This indicates the adaptive value of non-kin allocare may lie in buffering unpredictable risks. As non-kin allocare is never explored in such manner these results offer important new insights into the evolution of communal breeding, particularity when multiple mothers reduce simultaneously, as occurs in plural breeding systems. 


\section{Chapter Seven}

\section{Betwixt and Between: Social Networks Effect on Allocare and Reproductive Success}

There is significant evidence from non-human primates suggesting the adaptive nature of social ties, as they are associated with both increased longevity and fertility. However, the literature in humans, and in particular hunter-gatherers, has focused primarily on males' social status, hunting skills and fitness. Thus, while we know that indirect social ties (i.e. friends-of-friends) have implications for disease and information transmission, we know little about their role in female reproductive success. Here, I examine the adaptive function of direct (dyadic) and indirect social ties by exploring the role of social network position in soliciting allocare. I explored these questions by collecting reproductive histories, proximity networks, anthropometrics and disease data for 39 Agta mothers and 91 offspring living in six camps. I find that mothers aged over 25 with greater betweenness centrality (an individual with high betweenness links otherwise disparate parts of the network) had higher fertility and overall reproductive success at the cost of increasing poor health for both mothers and their offspring. The relationships appeared to be influenced by non-kin allocare: maternal betweenness is associated with increases in non-kin allocare, which is independently positively correlated with reproductive success while being negatively correlated with child quality. Therefore, Agta mothers appeared to focus on child quantity rather than quality in a high-risk environment vis-à-vis allocare. Furthermore, degree centrality (the number of dyadic ties an individual has) was negatively associated with reproductive success. I argue that simply having many direct ties equates to more competitors for resources, however being able to access disparate groups increases maternal ability to solicit allocare when facing childcare shortages, which increase with age. By expanding the cooperative breeding perspective to explore social networks I demonstrate that maternal centrality can be considered adaptive and a key component in ensuring successful reproduction of multiple offspring. 


\subsection{Introduction}

Sociality arises when it benefits individuals' fitness above and beyond the costs of living in a group (Silk 2007b). Thus, social status and bonds have long been associated with positive fitness outcomes including increased longevity, offspring survival and fertility (Brent 2015; Silk et al. 2006; Seeman 1996; Cameron et al. 2009). For instance, as explored in chapter 5, it is evident that childcare by non-kin significantly reduces the amount of energy a mother invests into childcare. This theoretically allows her to invest more energy into either child quality or her own fertility, increasing her overall reproductive success. As a result, individuals are expected to be able to manipulate their social worlds to maximise their fitness (Barrett et al. 2012). However, individuals' social networks are more complex than simple dyadic (direct) ties; social structure arises from the indirect ties between whole groups of individuals (Brent et al. 2011). For instance, your position in the network, who your friends are connected to (i.e. friends-of-friends) and the degree of network clustering are impossible to examine by only exploring dyadic relationships. These features play a central role in the transmission of information (Mann et al. 2012), disease (Weber et al. 2013; Keeling \& Eames 2005) and even emotions (Fowler \& Christakis 2008; Rosenquist et al. 2011). Thus, while we currently know much less about an individual's network position influence on fitness (Royle et al. 2012), the evidence suggests it could be under selective pressure and considered an extended phenotype requiring investigation (Formica et al. 2012).

Empirically, social ties appear to have a positive influence of fitness, however the evidence suggests that social bonds can be beneficial when they are few and strong as well as when they are many and weak (conceptualised as strength and breadth). In both yellow (Papio gynocephalus) and chacma baboons (Papio ursinus) from Botswana it was strong, consistent social bonds, particularly with female kin, that predicted survivorship of both the mother and her offspring (Silk et al. 2009; Silk et al. 2010; Silk et al. 2006). Similarly, Schulke and colleagues (2010) find that in Assamese macaques (Macaca assamensis) strongly bonded males had significantly higher dominance positions, promoting paternity success. However, the importance of a few strong social bonds is not consistently found. For instance, Cameron et al. (2009) showed that a composite measure of social integration in female feral horses (Equus sp.) was associated with increased fertility, while amount of grooming received from both male and female Amboseli baboons was associated with a significant increase in likelihood of females survival (Archie et al. 2014). Therefore, it may be the number of connections and degree of social integration which predicts fitness rather than 
strength of social ties, a finding also evident in Barbary macaques (Macaca sylvanus) and female vervet monkeys (Chlorocebus pygerythrus (Lehmann et al. 2015; McFarland \& Majolo 2013; McFarland et al. 2015)). Thus, large extended networks consisting of many weak ties may be as important as a few strong ties.

Consequentially current research indicates that some type of social ties has fitness implications, however which exact form seems highly variable. Murphy and colleagues (n.d.) specifically argue that weak and strong bonds may be more or less important given environmental hardship experienced in some baboon populations compared to others. Thus, in their sample, both strong and weak bonds are important as they allow mothers to maintain consistent support and flexible access to larger networks, which might be essential in less predictable environments. Examining the potential pathways by which sociality can promote fitness can shed more light on this issue. For instance, in chacma baboons smaller, more focused grooming groups have been associated with lower glucocorticoid levels due to grooming's stress relieving effects (Wittig et al. 2008; Crockford et al. 2008; Silk et al. 2009). As glucocorticoids are well known reproductive suppressants (Cameron 1997) as well as an immunosuppressant (Cohen et al. 1997; Tamashiro et al. 2005), this indicates the protective role of close-knit and consistent networks among some baboons. However, in horses the fitness-promoting effect appears to stem from group insulation from male harassment, which significantly reduced reproductive success. Thus, composition may matter less compared to how well connected individuals are (Cameron et al. 2009). Similarly, among barbary macaques more connections resulted in feeding tolerance and more coalition partners (Lehmann et al. 2015; McFarland \& Majolo 2013) while in vervet monkeys more partners meant better thermoregulatory ability (McFarland et al. 2015). Thus, as with any trait, different forms of social networks appear adaptive under different ecologies, constraints and selective pressures.

In humans (Homo sapiens sapiens), and in particular hunter-gatherers, the focus has remained on the direct benefits of cooperative action, rather than the influence of network structure and social position. For instance, there is extensive literature exploring why foragers engage in resource redistribution (Hooper et al. 2015; Jaeggi \& Gurven 2013a; Kaplan \& Gurven 2005; O'Connell et al. 1999) or who helps in childcare (Crittenden \& Marlowe 2008; Hrdy 2011; Kramer 2011; Meehan 2005). These traits are argued to be flexible and context dependent (Sear \& Mace 2008). However, it is unclear how individuals are able to manipulate this cooperation to optimise their fitness returns. Nonetheless, there is 
evidence that hunter-gatherer networks exhibit significant homophily (clustering according to similarity) allowing co-operators to preferentially connect to each other, thus facilitating redistribution (Apicella et al. 2012). At the same time, direct social ties are significantly correlated with human longevity (Holt-Lunstad et al. 2010; Giles 2005) while indirect ties predict the spread of obesity (Smith \& Christakis 2008), happiness (Fowler \& Christakis 2008) and depression (Rosenquist et al. 2011) throughout human social networks. Thus, network position has important implications for human wellbeing and survival; however the implications for fertility are less clear. As a result, I bring these approaches together and examine the adaptive function of direct and indirect network ties on maternal fitness by exploring the possible role of network position in soliciting allocare.

\subsection{Hypotheses and predictions}

\section{Box 1: Social network Analysis definitions}

- Node: independent actors who structure networks. Here nodes are individuals.

- Edges: the links that connect different nodes.

- Weighted network: edges are not binary (unweighted network) but weighted according to the number of interactions between nodes.

- Centrality: how important edges are in a network. Multiple measures:

○ Degree: number of edges attached to a node

- Strength: weighted version of degree, sum of the weights of all edges.

$\circ$ Betweenness: the number of geodesic (shortest) paths a node lies on between other nodes.

O Eigenvector centrality: the sum of the degree and strength of the nodes connected to ego

- Direct ties: A is directly connected to B

- Indirect ties: $\mathrm{A}$ is indirectly connected to $\mathrm{C}$ via a direct tie with $\mathrm{B}$
I have argued in section 2.4 and chapter 6 that hunter-gatherers, rather than rely on material resources such as property or wealth, buffer the risks of stochastic environments with relational wealth (Smith et al. 2010; Woodburn 1982; Chaudhary et al. 2015). As a result, I hypothesise that social networks function as a resource to facilitate cooperation. Thus, given humans' significant communal breeding and its implications in our evolution (Burkart et al. 2009; Sear \& Mace 2008; Crittenden \& Marlowe

2008; Hrdy 2011; Lancaster \& Kaplan 2009; Hawkes \& Coxworth 2013), maternal network position is expected to play an important role in the acquisition of allocare. I make the following two predictions: (i) measure/s of maternal network position will positively associate with child quality, maternal fertility and quality and (ii) more central mother's received more allocare from outside the household and/or the extended family.

Prediction (i) stems from the theoretical implications of cooperative breeding and life history theory. Allocare acts as a source of additional energy, lifting constraints off the mother. Thus, the mother is able to invest the additional energy into either current and/or 
future reproduction. Consequentially, increased allocare should be associated with either increased fertility and/or maternal quality if investment is placed in reproduction. Or, allocare may equally well be associated with increased parental effort as mothers produce higher quality offspring. As discussed in section 1.2.3, these energy allocations are weighed against one another given extrinsic risks (Charnov 1993). Mothers may invest in multiple low quality offspring when they cannot mitigate environmental risks or receive diminishing returns for their efforts (Quinlan 2007). As a result, if prediction (ii) holds and maternal network position is associated with increases in allocare, I expect increases in either: maternal fertility; maternal quality (as a proxy for future reproductive effort); or child quality.

Here, I explored these hypotheses and predictions by examining the relationship between four measures of maternal network position or centrality (betweenness; eigenvector centrality; degree; and strength (Gilby et al. 2013)) and fertility, survivorship to age 16 (proxy for reproductive success), childhood mortality (all deaths under 16 years), child and maternal nutritional quality (BMI and haemoglobin levels) as well as self-reported sickness over the last two weeks. I further explored the degree to which the same measures of network centrality correlated with the amount of care a mother's offspring received from kin, non-kin and non-household members over the duration of one week using motes to capture three-meter proximity. From this analysis I find two clear and consistent results: Firstly, maternal betweenness is positively associated with allocare from non-kin, fertility and reproductive success while negatively correlating with maternal and child quality. Secondly, degree centrality negatively predicts fertility, reproductive success and maternal BMI. Furthermore, non-kin allocare positively predicts reproductive success while negatively associating with child nutritional condition. Thus, it appears allocare received from non-kin may mediate the association between fertility and maternal betweenness.

\subsection{Methods}

Full data collection methods for motes deployment, reproductive histories, medical exams, anthropometrics and household characteristics have been described in the methodological chapter 3. 


\subsubsection{Social Network Analysis}

Social network analysis (SNA) is a useful tool for the exploration of the adaptive function of sociality as it quantifies the emergent properties of a social network (Barrett et al. 2012). Moving beyond dyadic or direct ties between individuals, SNA examines how the interaction between individuals creates a structure which impacts the behaviour and function of a system (Brent 2015; Newman 2010; Kurvers et al. 2014). Human social networks, similar to many other complex networks, are not homogeneous (i.e. each individual is connected to the same number of individuals). Rather some individuals have significantly more ties than others, resulting in a scale-free network (Keeling \& Eames 2005). The existence of highly central, well-connected individuals results in differential access to the currency moved throughout the network, be it influence, information, resources or even disease (Kurvers et al. 2014; Newman 2010; Meyers et al. 2005). Thus, different measures of centrality are predicted to result in differential outcomes (Lehmann et al. 2015; McFarland \& Majolo 2013; Murphy et al. n.d.). Here, I explore two different types of measures of centrality - direct and indirect - for 39 mothers. Each captures different pieces of information, which may be pertinent to an individual's fitness. As SNA entails its

own new language please refer to box 1 for definitions of terms used throughout this chapter.

The two direct measures of centrality are degree and strength. Degree is the number of edges (ties or links) connected to a node (an individual) thus represents the number of an individual's social ties. This is one of the most common measures of an individual's network position as arguably an individual with more connections may have more prestige and influence. Degree is particularly useful when a network is binary or unweighted (Newman 2010). However, when a network is weighted (i.e. edges have different weights according to the frequency of interaction) then strength centrality captures more information. An individual's strength is the sum of all the weights on the edges connected to it, thus captures the differences between strong and weak ties by measuring an individual's gregariousness (Whitehead 2008).

Indirect ties capture not only dyadic interactions but also an individual's network position given the positions of all other nodes. Here I choose two diverse measures of indirect centrality: betweenness and EC. Betweenness quantifies the degree to which a node is 'between' two clusters of other nodes which do not share edges, as demonstrated in Figure 7.1 (Freeman 1977). Betweenness then, is a measure of the degree to which a node lies on 
the path between other nodes, and is proportional to the number of geodesic (shortest) paths it lies on between any given two nodes (Newman 2010). Thus, it acts as a 'broker' in the network as they have a large influence on the flow of resources throughout the network (Newman 2004; Brent 2015; Whitehead 2008). Interestingly, individuals with high betweenness may have low other centrality measures as they may relatively peripheral to a couple of clusters. Betweenness, unlike other measures of centrality also exhibits a high range as it is significantly left-skewed (Newman 2010; Lusseau \& Newman 2004).

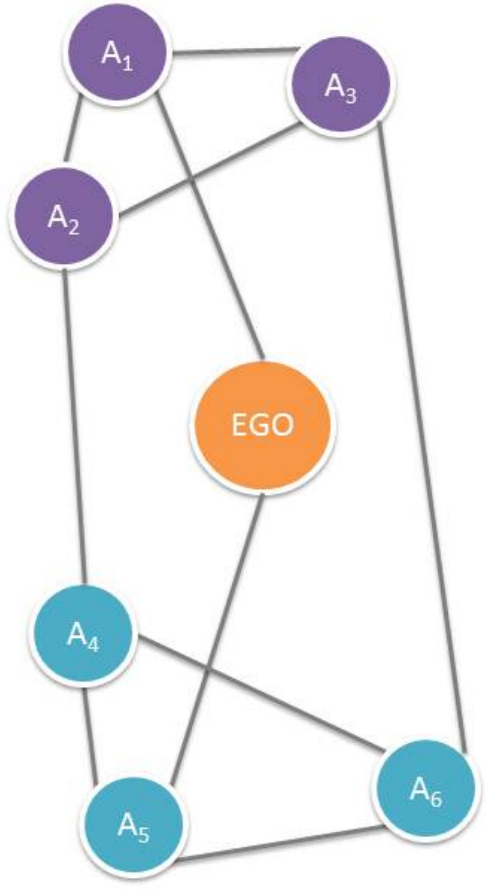

Low Betweenness Centrality

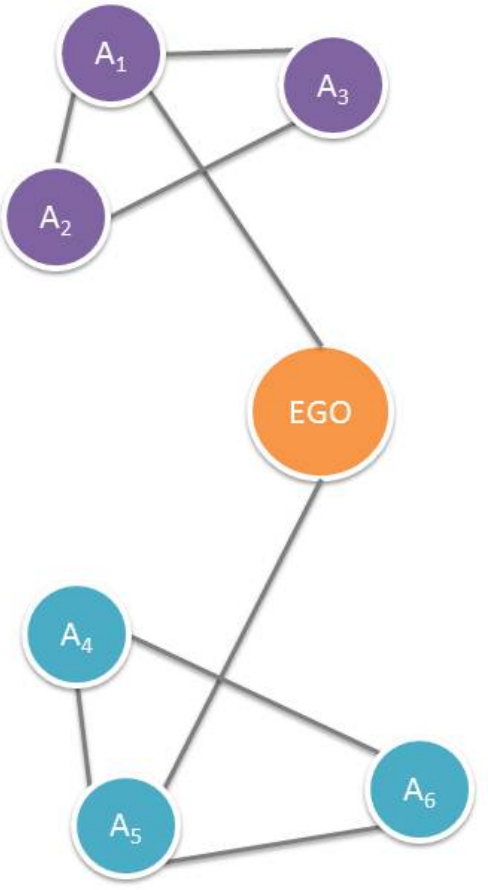

High Betweenness Centrality

Figure 7.1: Example network demonstrating low and high betweenness centrality. On the right ego (the orange node) has significantly higher betweenness as they sit on the only edge between the blue and purple clusters. Adapted from Brent (2015)

The second measure of indirect centrality is eigenvector centrality (EC), which develops degree centrality in a weighted network as some edges are more important than others (Newman 2004). An ego's EC is proportional to the sum of the strength centralities of the nodes connected to ego; thus it takes into account both the number and centrality of a node's edges. Nodes connected to other well-connected nodes have a higher EC centrality, as do individuals with many neighbours (Figure 7.2 (Newman 2010; Brent 2015)). Similar to 
betweenness, EC is often high for a few nodes and low for the reminder of the network (Whitehead 2008).

The network was comprised of only 'productive' individuals aged 12 years or older, following the cut-offs applied in throughout this thesis (section 3.5.3). This threshold was applied as after the age of eleven, hunter-gatherer children conduct significantly more caring and economic activities. They also require less care and provisioning themselves (Konner 2005; Kramer 2005). Thus, they are deemed to be no longer fully dependent children and included in the adult network.

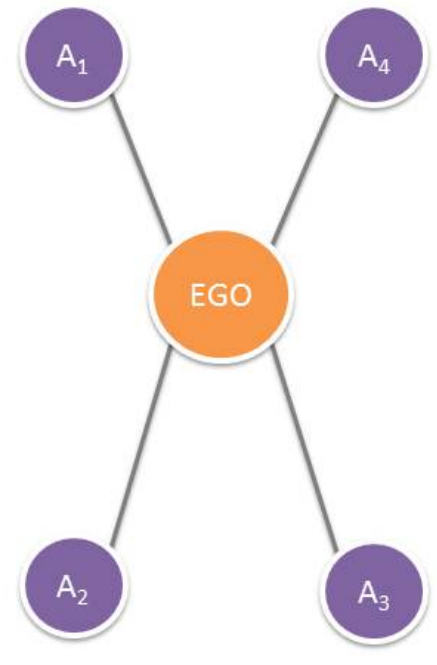

Low Eigenvector Centrality

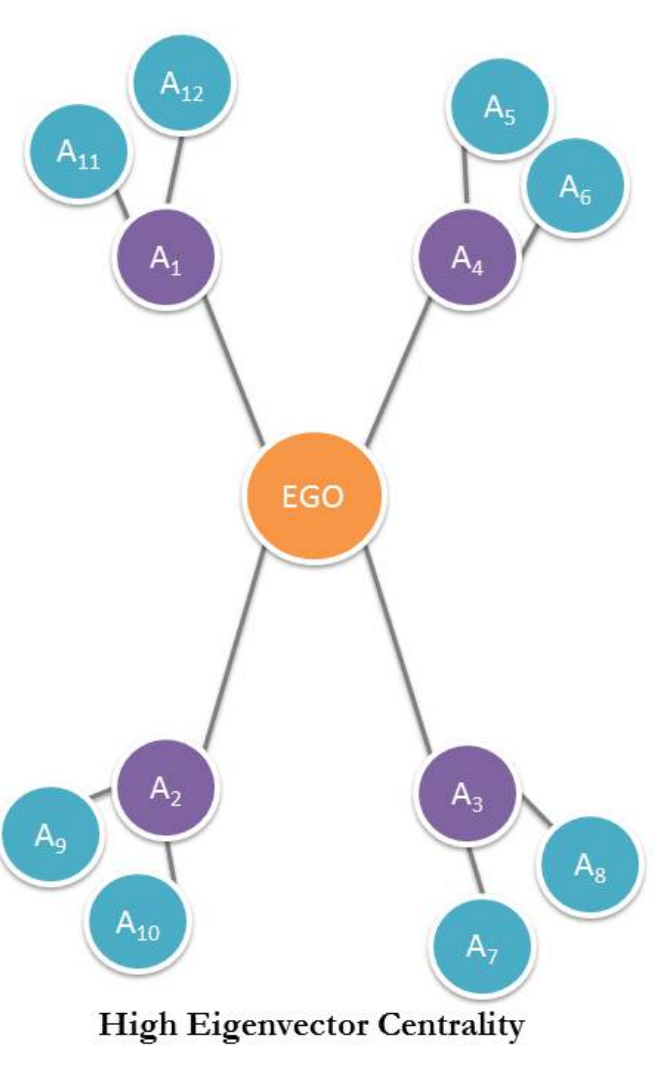

Figure 7.2: Example network demonstrating low and high eigenvector centrality (EC). On the right ego (the orange node) has significantly higher EC all their edges are shared with nodes who have many edges themselves. Adapted from Brent (2015).

\subsubsection{Proximity to kin, non-kin and non-household individuals}

As the effect of maternal network on her reproductive success and health is at the maternal level (all females over the age of 15) I also established an 'amount of care received' variable 
at the mother's level. For each child I summed the number of interactions they had with each individual who fell under the following categories: kin (coefficient of relatedness $\geq$ 0.125), non-kin (coefficient of relatedness $<0.125$ ) and non-household individuals (all individuals excluding the nuclear household, i.e. parents and dependent siblings) based on genealogical relatedness. This figure was then summed for each mother, producing one allocare score that reflects all the interactions all her children had with different types of allocarers. This figure was significantly affected by the number of children a mother had; mothers with more children necessarily had higher interaction scores with non-kin, kin and non-household individuals, thus exploring whether allocare was associated with fertility would be self-fulfilling. Therefore, the results were standardised per family size; mothers were given an allocare score depending on how many interactions their children experienced compared to the sample norm for this family size. For instance, mothers who received significantly more allocare from non-kin compared to the mean of the sample with four dependent offspring were given a positive score, while 0 represents the average amount of allocare received given the family size. As a result of this standardisation procedure there is no significant relationship between household size and the sum of number interactions mothers offspring had with non-kin $(p=0.92)$, kin $(p=0.94)$ and nonhousehold individuals $(p=0.9)$. Thus, I can explore the relationship between maternal network centrality, number of interactions and reproductive outcomes without confounding the analysis.

\subsubsection{BMI z-scores, haemoglobin levels and self-reported sickness.}

I used three outcome variables to measure maternal and child 'quality'. BMI is a measure of children's energetic balance and a proxy of overall nutritional quality and more generalised calorie intake. Haemoglobin levels are a measure of micronutritional quality, capturing variability in the quality of food. Children of poorer nutritional quality, or those who experience less investment or poorer quality investment are expected to experience some form of immune depression, thus likely to report more cases of sickness over the last two weeks. Child age has a significant and non-linear relationship with BMI. As a result, BMI was standardised by the smallest possible age groups given our sample size. These groups were as follows: infants aged less than one year; toddlers between one and two years; young children aged two to six years; and older children aged six to eleven years. 
Once the BMI scores had been standardised within these age groups no significant relationship remained between BMI and age $(p=0.95)$.

\subsubsection{Statistical analysis}

The main multilevel model analysis was conducted at the maternal level (network effects of fertility and care received) and contained two levels: level 1 being the mother and level 2 the camp they resided in. The analysis conducted at the child level (haemoglobin levels, self-reported sickness and BMI scores) contained three levels: the child (level 1), the household (level 2) and the camp (level 3). As many of the network centrality statistics covary each of these terms were ran in separate analyses (Brent 2015; Wey \& Blumstein 2012). Only theoretically informed variables were entered into the model and no terms were removed. All network measures were standardized by camp, thus represent whether or not a centrality score was high according to the camp average (Stanton \& Mann 2012; Sueur et al. 2011).

Age-specific residuals (described in section 3.5.1) were used in all multilevel models with demographic outcomes. Therefore, the influence of age has been removed completely. The fertility and allocare models contained no additional controls (given small sample sizes ${ }^{6}$ ), however each model was examined with the inclusion of an interaction between age and centrality. If the interaction was insignificant at $p<0.05$ then the interaction was removed from the model and the non-interaction model is reported.

Age in this analysis has been centred to 25 years, as I wanted to explore the relationship between centrality, fertility and allocaring according to reproductive career stage. Under 25 years the majority of mothers have 1 to 2 offspring (average $1.5 \pm 0.7$ ), less or equal to the number of adult carers present within the household (average $2.1 \pm 0.7$, a relationship expressed in Figure 6.2). Therefore, they are not in high 'need' of allocare and were abler to provide for the offspring themselves. However, at around 25 years this relationship switches as mothers on average have $3.7 \pm 0.98$ dependents versus $2.9 \pm 0.97$ carers, thus were more in need of allocare. As a result, this interaction with maternal age has been included to explore these relationships. The health models controlled for child and maternal age, child sex, whether the camp was 'settled' (binary, 1 being settled representing

${ }^{6}$ Originally the models were run with controls for degree of sedentism and household wealth. Their inclusion made little impact to the results, thus given the small sample size were removed from future analysis to increase the power of the analysis. 
a camp with permanent housing and a church and/or water pump) and 'household belongings' (a continuous measure quantifying wealth) as these have known relationships with health and wellbeing (see chapter 8). In the analysis on child outcomes I also controlled for number of siblings and maternal condition (i.e. maternal sickness, maternal haemoglobin concentration and maternal BMI in each of the relevant analyses).

Descriptive statistics for all variables can be found in Table 7.1. All null results not presented in the main text can be found in appendix $\mathrm{C}$, alongside intercept-only models. All multilevel analysis was conducted with the variables as continuous outcomes, however descriptive results demonstrating increases in reproductive success are expressed using the binary measure for centrality (i.e. high or low according to sample thresholds). This is simply to express the fertility results in a more useful manner for interpretation.

Table 7.1: Descriptive statistics for the sample for mothers $(n=39)$ and their children $(n=$ 91). All network centrality measures are $z$-scores to standardise the results per camp, as are amount of care received from in different categories of allocarers (controlling for household size), child BMI (controlling for the non-linear influence of age on BMI during childhood) and the reproductive measures.

\begin{tabular}{lrrrr}
\hline \multirow{2}{*}{ Variable } & \multicolumn{4}{c}{ Maternal Descriptive Statistics } \\
\cline { 2 - 5 } & Min. & Mean & Max & SD \\
\hline Maternal Age & 17 & 36.29 & 75 & 15.937 \\
Betweenness & -1.131 & -0.061 & 3.049 & 0.927 \\
Degree & -1.591 & 0.113 & 1.439 & 0.67 \\
Strength & -1.606 & 0.198 & 1.778 & 0.781 \\
EC & -1.332 & 0.178 & 1.766 & 0.856 \\
Mortality & -1.884 & -0.355 & 2.255 & 1.023 \\
Fertility & -1.292 & 0.159 & 1.734 & 0.676 \\
RS & -1.418 & 0.097 & 1.547 & 0.749 \\
Non-kin care & -1.689 & 0 & 2.169 & 0.939 \\
Kin care & -1.409 & 0 & 2.745 & 0.939 \\
Non-household care & -1.785 & 0 & 1.688 & 0.939 \\
BMI & 12.69 & 18.27 & 23.38 & 2.267 \\
Haemoglobin & 38 & 107.8 & 149 & 27.53 \\
Cases of sickness & 0 & 0.806 & 2 & 0.71 \\
& Child & Descriptive Statistics \\
\hline Child Age & 0.006 & 5.369 & 11 & 3.366 \\
BMI & -1.751 & 0 & 4.39 & 0.992 \\
Haemoglobin & 31 & 110.9 & 148 & 19.387 \\
Cases of sickness & 0 & 1.564 & 4 & 0.768 \\
Sibling number & 0 & 4.444 & 8 & 1.844 \\
\hline
\end{tabular}




\subsection{Results}

\subsubsection{Centrality correlates with fitness}

\subsubsection{Mothers with higher betweenness demonstrated higher fitness}

Overall the 39 mothers who took part in the motes experiment, distributed across six camps, had an average number of $5 \pm 3$ live births, ranging from 0 to 12 . Of these, the mean survival rate to age 16 was $4 \pm 2.6$, ranging from 6 to 10 mature offspring. Mother's with the highest betweenness had greater reproductive success (i.e. number of children surviving to 16 (Figure 7.3)) however this relationship was dependent on maternal age, revealed by the significant interaction between age (centred at 25 years) and betweenness. Thus, betweenness only significantly predicted fertility once a mother was 25 years or older, accounting for a sizable $27 \%$ of the maternal level variance in reproductive success. While betweenness had no significant relationship with mortality, it did with fertility. Again this relationship is dependent on age as only mothers over age 25 demonstrated increasing fertility with higher betweenness (Table 7.2). This model accounted for $19 \%$ of the maternal level variance in all live births.

Table 7.2: Multilevel models of maternal network characteristics, fertility and reproductive success (survivorship to age 16). Due to significant similarities between predictors each of the different network characteristics were included in separate models. VAR represents the amount of the individual level variance each predictor accounted for compared to the intercept-only model. The variance at the individual level in the intercept-only model was 0.4 for fertility and 0.5 for reproductive success. Models only included the measure of centrality and age centred at 25 years $(n=39)$.

\begin{tabular}{lccccc|ccccc}
\hline & \multicolumn{5}{c|}{ Fertility } & \multicolumn{5}{c}{ Reproductive Success } \\
\cline { 2 - 12 } Parameter & $\boldsymbol{B}$ & $\boldsymbol{p}$ & $\mathbf{2 . 5 \%}$ CI & $\mathbf{2 . 5 \%}$ CI & VAR & $\boldsymbol{B}$ & $\boldsymbol{p}$ & $\mathbf{2 . 5 \%}$ CI & $\mathbf{2 . 5 \%}$ CI & VAR \\
\hline EC & -0.16 & 0.18 & -0.37 & 0.09 & 0.12 & -0.19 & 0.15 & -0.47 & 0.08 & 0.04 \\
Betweenness & 0.10 & 0.34 & -0.12 & 0.32 & 0.27 & 0.02 & 0.77 & -0.23 & 0.28 & 0.19 \\
Betweenness*age & 0.02 & 0.04 & 0.00 & 0.03 & & 0.03 & 0.01 & 0.01 & 0.04 & \\
Degree & -0.48 & $<0.001$ & -0.88 & -0.20 & 0.32 & -0.43 & 0.01 & -0.76 & -0.10 & 0.16 \\
Strength & -0.23 & 0.07 & -6.67 & 0.04 & 0.19 & -0.24 & 0.10 & -0.53 & 0.05 & 0.07 \\
\hline
\end{tabular}


Age-controlled relationships are presented in Figure 7.3. However, as betweenness had no significant relationship with age $(B=-0.01, p=0.3)$, the raw survivorship (i.e. non-age controlled) results were used to describe the role of betweenness on demographic measures in more meaningful terms (i.e. not standardised residuals). By doing so, it is apparent that 'high' betweenness mothers (in or above the 3rd quartile; $0.98^{7}$ ) aged over 25 had 0.75 or $14.3 \%$ more offspring surviving to age 16 than mother's with 'low' betweenness (less than the $3^{\text {rd }}$ quartile, Table 7.3). These women with higher betweenness came from a range of camps (in terms of ecology and degree of settlement) and tended to be in peak reproductive years; women in the top quartile of betweenness have an average age of $33 \pm 10.8$ years.

\subsubsection{Maternal degree negatively associates with fertility and reproductive success}

No other network variable had a significant, positive relationship with fertility, reproductive success or mortality. However, degree centrality is associated with a significant decrease in reproductive success via reductions in fertility (Table 7.2; Figure 7.3). As above, non-aged controlled reproductive success rates revealed that mothers with low degree (below the mean; 0.11 ) had 1.45 or $30.9 \%$ more offspring survive to age 16 than did their peers with high degree (above the mean, Table 7.3). As with betweenness age does not have a significant relationship with degree confounding these results $(B=$ 0.003, $p=0.6$ ). The interaction between age and degree was insignificant for both reproductive success $(p=0.2)$ and fertility $(p=0.1)$ and removed from the final models.

Table 7.3: Non-age controlled fertility and reproductive rates for women aged 25 years and over according to high and low betweenness and degree centrality. Agecontrolled residuals are expressed visually in Figure 7.3. SD stands for standard deviation, SEM: standard error of the mean $(n=39)$.

\begin{tabular}{llrll}
\hline Measure & Level & Mean & SD & SEM \\
\hline Betweenness \& fertility & Low & 6.2 & 1.504 & 0.328 \\
& High & 6.571 & 2.122 & 0.802 \\
Betweenness \& RS & Low & 5.250 & 1.826 & 0.399 \\
& High & 6.0 & 1.870 & 0.707 \\
\multirow{2}{*}{ Degree \& fertility } & Low & 6.786 & 1.981 & 0.511 \\
& High & 5.769 & 1.211 & 0.336 \\
\multicolumn{1}{c}{ Degree \& RS } & Low & 6.143 & 1.845 & 0.476 \\
& High & 4.692 & 1.716 & 0.476 \\
\hline
\end{tabular}

\footnotetext{
${ }^{7}$ Betweenness has been separated consistently at the third quartile rather than mean due to the significant left skew in the data. Most individuals demonstrated little variance in betweenness while a few demonstrated a lot. Degree on the other hand was more evenly distributed, and hence separated at the mean (Whitehead 2008).
} 

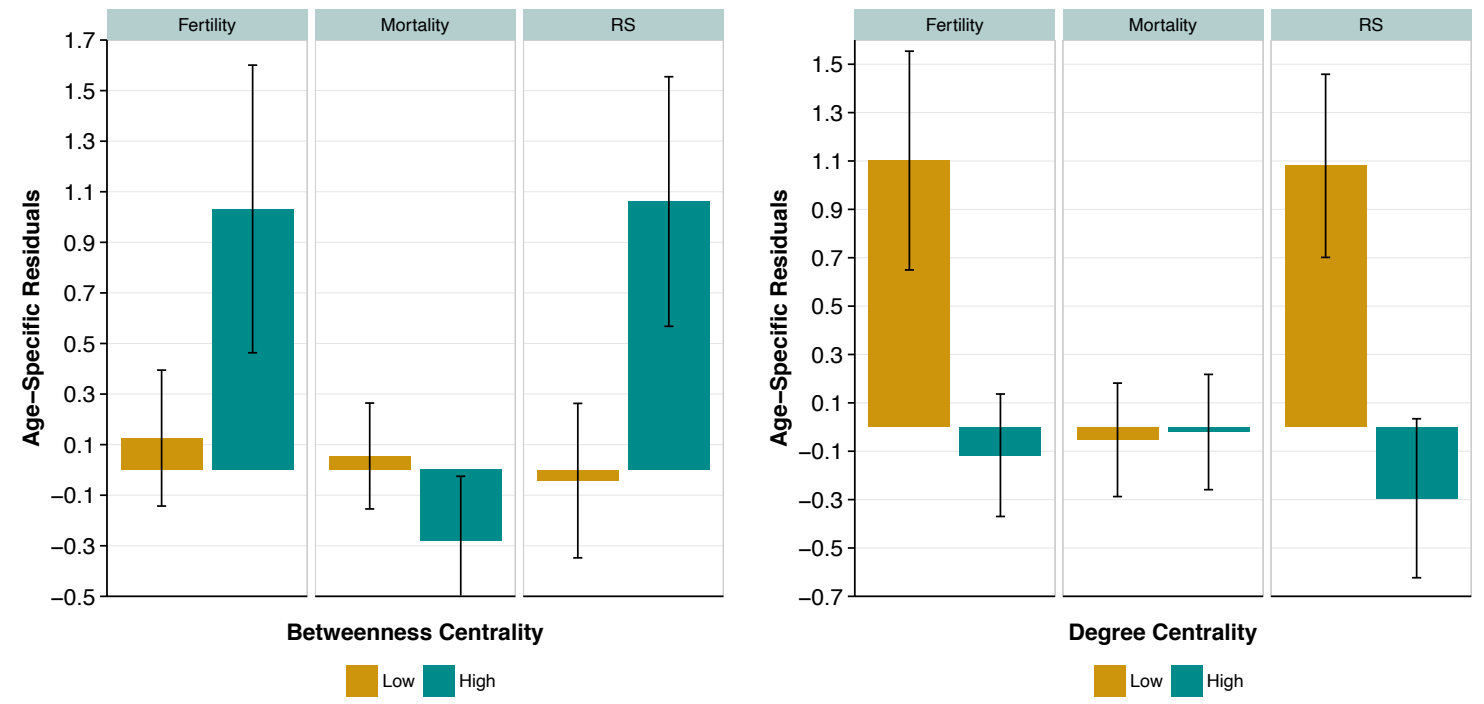

Figure 7.3: The relationship between betweenness, degree and age-specific residuals for fertility, mortality and reproductive success, $n=39$. Maternal centrality has been transformed into a binary variable. Individuals with betweenness below the third quartile (0.98) were coded as having 'low' betweenness. Individuals with betweenness of 0.98 or more had 'high' betweenness. For degree the standardised mean is 0.11 , above which is denoted as 'high' degree. Error bars represent standard errors of the mean. Fertility, mortality and reproductive success (survivorship to age 16) are all measured by age-specific residuals. RS stands for reproductive success.

Table 7.4: Multilevel models examining the relationship between the proportions of interactions between non-kin, non-household and kin proximity and maternal centrality. Significant variables $(p<0.05)$ are highlighted in bold, marginal predictors $(p<1)$ are in italics. The variance in the intercept-model was 0.94 for non-kin, 0.95 for non-household and 0.95 for kin. Interactions were only retained if significant at $p<0.05$, models contain age and the measure of centrality only $(n=29)$.

\begin{tabular}{c|ccc|ccccc|ccc}
\hline & \multicolumn{3}{c}{ Kin proximity } & \multicolumn{3}{c}{ Non-household proximity } & \multicolumn{3}{c}{ Non-kin proximity } \\
\cline { 2 - 10 } Parameter & $\boldsymbol{B}$ & $\mathbf{9 5 \%} \mathbf{C I}$ & VAR & \multicolumn{1}{c}{$\boldsymbol{B}$} & $\mathbf{9 5 \%} \mathbf{C I}$ & VAR & $\boldsymbol{B}$ & $\mathbf{9 5 \%}$ CI & VAR \\
\hline EC & 91.10 & $-0.5,0.4$ & 0.00 & -0.305 & $-0.8,0.2$ & 0.05 & -0.158 & $-0.7,0.3$ & 0.01 \\
Betweenness & -0.092 & $-0.4,0.3$ & 0.01 & 0.102 & $-0.3,0.5$ & 0.01 & $\mathbf{0 . 5 4 0}$ & $\mathbf{0 . 0 1 , 1 . 1}$ & $\mathbf{0 . 1 4}$ \\
Betweenness*age & - & - & - & - & - & - & $\mathbf{0 . 0 4 3}$ & $\mathbf{0 . 0 1 , 0 . 1}$ & \\
Degree & 0.274 & $-0.2,0.8$ & 0.04 & -0.468 & $-1,0.1$ & 0.09 & -0.310 & $-0.9,0.2$ & 0.04 \\
Strength & 0.103 & $-0.4,0.6$ & 0.01 & -0.102 & $-0.6,0.4$ & 0.01 & 0.016 & $-0.5,0.5$ & 0.00 \\
\hline
\end{tabular}




\subsubsection{Maternal betweenness predicts non-kin proximity}

Of the 39 mothers in the sample, 29 had living children $(n=81)$ who took part in the motes experiment. Maternal betweenness positively correlated with the amount of time children were in proximity with non-kin. No other network characteristics were significantly correlated with non-kin allocare or kin and non-household proximity (Table 7.4). Thus, maternal betweenness is only associated with non-kin proximity to their children.

\subsubsection{Mothers receiving more non-kin allocare demonstrate higher fitness}

Mothers whose children have more interactions with non-kin have significantly higher fertility and reproductive success: an effect that is again dependent on age (Table 7.5). The inclusion of non-kin proximity with the interaction with age decreased the maternal level variance by $38 \%$ for fertility and $33 \%$ for reproductive success. Overall then, it appears that among mothers older than 25 non-kin allocare is significantly correlated with increases in fertility and overall reproductive success. There is no significant relationship between the amount of contact received from non-kin and child mortality, and increases in nonhousehold or kin proximity are not associated with any reproductive outcomes. Overall then mothers aged 25 and over with high non-kin z-scores (more than the mean; 0.1) experienced 1.1 more offspring surviving to age 16 than their peers in receipt of fewer contacts from non-kin $(5.9 \pm 0.5$ versus $4.8 \pm 0.5$, respectively). This results in an increase in fitness, as measured by survival to maturity, of $21.9 \%$.

Table 7.5: Multilevel models examining the relationship between the proportions of interactions between non-kin, female fertility, and reproductive success. The variance in the intercept-model was 0.5 for fertility and 0.4 for reproductive success. Models contained no other controls and only significant interactions between age and allocare were retained $(n=29)$.

\begin{tabular}{|c|c|c|c|c|c|c|c|c|}
\hline & \multicolumn{4}{|c|}{ Fertility } & \multicolumn{4}{|c|}{ Reproductive Success } \\
\hline Parameter & $B$ & $p$ & $2.5 \% \mathrm{CI}$ & $2.5 \% \mathrm{CI}$ & $\bar{B}$ & $\bar{p}$ & $2.5 \% \mathrm{CI}$ & $2.5 \% \mathrm{CI}$ \\
\hline Intercept & 0.05 & 0.76 & -0.28 & 0.39 & 0.09 & 0.56 & -0.27 & 0.49 \\
\hline Non-Kin & -0.18 & 0.17 & -0.47 & 0.10 & -0.18 & 0.15 & -0.45 & 0.08 \\
\hline Non-kin*age & 0.03 & 0.00 & 0.01 & 0.05 & 0.03 & 0.01 & 0.01 & 0.05 \\
\hline Model Residual & \multicolumn{4}{|c|}{0.38} & \multicolumn{4}{|c|}{0.33} \\
\hline $\begin{array}{c}\text { Variance explained from } \\
\text { controls }\end{array}$ & \multicolumn{4}{|c|}{0.23} & \multicolumn{4}{|c|}{0.20} \\
\hline
\end{tabular}




\subsubsection{Centrality and allocare are associated with maternal and child quality}

\subsubsection{Betweenness positively correlates with maternal sickness, degree negatively correlates with maternal BMI}

Of the original 39 mothers who took part in the motes experiment I conducted a medical questionnaire on 36 to quantify bouts of sickness over the last two weeks. Mothers with higher betweenness reported significantly more bouts of sickness $(B=0.3, \mathrm{p}<0.01,95 \%$ CI $[0.1,0.5])$. The inclusion of betweenness into the model reduced the unexplained variance at the individual level by $22.6 \%$ compared to the model with controls. There is a significant relationship between fertility and sickness for more than two instances of sickness $(B=0.2, p=0.01,95 \%$ CI $[0.03,0.3])$, but not for fewer cases $(B=-0.3, p=0.39$, $95 \%$ CI $[-0.2,0.1])$. This suggests the association of betweenness with self-reported sickness is somewhat mediated by having more offspring. No other network measures were associated with increases in self-reported sickness or haemoglobin levels. However, maternal BMI is negatively associated with degree only $(B=-1.2, \mathrm{p}=0.01,95 \%$ CI [-2.1, $0.2]$ ), accounting for $11.6 \%$ of the unexplained variance compared to the control-only model. Thus, while mothers with higher betweenness suffer more sickness, mothers with higher degree demonstrate poorer nutritional condition.

\subsubsection{Maternal betweenness negatively correlates with child BMI and haemoglobin levels}

The 39 mothers who took part in the motes experiment had 91 children from whom I had anthropometrics for and 81 children I had collected blood samples from. Mother's betweenness is negatively and significantly associated with both her offspring's haemoglobin concentrations $(B=-5.0, \mathrm{p}=0.04,95 \%$ CI $[-9.8,0.3])$ and BMI $z$-scores $(B$ $=-0.2, \mathrm{p}=0.03,95 \% \mathrm{CI}[-0.4,-0.02])$. Thus, mothers with high betweenness had children who were in a worse nutritional condition and suffer from iron-deficient anaemia.

One hypothesis is that mothers with higher betweenness invested in fertility rather than child quality, increasing sibling competition and resulting in poorer outcomes for their children. Sibling number was of marginal significance as a predictor for child BMI $(p=$ 0.09 ) but not for haemoglobin levels $(p=0.5)$. As a result, I explored the influence of presence of younger and older siblings on BMI and haemoglobin levels with the full sample of under 12's ( $n=181$, Figure 7.4). This demonstrated that the association between BMI and maternal betweenness may be influenced by sibling competition effects, as the 
number of younger siblings is associated with a significant decrease in BMI z-score $(B=$ 0.17, $\mathrm{p}=0.01,95 \% \mathrm{CI}[-0.3,-0.04])$, resulting in a $35.8 \%$ decrease in household level variance compared to control-only models. However, the inclusion of this did not reduce the significance of maternal betweenness in the full model, but the beta did drop slightly (from -0.2 to -0.18). Therefore, mothers with higher betweenness may increase sibling competition due to increased fertility (i.e. quantity over quality). However, betweenness maintains an independent relationship with BMI despite the inclusion of sibling effects, suggesting other pathways. There is no relationship between haemoglobin concentrations and younger $(B=0.5, \mathrm{p}=0.7,95 \% \mathrm{CI}[-3,-2.3])$, or older sibling $(B=1, \mathrm{p}=0.2,95 \% \mathrm{CI}[-$ 0.6, 2.7]) presence, suggesting then that the relationship between haemoglobin concentrations and betweenness was wholly unrelated to sibling competition.
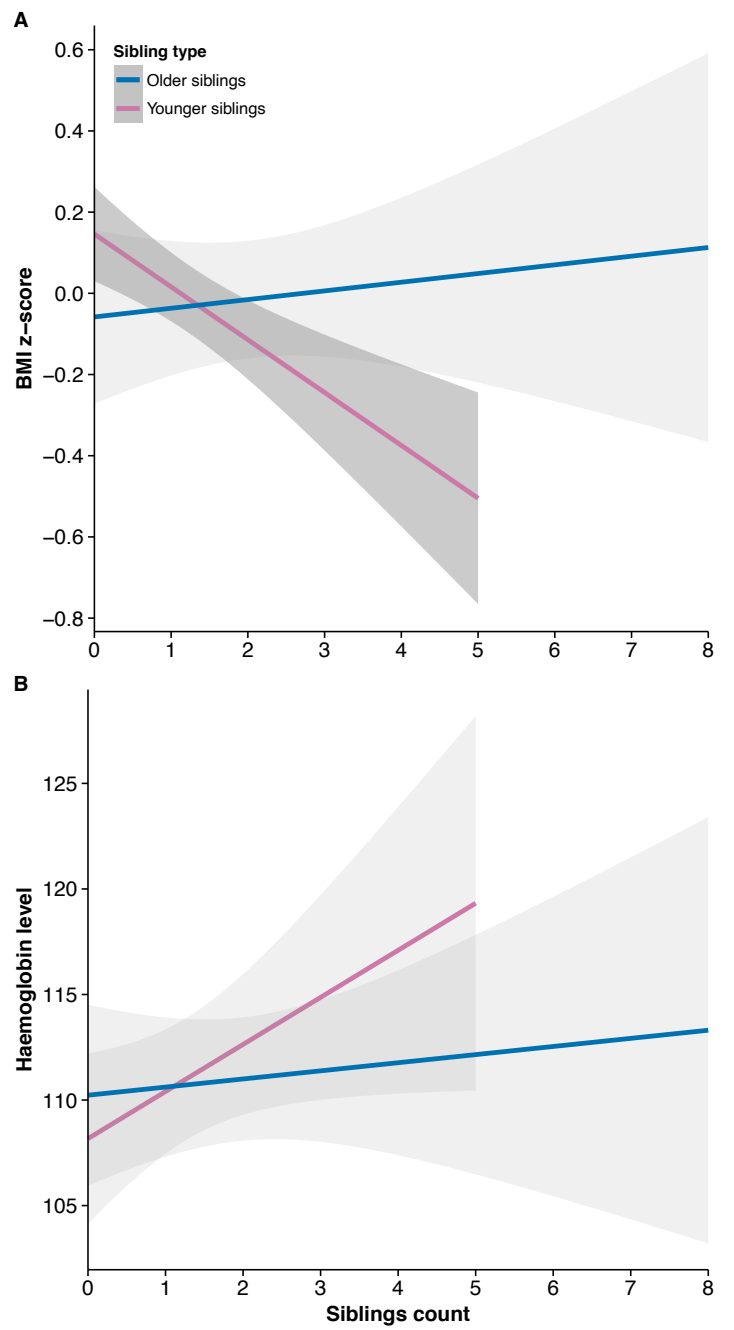

Figure 7.4: The relationship between number of younger and older siblings and (A) BMI $\mathrm{z}$-scores and (B) haemoglobin levels. Non-significant results are faded $(n=181)$. 


\subsubsection{Maternal degree positively predicts child haemoglobin levels}

While no measure of centrality significantly predicted child sickness, maternal degree was positively correlated with child haemoglobin levels $(B=10.2, \mathrm{p}=0.006,95 \%$ CI $[2.8$, 17.5]) but not with child BMI scores $(B=0.04, \mathrm{p}=0.8,95 \%$ CI $[-0.08,0.4])$, Figure 7.5).
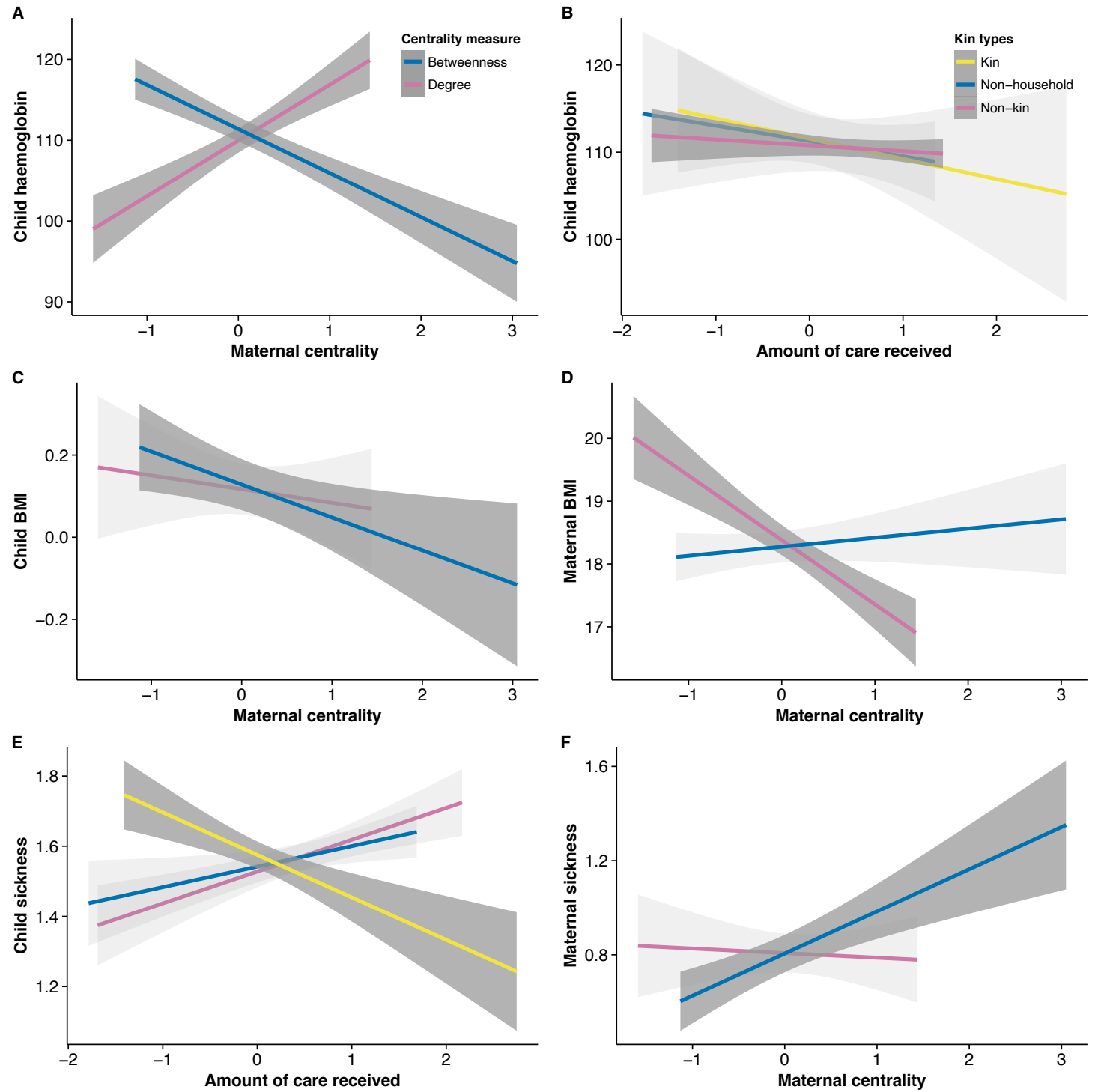

Figure 7.5: Maternal and child outcomes according to maternal centrality (degree and betweenness) and amount of care received from different categories (non-kin, kin and non-household). First row presents results for child haemoglobin levels by centrality (A) and amount of care (B). Second row presents results for child BMI (C) and maternal BMI (D) by centrality. Final rows presents results on child sickness by amount of care (E) and maternal sickness by centrality (F). Non-significant results have $95 \%$ confidence intervals faded. 


\subsubsection{Child outcomes by allocare}

Increased proximity with kin significantly decreased the instances of sickness over the last two weeks $(B=-0.23, \mathrm{p}=0.02,95 \% \mathrm{CI}[-0.4,-0.04])$. The inclusion of kin care decreased the individual level variance in sickness by $4.5 \%$ from the controlled model. The amount of care received from non-kin is associated with a reduction in a child's circulating haemoglobins $(B=-5.4, \mathrm{p}=0.05,95 \%$ CI $[-10.7,-0.02])$ and BMI z-scores $(B=-0.3, \mathrm{p}$ $=0.001,95 \%$ CI $[-0.5,-0.1])$. As above, the separation of younger and older siblings did slightly reduce the size of the effect for non-kin proximity (from -0.3 to -0.25 ), however it remained a significant predictor of BMI z-score demonstrating the independence of the relationships between increased fertility, sibling competition and BMI.

\subsection{Discussion}

While research into the adaptive consequences of network centrality and indirect social ties has received little empirical attention in humans, I have demonstrated that they have clear fitness implications (Table 7.6). These results suggest that mothers with greater betweenness have higher fertility and reproductive success at the cost of increasing poor health, while mothers with higher degree centrality demonstrate lower fitness. I hypothesised that the positive relationship between reproductive success and betweenness is related to the solicitation of non-kin allocare, further suggested by the relationship between fitness, betweenness, allocare and age. By centring age at 25 it is possible to capture reproductive career effects; under the age of 25 mothers had a $43.8 \%$ surplus of carers within the household, but over the age of 25 they face an average 30\% deficit in household childcare. As a result, non-kin childcare becomes increasingly important as mothers continue reproducing and the household is no longer a sufficient provider of childcare, particularly in the face of further unpredictable shortfalls. Thus, I hypothesised that mothers seek more non-kin allocare with increasing fertility, which reduces their workload and allows investment in fertility without diminishing overall reproductive success. Consequently, betweenness can be understood as a maternal behavioural strategy to increase access to allocare. Such a strategy has clear adaptive implications, particularly in high variance, high mortality environments like the Agta reside in. 


\subsubsection{Betweenness, non-kin allocare and fertility}

Individuals with high betweenness can be referred to as 'brokers' as they connect disparate parts of the network (Brent 2015). For instance, Lehmann and colleagues (2010) found that the simulated removal of high betweenness individuals in 11 Old World monkey species significantly reduced the connectivity of the networks (but did not destroy connectivity completely in bottlenose dolphins (Lusseau \& Newman 2004)). This trend is associated with 'small-world' networks; networks are more 'efficient' transmitters of information, disease or resources not when each individual is connected to one another (i.e. high degree) but when clusters are linked by a few central nodes (i.e. brokers (Brent et al. 2011)).

Table 7.6: Results summary. NE stands for no effect, / represents un-tested relationships, + a significant positive relationship, - a significant negative relationship. * represents a significant, positive interaction with age centred at 25 years.

\begin{tabular}{|c|c|c|c|c|c|c|c|}
\hline \multicolumn{5}{|c|}{ Measures of maternal centrality } & \multicolumn{3}{|c|}{ Measures of allocare } \\
\hline Dependent variable & Degree & Strength & Betweenness & EC & Kin & Household & Non-kin \\
\hline Fertility & - & $\mathrm{NE}$ & $+*$ & $\mathrm{NE}$ & $\mathrm{NE}$ & $\mathrm{NE}$ & $+*$ \\
\hline Mortality & NE & $\mathrm{NE}$ & $\mathrm{NE}$ & $\mathrm{NE}$ & $\mathrm{NE}$ & $\mathrm{NE}$ & $\mathrm{NE}$ \\
\hline RS & - & $\mathrm{NE}$ & $+^{*}$ & $\mathrm{NE}$ & $\mathrm{NE}$ & NE & $+*$ \\
\hline Kin proximity & $\mathrm{NE}$ & $\mathrm{NE}$ & $\mathrm{NE}$ & $\mathrm{NE}$ & & l & \\
\hline Household proximity & NE & $\mathrm{NE}$ & $\mathrm{NE}$ & $\mathrm{NE}$ & & / & \\
\hline Non-kin proximity & NE & $\mathrm{NE}$ & $+*$ & $\mathrm{NE}$ & & / & \\
\hline Maternal BMI & - & $\mathrm{NE}$ & $\mathrm{NE}$ & $\mathrm{NE}$ & & / & \\
\hline Maternal sickness & $\mathrm{NE}$ & $\mathrm{NE}$ & + & $\mathrm{NE}$ & & y & \\
\hline Maternal haemoglobin & $\mathrm{NE}$ & $\mathrm{NE}$ & $\mathrm{NE}$ & $\mathrm{NE}$ & & / & \\
\hline Child BMI & $\mathrm{NE}$ & $\mathrm{NE}$ & - & $\mathrm{NE}$ & $\mathrm{NE}$ & $\mathrm{NE}$ & - \\
\hline Child sickness & $\mathrm{NE}$ & $\mathrm{NE}$ & $\mathrm{NE}$ & $\mathrm{NE}$ & - & $\mathrm{NE}$ & $\mathrm{NE}$ \\
\hline Child haemoglobin & + & $\mathrm{NE}$ & - & $\mathrm{NE}$ & $\mathrm{NE}$ & $\mathrm{NE}$ & - \\
\hline
\end{tabular}

If foragers buffer risk and stochastity in unpredictable environments using extensive food sharing and cooperative breeding (Chaudhary et al., 2015; Jaeggi and Gurven, 2013; Meehan et al., 2014; Smith et al., 2010, section 1.2.3), then the structure of cooperation or insurance networks may impact the effectiveness of this method. For instance, if you only cooperate with one other household, this household would soon become overburdened or unable to help when required. In contrast, having ties to disparate parts of the network that 
are otherwise unconnected increases the number of possibilities. Furthermore, for communal breeding to function as an efficient means of risk pooling, 'exposure' units must be independent and unconnected to one another (Cashdan 1985). For instance, closely related households participated in the majority of food sharing; 88.3\% of Agta households shared food only with three other closely related households (mean coefficient of relatedness $=0.1$ (Dyble et al. n.d.)). Time spent foraging reduces the amount of time and/or quality of childcare, thus, if a mother relied on the same households to provide care and provisioning there would be a childcare deficit when the food-sharing cluster had a shortage of food. Exactly when a mother had to increase her foraging activities is when potential caretakers would also do the same, resulting in few available allocarers. Therefore, due to the lack of independence of potential caring units, mothers would not be able to receive all the care required to raise multiple costly offspring. Put simply, as suggested by Hrdy (2005), it is not wise to put all your eggs into one allocaring basket. This suggests why only non-kin allocare was significantly associated with both betweenness and fertility. This interpretation is further supported by the finding in section 6.4 .4 that non-kin allocare is associated with reductions in household wealth, suggestive that it plays a role in risk mitigation.

Overall, mothers connected to more disparate parts of the network maximised reproductive success, which may be due to increased access to allocare. Chapter 5 already revealed how increased non-kin interactions with children significantly reduced the amount of time mothers were proximate to their children. This indicates that, unlike care from siblings or fathers, individuals independent from the household provided substitutive care, hence reducing maternal energy burden. As mothers who acted as brokers in the network had higher fertility, perhaps this allocare allowed them to increase investment in foraging, producing more calories resulting in a faster resumption of fertile cycles (Sear et al. 2003). Or perhaps they simply reduced energy expenditure overall, and thus spent more time relaxing. The combination of reduced workload and increased nutritional condition has long been known to positively influence fecundity. For instance, $\mathrm{Au}$ foragerhorticulturalists resumed menses sooner after childbirth if they were better nourished (due to wage labour) independently of duration of breastfeeding (Tracer 1996). Similarly, in rural Ethiopia the addition of water pumps into the village significantly reduced women's workload (given they no longer had to walk long distances with heavy burdens). As a result there was an immediate reduction in interbirth interval as mothers were able to conceive again sooner (Gibson \& Mace 2002). This influence of decreased energy expenditure can 
function independently of nutritional intake; even if rural Polish women had a positive energy balance (the relationship between energy expenditure and intake), periods of intense labour were associated were ovarian suppression (Jasieńska \& Ellison 1998). Consequently, care from non-kin may have resulted in a significant fertility increases due to reductions in maternal workload. Further investigation on this topic should use time allocation data to explore mothers exact activity budgets given the amount of allocare received and from whom. This method was successful in the Aka foragers, where it was evident that caregivers significantly reduced mothers total energy expenditure (Meehan et al. 2013a).

Betweenness has also been associated with increased fertility in free-ranging chimpanzees, (Pan troglodytes schweinfurthii), albeit for different reasons; males with higher coalition network betweenness had increased rank and probability of siring offspring (Gilby et al. 2013). Male's preference for coalitions that were otherwise unconnected appeared to maximise connectivity in the network, with clear fitness implications, indicating the adaptiveness of third-party awareness in chimpanzees. Betweenness also appears important in other taxa; in terms of social transmission of ecological knowledge in bottlenose dolphins (Lusseau 2006) and in three tit species (Paridae spp. (Aplin et al. 2012)), as well as social position in long-tailed manakin (Chiroxiphia linearis (McDonald 2007)). Interestingly, among the long-tailed manakins it appeared that a male's betweenness earlier in life significantly predicted his social rise (McDonald 2007), a trend also noted in bottlenose dolphins (Stanton \& Mann 2012) and humans (Giles 2005). Perhaps this sheds further light on the significant interaction between network position and age; if the fitness effects of social networks are a product of lifetime centrality then their effects may accumulate over the life course. Thus, mothers with consistently high betweenness will ultimately have the highest reproductive success. Furthermore, betweenness is associated with significant heritability; in a twin study Fowler and colleagues (2009) found that genetic factors account for $29 \%$ of the variability in betweenness, a finding further supported by Brent and colleagues (2013) research on rhesus macaques. As a result, it appears that given the fitness benefits, variability and heritability of betweenness, it is likely that such traits like thirdparty awareness, social intelligence as well as social network qualities could be considered targets of natural selection.

7.5.2 Betwixt between child quantity and quality (including the mother's) 
It appears that both child BMI and haemoglobin levels decreased with rising levels of betweenness and non-kin allocare. A possibility is that non-kin allocare is of significantly lower quality. Certainly there is evidence among non-human primates, such as bonnet macaques (Macaca radiata), that forceful ('kidnapping') allocare of infants by mother's without offspring results in incompetent care and poor outcomes (Silk 1980). This reason, Hrdy (2011) suggests, may be a factor in the lack of allomothering in the other great apes and baboon species. In the case of the Agta it may be that non-kin allocare is also associated with meal sharing where non-related children are provided with less and/or lower quality foods resulting in a reduction of nutritional condition. Furthermore, maternal care would likely be of higher quality, and as non-kin allocare is associated with decreases in maternal interactions with a child, then perhaps it reduces a child's access to high quality childcare, particularly as the mother focuses on fertility (quantity) rather than quality. More in-depth data about the nature of allocare and provisioning is essential to explore the causal associations between child quality, betweenness and allocare. Nonetheless, it appears that allocare among the Agta may be an example of a maternal strategy of focusing on higher fertility, made possible by increased allocare and a reduction in workload, which results in lower quality offspring. Furthermore, this investment in fertility may simultaneously increase sibling competition resulting in further reductions in child quality (in this case BMI only). However, since child mortality is not increased by allocare or betweenness, this trade-off appears to pay-off in fitness terms.

Investment in quantity over quality makes 'life history sense' given the high morbidity, high mortality of the (particularly pygmy) hunter-gatherer lifestyle leading to faster life history strategies (Migliano et al. 2007). Our own data demonstrates that of 520 live births reported, the Agta experienced a childhood mortality rate of 388.5 per 1000 . Therefore, following life history predictions (Charnov 1993), I would expect Agta mothers to invest in quantity rather than quality as they will experience diminishing returns on their investment given the high pathogen and morbidity rate (Quinlan 2007) as well as the increased likelihood of offspring dying before they reach reproductive maturity, making increased investment redundant. Further exploration into the quality and consistency of maternal care according to extrinsic risks is paramount to exploring this interpretation further.

There is little indication that allocare has any positive effects on survival or wellbeing. This is surprising since much of the literature on cooperative childcare suggests that allocarers have a greater influence on child nutritional status than maternal fertility (Sear et al. 2003; 
Strassmann \& Gillespie 2002). However, as suggested above this may be related more to residence in a high risk, high pathogen and high mortality environment, rather than about the 'nature' of allocare and its consequences. Allocare simply frees up additional energy to be reinvested elsewhere. In a lower mortality environment allocare may be associated with increased child survival, however in a high mortality environment a mother may direct her energy to fertility instead. As with the variability in who helps according to context, it appears the consequences of this help are also dependent on the ecological and social conditions (Sear \& Mace 2008).

Nonetheless, the data does reveal that the total amount of allocare received by a household from kin is associated with a significant decrease in self-reported sickness. Thus, allocare from kin may go some way to improve children's condition, boosting their immunological defences and nutritional condition, in agreement with cooperative breeding literature in general (Sear et al. 2002; Sear \& Mace 2009; Sear \& Mace 2008; Meehan et al. 2014; Hawkes et al. 1997; Gibson \& Mace 2005). This may be related to the finding in section 5.5 which demonstrated that many types of kin increased the number of interactions a mother had with a child (including siblings, fathers, distant kin and paternal grandfathers). Therefore, children in receipt of more allocare from kin may be receiving more childcare overall, significantly improving their immunological condition. However, due to the focus on fertility and maternal network centrality I have not separated the developmental and health consequences of allocare from different type of kin (i.e. siblings, grandparents and aunts and uncles) nor looked at different age interactions (i.e. infancy, toddling and childhood). To truly explore these questions, these categories need to be separated as chapters 5 and 6 revealed that exactly who the allocarer is matters, as does the age of the child.

Interestingly, mothers with greater betweenness seem to suffer from more bouts of sickness. This finding is in line with much of the literature on disease transmission through networks which finds that 'brokers' in the network are both more likely to host a pathogen and transmit it widely throughout the network (Weber et al. 2013; Ueno \& Masuda 2008; Corner et al. 2003; Hamede et al. 2009). As individuals with high betweenness are those who lie on central ties, it follows that much of the disease transmission flows through them (Hamede et al. 2009). However, it also seems in our sample that the mothers who report being sick the most are those with more children, though this may be a trade-off between fertility and somatic maintenance (Stearns 1992; Lawson et al. 2012) or a product of 
children acting as 'super-spreaders' of disease (Glass \& Glass 2008; Keeling \& Eames 2005).

\subsubsection{The cost of social networks?}

Contrary to others findings (McFarland \& Majolo 2013; McFarland et al. 2015; Lehmann et al. 2015) degree has a significant negative relationship with fertility and reproductive success. Certainly, sociality comes at a cost of increased competition, risk of disease and social tensions (Krause \& Ruxton 2002; Silk 2007b), thus being highly connected to a network may have negative consequences. This finding is demonstrated in research with female yellow-bellied marmots (Marmota flaviventri) in which social affiliation and neighbour overlap were negatively correlated with reproductive success (Wey \& Blumstein 2012). Here, maternal BMI decreased with degree centrality, a likely factor impacting her fertility (Pike 2001; Lipson 2001; Ellison et al. 1993). This suggests that mothers with high degree have lower nutritional quality, perhaps related to the number of obligatory relationships they hold with others who compete for the same resources (Borgerhoff Mulder 2007; Sear 2008). This leaves unexplained why degree is positively correlated with child haemoglobin levels (but not BMI); if a high number of social ties due to resource competition has a negative effect on maternal BMI and fertility, then these individuals should also be in competition with the mother's offspring. This finding may be the consequence of uncontrolled relationships between degree and other predictors of child BMI, including the underlying causes of increased degree centrality. Or this may be the outcome of mothers with lower fertility increasing investment in child quality. These interpretations are mere speculation, therefore it is essential to expand these findings further to understand the effect of degree centrality on maternal and child outcomes.

\subsubsection{Limitations}

This study remains correlational and further research needs to be conducted into the processes underlying the relationship between degree and fertility and the relationship between betweenness and reported sickness. For instance, further exploration into the competition between individuals would be highly informative, as would observational data on the quality of allocare from different caretakers. Importantly, in this analysis I cannot distinguish whether more fertile mothers place themselves in a 'between' position to seek out more allocare to look after their offspring or whether mothers who are 'between' groups gain higher fertility due to the increased allocare from non-kin. However, this issue of causality makes no impact on the overall argument; both pathways demonstrate the 
advantages of network centrality. Perhaps more problematic, as argued by Gilby et al. (2013) is that betweenness may simply be a by-product of another trait which results in higher fertility. For instance, higher quality mothers may have higher betweenness. For this reason I explored the relationship with betweenness and allocare to understand these mechanisms (Brent 2015). However, future research should examine the role of social status and betweenness; individuals of high social status or relational wealth are known to have increased fertility (Chaudhary et al. 2015; Gurven \& von Rueden 2010; Smith 2004; von Rueden et al. 2011; Borgerhoff Mulder \& Beheim 2011).

\subsection{Conclusions}

I have shown that individual's network centrality has important implications for fitness outcomes among Agta hunter-gatherers. This reveals how weak, strong, direct and indirect ties have important influences on Agta reproduction and cooperation. In particular, by exploring the emergent features of social networks, rather than focusing on 'who cares', I find that a mother's network position can be considered an extended phenotype given its importance in gaining access to a dispersed networks of allocare which significantly boost maternal reproductive success. The importance of a measure like betweenness may then lie with highly cooperative groups living in risky niches. Given the variable and unpredictable hunter-gatherer environment, on top of an existing childcare deficit (section 5.4), the ability to manipulate one's social network to ensure the household is buffered from care shortages may offer an important insight into the evolution of sociality and cooperation (Wey et al. 2013; Hill et al. 2009). This further reveals the evolutionary importance of third-party knowledge; if being a broker to disparate parts of the network is beneficial, it follows that individual's maintain an awareness of not only who they are friends with, but who are friends of friends (Brent 2015). Such dynamics take considerable social intelligence, and indicate one possible selective pressure for brain expansion in primates (Silk 2007a). These results are suggestive of the evolutionary importance of encephalisation in facilitating management of complex and diverse social networks since an individual's centrality depends not only on their direct ties but also indirect ties throughout the population (Formica et al. 2012). Further research using social network analysis to explore these indirect properties' influence on human fitness is essential, as they may play a major role in our social and behavioural evolution. 


\title{
Chapter eight \\ The Cost of Cultivation and Sedentism: Reflections on the Neolithic Epidemiological and Demographic Transition ${ }^{8}$
}

\begin{abstract}
Evidence from bioarchaeology presents a Neolithic paradox, as the transition to agriculture $13,000-10,000$ years ago is associated with both positive effects, such as higher fertility and population growth, and negative effects, such as increased morbidity and mortality rates. From a Darwinian perspective on human behaviour and reproduction, which focuses on lifetime fitness rather than shorter-term wellbeing alone, trends of increasing fertility and morbidity can be considered adaptive if their trade-off ultimately increases reproductive success. Here, I test this hypothesis, and by doing so, reconcile the conflicting evidence stemming from bioarchaeology. I propose that increased cultivation and sedentism involves a quality-quantity trade-off as mothers traded offspring survival for increased fertility, achieving greater reproductive success in spite of deteriorating health. I test this hypothesis by investigating fertility, mortality and health in Agta hunter-gatherers whose camps exhibit variable levels of sedentarization, mobility and cultivation. I conducted blood composition tests with 345 Agta and found that viral and helminthic infections as well as child mortality rates are significantly increased with sedentism. Despite this, age-controlled fertility and overall reproductive success are both positively affected by sedentarisation and participation in cultivation. Thus, I provide evidence how an adaptive mechanism can reconcile the decline in health and child survival with the observed demographic expansion during the Neolithic.
\end{abstract}

\footnotetext{
8 A condensed version of a paper currently in press as: Page, A. E., Viguier, S., Dyble, M., Smith, D., Chaudhary, N., Salali, G., Thompson, J., Vinicius, L., Mace, R., and Migliano, A. B. (In press) Reproductive trade-offs in extant hunter-gatherers suggest adaptive mechanisms for the Neolithic expansion. PNAS (appendix E)
} 


\subsection{Introduction}

The onset of the Holocene epoch around 12,000 BP (before present) was marked by the end of the last glacial maximum in which temperatures rose and the climate stabilised (Zheng et al. 2012; Zahid et al. 2015), producing abundant environments ideal for the onset and intensification of food production. Agriculture arose in the Fertile Crescent in the current day Middle East around 10-12,000 BP and expanded into northern Europe by 6,000 BP, while independently arising in China, New Guinea and Mesoamerica (Bellwood \& Oxenham 2008; Diamond \& Bellwood 2003; Gupta 2004; Shennan et al. 2013). Thus, agriculture was firmly established as the dominant subsistence system by 5000 BP (BarYosef \& Meadow 1995; Bollongino et al. 2013; Piperno \& Pearsall 1998; Price \& Gebauer 1995; Richerson et al. 2001). The rise of agriculture is not only associated with a revolution in subsistence, but extended to changes in social and political organisation, degrees of sedentarisation, food storage, wealth accumulation and inequality as well as increasing population size (Cohen \& Crane-Kramer 2007; Hayden 1995; Piperno \& Pearsall 1998; Price \& Gebauer 1995). Hence the term 'Neolithic Revolution' to denote a turning point in human sociocultural evolution. However, as revolutions go the Neolithic one was particularly slow; archaeological evidence suggests that farming and foraging populations lived side-by-side (Kelly 1992; Inomata et al. 2015) as well as evidence of long-term persistence of low-level food production as the uptake of cultigens was patchy over five millennia (Bowles 2011). Similarly, it appears that the transition to greater settlement sizes and sedentism ${ }^{9}$ was gradual over a period of several thousand years (Ruff et al. 2015). Nevertheless, it seems that the onset of food production and sedentism produced significant population expansions (Diamond 2002), often referred to as the Neolithic Demographic Transition (Bocquet-Appel 2011) due to significant increases in fertility over the Neolithic period (Guerrero et al. 2008).

Settled, food producing women are argued to experience increased energy availability allowing them to re-invest this into fertility (Starling \& Stock 2007; Ellison 2003; BocquetAppel 2008). Domesticated grains represent a concentrated source of carbohydrates, which improves nutritional condition and consequentially increases reproductive value (Herrera 2000; Hardy et al. 2015; Hausman \& Wilmsen 1985). Furthermore, agricultural produce are

\footnotetext{
${ }^{9}$ Here I take Kelly's (1992) definition of sedentism as a relative rather than absolute condition; it refers to a more settled way of life relative to previous conditions. Furthermore, I do not argue that this sedentism is permanent or unidirectional, only that it is commonly associated with food production while it can also occur without the present of cultivation (Hitchcock \& Ebert 1984; Kelly 2013).
} 
often used as supplementary foods to speed up weaning and shorten interbirth intervals (Sellen 2006). In extant hunter-gatherers more acculturated groups are noted to significantly reduce breastfeeding and demonstrate higher fertility (Hitchcock 1982). Early research certainty took a Hobbesian ${ }^{10}$ view of hunter-gatherers in which farmers were assumed to have evolved more efficient, less demanding methods of subsistence, marking a progression in human economic development (Larsen 2006; Kelly 2013). Thus, fertility was also proposed to increase due to reductions in maternal energy expenditure, as food production was 'less of a toll'. However, current evidence suggests that the first farmers were no more productive than foragers (Bowles 2011), as foragers appeared to switch back and forth between modes of subsistence and developed stable mixed-subsistence regimes which remains true of populations today (Hitchcock \& Ebert 1984). Others have argued it is the changes in the types of work women conduct which results in fertility promotion, such as increased time in food processing and a reduction of foraging minimising aerobic exercise, rather than productivity per se (Kelly 2013). Overall then, the Neolithic transition is defined by increases in energy availability producing average population growth rates which rose from $<0.001 \%$ per year to $0.04 \% 0^{11}$ during the early Neolithic (Bandy, 2005; Bellwood and Oxenham, 2008; Cohen and Crane-Kramer, 2007; Downey et al., 2014; Hershkovitz and Gopher, 2008; Wells and Stock, 2007; Willis and Oxenham, 2013; Zahid et al., 2015).

While the Neolithic revolution occurred during a period of population growth, the causal relationship between agriculture and fertility increases remains controversial (Cohen 2009). Comparing population growth rates using summed calibrate radiocarbon date distributions demonstrates very similar growth rates for populations transitioning to agriculture and those that were still foraging (Zahid et al. 2015; Shennan et al. 2013). Furthermore, in North America where farming arose around 5000-3000 BP, there is no further increase in the population growth rate beyond a constant long-term trend (Zahid et al. 2015). This is consistent with genomic and archaeological evidence which suggests human population expansion occurred before the Neolithic revolution (Zheng et al. 2012). Perhaps then the same climatic improvements that opened the door for cultivation allowed an earlier human expansion and sedentarisation (Mellars 2006; Aimé et al. 2013). Simply put, it is unlikely

\footnotetext{
10 As in 'nasty, brutish and short' (Hobbes 1651, vol.XIII: 9).

11 While some (Bocquet-Appel and Naji, 2006) argue this figure is closer to $0.1 \%$, current archaeological and genetic evidence is supportive of $0.04 \%$ given an small effective population size around 50,000 years ago resulting in known world population sizes at AD 1800 (Zahid et al. 2015). Nonetheless, the evidence is supportive of a significant increase in growth rate regardless of exact figures.
} 
that immediate return foragers (as we envision them today) transformed into subsistence agriculturalists overnight with a higher fertility rate. Rather, there were prolonged intermediate stages in which fertility steadily rose due to increased settlement, storage and technological developments, which may have been further escalated by the rise of agriculture from 10,000 BP (Zheng et al. 2012). Supportive of the role of settlement in population expansion, Aimé et al. (2013) found that among 66 African and Eurasian populations from different subsistence regimes (foragers, pastoralists and farmers) that farmers consistently experienced expansion events while hunter-gatherers demonstrated constant population sizes. However, farmers experienced greater population expansion than pastoralists, indicating it was the shared mobility of foragers and pastoralists which kept population growth in check, rather than mode of subsistence (Aimé et al. 2013). Consequentially, it remains of interest to explore the reproductive consequences of settlement and subsistence change.

The Neolithic behavioural revolution also stimulated the first epidemiological transition, which was, on the whole, not beneficial of early farmers (Cohen \& Armelagos 1984; Cohen 2000; Barrett et al. 1998). Multiple paleopathological studies reveal significant increases in morbidity and mortality associated with a reduction in dietary breadth and sedentarisation (Armelagos et al. 2009; Cohen \& Armelagos 1984; Cohen \& Crane-Kramer 2007; Cohen 1989; Kohler \& Varien 2010; Richards 2002). Due to specialisation in a few staple crops such as maize, wheat and rice (Cohen \& Armelagos 1984; Richards 2002; Armelagos et al. 2005), while increasing overall glucose intake (Hardy et al. 2015), essential micro- and macronutrients were lost, leading to increases in anaemia and chronic malnutrition (Cordain 1999; Barrett et al. 1998). In the archaeological record such a diet is reflected in increases in dental defects, such as caries indicating high carbohydrate consumption (Pechenkina et al. 2002; Lukacs 2007) and linear enamel hypoplasia indicating periods of malnutrition and/or illness and stress (Morfin \& Storey 2007; Starling \& Stock 2007). Thus, it has been argued that the foragers diet high in meat, fruit and vegetable fibres and micronutrients was more varied, and thus, healthier (Eaton \& Eaton 2003; Larsen 2008). Furthermore, loss of mobility appears associated with declining skeletal robusticity from the Upper Palaeolithic to the $20^{\text {th }}$ century (Ruff et al. 2015). Consequently, earlier farmers have been portrayed as shorter, malnourished and less robust compared to huntergatherers (Willis \& Oxenham 2013; Pechenkina et al. 2002; Danford et al. 2007; Cohen \& Crane-Kramer 2007; Ruff et al. 2015; Larsen 2006). 
Research further reveals increased prevalence of tuberculosis, syphilis and the plague (Cohen \& Crane-Kramer 2007; Hershkovitz et al. 2008; Greenblatt \& Spigelman 2003; Rasmussen et al. 2015) as well as overall immunological stress (Mathieson et al. 2015). Hunter-gatherers' typically reside at low population densities of around one person per square kilometre (Froment 2008), which are protective from epidemic disease organisms (McElory \& Townsend 2008; Cockburn 1977). As such, increasing mortality has been associated with increased population density (Waguespack 2002). As communities expanded and came into frequent contact with one another, domesticated animals and rodents, they became the perfect reservoir for fast-moving and acute infectious diseases (Barrett et al. 1998; Cockburn 1977). For instance, genetic analysis on plasmodium DNA indicates that it arose less than 6,000 years ago concurrently with slash-and-burn agriculture in Africa (Armelagos \& Harper 2005). Many RNA viruses have a high mutation rate, genome re-assortment and virulence and, as such, are frequently transmitted between species (Van Blerkom 2003). Many of these viruses rely on high population densities (i.e. Paramyxoviruses such as mumps and measles rely on populations of over 500,000 (Norrby et al. 1992)), and depend of frequent interspecies exchanges common with the domestication of animals (Goral et al. 1996; van der Poel et al. 2000; Stephenson et al. 1999). As such, RNA viruses appeared to have evolved relatively recently as humans congregated with domesticated animals (Van Blerkom 2003)), while bacterial genomes reveal dramatic periods of adaptation associated with the Neolithic and it's new selective pressures (Mira et al. 2006).

The same pressures which facilitated virulent bacterial and viral pathogens (higher population densities, sedentarization, domestication and faecal pollution (Barrett et al. 1998; Cockburn 1977; Froment 2014)) equally well supported numerous soil-borne helminths Jackson et al. 2009; Leles et al. 2010; Bethony et al. 2006; Dounias \& Froment 2006; Hotez et al. 2005; Hurtado et al. 2008; Van Blerkom 2003)). Forgers ability to move significantly reduced exposure to communicable diseases transmitted due to lack of sanitation, vectorpresence and parasitic load (Dounias \& Froment 2011). When previously mobile groups settle, faecal pollution becomes excessive which increases helminth presence (Dounias \& Froment 2006). Helminths are parasitic worms, many of which reside in humans' intestines, living off their hosts for years. In particular, roundworm species from the family Nematoda include three soil-transmitted helminths which infect millions worldwide; whipworm (Trichuris trichiura), hookworm (Ancylostoma duodenale or Necator americanus) and 
giant roundworm (Ascaris Lumbricoides (Bethony et al. 2006)). While individuals, particularly children can maintain chronic polyparasitic infections, the physical cost is substantial; causing anaemia, malnutrition, chronic diarrhoea and vomiting, persistent inflammatory response as well as growth and intellectual retardation (Hurtado et al. 2008; Bethony et al. 2006; Hotez et al. 2005). Both whipworm and roundworm larvae survive in faecal contaminated soil and are digested when food or unwashed hands come into contact with this soil. Hookworm, is transmitted after the ova develop in the soil and pierce the sole of bare feet and work their way into the digestive system (Froment 2014; Dunn 1977; Bethony et al. 2006; Hotez et al. 2005). As such, poor sanitation is a major cause of persistent helminth infections.

Some argue that Palaeolithic foragers experienced high helminths loads (Hurtado et al. 2008; Rook 2009; Stearns 2012) and certainly some parasites are 'heirloom species' (Sprent 1969) which have long-standing co-evolutionary relationships with hominids. For instance, genomic evidence indicates tapeworm (Taenia spp.) has existed since the Palaeolithic (Armelagos \& Harper 2005; Hoberg et al. 2001; Perry 2014). Similarly, pinworm (Enterobius vermicularis) which is transmitted directly via touching, is likely to have evolved with the gregarious behaviour of hominoid primates and remains common even in industrial countries today (Barrett et al. 1998). Furthermore, roundworm has been found in human coprolites from one site in France from around 30,000 BP (Gonçalves et al. 2003), thus pre-dating agriculture. However, the evidence for extreme soil-transmitted helminths burden before agriculture is lacking (Reinhard 1988; Dunn 1977) as archaeological data shows instead an increase in helminths associated with farming (Hugot et al. 1999; Reinhard et al. 1985; Reinhard \& Araujo 2012; Reinhard 1988; Santoro et al. 2003; Leles et al. 2010) compared to mobile, low-density hunter-gatherers. With such conflicting evidence, exploring the predictors of helminth infection will be highly informative about humans' immunological evolution.

In summary, the overall effect of agriculture on health was a trend towards increasing morbidity and mortality (Cohen \& Armelagos 1984; Cohen 2009; Danford et al. 2007; Willis \& Oxenham 2013), although its intensity exhibits some regional variation and inconsistent trends (Hutchinson et al. 2007; Pinhasi \& Stock 2011; Douglas 2006; Humphrey et al. 2014; Lukacs 1992; Oxenham \& Tayles 2006; Oxenham et al. 2008; Pietrusewsky \& Ikehara-Quebral 2007; Tayles et al. 2009; Walker \& Hewlett 1990)). Thus, 
based on current archaeological evidence and epidemiological theory the following hypotheses were developed: (i) individuals residing in larger, settled camps consuming increased cultigens will suffer from higher rates of viral and bacterial diseases; (ii) helminth disease burden will be greater in more settled camps with more permanent structures; and (iii) as a result child mortality will be greater in settled camps.

Given this significant increase in disease burden, why farming arose independently and expanded in diverse geographical regions remains a puzzle (Diamond 2002; Lambert 2009). Here I propose that agriculture was accompanied by a life history trade-off, whereby mothers change strategy to invest in offspring quantity rather than quality and thereby increase their reproductive fitness. At the same time as women reduced their energy expenditure via reductions in foraging and mobility (Kelly 2013) the simultaneously increases in pathogen load would produce diminishing returns to maternal investment (Quinlan 2007). As environments become risky and extrinsic mortality rates grow, a mother's ability to buffer her children from these risks reduces. When this occurs life history theory predicts that organisms that switch to faster life history strategies and move investment away from a child and into increased fertility will optimises lifetime fitness. As a result, we can expect that the combination of increased energy availability and pathological risk to result in an adaptive switch to quantity rather than quality. Thus, my final two predictions are that (iv) settled mothers who conduct less foraging have a positive energy balance resulting in increased fertility and consequentially $(v)$ overall reproductive success.

By proposing that a quantity-quality trade-off, I provide an adaptive mechanism that reconciles the decline in population health with increases in fertility during the Neolithic transition. I test this hypothesis by measuring fertility, mortality alongside viral, bacterial and helminth markers in different Agta camps. I found that sedentarization was associated with increased morbidity and mortality, yet both sedentarization and involvement in nonforaging activities are both associated with even further increases in fertility, resulting in higher reproductive fitness. My results provide evidence that a quality-quantity trade-off was adaptive and suggests a pathway through which, despite poor health, farming could have become the dominant subsistence system after its origin in the Neolithic. Thus, I demonstrate how exploring the mechanisms apparent in extant foragers is a theoretically informative approach to test key archaeological predictions (Bocquet - Appel \& Naji 2006; Cohen \& Armelagos 1984; Cohen \& Crane-Kramer 2007). 


\subsection{Methods}

By examining the patterning of health within a extant foraging group it is possible to avoid problematic comparisons with neighbouring farmers or urban residents and removes the need to treat foragers as prehistoric 'relics' (Dunn 1977; Stock \& Pinhasi 2011). Modern day foragers heavily interact with their neighbours, but are socially and economically worse off. Therefore, in direct comparison foragers health will often be found lacking (Froment 2014; Dounias \& Froment 2006; Hill et al. 2007; Tanner et al. 2009; Vasunilashorn et al. 2010; Vasunilashorn et al. 2011; Gurven et al. 2008; McDade et al. 2005; Gurven et al. 2007). Furthermore, health is impacted by such a range of factors (Willis \& Oxenham 2013; Hershkovitz et al. 2008; Danford et al. 2007; Hutchinson et al. 2007) that drawing a simple dichotomy between farmers and hunter-gatherers obscures many important trends making comparisons arbitrary (Stock \& Pinhasi 2011). For instance, extant foragers may have lower body mass index (BMI) than neighbouring farmers, such as Bakola pygmies and Bantu farmers (Froment et al. 1996), however it is difficult to separate the pathologic (i.e. malnutrition), adaptive (i.e. faster life histories (Migliano et al. 2007)), and the physiological (i.e. different body composition) explanations. Likewise, when comparing total fertility rates between different subsistence groups Bentley et al. (1993) conclude that it is impossible to predict fertility on the basis of subsistence alone due to degree of variability and overlap between classifications.

Simply taking small-scale societies today as reflective of our Palaeolithic past is equally as problematic. For instance, research from South America settled horticulturalist-foragers finds they live in highly infectious environments, with high mortality rates from disease at all ages (Hill et al. 2007; Gurven et al. 2007) and disease profiles are comparable to $19^{\text {th }}$ century industrial Europe (Gurven et al. 2008). The Tsimane from Bolivia have high helminth loads (Hurtado et al. 2008), elevated levels of C-reactive protein (CRP) and IL-6 (markers of systemic inflammation) and white blood cell counts (indicative of immune response (Vasunilashorn et al. 2011; Vasunilashorn et al. 2010)). Thus, one could be mistaken to believe that hunter-gatherer lives were indeed 'nasty, brutish and short' (Hobbes 1651, vol.XIII: 9). However, as a minority peoples in a developing country this may highlight more about the difficulties facing many previously nomadic foragers, than our ancient past. Yet, by comparing variance within one population it is possible to control for a myriad of other factors, and meaningfully explore the effects of patterns and trends associated with the Neolithic transition (reduction in foraging, larger camp size, 
lower mobility and more belongings (Hayden 1995; Piperno \& Pearsall 1998)) on health and fertility without make large generalisations about how well the Agta represent 'real hunter-gatherers'. The Agta have had extensive contact with outsiders for generations; they are neither isolated nor untouched. Furthermore, Agta 'transition' to agriculture has been ethnographically reported since the 1960's, and their extinction predicted for even longer (Minter 2010; Rai 1990; Headland 1989). Thus, I am not arguing that this process is sudden on any particular time scale. However, a quantification of the effects of decreases in foraging and increases in settlement do not require 'transition' to be recent, only that there exists significant variability among the Agta.

\subsubsection{Measuring health}

Full methodological procedures of the health survey are explained at length in section 3.4. In this analysis health has been measured using white blood cell analysis to capture differences in viral, bacterial and helminthic infections (Table 8.1). Parasite burden was further explored using faecal analysis to examine parasite load. A medical questionnaire was also conducted to explore the predictors of vaccination rates and the receipt of medical assistance.

Table 8.1: White blood cell types, medically abnormal thresholds and health implications.

\begin{tabular}{|c|c|c|c|}
\hline Measure & $\begin{array}{c}\text { Adult abnormal } \\
\text { values }(12+)\end{array}$ & Child abnormal values & Indication of \\
\hline Lymphocytosis & $>3 \times 10^{9} / \mathrm{L}$ & $\begin{array}{c}8.0 \times 10^{9} / \mathrm{L} \text { children under } 12 \text { years } 4-12 \times \\
10^{9} / \mathrm{L} \text { children under } 2 \text { years; } 9 \times 10^{9} / \mathrm{L} \text { children } \\
\text { aged between } 2 \text { - } 2 \text { years; } 1-5 \times 10^{9} / \mathrm{L} \text { children } \\
\text { aged } 6 \text { to } 12 .\end{array}$ & Viral infection \\
\hline Neutrophilia & $>7 \times 10^{9} / \mathrm{L}$ & $8.0 \times 10^{9} / \mathrm{L}$ children under 12 years & $\begin{array}{l}\text { Bacterial } \\
\text { infection }\end{array}$ \\
\hline Eosinophilia & $>0.5 \times 10^{9} / \mathrm{L}$ & $\begin{array}{c}>1 \times 10^{9} / \mathrm{L} \text { children aged under } 12 \text { years. } \\
\text { Moderate at }>1.5 \&<5 \times 10^{9} / \mathrm{L} \\
\text { Severe at }>5 \times 10^{9} / \mathrm{L}\end{array}$ & $\begin{array}{l}\text { Helminthic } \\
\text { infection }\end{array}$ \\
\hline
\end{tabular}

Full details are in the methodological section 3.4.4.2. 


\subsubsection{Measuring transition}

Two different measures of mobility were gathered: household mobility and out-of-camp mobility. For camp-level mobility I conducted a survival analysis that quantifies the proportion of individuals leaving camps. If all individuals who had been present on my first visit remained so during later visits, the camp had a survival rating of 1 . If, however, camp composition completely changed the camp had a survival rating of 0 . Therefore, this measure quantifies out-of-camp mobility. Leaving was defined as any departure from camp which was longer than overnight. At the household level, individuals were either allocated as mobile (0) or settled (1) depending on whether or not I had ever witnessed them to move (again for longer than one night) at least once during my fieldwork.

While these mobility variables capture peoples' movement, the degree of camp sedentarization was also coded according to housing type. The camp bousing type variable is on a three-point scale, 0 being the most temporary and includes camps with lean-to shelters, which frequently change in either location or position. Camps allocated to 2 on the scale were fully settled camps in which the houses were permanent (wooden huts with metal roofs) and unable to move. Camps with a mixture of both of these features had a temporary measure of 1 . Finally, for a binary analysis the camps were simply separated into a category of sedentarized camp: 0 being a mobile camp and 1 a sedentarized camp based on the presence of permanent housing, churches and infrastructure such as water pumps. Therefore, with these measures I have both a sense of the permanence of camps as well as individual's mobility in and out of them.

Table 8.2: Descriptive statistics for categorical independent variables

\begin{tabular}{clrl}
\hline & Class & $\boldsymbol{n}$ & $\mathbf{0}$ \\
\hline \multirow{2}{*}{ Sex } & Female & 182 & 43.9 \\
& Male & 233 & 56.1 \\
Settled Agta & Mobile & 114 & 27.5 \\
& Settled & 300 & 72.5 \\
\multirow{2}{*}{ Agta in Sedentary camps } & Mobile & 111 & 26.8 \\
& Sedentary & 304 & 73.3 \\
& Temporary & 81 & 19.5 \\
Maternal Foraging type & Semi-permanent & 147 & 35.4 \\
& Permanent & 187 & 45.0 \\
& Less than $75 \%$ & 358 & 91.5 \\
& More than $75 \%$ & 33 & 0.09 \\
\hline
\end{tabular}


While these settlement and mobility indices may appear similar, they make important distinctions. For instance, the camp housing measures are expected to be more important for soil-transmitted helminths which reside in fecally contaminated soil, of which there is a lot more of in permanent camps. A camp may have many of the same people residing in it (thus high out-of-camp mobility score), but if they actively move around an area this may significantly reduce helminth presence. This frequently happens in camps comprised of lean-to shelters, which often change location and position during the night. When asked the Agta stated they moved short distance because it was dirty, smelly or too many insects at the previous location. Even these short distance moves are sufficient to break up the transmission routes of soil-transmitted helminths (Bethony et al. 2006; Dounias \& Froment 2011). Out-of-camp mobility, on the other hand, reflects more about increasing camp size and population density: when few people leave a camp its size necessarily gets larger. Therefore, out-of-camp mobility is likely an important predictor for viral infections which depend on larger population sizes (Van Blerkom 2003). Finally, the binary sedentarized camp measure focuses on external influences on the camps: camps with churches and/or water pumps are coded a sedentarized, while camps with no intervention are coded as mobile. Thus, if a disease marker is affected by medical intervention this variable is likely to be an important predictor.

Table 8.3: Descriptive statistics for the continuous dependent and independent variables $(n=345)$.

\begin{tabular}{lrrrr}
\hline & Min & Max & Mean & SD \\
\hline Age & 0 & 80 & 20.27 & 18.89 \\
Camp size & 5 & 77 & 49.62 & 22.27 \\
Household belongings & 0.18 & 5.5 & 2.01 & 1.12 \\
Mean relatedness & 0 & 0.4 & 0.12 & 0.07 \\
Out-of-camp mobility & 0 & 0.86 & 0.59 & 0.23 \\
Proportion of foraging & 0 & 1 & 0.75 & 0.36 \\
Lymphocytes & 1.2 & 10.7 & 4.015 & 1.677 \\
Neutrophils & 0.9 & 15.5 & 4.298 & 1.74 \\
Eosinophils & 0 & 7.2 & 1.419 & 1.09 \\
\hline
\end{tabular}


Finally, transition was also measured by the proportion of time spent foraging. As this research focuses on maternal fertility I took the proportion of economic activities the mother spent in foraging (compared to cash labour, cultivation and so forth) as the household measure. Given that the majority of individuals did spend a significant amount of time foraging I set the cut-off at the mean of the sample (0.75), thus individuals higher than the mean were considered to conduct a large amount of foraging. Full descriptive statistics are given in Table 8.2 and Table 8.3.

Table 8.4: Selection process for camp sedentarization variables to be entered into each model. Models with the lowest AIC are highlighted in italics, which were entered into the final model.

\begin{tabular}{clrr}
\hline Dependent & Predictor & \multicolumn{1}{l}{$\boldsymbol{p}$} & \multicolumn{1}{c}{ AIC } \\
\hline \multirow{4}{*}{ Lymphocytosis } & Out-of-camp mobility & 0.001 & 422.71 \\
& Camp housing type & 0.375 & 433.156 \\
& Settled camp & 0.03 & 427.339 \\
\cline { 2 - 4 } Neutrophilia & Out-of-camp mobility & 0.897 & 140.871 \\
& Camp housing type & 0.383 & 142.143 \\
\cline { 2 - 4 } Eosinophils & Settled camp & 0.253 & 139.527 \\
& Out-of-camp mobility & 0.823 & 1024.894 \\
\cline { 2 - 4 } Fertility & Camp housing type & 0.003 & 1018.366 \\
& Settled camp & 0.025 & 1020.096 \\
\cline { 2 - 4 } Reproductive Success & Out-of-camp mobility & 0.07 & 504.849 \\
& Camp housing type & 0.26 & 463.14 \\
\cline { 2 - 4 } & Settled camp & 0.256 & 506.815 \\
& Out-of-camp mobility & 0.518 & 486.548 \\
\cline { 2 - 4 } Childhood mortality housing type & 0.485 & 486.372 \\
& Settled camp & 0.666 & 486.775 \\
\cline { 2 - 4 } & Out-of-camp mobility & 0.244 & 324.849 \\
& Camp housing type & 0.011 & 322.549 \\
\cline { 2 - 4 } & Settled camp & 0.066 & 323.387 \\
\hline
\end{tabular}

I used these 'transition' variables in multilevel analyses to examine pathogen load, fertility, morbidity and mortality. Due to covariance two models were produced for each analysis: one exploring the effects of household mobility (whether a household moved camp at least once or not) and the second, exploring the effects of household foraging (binary measure, $1=$ more than $75 \%$ of maternal time spent in foraging). Both these models included one camp 
settlement variable, chosen based on the lowest AIC score in univariate regressions (Table 8.4), and included: i) camp bousing type (temporary, semi-permanent or permanent); ii) a binary sedentarized camp variable denoted by the presence of permanent camp infrastructures; and iii) out-of-camp mobility over two years, 0 representing camp abandonment. Only theoretically informed variables were entered into the models and all models contained appropriate controls (age, sex, mean relatedness and household dependents and belongings) and the best predictor variable for camp sedentarization. Due to the smaller sample size, non-parametric Kruskal-Wallis tests were used to examine the data on polyparasitic infections. All demographic models used age-specific residuals (section 3.5.1) as dependent variables. All model betas are reported alongside standard errors. Full model result tables can be found in appendix D.

\subsection{Results}

\subsubsection{Settled Agta exhibit increased fertility}

For 117 women I interviewed (aged between 16-75 years), the mean number of live births was 4.4 (ranging from 0 to 13). Settled mothers had significantly higher fertility than women who moved camp at least once $(\beta=1.3 \pm 0.6, p=0.04, n=90)$. Correspondingly, increases in household belongings were positively associated with fertility $(\beta=0.5+0.2, p$ $=0.005)$. This relationship is further confirmed by examining TFR (total fertility rate): settled mothers had 16.7\% higher TFR (7.7) compared to mobile mothers (6.6). Similarly, for all live births settled mothers had 20.5\% more offspring (5.3) than mobile mothers (4.4; Table 8.5). A possible pathway may be that settled mothers are able to accumulate more somatic resources for reproduction. Accordingly, settlement was a significant predictor of maternal BMI (i.e. mothers never witnessed to move camp, $\beta=1.7 \pm 0.6, p=0.004$ ), and BMI positively predicts fertility $(\beta=0.3 \pm 0.1, p=0.02)$.

\subsubsection{Mothers who forage more exhibit lower fertility}

A high degree of foraging also significantly predicts fertility $(B=-1.4 \pm 0.7, p=0.04)$. As a result, mothers who spent less than $75 \%$ of their time foraging experience 0.23 higher fertility residuals than expected for their age. Mothers who spent more than $75 \%$ of their time foraging had 0.85 less offspring, given their age. Women who spent more time foraging also had marginally lower BMI $(B=-1.5 \pm 0.9, p=0.08)$. Therefore, transition to 
farming as measured by both increasing cultivation and sedentarization are positively associated with fertility, perhaps due to increased somatic resources.

Table 8.5: Fertility, mortality and survivorship to age 16 figures averaged by mobility and camp type $(n=117)$.

\begin{tabular}{llrrr|rrr}
\hline \multirow{2}{*}{ Measures } & \multicolumn{3}{c|}{ Actual rates } & \multicolumn{3}{c}{ Age controlled residuals } \\
\cline { 3 - 8 } Fertility & \multicolumn{1}{c}{ Condition } & Mean & \multicolumn{1}{c}{ SD } & SEM & Mean & \multicolumn{1}{c}{ SD } & SEM \\
\hline \multirow{3}{*}{ Mortality } & Nomad & 4.381 & 2.889 & 0.63 & -0.408 & 2.09 & 0.456 \\
& Mobile in settled camp & 3.185 & 3.026 & 0.582 & -0.998 & 2.129 & 0.41 \\
& Settled & 5.246 & 3.296 & 0.397 & 0.515 & 2.535 & 0.305 \\
\hline \multirow{3}{*}{ RS } & Nomad & 0.571 & 0.978 & 0.213 & -0.275 & 1.127 & 0.246 \\
& Mobile in settled camp & 0.727 & 0.883 & 0.188 & -0.04 & 0.649 & 0.138 \\
& Settled & 0.926 & 1.331 & 0.161 & 0.098 & 1.108 & 0.134 \\
\hline & Nomad & 3.81 & 2.562 & 0.559 & -0.304 & 1.69 & 0.369 \\
& Mobile in settled camp & 3.182 & 2.343 & 0.5 & -0.729 & 1.842 & 0.393 \\
\hline & Settled & 4.397 & 2.632 & 0.319 & 0.33 & 2.242 & 0.272 \\
\hline
\end{tabular}

Note: Actual rates are presented alongside the age-controlled residuals. A residual 0 is the average rate of any given age group. Thus, a positive residual is above average, while a negative residual is below average. I have provided both the age controlled and non-age controlled results as there is no significant relationship between age and degree of sedentarization. SD stands for standard deviation; SEM: standard error of the mean.

\subsubsection{Sedentarization is associated with health deterioration}

I examined the effects of cultivation and sedentarization on three white blood cell types lymphocytes, neutrophils and eosinophils - as proxies for different immunological attacks: viral, bacterial and helminthic, respectively. Our sample consisted of 345 Agta, 140 of whom were adults (48.6\% males) and 205 children under 16 (60\% males). Due to inconclusive test results, missing data reduced the sample to 293, consisting of 117 adults (47\% males) and 176 children (59\% males). Table 8.6 shows that people in large camps with low out-of-camp mobility had 2.8 times higher chances of presenting with lymphocytosis compared to mobile camps (Figure 8.1). People residing in camps with permanent and semi-permanent houses had significantly higher eosinophil concentrations than individuals in temporary camps. Severe eosinophilia $\left(>5 \times 10^{9} / \mathrm{L}\right)$ is indicative of extreme helminth infestations and only found in sedentarized camps (Figure 8.2). In contrast, people residing in sedentarized camps with some form of infrastructure (i.e. church or water pump) had lower odds (by $80 \%$ ) of neutrophilia. One major bacterial disease among the Agta is Mycobacterium tuberculosis (TB), demonstrated by the fact that 
$41.7 \%$ of the 24 individuals suffering from neutrophilia had clinically diagnosed or suspected TB. In contrast, percentage of time spent foraging had little effect on health. Individuals in households who foraged more had a $76 \%$ reduction in odds of presenting with lymphocytosis than households who foraged less. However, indicators of helminthic or bacterial infections were not affected by percentage of time spent foraging.

Table 8.6: White blood cell types, medically abnormal thresholds, frequency, predictors and health implications.

\begin{tabular}{|c|c|c|c|c|}
\hline Measure & $\begin{array}{c}\text { Abnormal } \\
\text { values }\end{array}$ & $\begin{array}{c}\% \\
\text { affected }\end{array}$ & Explanatory variables & Indication of \\
\hline Lymphocytosis & $\begin{array}{l}\text { Lymphocytes } \\
>3 \times 10^{9} / \mathrm{L}\end{array}$ & $67 \%$ & $\begin{array}{l}\text { Out-of-camp mobility } \mathrm{OR}=2.8 \\
\mathrm{p}=0.03 ; \\
>75 \% \text { foraging: } \mathrm{OR}=0.24, \mathrm{p}= \\
0.04 \text { (baseline }<75 \% \text { foraging })\end{array}$ & Viral infection \\
\hline Neutrophilia & $\begin{array}{l}\text { Neutrophils } \\
>7 \times 10^{9} / \mathrm{L}\end{array}$ & $6.5 \%$ & $\begin{array}{l}\text { Settled camp: } \mathrm{OR}=0.2 \mathrm{p}=0.01 \\
\text { baseline: } \text { mobile camp }\end{array}$ & $\begin{array}{l}\text { Bacterial } \\
\text { infection }\end{array}$ \\
\hline Eosinophilia & $\begin{array}{c}\text { Eosinophils } \\
>0.5 \mathrm{x} \\
10^{9} / \mathrm{L}\end{array}$ & $71.3 \%$ & $\begin{array}{l}\text { Semi-permanent housing: } B \\
=0.53+0.2, \mathrm{p}=0.004 ; \\
\text { Permanent housing: } \beta=0.45+ \\
0.2 \mathrm{p}=0.007 \\
\text { baseline: temporary }\end{array}$ & $\begin{array}{l}\text { Helminthic } \\
\text { infection }\end{array}$ \\
\hline
\end{tabular}

Note: Eosinophilia is a continuous analysis so to differentiate between individuals with mild eosinophilia, medium and extreme eosinophilia, as these reflect significant variance in helminth load. All other analysis are logistic. Adult abnormal values are given in the table, however there is slight variation in thresholds for children aged under 12 years. Full details are in the methodological section 3.4.4.2.

To explore helminth burden further, I collected 30 faecal samples from Agta who had presented with eosinophilia. All 30 samples tested positive for helminth ova. The mean number of species was $1.6 \pm 0.7$ and included: roundworm (Ascaris Lumbricoides, 83.3\%); hookworm (Ancylostoma duodenale or Necator americanus, 46.7\%); and whipworm (Trichuris trichiura, 30\%). Due to the small sample size non-parametric Kruskal-Wallis tests were run on three measures of transition. Living in a sedentarized camp was associated with a 0.65 mean increase in parasite species number compared to living in mobile camps $\left(\chi^{2}=5.9, \mathrm{df}=1, p=0.02\right)$. Household participation in cash labour decreased helminth loads by $37.4 \%\left(\chi^{2}=8.9, \mathrm{df}=1, p=0.003\right)$ indicating that families who interacted with the wider economy may be more able, financially and/or behaviourally, to acquire anthelmintic medicines. While cash labour is a positive predictor, the proportion of time spent foraging 
is not $\left(\chi^{2}=3.3 \mathrm{df}=1, p=0.2\right)$. My results indicate that it is settlement, rather than cultivation, which predicts helminth infestation.

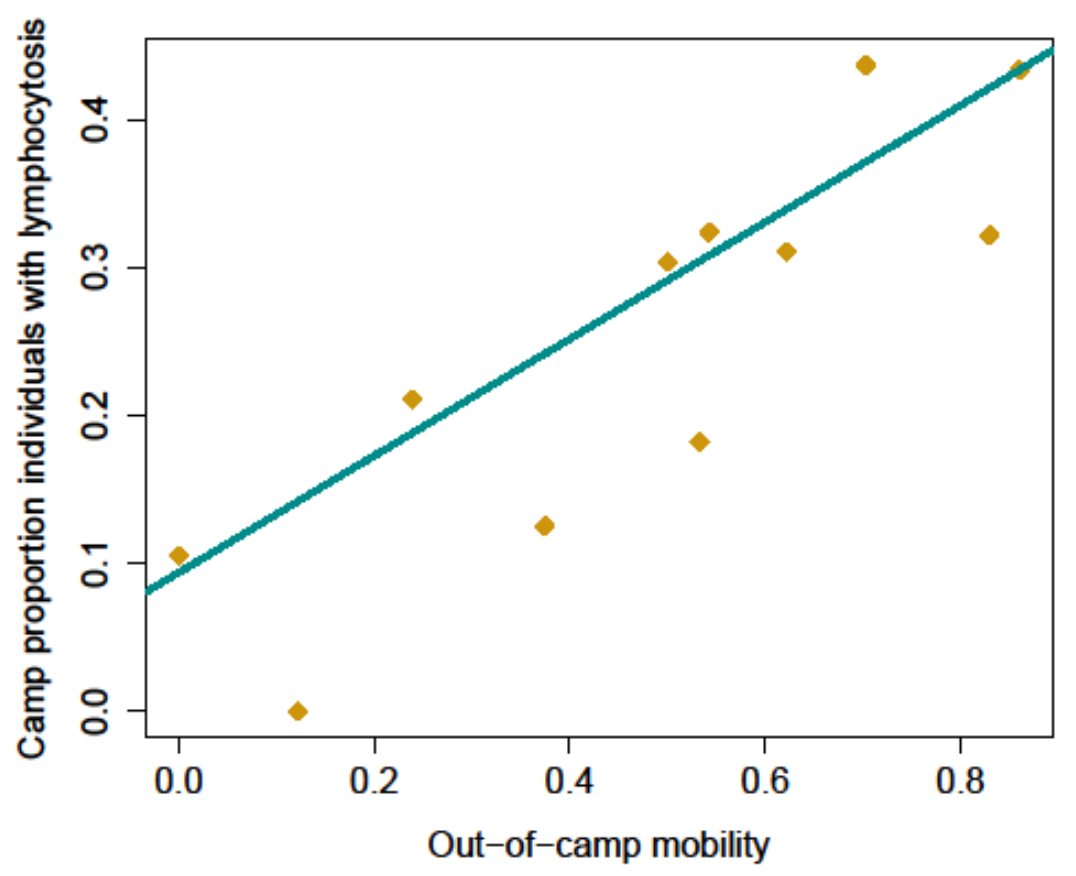

Figure 8.1: Lymphocytosis by out-of-camp mobility $(n=293)$. The dependent variable (presenting with lymphocytosis or not) is binary, thus each point represents the proportion of individuals presenting with lymphocytosis in a camp with that out-of-mobility score, ranging from 0 to $43 \%$. Out-of-camp mobility is a measure of camp composition; if a camp has a score of 1 no one left over the two-year study period. A score of zero indicates an abandoned camp.

\subsubsection{Settlement increases child mortality}

I examined whether the negative effects of settlement on health also extended to child mortality rates (including all post-natal deaths until age 16). Mothers residing in camps with permanent housing experienced significantly higher childhood mortality rates, compared to mothers living in camps with temporary housing $(B=0.7 \pm 0.3, p=0.005)$. The relationship for semi-permanent camps was in the same direction but of marginal significance $(B=0.5 \pm 0.3, p=0.08)$. This relationship results in a $63.2 \%$ increase in mortality rates for settled mothers living in sedentarized camps (0.93) when compared to nomadic mothers in temporary camps (0.57). Thus, the sedentarized camps with the highest helminth and viral disease rates also experience the highest childhood mortality 
rates. Following the morbidity results, proportion of time spent in cultivation had no significant relationship with child mortality $(B=0.01 \pm 0.04, p=0.8)$.

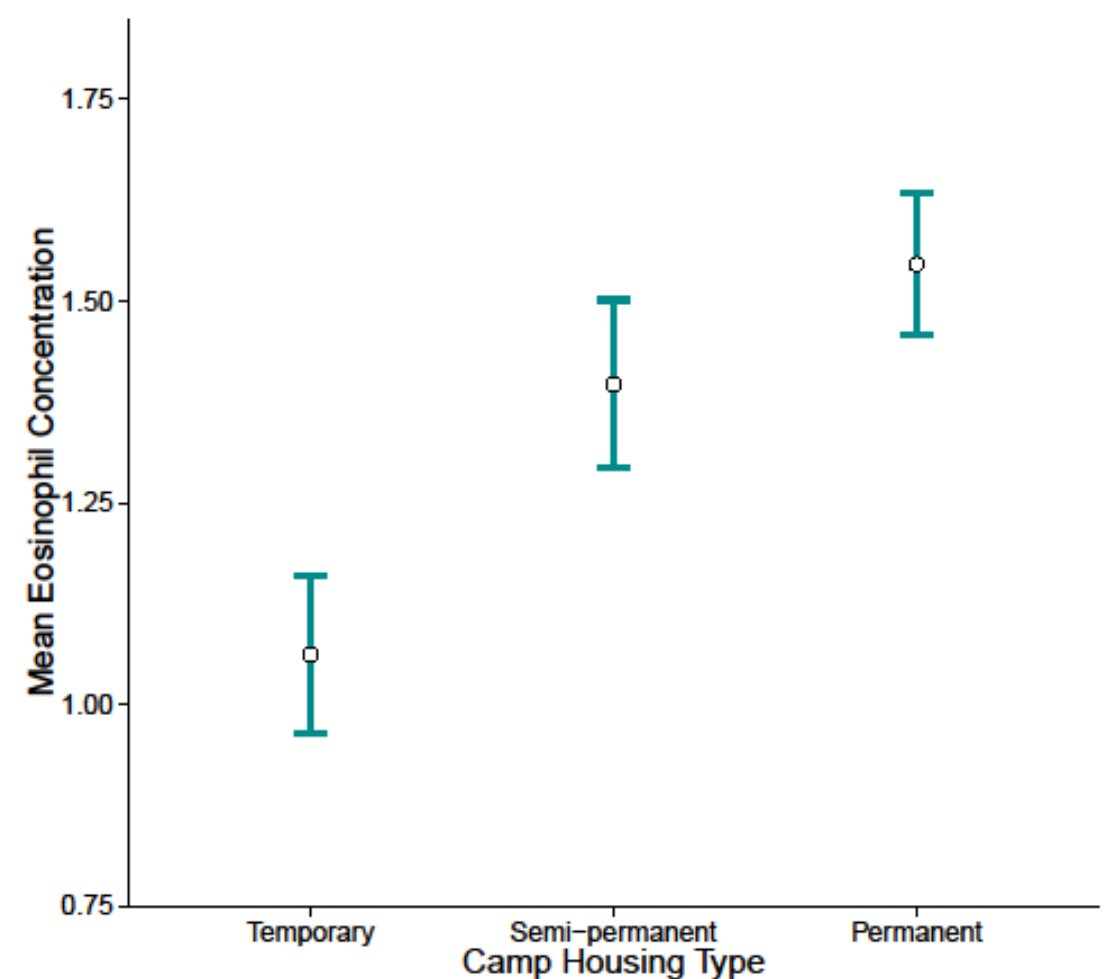

Figure 8.2: Mean eosinophil concentration by camp housing type $(n=293)$. Camps with temporary houses (highly mobile lean-tos) have the lowest eosinophil concentrations while camps with permanent housing (wooden huts with metal roofs) have the highest. Bars reflect standard errors.

\subsubsection{A quantity-quality trade-off explains higher reproductive success in} transitioning Agta

My results show that women who settle down trade child survival for fertility, increasing their overall reproductive success (Figure 8.3). Settled women $(B=0.8 \pm 0.4, p=0.03$ ) had significantly more children surviving to age 16 than mobile women. Although these settled women faced higher child mortality rates, they also experienced extremely high fertility. As a result of the negative morbidity effect of sedentarized camps, compared to the positive fertility effect of settled households, women residing in permanent camps suffered lower child survival $(B=-1.2 \pm 0.5, p=0.01)$ compared to temporary camps, once household mobility has been controlled for. Figure 8.3 demonstrates that mobile women living in sedentarized camps faced the worst situation as they experienced both higher mortality 
rates associated with permanent camps and the lower fertility rates associated with high mobility. Thus, they experienced 0.73 lower reproductive fitness given their age. Overall, my results confirm that settled mothers, in spite of the higher mortality rates, had an extra 0.59 offspring (4.4) surviving to age 16 compared to mobile women (3.8), a relative increase of $15.8 \%$ in reproductive fitness.

A

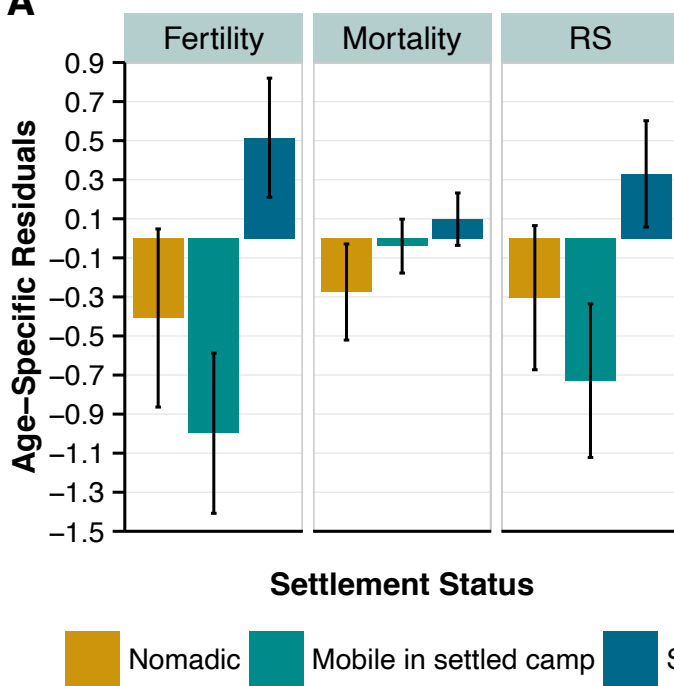

B

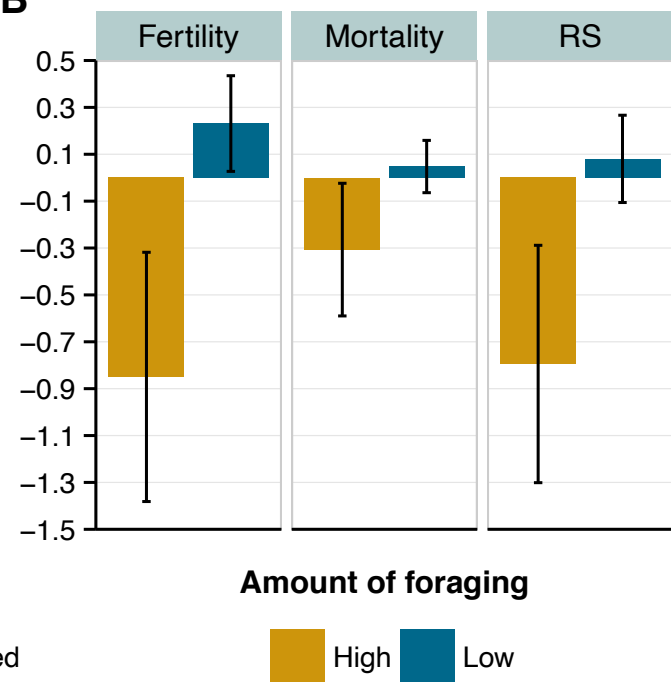

Figure 8.3: Relationship between measures of transition and residuals of age-specific fertility, mortality and reproductive success (RS) separated by (A) individual settlement status and (B) degree of foraging. Error bars represent standard errors of the mean $(n=117)$.

Matching my fertility results, I found that maternal foraging $(B=-1.2 \pm 0.6, p=0.05)$ also predicted survivorship to age 16. Mothers who foraged more than $75 \%$ of the time had 0.74 less children surviving to age 16 given their age, while mothers who spent less time foraging experienced 0.04 more children surviving to age 16 (Figure 8.3). Thus, while abandoning foraging does not significantly affect child mortality rates, it does increase fertility, and consequently mother's reproductive success. Consequently, while cultivation is associated with increased fertility, sedentarization is associated with both increased fertility and increased morbidity and mortality. The overall consequence of this combined process is a reproductive trade-off where settled mothers involved in cultivation have the highest reproductive fitness, despite increased morbidity and mortality. 


\subsubsection{External involvement}

The Agta have long-standing interactions with their neighbours, a common feature of many Agta and Aeta groups in the Philippines. However, as there is no road or easy route to Palanan, external pressures (extractive industries, land grabbing and clearing, resource destruction and migration) have been significantly reduced for the Palanan Agta compared to populations to the south of the NSMNP (Early \& Headland 1998; Headland 1989; Headland et al. 1989). Nonetheless, this does not negate the fact that the Agta do have some access to basic forms of modern medical care, occasionally attend school (however, this remains a minority of children in limited areas and not yet consistently), and a couple of the most settled camps are influenced by the Evangelical church.

I examined the relationship between 'transition' and access to medical care. Vaccination rates are very low in the population in general, with a mean vaccination rate of $0.18 \pm 0.21$. In a linear regression on camp vaccination rate $(n=13)$ the major predictors of having a vaccination is living in a sedentarized camp $(B=0.19 \pm 0.07, p=0.02)$ close to town $(B=$ $-0.013 \pm 0.005, p=0.01)$, which accounts for $76 \%$ of the variance. This is because any religious charity or government intervention is focused on the easy to find, easy to access Agta camps. Mobile camps the furthest from town reported no contact with nurses, doctors or other medical providers. I found that only $34 \%$ out of 325 cases of sickness received medical treatment, but this was dependent on acculturation. Receiving medical treatment was predicted by living in a sedentarised camp $(B=0.36 \pm 0.18, p=0.04)$ and household participation in cash labour $(B=0.19 \pm 0.08, p=0.02)$ in MLM controlling for sex and age $(n=325)$. This implies that households in more integrated camps with access to cash are better able to access medical care. Thus, I would expect health to be improved in the sedentarized camps closer to town. However, I find the opposite (expect in the case of neutrophilia), implying it has little protective effect, perhaps since the care is inconsistent and/or of poor quality (the resources are rudimentary, medication often expensive and/or unavailable, and cultural barriers are extensive between Agta and nonAgta). Thus, individuals who would be likely to receive medical assistance do not seem to significantly benefit from this, or if they do, it is not sufficient to buffer the ill effects of settlement. 


\subsection{Discussion}

The variability of lifestyles currently observed within the Agta allows the testing of hypothesis proposed by archaeologists to explain the demographic processes taking place during the Neolithic transition (Barrett et al., 1998; Bocquet-Appel and Naji, 2006). Current evidence from archaeology and other sources suggests a Neolithic paradox as fertility increases alongside morbidity. Leaving researchers asking why was farming so successful if quality and duration of life shortened dramatically (Lambert 2009). Using a human behavioural ecology perspective, which focuses on reproductive success rather than health, reveals that behavioural strategies, such as increasing cultivation and settlement, can be considered adaptive even in the face of mounting ill health. My results support the hypothesis that a quantity-quality trade-off can explain the demographic and health changes associated with transition from mobile foraging to sedentarized food production. In so far that variability observed within the Agta is reflective of Neolithic trends, these results support the hypothesis that fertility increases with cultivation and sedentarisation, outstripping mortality increases, resulting in population growth. Thus, this ultimately suggests that despite the increased disease burden and lower quality of life, sedentarised cultivation was and is a successful behavioural adaptation.

\subsubsection{Fertility, sedentism and cultivation}

Agriculture and sedentism has long been associated with fertility increases (Bentley et al. 1993; Sellen \& Mace 1997; Roth \& Ray 1985; Roth 1981; Hitchcock 1982; Kelly 2013) as the reduction in energy expenditure with settlement (Blurton Jones 1987) and increased carbohydrate consumption (Herrera 2000; Hardy et al. 2015) are associated with increased BMI, which correlates with shorter inter-birth intervals and higher fertility (Tracer 1991). Human fertility is particularly responsive to energetics via hormonal pathways (PanterBrick \& Pollard 1999). If energy intake is low or energy expenditure high (i.e. a negative energy balance) the body will suppress ovulation and invest in somatic effort until conditions improve (Pike 2001; Bentley et al. 1998). Metabolic hormones (insulin and insulin-like-growth-factor) function as physiological signals of environmental conditions and their receptors are found in ovarian cells (Lipson 2001). Metabolic hormones increase in good conditions stimulating gonadal hormones, oestradiol and progesterone, which prompt ovulation (Pollard 2008). For instance, weight loss as low as two kilograms is sufficient to reduce progesterone among the Lese resulting in anovulation (Ellison 2001) 
while increased work load alone was sufficient to decrease progesterone among Polish farm workers without any significant decrease in nutritional condition (Jasieńska \& Ellison 1998). Therefore, a positive energy balance, can be associated with increased fertility: Gibson and Mace (2002) found that the introduction of water taps in rural communities in Ethiopia significantly reduced first birth interval length resulting in population growth due to a reduction in maternal workload.

Therefore, agriculture has been understood to increase total fertility rate due to energetic factors (Bentley et al. 1993; Sellen \& Mace 1997). However, here I suggest that part of the fertility increase may be associated with combatant increases in childhood mortality. Mothers residing in sedentarised camps face significantly higher child mortality, the causes of which may be considered extrinsic if increasing parental investment does nothing to diminish their effect on child survival (Quinlan 2007). As a result mothers may switch to a quantity strategy and emphasise fertility over parental care. Supportive of this hypothesis is evidence across sub-Saharan African countries that the presence of a trade-off between child quantity and quality becomes increasingly apparent with socioeconomic development (Lawson et al. 2012; Gibson \& Lawson 2011). While this relationship with 'modernisation' functions in the opposite direction, it expresses the same rule. For instance, Gibson and Lawson (2011) found that as modernisation decreased extrinsic risk in Ethiopia, parental investment started to have a larger influence on child outcomes, thus changing the risk from extrinsic to intrinsic. As a result, the evidence of increasing offspring competition for parental resources becomes increasingly present. Here I argue that the increase in pathogen load with sedentism can be considered an extrinsic risk after a point at which maternal care receives diminishing returns in child survival (Quinlan 2007). Consequentially, mothers switch to investment in fertility to mitigate higher losses in child mortality. Future exploration of this question should explore to what degree the viral and helminthic disease load can truly be considered extrinsic risks, however in a population with very little access to medicine and resources, maternal influence over disease is likely limited.

Kramer and Boone (2002) approach the apparent fertility increase in agriculturalists from a different angle. They highlight Bentley's et al. (1993) finding that while agriculturalists experience significantly higher fertility rates than foragers, horticulturalists demonstrated slightly (but non-significant) lower rates, suggesting that perhaps it is not sedentism or agricultural production which results in increasing fertility but alterations to the cost of 
children. For instance, Draper and Cashdan (1988) argued that hunter-gatherer childcare is defined by parental care rather than allocarers; it was increasing settlement and nonforaging activities which separated parents from offspring, resulting in increased peerrearing. Following the economic argument then (Cain 1977; Lee \& Kramer 2002) the cost of children reduces as they are better able to support themselves as well as their siblings, thus increasing fertility as mothers can afford more children.

However, Draper and Cashdan's (1988) conclusions were based on very short observational periods (31 hours) in a small sample (19 children in 'bush' camps and 21 in sedentary camps), thus certainly require revisiting. More recent anthropological theory and evidence is supportive of significant amounts of allocare from around two years (Konner 2005). As demonstrated in section 5.4, mothers care is quickly overtaken by other types of allocarers, which is consistent with results found in many of foraging populations (Meehan 2009; Crittenden \& Marlowe 2008; Ivey 2000). Furthermore, I revealed that increases in amount of time spent in cultivation and/or wage labour is positively associated with time spent with mothers, contra Draper and Cashdan's (1988) thesis. In fact, I would argue that cultivation close to camp means that mothers are never far from their offspring and are more able to take toddlers with them. Furthermore, with increased cultivation comes more food processing (primarily rice husking) which takes a significant amount of time and is conducted in camp. Finally, Kramer and Boone (2002) report that child economic activities may be increased in agricultural populations due to the changing nature of subsistence, such as harvesting, processing and caring for animals. However, recent evidence demonstrates that unlike earlier assumptions (Kaplan et al. 2000; Draper \& Cashdan 1988) hunter-gatherer children are particularly active food producers and significantly underwrite their own costs (Crittenden et al. 2013). As a result, in a follow-up analysis Kramer (2005) finds little consistency between children's economic roles and modes of subsistence. Accordingly, I find little support for this hypothesis in both the literature and my own data.

\subsubsection{The epidemiological downside}

The data indicates that sedentarisation in the Agta causes increased risk of infectious disease. Viral infections were higher in the larger, less mobile camps with increased cultivation. Camp size and population density (measured here through out-of-camp mobility) were significantly correlated with viral infections in my data. This follows 
expectations of transmission of viruses such as Caliciviruses and Rotoviruses, causing gastroenteritis and influenza (Van Blerkom 2003), both common among settled Agta and dependent on higher population densities (Norrby et al. 1992). This mirrors the finding of increased risk of high C-reactive protein concentrations (indicative of an inflammatory response) in children among more acculturated Tsimane foragerhorticulturalists (McDade et al. 2005). In particular, cultivation may independently predict increased viral load (indicated by the association between lymphocytosis and foraging) due to poorer nutritional quality of agricultural staples. Anthropological studies have found that !Kung foragers engaged in food production experienced more signs of malnutrition (Hitchcock \& Ebert 1984) as increased consumption of rice with cultivation entails a loss of dietary breadth and macro- and micro-nutritional quality (Hurrell 2003; Mcdonell \& Oxenham 2014) leading to malnutrition, which is a significant predictor of disease and mortality (WHO, 2012).

As with viral infections, Agta living in more permanent camps (as predicted by camp housing type) are also worse off in terms of helminthic infections, matching theoretical predictions (Barrett et al. 1998; Leles et al. 2010; Mascie-Taylor 1993; Reinhard \& Araujo 2012; Reinhard 1988). Contrary to this, some studies have argued the high helminth burden was a major selective pressure before the Neolithic (Cooper 2004; Hurtado et al. 2008; Rook 2009) due to extreme helminth rates in many semi-sedentarized, farmer-foragers (Froment 2014; Hurtado et al. 2008; Tanner et al. 2009). However, these groups are no longer fully mobile. Supporting my interpretation and findings, researchers observed a near absence of helminths in an isolated Amazonian group (London \& Hruschka 2014), Hadza children (Jelliffe 1962) and lower helminth loads among isolated and mobile Saluma and Yanomama groups (Confalonieri et al. 1991). While some parasitic species were hosted by our hominid ancestors (Barrett et al. 1998; Gonçalves et al. 2003; Sprent 1969), there is little archaeological evidence for an extreme intestinal parasite burden predating agriculture (Gonçalves et al. 2003; Hugot et al. 1999; Leles et al. 2010; Reinhard et al. 1985; Reinhard \& Araujo 2012; Santoro et al. 2003). For instance, when comparing coprolites (desiccated faeces) from one hunter-gatherer site and two agricultural villages in Utah Reinhard (1988) found an absence of parasites among the foragers while the farmers suffered from at least four species (pinworms, tapeworms, hookworm and threadworm (Strongyloides stercoralis)). Thus, it appeared that mobility, group size and agricultural practices were associated with increased parasitic loads, a finding repeated in parasitological analysis of coprolites from 
the Inca conquest of Northern Chile (Santoro et al. 2003). Consequentially, both increased viral and helminth burdens are likely costs associated with sedentarization.

It should be noted that settled Agta did not demonstrate an increased incidence of neutrophilia, which in this sample was often caused by TB. Chronic bacterial infections, such as TB, have evolved to survive at low population densities, thus likely predate agriculture (Armelagos \& Harper 2005; Sreevatsan et al. 1997). The lower incidence of neutrophilia in settled Agta camps is likely due to the limited access to medical care, which might also explain the finding that cash labour decreased helminth loads by $37.4 \%$. Since westernized medicine is a recent occurrence, the overall increased helminthic load in sedentarized camps may be underestimating the effect that settlement had on human populations during the Neolithic transition.

More permanent camps experienced significantly higher childhood mortality rates, matching archaeological evidence from the Neolithic (Cohen \& Crane-Kramer 2007). Given the exceptional high mortality rates reported for the Agta (Early \& Headland 1998), Ache (Hill \& Hurtado 1996), Hiwi (Hill et al. 2007) to name a few, hunter-gatherers were commonly seen to maintain steady population sizes by matching high fertility with high mortality (Larsen 2006; Foley \& Lahr 2001; Pennington 2001). However, there is sufficient theoretical and empirical evidence which suggests this is not the case (Kelly 2013; Hill \& Hurtado 1996; Hitchcock 1982), which has been further supported by this study. Given that consistent major causes of death across foraging populations include pneumonia, bronchitis and gastro-intestinal disorders (Kelly 2013) which increase with population size and sedentism, it is not surprising that the most settled Agta experienced significantly higher rates of childhood mortality. In some populations increased sedentism results in increased acculturation and access to medical resources to such a degree that mortality is decreased overall (Kelly 2013). For instance, among the Pumé (Kramer \& Greaves 2007) and Tsimane (Gurven et al. 2007) horticulturalist-foragers, more settled groups living closer to towns suffered lower mortality rates while facing higher prevalence of respiratory tract infections. Thus, in extant populations a key driver of decreased mortality is increased access to health care, as well as government intervention and better living standards due to infrastructure and hygiene (Kramer \& Greaves 2007). This is not the case for the Agta. From my experience, when medical intervention does occur it was haphazard and low quality. Furthermore, when governmental interventions are directed at them it is within 
the 'farming' paradigm, which attempts to settle the Agta close to town centres. But when this occurs the camps frequently lack sanitation or clean water supplies, or if present they are rarely functional. For instance, Minter (personal communication) reports that many toilets are incomplete as there are no holes underneath them. Thus, quiet simply the Agta are often encouraged to settle without any move made to improve their standards of living. Thus, morbidity and mortality rates increase significantly.

\subsubsection{Archaeological insights and human behavioural ecology}

Reverting back to the Neolithic transition once more, these results suggest, following Bocquet-Appel and Naji (2006), that one reason population growth did not explode during the Neolithic was because it was matched by high mortality. By examining the number of juvenile skeletons present in cemeteries it appears that farming was associated with two additional births per woman (Bocquet-Appel 2011). However, if this was the case then the population growth really would have experienced a 'revolution', rather than a steady increase apparent from the archaeological record. Recent studies using radiocarbon analysis of population growth in both North America and Europe are supportive of this hypothesis (Shennan et al. 2013; Zahid et al. 2015). Zahid and colleagues (2015) argue that population growth in Wyoming and Colorado is characterised by long-term growth punctuated by short-term fluctuations, matching boom-and-bust pattern reported in Europe (Shennan et al. 2013). Given the long-term growth, a sudden 'explosion' in fertility would require mortality to catch up very quickly to fit in with this trend (Zahid et al. 2015). As mortality is significantly harder to explore archaeologically (Zahid et al. 2015; Cohen 1989) this dynamic has gone comparatively unexplored, however the current research among extant foragers does suggest that such trends are possible.

A limitation of this study is its correlative nature, which leaves unanswered the question of the causal direction between fertility, sedentarization and food production. Since sedentarization correlates with food production in the Agta, with more sedentarized households abandoning foraging, it seems that these two process are interdependent, and thus are treated here as part of a process that involved a cluster of 'transition' traits. This is likely to be the case for the Neolithic transition as well, as many have reported the interplay between sedentarization and food production leading to the demographic changes associated in the origins of agriculture (Hayden 1995; Price \& Gebauer 1995). It may be that sedentism is a more important factor due to reductions in energy expenditure, falling 
into line with recent archaeological observation of increases in population growth prior to the Neolithic revolution. However, separating these processes requires in-depth observations about maternal energy expenditure in contrasting scenarios (i.e. settled, mobile, foraging and farming). By exploring the causal pathways, it will be easier to draw a distinction between these interdependent processes. Additionally, a more detailed analysis of dietary changes is also important to explore changes in nutritional condition as well as pathological state as the are greatly interlinked. Further limitations stem from using a crosssectional study design to explore longer-term trends, thus, it is unclear to what degree sedentism is reflective of mobility trends beyond our two-year sample period. However, a cross-sectional study design does permit the exploration of intra-population variability without requiring this variability to be recent or unilineal. Furthermore, foraging and mobility behaviors are likely altered during the heavy rainy and typhoon season not captured here as data collection occurred during the dry period. However, this does mean there is no systemic temporal bias in the data as camps were visited within the same season.

Finally, these results must be considered within the context of modernity and within one population. Thus, I can only reflect on past behavioural processes with current ones, but I believe my approach here reduces the error inherent in assuming one population of foragers are the 'true hunter-gatherers'. Therefore, there results demonstrate how we must be weary of using modern hunter-gatherers as direct mirrors into the Palaeolithic. The Agta, like many foraging groups live on the outskirts of societies with modern medical care and developmental directives; they are not isolated or untouched. Methodologically, this is why it is essential to examine patterning within populations (Dunn 1977; Stock \& Pinhasi 2011). Modern day foragers are culturally and social discriminated against, as their nonforaging neighbours see them as less than human. It is for this reason it is so important to examine how the degree of sedentarisation exactly impacts the Agta's health, especially as many developmental policies actively promote settlement. Nonetheless, I argue, following Kelly (2013) that while the Agta, like any hunter-gatherer, are not 'untouched' they still must make decisions based on a set of principles grounded in the fact they survive in a foraging niche, primarily without food storage or wealth. As a result it is possible to develop and test hypothesis rooted in human behavioural ecology to explore how adaptive behaviour, and in this context reproductive trade-offs, truly are. This allows the testing of hypothesis developed from the archaeological literature, which remains a useful avenue of research as long as one bears in mind that Agta are not living 10,000 years ago. 


\subsection{Conclusions}

In summary, hypotheses developed from archaeology imply that agriculture and sedentism has been associated with paradoxically both increases in fertility and increases in morbidity. However this paradox can be accounted for by applying a life history model which considers the optimisation of maternal investment in parental care and fertility according to prevailing conditions. Testing this model with data from the Agta provides evidence supporting the hypothesis that while the transition to agriculture in the Neolithic significantly depressed health, the overall fitness payoff was greater. Therefore, the proposed quality-quantity trade-off provides an adaptive mechanism that reconciles declines in health, increased mortality and demographic expansion following the spread of agriculture in the Neolithic. Finally, since high fertility rates were accompanied by relatively high mortality rates, the trade-off also suggests why population numbers did not explode during the Neolithic but instead increased relatively slowly (Bocquet - Appel \& Naji 2006). 


\section{Chapter Nine \\ Discussion and Conclusions}

This thesis furthers our understanding of cooperation and life history in an extant foraging group. I have presented innovative ways to explore allocare and the fitness consequences of social networks in small-scale societies. I have robustly examined the role of kin selection and reciprocity in determining allocare, highlighting the importance of cooperation as a form of risk mitigation and presented an original study focused on examining within population variance and how this influences life history trade-offs. By doing so I make a methodological point about the use of extant hunter-gatherer to make inferences about our past. Finally, I offer an adaptive mechanism by which to understand the apparent paradox between a Neolithic pattern of disease and demography by examining life history trade-offs. In this chapter I will briefly summarise the main findings from this research. Then I will offer some thoughts about the implications of my research for the wider literature on cooperative breeding and hunter-gather studies.

\subsection{Overview of findings}

In chapter five I demonstrated that fathers, siblings and non-kin are more significant allocarers than grandmothers by exploring who interacts with dependent children across a range of ages. This is in contradiction to Hawkes et al. (2000) hypothesis that the evolution of a prolonged post-menopause lifespan in humans is related to the beneficial role grandmothers play in cooperative breeding. However, this is supported by the majority of direct allocare studies among hunter-gatherers which find little investment by grandmothers (Kramer 2010; Kramer 2005; Crittenden \& Marlowe 2008; Goodman et al. 1985; Hill \& Hurtado 2009). Others have argued that grandmothers are a poor evolutionary strategy in foraging populations given their high mortality and flexible mobility (Hill \& Hurtado 2009). My data supports this statement. The role of allocarer is context dependent and likely reactive to age-specific mortality schedules. Grandmothers may have had a smaller role in populations with high mortality and early first births, and perhaps grow in importance with slower life history strategies. 
In chapter six I presented the first detailed hypothesis test of why individuals provide costly allocare in the Agta. Competing explanations of kin and reciprocity are rarely examined in a multivariate analysis within the human literature, thus my conclusions have important implications for understanding the evolution of communal breeding. The results are supportive of the influence of both kin selection and reciprocity in communal breeding. Following increasing evidence supportive of the role of reciprocity (Nolin 2010; AllenArave et al. 2008; Jaeggi \& Gurven 2013b; Kasper \& Borgerhoff Mulder 2015; Alvard 2009) my work demonstrates that distant kin and non-kin provide a significant amount of proximity care predicted by contingency, benefits and costs. This brings this analysis of cooperative breeding in line with the larger cooperative literature which systematically explores the roles of $B$ and $C$ rather than simply varying $r$ (Gurven 2004b; Hooper 2015; Kasper \& Borgerhoff Mulder 2015; Jaeggi \& Gurven 2013b). However, these dynamics are influenced by subsistence economy as increasing wealth and decreasing foraging emphasises the role of close kin over that of more distant and unrelated individuals. Recent research has focused on cooperation as forms of insurance against risk following needbased transfers (Aktipis et al. 2011; Hao et al. 2015). My work extends this by testing these hypotheses among the Agta, highlighting the usefulness of these perspectives.

Chapter seven demonstrated that communal breeding and maternal social networks have implications for fitness. Mothers with greater betweenness centrality had higher reproductive success and received more allocare from non-kin at the cost of poorer health for both the mothers and their offspring. Degree centrality on the other hand negatively influenced maternal reproductive success. Following literature from primatology and behavioural ecology, it appears then that the formation of social ties has implications for fitness revealing that being aware of 'who is friends with whom' is an important behavioural strategy (Murphy et al. n.d.; Gilby et al. 2013; Brent 2015; McFarland et al. 2015; Lehmann et al. 2015; Stanton \& Mann 2012). Chapter seven further revealed that cooperative breeding has an adaptive function as care from non-kin (who have been demonstrated to substitute maternal investment in chapter five) increases reproductive success. Contradictory to the majority of literature on human cooperative breeding it appears that Agta allocare is associated with increased fertility and decreased child quality. Future research should separate different types of kin since it appears that grandmothers, aunts and uncles and cousins may not always be cooperative. Following this, the beneficial effects of betweenness may lay in the linking of disparate parts of the network which from 
the risk buffering perspective may have important implications for ensuring they are in receipt for care from non-kin when they have a care shortage.

Chapter eight stayed on the topic of quality-quantity trade-offs but turned its perspective to the influence of subsistence change and mobility. Settlement and foraging increased fertility above and beyond combatant increases in disease and mortality as settled mothers experienced higher reproductive success than mobile mothers. Thus, as disease increases mothers may switch investment to fertility and given the increases in BMI with settled farming (due to either reductions in energy expenditure and/or increased energy intake) fertility increased even further, resulting in higher reproductive success. This application of life history trade-offs to the apparent paradox of population growth at a time of large increases in mortality and morbidity (Lambert 2009) offers important insights into archaeological questions about the Neolithic transition (Bocquet-Appel and Naji, 2006; Larsen, 2008; Zahid et al., 2015).

\subsection{Implications for cooperation, risk and hunter-gatherers}

\subsubsection{Moving beyond kin and their selection}

The exploration of communal breeding in humans has been defined by the role of kin. Thus, anthropological and demographic literature has been particularly good at revealing the adaptive function of allocare between kin (Voland et al. 2005; Sear \& Mace 2009; Sear \& Mace 2008; Kramer 2005). Thus, rather than demonstrating that humans are cooperative breeders as cooperation subsides our rapid reproduction of costly infants (Mace \& Sear 2005), the literature, by-and-large demonstrates that humans cooperatively breed with kin (Sear \& Mace 2008; Snopkowski \& Sear 2013; Tymicki 2004), which are theoretically speaking, two different things. Understanding humans to be communal, or plural breeders, opens up the door to cooperation not driven by relatedness but based on direct benefits. Without suppression of ovulation, mothers breed together and they (and their children) are able to assist based on reciprocity, allowing for unrelated individuals to cooperate within the inclusive fitness paradigm. Hunter-gatherers consistently reside in camps with a higher proportion of non-kin than kin (Dyble et al. 2015; Hill et al. 2011), and thus are less related to their group than other small-scale societies who do not practice foraging (Walker 2014). Therefore, given the fluidity of camp composition as individuals and households regularly move between groups (Kelly 2013), the most reliable and consistent 'group' of co- 
operators outside the household is likely unrelated. As a result, co-residence with non-kin in fluid groups produces an environment in which humans are hyper-cooperative with individuals they do not receive indirect fitness benefits from (Burkart et al. 2014). Here, I have argued that this may be advantageous in evolutionary terms due to an increased ability to buffer risks in the environment when a population is mobile and lacks substantial wealth; a wide and dispersed social network produces multiple allocare options which are necessarily limited among kin. From this unique perspective I have produced analyses that highlight the importance of non-kin in subsidising maternal fertility by providing a significant amount of allocare to dependent children.

The primacy of kin in the literature has resulted in a void of research on allocare from nonkin and how allocare is maintained among 'selfish' individuals. I know of no other study which examines the question of communal breeding in this manner. However, in contrast the literature on food sharing and wider cooperation in small-scale societies is brimming with examples of robust, multivariate analysis of competing hypothesis exploring cooperation between both kin and non-kin (Jaeggi \& Gurven 2013b; Kasper \& Borgerhoff Mulder 2015; Alvard 2009; Nolin 2010). In particular, recent analysis has sought to break down the data and separate the confounding effects associated with costs and benefits (Hooper 2015), relatedness and proximity (Alvard 2009; Nolin 2010), reciprocity between kin and non-kin (Allen-Arave et al. 2008; Kasper \& Borgerhoff Mulder 2015), and by doing so greatly furthers our understanding of the complex exchanges between individuals. By following the theoretical path set by the cooperative literature at large it is possible to understand the ultimate function of communal breeding and how it varies according to the individual and context. Previously, sample sizes and age controls have been poor in huntergatherer studies (Mace \& Sear 2005), limiting the ability to conduct such explorations. However, hunter-gatherer studies have the benefit of often capturing the totality of interactions; communal breeding is the product of provisioning and food production, domestic help and direct care. Larger scale demographic studies struggle to capture these pathways which confound relationships. Consequentially, future work should focus on bringing these perspectives together, increasing the sample sizes of hunter-gatherer studies and explaining the totality of cooperative interactions. This approach might provide a better framework to under the diversity of results often apparent in the literature. 


\subsubsection{Relational wealth and its relation with risk}

A second theme throughout this thesis surrounds the concepts of risk, its mitigation and the role of wealth. Borgerhoff Mulder and colleagues (Borgerhoff Mulder \& Beheim 2011; Borgerhoff Mulder et al. 2009) developed a framework of expanding 'wealth' beyond the confines of maternal objects, into embodied or relational wealth. It is this relational wealth that I have discussed throughout this thesis; "the social ties on which an individual can draw, ties that derive from social position, trust, reputation, kinship and symbolic goods"(Borgerhoff Mulder \& Beheim 2011: 345). Thinking about wealth in this manner offers, I believe, informative insights into how small-scale societies use behavioural strategies to adapt in any given environment. Given that this framework highlights the comparable roles of 'wealth in people' with 'wealth in things' it has direct relevance to hunter-gatherer research.

Traditionally hunter-gatherer research has often attributed behavioural strategies to their residence in risky, unpredictable environments without wealth, thus their use of social insurance via cooperation was understood to mitigate unforeseen losses (Kelly 2013). Certainly Winterhalder (1986) original model is robust and demonstrates that even among six to eight hunters, if returns are asynchronised, variability in food returns is effectively dealt with by food pooling. Thus, theoretically relational wealth appears to play an important role, leading Kaplan et al. (2009) to argue that risk-reduction strategies have major implications for the evolution of human social organisation. Due to large and variable package sizes being the 'basis' of foraging subsistence, cooperation produces reliable ties between households and non-kin, extensively broadening our social networks. I do not disagree with this theory, and believe that risk is an informative way of examining human cooperation. However, this theory is rarely tested, rather simply applied as an interpretation of results (as I have done in chapter 7). For instance, many studies imply that the ultimate why behind humans extensive cooperation, social capital, food sharing is due to survival in risky foraging niche (Jaeggi \& Gurven 2013a; Salali \& Migliano 2015; Chaudhary et al. 2015; Gurven \& Hill 2009). This is not problematic ipso facto; however not all foraging environments are the same. Kelly (2013) warns about using 'hunting-gathering' as an explanatory variable, stating we should explore variability within and between populations to test these relationships. It is evident that there is significant variability in hunter-gatherer foraging returns, ranging from the Hadza who acquired meat on less than 3\% of hunting trips (Hawkes 1991), to the Ache who faced a 60\% failure rate (Hill et al. 1987) and as high 
as Agta fishing trips which were successful on $89 \%$ of trips. Surely then, such variability and range in package sizes (from the Hadza big game, Ache smaller jungle creatures to the small river fish consumed by the Agta) would be predicted to result in variability in cooperative strategies.

Consequently, future work should explore the relationship between cooperative behaviours, variability in returns and different types of risk environments, including disease risk (Sugiyama \& Chacon 2000) and fitness outcomes. This would go further in exploring the adaptive value of cooperation. For instance, here I have argued that communal breeding and a high betweenness centrality is adaptive as it increases fitness. I speculate that this is due to betweenness allowing individuals to link to disparate parts of the network ensuring that allocare remains constant in an environment when unpredictable shortfalls due to illness or foraging failure mean that childcare cannot be guaranteed within one 'exposure' unit. Care from non-kin is important as it can meet childcare demands when other kin cannot, particularly when there is little material wealth to buffer a household from risk. Howell (2010), in her work on the life history of the Dobe !Kung comes to a similar conclusion that a wide network of providers act as insurance, rather than simply 'kin as wealth'. However, these statements need further testing. For instance, future research should explore how the importance of 'betweenness' and non-kin allocare varies according to different measures of risk in the environment. Furthermore, I demonstrated that relational wealth holds the predicted relationship with material wealth, suggesting that different forms of risk mitigation, such as material accumulation and subsistence diversification, may mitigate the association between risk and cooperation. Therefore, do global properties of social networks change with ecology, risk and subsistence patterns? It is questions like these which will further our understanding of how groups behavioural strategies adapt to different environmental pressures.

\subsection{Hunter-gatherers and human evolution}

"Long before anthropologists arrived on the scene bunter-gatherers had already been given disease, shot at, traded with, employed and exploited by colonial powers or agricultural neighbors" (Kelly 2013: 16) 
It would be remiss of me not to discuss the role of hunter-gatherer studies in understanding human behavioural evolution. It is a contentious issue and the arguments for and against have been well rehearsed (O'Connell (1995) says the same and the debate remains by-andlarge unchanged). However, in conclusion to this thesis I would like to offer my own interpretation of the issue, particularly due to the relevance to chapter 8 . We have, of course, moved a long way from early anthropological thought, which saw present day foragers as analogous to our Palaeolithic ancestors (Wilson 1978; Kelly 2013). Predominantly, the study of human behavioural ecology has focused on the variability of foraging groups alive today, indicating that it is not possible to define a 'pure' or 'model' hunter-gatherer group. Certainty I would not attempt to do so for the Agta, but not because I believe they are any more, or any less of a 'foraging group' than any other population. Rather I argue that this line of argument leads to erroneous perspectives as it implies that hunter-gatherers were static before 150 - 200 years ago, so if we "carefully...account for the effects of contact on their way of life" (Lee 1979: 2) we can get at the 'reality' of foraging. Foragers have not lived in a world of only foragers since 13,000 - 6,000 (depending of course on geographic location (Diamond \& Bellwood 2003)), and the archaeological, linguistic and genetic record is rife with evidence of significant amounts of interaction, trade, and stable periods of low-level food production (Smith 2001; Price \& Gebauer 1995; Piperno \& Pearsall 1998; Inomata et al. 2015; Bar-Yosef \& Meadow 1995; Bollongino et al. 2013). As a result it is unclear why or how one could 'remove' these influences which necessarily have shaped foragers behavioural strategies since their occurrence.

Consequentially, I find Marlowe's (2005: 54) justification of the Hadza being 'model huntergatherers" as they have changed little in the last century since "all we can do is perhaps give extra weight to those with less contact" problematic. What about the centuries or millennia before this? Arguments by both Foley (1988) and Kuhn and Stiner (2001) point out the diversity in hominid and human evolution, respectively, indicating that saying humans spent 99\% of their history as hunter-gatherers lacks meaning given there is no one single time prior to the Neolithic revolution 10,000 - 13,000 years ago. Given that the Palaeolithic is marked by significant climatic changes, such as several glacial maxima, it is not surprising the archaeological record is one of considerable variability (Kuhn \& Stiner 2001). Thus, what we consider to be the 'hunter-gatherer package' has not consistently been present, but is the product of adaptation to various selective pressures (Foley 1988; Kelly 2013). 
Consequently, even if we can infer that hunter-gatherers today are 'pre-Neolithic', then we are only representing "just a few late chapters in a long and complicated evolutionary story that began more than 2.5 million years ago" (Kuhn \& Stiner 2001: 128). So where does that leave us - what meaning can we infer from extant hunter-gatherer studies about human and hominid prehistory and evolution?

Broadly speaking I am in agreement with the theoretical frameworks put forward by Kelly (2013), O’Connell (1995), Winterhalder and Kennett (2006) and Kuhn and Stiner (2001), which I have applied in chapter 8. Through the lens of human behavioural ecology we can understand behavioural strategies as optimising processes dependent on environmental, historical and social constraints (Kappeler et al. 2013). Thus, with the emphasis on individual decision-making and optimisation it is possible to develop and test predictions following inferences from archaeology. Rather than argue that one type of forager is 'ancient' or 'pure' we should be exploring how hunter-gatherers react to selective pressures, which may reveal parallels from prehistory. Thus, understanding individual decision-making following evolutionary principles adds new pathways of investigation to the study of archaeological periods (Winterhalder \& Kennett 2006). Such approaches lie at the heart of behavioural ecology and should not be dismissed from understanding human evolution. As previously argued (Kelly 2013; Blurton Jones et al. 2002) hunter-gatherers today still must make allocation trade-offs based on their mode of subsistence, degree of mobility and social structures. Examining how this occurs and how this varies according to key variables provides a theoretical structure to exploring the behavioural ecology of human evolution (Kuhn \& Stiner 2001). Therefore, I argue that it is by examining variability within a population, according to predictions from archaeology, that it is possible to reveal the roles of decision-making and optimisation, allowing human behaviour ecology to create a framework to understand human evolution. Under this framework, inferences about human behaviour from an evolutionary perspective are by no way limited to hunter-gatherers; in complete agreement with Nettle et al. (2013) if human behavioural ecology is how behaviour evolves in response to ecological conditions, then all societies tell us something about behavioural evolution. 


\section{References}

Agarwal, R. \& Heinz, T., 1992. Bedside hemoglobinometry in hemodialysis patients: lessons from point-ofcare testing. American Society for Artificial Internal Organs, 47(3), pp.240-3.

Aiello, L. \& Key, C., 2002. Energetic consequences of being a Homo erectus female. American Journal of Human Biology, 14(5), pp.551-565.

Aiello, L. \& Wheeler, P., 1995. The expensive tissue hypothesis: the brain and the digestive system in human and primate evolution. Current Anthropology, 36, pp.199 - 221.

Aimé, C. et al., 2013. Human genetic data reveal contrasting demographic patterns between sedentary and nomadic populations that predate the emergence of farming. Molecular biology and evolution, 30(12), pp.2629-44.

Akhtar, K. et al., 2008. HemoCue photometer: a better alternative of hemoglobin estimation in blood donors? Indian Journal of Hematology and Blood Transfusion, pp.170-172.

Aktipis, C.A., Cronk, L. \& de Aguiar, R., 2011. Risk-Pooling and Herd Survival: An Agent-Based Model of a Maasai Gift-Giving System. Human Ecology, 39(2), pp.131-140.

Alam, N., 1995. Birth Spacing and Infant and Early Childhood Mortality in a High Fertility Area of Bangladesh: Age-Dependent and Interactive Effects. Journal of Biosocial Science, 27(4), pp.393-404.

Allal, N. et al., 2004. An evolutionary model of stature, age at first birth and reproductive success in Gambian women. Proceedings. Biological sciences / The Royal Society, 271(1538), pp.465-470.

Allen, S.M., 1985. The Rain Forest of Northeast Luzon and Agta Foragers. In P. B. Griffin \& a EstiokoGriffin, eds. The Agta of Northeastern Luzon: Recent Studies. Cebu City: San Carlos Publications, pp. 45-68.

Allen-Arave, W., Gurven, M. \& Hill, K., 2008. Reciprocal altruism, rather than kin selection, maintains nepotistic food transfers on an Ache reservation. Evolution and Human Behavior, 29(5), pp.305-318.

Alvard, M., 2009. Kinship and Cooperation: The axe fight revisited. Human Nature, 20(4), pp.394-416.

Alvarez, H.P., 2000. Grandmother hypothesis and primate life histories. American Journal of Physical Anthropology, 113(3), pp.435-450.

Apicella, C.L. et al., 2012. Social networks and cooperation in hunter-gatherers. Nature, 481(7382), pp.497501.

Apicella, C.L. \& Crittenden, A.N., 2013. Hunter-Gatherer Families and Parenting. The Handbook of Evolutionary Psychology, IV(23), pp.1-20.

Aplin, L.M. et al., 2012. Social networks predict patch discovery in a wild population of songbirds. Proceedings of the Royal Society B: Biological Sciences, 279(1745), pp.4199-4205.

Archie, E.A. et al., 2014. Social affiliation matters: both same-sex and opposite-sex relationships predict survival in wild female baboons. Proceedings. Biological sciences / The Royal Society, 281(1793), p.20141261-.

Armelagos, G.J. et al., 2009. Enamel hypoplasia and early mortality: Bioarcheological support for the Barker hypothesis. Evolutionary Anthropology, 18(6), pp.261-271.

Armelagos, G.J., Brown, P.J. \& Turner, B., 2005. Evolutionary, historical and political economic perspectives on health and disease. Social Science and Medicine, 61(4 SPEC. ISS.), pp.755-765.

Armelagos, G.J. \& Harper, K.N., 2005. Genomics at the Origins of Agriculture. Evolutionary Anthropology, 14, pp.109-121. 
van Assendelft, O.W., 2002. Calibration, control of hematology analyzers. Adv Administrators Lab, 22, pp.4347.

Axelrod, R. \& Hamilton, W.D., 1981. The Evolution of Cooperation. Science, 211(4489), pp.1390-1396.

Baden, A.L. et al., 2013. Communal nesting, kinship, and maternal success in a social primate. Behavioral Ecology and Sociobiology, 67, pp.1939-1950.

Baker, A.J. \& Wood, F., 1992. Reproduction of the emperor tamarin (Saguinus imperator) in captivity, with comparison to the cotton top and golden lion tamarins. American Journal of Primatology, 26, pp.1-10.

Baker, D., 1991. Evolution of the social system of the golden lion tamarin (Leontopithecus rosalia). University of Maryland.

Bales, K. et al., 2000. Effects of allocare-givers on fitness of infants and parents in callitrichid primates. Folia primatologica; international journal of primatology, 71(1-2), pp.27-38.

Bandy, M.S., 2005. New World settlement evidence for a two-stage Neolithic Demographic Transition. Current Anthropology, 46(S), pp.S109-S115.

Barclay, P. \& Reeve, H.K., 2012. The varying relationship between helping and individual quality. Behavioral Ecology, 23(4), pp.693-698.

Barrat, A. \& Cattuto, C., Temporal networks of face-to-face human interactions, pp.1-28. Available at: http://arxiv.org/pdf/1305.3532v1.pdf.

Barrett, L., Henzi, S.P. \& Lusseau, D., 2012. Taking sociality seriously: the structure of multi-dimensional social networks as a source of information for individuals. Philosophical Transactions of the Royal Society B: Biological Sciences, 367(1599), pp.2108-2118.

Barrett, L. \& Stulp, G., 2013. Four more reasons why ethology matters: Tinbergen and the human sciences. Human ethology bulletin, 4(4), pp.39-49.

Barrett, R. et al., 1998. Emerging and re-emerging infectious diseases: The Third Epidemiologic Transition. Annual Review of Anthropology, 27(1), pp.247-271.

Bar-Yosef, O. \& Meadow, R.H., 1995. The origins of argiculture in the near east. In D. Price \& A. B. Gebauer, eds. Last Hunters-First Farmers. Santa Fe: School of American Research Press, pp. 39-94.

Bates, D. et al., 2013. lme4 R Package. Available at: http://lme4.r-forge.r-project.org/.

Beck, N., 2008. Diagnostic Hematology, London: Springer.

Beise, J., 2005. The helping grandmother and the helpful grandmother: The role of maternal and paternal grandmothers in child mortality in the 17th and 18th century population of French settlers in Quebec, Canada. In E. Voland, A. Chasiotis, \& W. Schiefenhovel, eds. Grandmotherhood: The evolutionary significance of the second half of the female life. New Brunswick: Rutgers University Press, pp. 215-238.

Beise, J. \& Voland, E., 2002. A multilevel event history analysis of the effects of grandmothers on child mortality in a historical German population,

Bell, B. et al., 2010. The impact of small cluster size on multilevel models: a Monte Carlo examination of twolevel models with binary and continuous predictors. JSM Proceedings, Section on Survey Research Methods, pp.4057-4067.

Bellwood, P., 2010. Archaeology of Southeast Asian hunters and gatherers. In R. B. Lee \& R. Daly, eds. The Cambridge Encyclopedia of Hunters and Gatherers. Cambridge: Cambridge University Press, pp. 284-288.

Bellwood, P. \& Oxenham, M., 2008. The expansions of farming societies and the role of the Neolithic Demographic Transition. In J. Bocquet-Appel \& O. Bar-Yosef, eds. The Neolithic Demographic Transition and its Consequences. London: Springer, pp. 13-34.

Bentley, G.R., Goldberg, T. \& Jasieńska, G., 1993. The Fertility of Agricultural and Non-Agricultural Traditional Societies. Population Studies, 47(2), pp.269-281. 
Bentley, G.R., Harrigan, a M. \& Ellison, P.T., 1998. Dietary composition and ovarian function among Lese horticulturalist women of the Ituri Forest, Democratic Republic of Congo. European journal of clinical nutrition, 52(4), pp.261-70.

Bentley, G.R., Jasienska, G. \& Goldberg, T., 1993. Is the Fertility Higher Than Agriculturalists That of Nonagriculturalists ?'. Current Anthropology, 34(5), pp.778-785.

Bentley, S., Johnson, A. \& Bishop, C., 1993. A parallel evaluation of four automated hematology analyzers. American Journal of Clinical Pathology, 100, pp.626-632.

Bereczkei, T., 1998. Kinship Network, Direct Childcare, and Fertility Among Hungarians and Gypsies. Evolution and Human Behavior, 19, pp.283-298.

Bereczkei, T. \& Dunbar, R.I.M., 2002. Helping at the Nest and Sex Biased Parental Investment in a Hungarian Gypsy Population. Current Anthropology, 43, pp.804-809.

Bethony, J. et al., 2006. Soil-transmitted helminth infections: ascariasis, trichuriasis, and hookworm. Lancet, 367(9521), pp.1521-1532.

Bhaskaram, P. et al., 2003. Validation of hemoglobin estimation using Hemocue. Indian journal of pediatrics, 70(1), pp.25-8.

Bion, G.P. \& B.Griffin, M., 1997. Agta Foragers: Alternative histories, and cultural autonomy in... Australian Journal of Anthropology, 8, p.259.

Van Blerkom, L.M., 2003. Role of Viruses in Human Evolution. American Journal of Physical Anthropology, 122(SUPPL. 46), pp.14-46.

Blurton Jones, N., 1987. Bushman birth spacing: Direct tests of some simple predictions. Ethology and Sociobiology, 8(3), pp.183-203.

Blurton Jones, N.G. et al., 1992. Demography of the Hadza, an increasing and high density population of Savanna foragers. American journal of physical anthropology, 89(2), pp.159-181.

Blurton Jones, N.G. et al., 2000. Paternal Investment and Hunter-Gatherer Divorce Rate. In L. Cronk, N. A. Chagnon, \& W. Irons, eds. Adaptation and Human Behavior. New York: Aldine de Gruyter.

Blurton Jones, N.G., Hawkes, K. \& O’Connell, J.F., 2002. Antiquity of postreproductive life: Are there modern impacts on hunter-gatherer postreproductive life spans? American Journal of Human Biology, 14(2), pp.184-205.

Bocquet-Appel, J. \& Naji, S., 2006. Testing the Hypothesis of a Worldwide Neolithic Demographic Transition: Corroboration from American Cemeteries. Current Anthropology, 47(2), pp.341-365.

Bocquet-Appel, J.P., 2008. Explaining the Neolithic Demographic Transition. In J. Bocquet-Appel \& O. BarYosef, eds. The Neolithic Demographic Transition and its Consequences. London: Springer, pp. 35-56.

Bocquet-Appel, J.-P., 2011. When the world's population took off: the springboard of the Neolithic Demographic Transition. Science (New York, N.Y.), 333(6042), pp.560-561.

Bogin, B., 1997. Evolutionary Hypotheses for Human Childhood. Yearbook of Physical Anthropology, 40: .63-89.

Bohler, E. \& Bergstrom, S., 1995. Subsequent pregnancy affects morbidity of previous child. Journal of Biosocial Science, 27(4), pp.431-442.

Bollongino, R. et al., 2013. Stone Age Central Europe. Science, 342(October), pp.479-481.

Borgerhoff Mulder, M., 1998. Brothers and Sisters: How Sibling Interactions Affect Optimal Parental Allocations. Human Nature, 9(2), pp.119-162.

Borgerhoff Mulder, M., 2007. Hamilton's rule and kin competition: the Kipsigis case. Evolution and Human Behavior, 28(5), pp.299-312.

Borgerhoff Mulder, M. et al., 2009. Intergenerational Wealth Transmission and the Dynamics of Inequality in 
small-scale societies. Science, 326(5953), pp.682-688.

Borgerhoff Mulder, M., 1989. Menarche, menopause and reproduction in the Kipsigis of Kenya. Journal of biosocial science, 21(2), pp.179-192.

Borgerhoff Mulder, M., 2000. Optimizing Offspring: The Quantity-Quality Trade-off in Agropastoral Kipsigis. Evolution and Human Behaviour, 21(6), pp.391-410.

Borgerhoff Mulder, M. et al., 2011. Understanding the nature of wealth and its effects on human fitness. Philosophical transactions of the Royal Society of London. Series B, Biological sciences, 366(1563), pp.344-356.

Borgerhoff Mulder, M. \& Beheim, B. a, 2011. Understanding the nature of wealth and its effects on human fitness. Philosophical transactions of the Royal Society of London. Series B, Biological sciences, 366, pp.344-356.

Borgerhoff Mulder, M. \& Caro, T.M., 1985. The use of quantitative observational techniques in anthropology. Current Anthropology, 26, pp.323-335.

Bowles, S., 2011. Cultivation of cereals by the first farmers was not more productive than foraging. Proceedings of the National Academy of Sciences of the United States of America, 108, pp.4760-4765.

Brandes, U. et al., 2007. On Modularity Clustering. Knowledge and Data Engineering, IEEE Transactions on, 20(2), pp.172-188.

Brent, L.J.N. et al., 2015. Ecological knowledge, leadership, and the evolution of menopause in killer whales. Current Biology, 25(6), pp.746-750.

Brent, L.J.N., 2015. Friends of friends: are indirect connections in social networks important to animal behaviour? Animal Behaviour, 103, pp.211-222.

Brent, L.J.N. et al., 2013. Genetic origins of social networks in rhesus macaques. Scientific reports, 3, p.1042.

Brent, L.J.N., Lehmann, J. \& Ramos-Fernández, G., 2011. Social network analysis in the study of nonhuman primates: A historical perspective. American Journal of Primatology, 73(8), pp.720-730.

Briggs, C., Kimber, S. \& Green, L., 2012. Where are we at with point- of- care testing in haematology? British Journal of Haematology, 158(6), pp.679-690.

Broek, I. et al., 2013. Clinical Guidelines Diagnosis and Treatment Manual, Medecins San Frontiere.

Brown, J.L., 2014. Helping Communal Breeding in Birds: Ecology and Evolution, London: Princeton University Press.

Burkart, J.M. et al., 2014. The evolutionary origin of human hyper-cooperation. Nature communications, 5, p.4747.

Burkart, J.M., Hrdy, S.B. \& Van Schaik, C.P., 2009. Cooperative breeding and human cognitive evolution. Evolutionary Anthropology, 18(5), pp.175-186.

Burnham, K.P. \& Anderson, D.R., 2004. Multimodel Inference: Understanding AIC and BIC in Model Selection. Sociological Methods \& Research, 33, pp.261-304.

Cain, M.T., 1977. The economic activities of children in a village in Bangladesh. Population and Development Review, 3(3), pp.201-227.

Cameron, E.Z., Setsaas, T.H. \& Linklater, W.L., 2009. Social bonds between unrelated females increase reproductive success in feral horses. Proceedings of the National Academy of Sciences of the United States of America, 106(33), pp.13850-13853.

Cameron, J.L., 1997. Stress and behaviorally induced reproductive dysfunction in primates. In Seminars in Reproductive Endocrinology. pp. 37-45.

Campbell, C. \& Lee, J.Z., 2002. When husbands and parents die: widowhood and orphanhood in late Imperial Liaoning, 1789-1909. In When Dad Died: Individuals and Families Coping with Distress in Past Societies. pp. 301-322. 
Campbell, K.L. \& Wood, J.W., 1988. Fertility in traditional societies. In P. Diggory, S. Teper, \& M. Potts, eds. Natural Human Fertility. London: Macmillan Publishing, pp. 39-69.

Caraco, T. \& Brown, J.L., 1986. A game between communal breeders: When is food-sharing stable? Journal of Theoretical Biology, 118(4), pp.379-393.

Cashdan, E. a, 1985. Coping with Risk: Reciprocity Among the Basarwa of Northern Botswana. Man, 20(3), pp. $454-474$.

Cassidy, C.M., 1980. Benign neglect and toddler malnutrition. In Social and Biological Predictors of Nutritional Status, Physical Growth and Neurological Development. pp. 109-139.

Cattuto, C. et al., 2010. Dynamics of person-to-person interactions from distributed RFID sensor networks. PLOS ONE, 5(7), pp.1-9.

Caudell, M., Rotolo, T. \& Grima, M., 2015. Informal lending networks in rural Ethiopia. Social Networks, 40, pp.34-42.

Chagnon, N.A., 1974. Studying the Yanomamö, New York: Holt, Rinehard and Winston.

Charnov, E., 1993. Life history invariants: some explorations of symmetry in evolutionary ecology, New York City: Oxford University Press.

Charnov, E. \& Berrigan, D., 1993. Why do female primates have such long lifespans and so few babies? or Life in the slow lane. Evolutionary Anthropology, 1, pp.191-194.

Chaudhary, N. et al., 2015. Polygyny without wealth : popularity in gift games predicts polygyny in BaYaka Pygmies. Royal Society Open Science, 2.

Clarke, P. \& Wheaton, B., 2007. Addressing Data Sparseness in Contextual Population Research: Using Cluster Analysis to Create Synthetic Neighborhoods. Sociological Methods \& Research, 35(3), pp.311-351.

Clutton-Brock, T., 2002. Breeding together: kin selection and mutualism in cooperative vertebrates. Science, 296(2002), pp.69-72.

Clutton-Brock, T., 2009. Cooperation between non-kin in animal societies. Nature, 462(7269), pp.51-57.

Clutton-Brock, T.H., Brotherton, P.N.M., et al., 2001. Contributions to cooperative rearing in meerkats. Animal Behaviour, 61(4), pp.705-710.

Clutton-Brock, T.H., Russell, a F., et al., 2001. Effects of helpers on juvenile development and survival in meerkats. Science, 293(2001), pp.2446-2449.

Clutton-Brock, T.H. et al., 2000. Individual contributions to babysitting in a cooperative mongoose, Suricata suricatta. Proceedings. Biological sciences / The Royal Society, 267(1440), pp.301-305.

Clutton-Brock, T.H., Russell, A.F. \& Sharpe, L.L., 2004. Behavioural tactics of breeders in cooperative meerkats. Animal Behaviour, 68(5), pp.1029-1040.

Cockburn, T.A., 1977. Infectious Diseases in Ancient Populations. In D. Landy, ed. Culture, Disease and Healing. Studies in Medical Anthropology. New York: Macmillan Publishing, pp. 83-96.

Cohen, M.L., 2000. Changing patterns of infectious disease. Nature, 406(6797), pp.762-767.

Cohen, M.N., 1989. Health and the rise of civilisation, London: Yale University Press.

Cohen, M.N., 2009. Introduction: Rethinking the Origins of Agriculture. Current Anthropology, 50(5), pp.591595.

Cohen, M.N. \& Armelagos, G.J., 1984. Paleopathology at the Origins of Agriculture, Gainesville: Academic Press.

Cohen, M.N. \& Crane-Kramer, G.M.M., 2007. Ancient Health: Skeletal indicators of agricultural and economic intensification, Gainesville: University Press of Florida. 
Cohen, S. et al., 1997. Chronic social stress, social status, and susceptibility to upper respiratory infections in nonhuman primates. Psychosomatic medicine, 59(3), pp.213-221.

Coleman-Jensen, A., Nord, M. \& Singh, A., 2014. Household food security in the United States in 2013, ERR-173, Available at: http://162.79.45.209/media/1183208/err-155.pdf.

Confalonieri, U., Ferreira, L.F. \& Araujo, A., 1991. Intestinal Helminths in Lowland South American Indians: Some Evolutionary Interpretations. Human Biology, 63(6), pp.863-873.

Cooper, P.J., 2004. Intestinal worms and human allergy. Parasite Immunology, 26(11-12), pp.455-467.

Cordain, L., 1999. Cereal grains: Humanity s double-edged sword. World Rev Nutr Diet., 84, pp.19-73.

Corner, L.A.., Pfeiffer, D.. \& Morris, R.., 2003. Social-network analysis of Mycobacterium bovis transmission among captive brushtail possums (Trichosurus vulpecula). Preventive Veterinary Medicine, 59(3), pp.147_ 167.

Coster, A., 2012. Pedigree 1.4.

Crittenden, A.N., 2009. Allomaternal care and juvenile foraging among the Hadra: Implications for the evolution of cooperative breeding in humans. University of California San Diego.

Crittenden, A.N. et al., 2013. Juvenile foraging among the Hadza: Implications for human life history. Evolution and Human Behavior, 34(4), pp.299-304.

Crittenden, A.N. \& Marlowe, F.W., 2008. Allomaternal care among the Hadza of Tanzania. Human Nature, 19(July), pp.249-262.

Crittenden, A.N. \& Zes, D.A., 2015. Food Sharing among Hadza Hunter-Gatherer Children. PloS one, 10(7), p.e0131996.

Crockford, C. et al., 2008. Social stressors and coping mechanisms in wild female baboons (Papio hamadryas ursinus). Hormones and Behavior, 53(1), pp.254-265.

Crognier, E., Baali, A. \& Hilali, M.K., 2001. Do “Helpers at the nest” increase their parents' reproductive success? American Journal of Human Biology, 13(3), pp.365-373.

Crognier, E., Villena, M. \& Vargas, E., 2002. Helping patterns and reproductive success in Aymara communities. American Journal of Human Biology, 14(3), pp.372-379.

Csardi, G., 2015. igraph 1.0.1.

Danford, M.E. et al., 2007. Health and the transition to Horticulture in the south-central United States. In M. N. Cohen \& G. M. M. Crane-Kramer, eds. Ancient Health: Skeletal indicators of agricultural and economic intensification. Gainesville: University Press of Florida, pp. 65-79.

Danielsbacka, M. et al., 2011. Grandparental child care in Europe: Evidence for preferential investment in more certain kin. Evolutionary Psychology, 9(1), pp.3-24.

Darwin, C., 1859. On the Origin of the Species, London: Random House.

Darwin, C., 1871. The Descent of Man, London: Prometheus Books.

Davies, N.B., Krebs, J.R. \& West, S., 2012. An Introduction to Behavioural Ecology 4th ed., London: WileyBlackwell.

Dawkins, R., 1989. The Selfish Gene, Oxford: Oxford University Press.

Delfin, F. et al., 2011. The Y-chromosome landscape of the Philippines: extensive heterogeneity and varying genetic affinities of Negrito and non-Negrito groups. European journal of human genetics: EJHG, 19(July 2010), pp.224-230.

Denham, W., 1974. Infant Transport among the Alyawara Tribe. Oceania, 44(4), pp.253-277. 
Desai, S., 1995. When Are Children from Large Families Disadvantaged? Evidence from Cross-National Analyses. Population Studies, 49(2), pp.195-210.

Diamond, J., 2002. Evolution, consequences and future of plant and animal domestication. Nature, 418(6898), pp.700-7.

Diamond, J., 2014. Human melting pots in southeast Asia. Nature, pp.10-11.

Diamond, J. \& Bellwood, P., 2003. Farmers and their languages: the first expansions. Science (New York, N.Y.), 300(5619), pp.597-603.

Dickinson, J.L. \& Hatchwell, B.J., 2004. Fitness consequences of helping. In W. D. Koenig \& J. L. Dickinson, eds. Ecology and Evolution of Cooperative Breeding in Birds. Cambridge: Cambridge University Press.

Douglas, M.T., 2006. Subsistence change and dental health in the people of Non Nok Tha, northeast Thailand. In M. F. Oxenham \& N. Tayles, eds. Bioarchaeology of Southeast Asia. Cambridge: Cambridge University Press, pp. 191-219.

Dounias, E. \& Froment, a., 2011. From foraging to farming among present-day forest hunter-gatherers: consequences on diet and health. International Forestry Review, 13(3), pp.294-304.

Dounias, E. \& Froment, A., 2006. When forest-based hunter-gatherers become sedentary: Consequences for diet and health. Unasylva, 57, pp.26-33.

Downey, S.S. et al., 2014. The Neolithic Demographic Transition in Europe: Correlation with Juvenility Index Supports Interpretation of the Summed Calibrated Radiocarbon Date Probability Distribution (SCDPD) as a Valid Demographic Proxy. PloS one, 9(8), p.e105730.

Draper, P. \& Cashdan, E., 1988. Technological change and child behavior among the! Kung. Ethnology, 27(4), pp.339-365.

Dunbar, R.I.M., 2003. The Social Brain: Mind, Language, and Society in Evolutionary Perspective. Annual Review of Anthropology, 32.

Dunn, F.L., 1977. Health and Disease in Hunter-gatherers: Epidemiological factors. In D. Landy, ed. Culture, Disease and Healing. Studies in Medical Anthropology. New York: Macmillan Publishing, pp. 99-106.

Dyble, M. et al., Food sharing and multilevel organization among two hunter-gatherer societies.

Dyble, M. et al., 2015. Sex equality can explain the unique social structure of hunter-gatherer bands. Science, 348(6236), pp.796-798.

Eagle, N. \& Pentland, A., 2006. Reality mining: Sensing complex social systems. Personal and Ubiquitous Computing, 10(4), pp.255-268.

Early, J.D. \& Headland, T.N., 1998. Population Dynamics of a Philippine Rain Forest People: The San Ildefonso Agta, Gainesville: University Press of Florida.

Eaton, S.B. \& Eaton, S.B., 2003. Hunter-gatherers and human health. In R. B. Lee \& R. Daly, eds. Cambridge Encyclodepida of Hunter-Gatherers. Cambridge: Cambridge University Press, pp. 449-456.

Eberle, M. \& Kappeler, P.M., 2006. Family insurance: Kin selection and cooperative breeding in a solitary primate (Microcebus murinus). Behavioral Ecology and Sociobiology, 60, pp.582-588.

Ellison, P.T., 2003. Energetics and reproductive effort. American Journal of Human Biology, 15(3), pp.342-351.

Ellison, P.T., 2001. On Fertile Ground. In Balancing Act. pp. 167-181.

Ellison, P.T. et al., 1993. Population variation in ovarian function. The Lancet, 342(SEPTEMBER), pp.433434.

Emlen, S.T., 1995. An evolutionary theory of the family. Proceedings of the National Academy of Sciences of the United States of America, 92(18), pp.8092-8099. 
Emlen, S.T., 1982. The Evolution of Helping. I An Ecological Constraints Model. The American Naturalist, $119(1)$, pp.29-39.

Endicott, K. \& Endicott, K., 2008. The Headmand was a woman, Long Grove, Ill.: Waveland Press.

Endicott, K.M. \& Endicott, K.L., 2008. The headman was a woman: The gender egalitarian Batek of Malaysia, Long Grove, Ill.: Waveland Press.

Endicott, P., 2013. Introduction: revisiting the "negrito" hypothesis: a transdisciplinary approach to human prehistory in southeast Asia. Human biology, 85(1), pp.7-20.

Estiko, A.A. \& Griffin, P.B., 1981. Woman the hunter. In F. Dahlberg, ed. Woman the Gatherer. New Haven: Yale University Press.

Estioko-Griffin, A.A. \& Griffin, P.B., 1981. The beginning of cultivation among Agta hunter-gatherers in Northeast Luzon. In H. Olofsen, ed. Adaptive strategies and change in Philippine swidden-based societies. Laguna: Forest Research Institute, pp. 55-72.

Estioko-Griffin, A.A. \& Griffin, P.B., 1985. Women hunters: The implications for Pleistocene pre- history and contemporary ethnography. In M. J. Goodman, ed. Women in Asia and the Pacific: Towards an EastWest Dialogue. Honolulu: University of Hawaii Press.

Fafchamps, M., 1992. Solidarity Networks in Preindustrial Societies: Rational Peasants with a Moral Economy. Economic Development and Cultural Change, 41(1), pp.147-174.

Faulkes, C.G. et al., 1997. Ecological constraints drive social evolution in the African mole-rats. Proceedings. Biological sciences / The Royal Society, 264(November), pp.1619-1627.

La Ferrara, E., 2002. Inequality and group participation: theory and evidence from rural Tanzania. Journal of Public Economics, 85, p.235?273.

Fisher, R.A., 1930. The Genetical Theory of Natural Selection, Oxford: Clarendon Press.

Fletcher, A.W., 2001. Development of infant independence from the mother in wild mountain gorillas. In Mountain Gorillas: Three Decades of Research. In M. M. Robbins, P. Sicotte, \& K. Stewart, eds. Mountain Gorillas: Three Decades of Research in Karisoke. Cambridge: Cambridge University Press, pp. 158182.

Foley, R.A., 1988. Hominids, Humans and Hunter-Gatherers: An Evolutionary Perspective. In T. Ingold, D. Riches, \& J. Woodburn, eds. Hunters and Gatherers 1: History, Evolution and Social Change. Oxford: Berg, pp. 207-221.

Foley, R.A. \& Lahr, M.M., 2001. Anthropological, demographic and archeaological context of evolutionary genetics. In P. Donnelly, ed. Genes, Fossils, and Behaviour: An Integrated Approach to Human Evolution. Oxford: IOS Press, pp. 223-245.

Foote, A.D., 2008. Mortality rate acceleration and post-reproductive lifespan in matrilineal whale species. Biology letters, 4(2), pp.189-91.

Formica, V. a. et al., 2012. Fitness consequences of social network position in a wild population of forked fungus beetles (Bolitotherus cornutus). Journal of Evolutionary Biology, 25(1), pp.130-137.

Foster, K.R., Wenseleers, T. \& Ratnieks, F.L.W., 2006. Kin selection is the key to altruism. Trends in Ecology \& Evolution, 21(2), pp.57-60.

Fouts, H.N., Hewlett, B.S. \& Lamb, M.E., 2005. Parent-Offspring Weaning Conflicts among the Bofi Farmers and Foragers of Central Africa. Current Anthropology, 46(1), pp.29-50.

Fowler, J.H. \& Christakis, N. a, 2008. Dynamic spread of happiness in a large social network: longitudinal analysis over 20 years in the Framingham Heart Study. BMJ (Clinical research ed.), 337(February 2009): 2338.

Fowler, J.H., Dawes, C.T. \& Christakis, N. a., 2009. Model of genetic variation in human social networks. Proceedings of the National Academy of Sciences of the United States of America, 106(6), pp.1720-1724. 
Franzen, M., 2004. Key variables in tests of food sharing. Open peer commentary to Gurven 2004. Behavioral and Brain Sciences, 27, pp.543-583.

Freeman, L.C., 1977. A set of measures of centrality based upon betweenness. Sociometry, 40, pp.35-41.

Froment, A., 2008. Biodiversity, environment and health among rainforest-dwellers: an evolutionary perspective. In C. J. . P. Colfer, ed. Human health and Forests: A global overview of issues, practice and policy. London: Earthscan, pp. $259-274$.

Froment, A., 2014. Human Biology and Health of African Rainforest Inhabitants. In B. S. Hewlett, ed. Hunter-Gatherers of the Congo Basin: Cultures, Histories and Biology of African Pygmies. London: Transaction Publishers, pp. 117-164.

Froment, A., Koppert, G.J.A. \& Loung, J., 1996. Bien Manger, Vivre Bien: Etat nutritionnel et sante des populations forestieres du Cameroun. In C. M. Hladik et al., eds. L'alimentation en foret tropicale: interactions bioculturelles et perspectives de developpement. UNESCO, p. 1406.

Gelman, A., 2006. Prior distribution for variance parameters in hierarchical models. Bayesian Analysis, 1(3), pp.515-533.

Gibson, M. a \& Mace, R., 2003. Strong mothers bear more sons in rural Ethiopia. Proceedings. Biological sciences / The Royal Society, 270 Suppl, pp.S108-S109.

Gibson, M. a. \& Mace, R., 2005. Helpful grandmothers in rural Ethiopia: A study of the effect of kin on child survival and growth. Evolution and Human Behavior, 26(6), pp.469-482.

Gibson, M. \& Mace, R., 2002. The impact of labor-saving technology on first birth intervals in rural Ethiopia. Human biology; an international record of research, 74(1), pp.111-128.

Gibson, M.A. \& Lawson, D.W., 2011. “Modernization” increases parental investment and sibling resource competition: Evidence from a rural development initiative in Ethiopia. Evolution and Human Behavior, 32(2), pp.97-105.

Gilby, I.C. et al., 2013. Fitness benefits of coalitionary aggression in male chimpanzees. Behavioral Ecology and Sociobiology, 67(3), pp.373-381.

Giles, L.C., 2005. Effect of social networks on 10 year survival in very old Australians: the Australian longitudinal study of aging. Journal of Epidemiology \& Community Health, 59(7), pp.574-579.

Glass, L.M. \& Glass, R.J., 2008. Social contact networks for the spread of pandemic influenza in children and teenagers. BMC public bealth, 8(1), p.61.

Gonçalves, M.L.C., Araújo, A. \& Ferreira, L.F., 2003. Human Intestinal Parasites in the Past: New Findings and a Review. Memorias do Instituto Oswaldo Cruz, 98(SUPPL. 1), pp.103-118.

González, M.C., Hidalgo, C. a \& Barabási, A.-L., 2008. Understanding individual human mobility patterns. Nature, 453(7196), pp.779-782.

Goodman, M.J. et al., 1985. The compatibility of hunting and mothering among the agta hunter-gatherers of the Philippines. Sex Roles, 12, pp.1199-1209.

Goral, M.I., Mochow-Grundy, M. \& Demody, T.S., 1996. Sequence diversity within the reovirus S3 gene: reoviruses evolve independently of host species, geographic locale, and date of isolation. Virology, 216, pp.265-271.

Grafen, A., 2002. A first formal link between the price equation and an optimization program. Journal of theoretical biology, 217(1), pp.75-91.

Greenblatt, C.L. \& Spigelman, M., 2003. Emerging pathogens: archaeology, ecology and evolution of infectious disease C. L. Greenblatt \& M. Spigelman, eds., Oxford: Oxford University Press.

Greer, P.J. et al., 2013. Wintrobes Clinical Hemaology Thirteenth., Lippincott Williams \& Wilkins.

Griffin, A.S. \& West, S.A., 2002. Kin selection: Fact and fiction. Trends in Ecology and Evolution, 17(1): 15-21. 
Griffin, A.S., West, S.A. \& Buckling, A., 2004. Cooperation and competition in pathogenic bacteria. Nature, 430(7003), pp.1024-7.

Griffin, B.M., 1996. Change and stability; Agta kinship in a history of uncertainty. University of Illinois.

Griffin, P. \& Griffin, M., 2000. Agta hunting and sustainability of resource use in north-eastern Luzon, Philippines. In Hunting for sustainability in tropical forests. pp. 325-335.

Griffin, P.B., 1985. A contemporary view of the shift from hunting to horticulture. In V. N. Misra \& P. Bellwood, eds. Recent advances in Indo-Pacific prehistory. New Delhi: Oxford IBH Publishing, pp. 349-352.

Griffin, P.B. \& Griffin, M.B., 1992. Fathers and Childcare among the Cagayan Agta. In B. S. Hewlett, ed. Father-Child Relations: Cultural and Biosocial Contexts. New Brunswick: Transaction Publishers, pp. $297-$ 320.

Griffin, P.B. \& Griffin, M.B., 2010. The Agta of eastern Luzon, Philippines. In R. B. Lee \& R. Daly, eds. The Cambridge Encyclopedia of Hunters and Gatherers. Cambridge: Cambridge University Press, pp. 289-293.

Griffin, P.B. \& Headland, T.N., 1994. The Agta of the Philippines. In G. Bruenhault, ed. Traditional peoples today; The illustrated history of mankind. New York: Harper Collins, pp. 71-73.

Gross, M.R., 2005. The Evolution of parental care. The Quarterly Review of Biology, 80(1), pp.37-45.

Gu, Z., 2012. CePa: Centrality-based pathway enrichment.

Guerrero, E., Naji, S. \& Bocquet-Appel, J., 2008. The signal of the Neolithic Demographic Transition in the Levant. In J. Bocquet-Appel \& O. Bar-Yosef, eds. The Neolithic Demographic Transition and its Consequences. London: Springer, pp. 57-80.

Gupta, A.K., 2004. Origin of agriculture and domestication of plants and animals linked to early Holocene climate amelioration. Current Science, 87(1), pp.54-59.

Gurven, M. et al., 2008. Aging and inflammation in two epidemiological worlds. The journals of gerontology. Series A, Biological sciences and medical sciences, 63(2), pp.196-199.

Gurven, M. et al., 2015. Does Market Integration Buffer Risk, Erode Traditional Sharing Practices and Increase Inequality? A Test among Bolivian Forager-Farmers. Human Ecology, (August).

Gurven, M. et al., 2000. “It's a Wonderful Life”. signaling generosity among the Ache of Paraguay. Evolution And Human Behavior, 21(4), pp.263-282.

Gurven, M., 2004a. Reciprocal altruism and food sharing decisions among Hiwi and Ache hunter?gatherers. Behavioral Ecology and Sociobiology, 56(4), pp.366-380.

Gurven, M. et al., 2001. Reservation food sharing among the Ache of Paraguay. Human Nature, 12(4), pp.273297.

Gurven, M., 2006. The Evolution of Contingent Cooperation. Current Anthropology, 47(1), pp.185-192.

Gurven, M., 2004b. To give and to give not: The behavioral ecology of human food transfers. Behavioral and Brain Sciences, 27(4), pp.543-583.

Gurven, M. \& Hill, K., 2010. Moving beyond Stereotypes of Men’s Foraging Goals. Current Anthropology, 51(2), pp.265-267.

Gurven, M. \& Hill, K., 2009. Why Do Men Hunt? Current Anthropology, 50(1), pp.51-74.

Gurven, M. \& Kaplan, H., 2006. Determinants of time allocation across the lifespan: A theoretical model and an application to the Machiguenga and Piro of Peru. Human Nature, 17(1), pp.1-49.

Gurven, M., Kaplan, H. \& Supa, A.Z., 2007. Mortality experience of Tsimane Amerindians of Bolivia: Regional variation and temporal trends. American Journal of Human Biology, 19(3), pp.376-398.

Gurven, M. \& von Rueden, C., 2010. Hunting, social status and biological fitness. Social biology, 53(1-2), 
pp.81-99.

Gwetu, P. et al., 2014. Hemocue Validation for the Diagnosis of Anaemia in Children: A Semi- Systematic Review. Pediatrics \& Therapeutics, 4(1), pp.1-4.

Hadley, C., 2004. The Costs and Benefits of Kin: Kin Networks and Children's Health among the Pimbwe of Tanzania. Human Nature, 15(4), pp.377-395.

Hagen, E.H. \& Barrett, H.C., 2009. Cooperative Breeding and Adolescent Siblings: Evidence for the Ecological Constraints Model? Current Anthropology, 50, pp.727-737.

Hamede, R.K. et al., 2009. Contact networks in a wild Tasmanian devil (Sarcophilus harrisii) population: Using social network analysis to reveal seasonal variability in social behaviour and its implications for transmission of devil facial tumour disease. Ecology Letters, 12(11), pp.1147-1157.

Hames, R., 1988. The allocation of parental care among the Ye'Kawana. In L. Betzig, M. Borgerhoff Mulder, \& P. Turke, eds. Human Reproductive Behavior: A Darwinian Perspective. Cambridge: Cambridge University Press, pp. 237-251.

Hames, R., 2004. The purpose of exchange helps shape the mode of exchange. Behavioral and Brain Sciences, 27(4), pp.543-583.

Hames, R. \& Draper, P., 2004. Women's work, child care, and helpers-at-the-nest in a hunter-gatherer society. Human Nature, 15(4), pp.319-341.

Hamilton, W.D., 1967. Extraordinary Sex Ratios. Science, 156, pp.477-488.

Hamilton, W.D., 1964. The genetical evolution of social behaviour. I. Journal of Theoretical Biology, 7(1), pp.116.

Hao, Y. et al., 2015. Need-based transfers on a network: a model of risk-pooling in ecologically volatile environments. Evolution and Human Behavior, 36(4), pp.265-273.

Hardy, K. et al., 2015. The importance of dietary carbohydrate in human evolution. The Quarterly Review of Biology, 90(3), pp.251-268.

Hatchwell, B.J., 2007. Avian Reproduction: Role of Ecology in the Evolution of Cooperative Breeding. Current Biology, 17(19).

Hausman, A.J. \& Wilmsen, E.N., 1985. Economic Change and Secular Trends in the Growth of San Children. Human Biology, 57(4), pp.563-571.

Hawkes, K., 2000. Hunting and the evolution of egalitarian societies: lessons from the Hadza. Hierarchies in action: cui bono, (27), pp.59-83.

Hawkes, K., 1991. Hunting income patterns among the Hadza: big game, common goods, foraging goals and the evolution of the human diet. Philosophical Transactions: Biological Sciences, 334(1270), pp.243-250.

Hawkes, K. et al., 2000. The Grandmother Hypothesis and Human Evolution. In Adaptation and Human Behavior. pp. 237-258.

Hawkes, K. \& Coxworth, J.E., 2013. Grandmothers and the evolution of human longevity: A review of findings and future directions. Evolutionary Anthropology, 22(6), pp.294-302.

Hawkes, K. \& O’Connell, J., 1998. Grandmothering, menopause, and the evolution of human life histories. Proceedings of the National Academy of Sciences, 95(3), pp.1336-1339.

Hawkes, K., O’Connell, J.F. \& Blurton Jones, N.G., 1997. Hadza Women's Time Allocation, Offspring Provisioning and the Evolution of Long Postmenopausal Life Spans. Current Anthropology, 38(4), pp.551-577.

Hawkes, K., O’Connell, J.F. \& Blurton Jones, N.G., 2003. Human Life Histories: Primate trade-offs, grandmothering, socioecology and the fossil record. In P. M. Kappeler \& M. E. Pereira, eds. Primate life histories and socioecology. London: The University of Chicago Press, pp. 204-232. 
Hayden, B., 1995. A new overview of domestication. In D. Price \& A. B. Gebauer, eds. Last Hunters-First Farmers. Santa Fe: School of American Research Press, pp. 215-242.

Headland, T.N., 1984. Agta Negritos of the Philippines. Cultural Survival Quarterly, 8(3), pp.29-31.

Headland, T.N., 1988. Ecosystemic change in a Philippine tropical rainforest and its effects on a Negrito foraging society. Tropical Ecology, 29, pp.121-35.

Headland, T.N. et al., 1989. Hunter-Gatherers and Their Neighbors from to the Prehistory Present' by Thomas N. Headland and. Current Anthropology, 30, pp.43-66.

Headland, T.N., 1987. Kinship and Social Behavior among Agta Negrito Hunter-Gatherers. Ethnology, 26(4), pp.261-280.

Headland, T.N., 1989. Population Decline in a Philippine Negrito Hunter-Gatherer Society. American Journal of Human Biology, 72, pp.59-72.

Headland, T.N., 1986. Why foragers do not become farmers; A historical study of a changing ecosystem and its effect on a Negrito bunter-gatherer group in the Philippines. University of Hawaii.

Headland, T.N., Headland, J.D. \& Uehara, R., 2011. Agta Demographic Database. Available at: http://www.sil.org/resources/publications/entry/9299.

HemoCue, 2014a. HemoCue Hb 301 Operating Manual,

HemoCue, 2014b. HemoCue WBC DIFF Operating Manual,

Henrich, J., 1997. Market Incorporation, Agricultural Change, and Sustainability Among the Machiguenga Indians of the Peruvian Amazon. Human Ecology, 25(2), pp.319-351.

Herrera, E., 2000. Metabolic adaptations in pregnancy and their implications for the availability of substrates to the fetus. European journal of clinical nutrition, 54 Suppl 1, pp.S47-S51.

Hershkovitz, I. et al., 2008. Detection and molecular characterization of 9000-year-old Mycobacterium tuberculosis from a neolithic settlement in the Eastern mediterranean. PLOS ONE, 3(10), pp.1-6.

Hershkovitz, I. \& Gopher, A., 2008. Demographic, Biological and Cultural Aspects of the Neolithic revolution: A view from the southern Levant. In J. Bocquet-Appel \& O. Bar-Yosef, eds. The Neolithic Demographic Transition and its Consequences. London: Springer, pp. 441-482=0.

Hewlett, B.S., 1991. Demography and childcare in preindustrial societies. Journal of anthropological research, 47(1), pp.1-37.

Hewlett, B.S. et al., 2000. Parental investment strategies among Aka foragers, Ngandu farmers and EuroAmerican urban-industrialists. In Adaptation and Human Behaviour: an Anthropological Perspective. pp. 155177.

Hewlett, B.S., 1988. Sexual selection and paternal investment among Aka pygmies. In L. Betzig, M. Borgerhoff Mulder, \& P. Turke, eds. Human Reproductive Behavior: A Darwinian Perspective. Cambridge: Cambridge University Press, pp. 263-276.

Heyer, E. et al., 2013. Genetic diversity of four Filipino negrito populations from Luzon: comparison of male and female effective population sizes and differential integration of immigrants into Aeta and Agta communities. Human biology, 85(1), pp.189-208.

Higham, C., 2013. Hunter-Gatherers in Southeast Asia: From Prehistory to the Present. Human Biology, 85(1), pp.21-44.

Hill, K. et al., 1987. Foraging decisions among Aché hunter-gatherers: New data and implications for optimal foraging models. Ethology and Sociobiology, 8(1), pp.1-36.

Hill, K., 1993. Life history theory and evolutionary anthropology. Evolutionary Anthropology, 2(3), pp.78-88.

Hill, K. et al., 2001. Mortality rates among wild chimpanzees. Journal of human evolution, 40, pp.437-450. 
Hill, K., Barton, M. \& Hurtado, A.M., 2009. The emergence of human uniqueness: Characters underlying behavioral modernity. Evolutionary Anthropology: Issues, News, and Reviews, 18(5), pp.187-200.

Hill, K., Hurtado, a. M. \& Walker, R.S., 2007. High adult mortality among Hiwi hunter-gatherers: Implications for human evolution. Journal of Human Evolution, 52, pp.443-454.

Hill, K. \& Hurtado, A.M., 1996. Aché Life History: The Ecology and Demography of a Foraging People, New Haven: Aldine de Gruyter.

Hill, K. \& Hurtado, A.M., 2009. Cooperative breeding in South American hunter-gatherers. Proceedings. Biological sciences / The Royal Society, 276(1674), pp.3863-3870.

Hill, K. \& Kaplan, H., 1999. Life history traits in humans: Theory and Empirical Studies. Annual Review of Anthropology, 28, pp.397-430.

Hill, K.R. et al., 2011. Co-residence patterns in hunter-gatherer societies show unique human social structure. Science, 331(6022), pp.1286-1289.

Hinds, L.E., Brown, C.L. \& Clark, S.J., 2007. Point of care estimation of haemoglobin in neonates. Archives of disease in childhood. Fetal and neonatal edition, 92(5), pp.F378-80.

Hitchcock, R.K., 1982. Patterns of Sedentism among the Basarwa of Botswana. In E. Leacock \& R. Lee, eds. Politics and History in Band Societies. Cambridge: Cambridge University Press, pp. 223-267.

Hitchcock, R.K. \& Biesele, M., 2000. Introduction. In P. P. Schweitzer, M. Biesele, \& R. K. Hitchcock, eds. Hunter-Gatherers in the Modern World. Oxford: Berghahn Books, pp. 1-28.

Hitchcock, R.K. \& Ebert, J.I., 1984. Foraging and food production among Kalahari Hunter/Gatherers. In C. J. Desmond \& S. A. Brandt, eds. From Hunters to Farmers: The causes and consequences of food production in Africa. London: University of California Press, pp. 328-348.

Hobbes, T., 1651. Leviathan, Oxford: Oxford University Press.

Hoberg, E.P. et al., 2001. Out of Africa: origins of the Taenia tapeworms in humans. Proceedings of the Royal Society B: Biological Sciences, 268(1469), pp.781-787.

Holt-Lunstad, J., Smith, T.B. \& Bradley Layton, J., 2010. Social relationships and mortality risk: a metaanalytic review. PLoS Medicine, 7(7), p.859.

Hooper, P., 2015. Inclusive fitness and differential productivity across the life course determine intergenerational transfers in a small-scale human society. Proceedings of the Royal Society B: Biological Sciences, 282(1803), p.20142808.

Hooper, P.L., Gurven, M., et al., 2015. Inclusive fitness and differential productivity across the life course determine intergenerational transfers in a small-scale human society. Proceedings. Biological sciences / The Royal Society, 282(1803), p.20142808.

Hooper, P.L., Demps, K., et al., 2015. Skills, division of labour and economies of scale among Amazonian hunters and South Indian honey collectors. Philosophical transactions of the Royal Society of London. Series B, Biological sciences, 370(1683).

Hotez, P.J. et al., 2005. Hookworm: “The great infection of mankind.” PLoS Medicine, 2, pp.0187-0191.

Howell, N., 1979. Demography of the Dobe!Kung, London: Aldine.

Howell, N., 2010. Life Histories of the Dobe!Kung: Food, fatness, and well-being over the life span, London: University of California Press.

Hrdy, S.B., 2005. Cooperative breeders with an ace in the hole. In Grandmotherhood: The evolutionary significance of the second half of the female life. New Brunswick: Rutgers University Press, pp. 295-317.

Hrdy, S.B., 2008. Cooperative breeding and the paradox of facultative fathering. In R. S. Bridges, ed. Neurobiology of the Parental Mind. London: Academic Press, pp. 405-415. 
Hrdy, S.B., 2011. Mothers and Others: The evolution origins of mutual understanding, London: Harvard University Press.

Hrdy, S.B., 2009. Mothers and Others: The evolutionary origins of mutual understanding, London: Harvard University Press.

Hudson-Thomas, M., Bingham, K.C. \& Simmons, W.K., 1994. An evaluation of the HemoCue for measuring haemoglobin in field studies in Jamaica. Bulletin of the World Health Organization, 72(3), pp.423-6.

Hugot, J.P. et al., 1999. Human enterobiasis in evolution: origin, specificity and transmission. Parasite (Paris, France), 6(3), pp.201-8.

Humphrey, L.T. et al., 2014. Earliest evidence for caries and exploitation of starchy plant foods in Pleistocene hunter-gatherers from Morocco. Proceedings of the National Academy of Sciences, 111(3), pp.954-959.

Hurrell, R.F., 2003. Influence of vegetable protein sources on trace element and mineral bioavailability. The Journal of nutrition, 133(9), p.2973S-7S.

Hurtado, a. M. et al., 2008. The role of helminthes in human evolution. In S. Elton \& P. O’Higgins, eds. Medicine and Evolution: Current Applications, Future Prospects. London: CRC Press, pp. 153-180.

Hurtado, M. \& Hill, K.R., 1987. Early dry season subsistence ecology cuiva (hiwi) forages of Venezuela. Human Ecology, 15, pp.163-187.

Hutchinson, D.L., Norr, L. \& Teaford, M.F., 2007. Outer Coast Foragers and Inner Coast Farmers in Late Prehistoric North Carolina. In M. N. Cohen \& G. M. M. Crane-Kramer, eds. Ancient Health: Skeletal indicators of agricultural and economic intensification. Gainesville: University Press of Florida, pp. 52-64.

Inomata, T. et al., 2015. Development of sedentary communities in the Maya lowlands: Coexisting mobile groups and public ceremonies at Ceibal, Guatemala. Proceedings of the National Academy of Sciences, p.201501212.

Isella, L. et al., 2011. What's in a crowd? Analysis of face-to-face behavioral networks. Journal of Theoretical Biology, 271(1), pp.166-180.

Ivey, P.K., 2000. Cooperative reproduction in Ituri forest Hunter-Gatherers: Who cares for Efe infants? Current Anthropology, 41(5), pp.856-866.

Jackson, J. a. et al., 2009. Review series on helminths, immune modulation and the hygiene hypothesis: Immunity against helminths and immunological phenomena in modern human populations: Coevolutionary legacies? Immunology, 126(1), pp.18-27.

Jaeggi, A. V \& Gurven, M., 2013a. Natural cooperators: Food sharing in humans and other primates. Evolutionary Anthropology, 22(4), pp.186-195.

Jaeggi, A. V \& Gurven, M., 2013b. Reciprocity explains food sharing in humans and other primates independent of kin selection and tolerated scrounging: a phylogenetic meta-analysis. Proceedings of the Royal Society B: Biological Sciences, 280(1768), pp.1-8.

Jasieńska, G. \& Ellison, P.T., 1998. Physical work causes suppression of ovarian function in women. Proceedings. Biological sciences / The Royal Society, 265(1408), pp.1847-51.

Jelliffe, D.B., 1962. The Children of the Hadza Hunters. Tropical Pediatrics, 60, pp.907-913.

Jennions, M.D. \& Macdonald, D.W., 1994. Cooperative breeding in mammals. Trends in Ecology \& Evolution, 9(3), pp.89-93.

Jetz, W. \& Rubenstein, D.R., 2011. Environmental uncertainty and the global biogeography of cooperative breeding in birds. Current Biology, 21(1), pp.72-78.

Jones, N.B., 1986. Bushman birth spacing: A test for optimal interbirth intervals. Ethology and Sociobiology, 7(2), pp.91-105.

Kaplan, H., Hill, K., et al., 2000a. A Theory of Human Life History Evolution: Diet, Intelligence, and 
Longevity. Evolutionary Anthropology, 9(4), pp.156-185..

Kaplan, H., Hill, K., et al., 2000b. A Theory of Human Life History Evolution: Diet, Intelligence, and Longevity. Evolutionary Anthropology, 9(4), pp.156-185.

Kaplan, H., Hill, J., et al., 2000. A theory of human life history evolution: diet, intelligence, and longevity. Evolutionary Anthropology, 9, pp.156-185.

Kaplan, H., 1994. Evolutionary and Wealth Flows Theories of Fertility: Empirical Tests and New Models. Population and Development Review, 20(4), pp.753-791.

Kaplan, H. \& Gurven, M., 2005. The natural history of human food sharing and cooperation: a review and a new multi-individual approach to the negotiation of norms. Moral sentiments and material interests: The foundations of cooperation in economic life, pp.75-113.

Kaplan, H. \& Hill, K., 1985. Food Sharing Among Ache Foragers: Tests of Explanatory Hypotheses. Current Anthropology, 26, p.223.

Kaplan, H., Lancaster, J. \& Robson, A., 2003. Embodied Capital and the Evolutionary Economics of the Human Life Span. Population and Development Review, 29, pp.152-182.

Kaplan, H.S., Hooper, P.L. \& Gurven, M., 2009. The evolutionary and ecological roots of human social organization. Philosophical transactions of the Royal Society of London. Series B, Biological sciences, 364(1533), pp.3289-3299.

Kappeler, P.M. et al., 2013. Constraints and flexibility in mammalian social behaviour: introduction and synthesis. Philosophical transactions of the Royal Society of London. Series B, Biological sciences, 368(1618), p.20120337.

Kasper, C. \& Borgerhoff Mulder, M., 2015. Who Helps and Why? Current Anthropology, 56(5), pp.701-735.

Kazandjieva, M. A. et al., 2010. Experiences in measuring a human contact network for epidemiology research. Proceedings of the 6th Workshop on Hot Topics in Embedded Networked Sensors - HotEmNets '10, p.1. Available at: http://dl.acm.org/citation.cfm?id=1978642.1978651.

Keeling, M.J. \& Eames, K.T.D., 2005. Networks and epidemic models. Journal of The Royal Society Interface, 2(4), pp.295-307.

Kelly, R.L., 1992. Mobility/Sedentism: Concepts, Archaeological Measures and Effects. Annual Review of Anthropology, 21, pp.43-66.

Kelly, R.L., 2013. The Lifeways of Hunter-Gatherers: The Foraging Specturm second., Cambridge: Cambridge University Press.

Kennedy, G., 2005. From the ape's dilemma to the weanling's dilemma: early weaning and its evolutionary context. Journal of Human Evolution, 48(2), pp.123-145.

Kim, M.J. et al., 2013. Comparison of the accuracy of noninvasive hemoglobin sensor (NBM-200) and portable hemoglobinometer (hemocue) with an automated hematology analyzer (LH500) in blood donor screening. Annals of Laboratory Medicine, 33(4), pp.261-267.

Kleiman, D.G. \& Malcolm, J.R., 1981. The evolution of male parental investment in mammals. In D. Gubernick \& P. H. Klopfer, eds. Parental care in mammals. New York: Plenum, pp. 347-387.

Koenig, W.D. \& Dickinson, J.L., 2004. Ecology and Evolution of Cooperative Breeding in Birds W. D. Koenig \& J. L. Dickinson, eds., Cambridge: Cambridge University Press.

Kohler, T.A. \& Varien, M.D., 2010. A Scale Model of Seven Hundred Years of Farming Settlements in Southwestern Colorado. In M. S. Bandy \& J. R. Fox, eds. Becoming Villagers. Tucson: University of Arizona Press, pp. 37-61.

Konner, M., 2005. Hunter-Gatherer Infancy and Childhood: The !Kung and Others. In B. S. Hewlett \& M. E. Lamb, eds. Hunter-Gatherer Childhoods: Cultural, Developmental, \& Evolutionary Perspectives. New Brunswick: Aldine Transaction, pp. 19-64. 
Koster, J. et al., 2015. Multilevel modeling analysis of dyadic network data with an application to Ye'kwana food sharing. American journal of physical anthropology, 157(3), pp.507-512.

Koster, J.M. \& Leckie, G., 2014. Food sharing networks in lowland Nicaragua: An application of the social relations model to count data. Social Networks, 38, pp.100-110.

Kramer, K. \& Greaves, R., 2007. Changing Patterns of Infant Mortality and Maternal Fertility among Pumé Foragers and Horticulturalists. American Anthropologist, 109(4), pp.713-726.

Kramer, K.L., 2005. Children's help and the pace of reproduction: Cooperative breeding in humans. Evolutionary Anthropology, 14, pp.224-237.

Kramer, K.L., 2010. Cooperative Breeding and its Significance to the Demographic Success of Humans. Annual Review of Anthropology, 39(1), pp.417-436.

Kramer, K.L., 2008. Early sexual maturity among pume foragers of Venezuela: Fitness implications of teen motherhood. American Journal of Physical Anthropology, 136(April), pp.338-350.

Kramer, K.L., 2002. Helping Behavior among Maya Children. , 13(2), pp.299-325.

Kramer, K.L., 2011. The evolution of human parental care and recruitment of juvenile help. Trends in Ecology and Evolution, 26(10), pp.533-540.

Kramer, K.L. \& Boone, J.L., 2002. Why Intensive Agriculturalists Have Higher Fertility: A household energy budget approach. Current Anthropology, 43(3), pp.511-517.

Kramer, K.L., Greaves, R.D. \& Ellison, P.T., 2009. Early reproductive maturity among pume foragers: Implications of a pooled energy model to fast life histories. American Journal of Human Biology, 21(November 2008), pp.430-437.

Krause, J. \& Ruxton, G.D., 2002. Living in Groups. Living in groups, I(4), p.210.

Kuhn, S.L. \& Stiner, M.C., 2001. The Antiquity of Hunter-gatherers. In C. Panter-Brick, R. Layton, \& P. Rowley-Conwy, eds. Hunter-gatherers: Interdisciplinary Perspectives. Cambridge: Cambridge University Press, pp. 99-142.

Kurvers, R.H.J.M. et al., 2014. The evolutionary and ecological consequences of animal social networks: Emerging issues. Trends in Ecology and Evolution, 29(6), pp.326-335.

Lack, D., 1954. The Significance of Migration BT - The Natural Regulation of Animal Numbers. In The Natural Regulation of Animal Numbers. Oxford: Oxford University Press.

Lambert, P.M., 2009. Health versus fitness: competing themes in the origins and spread of agriculture? Current anthropology, 50(5), pp.603-608.

Lancaster, J.B., 1971. Play-mothering: the relations between juvenile females and young infants among freeranging vervet monkeys (Cercopithecus aethiops). Folia primatologica; international journal of primatology, 15, pp.161-182.

Lancaster, J.B. \& Kaplan, H.S., 2009. The Endocrinology of the Human Adaptive Complex. The Endocrinology of Social Relationships, (7), pp.95-118.

Larsen, C.S., 2008. Foraging to Farming Transition: Global Health Impacts, Trends, and Variation. In C. Smith, ed. Encyclopedia of Global Archaeology. New York: Springer, pp. 2818-2824.

Larsen, C.S., 2006. The agricultural revolution as environmental catastrophe: Implications for health and lifestyle in the Holocene. Quaternary International, 150(1), pp.12-20.

Lawson, D.W., Alvergne, a. \& Gibson, M. a., 2012. The life-history trade-off between fertility and child survival. Proceedings of the Royal Society B: Biological Sciences, 279(1748), pp.4755-4764.

Lawson, D.W. \& Mace, R., 2011. Parental investment and the optimization of human family size. Philosophical transactions of the Royal Society of London. Series B, Biological sciences, 366(1563), pp.333-343. 
Lee, P.C., Majluf, P. \& Gordon, I.J., 1991. Growth, weaning and maternal investment from a comparative perspective. Journal of Zoology, 225(1), pp.99-114.

Lee, R., 1979. The !Kung San, Cambridge: Cambridge University Press.

Lee, R.B., 1968. What hunters do for a living. In R. B. Lee \& I. Devore, eds. Man the Hunter. Chicago: Aldine Publishing Company, pp. 30-48.

Lee, R.B. \& DeVore, I., 1968. Introduction. In R. B. Lee \& I. DeVore, eds. Man the Hunter. Chicago: Aldine Publishing Company, pp. 3-12.

Lee, R.D. \& Kramer, K.L., 2002. Children's Economic Roles in the Maya Family Life Cycle: Cain, Caldwell, and Chayanov Revisited. Population and development review, 28(3), pp.475-499.

Lehmann, J., Majolo, B. \& McFarland, R., 2015. The effects of social network position on the survival of wild Barbary macaques, Macaca sylvanus. Behavioral Ecology, 00, p.arv169.

Leles, D. et al., 2010. A parasitological paradox: Why is ascarid infection so rare in the prehistoric Americas? Journal of Archaeological Science, 37(7), pp.1510-1520.

Lewis, S.E. \& Pusey, A.E., 1997. Factors influencing the Occurrence of Communal Care in Plural Breeding Mammals. In N. G. Solomon \& J. A. French, eds. Cooperative Breeding in Mammals. Cambridge: Cambridge University Press, pp. 335-363.

Lipson, M. et al., 2014. Reconstructing Austronesian population history in Island Southeast Asia. Nature communications, 5, p.4689.

Lipson, S.F., 2001. Metabolism, Maturation and Ovarian function. In P. T. Ellison, ed. Reproductive Ecology and Human Evolution. New York: Aldine de Gruyter, pp. 235-248.

Little, P.D. et al., 2001. Avoiding Disaster: Diversification and Risk Management among East African Herders. Development and Change, 32, pp.401-433.

London, D. \& Hruschka, D., 2014. Helminths and human ancestral immune ecology: What is the evidence for high helminth loads among foragers? American Journal of Human Biology, 26(January), pp.124-129.

Lovejoy, C.O., 1981. The Origin of Man. Science, 211(4480), pp.341-350.

Lukacs, J.R., 2007. Climate, subsistence and health in Prehistoric India: the biological impact of a short-term subsistence shift. In M. N. Cohen \& G. M. M. Crane-Kramer, eds. Ancient Health: Skeletal indicators of agricultural and economic intensification. Gainesville: University Press of Florida, pp. 237-249.

Lukacs, J.R., 1992. Dental paleopathology and agricultural intensification in south Asia: new evidence from Bronze Age Harappa. American journal of physical anthropology, 87(2), pp.133-150.

Lukas, D. \& Clutton-Brock, T., 2012. Life histories and the evolution of cooperative breeding in mammals. Proceedings of the Royal Society B: Biological Sciences, 279(August), pp.4065-4070.

Lusseau, D., 2006. Evidence for social role in a dolphin social network. Evolutionary Ecology, 21(3), pp.357366.

Lusseau, D. \& Newman, M.E.J., 2004. Identifying the role that animals play in their social networks. Proceedings of the Royal Society B: Biological Sciences, 271(Suppl_6), pp.S477-S481.

Maas, C.J.M. \& Hox, J.J., 2005. Sufficient sample sizes for multilevel modeling. Methodology, 1(3), pp.86-92.

Mace, R., 1996. Biased parental investment and reproductive success in Gabbra pastoralists. Behavioral ecology and sociobiology, 38(2), pp.75-81.

Mace, R. \& Sear, R., 2005. Are Humans Cooperative Breeders? In E. Voland, A. Chasiotis, \& W. Schiefenhovel, eds. Grandmotherhood: The evolutionary significance of the second half of the female life. London: Rutgers University Press, pp. 143-159.

Magvanjav, O. et al., 2012. Sibling composition and children's anthropometric indicators of nutritional status: 
Evidence from native Amazonians in Bolivia. Annals of Human Biology, 40(February), p.121024073448002.

Mann, J. et al., 2012. Social networks reveal cultural behaviour in tool-using using dolphins. Nature Communications, 3, p.980.

Marlowe, F.W., 2003. A critical period for provisioning by Hadza men. Implications for pair bonding. Evolution and Human Behavior, 24, pp.217-229.

Marlowe, F.W., 2005. Hunter-gatherers and human evolution. Evolutionary Anthropology, 14, pp.54-67.

Marlowe, F.W., 2004. Marital Residence among Foragers. Current Anthropology, 45(2), pp.277-284.

Marlowe, F.W., 2010. The Hadza: Hunter-gatherers of Tanzania, Berkeley: University of California Press.

Mascie-Taylor, C.G.N., 1993. The Biological Anthropology of Disease. In C. G. N. Mascie-Taylor, ed. The Anthropology of Disease. Oxford: Oxford University Press, pp. 1-72.

Mathews, P. \& Sear, R., 2013. Family and Fertility: Kin Influence on the Progression to a Second Birth in the British Household Panel Study. PLOS ONE, 8(3).

Mathieson, I. et al., 2015. Genome-wide patterns of selection in 230 ancient Eurasians. Nature, advance on.

McDade, T.W. et al., 2005. Predictors of C-reactive protein in Tsimane' 2 to 15 year-olds in lowland Bolivia. American Journal of Physical Anthropology, 128(4), pp.906-913.

McDonald, D.B., 2007. Predicting fate from early connectivity in a social network. Proceedings of the National Academy of Sciences, 104(26), pp.10910-10914.

Mcdonell, A. \& Oxenham, M.F., 2014. Localised Primary Canine Hypoplasia: Implications for Maternal and Infant Health at Man Bac, Vietnam, 4000-3500years BP. International Journal of Osteoarchaeology, 24(4), pp.531-539.

McElory, A. \& Townsend, P.K., 2008. Medical Anthropology in Ecological Perspective Fifth., Oxford: Westview Press.

McFarland, R. et al., 2015. Social integration confers thermal benefits in a gregarious primate. Journal of Animal Ecology, pp.871-878.

McFarland, R. \& Majolo, B., 2013. Coping with the cold: predictors of survival in wild Barbary macaques, Macaca sylvanus. Biology letters, 9, p.20130428.

Medina Lara, A., 2005. Evaluation and costs of different haemoglobin methods for use in district hospitals in Malawi. Journal of Clinical Pathology, 58(1), pp.56-60.

Meehan, C.L., 2009. Maternal Time Allocation in Two Cooperative Childrearing Societies. Human Nature, 20, pp.375-393.

Meehan, C.L., 2005. The effects of residential locality on parental and alloparental investment among the Aka foragers of the central African Republic. Human Nature, 16(1), pp.58-80.

Meehan, C.L., Helfrecht, C. \& Quinlan, R.J., 2014. Cooperative breeding and Aka children's nutritional status: Is flexibility key? American Journal of Physical Anthropology, 153(October 2013), pp.513-525.

Meehan, C.L., Quinlan, R. \& Malcom, C.D., 2013a. Cooperative breeding and maternal energy expenditure among aka foragers. American Journal of Human Biology, 25(1), pp.42-57.

Meehan, C.L., Quinlan, R. \& Malcom, C.D., 2013b. Cooperative breeding and maternal energy expenditure among aka foragers. American Journal of Human Biology, 25(October 2012), pp.42-57.

Mellars, P., 2006. Why did modern human populations disperse from Africa ca. 60,000 years ago? A new model. Proceedings of the National Academy of Sciences, 103(25), pp.9381 -9386.

Meyers, L.A. et al., 2005. Network theory and SARS: predicting outbreak diversity. Journal of Theoretical Biology, 
232(1), pp.71-81.

Migliano, A.B. et al., 2013. Evolution of the Pygmy Phenotype: Evidence of Positive Selection from Genome-wide Scans in African, Asian, and Melanesian Pygmies. Human Biology, 85(1-3), pp.251-284.

Migliano, A.B. et al., 2016. High-resolution maps of hunter-gatherer social networks reveal human adaptation for cultural exchange, Cold Spring Harbor Labs Journals.

Migliano, A.B., Vinicius, L. \& Lahr, M.M., 2007. Life history trade-offs explain the evolution of human pygmies. Proceedings of the National Academy of Sciences of the United States of America, 104(51), pp.2021620219.

Mills, A.F. \& Meadows, N., 1989. Screening for anaemia: evaluation of a haemoglobinometer. Archives of disease in childhood, 64(10), pp.1468-71.

Minter, T., 2010. The Agta of the Northern Sierra Madre: Livelihood strategies and resilience among Philippine huntergatherers. Leiden University.

Mira, A., Rushker, R. \& Rodriguez-Valera, F., 2006. The Neolithic revolution of bacterial genomes. Trends in Microbiology, 14(5), pp.200-206.

Mitaishvili, R., 2010. The Human Blood: Composition, Typing, Lab Test Interpretation, RM Global Health.

Mitani, J.C. \& Watts, D., 1997. The evolution of non-maternal caretaking among anthropoid primates: Do helpers help? Behavioral Ecology and Sociobiology, 40, pp.213-220.

Center of Multilevel Modelling., 2015. LEMMA: Learning envrionment for multilevel methodology and applications. Available at: http://www.bristol.ac.uk/cmm/learning/online-course/index.html.

Morfin, L.M. \& Storey, R., 2007. From Early Village to Regional Center in Mesoamerica: An Investigation of Lifestyles and Health. In M. N. Cohen \& G. M. M. Crane-Kramer, eds. Ancient Health: Skeletal indicators of agricultural and economic intensification. Gainesville: University Press of Florida, pp. 80-91.

Morris, S.S. et al., 1999. Precision, accuracy, and reliability of hemoglobin assessment with use of capillary blood. The American journal of clinical nutrition, 69(6), pp.1243-1248.

Muñoz, M. et al., 2005. Utility of point-of-care haemoglobin measurement in the HemoCue-B haemoglobin for the initial diagnosis of anaemia. Clinical and laboratory baematology, 27(2), pp.99-104. Available at: http://www.ncbi.nlm.nih.gov/pubmed/15784124.

Murphy, D. et al., The Complex Benefits of social capital: Drivers of female Baboon reproductive success,

Nettle, D., 2010. Dying young and living fast: Variation in life history across English neighborhoods. Behavioral Ecology, 21(2), pp.387-395.

Nettle, D., 2011. Flexibility in reproductive timing in human females: integrating ultimate and proximate explanations. Philosophical transactions of the Royal Society of London. Series B, Biological sciences, 366(1563), pp.357-65.

Nettle, D. et al., 2012. Human Behavioral Ecology : Current research and future prospects. Behavioral Ecology, pp.1-19.

Nettle, D. et al., 2013. Human behavioral ecology: current research and future prospects. Behavioral Ecology, 24(5), pp.1031-1040.

Neufeld, L. et al., 2002. Hemoglobin measured by Hemocue and a reference method in venous and capillary blood: A validation study. Salud pública de México, 44(3), pp.219-227.

Neville, R.G., 1987. Evaluation of portable haemoglobinometer in general practice. British medical journal (Clinical research ed.), 294(6582), pp.1263-1265.

Newman, M.E.J., 2004. Analysis of weighted networks. Physical Review E - Statistical, Nonlinear, and Soft Matter Physics, 70(5 2), pp.1-9. 
Newman, M.E.J., 2010. Networks: An introduction, Oxford: Oxford University Press.

Nolin, D. a., 2010. Food-Sharing Networks in Lamalera, Indonesia: Reciprocity, Kinship, and Distance. Human Nature, 21(3), pp.243-268.

Nolin, D. a., 2012. Food-sharing networks in Lamalera, Indonesia: Status, sharing, and signaling. Evolution and Human Behavior, 33(4), pp.334-345.

Nolin, D.A., 2015. Open peer commentary to Kasper and Borgerhoff Mulder (2015) Who helps and why? Current Anthropology, 56(5), pp.701-735.

Norrby, E. et al., 1992. Humanized animal viruses with special reference to the primate adaptation to morbillivirus. Vet Microbiol, 33, pp.275-286.

Nowak, M. a, 2006. Five rules for the evolution of cooperation. Science, 314, pp.1560-1563.

Nowell, A.A. \& Fletcher, A.W., 2007. Development of Independence from the Mother in Gorilla gorilla gorilla. International Journal of Primatology, 28(2), pp.441-455.

O'Connell, J.F., 1995. Ethnoarchaeology needs a general theory of behavior. Journal of Archaeological Research, 3(3), pp.205-255.

O’Connell, J.F., Hawkes, K. \& Blurton Jones, N.G., 1999. Grandmothering and the evolution of homo erectus. Journal of human evolution, 36, pp.461-485.

O’Neill, E. et al., 2006. Instrumenting the City: Developing Methods for Observing and Understanding the Digital Cityscape. In Proceedings of the 8th International Conference of Ubiquitous Computing (UbiComp'06). pp. 315-332.

Omoto, K., 1984. The Negritos: genetic origins and microevolution. Acta anthropogenetica, 8(1-2), pp.137-47.

Oota, H. et al., 2005. Recent origin and cultural reversion of a hunter-gatherer group. PLoS Biology, 3(3), pp.0536-0542.

Orr, H.A., 2009. Fitness and its role in evolutionary genetics. Nature Reviews Genetics, 10(8), pp.531-539.

Oxenham, M.F., Nguyen, L.C. \& Nguyen, K.D., 2008. Oral health in northern Vietnam. Bull Indo-Pacific Prehist Assoc, 22, pp.121-134.

Oxenham, M.F. \& Tayles, N., 2006. Synthesising Southeast Asian population history and palaeohealth. In M. F. Oxenham \& N. Tayles, eds. Bioarchaeology of Southeast Asia. Cambridge: Cambridge University Press, pp. 335-349.

Pagel, M. \& Harvey, P.H., 2002. Evolution of the juvenile period in mammals. In M. E. Pereira \& L. A. Fairbanks, eds. Juvenile Primates: Life history, development and behaviour. London: University of Chicago Press, pp. 28-38.

Panisson, A. et al., 2012. On the dynamics of human proximity for data diffusion in ad-hoc networks. Ad Hoc Networks, 10(8), pp.1532-1543.

Panter-Brick, C. \& Pollard, T.M., 1999. Work and hormonal variation in subsistence and industrial contexts. In C. Panter-Brick \& C. M. Worthman, eds. Hormones, Health and Behaviour: A Social-ecological and Lifespan Perspective. Cambridge: Cambridge University Press, pp. 139-183.

Patton, J.Q., 2005. Meat sharing for coalitional support. Evolution and Human Behavior, 26(2), pp.137-157.

Pechenkina, E. a., Benfer, R. a. \& Zhijun, W., 2002. Diet and health changes at the end of the Chinese Neolithic: The Yangshao/Longshan transition in Shaanxi province. American Journal of Physical Anthropology, 117(1), pp.15-36.

Pennington, R., 2001. Hunter-gatherer demography. In C. Panter-Brick, R. Layton, \& P. Rowley-Conwy, eds. Hunter-Gatherers: An Interdisciplinary Perspective. Cambridge: Cambridge University Press, pp. 170-204.

Pennington, R. \& Harpending, H., 1988. Fitness and fertility among Kalahari!Kung. American journal of physical 
anthropology, 77(3), pp.303-319.

Perry, G.H., 2014. Parasites and human evolution. Evolutionary anthropology, 23(6), pp.218-28.

Peterson, J.T., 1978. The Ecology of Social Boundaries: Agta Foragers of the Philippines, London: University of Illinois Press.

Pietrusewsky, M. \& Ikehara-Quebral, R., 2007. The bioarchaeology of the Vat Kommou cemetry, Angkor Borei, Cambodia. Bull Indo-Pacific Prehist Assoc, 26, pp.86-97.

Pike, L., 2001. The Evolutionary and ecological context of human pregnancy. In P. T. Ellison, ed. Reproductive Ecology and Human Evolution. New York: Aldine de Gruyter, pp. 39-58.

Pinhasi, R. \& Stock, J.T., 2011. Human Bioarchaeology of the Transition to Agriculture,

Piperno, D. \& Pearsall, D.M., 1998. The origins of agriculture in the Lowland Neotropics, London: Academic Press.

van der Poel, W.H.M. et al., 2000. Norwalk-like calicivirus genes in farm animals. Emerging Infectious Diseases, 6.

Pollard, T.M., 2008. Western Disease: An Evolutionary Perspective, Cambridge: Cambridge University Press.

Pollet, T. V. et al., 2015. Taking the aggravation out of data aggregation: A conceptual guide to dealing with statistical issues related to the pooling of individual-level observational data. American Journal of Primatology, (January 2014)

Price, D.T. \& Gebauer, A.B., 1995. New perspectives on the transition to agriculture. In D. T. Price \& A. B. Gebauer, eds. Last Hunters-First Farmers. Santa Fe: School of American Research Press, pp. 3-20.

Promislow, D.E.L., 1991. Senescence in Natural Populations of Mammals: A Comparative Study. Evolution, 45(8), pp.1869-1887.

Promislow, D.E.L. \& Harvey, P.H., 1990. Living fast and dying young: a comparative analysis of life-history variation among mammals. Journal of Zoology, London, 220, pp.417-437.

Quinlan, R.J., 2007. Human parental effort and environmental risk. Proceedings of the Royal Society B: Biological Sciences, 274(1606), pp.121-125.

Radtke, H. et al., 2005. Hemoglobin screening in prospective blood donors: Comparison of different blood samples and different quantitative methods. Transfusion and Apheresis Science, 33(1), pp.31-35.

Rai, N.K., 1982. From Forest to Field: A study of philippine Negrito Foragers in Transition. Hawaii.

Rai, N.K., 1990. Living in a lean-to: Philippine Negrito foragers in transition, Ann Arbor: University of Michigan.

Rand, D.G. \& Nowak, M. a., 2013. Human cooperation. Trends in Cognitive Sciences, 17(8), pp.413-425.

Rasmussen, S. et al., 2015. Early Divergent Strains of Yersinia pestis in Eurasia 5,000 Years Ago. Cell, 163(3), pp.571-582.

Rechner, I.J. et al., 2002. Evaluation of the HemoCue compared with the Coulter STKS for measurement of neonatal haemoglobin. Archives of disease in childhood. Fetal and neonatal edition, 86(3), pp.F188-9.

Reid, L. a, 2013. Who are the Philippine negritos? Evidence from language. Human biology, 85(1), pp.329-58.

Reinhard, K., Ambler, J.R. \& Mcguffie, M., 1985. Diet and Parasitism at Dust Devil cave. American Antiquity, 50(4), pp.819-824.

Reinhard, K.J., 1988. Cultural ecology of prehistoric parasitism on the Colorado Plateau as evidenced by coprology. American journal of physical anthropology, 77(3), pp.355-366.

Reinhard, K.J. \& Araujo, A., 2012. Synthesining parasitology with archaeology in paleopathology. In J. E. Buikstra \& C. Roberts, eds. The global history of Paleapathology. Oxford: Oxford University Press, pp. 751764. 
Richards, M.P., 2002. A brief review of the archaeological evidence for Palaeolithic and Neolithic subsistence. European journal of clinical nutrition, 56(12), p.16 p following 1262.

Richerson, P.J., Boyd, R. \& Bettinger, R.L., 2001. Was Agriculture Impossible during the Pleistocene but Mandatory during the Holocene? A Climate Change Hypothesis. American Antiquity, 66(3), pp.387-411.

Riehl, C., 2013. Evolutionary routes to non-kin cooperative breeding in birds. Proceedings of the Royal Society B: Biological Sciences, 280, p.20132245.

Rippmann, C.E. et al., 1997. Hemocue, an accurate bedside method of hemoglobin measurement? Journal of clinical monitoring, 13(6), pp.373-7.

Robson, S.L. \& Wood, B., 2008. Hominin life history: reconstruction and evolution. Journal of Anatomy, 212(4), pp.394-425.

Rook, G.A.W., 2009. Review series on helminths, immune modulation and the hygiene hypothesis: The broader implications of the hygiene hypothesis. Immunology, 126(1), pp.3-11.

Rosenblit, J. et al., 1999. Evaluation of three methods for hemoglobin measurement in a blood donor setting. São Paulo medical journal $=$ Revista paulista de medicina, 117(3), pp.108-12.

Rosenquist, J.N., Fowler, J.H. \& Christakis, N. a, 2011. Social network determinants of depression. Molecular psychiatry, 16(3), pp.273-281.

Roth, E.A., 1981. Sedentism and Changing Fertility Patterns in a Northern Athapascan Isolate. Journal of Human Evolution, 10(5), pp.413-425.

Roth, E.A. \& Ray, A.K., 1985. Demographic Patterns of Sedentary and Nomadic Juang of Orissa. Human Biology, 57(3), pp.319-325.

Royle, N.J. et al., 2012. Offspring social network structure predicts fitness in families. Proceedings of the Royal Society B: Biological Sciences, (October), pp.4914-4922.

Rubenstein, D.R. \& Lovette, I.J., 2007. Temporal Environmental Variability Drives the Evolution of Cooperative Breeding in Birds. Current Biology, 17, pp.1414-1419.

von Rueden, C., Gurven, M. \& Kaplan, H., 2011. Why do men seek status? Fitness payoffs to dominance and prestige. Proceedings. Biological sciences / The Royal Society, 278(1715), pp.2223-2232.

Ruff, C.B. et al., 2015. Gradual decline in mobility with the adoption of food production in Europe. Proceedings of the National Academy of Sciences, 112(23), p.201502932.

Russell, A.F., 2004. Mammals: Comparisons and Contrasts. In W. D. Koenig \& J. L. Dickinson, eds. Ecology and Evolution of Cooperative Breeding in Birds. Cambridge: Cambridge University Press, pp. 210-227.

Sahlins, M., 1968. Notes on the original affluent society. In R. Lee \& I. DeVore, eds. Man the Hunter. Chicago: Aldine, pp. 85-89.

Salali, G.D. \& Migliano, A.B., 2015. Future Discounting in Congo Basin Hunter-Gatherers Declines with Socio-Economic Transitions. PLoS ONE, 10(9), pp.1-10.

Sanchis-Gomar, F. et al., 2013. Hemoglobin Point-of-Care Testing: The HemoCue System. Journal of Laboratory Automation, 18(3), pp.198-205.

Santoro, C., Dorsey Vinton, S. \& Reinhard, K.J., 2003. Inca Expansion and Parasitism in the Lluta Valley: Preliminary Data. Memorias do Instituto Oswaldo Crur, 98(SUPPL. 1), pp.161-163.

Sari, M. et al., 2001. Estimating the prevalence of anaemia: A comparison of three methods. Bulletin of the World Health Organization, 79(6), pp.506-511.

Saxena, R. \& Malik, R., 2003. Comparison of HemoCue method with the cyanmethemoglobin method for estimation of hemoglobin. Indian pediatrics, 40(9), p.917.

Scelza, B. a., 2009. The grandmaternal niche: Critical caretaking among Martu Aborigines. American Journal of 
Human Biology, 21(November 2008), pp.448-454.

Schalk, E. et al., 2007. Use of capillary blood count parameters in adults. Vox sanguinis, 93(4), pp.348-53.

von Schenck, H., Falkensson, M. \& Lundberg, B., 1986. Evaluation of "HemoCue," a new device for determining hemoglobin. Clinical chemistry, 32(3), pp.526-9.

Scholes, C. et al., 2011. Genetic diversity and evidence for population admixture in Batak Negritos from Palawan. American Journal of Physical Anthropology, 146(1), pp.62-72.

Schülke, O. et al., 2010. Social Bonds Enhance Reproductive Success in Male Macaques. Current Biology, 20(24), pp.2207-2210.

Sear, R., 2015. Evolutionary Contributions to the Study of Human Fertility. Population Studies, 69, pp.S39-S55.

Sear, R., 2008. Kin and child survival in rural Malawi: Are matrilineal kin always beneficial in a matrilineal society? Human Nature, 19(Hrdy 1999), pp.277-293.

Sear, R. et al., 2002. The effects of kin on child mortality in rural Gambia. Evolution and Human Behavior, 24(1), pp.25-42.

Sear, R. \& Coall, D., 2011a. How Much Does Family Matter? Cooperative Breeding and the Demographic Transition. Population and Development Review, 37(March), pp.81-112.

Sear, R. \& Coall, D., 2011b. How Much Does Family Matter? Cooperative Breeding and the Demographic Transition. Population and Development Review, 37, pp.81-112.

Sear, R. \& Mace, R., 2009. Family matters : kin, demography and child health in a rural Gambian population. , pp.1-25.

Sear, R. \& Mace, R., 2008. Who keeps children alive? A review of the effects of kin on child survival. Evolution and Human Behavior, 29(1), pp.1-18.

Sear, R., Mace, R. \& McGregor, I. a, 2000. Maternal grandmothers improve nutritional status and survival of children in rural Gambia. Proceedings. Biological sciences / The Royal Society, 267(1453), pp.1641-1647.

Sear, R., Mace, R. \& McGregor, I.A., 2003. The effects of kin on female fertility in rural Gambia. Evolution and Human Behavior, 24, pp.25-42.

Seeman, T.E., 1996. Social ties and health: the benefits of social integration. Annals of epidemiology, 6(5), pp.442-451.

Seguin, P. et al., 2011. Determination of capillary hemoglobin levels using the HemoCue system in intensive care patients. Journal of Critical Care, 26(4), pp.423-427.

Sellen, D., 2006. Lactation, complementary feeding, and human life history. In K. Hakes \& R. R. Paine, eds. The Evolution of Human Life History. Santa Fe: SAR Press, pp. 155-196.

Sellen, D. \& Mace, R., 1997. Fertility and mode of subsistence: a phylogenetic analysis. Current Anthropology, 3(5), pp.878-889.

Shahshahani, H.J., Meraat, N. \& Mansouri, F., 2013. Evaluation of the validity of a rapid method for measuring high and low haemoglobin levels in whole blood donors. Blood transfusion = Trasfusione del sangue, 11(3), pp.385-90.

Shenk, M. \& Mattison, S.M., 2011. The rebirth of kinship. Human Nature, 22(1), pp.1-15.

Shennan, S. et al., 2013. Regional population collapse followed initial agriculture booms in mid-Holocene Europe. Nature Communications, 4, pp.1-8.

Silk, J.B. et al., 2010. Female chacma baboons form strong, equitable, and enduring social bonds. Behavioral Ecology and Sociobiology, 64(11), pp.1733-1747.

Silk, J.B., 1980. Kidnapping and female competition among captive bonnet macaques. Primates, 21:100-110. 
Silk, J.B., 2007a. Social components of fitness in primate groups. Science, 317(5843), pp.1347-1351.

Silk, J.B., 2007b. The adaptive value of sociality in mammalian groups. Philosophical transactions of the Royal Society of London. Series B, Biological sciences, 362(January), pp.539-559.

Silk, J.B. et al., 2009. The benefits of social capital: close social bonds among female baboons enhance offspring survival. Proceedings. Biological sciences / The Royal Society, 276(1670), pp.3099-3104.

Silk, J.B., Alberts, S.C. \& Altmann, J., 2006. Social relationships among adult female baboons (Papio cynocephalus) II. Variation in the quality and stability of social bonds. Behavioral Ecology and Sociobiology, 61(2), pp.197-204.

Smith, B.D., 2001. Low-level food production. Journal of Archaeological Research, 9(1), pp.1-43.

Smith, E.A. et al., 2010. Wealth Transmission and Inequality among Hunter-Gatherers. Current Anthropology, 51(1), pp.19-34.

Smith, E.A., 2004. Why do good hunters have higher reproductive success? Human Nature, 15(4), pp.343364.

Smith, K.P. \& Christakis, N. a., 2008. Social Networks and Health. Annual Review of Sociology, 34(1), pp.405429.

Snijders, T. a. B., 2005. Power and sample size in multilevel linear models. Encyclopedia of Statistics in Behavioral Science, 3, pp.1570-1573.

Snijders, T.A.B. \& Bosker, R.J., 2012. Multilevel Analysis: An Introduction to Basic and Advanced Multilevel Modeling 2nd ed., London: Sage.

Snopkowski, K. \& Sear, R., 2015. Grandparental help in Indonesia is directed preferentially towards needier descendants: a potential confounder when exploring grandparental influences on child health. Social Science \& Medicine, 128, pp.105-14.

Snopkowski, K. \& Sear, R., 2013. Kin influences on fertility in Thailand: Effects and mechanisms. Evolution and Human Behavior, 34(2), pp.130-138.

Solomon, N.G. \& French, J.A., 1997. The Study of Mammalian Cooperative Breeding. In N. G. Solomon \& J. A. French, eds. Cooperative Breeding in Mammals. Cambridge: Cambridge University Press, pp. 1-10.

Solomon, N.G. \& Hayes, L.D., 2009. The Biological Basis of Alloparental Behaviour in Mammals. In G. Bentley \& R. Mace, eds. Substitute Parents: Biological and Social perspective on alloparenting across human societies. Oxford: Berghahn Books, pp. 13-49.

De Souza, R.G., 2006. Body size and growth: The significance of chronic malnutrition among the Casiguran Agta. Annals of Human Biology, 33(5-6), pp.604-619.

Spielmann, N. et al., 2012. Accuracy and precision of hemoglobin point-of-care testing during major pediatric surgery. International journal of laboratory hematology, 34(1), pp.86-90.

Sprent, J.F.A., 1969. Helminth “zoonoses”: an analysis. Helminthol. Abstr., 38, pp.333-351.

Sreevatsan, S. et al., 1997. Restricted structural gene polymorphism in the Mycobacterium tuberculosis complex indicates evolutionarily recent global dissemination. Proceedings of the National Academy of Sciences of the United States of America, 94(18), pp.9869-74.

Srivastava, T. et al., 2014. Methods for Hemoglobin Estimation: A Review of “ What Works ." Journal of Hematology and Transfusion, 2(3), pp.2005-2006.

Stanton, M. a. \& Mann, J., 2012. Early Social Networks Predict Survival in Wild Bottlenose Dolphins. PLoS ONE, 7(10), pp.1-6.

Starling, A.P. \& Stock, J.T., 2007. Dental indicators of health and stress in early Egyptian and Nubian agriculturalists: A difficult transition and gradual recovery. American Journal of Physical Anthropology, 134(4), pp.520-528. 
Stearns, S., 1992. The evolution of life histories, Oxford: Oxford University Press.

Stearns, S.C., 2012. Evolutionary medicine: its scope, interest and potential. Proceedings of the Royal Society B: Biological Sciences, 279(1746), pp.4305-4321.

Stehlé, J., Voirin, N., Barrat, A., Cattuto, C., Isella, L., et al., 2011. High-Resolution Measurements of Face-toFace Contact Patterns in a Primary School. PLoS ONE, 6(8), p.e23176.

Stehlé, J., Voirin, N., Barrat, A., Cattuto, C., Colizza, V., et al., 2011. Simulation of an SEIR infectious disease model on the dynamic contact network of conference attendees. BMC medicine, 9(1), p.87.

Stephenson, C.B., Casebolt, D.B. \& Gangopadhyay, N.N., 1999. Phylogenetic analysis of a highly conserved region of the polymerasegene from 11 conronaviruses and development of a consensus polymerase chain reaction assay. Virus Research, 60, pp.181-189.

Stock, J. \& Migliano, A.B., 2009. Stature, Mortality, and Life History among Indigenous Populations of the Andaman Islands, 1871-1986. Current Anthropology, 50(5), pp.713-725.

Stock, J.T. \& Pinhasi, R., 2011. Changing paradigms in our understnading of the transition to agriculture: human bioarchaeology, behavoiur and adaptation. In Human Bioarchaeology of the Transition to Agriculture. Oxford: Wiley-Blackwell, pp. 1 - 16.

Strassmann, B.I., 2011. Cooperation and competition in a cliff-dwelling people. Proceedings of the National Academy of Sciences of the United States of America, 108, pp.10894-10901.

Strassmann, B.I. \& Garrard, W.M., 2011. Alternatives to the Grandmother Hypothesis. Human Nature, 22, pp.201-222.

Strassmann, B.I. \& Gillespie, B., 2002. Life-history theory, fertility and reproductive success in humans. Proceedings of the Royal Society B: Biological Sciences, 269(1491), pp.553-562.

Sueur, C. et al., 2011. How can social network analysis improve the study of primate behavior? American Journal of Primatology, 73(8), pp.703-719.

Sugiyama, L.S., 2004. Illness, injury, and disability among Shiwiar forager-horticulturalists: Implications of health-risk buffering for the evolution of human life history. American Journal of Physical Anthropology, 123(4), pp.371-389.

Sugiyama, L.S. \& Chacon, R., 2000. Effects of illness and injury on foraging among the Yora and Shiwiar: pathology risk as adaptive problem. Human behavior and adaptation, pp.371-395.

Tamashiro, K.L.K., Nguyen, M.M.N. \& Sakai, R.R., 2005. Social stress: From rodents to primates. Frontiers in Neuroendocrinology, 26(1), pp.27-40.

Tanner, S. et al., 2009. Influence of helminth infections on childhood nutritional status in lowland Bolivia. American Journal of Human Biology, 21(5), pp.651-656.

Tardif, S.D., 1997. The Bioenergetics of Parental Behaviour and the Evolution of Alloparent Care in Marmosets and Tamarins. In N. G. Solomon \& J. A. French, eds. Cooperative Breeding in Mammals. Cambridge: Cambridge University Press, pp. 11-33.

Tatsumi, N., Miwa, S. \& Lewis, S.M., 2002. Specimen Collection, Storage, and Transmission to the Laboratory for Hematological Tests. International Journal of Hematology, 75(3), pp.261-268.

Tayles, N., Domett, K.M. \& Halcrow, S., 2009. Can dental caries be interpreted as evidence of farming? The Asian experience. In T. Koppe, G. Meyer, \& K. W. Alt, eds. Comparative Dental Morpohology. Basel: Karger, pp. 162-166.

Tecot, S.R. et al., 2012. Infant parking and nesting, not allomaternal care, influence Malagasy primate life histories. Behavioral Ecology and Sociobiology, 66, pp.1375-1386.

Teli, M., Ng, Y. \& Ingram, R., 2002. Evaluation of the Hemocue portable hemoglobinometer after major joint arthroplasty. The Journal of arthroplasty, 17(2), pp.224-6. 
Therneau, T. et al., 2015. Kinship2 1.6.4.

Tinbergen, N., 1963. On aims and methods of Ethology. Zeitschrift für Tierpsychologie, 20, pp.410-433.

Tracer, D., 1991. Fertility related changes in maternal body composition among the Au of Papua New Guinea. American Journal of Physical Anthropology, 85(4), pp.393-406.

Tracer, D., 1996. Lactation, nutrition, and postpartum amenorrhea in lowland Papua New Guinea. Human biology; an international record of research, 68(2), pp.277-292.

Trivers, R.L., 1971. The Evolution of Reciprocal Altruism. Chicago Journals, 46(1), pp.35-57.

Trivers, R.L.L., 1972. Parental investment and sexual selection B. Campbell, ed., Chicago: Aldine.

Tucker, B. \& Young, A.G., 2005. Growing up Mikea: children's time allocation and tuber foraging in southwestern Madagascar. In B. Hewlett \& M. E. Lamb, eds. Hunter-gatherer childhoods. New York: Aldine de Gruyter, pp. 147-171.

Tymicki, K., 2004. Kin influence on female reproductive behavior: The evidence from reconstitution of the Bejsce parish registers, 18th to 20th centuries, Poland. American Journal of Human Biology, 16(October 2003), pp.508-522.

Ueno, T. \& Masuda, N., 2008. Controlling nosocomial infection based on structure of hospital social networks. Journal of Theoretical Biology, 254(3), pp.655-666.

Valeggia, C.R., 2009. Flexible caretakers: responses of Toba families in transition. In G. R. Bentley \& R. Mace, eds. Substitute Parents: Biological and Social perspective on alloparenting across buman societies. New York: Berghahn Books, pp. 100-114.

Vasunilashorn, S. et al., 2010. Blood lipids, infection, and inflammatory markers in the Tsimane of Bolivia. American Journal of Human Biology, 22(6), pp.731-740.

Vasunilashorn, S. et al., 2011. Inflammatory gene variants in the Tsimane, an indigenous Bolivian population with a high infectious load. Biodemography and social biology, 57(September 2013), pp.33-52.

Voland, E., Chasiotis, A. \& Schiefenhovel, W., 2005. Grandmotherhood: The evolutionary significance of the second half of female life, London: Rutgers University Press.

Waguespack, N.M., 2002. Colonization of the Americas: Disease Ecology and the Paleoindian Lifestyle. Human Ecology, 30(2), pp.227-243.

Wahed, A. \& Dasgupta, A., 2015. Hematology and Coagulations: A Comprehensive Review for Board Preparation, Certification and Clinical Practice., London: Elsevier.

Walker, P. \& Hewlett, B., 1990. Dental health diet and social status among Central African foragers and farmers. American Anthropologist, 92(2), pp.383-398.

Walker, R., Burger, O., et al., 2006. Evolution of brain size and juvenile periods in primates. Journal of Human Evolution, 51(5), pp.480-489.

Walker, R., Hill, K.R., et al., 2006. Life in the slow lane revisited: Ontogenetic separation between chimpanzees and humans. American Journal of Physical Anthropology, 129, pp.577-583.

Walker, R.S., 2014. Amazonian horticulturalists live in larger, more related groups than hunter-gatherers. Evolution and Human Behavior, 35(5), pp.384-388.

Wamboldt, M.Z., Gelhard, R.E. \& Insel, T.R., 1988. Gender differences in caring for infant Cebuella pygmaea: the role of infant age and relatedness. Developmental psychobiology, 21(2), pp.187-202.

Washburn, S.L. \& Lancaster, C.S., 1968. The evolution of hunting. In R. B. Lee \& I. Devore, eds. Man the Hunter. Chicago: Aldine Publishing Company, pp. 293-303.

Weber, N. et al., 2013. Badger social networks correlate with tuberculosis infection. Current Biology, 23(20), pp.R915-R916. 
Wells, J.C.K. \& Stock, J.T., 2007. The biology of the colonizing ape. Yearbook of Physical Anthropology, 50, pp.191-222.

West, S.A. et al., 2001. Testing Hamilton's rule with competition between relatives. Nature, 409(6819), pp.510-513.

West, S.A., Griffin, A.S. \& Gardner, A., 2007. Social semantics: altruism, cooperation, mutualism, strong reciprocity and group selection. Journal of Evolutionary Biology, 20(2), pp.415-432.

West, S.A., El Mouden, C. \& Gardner, A., 2011. Sixteen common misconceptions about the evolution of cooperation in humans. Evolution and Human Behavior, 32(4), pp.231-262.

Wey, T.W. et al., 2013. Reproductive correlates of social network variation in plurally breeding degus (Octodon degus). Animal Behaviour, 85(6), pp.1407-1414.

Wey, T.W. \& Blumstein, D.T., 2012. Social attributes and associated performance measures in marmots: Bigger male bullies and weakly affiliating females have higher annual reproductive success. Behavioral Ecology and Sociobiology, 66(7), pp.1075-1085.

Whitehead, H., 2008. Analyzing Animal Socities: Quantitative Methods for Vertebrate Social Analysis, London: The University of Chicago Press.

Whitehead, H., 2015. Life history evolution: What does a menopausal killer whale do? Current Biology, 25(6), pp.R225-R227.

Who, 2012. WHO Children reducing mortality. WHO Media centre, p.1. Available at: http://www.who.int/mediacentre/factsheets/fs178/en/.

Wich, S.A. et al., 2004. Life history of wild Sumatran orangutans (Pongo abelii). Journal of Human Evolution, 47(6), pp.385-398.

Wiessner, P., 1996. Levelling the Hunter: constraints on the status quest in foraging societies. In P. Wiessner \& W. Schiefenhovel, eds. Food and the status quest. Providence: Berghahn Books, pp. 171-192.

Wiessner, P., 2002. The Vines of Complexity: Egalitarian Structures and the Institutionalization of Inequality among the Enga. Current Anthropology, 43(2), pp.233-269.

Wilke, C. \& Wickham, H., 2015. cowplot.

Williams, G.C., 1957. Pleiotropy, Natural Selection, and the Evolution of Senescence. Evolution, 11, pp.398411.

Willis, A. \& Oxenham, M.F., 2013. The neolithic demographic transition and oral health: The Southeast Asian experience. American Journal of Physical Anthropology, 152(2), pp.197-208.

Wilson, E.O., 1978. On Human Nature, Cambridge (MA): Harvard University Press.

Wilson, E.O., 2008. One Giant Leap: How Insects Achieved Altruism and Colonial Life. BioScience, 58: 17.

Winterhalder, B., 1986. Diet choice, risk, and food sharing in a stochastic environment. Journal of Anthropological Archaeology, 5(4), pp.369-392.

Winterhalder, B., 1996. Social foraging and the behavioral ecology of intragroup resource transfers. Evolutionary Anthropology, 5, pp.46-57.

Winterhalder, B. \& Kennett, D.J., 2006. Behavioral Ecology and the transition from huntering and gathering to agriculture. In D. J. Kennett \& B. Winterhalder, eds. Behavioral ecology and the transition to agriculture. London: University of California Press, pp. 1-21.

Winterhalder, B., Lu, F. \& Tucker, B., 1999. Risk-senstive adaptive tactics: models and evidence from subsistence studies in biology and anthropology. Journal of Archaeological Research, 7(4), pp.1-3.

Winterhalder, B. \& Smith, E.A., 2000. Analyzing adaptive strategies: Human behavioral ecology at twentyfive. Evolutionary Anthropology: Issues, News and Reviews, 9(2), pp.51-72. 
Wittig, R.M. et al., 2008. Focused grooming networks and stress alleviation in wild female baboons. Hormones and behavior, 54(1), pp.170-7.

Wood, J.W., Johnson, P.L. \& Campbell, K.L., 1985. Demographic and endocrinological aspects of low natural fertility in Highland New Guinea. Journal of biosocial science, 17(1), pp.57-79.

Woodburn, J., 1982. Egalitarian Societies. Man, 17, pp.431-451.

Yellen, J.E., 1990. The Transformation of the Kalahari !Kung. Scientific American, 262(4), pp.96-105.

Zahid, H.J., Robinson, E. \& Kelly, R.L., 2015. Agriculture, population growth, and statistical analysis of the radiocarbon record. $P N A S$, p.1517650112

Zheng, H.-X. et al., 2012. MtDNA analysis of global populations support that major population expansions began before Neolithic Time. Scientific Reports, 2(745), pp.1-8.

Ziker, J. \& Schnegg, M., 2005. Food sharing at meals. Human Nature, 16(2), pp.178-210.

Zuur, A.F., Ieno, E.N. \& Elphick, C.S., 2010. A protocol for data exploration to avoid common statistical problems. Methods in Ecology and Evolution, 1(1), pp.3-14. 


\section{Appendices}

\section{Appendix A}

\section{Data collection documents}

This appendix contains the English-language originals of the information sheets, consent form, data collection protocols and data collection forms used in this research. 


\section{Participant Information Sheet}

Hunter-gatherer resilience: A project with University

College, London

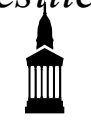

This project will be developed at University College London, by Dr Andrea Bamberg Migliano and participants of the "Hunter-Gatherer Resilience" project including $\mathrm{PhD}$ students and post-doctoral researchers.

The project aims to help us understand how hunter-gatherers live, and in what ways they are different from farmers. To help understand these differences, we will:

- Measure and weigh people to understand how people grow and change as they get older;

- Understand how the food people eat affects how they grow;

- Take sample of saliva to get DNA. DNA can tell us how you are related to other pygmies, why you are different from farmers, and why some people get sickness like malaria more often than others.

- Ask about recent illness and conduct medical tests to understand how healthy you and your family is;

- Collect information on who you and your children interact with;

- Ask about your family and way of life, to understand how you are related to each other.

Getting the sample of saliva is simple, and safe. If you agree to help us, after signing the consent form; you will be given a container in which to collect your own saliva. You will need to spit in it until it is half full, close it and return it to the researcher. The researcher will give it a number and date and take note of the number, your sex and village name.

This project is run through University College London, England, and is therefore in accordance with English Law. Data and any information will be treated as strictly confidential and handled in accordance with the provisions of the Data Protection Act, UK, 1998. 


\section{Participant Consent Form}

Hunter-gatherer resilience: A project with University College, London

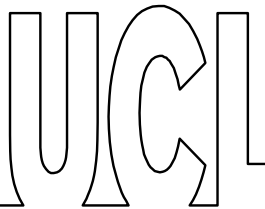

I have read (or, where appropriate, have had read to me) and understood the information above, and any questions I have asked have been answered to my satisfaction.

I agree to participate in the project, realising that I may physically withdraw from the study at any time and may request that no data arising from my participation are used, up to four weeks following the completion of my participation in the research.

I agree that research data provided by me or with my permission during the project may be included in a thesis, presented at conferences and published in journals on the condition that neither my name nor any other identifying information is used.

Name of participant:

Date:

$/ 2014$

Signature:

Name of authorised representative:

Date: $/ 2014$

Signature:

Name of researcher:

Date: $/ 2014$

Signature: 


\section{Field Protocols}

\section{Upon Arrival in Camp}

1. Introduce ourselves and display/explain posters to everyone so they know; 1) Why it is interesting; 2) How we will collect the data; and 3) What we want to find out

- Act this out ourselves first (1 person reads, other 2 act)

- Measurements; 1) Why? See how you grow and develop, 2) How? Measure and weight each of you, 3) Find out? How growth is related to diet and health

- Saliva; 1) Why? Everyone’s saliva is different (like fingerprints), 2) How? Spit into pot, 3) Find out? How you are related to other people/populations, and why some people get ill and others don't (also doing genealogies to look at this)

2. Do community mapping with knowledgeable informant(s) in two stages

$\circ$ 1) Inter-community information (where other villages are (inc. size and distance), where nonAgta/Mbendjele reside (inc. size and distance), nearby resources, paths/roads)

- 2) Intra-community information (number of households, size of households, names of everyone, approximate ages and life stages of all in camp)

3. At this time, we can also ask informant(s) for any important events which would be useful for aging (e.g., breakout of war, disease epidemics, other previous researchers)

4. Go around the camp introducing ourselves to everyone, taking pictures, and GPS coordinates (of both village and households - if resolution permits)

- write names (inc. all aliases)/ID/village/age cohort on back of photo

5. Explain that we will give compensation for their time and cooperation (rice, coffee, etc.)

○ For measuring children, offer honey stick/biscuit/sweet treat

\section{Order of Interviews}

1) Repeat information again and get consent from everyone (for children, obtain consent from parents/guardians)

2) Genealogies

3) Relative Aging

4) Saliva Samples and Measurements/Questions

\section{Genealogies}

\section{- $\quad$ Step-by-step Guide}

1. Explain what we are going to do/ask, and obtain informed consent from everyone by either a fingerprint or ' $\mathrm{X}$ ' on sheet (parents can do this on behalf of their children) 
2. On A4 paper, begin with ego (or matriarch/patriarch of family if conducted in groups), and below symbol write ID, name (and other aliases), and age (if known)

3. Another person who has the booklet then notes extra information (date of birth/death, cause of death, village/clan, etc.)

4. Repeat process with spouse/previous spouses (begin with first spouse, and end with current/most recent spouse)

5. Ask who their first child is, then second, then third, etc. and get the same information

a. Probe - To estimate the birth intervals, ask whether $x$ was walking/crawling/talking when next child was born

6. After have all children, go over again and ask whether anyone died young/miscarried between each pair, or whether they forgot anyone

7. Continue down order of kin using the same method (first/second/third-born sister/maternal cousin/paternal second cousins, followed by checking for any gaps of forgotten/dead people)

\section{- Order of questions}

- 1) Ego and spouse (including previous spouses)

- 2) Children and their offspring

- 3) Siblings and their offspring

- 4) Maternal relations (mother, mother's siblings, maternal cousins, maternal grandparents, etc.)

- 5) Paternal relations (father, father's siblings, paternal cousins, paternal grandparents, etc.)

\section{Accuracy, Consistency, and Detail Checks}

- To check for accuracy/consistency, each night after completing genealogies compare them to other overlapping ones, to make sure info is similar

- Any discrepancies can then be asked about the next day

- As more distant information is less likely to be accurate, best to try and find these distant kin and compare genealogies

- If different, give more weight to closer relations

- Match photos to genealogies, to ensure we have all pictures

\section{Relative Aging}

\section{- $\quad$ First Stage - Approximate Groupings}

1. Prepare photos of people in current camp and 2 or 3 of the nearest camps

a. Doesn't apply to first camps, but can go back over them at the end

2. Group photos into approximate ages based on rough age groupings (infant, child, teenager, young adult, middle-aged, old), or by approximate age (e.g., 0-5, 5-10, 10-15, 15-25, 25-35, 35$50,50+)$

a. Knowledge individual(s) could assist this to begin with before interviews 


\section{- Second Stage - Relative Aging}

1. Beginning with within-camp photos, get individuals (or groups) to arrange pictures in order of age centred around a target, one at a time

- First comparison: 'Is $x$ older or younger than you?'

- Second comparison: 'Is $y$ older or younger than you?', followed by, 'Is $y$ older or younger than $x$ ?'

- Continue until chain is built

2. Begin with within-camp comparisons, then add in between-camp comparisons after preliminary within-camp list has been made

- Also include photos of people from birth camp

- Need to make sure that Ego knows all of the targets

- Add open question of "Is there anyone else you know and would like to add to this list?"

3. Once list has been finalised, transcribe order into booklet

- Parents will make relative age lists of children, using the same method

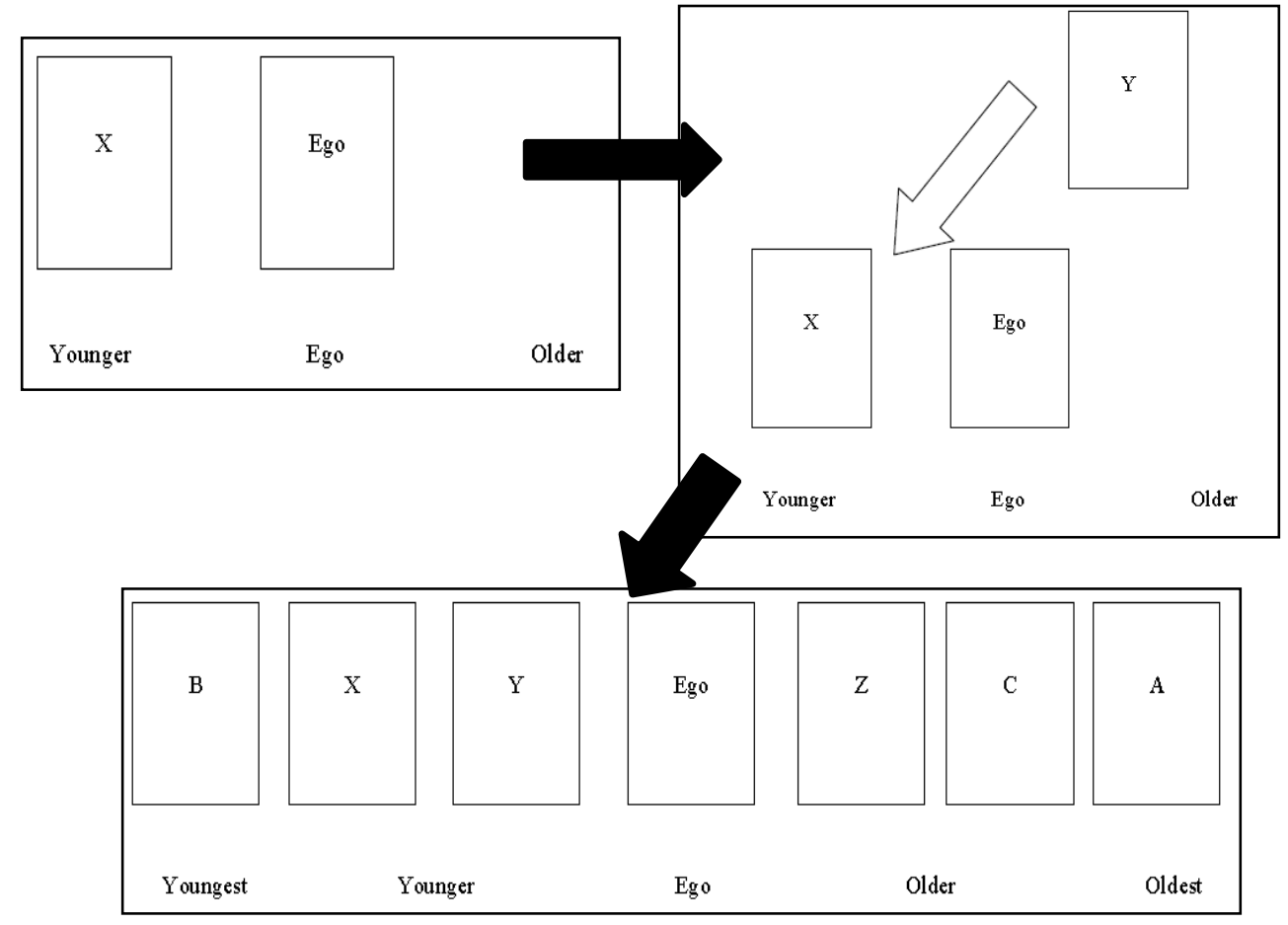

Figure 2: Example of relative aging process

\section{Deceased Individuals}

- Ask mother/siblings whether target was older/younger/contemporaneous with a known individual/event when they were born/died (Triangle method used in Ache)

- E.g., $x$ was born between $a$ and $b$, and died around the same time $c$ was born 


\section{Checking for Consistency and Accuracy}

- Consistency: As with Ache method, could take the most common arrangement to be the most likely, while using the amount of disagreement to assess probability of order (e.g., if 9/10 say $a$ $>b$, we can be fairly sure, but if only $6 / 10$ say $a>b$, we are less sure)

- Accuracy: Need records which can be dated absolutely and show birth orders (which may not be possible)

Physical measurement protocols

Jed Stevenson, Jimma Child Development Study (2007 / 2008)

\section{Weight}

1. Put the scale on a flat place on the floor. Turn on the scale.

2. Enter the height, sex, and age of the person. The scale is now ready to weigh a person.

3. Ask the individual to stand on the scale. You will see the weight displayed in kilograms (e.g. 68.3). Body fat, body water, and bone mass will then scroll through

4. For infants, weigh the Mother alone, then Mother with child, and subtract the two to get infant's weight.

\section{Length (for infants)}

1. Lay the infant flat on the ground (flat bit of ground, wooden board, etc.).

2. Position the child correctly:

a. One person should hold the child's head against the headboard. The child should look directly upward, and the crown of his/her head should touch the headboard.

b. Another person should straighten the child's legs. The child's toes should point directly upwards.

3. Record the measurement in centimeters to the nearest $0.1 \mathrm{~cm}$ using the anthropometer/tape measure.

\section{Height}

1. Put the anthropometer on a flat place on the ground.

2. Tell the individual to stand with the rod to his / her back.

3. Position the individual correctly:

a. One person should check the person's position:

i. The individual should look directly forward.

ii. The individual's toes should point directly forwards.

iii. Their back should be straight.

4. Another person should take the measurement:

a. Ask the person to breathe in, and move the gauge on the anthropometer down until level with the crown of the child's head.

i. Another person makes sure that the anthropometer is straight, and not tilted forwards or backwards.

5. Record the measurement in centimeters to the nearest $0.1 \mathrm{~cm}$. 


\section{Data collection forms}

1. To the following questions please answer always, sometimes or never true - ask the mother

\begin{tabular}{|l|l|l|l|}
\hline Question & Always & Sometimes & Never \\
\hline $\begin{array}{l}\text { 2. How often do you have food in your } \\
\text { household for more than the next day? }\end{array}$ & & & \\
\hline 3. We did not have enough to eat & & & \\
\hline 4. I was worried our food would run out & & & \\
\hline 5. I was not eating enough & & & \\
\hline $6 . \quad$ The children were not eating enough & & & \\
\hline 7. The children did not eat for one day & & & \\
\hline 8. I did not eat for one day & & & \\
\hline $\begin{array}{l}\text { 9. } \quad \text { I had to eat less/miss meals so my children } \\
\text { could eat }\end{array}$ & & & \\
\hline $\begin{array}{l}\text { 10. I am hungry but didn't eat because there } \\
\text { was not enough food }\end{array}$ & & & \\
\hline 11. $\begin{array}{l}\text { The children are hungry but didn't eat } \\
\text { because there was not enough food }\end{array}$ & & & \\
\hline
\end{tabular}

\section{Medical questionnaire}

\section{GASTRO-INTESTINAL DISEASE}

How many times in the last week have members of your family suffered from diarrhoea?

\begin{tabular}{|l|l|l|l|l|}
\hline Name & Not at all & Once & $\mathbf{3 - 4}$ times & Daily \\
\hline Mother & & & & \\
\hline Father & & & & \\
\hline & & & & \\
\hline & & & &
\end{tabular}

For individuals with severe diarrhoea are there any additional symptoms (please circle):

1.vomiting nausea mucus blood bloating fever cramps

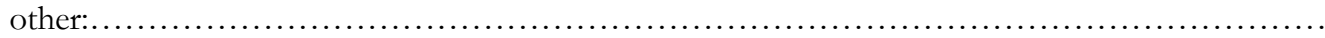

2.vomiting nausea mucus blood bloating fever cramps

3.vomiting nausea mucus blood bloating fever cramps

other:.

blood bloating fever

4.vomiting nausea mucus blood bloating fever cramps

other: ....

Did you seek any

treatment?.

\section{FLUS AND FEVERS}

How many times in the last two weeks have members of your family suffered from fever/flu?

\begin{tabular}{|l|l|l|l|l|}
\hline Name & Not at all & Once & $3-4$ times & Daily \\
\hline Mother & & & & \\
\hline Father & & & & \\
\hline & & & & \\
\hline & & & & \\
\hline
\end{tabular}

For individuals with recurrent fever are there any additional symptoms (please circle): 


\begin{tabular}{|c|c|c|c|c|}
\hline $\begin{array}{l}\text { 1.vomiting } \\
\text { other:........ }\end{array}$ & boils/wounds & $\begin{array}{c}\text { cough } \\
\ldots \ldots \ldots\end{array}$ & swollen gland & disorientation \\
\hline $\begin{array}{l}\text { 2.vomiting } \\
\text { other:........ }\end{array}$ & boils/wounds & cough & swollen gland & disorientation \\
\hline $\begin{array}{l}\text { 3.vomiting } \\
\text { other:....... }\end{array}$ & boils/wounds & cough & swollen gland & disorientation \\
\hline $\begin{array}{l}\text { 4.vomiting } \\
\text { other:....... }\end{array}$ & boils/wounds & cough & swollen gland & disorientation \\
\hline
\end{tabular}

\section{Did you seek any}

treatment?

\section{COUGHS}

Do any of your family members suffer from recurrent or persistent coughs? Are they characterised by:

\begin{tabular}{|c|c|c|c|c|c|c|c|c|c|}
\hline Name & $\begin{array}{l}\text { Dry or } \\
\text { cracking }\end{array}$ & $\begin{array}{l}\text { Wet or } \\
\text { phlegm }\end{array}$ & $\begin{array}{l}\text { Wheezing } \\
\text { or trouble } \\
\text { breathing }\end{array}$ & $\begin{array}{l}\text { Yellow } \\
\text { or green } \\
\text { phlegm }\end{array}$ & Blood & $\begin{array}{l}\text { Chest } \\
\text { or } \\
\text { back } \\
\text { pain }\end{array}$ & Thin & weak & $\begin{array}{l}\text { Chronic } \\
(>2 \\
\text { weeks })\end{array}$ \\
\hline \multicolumn{10}{|l|}{ Mother } \\
\hline \multicolumn{10}{|l|}{ Father } \\
\hline & & & & & & & & & \\
\hline & & & & & & & & & \\
\hline
\end{tabular}

Did you seek any

treatment?

\section{PARASITES}

Do any of your family members suffer from the following:

\begin{tabular}{|l|l|l|l|l|l|l|}
\hline Name & $\begin{array}{l}\text { Swollen } \\
\text { belly }\end{array}$ & $\begin{array}{l}\text { Sore } \\
\text { belly/indigestion }\end{array}$ & visual & $\begin{array}{l}\text { Itchy } \\
\text { anus }\end{array}$ & $\begin{array}{l}\text { Pain } \\
\text { urinating/blood }\end{array}$ & $\begin{array}{l}\text { Anaemic or very } \\
\text { pale/weak }\end{array}$ \\
\hline Mother & & & & & & \\
\hline Father & & & & & & \\
\hline & & & & & & \\
\hline & & & & & & \\
\hline
\end{tabular}

Did you seek any

treatment? 


\section{SKIN CONDITIONS}

Do any of your family members suffer from the following:

\begin{tabular}{|l|l|l|l|l|l|l|}
\hline Name & $\begin{array}{l}\text { Small } \\
\text { itchy } \\
\text { sores }\end{array}$ & $\begin{array}{l}\text { Sores } \\
\text { with pus }\end{array}$ & Boils & $\begin{array}{l}\text { Large dark } \\
\text { spots or patches }\end{array}$ & $\begin{array}{l}\text { Swollen } \\
\text { feet/hands/face }\end{array}$ & $\begin{array}{l}\text { Rings which } \\
\text { spread and itch }\end{array}$ \\
\hline Mother & & & & & & \\
\hline Father & & & & & & \\
\hline & & & & & & \\
\hline & & & & & & \\
\hline
\end{tabular}

Did you seek any

treatment?

\section{MALNUTRITION}

Signs of malnutrition:

\begin{tabular}{|l|l|l|l|l|l|}
\hline Name & Thinness & Potbelly & Wasting & Goitre & Vit A \\
\hline Mother & & & & & \\
\hline Father & & & & & \\
\hline & & & & & \\
\hline & & & & & \\
\hline & & & & & \\
\hline & & & & & \\
\hline & & & & & \\
\hline
\end{tabular}

\section{Food diary}

\begin{tabular}{|l|l|l|l|}
\hline Mother & & Follect/Give/Trade/Buy/Garden & Who? \\
\hline What time did you last eat? & & Collect/Give/Trade/Buy/Garden & Who? \\
\hline Food 1 & & Collect/Give/Trade/Buy/Garden & Who? \\
\hline Food 2 & \multicolumn{3}{|l|}{} \\
\hline Food 3 & $\begin{array}{l}\text { Fish/hunt/gather/garden/CL/None/ } \\
\text { What did you do today }\end{array}$ & Details (i.e. weight): \\
\hline Mother & $\begin{array}{l}\text { Fish/hunt/gather/garden/CL/None/ } \\
\text { Fick }\end{array}$ & Details (i.e. weight): \\
\hline
\end{tabular}




\section{Funding and ethics}

This research and fieldwork was approved by UCL Ethics Committee (UCL Ethics code 3086/003) and carried out with permission from local government and tribal leaders in Palanan. Informed consent was obtained from all participants, and parents signed the informed consents for their children (after group and individual consultation and explanation of the research objectives in the Agta language). As part of the process, people identified as having any diseases, were provided with medical care, in partnership with the local government and field hospital. 


\section{Camp Scan form}

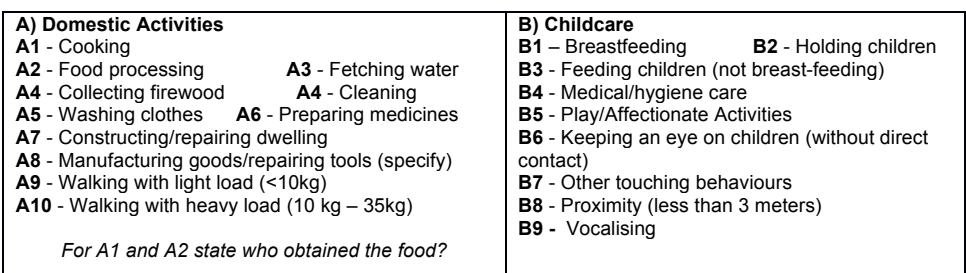
$\begin{array}{ll}\text { C) Out of Camp } & \text { C2 - Fishing } \\ \text { C1 - Hunting } & \text { C3 - Gathering wild foods } \\ \text { C4 - Collecting }\end{array}$
C5 - Collecting honey
C6 - Agricultural work on own land
C7 - Wage labour (specify which)
Cocity where and item traded)
- At school or accompanying child to school
(specify trade/own use for $\mathrm{C} 1, \mathrm{C} 2, \mathrm{~A} 8$ )
- Logging

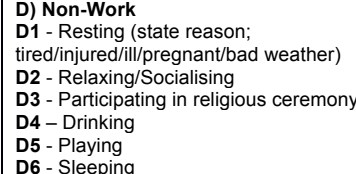

D3 - Participating in religious ceremony

D4 - Drinking

D5 - Playing

\begin{tabular}{|c|c|c|c|c|c|}
\hline \multicolumn{3}{|l|}{ Time: } & \multicolumn{3}{|l|}{ Time: } \\
\hline Activ. & Grp & Whose & Activ. & Grp & Whose \\
\hline & & & & & \\
\hline & & & & & \\
\hline & & & & & \\
\hline & & & & & \\
\hline & & & & & \\
\hline & & & & & \\
\hline & & & & & \\
\hline & & & & & \\
\hline & & & & & \\
\hline & & & & & \\
\hline & & & & ـ & \\
\hline & & & & 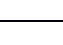 & \\
\hline & & & & 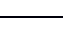 & \\
\hline & & & & & \\
\hline & & & & & \\
\hline & & & & & \\
\hline & & & & & \\
\hline & & & & & \\
\hline & & & & & \\
\hline & & & & & \\
\hline & & & & & \\
\hline & & & & & \\
\hline & & & & & \\
\hline
\end{tabular}




\section{Appendix B}

\section{Additional motes and aging methods from chapter three}

This appendix presents data collection and analysis collected by other members of the hunter-gatherer team which was used in this thesis. 


\section{Motes: Software and data recovery}

The embedded software developed in $\mathrm{C}$ and nes $\mathrm{C}$ for the experiment was written following an iterative process with many testing phases to adjust the parameters (frequency of messages, strength of wireless communications, length of phases of sleep to save battery and so forth) to their optimum values. In our application, each device sends messages every two minutes, receives messages from other devices within the programmed distance and stores them in a long-term memory. At the end of the experiment the device's memory can be downloaded to a computer via a PC side application written in JAVA. A message frequency of every two minutes was chosen due to the increased duration of the experiment of between five to six days (120 to 144 hours). We calculated that if each individual were in proximity to, on average, ten individuals then they would receive a maximum of 43,200 messages. At 12-bytes per message in our study this equates to 500 kilobytes or $25 \%$ of the $2 \mathrm{MB}$ capacity of the devices. This was a cautious estimate since we were unsure of the density of the camps. After the completion of the experiment we soon realised that we had only utilised on average $10-20 \%$ of the $2 \mathrm{MB}$ memory since individuals were in close contact with far fewer neighbours than we originally expected. Future deployments should include an altered parameter for messages to be sent and received each 30 seconds. Nonetheless, even with the message transmitted every two minutes, over the duration of a week we still collected an average of 3,150 message packets per individual, with a maximum of 20,876 . Such fine-grained spatial social network data has never been collected previously, thus remains a significantly amount of data.

Once the data from each mote have been recovered and stored on a computer, the raw data was then run through a stringent data processing system written in Python and leveraging the filtering power of MySQL databases. This ensured that the used data was free from corruption due to devices shock damage or water exposure. As each mote was recording data from an origin of time set at its start-up, all messages had to be put back on the real timescale after the experiment. To do this we recorded the start and stop time of each device and characterised the linear clock drift arising from using a basic crystal oscillator in the device for time keeping purposes. Then, we analysed all the messages received by each device and when we detected a reboot - time reset at zero - we used the 
time of the motes communicating with the device which did not reboot during this period to re-synchronise the focal device. Where most motes did not have reboots in their recorded data, the ones that did usually had many as they are often created by permanent damage to the device creating electronic instability of the systems. Once the initial filtering of the raw data was conducted the data is matched with individuals' ID and each interaction between dyads recorded in a database. The latter is finally transformed into a matrix containing the frequency of interactions between each dyad that took part in the experiment.

\section{Aging methodology}

In the Gibbs sampling Markov chain Monte Carlo (MCMC) algorithm each individual is allocated with an a priori age distribution; such as no younger than $i$ and no older than $u$ and between these two points, all ages are equally probable. This seeks to generate a posterior age probability distribution per individual which allows downstream analysis to incorporate the uncertainties associated with point estimates. As each individual is given a random age within their bracket, inconsistency occur which mean they cannot be allocated. For instance, if a younger individual has been estimated an age of 3-7, and the algorithm thus places ego at 6 years old, but the next individual has an known age of 5.5 but considered as older, the list is now inconsistent and the run is discarded. This process is repeated millions of times until only the runs in which all individuals in an age rank are assigned as age are kept.

This procedure can generate as many samples as desired; the more reduce the sampling error and thus, better approximate the underlying distribution. From this it is possible to compute summary statistics for each individual's age (mean, median and mode), since the data for each individual originates from multiple relative age lists. In order to assess the efficiency of this Gibbs sampler age estimation method, we compared it with third-order (Blurton Jones et al. 1992) and fifth-order (Hill \& Hurtado 1996) polynomial, and local regression approaches (Figure 1). We used a cohort of 65 Agta (Headland et al. 2011) for which we had both relative rankings and date of births. 
The cohort was partitioned into $\mathrm{z}=5$ partitions of $\mathrm{w}=13$ randomly sampled individuals (so that $\left.\mathrm{w}^{*} \mathrm{z}=65\right)$. Within one partition $\mathrm{z}_{\mathrm{i}}$, the date of birth of these 13 individuals were used to estimate the coefficients of the regression equations, where the date of births and the ranks were the independent and dependent variables, respectively. Once the equations had been obtained, the ages of the remaining $\mathrm{v}=52$ individuals (where $\mathrm{v}=65-\mathrm{w}$ ) were determined using the equations on the $\mathrm{v}$ ranks. The same $\mathrm{z}$ partitions and random set of $\mathrm{w}$ individuals were used with the Gibbs sampler to estimate the ages of the remaining $\mathrm{v}$ individuals. These procedures were repeated for the $z$ partitions, which provided $z-1$ age estimates for each individual. Since the dates of birth of the 65 individuals were known, we calculated the mean difference between the known and the estimated ages under each approach (three regression methods and the Gibbs sampler approaches). The best method would be the one that provides the lowest mean difference between the known and the estimated ages (Figure 1).

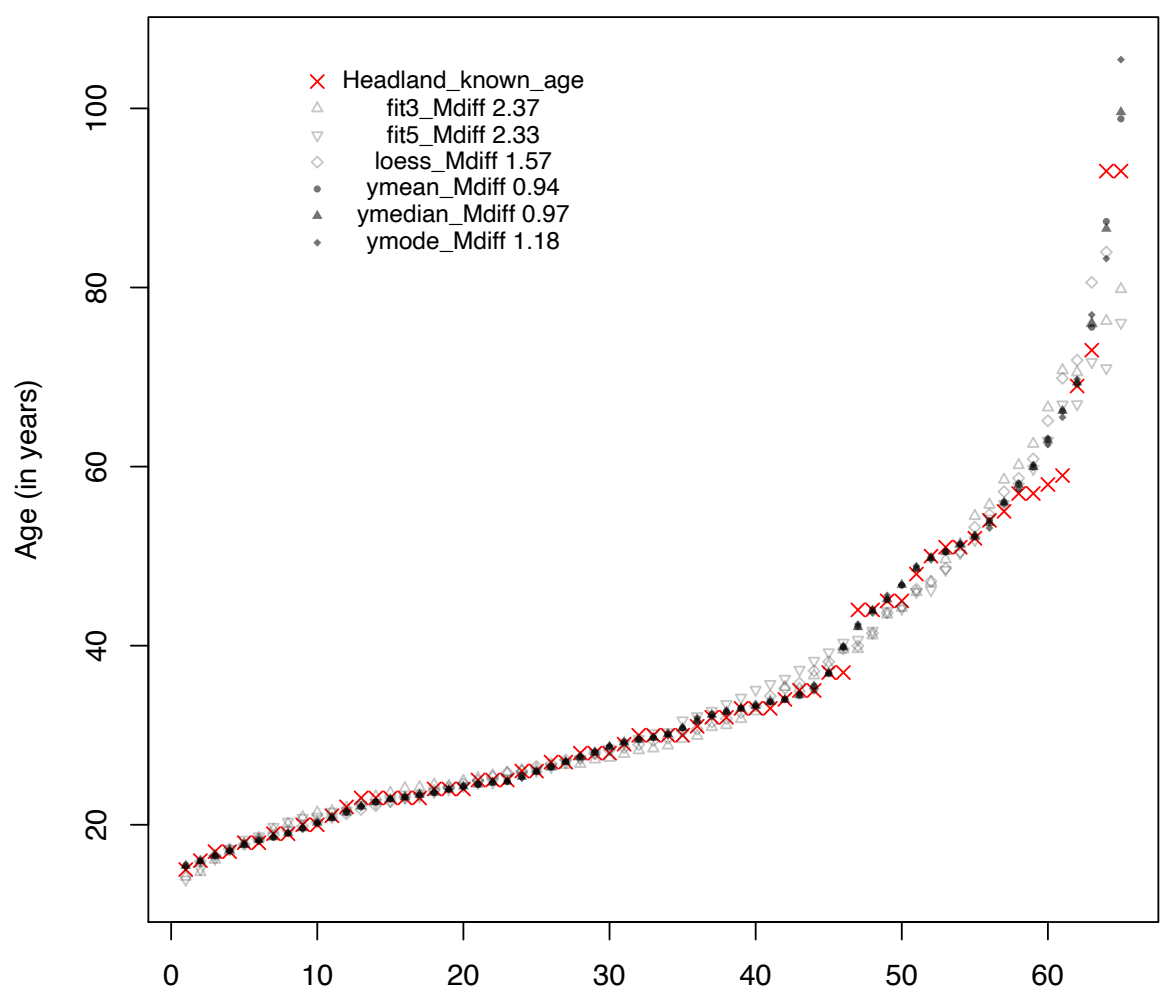

Figure 1: Quantification of aging techniques. Relationship between Headland's study population of known ages (red crosses) compared with previous methodologies of estimating ages in the literature (clear triangle pointing up, third-order polynomial regression; clear triangle point down, fifthorder polynomial regression; clear circle, local regression) and the Gibbs sampling method mean age (black circles). The mean difference between known and estimated ages for each of these methods is given in the top right-hand corner of the plot, being lowest (0.94) using the mean figure produced by the Gibbs sampler age model. 
Appendix C

Model outputs for chapter seven: Betwixt and between. 


\section{Model results}

In the following I have included models for null results not directly reported in the chapter. I have also included the intercept-only model for the computation of variance explained for each of the dependent variables. The proportion of variance which is attributed to each level is labelled VCP while the proportion reduction in variance comparative to the null-model is labelled as VAR. Significance values are given it $z$-scores; $z$-scores above 1.96 are taken as significant at $p=0.05$, while a $z$-score of 2.58 represents $p=0.01$ and a $z$-score of 3.33 or higher represents a $p$-value lower than 0.001 (Modelling 2015). The following models follow the order of results in the chapter: demographic results by centrality (fertility, reproduction and mortality); demographic results by kin and non-household care; mortality results by non-kin care; maternal haemoglobin levels, BMI and reported sickness by centrality; child haemoglobin levels, BMI and reported sickness by maternal centrality; allocare measures and child haemoglobin levels, BMI and reported sickness. 
Centrality and reproductive success

\begin{tabular}{|c|c|c|c|}
\hline \multicolumn{4}{|c|}{ Reproductive success Intercept-only model } \\
\hline AIC & BIC & & \\
\hline 90.1 & 95 & & \\
\hline \multicolumn{4}{|c|}{ Random effects: } \\
\hline Levels & Variance & SD & $\mathrm{VCP}$ \\
\hline Camp & 0.05492 & 0.2344 & 0.1 \\
\hline Residual & 0.49338 & 0.7024 & 0.9 \\
\hline \multicolumn{4}{|c|}{ Fixed effects: } \\
\hline & Estimate & SE & Z-score \\
\hline (Intercept) & 0.1001 & 0.153 & 0.654 \\
\hline
\end{tabular}

\begin{tabular}{|l|r|r|r|r|r|}
\hline \multicolumn{5}{|c|}{ Reproductive success EC model } \\
\hline AIC & \multicolumn{1}{|l|}{ BIC } & & & & \\
\hline 92.100 & 100.300 & & & & \\
\hline Random effects & Variance & \multicolumn{1}{l|}{ SD } & \multicolumn{1}{l|}{ VCP } & \multicolumn{1}{l|}{ VAR } & \\
\hline Levels & & & & & \\
\hline Camp & 0.042 & 0.204 & 0.081 & 0.242 & \\
\hline Residual & 0.475 & 0.689 & 0.919 & 0.038 & \\
\hline Fixed effects & & & & \\
\hline & Estimate & SE & Z-score & Lower CI & Upper CI \\
\hline Intercept) & 0.137 & 0.164 & 0.835 & -0.228 & 0.548 \\
\hline EC & -0.191 & 0.133 & -1.434 & -0.468 & 0.077 \\
\hline Age & -0.001 & 0.007 & -0.078 & -0.015 & 0.014 \\
\hline
\end{tabular}

\begin{tabular}{|l|r|r|r|r|r|}
\hline \multicolumn{5}{|c|}{ Reproductive success strength model } \\
\hline AIC & \multicolumn{1}{|l|}{ BIC } & & & & \\
\hline 91.400 & 99.600 & & & & \\
\hline Random effects & Variance & \multicolumn{1}{|l|}{ SD } & \multicolumn{1}{l|}{ VCP } & \multicolumn{1}{l|}{ VAR } & \\
\hline Levels & & & & & \\
\hline Camp & 0.054 & 0.233 & 0.106 & 0.009 & \\
\hline Residual & 0.458 & 0.677 & 0.894 & 0.071 & \\
\hline Fixed effects & & & & \\
\hline & Estimate & SE & Z-score & Lower CI & Upper CI \\
\hline (Intercept) & 0.157 & 0.171 & 0.922 & -0.222 & 0.593 \\
\hline Strength & -0.238 & 0.144 & -1.648 & -0.531 & 0.052 \\
\hline Age & -0.001 & 0.007 & -0.153 & -0.014 & 0.013 \\
\hline
\end{tabular}




\section{Centrality and fertility}

\begin{tabular}{|c|c|c|c|}
\hline \multicolumn{4}{|c|}{ Fertility Intercept-only model } \\
\hline AIC & BIC & & \\
\hline 90.100 & 95.000 & & \\
\hline \multicolumn{4}{|c|}{ Random effects: } \\
\hline Levels & Variance & SD & VCP \\
\hline Camp & 0.035 & 0.186 & 0.077 \\
\hline Residual & 0.416 & 0.645 & 0.923 \\
\hline \multicolumn{4}{|c|}{ Fixed effects: } \\
\hline & Estimate & SE & Z-score \\
\hline (Intercept) & 0.1581 & 0.1326 & 1.193 \\
\hline
\end{tabular}

\begin{tabular}{|c|c|c|c|c|c|}
\hline \multicolumn{6}{|c|}{ Fertility EC model } \\
\hline$\overline{\text { AIC }}$ & BIC & & & & \\
\hline 83.400 & 91.600 & & & & \\
\hline \multicolumn{6}{|c|}{ Random effects } \\
\hline Levels & Variance & $\mathrm{SD}$ & VCP & VAR & \\
\hline Camp & 0.121 & 0.348 & 0.264 & -2.502 & \\
\hline Residual & 0.338 & 0.581 & 0.736 & 0.187 & \\
\hline \multicolumn{6}{|c|}{ Fixed effects } \\
\hline & Estimate & SE & Z-score & Lower CI & Upper CI \\
\hline (Intercept) & 0.158 & 0.189 & 0.833 & -0.277 & 0.648 \\
\hline $\mathrm{EC}$ & -0.232 & 0.126 & -1.845 & 6.666 & 0.040 \\
\hline Age & 0.005 & 0.006 & 0.897 & -0.006 & 0.018 \\
\hline
\end{tabular}

\begin{tabular}{|l|r|r|r|r|r|}
\hline \multicolumn{5}{|c|}{ Fertility strength model } \\
\hline AIC & \multicolumn{1}{|l|}{ BIC } & & & & \\
\hline 84.600 & 92.800 & & & & \\
\hline Random effects & Variance & \multicolumn{1}{l|}{ SD } & \multicolumn{1}{l|}{ VCP } & \multicolumn{1}{l|}{ VAR } & \\
\hline Levels & 0.078 & 0.279 & 0.175 & -1.257 & \\
\hline Camp & & & & & \\
\hline Residual & 0.367 & 0.606 & 0.825 & 0.117 & \\
\hline Fixed effects & Estimate & SE & Z-score & Lower CI & Upper CI \\
\hline \multicolumn{1}{|l|}{} & & & & & \\
\hline Intercept) & 0.128 & 0.171 & 0.752 & -0.269 & 0.580 \\
\hline Strength & -0.157 & 0.118 & -1.328 & -0.369 & 0.090 \\
\hline Age & 0.006 & 0.006 & 0.919 & -0.007 & 0.018 \\
\hline
\end{tabular}


Centrality and mortality

\begin{tabular}{|c|c|c|c|c|c|}
\hline \multicolumn{6}{|c|}{ Mortality EC model } \\
\hline AIC & BIC & & & & \\
\hline 120.1 & 133.2 & & & & \\
\hline \multicolumn{6}{|c|}{ Random effects } \\
\hline Levels & Variance & SD & $\mathrm{VCP}$ & VAR & \\
\hline Camp & 0.000 & 0.000 & 0.000 & 1.000 & \\
\hline Residual & 0.906 & 0.952 & 1.000 & 0.071 & \\
\hline \multicolumn{6}{|c|}{ Fixed effects } \\
\hline & Estimate & SE & Z-score & Lower CI & Upper CI \\
\hline (Intercept) & -0.306 & 0.552 & -0.554 & -1.415 & 0.804 \\
\hline EC & 0.295 & 0.192 & 1.534 & -0.092 & 0.681 \\
\hline Age & 0.012 & 0.010 & 1.169 & -0.008 & 0.030 \\
\hline Fertility & 0.064 & 0.106 & 0.603 & -0.149 & 0.277 \\
\hline
\end{tabular}

\begin{tabular}{|l|l|l|l|}
\hline \multicolumn{4}{|c|}{ Mortality Intercept-only model } \\
\hline AIC & \multicolumn{1}{|l|}{ BIC } & & \\
\hline 115.700 & 120.600 & & \\
\hline Random effects: & \multicolumn{1}{|l|}{} \\
\hline Levels & Variance & SD & VCP \\
\hline Camp & 0.097 & 0.311 & 0.090 \\
\hline Residual & 0.975 & 0.987 & 0.910 \\
\hline Fixed effects: & & \\
\hline & Estimate & SE & Z-score \\
\hline (Intercept) & -0.024 & 0.210 & -0.116 \\
\hline
\end{tabular}

\begin{tabular}{|l|r|l|l|l|l|l|}
\hline \multicolumn{5}{|c|}{ Mortality betweenness model } \\
\hline AIC & BIC & & & & \\
\hline 121.900 & 135.000 & & & & \\
\hline Random effects & Variance & SD & VCP & VAR & \\
\hline Levels & 0.000 & 0.000 & 0.000 & 1.000 & \\
\hline Camp & 0.951 & 0.975 & 1.000 & 0.024 & \\
\hline Residual & & & & \\
\hline Fixed effects & & & & \\
\hline & Estimate & SE & Z-score & Lower CI & Upper CI \\
\hline (Intercept) & -0.166 & 0.571 & -0.290 & -1.315 & 0.983 \\
\hline Betweenness & -0.115 & 0.180 & -0.641 & -0.477 & 0.246 \\
\hline Age & 0.010 & 0.010 & 0.971 & -0.011 & 0.031 \\
\hline Fertility & 0.055 & 0.110 & 0.496 & -0.167 & 0.277 \\
\hline
\end{tabular}




\begin{tabular}{|c|c|c|c|c|c|}
\hline \multicolumn{6}{|c|}{ Mortality Strength model } \\
\hline AIC & BIC & & & & \\
\hline 120.300 & 133.400 & & & & \\
\hline \multicolumn{6}{|c|}{ Random effects } \\
\hline Levels & Variance & $\mathrm{SD}$ & $\mathrm{VCP}$ & VAR & \\
\hline Camp & 0.000 & 0.000 & 0.000 & 1.000 & \\
\hline Residual & 0.951 & 0.975 & 1.000 & 0.024 & \\
\hline \multicolumn{6}{|c|}{ Fixed effects } \\
\hline & Estimate & $\mathrm{SE}$ & Z-score & Lower $\mathrm{CI}$ & Upper CI \\
\hline (Intercept) & -0.328 & 0.555 & -0.591 & -1.445 & 0.788 \\
\hline Strength & 0.311 & 0.214 & 1.453 & -0.119 & 0.741 \\
\hline Age & 0.010 & 0.010 & 1.025 & -0.009 & 0.029 \\
\hline Fertility & 0.064 & 0.106 & 0.606 & -0.149 & 0.278 \\
\hline
\end{tabular}

\begin{tabular}{|c|c|c|c|c|c|}
\hline \multicolumn{6}{|c|}{ Mortality degree model } \\
\hline AIC & BIC & & & & \\
\hline 121.800 & 134.900 & & & & \\
\hline \multicolumn{6}{|c|}{ Random effects } \\
\hline Levels & Variance & $\mathrm{SD}$ & $\mathrm{VCP}$ & VAR & \\
\hline Camp & 0.000 & 0.000 & 0.000 & 1.000 & \\
\hline Residual & 0.947 & 0.973 & 1.000 & 0.029 & \\
\hline \multicolumn{6}{|c|}{ Fixed effects } \\
\hline & Estimate & SE & Z-score & Lower $\mathrm{CI}$ & Upper CI \\
\hline (Intercept) & -0.242 & 0.562 & -0.431 & -1.372 & 0.888 \\
\hline Degree & -0.221 & 0.286 & -0.772 & -0.797 & 0.355 \\
\hline Age & 0.011 & 0.010 & 1.063 & -0.010 & 0.031 \\
\hline Fertility & -0.011 & 0.124 & -0.087 & -0.260 & 0.238 \\
\hline
\end{tabular}


Allocare and mortality

\begin{tabular}{|l|r|r|r|r|r|r|}
\hline \multicolumn{7}{|c|}{ Mortality non-kin model } \\
\hline AIC & \multicolumn{1}{|l|}{ BIC } & & & & \\
\hline 97.500 & 109.700 & & & & \\
\hline Random effects & Variance & SD & VCP & \multicolumn{1}{l|}{ VAR } & \\
\hline Levels & 0.000 & 0.000 & 0.000 & 1.000 & \\
\hline Camp & 0.643 & 0.802 & 1.000 & 0.341 & \\
\hline Residual & & & & \\
\hline Fixed effects & Estimate & SE & Z-score & Lower CI & Upper CI \\
\hline & 0.111 & 0.501 & 0.222 & -0.900 & 1.122 \\
\hline (Intercept) & 0.067 & 0.155 & 0.430 & -0.246 & 0.380 \\
\hline Non-kin & 0.034 & 0.090 & 0.381 & -0.147 & 0.215 \\
\hline Fertility & -0.008 & 0.011 & -0.735 & -0.031 & 0.014 \\
\hline Age & & & & \\
\hline
\end{tabular}

\begin{tabular}{|c|c|c|c|c|c|}
\hline \multicolumn{6}{|c|}{ Mortality non-household model } \\
\hline AIC & BIC & & & & \\
\hline 97.200 & 109.400 & & & & \\
\hline \multicolumn{6}{|c|}{ Random effects } \\
\hline Levels & Variance & $\mathrm{SD}$ & $\mathrm{VCP}$ & VAR & \\
\hline Camp & 0.000 & 0.000 & 0.000 & 1.000 & \\
\hline Residual & 0.638 & 0.799 & 1.000 & 0.346 & \\
\hline \multicolumn{6}{|l|}{ Fixed effects } \\
\hline & Estimate & $\mathrm{SE}$ & Z-score & Lower CI & Upper CI \\
\hline (Intercept) & 0.094 & 0.499 & 0.188 & -0.914 & 1.102 \\
\hline non-household & 0.104 & 0.154 & 0.677 & -0.206 & 0.414 \\
\hline Fertility & 0.031 & 0.089 & 0.348 & -0.149 & 0.212 \\
\hline Age & -0.006 & 0.011 & -0.542 & -0.029 & 0.016 \\
\hline
\end{tabular}

\begin{tabular}{|l|r|r|r|r|r|r|}
\hline \multicolumn{5}{|c|}{ Mortality kin model } \\
\hline AIC & \multicolumn{1}{|c|}{ BIC } & & & & \\
\hline 99.000 & 111.200 & & & & \\
\hline Random effects & & & & \\
\hline Levels & Variance & SD & VCP & \multicolumn{1}{|l|}{ VAR } & \\
\hline Camp & 0.000 & 0.000 & 0.000 & 1.000 & \\
\hline Residual & 0.673 & 0.820 & 1.000 & 0.310 & \\
\hline Fixed effects & & & & \\
\hline & Estimate & SE & Z-score & Lower CI & Upper CI \\
\hline (Intercept) & -0.011 & 0.524 & -0.021 & -1.070 & 1.048 \\
\hline Kin & 0.013 & 0.162 & 0.081 & -0.314 & 0.341 \\
\hline Fertility & 0.054 & 0.091 & 0.595 & -0.129 & 0.237 \\
\hline Age & -0.008 & 0.011 & -0.702 & -0.030 & 0.012 \\
\hline
\end{tabular}


Allocare and reproductive success

\begin{tabular}{|l|r|r|r|r|l|l|}
\hline \multicolumn{7}{|c|}{ RS kin model } \\
\hline AIC & BIC & & & & \\
\hline 139.600 & 150.200 & & & & \\
\hline Random effects & & & & \\
\hline Levels & Variance & SD & VCP & VAR & \\
\hline Camp & 0.000 & 0.000 & 0.000 & 1.000 & \\
\hline Residual & 2.352 & 1.534 & 1.000 & 0.049 & \\
\hline Fixed effects & & & & \\
\hline & Estimate & SE & Z-score & Lower CI & Upper CI \\
\hline (Intercept) & 0.226981 & 0.967173 & 0.235 & -1.723465359 & 2.1774266 \\
\hline Kin & -0.004775 & 0.302004 & -0.016 & -0.613810207 & 0.6042607 \\
\hline Age & 0.02811 & 0.01927 & 1.458 & -0.0107572 & 0.06698053 \\
\hline
\end{tabular}

\begin{tabular}{|l|c|c|c|c|c|c|}
\hline \multicolumn{7}{|c|}{ RS non-household model } \\
\hline AIC & BIC & & & & \\
\hline 138.900 & 149.600 & & & & \\
\hline Random effects & Variance & SD & VCP & VAR & \\
\hline Levels & 0.000 & 0.000 & 0.000 & 1.000 & \\
\hline Camp & 2.306 & 1.519 & 1.000 & 0.068 & \\
\hline Residual & Estimate & SE & Z-score & Lower CI & Upper CI \\
\hline Fixed effects & 0.19339 & 0.9418 & 0.205 & -1.705879939 & 2.09266983 \\
\hline & 0.22106 & 0.28994 & 0.762 & -0.363649636 & 0.8057778 \\
\hline Intercept) & 0.02743 & 0.01833 & 1.496 & -0.01081878 & 0.06318491 \\
\hline non-household & Age & & & \\
\hline
\end{tabular}


Allocare and fertility

\begin{tabular}{|l|r|r|r|r|r|r|}
\hline \multicolumn{7}{|c|}{ Fertility kin model } \\
\hline AIC & BIC & & & & \\
\hline 140.300 & 151.000 & & & & \\
\hline Random effects & \multicolumn{5}{|l|}{} \\
\hline Levels & Variance & SD & VCP & \multicolumn{1}{l|}{ VAR } & \\
\hline Camp & 0.000 & 0.000 & 0.000 & 0.000 & \\
\hline Residual & 2.401 & 1.550 & 1.000 & 0.005 & \\
\hline Fixed effects & & & & \\
\hline & Estimate & SE & Z-score & Lower CI & Upper CI \\
\hline (Intercept) & -0.942 & 0.977 & -0.964 & -4.399 & 1.028 \\
\hline Kin & 0.184 & 0.305 & 0.601 & -0.432 & 0.799 \\
\hline Age & 0.010 & 0.009 & 1.134 & -0.007 & 0.027 \\
\hline
\end{tabular}

\begin{tabular}{|l|r|l|l|l|l|l|}
\hline \multicolumn{7}{|c|}{ Fertility non-household model } \\
\hline AIC & BIC & & & & \\
\hline 138.900 & 149.600 & & & & \\
\hline Random effects & Variance & SD & VCP & VAR & \\
\hline Levels & 0.000 & 0.000 & 0.000 & 0.000 & \\
\hline Camp & 2.343 & 1.531 & 1.000 & 0.029 & \\
\hline Residual & Estimate & SE & Z-score & Lower CI & Upper CI \\
\hline Fixed effects & -0.676 & 0.949 & -0.712 & -2.590 & 1.238 \\
\hline & 0.202 & 0.292 & 0.691 & -0.387 & 0.791 \\
\hline (Intercept) & 0.013 & 0.012 & 1.037 & -0.012 & 0.037 \\
\hline non-household & & & &
\end{tabular}




\section{Centrality and maternal sickness}

\begin{tabular}{|c|c|c|c|}
\hline \multicolumn{4}{|c|}{ Maternal sickness Intercept-only model } \\
\hline AIC & BIC & & \\
\hline 82.5 & 87.2 & & \\
\hline \multicolumn{4}{|c|}{ Random effects: } \\
\hline Levels & Variance & SD & $\mathrm{VCP}$ \\
\hline Camp & 0 & 0 & 0 \\
\hline Residual & 0.49 & 0.7 & 1 \\
\hline \multicolumn{4}{|c|}{ Fixed effects: } \\
\hline & Estimate & SE & Z-score \\
\hline (Intercept) & 0.8056 & 0.1167 & 6.905 \\
\hline
\end{tabular}

\begin{tabular}{|c|c|c|c|c|c|}
\hline \multicolumn{6}{|c|}{ Maternal sickness EC } \\
\hline AIC & BIC & & & & \\
\hline 82.400 & 95.000 & & & & \\
\hline \multicolumn{6}{|l|}{ Random effects } \\
\hline Levels & Variance & SD & $\mathrm{VCP}$ & VAR & \\
\hline Camp & 0.000 & 0.000 & 0.000 & 0.000 & \\
\hline Residual & 0.370 & 0.608 & 1.000 & 0.245 & \\
\hline \multicolumn{6}{|l|}{ Fixed effects } \\
\hline & Estimate & SE & Z-score & Lower CI & Upper CI \\
\hline (Intercept) & -0.942 & 0.977 & -0.964 & -4.399 & 1.028 \\
\hline EC & 0.184 & 0.305 & 0.601 & -0.432 & 0.799 \\
\hline Age & 0.010 & 0.009 & 1.134 & -0.007 & 0.027 \\
\hline Settled & 0.184 & 0.305 & 0.601 & -0.432 & 0.799 \\
\hline Household wealth & 0.010 & 0.009 & 1.134 & -0.007 & 0.027 \\
\hline
\end{tabular}

\begin{tabular}{|l|r|l|l|l|l|}
\hline \multicolumn{5}{|c|}{ Maternal sickness Strength } \\
\hline AIC & BIC & & & & \\
\hline \multicolumn{7}{|c|}{83.300} & 96.000 & & & & \\
\hline Random effects & Variance & SD & VCP & VAR & \\
\hline Levels & 0.000 & 0.000 & 0.000 & 0.000 & \\
\hline Camp & 2.343 & 1.531 & 1.000 & 0.029 & \\
\hline Residual & & & & \\
\hline Fixed effects & Estimate & SE & Z-score & Lower CI & Upper CI \\
\hline \multicolumn{7}{|c|}{0.291} & 0.367 & 0.794 & -0.449 & 1.031 \\
\hline (Intercept) & -0.083 & 0.139 & -0.594 & -0.363 & 0.197 \\
\hline Strength & 0.016 & 0.007 & 2.369 & 0.002 & 0.029 \\
\hline Age & 0.079 & 0.218 & 0.363 & -0.359 & 0.517 \\
\hline Settled & 0.056 & 0.167 & 0.332 & -0.281 & 0.392 \\
\hline Household wealth & & & & \\
\hline
\end{tabular}

\begin{tabular}{|c|c|c|c|c|c|}
\hline \multicolumn{6}{|c|}{ Maternal sickness degree } \\
\hline AIC & BIC & & & & \\
\hline 83.700 & 96.300 & & & & \\
\hline \multicolumn{6}{|l|}{ Random effects } \\
\hline Levels & Variance & $\mathrm{SD}$ & $\mathrm{VCP}$ & VAR & \\
\hline Camp & 0.000 & 0.000 & 0.000 & 0.000 & \\
\hline Residual & 0.383 & 0.619 & 1.000 & 0.218 & \\
\hline \multicolumn{6}{|l|}{ Fixed effects } \\
\hline & Estimate & SE & Z-score & Lower CI & Upper CI \\
\hline (Intercept) & 0.268517 & 0.367034 & 0.732 & -0.471627574 & 1.00865925 \\
\hline Degree & 0.009108 & 0.152394 & 0.06 & -0.297725897 & 0.31594147 \\
\hline Age & 0.015843 & 0.00661 & 2.397 & 0.002533823 & 0.02915241 \\
\hline Settled & 0.044457 & 0.210884 & 0.211 & -0.380802247 & 0.46971545 \\
\hline Household wealth & 0.036124 & 0.164903 & 0.219 & -0.295896146 & 0.36814353 \\
\hline
\end{tabular}




\section{Centrality and maternal BMI}

\begin{tabular}{|l|l|l|l|}
\hline \multicolumn{5}{|c|}{ Maternal BMI Intercept-only model } \\
\hline AIC & \multicolumn{1}{|l|}{ BIC } & & \\
\hline 179.500 & 184.500 & & \\
\hline Random effects: & \multicolumn{5}{|c|}{} \\
\hline Levels & Variance & SD & VCP \\
\hline Camp & 0.000 & 0.000 & 0.000 \\
\hline Residual & 5.008 & 2.238 & 1.000 \\
\hline Fixed effects: & Estimate & SE & Z-score \\
\hline & 18.266 & 0.358 & 50.970 \\
\hline (Intercept)
\end{tabular}

\begin{tabular}{|c|c|c|c|c|c|}
\hline \multicolumn{6}{|c|}{ Maternal BMI Strength } \\
\hline AIC & BIC & & & & \\
\hline 182.600 & 195.900 & & & & \\
\hline \multicolumn{6}{|l|}{ Random effects } \\
\hline Levels & Variance & SD & $\mathrm{VCP}$ & VAR & \\
\hline Camp & 0.000 & 0.000 & 0.000 & 0.000 & \\
\hline Residual & 4.193 & 2.048 & 1.000 & 0.163 & \\
\hline \multicolumn{6}{|l|}{ Fixed effects } \\
\hline & Estimate & SE & Z-score & Lower CI & Upper CI \\
\hline (Intercept) & 16.672 & 1.190 & 14.007 & 14.277 & 19.279 \\
\hline Strength & -0.304 & 0.452 & -0.672 & -1.211 & 0.604 \\
\hline Age & -0.045 & 0.021 & -2.118 & -0.088 & -0.002 \\
\hline Settled & 1.044 & 0.701 & 1.489 & -0.365 & 2.452 \\
\hline Household wealth & 0.305 & 0.549 & 0.555 & -0.798 & 1.407 \\
\hline
\end{tabular}

\begin{tabular}{|c|c|c|c|c|c|}
\hline \multicolumn{6}{|c|}{ Maternal BMI EC } \\
\hline AIC & BIC & & & & \\
\hline 182.800 & 196.100 & & & & \\
\hline \multicolumn{6}{|l|}{ Random effects } \\
\hline Levels & Variance & $\mathrm{SD}$ & $\mathrm{VCP}$ & VAR & \\
\hline Camp & 0.000 & 0.000 & 0.000 & 0.000 & \\
\hline Residual & 4.217 & 2.054 & 1.000 & 0.158 & \\
\hline \multicolumn{6}{|l|}{ Fixed effects } \\
\hline & Estimate & SE & Z-score & Lower CI & Upper CI \\
\hline (Intercept) & 16.620 & 1.189 & 13.977 & 13.202 & 19.106 \\
\hline $\mathrm{EC}$ & -0.192 & 0.409 & -0.470 & -1.014 & 0.630 \\
\hline Age & -0.045 & 0.021 & -2.086 & -0.087 & -0.002 \\
\hline Settled & 1.008 & 0.702 & 1.436 & -0.402 & 2.417 \\
\hline Household wealth & 0.273 & 0.547 & 0.499 & -0.826 & 1.372 \\
\hline
\end{tabular}

\begin{tabular}{|c|c|c|c|c|c|}
\hline \multicolumn{6}{|c|}{ Maternal BMI betweenness } \\
\hline AIC & BIC & & & & \\
\hline 83.700 & 96.300 & & & & \\
\hline \multicolumn{6}{|l|}{ Random effects } \\
\hline Levels & Variance & SD & $\mathrm{VCP}$ & VAR & \\
\hline Camp & 0.000 & 0.000 & 0.000 & 0.000 & \\
\hline Residual & 4.229 & 2.057 & 1.000 & 0.184 & \\
\hline \multicolumn{6}{|l|}{ Fixed effects } \\
\hline & Estimate & $\mathrm{SE}$ & Z-score & Lower CI & Upper CI \\
\hline (Intercept) & 16.512 & 1.189 & 13.885 & 14.122 & 18.901 \\
\hline Betweenness & 0.124 & 0.374 & 0.331 & -0.628 & 0.875 \\
\hline Age & -0.043 & 0.022 & -1.995 & -0.087 & 0.000 \\
\hline Settled & 0.971 & 0.694 & 1.399 & -0.423 & 2.365 \\
\hline Household wealth & 0.215 & 0.540 & 0.398 & -0.870 & 1.300 \\
\hline
\end{tabular}




\section{Centrality and maternal haemoglobin}

\begin{tabular}{|c|c|c|c|c|c|}
\hline \multicolumn{6}{|c|}{ Maternal RBC EC } \\
\hline AIC & BIC & & & & \\
\hline 345.8 & 350.6 & & & & \\
\hline \multicolumn{6}{|l|}{ Random effects } \\
\hline Levels & Variance & SD & $\mathrm{VCP}$ & VAR & \\
\hline Camp & 0.000 & 0.000 & 0.000 & 1.000 & \\
\hline Residual & 627.500 & 25.050 & 1.000 & 0.134 & \\
\hline \multicolumn{6}{|l|}{ Fixed effects } \\
\hline & Estimate & SE & Z-score & Lower CI & Upper CI \\
\hline (Intercept) & 87.84935 & 15.24165 & 5.764 & 57.1614172 & 118.5372843 \\
\hline $\mathrm{EC}$ & 1.77594 & 5.12868 & 0.346 & -8.5502775 & 12.1021631 \\
\hline Age & -0.37339 & 0.27081 & -1.379 & -0.9186441 & 0.1718738 \\
\hline Settled & -2.72238 & 8.80246 & -0.309 & -20.4454848 & 15.0007259 \\
\hline Household wealth & 11.47289 & 6.84307 & 1.677 & -2.3051285 & 25.2509083 \\
\hline
\end{tabular}

\begin{tabular}{|l|l|l|l|}
\hline \multicolumn{5}{|c|}{ Maternal RBC Intercept-only model } \\
\hline AIC & BIC & & \\
\hline 179.500 & 184.500 & & \\
\hline Random effects: & \multicolumn{5}{l|}{} \\
\hline Levels & Variance & SD & VCP \\
\hline Camp & 12.900 & 3.591 & 0.018 \\
\hline Residual & 724.200 & 26.911 & 0.982 \\
\hline Fixed effects: & Estimate & SE & Z-score \\
\hline & 107.526 & 4.761 & 22.59 \\
\hline (Intercept)
\end{tabular}

\begin{tabular}{|c|c|c|c|c|c|}
\hline \multicolumn{6}{|c|}{ Maternal RBC Strength } \\
\hline AIC & BIC & & & & \\
\hline 349.000 & 361.700 & & & & \\
\hline \multicolumn{6}{|l|}{ Random effects } \\
\hline Levels & Variance & SD & $\mathrm{VCP}$ & VAR & \\
\hline Camp & 0.000 & 0.000 & 0.000 & 1.000 & \\
\hline Residual & 610.000 & 24.700 & 1.000 & 0.158 & \\
\hline \multicolumn{6}{|l|}{ Fixed effects } \\
\hline & Estimate & $\mathrm{SE}$ & Z-score & Lower CI & Upper CI \\
\hline (Intercept) & 86.628 & 15.022 & 5.767 & 56.383 & 116.873 \\
\hline Strength & 6.012 & 5.586 & 1.076 & -5.235 & 17.258 \\
\hline Age & -0.357 & 0.267 & -1.336 & -0.895 & 0.181 \\
\hline Settled & -4.438 & 8.746 & -0.507 & -22.048 & 13.172 \\
\hline Household wealth & 10.506 & 6.792 & 1.547 & -3.168 & 24.181 \\
\hline
\end{tabular}

\begin{tabular}{|c|c|c|c|c|c|}
\hline \multicolumn{6}{|c|}{ Maternal RBC betweenness } \\
\hline AIC & BIC & & & & \\
\hline 350.100 & 362.800 & & & & \\
\hline \multicolumn{6}{|l|}{ Random effects } \\
\hline Levels & Variance & SD & VCP & VAR & \\
\hline Camp & 0.000 & 0.000 & 0.000 & 1.000 & \\
\hline Residual & 628.100 & 25.060 & 1.000 & 0.133 & \\
\hline \multicolumn{6}{|l|}{ Fixed effects } \\
\hline & Estimate & SE & Z-score & Lower CI & Upper CI \\
\hline (Intercept) & 89.028 & 15.261 & 5.834 & 58.302 & 119.754 \\
\hline Degree & -1.423 & 4.779 & -0.298 & -11.044 & 8.198 \\
\hline Age & -0.387 & 0.276 & -1.403 & -0.942 & 0.168 \\
\hline Settled & -2.513 & 8.728 & -0.288 & -20.086 & 15.061 \\
\hline Household wealth & 11.785 & 6.785 & 1.737 & -1.875 & 25.445 \\
\hline
\end{tabular}




\begin{tabular}{|l|r|r|r|r|r|}
\hline \multicolumn{7}{|c|}{ Maternal RBC Degree } \\
\hline AIC & \multicolumn{1}{|c|}{ BIC } & & & & \\
\hline \multicolumn{7}{|c|}{349.200} & 361.800 & & & & \\
\hline Random effects \\
\hline Levels & Variance & SD & VCP & VAR & \\
\hline Camp & 0.000 & 0.000 & 0.000 & 1.000 & \\
\hline Residual & 612.000 & 24.740 & 1.000 & 0.155 & \\
\hline Fixed effects & & & & & \\
\hline & Estimate & SE & Z-score & Lower CI & Upper CI \\
\hline (Intercept) & 88.593 & 14.950 & 5.926 & 58.492 & 118.693 \\
\hline Degree & 6.239 & 6.136 & 1.017 & -6.115 & 18.593 \\
\hline Age & -0.345 & 0.269 & -1.283 & -0.886 & 0.196 \\
\hline Settled & -2.496 & 8.483 & -0.294 & -19.576 & 14.584 \\
\hline Household wealth & 11.279 & 6.716 & 1.679 & -2.243 & 24.802 \\
\hline
\end{tabular}




\section{Centrality and child haemoglobin}

\begin{tabular}{|l|l|l|l|}
\hline \multicolumn{4}{|c|}{ Child RBC Intercept-only model } \\
\hline AIC & \multicolumn{1}{|l|}{ BIC } & & \\
\hline 733.000 & 742.600 & & \\
\hline Random effects: & & \\
\hline Levels & Variance & SD & VCP \\
\hline Camp & 0.000 & 0.000 & 0.000 \\
\hline Household & 69.460 & 8.334 & 0.150 \\
\hline Residual & 392.460 & 19.811 & 0.850 \\
\hline Fixed effects: & & \\
\hline \multicolumn{4}{|r|}{} \\
\hline
\end{tabular}

\begin{tabular}{|c|c|c|c|c|c|}
\hline \multicolumn{6}{|c|}{ Child RBC EC } \\
\hline AIC & BIC & & & & \\
\hline 669.100 & 696.600 & & & & \\
\hline \multicolumn{6}{|l|}{ Random effects } \\
\hline Levels & Variance & SD & $\mathrm{VCP}$ & VAR & \\
\hline Camp & 0.000 & 0.000 & 0.000 & 1.000 & \\
\hline Household & 0.000 & 0.000 & 0.000 & 1.000 & \\
\hline Residual & 403.300 & 20.080 & 1.000 & -0.028 & \\
\hline \multicolumn{6}{|l|}{ Fixed effects } \\
\hline & Estimate & SE & Z-score & Lower CI & Upper CI \\
\hline (Intercept) & 103.133 & 18.668 & 5.525 & 64.940 & 141.025 \\
\hline Maternal EC & 4.258 & 4.036 & 1.055 & -3.868 & 12.417 \\
\hline Age & 0.948 & 0.759 & 1.250 & -0.559 & 2.456 \\
\hline Maternal RBC & -0.196 & 0.122 & -1.605 & -0.439 & 0.047 \\
\hline Child sex & 6.007 & 4.998 & 1.202 & -3.919 & 15.934 \\
\hline Settlement & 7.719 & 5.421 & 1.424 & -3.047 & 18.484 \\
\hline Dependents & 0.689 & 1.955 & 0.353 & -3.193 & 4.571 \\
\hline Household wealth & 4.441 & 3.527 & 1.259 & -2.563 & 11.445 \\
\hline
\end{tabular}

\begin{tabular}{|c|c|c|c|c|c|}
\hline \multicolumn{6}{|c|}{ Child RBC Strength } \\
\hline AIC & BIC & & & & \\
\hline 669.300 & 696.800 & & & & \\
\hline \multicolumn{6}{|l|}{ Random effects } \\
\hline Levels & Variance & SD & $\mathrm{VCP}$ & VAR & \\
\hline Camp & 0.000 & 0.000 & 0.000 & 1.000 & \\
\hline Household & 0.000 & 0.000 & 0.000 & 1.000 & \\
\hline Residual & 404.100 & 20.100 & 1.000 & -0.030 & \\
\hline \multicolumn{6}{|l|}{ Fixed effects } \\
\hline & Estimate & SE & Z-score & Lower CI & Upper $\mathrm{Cl}$ \\
\hline (Intercept) & 108.155 & 19.404 & 5.574 & 68.825 & 146.709 \\
\hline Maternal strength & 4.061 & 4.137 & 0.982 & -4.155 & 12.277 \\
\hline Maternal BMI & -0.225 & 0.131 & -1.721 & -0.485 & 0.035 \\
\hline Child age & 0.955 & 0.760 & 1.257 & -0.554 & 2.465 \\
\hline Child sex & 6.057 & 5.006 & 1.210 & -3.884 & 15.998 \\
\hline Settlement & 7.346 & 5.638 & 1.303 & -3.890 & 18.547 \\
\hline Dependents & 0.237 & 1.790 & 0.132 & -3.318 & 3.934 \\
\hline Household wealth & 4.569 & 3.516 & 1.300 & -2.413 & 11.551 \\
\hline
\end{tabular}


Centrality and child BMI

\begin{tabular}{|c|c|c|c|}
\hline \multicolumn{4}{|c|}{ Child BMI Intercept-only model } \\
\hline AIC & BIC & & \\
\hline 222.300 & 232.300 & & \\
\hline \multicolumn{4}{|c|}{ Random effects: } \\
\hline Levels & Variance & SD & $\mathrm{VCP}$ \\
\hline Camp & 0.164 & 0.405 & 0.228 \\
\hline Household & 0.000 & 0.000 & 0.000 \\
\hline Residual & 0.553 & 0.743 & 0.772 \\
\hline \multicolumn{4}{|c|}{ Fixed effects: } \\
\hline & Estimate & SE & Z-score \\
\hline (Intercept) & 0.093 & 0.184 & 0.506 \\
\hline
\end{tabular}

\begin{tabular}{|c|c|c|c|c|c|}
\hline \multicolumn{6}{|c|}{ Child BMI EC } \\
\hline AIC & BIC & & & & \\
\hline 227.600 & 257.800 & & & & \\
\hline \multicolumn{6}{|l|}{ Random effects } \\
\hline Levels & Variance & SD & $\mathrm{VCP}$ & VAR & \\
\hline Camp & 0.074 & 0.273 & 0.127 & 0.546 & \\
\hline Household & 0.000 & 0.000 & 0.000 & 0.000 & \\
\hline Residual & 0.509 & 0.713 & 0.873 & 0.079 & \\
\hline \multicolumn{6}{|l|}{ Fixed effects } \\
\hline & Estimate & SE & Z-score & Lower CI & Upper CI \\
\hline (Intercept) & -1.118 & 0.910 & -1.228 & -2.968 & 0.748 \\
\hline Maternal EC & 0.015 & 0.128 & 0.114 & -0.240 & 0.270 \\
\hline Maternal BMI & 0.109 & 0.042 & 2.611 & 0.026 & 0.191 \\
\hline Settlement & -0.350 & 0.312 & -1.122 & -1.015 & 0.554 \\
\hline Child sex & -0.145 & 0.166 & -0.875 & -0.473 & 0.184 \\
\hline Child age & -0.025 & 0.024 & -1.012 & -0.073 & 0.024 \\
\hline Dependents & 0.063 & 0.066 & 0.956 & -0.082 & 0.196 \\
\hline Household wealth & -0.098 & 0.151 & -0.650 & -0.447 & 0.335 \\
\hline
\end{tabular}




\begin{tabular}{|c|c|c|c|c|c|}
\hline \multicolumn{6}{|c|}{ Child BMI Strength } \\
\hline AIC & BIC & & & & \\
\hline 227.500 & 257.600 & & & & \\
\hline \multicolumn{6}{|l|}{ Random effects } \\
\hline Levels & Variance & SD & $\mathrm{VCP}$ & VAR & \\
\hline Camp & 0.070 & 0.265 & 0.121 & 0.570 & \\
\hline Household & 0.000 & 0.000 & 0.000 & 0.000 & \\
\hline Residual & 0.509 & 0.714 & 0.879 & 0.079 & \\
\hline \multicolumn{6}{|l|}{ Fixed effects } \\
\hline & Estimate & SE & Z-score & Lower CI & Upper CI \\
\hline (Intercept) & -1.206 & 0.909 & -1.327 & -3.059 & 0.655 \\
\hline Maternal strength & 0.056 & 0.128 & 0.439 & -0.199 & 0.312 \\
\hline Maternal BMI & 0.113 & 0.042 & 2.673 & 0.029 & 0.197 \\
\hline Settlement & -0.385 & 0.309 & -1.249 & -1.040 & 0.508 \\
\hline Child sex & -0.145 & 0.166 & -0.877 & -0.473 & 0.184 \\
\hline Child age & -0.024 & 0.024 & -0.977 & -0.073 & 0.025 \\
\hline Dependents & 0.073 & 0.062 & 1.174 & -0.064 & 0.198 \\
\hline Household wealth & -0.121 & 0.152 & -0.799 & -0.469 & 0.314 \\
\hline
\end{tabular}

\begin{tabular}{|c|c|c|c|c|c|}
\hline \multicolumn{6}{|c|}{ Child BMI Degree } \\
\hline AIC & BIC & & & & \\
\hline 227.600 & 257.700 & & & & \\
\hline \multicolumn{6}{|l|}{ Random effects } \\
\hline Levels & Variance & SD & $\mathrm{VCP}$ & VAR & \\
\hline Camp & 0.067 & 0.260 & 0.116 & 0.588 & \\
\hline Household & 0.000 & 0.000 & 0.000 & 0.000 & \\
\hline Residual & 0.511 & 0.715 & 0.884 & 0.075 & \\
\hline \multicolumn{6}{|l|}{ Fixed effects } \\
\hline & Estimate & SE & Z-score & Lower CI & Upper $\mathrm{Cl}$ \\
\hline (Intercept) & -1.144 & 0.901 & -1.270 & -2.947 & 0.740 \\
\hline Maternal degree & 0.037 & 0.158 & 0.233 & -0.081 & 0.414 \\
\hline Maternal BMI & 0.111 & 0.043 & 2.565 & -0.016 & 0.203 \\
\hline Child age & -0.365 & 0.298 & -1.225 & 0.000 & -0.219 \\
\hline Child sex & -0.131 & 0.175 & -0.749 & -0.149 & 0.219 \\
\hline Settlement & -0.025 & 0.024 & -1.028 & -0.060 & 0.015 \\
\hline Dependents & 0.062 & 0.055 & 1.137 & -0.042 & 0.170 \\
\hline Household wealth & -0.104 & 0.143 & -0.728 & 0.000 & -0.034 \\
\hline
\end{tabular}


Centrality and child reported sickness

\begin{tabular}{|c|c|c|c|}
\hline \multicolumn{4}{|c|}{ Child sickness Intercept-only model } \\
\hline AIC & BIC & & \\
\hline 194.500 & 221.400 & & \\
\hline \multicolumn{4}{|c|}{ Random effects: } \\
\hline Levels & Variance & SD & VCP \\
\hline Camp & 0.103 & 0.322 & 0.175 \\
\hline Household & 0.011 & 0.105 & 0.019 \\
\hline Residual & 0.477 & 0.691 & 0.806 \\
\hline \multicolumn{4}{|c|}{ Fixed effects: } \\
\hline & Estimate & SE & Z-score \\
\hline (Intercept) & 0.093 & 0.184 & 0.506 \\
\hline
\end{tabular}

\begin{tabular}{|c|c|c|c|c|c|}
\hline \multicolumn{6}{|c|}{ Child Sickness EC } \\
\hline AIC & BIC & & & & \\
\hline 196.400 & 225.700 & & & & \\
\hline \multicolumn{6}{|l|}{ Random effects } \\
\hline Levels & Variance & SD & VCP & VAR & \\
\hline Household & 0.000 & 0.000 & 0.000 & 1.000 & \\
\hline Camp & 0.000 & 0.000 & 0.000 & 1.000 & \\
\hline Residual & 0.445 & 0.667 & 1.000 & 0.067 & \\
\hline \multicolumn{6}{|l|}{ Fixed effects } \\
\hline & Estimate & SE & Z-score & Lower CI & Upper CI \\
\hline (Intercept) & 1.145 & 0.343 & 3.343 & 0.466 & 1.825 \\
\hline Child age & -0.015 & 0.024 & -0.622 & -0.061 & 0.032 \\
\hline Maternal EC & 0.049 & 0.120 & 0.411 & -0.189 & 0.288 \\
\hline Maternal sickness & 0.419 & 0.133 & 3.159 & 0.156 & 0.681 \\
\hline Settlement & 0.268 & 0.167 & 1.603 & -0.063 & 0.599 \\
\hline Child sex & -0.090 & 0.156 & -0.579 & -0.399 & 0.219 \\
\hline Dependents & 0.013 & 0.064 & 0.198 & -0.114 & 0.139 \\
\hline Household wealth & -0.246 & 0.106 & -2.324 & -0.456 & -0.036 \\
\hline
\end{tabular}




\begin{tabular}{|c|c|c|c|c|c|}
\hline \multicolumn{6}{|c|}{ Child Sickness Strength } \\
\hline AIC & BIC & & & & \\
\hline 196.400 & 225.800 & & & & \\
\hline \multicolumn{6}{|l|}{ Random effects } \\
\hline Levels & Variance & SD & $\mathrm{VCP}$ & VAR & \\
\hline Camp & 0.000 & 0.000 & 0.000 & 1.000 & \\
\hline Household & 0.000 & 0.000 & 0.000 & 1.000 & \\
\hline Residual & 0.445 & 0.667 & 1.000 & 0.066 & \\
\hline \multicolumn{6}{|l|}{ Fixed effects } \\
\hline & Estimate & SE & Z-score & Lower CI & Upper CI \\
\hline (Intercept) & 1.162 & 0.337 & 3.452 & 0.495 & 1.830 \\
\hline Maternal strength & 0.037 & 0.115 & 0.323 & -0.191 & 0.265 \\
\hline Maternal sickness & 0.414 & 0.133 & 3.108 & 0.150 & 0.677 \\
\hline Child age & -0.015 & 0.024 & -0.616 & -0.061 & 0.032 \\
\hline Settlement & 0.274 & 0.166 & 1.645 & -0.056 & 0.603 \\
\hline Child sex & -0.089 & 0.156 & -0.569 & -0.399 & 0.221 \\
\hline Dependents & 0.008 & 0.061 & 0.124 & -0.113 & 0.128 \\
\hline Household wealth & -0.245 & 0.107 & -2.281 & -0.458 & -0.032 \\
\hline
\end{tabular}

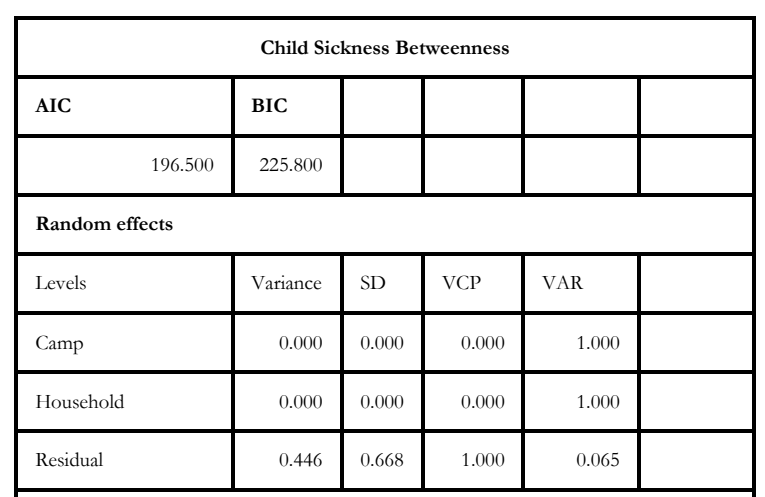

\begin{tabular}{|l|r|l|r|r|r|}
\hline Fixed effects & Estimate & \multicolumn{1}{c|}{ SE } & Z-score & Lower CI & Upper CI \\
\hline (Intercept) & 1.174 & 0.335 & 3.500 & 0.509 & 1.839 \\
\hline Maternal betweenness & 0.011 & 0.085 & 0.126 & -0.158 & 0.180 \\
\hline Maternal sickness & 0.414 & 0.136 & 3.042 & 0.144 & 0.683 \\
\hline Child age & -0.014 & 0.024 & -0.598 & -0.061 & 0.033 \\
\hline Settlement & 0.295 & 0.158 & 1.863 & -0.019 & 0.608 \\
\hline Child sex & -0.094 & 0.156 & -0.601 & -0.403 & 0.216 \\
\hline Dependents & -0.003 & 0.054 & -0.060 & -0.110 & 0.104 \\
\hline Household wealth & -0.234 & 0.102 & -2.297 & -0.435 & -0.032 \\
\hline
\end{tabular}

\begin{tabular}{|c|c|c|c|c|c|}
\hline \multicolumn{6}{|c|}{ Child Sickness Degree } \\
\hline AIC & BIC & & & & \\
\hline 195.100 & 224.400 & & & & \\
\hline \multicolumn{6}{|l|}{ Random effects } \\
\hline Levels & Variance & SD & $\mathrm{VCP}$ & VAR & \\
\hline Camp & 0.000 & 0.000 & 0.000 & 1.000 & \\
\hline Household & 0.000 & 0.000 & 0.000 & 1.000 & \\
\hline Residual & 0.438 & 0.662 & 1.000 & 0.081 & \\
\hline \multicolumn{6}{|l|}{ Fixed effects } \\
\hline & Estimate & $\mathrm{SE}$ & Z-score & Lower CI & Upper CI \\
\hline (Intercept) & 1.225 & 0.332 & 3.690 & 0.567 & 1.883 \\
\hline Maternal degree & -0.154 & 0.127 & -1.215 & -0.407 & 0.098 \\
\hline Maternal sickness & 0.427 & 0.132 & 3.238 & 0.165 & 0.688 \\
\hline Child age & -0.015 & 0.023 & -0.626 & -0.061 & 0.032 \\
\hline Settlement & 0.318 & 0.157 & 2.029 & 0.007 & 0.628 \\
\hline Child sex & -0.160 & 0.164 & -0.974 & -0.485 & 0.166 \\
\hline Dependents & -0.018 & 0.054 & -0.333 & -0.126 & 0.090 \\
\hline Household wealth & -0.243 & 0.101 & -2.401 & -0.443 & -0.042 \\
\hline
\end{tabular}


Allocare and child RBC

\begin{tabular}{|c|c|c|c|c|c|c|c|c|c|c|c|}
\hline \multicolumn{6}{|c|}{ Child RBC household care } & \multicolumn{6}{|c|}{ Child RBC kin care } \\
\hline AIC & BIC & & & & & AIC & BIC & & & & \\
\hline 690.100 & 718.100 & & & & & 703.100 & 731.400 & & & & \\
\hline \multicolumn{6}{|l|}{ Random effects } & \multicolumn{6}{|l|}{ Random effects } \\
\hline \multirow[t]{2}{*}{ Levels } & Variance & $\mathrm{SD}$ & VCP & VAR & & Levels & Variance & SD & $\mathrm{VCP}$ & VAR & \\
\hline & & & & & & Camp & 0.000 & 0.000 & 0.000 & 1.000 & \\
\hline Camp & 0.000 & 0.000 & 0.000 & 1.000 & & Household & 11.800 & 3.435 & 0.033 & 0.515 & \\
\hline Household & 0.000 & 0.000 & 0.000 & 1.000 & & Residual & 342.500 & 18.510 & 0.967 & 0.105 & \\
\hline Residual & 375.100 & $\begin{array}{r}19.37 \\
0\end{array}$ & 1.000 & 0.020 & & \multicolumn{6}{|l|}{ Fixed effects } \\
\hline \multicolumn{6}{|l|}{ Fixed effects } & & Estimate & SE & Z-score & Lower CI & Upper CI \\
\hline & $\begin{array}{l}\text { Estimat } \\
\mathrm{e}\end{array}$ & SE & $\begin{array}{l}Z- \\
\text { score }\end{array}$ & $\begin{array}{l}\text { Lower } \\
\text { CI }\end{array}$ & $\begin{array}{l}\text { Upper } \\
\text { CI }\end{array}$ & (Intercept) & 99.061 & 17.340 & 5.713 & 63.216 & 133.701 \\
\hline (Intercept) & 99.585 & $\begin{array}{r}17.45 \\
2\end{array}$ & 5.706 & 64.626 & 134.191 & Kin care & -1.104 & 2.614 & -0.422 & -6.293 & 4.314 \\
\hline $\begin{array}{l}\text { Non-household } \\
\text { care }\end{array}$ & -5.796 & 2.577 & -1.249 & -11.006 & 0.053 & Maternal RBC & -0.092 & 0.119 & -0.775 & -0.337 & 0.156 \\
\hline Maternal RBC & -0.169 & 0.115 & -1.466 & -0.398 & 0.060 & Child age & 0.820 & 0.690 & 1.188 & -0.528 & 2.193 \\
\hline Child age & 0.968 & 0.722 & 1.342 & -0.464 & 2.400 & Child sex & 4.530 & 4.521 & 1.002 & -4.562 & 13.652 \\
\hline Child sex & 5.636 & 4.722 & 1.194 & -3.737 & 15.008 & Settlement & 9.020 & 4.849 & 1.860 & -1.106 & 18.964 \\
\hline Settlement & 13.848 & 5.054 & 2.740 & 3.816 & 23.879 & Household wealth & 3.820 & 3.417 & 1.118 & -3.127 & 13.102 \\
\hline Household wealth & 6.655 & 3.413 & 1.950 & -0.121 & 13.431 & Dependents & -0.013 & 1.520 & -0.009 & -3.029 & 3.150 \\
\hline Dependents & 0.324 & 1.485 & 0.218 & -2.623 & 3.270 & & & & & & \\
\hline
\end{tabular}


Allocare and child sickness

\begin{tabular}{|c|c|c|c|c|c|}
\hline \multicolumn{6}{|c|}{ Child sickness non-kin care } \\
\hline AIC & BIC & & & & \\
\hline 186.500 & 212.700 & & & & \\
\hline \multicolumn{6}{|l|}{ Random effects } \\
\hline Levels & Variance & SD & $\mathrm{VCP}$ & VAR & \\
\hline Camp & 0.000 & 0.000 & 0.000 & 1.000 & \\
\hline Household & 0.000 & 0.000 & 0.000 & 1.000 & \\
\hline Residual & 0.458 & 0.676 & 1.000 & 0.040 & \\
\hline \multicolumn{6}{|l|}{ Fixed effects } \\
\hline & Estimate & SE & Z-score & Lower CI & Upper CI \\
\hline (Intercept) & 1.236 & 0.304 & 4.068 & 0.633 & 1.839 \\
\hline Non-kin & 0.023 & 0.094 & 0.243 & -0.164 & 0.210 \\
\hline Maternal sickness & 0.402 & 0.140 & 2.884 & 0.126 & 0.679 \\
\hline Child age & -0.014 & 0.025 & -0.565 & -0.063 & 0.035 \\
\hline Child sex & -0.088 & 0.163 & -0.539 & -0.411 & 0.235 \\
\hline Settlement & 0.315 & 0.168 & 1.878 & -0.018 & 0.649 \\
\hline Household wealth & -0.233 & 0.108 & -2.166 & -0.447 & -0.020 \\
\hline Dependents & 0.324 & 1.485 & 0.218 & -2.623 & 3.270 \\
\hline
\end{tabular}

\begin{tabular}{|c|c|c|c|c|c|}
\hline \multicolumn{6}{|c|}{ Child sickness non-household care } \\
\hline AIC & BIC & & & & \\
\hline 703.100 & 731.400 & & & & \\
\hline \multicolumn{6}{|l|}{ Random effects } \\
\hline Levels & Variance & SD & VCP & VAR & \\
\hline Camp & 0.000 & 0.000 & 0.000 & 1.000 & \\
\hline Household & 0.000 & 0.000 & 0.000 & 1.000 & \\
\hline Residual & 0.458 & 0.677 & 1.000 & 0.040 & \\
\hline \multicolumn{6}{|l|}{ Fixed effects } \\
\hline & Estimate & SE & Z-score & Lower CI & Upper CI \\
\hline (Intercept) & 1.244 & 0.352 & 3.535 & 0.546 & 1.942 \\
\hline Non-household care & 0.002 & 0.089 & 0.028 & -0.173 & 0.178 \\
\hline Maternal sickness & 0.418 & 0.146 & 2.865 & 0.129 & 0.708 \\
\hline Child age & -0.014 & 0.025 & -0.555 & -0.063 & 0.036 \\
\hline Child sex & -0.091 & 0.165 & -0.550 & -0.418 & 0.236 \\
\hline Settlement & 0.324 & 0.167 & 1.935 & -0.008 & 0.656 \\
\hline Household wealth & -0.227 & 0.107 & -2.122 & -0.439 & -0.011 \\
\hline Dependents & -0.001 & 0.054 & -0.025 & -0.109 & 0.106 \\
\hline
\end{tabular}


Allocare and BMI

\begin{tabular}{|c|c|c|c|c|c|c|c|c|c|c|c|}
\hline \multicolumn{6}{|c|}{ Child BMI non-household care } & \multicolumn{6}{|c|}{ Child BMI kin care } \\
\hline AIC & BIC & & & & & AIC & BIC & & & & \\
\hline 210.900 & 240.300 & & & & & 222.200 & 251.900 & & & & \\
\hline \multicolumn{6}{|l|}{ Random effects } & \multicolumn{6}{|l|}{ Random effects } \\
\hline Levels & $\begin{array}{l}\text { Varianc } \\
\mathrm{e}^{\text {Van }}\end{array}$ & $\mathrm{SD}$ & $\mathrm{VCP}$ & VAR & & Levels & Variance & SD & $\mathrm{VCP}$ & VAR & \\
\hline Camp & 0.000 & $\begin{array}{r}0.00 \\
0\end{array}$ & 0.000 & 1.000 & & & & & & & \\
\hline Household & 0.000 & $\begin{array}{r}0.00 \\
0\end{array}$ & 0.000 & 1.000 & & Camp & 0.056 & 0.236 & 0.096 & 0.659 & \\
\hline Residual & 0.514 & $\begin{array}{r}0.71 \\
7\end{array}$ & 1.000 & 0.069 & & Household & 0.000 & 0.000 & 0.000 & 1.000 & \\
\hline \multicolumn{6}{|l|}{ Fixed effects } & Residual & 0.523 & 0.723 & 0.904 & 0.054 & \\
\hline & $\begin{array}{l}\text { Estimat } \\
\mathrm{e}\end{array}$ & SE & $\begin{array}{l}Z \text { - } \\
\text { score }\end{array}$ & $\begin{array}{l}\text { Lower } \\
\text { CI }\end{array}$ & $\begin{array}{l}\text { Upper } \\
\mathrm{CI}\end{array}$ & \multicolumn{6}{|l|}{ Fixed effects } \\
\hline (Intercept) & -0.981 & $\begin{array}{r}0.79 \\
0\end{array}$ & -1.243 & -2.547 & 0.584 & & Estimate & SE & Z-score & Lower CI & Upper CI \\
\hline $\begin{array}{l}\text { Non-household } \\
\text { care }\end{array}$ & -0.282 & $\begin{array}{r}0.09 \\
0\end{array}$ & -1.147 & -0.460 & 0.014 & (Intercept) & -0.878 & 0.887 & -0.990 & -2.718 & 0.899 \\
\hline Maternal BMI & 0.117 & $\begin{array}{r}0.03 \\
9\end{array}$ & 2.969 & 0.039 & 0.196 & Kin care & -0.067 & 0.098 & -0.682 & -0.281 & 0.138 \\
\hline Child age & -0.019 & $\begin{array}{r}0.02 \\
5\end{array}$ & -0.771 & -0.069 & 0.031 & Maternal BMI & 0.100 & 0.042 & 2.392 & 0.063 & 0.166 \\
\hline Child sex & -0.131 & $\begin{array}{r}0.17 \\
0\end{array}$ & -0.770 & -0.468 & 0.206 & Child age & -0.032 & 0.025 & -1.263 & -0.070 & 0.008 \\
\hline Settlement & -0.428 & $\begin{array}{r}0.17 \\
5\end{array}$ & -2.445 & -0.775 & -0.081 & Child sex & -0.130 & 0.170 & -0.767 & -0.247 & 0.177 \\
\hline Household wealth & -0.222 & $\begin{array}{r}0.11 \\
1\end{array}$ & -2.003 & -0.441 & -0.002 & Settlement & -0.438 & 0.280 & -1.563 & -1.042 & 0.010 \\
\hline Dependents & 0.066 & $\begin{array}{r}0.04 \\
9\end{array}$ & 1.352 & -0.031 & 0.162 & Household wealth & -0.151 & 0.142 & -1.059 & -0.498 & 0.304 \\
\hline & & & & & & Dependents & 0.060 & 0.056 & 1.080 & -0.068 & 0.174 \\
\hline
\end{tabular}

\author{
Appendix D
}

Model outputs for chapter eight: The cost of cultivation. 


\section{Multi-level model result tables}

All predictive variables are reported, '- ' refers to when variables were removed due to multicollinearity and are contained in the other model. The 'camp variable' included in the model was based on best AIC scores in single level regressions to choose between the binary measure of 'settled' or 'mobile', the housing measure (temporary, semi-permanent and permanent housing), and the continuous variable of 'out-of-camp mobility'. The demographic, BMI and eosinophil models are all continuous multilevel models and lymphocytosis and neutrophilia models are logistic multilevel models, all with individual (level 1) and camp levels (level 2). Significant results are highlighted in bold, marginally significant results $(p<0.1)$ in italics. 'Ref' refers to the baseline or comparison category, temporal housing. VPC stands for variance partition coefficients which represent the proportion of variance which each level accounts for. Variance explained is the percentage reduction in the variance at each of the levels compared to the intercept-only level with the inclusion of all fixed-effects into the model (Koster et al. 2015). In the logistic models explained variance is computed by establishing the variance of the predicted probability from the model and again allocating percentage variance explained at each level (the individual, the camp and the total variance explained (Snijders \& Bosker 2012)).

Table 1: MLM results for fertility. $n=90$, sample size reduced from 117 due to missing data. 


\begin{tabular}{|c|c|c|c|c|c|c|c|}
\hline & & \multicolumn{3}{|c|}{ Foraging Model } & \multicolumn{3}{|c|}{ Settlement Model } \\
\hline Variable group & Variable & $\bar{B}$ & $\mathrm{SE}$ & $p$ & $\bar{B}$ & SE & $p$ \\
\hline- & Intercept & -0.893 & 0.700 & 0.202 & -1.346 & 0.723 & 0.063 \\
\hline \multirow[t]{3}{*}{ Household variables } & Settled household & - & - & - & 0.934 & 0.441 & 0.034 \\
\hline & High forager & -1.399 & 0.680 & 0.040 & - & - & - \\
\hline & Household belongings & 0.234 & 0.174 & 0.177 & 0.463 & 0.164 & 0.005 \\
\hline \multirow[t]{3}{*}{ Camp variables } & Temporary housing & ref & ref & ref & ref & ref & ref \\
\hline & Semi-permanent housing & 0.087 & 0.554 & 0.874 & -0.280 & 0.556 & 0.614 \\
\hline & Permanent housing & -0.240 & 0.530 & 0.650 & -0.653 & 0.547 & 0.232 \\
\hline \multirow[t]{5}{*}{-} & Model AIC & & 358.4 & & & 386.7 & \\
\hline & Camp VPC & & 0 & & & 0 & \\
\hline & Individual VPC & & 1 & & & 1 & \\
\hline & Camp variance reduction & & $0.00 \%$ & & & $0.00 \%$ & \\
\hline & Individual variance reduction & & $12.20 \%$ & & & $11.70 \%$ & \\
\hline
\end{tabular}

Table 2: MLM results for childhood mortality (under 16 years), $n=90$

\begin{tabular}{|c|c|c|c|c|c|c|c|}
\hline & & \multicolumn{3}{|c|}{ Foraging Model } & \multicolumn{3}{|c|}{ Settlement Model } \\
\hline Variable group & Variable & $B$ & $\mathrm{SE}$ & $p$ & $\bar{B}$ & SE & $p$ \\
\hline- & Intercept & -0.411 & 0.329 & 0.212 & -0.277 & 0.371 & 0.456 \\
\hline \multirow[t]{4}{*}{ Household variables } & Settled household & - & - & - & -0.170 & 0.214 & 0.425 \\
\hline & High forager & 0.098 & 0.322 & 0.761 & - & - & - \\
\hline & Age-specific fertility & 0.191 & 0.04 & $<0.01$ & 0.197 & 0.04 & $<0.01$ \\
\hline & Household belongings & 0.034 & 0.081 & 0.672 & -0.024 & 0.083 & 0.770 \\
\hline \multirow[t]{3}{*}{ Camp variables } & Temporary housing & ref & ref & ref & ref & ref & ref \\
\hline & Semi-permanent housing & 0.492 & 0.28 & 0.083 & 0.604 & 0.36 & 0.096 \\
\hline & Permanent housing & 0.727 & 0.26 & 0.01 & 0.627 & 0.313 & 0.045 \\
\hline \multirow[t]{4}{*}{-} & Model AIC & & 229.9 & & & 251.3 & \\
\hline & Camp VPC & & 0.021 & & & 0.081 & \\
\hline & Individual VPC & & 0.979 & & & 0.919 & \\
\hline & Camp variance reduction & & $86.54 \%$ & & & $44.40 \%$ & \\
\hline
\end{tabular}


Table 3: MLM results for reproductive success (survivorship to 16 years), $n=90$.

\begin{tabular}{|c|c|c|c|c|c|c|c|}
\hline & & \multicolumn{3}{|c|}{ Foraging Model } & \multicolumn{3}{|c|}{ Settlement Model } \\
\hline Variable group & Variable & $\bar{B}$ & $\mathrm{SE}$ & $p$ & $\boldsymbol{B}$ & $\mathrm{SE}$ & $p$ \\
\hline- & Intercept & -0.136 & 0.599 & 0.820 & -0.600 & 0.631 & 0.342 \\
\hline \multirow[t]{3}{*}{ Household variables } & Settled household & - & - & - & 0.824 & 0.385 & 0.032 \\
\hline & High forager & -1.151 & 0.581 & 0.048 & - & - & - \\
\hline & Household belongings & 0.099 & 0.149 & 0.505 & 0.335 & 0.144 & 0.020 \\
\hline \multirow[t]{3}{*}{ Camp variables } & Temporary housing & ref & ref & ref & ref & ref & ref \\
\hline & Semi-permanent housing & -0.367 & 0.473 & 0.438 & -0.711 & 0.485 & 0.143 \\
\hline & Permanent housing & -1.010 & 0.453 & 0.026 & -1.233 & 0.478 & 0.010 \\
\hline \multirow[t]{5}{*}{ - } & Model AIC & & 332.4 & & & 362.3 & \\
\hline & Camp VPC & & 0 & & & 0 & \\
\hline & Individual VPC & & 1 & & & 1 & \\
\hline & Camp variance reduction & & $0.00 \%$ & & & $0.00 \%$ & \\
\hline & Individual variance reduction & & $12.76 \%$ & & & $11.25 \%$ & \\
\hline
\end{tabular}


Table 4: Logistic MLM results for Neutrophilia. $n=293$

\begin{tabular}{|c|c|c|c|c|c|c|c|c|c|}
\hline & & \multicolumn{4}{|c|}{ Foraging Model } & \multicolumn{4}{|c|}{ Settlement Model } \\
\hline Variable group & Variable & $\bar{B}$ & $\mathrm{SE}$ & $\overline{\text { OR }}$ & $p$ & $\bar{B}$ & $\mathrm{SE}$ & OR & $\bar{p}$ \\
\hline- & Intercept & -2.60 & 1.00 & 0.24 & 0.00 & -1.95 & 1.14 & 0.14 & 0.09 \\
\hline \multirow[t]{3}{*}{ Individual variable } & Age & -0.27 & 0.32 & 0.98 & 0.33 & -0.01 & 0.01 & 0.99 & 0.59 \\
\hline & Sex & -0.24 & 0.57 & 0.78 & 0.67 & -0.44 & 0.56 & 0.64 & 0.43 \\
\hline & Mean relatedness & -7.73 & 5.70 & 0.00 & 0.18 & -7.68 & 5.81 & 0.00 & 0.18 \\
\hline \multirow[t]{4}{*}{$\begin{array}{c}\text { Household } \\
\text { variables }\end{array}$} & Settled household & - & - & - & - & 1.275 & 0.71 & $\begin{array}{r}3.57 \\
8\end{array}$ & $\begin{array}{r}0.07 \\
4\end{array}$ \\
\hline & High forager & 1.14 & 0.71 & 3.9 & 0.11 & - & - & - & - \\
\hline & Dependents & -0.17 & 0.16 & 0.85 & 0.30 & -0.25 & 0.15 & 0.76 & 0.1 \\
\hline & $\begin{array}{l}\text { Household } \\
\text { belongings }\end{array}$ & 0.106 & $\begin{array}{r}0.34 \\
9\end{array}$ & $\begin{array}{r}0.90 \\
0\end{array}$ & $\begin{array}{r}0.76 \\
2\end{array}$ & $0.024^{-}$ & $\begin{array}{r}0.32 \\
3\end{array}$ & 0.98 & 0.94 \\
\hline Camp variables & Permanent Camp & -1.27 & 0.7 & 0.22 & 0.07 & -1.78 & 0.69 & 0.17 & 0.01 \\
\hline \multirow[t]{2}{*}{-} & Model AIC & \multicolumn{4}{|c|}{133.8} & \multicolumn{4}{|c|}{136.4} \\
\hline & Model residual & \multicolumn{4}{|c|}{0.723} & \multicolumn{4}{|c|}{0.678} \\
\hline
\end{tabular}


Table 5: Logistic MLM results for Lymphocytosis $(n=293)$

\begin{tabular}{|c|c|c|c|c|c|c|c|}
\hline & & \multicolumn{3}{|c|}{ Foraging Model } & \multicolumn{3}{|c|}{ Settlement Model } \\
\hline Variable group & Variable & $\bar{B}$ & OR & $p$ & $\bar{B}$ & $\overline{\text { OR }}$ & $p$ \\
\hline \multirow[t]{2}{*}{ - } & Intercept & -3.315 & 0.036 & $<0.001$ & -3.656 & 0.026 & $<0.001$ \\
\hline & Age & 0.017 & 1.018 & 0.034 & 0.010 & 1.010 & 0.020 \\
\hline \multirow[t]{2}{*}{ Individual variable } & Sex & 0.036 & 1.037 & 0.897 & 0.117 & 1.124 & 0.674 \\
\hline & Mean relatedness & -1.180 & 0.307 & 0.683 & -2.270 & 0.103 & 0.455 \\
\hline \multirow{4}{*}{ Household variables } & Settled household & - & - & - & -0.152 & 0.859 & 0.699 \\
\hline & High forager & -1.420 & 0.242 & 0.045 & - & - & - \\
\hline & Dependents & 0.067 & 1.070 & 0.368 & 0.118 & 1.126 & 0.122 \\
\hline & Household belongings & -0.063 & 0.939 & 0.622 & 0.023 & 1.024 & 0.882 \\
\hline \multirow[t]{2}{*}{ Camp variables } & Out-of-camp mobility & 1.871 & 6.496 & 0.019 & 2.194 & 8.967 & 0.030 \\
\hline & Model AIC & & 340.3 & & & 351.5 & \\
\hline \multirow[t]{2}{*}{ - } & Model residual & & 0.754 & & & 0.763 & \\
\hline & Explained from null & & 0.246 & & & 0.212 & \\
\hline
\end{tabular}


Table 6: Continuous MLM results for maternal BMI $(n=57)$.

\begin{tabular}{|c|c|c|c|c|c|c|c|}
\hline & & \multicolumn{3}{|c|}{ Foraging Model } & \multicolumn{3}{|c|}{ Settlement Model } \\
\hline Variable group & Variable & $\boldsymbol{B}$ & SE & $p$ & $\boldsymbol{B}$ & SE & $p$ \\
\hline- & Intercept & 18.45 & 1.15 & $<0.001$ & 18.58 & 1.11 & $<0.001$ \\
\hline Individual variable & Age & -0.008 & 0.02 & 0.680 & -0.027 & 0.02 & 0.138 \\
\hline \multirow{3}{*}{ Household variables } & Settled household & - & - & - & 1.733 & 0.606 & 0.004 \\
\hline & High forager & -1.513 & 0.88 & 0.08 & - & - & - \\
\hline & Household belongings & 0.429 & 0.24 & 0.07 & 0.500 & 0.23 & 0.026 \\
\hline \multirow{3}{*}{ Camp variables } & Temporary housing & ref & ref & ref & ref & ref & ref \\
\hline & Semi-permanent housing & 0.273 & 0.71 & 0.702 & -0.375 & 0.7 & 0.591 \\
\hline & Permanent housing & -0.005 & 0.71 & 0.994 & -0.672 & 0.71 & 0.347 \\
\hline \multirow{4}{*}{ Health variables } & Anaemia & 0.645 & 0.55 & 0.242 & 0.168 & 0.56 & 0.763 \\
\hline & Neutrophilia & -0.228 & 0.17 & 0.171 & -0.283 & 0.16 & 0.080 \\
\hline & Eosinophilia & -0.183 & 0.19 & 0.324 & -0.104 & 0.18 & 0.562 \\
\hline & Lymphocytosis & 0.042 & 0.06 & 0.472 & 0.025 & 0.06 & 0.666 \\
\hline
\end{tabular}




\begin{tabular}{llc|c}
\hline & Model AIC & 256.1 & 251.3 \\
& Camp VPC & 0.000 & 0.000 \\
- & Individual VPC & 1.000 & 1.000 \\
& Camp variance reduction & $100.00 \%$ & $100.00 \%$ \\
& Individual variance reduction & $18.70 \%$ & $25.17 \%$ \\
\hline
\end{tabular}

Table 7: Continuous MLM results for eosinophil concentrations, $n=293$

\begin{tabular}{|c|c|c|c|c|c|c|c|}
\hline & & \multicolumn{3}{|c|}{ Foraging Model } & \multicolumn{3}{|c|}{ Settlement Model } \\
\hline Variable group & Variable & $\bar{B}$ & $\mathrm{SE}$ & $\bar{p}$ & $\bar{B}$ & $\mathrm{SE}$ & $\bar{p}$ \\
\hline \multirow[t]{2}{*}{-} & Intercept & 0.541 & 0.237 & 0.022 & 0.620 & 0.259 & 0.017 \\
\hline & Age & -0.012 & 0.003 & $<0.001$ & -0.009 & 0.003 & 0.003 \\
\hline \multirow[t]{2}{*}{ Individual variable } & Sex & -0.207 & 0.112 & 0.063 & -0.241 & 0.110 & 0.029 \\
\hline & Mean relatedness & 0.645 & 0.951 & 0.497 & 0.905 & 0.974 & 0.353 \\
\hline \multirow{4}{*}{ Household variables } & Settled Household & - & - & - & 0.027 & 0.139 & 0.845 \\
\hline & High forager & 0.053 & 0.214 & 0.805 & - & - & - \\
\hline & Dependents & 0.049 & 0.031 & 0.107 & 0.035 & 0.031 & 0.257 \\
\hline & Household belongings & -0.025 & 0.053 & 0.638 & -0.036 & 0.054 & 0.498 \\
\hline \multirow{3}{*}{ Camp variables } & Temporary housing & ref & ref & ref & ref & ref & ref \\
\hline & Semi-permanent housing & 0.525 & 0.181 & 0.004 & 0.435 & 0.194 & 0.025 \\
\hline & Permanent housing & 0.448 & 0.166 & 0.007 & 0.371 & 0.180 & 0.039 \\
\hline
\end{tabular}




\begin{tabular}{llc|c}
\hline & Model AIC & 792.7 & 818.4 \\
& Camp VPC & 0.007 & 0.015 \\
- & Individual VPC & 0.993 & 0.985 \\
& Camp variance reduction & $57.73 \%$ & $5.30 \%$ \\
& Individual variance reduction & $22.92 \%$ & $21.17 \%$ \\
\hline
\end{tabular}




\section{Appendix E}

Reproductive Trade-offs in Extant Hunter-

Gatherers Suggest Adaptive Mechanisms for the Neolithic Expansion.

This appendix presents the manuscript in press at PNAS which stems from work presented in chapter eight. 


\section{Reproductive trade-offs in extant hunter-gatherers suggest adaptive mechanisms for the Neolithic expansion}

Abigail E. Page, Sylvain Viguier, Mark Dyble, Daniel Smith, Nikhil Chaudhary, Gul. D. Salali, James Thompson, Lucio Vinicius, Ruth Mace and Andrea. B. Migliano

Department of Anthropology, University College London, 14 Taviton Street, London WC1H OBW, UK.

Submitted to Proceedings of the National Academy of Sciences of the United States of America

The Neolithic demographic transition remains a paradox, as it is associated with both positive effects, such as higher population growth as well as negative effects, such as increased morbidity and mortality rates. Here we reconcile the conflicting evidence by proposing that the spread of agriculture involved a life history quality-quantity trade-off whereby mothers traded offspring survival for increased fertility, achieving greater reproductive success in spite of deteriorating health. We test this hypothesis by investigating fertility, mortality, health and overall reproductive success in Agta hunter-gatherers whose camps exhibit variable levels of sedentarization, mobility and involvement in agricultural activities. We conducted blood composition tests with 345 Agta and found that viral and helminthic infections as well as child mortality rates were significantly increased with sedentarization. Despite this, age-controlled fertility and overall reproductive success are both positively affected by sedentarization and participation in cultivation. Thus, we provide the first empirical evidence of an adaptive mechanism in foragers which reconciles the decline in health and child survival with the observed demographic expansion during the Neolithic.

Hunter-gatherers | quality-quantity trade-off $\mid$ Neolithic revolution epidemiological transition

\section{Introduction}

Agriculture appeared in multiple locations from around 10-12,000 BP before slowly spreading to become the dominant subsistence system by $5000 \mathrm{BP}(1-5)$. The Neolithic transition was associated with sedentarization, food storage, wealth accumulation and inequality as well as increasing population size $(3,4,6,7)$. It has been suggested that an increased availability of calories combined with a reduction in energy expenditure due to sedentarization led to increased eneroy availability for reproduction (8-10). As a result, while exact estimates vary, it has been argued that average population growth rates rose from $<0.001 \%$ per year to approximately $0.04 \%$ during the early Neolithic $(6,8,11-17)$.

On the other hand, studies reveal significant increases in morbidity and mortality associated with reduction in dietary breadth and sedentarization $(6,18-22)$. Research reveals increased prevalence of tuberculosis, syphilis and the plague $(6,23-25)$, overall immunological stress (26) and a deterioration in oral health (16, $27,28)$. Farming led to higher population densities, sedentarization, increased contact with neighboring populations, presence of rodents attracted by food stores, domestication and fecal pollution (29-31). All those factors facilitated virulent bacterial and viral pathogens as well as soil-borne helminths (roundworm, hookworm and whipworm (32-38)). While some argue that Paleolithic foragers experienced high helminths loads $(37,39,40)$, archaeological data shows instead an increase in helminths associated with farming $(33,41-45)$ compared to mobile, low-density hunter-gatherers. In summary, the overall effect of agriculture on health was a trend towards increasing morbidity and mortality
$(16,19,28,46)$, although its intensity exhibits some regional variation and inconsistent trends (47-56)).

Given this significant increase in disease burden, why farming arose independently and expanded in diverse geographical regions $(57,58)$ remains a puzzle. Here we propose that settled agriculture was accompanied by a life history trade-off, whereby mothers switch strategy to invest in offspring quantity rather 8 than quality and thereby increase their reproductive fitness. A quantity-quality trade-off is an adaptive process based on a principle of optimal distribution of limited resources: parents must either allocate finite resources to a few higher quality offspring or to multiple lower quality ones (59-64). By proposing that a quantity-quality trade-off provides an explanation for the transition to agriculture, we provide an adaptive mechanism that reconciles the decline in population health with increases in mortality during the Neolithic transition. We test this hypothesis with data from extant Palanan Agta foragers in the Philippines (hence forth referred to as Agta), a diverse group varying in mobility, foraging, wealth accumulation and camp permanence; traits all attributed to the Neolithic revolution $(1,3,6,7)$. Residing completely within the Northern Sierra Madre Natural Park the Palanan Agta have remained mostly separated from other Agta populations who reside outside the park limits and further south in Casiguran (65-67). By measuring fertility, mortality alongside viral, bacterial and helminth markers in different Agta camps, we found that sedentarization is associated with increased morbidity and mortality, yet both sedentarization and involvement in non-

\section{Significance}

The rise of agriculture during the Neolithic period has paradoxically been associated with worldwide population growth in spite of increases in disease and mortality. We examine the effects of sedentarization and cultivation on disease load, mortality and fertility among Agta foragers. We report increased disease and mortality rates associated with sedentarization alongside an even larger increase in fertility associated with both participation in cultivation and sedentarization. Thus, mothers who transition to agriculture have higher reproductive fitness. We provide the first empirical evidence of an adaptive mechanism behind the expansion of agriculture, explaining how we can reconcile the Neolithic increase in morbidity and mortality with the observed demographic expansion.

Reserved for Publication Footnotes 


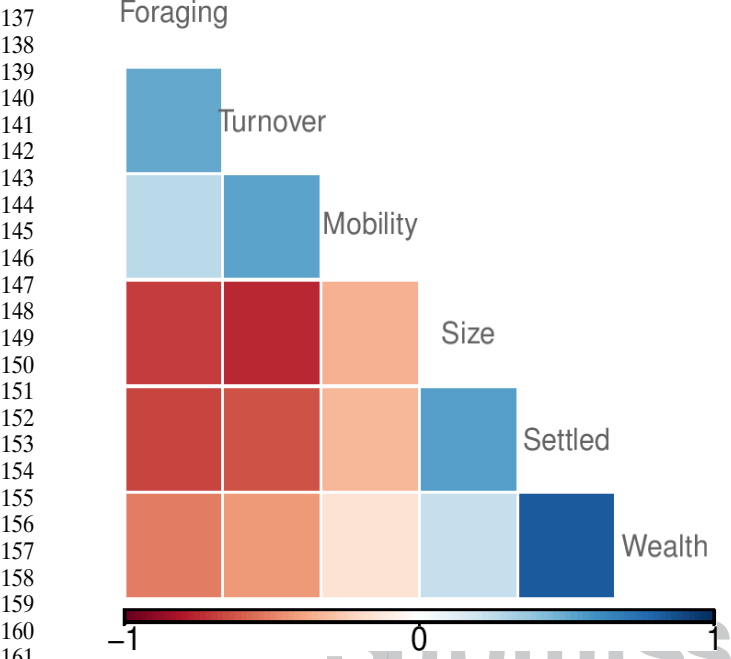

161

Fig. 1. Correlation plot for six key predictor variables $(n=345)$. Pos tive correlations are marked in blue shades, negative in red shades. All correlations are statistically significant at $p<0.05$. Variables are: settled camp (number of permanent structures within a camp); wealth (an index quantifying household belongings); mobility (whether an individual was ever witnessed to move camp over a two year period, $0=$ never moved); turnover (number of people leaving a camp); and foraging (proportion of food that individuals acquired from foraging activities versus food production and wage labor).

foraging activities are both associated with even further increase in fertility, resulting in higher reproductive fitness. Our result provide evidence that a quality-quantity trade-off was adaptive and suggests a pathway through which, despite poor health, farming could have become the dominant subsistence system after its origin in the Neolithic. Thus, we demonstrate how exploring the mechanisms that lead extant foragers to seek increased settlement and food production is a theoretically informative approach to test key archaeological predictions $(6,8,19)$.

\section{Results}

Palanan Agta exhibit a suite of traits attributed to the Neolithic transition. Archaeological studies have shown that the Neolithic transition from hunter-gathering to agriculture is associated with a suite of traits $(3,7)$ that are also present across Agta camps. Figure 1 shows significant correlations between proportion of food produced in cultivation and key 'transition' traits. These correlations indicate that while extensive foraging, small size, high mobility and few belongings characterize some Agta camps; others exhibit more food production, larger camp size, little mobility and many belongings. Thus, the variability present within the Agta today allows us to test to influence of 'transition' on fitness related outcomes.

We used these 'transition' variables associated with the $\mathrm{Ne}$ olithic revolution in multilevel analysis to examine fertility, morbidity and mortality. Due to covariance two models were produced for each analysis: one exploring the effects of household mobility (whether an household moved camp at least once or not during two years of fieldwork) and the second, exploring the effects of household foraging (binary measure, $1=$ more than $75 \%$ of maternal time spent in foraging as opposed to cultivation and wage labor). Both these models included one camp settlement variable, chosen based on lowest AIC score in univariate regressions (SI appendix table S4), and included: $i$ ) camp housing type (temporary, semi-permanent or permanent); ii) a binary sedentarized camp variable denoted by the presence of permanent camp infrastructures; and iii) out-of-camp mobility over two years, 0 representing camp abandonment. AICs and full model results are reported in the SI appendix tables S8-14.

Settled Agta exhibit increased fertility. The total fertility rate (TFR) among the Agta based on 41 live births in 2013-2014 was 7.7 , one of the highest reported in foragers (68). For 117 women we interviewed (aged between 16-75 years), the mean number of live births was 4.4 (ranging from 0 to 13 ). Residuals from a linear regression of offspring count and maternal age, including a logarithmic term, were used to control for age effects on fertility and reproductive success, while an exponential term was included for child mortality (see SI appendix). Settled mothers had significantly higher fertility than women who moved camp at least once $(\beta=1.3+0.6, p=0.04)$. Correspondingly, increases in household belongings were positively associated with fertility $(\beta=0.5+0.2$, $p=0.005)$. This relationship is further confirmed by examining TFR: settled mothers had $16.7 \%$ higher TFR (7.7) compared to mobile mothers TFR (6.6). Similarly, for all live births settled mothers had $20.5 \%$ more offspring (5.3) than mobile mothers 4.4: SI appendix table S6). A possible pathway may be that settled mothers are able to accumulate more somatic resources for reproduction. Accordingly, settlement was a significant predictor of maternal BMI (i.e. mothers never witness to move camp, $\beta=$ $1.7+0.6, p=0.004)$, and BMI positively predicts fertility $(\beta=$ $0.3+0.1, p=0.02$ )

Mothers who forage more exhibit lower fertility. A high degree of foraging also significantly predicts fertility $(\beta=-1.4+0.7, p=$ $0.04)$. As a result, mothers who spent less than $75 \%$ of their time foraging experience 0.23 higher fertility residuals than expected for their age. Mothers who spent more than $75 \%$ of their time foraging had 0.85 less offspring, given their age (SI appendix table S7). Women who spent more time foraging also had marginally lower BMI $(\beta=-1.5+0.9, p=0.08)$. Therefore, transition to farming as measured by both increasing cultivation and sedentarization are positively associated with fertility, perhaps due to increased somatic resources.

Sedentarization is associated with health deterioration. We examined the effects of cultivation and sedentarization on three white blood cell types - lymphocytes, neutrophils and eosinophils - as proxies for different immunological attacks: viral, bacterial and helminthic, respectively (see SI appendix). Our sample consisted of 345 Agta, 140 of which were adults ( $48.6 \%$ males) consisted of 345 Agta, 140 of which were adults (48.6\% males)
and 205 children under 16 (60\% males). Due to inconclusive and 205 children under 16 (60\% males). Due to inconclusive
test results missing data reduced the sample to 293 , consisting test results missing data reduced the sample to 293 , consisting
of 117 adults ( $47 \%$ males) and 176 children (59\% males). Table 1 shows that people in large camps with low out-of-camp mobility had 2.8 times higher chances of presenting with lymphocytosis compared to mobile camps. People residing in camps with permanent and semi-permanent houses had significantly higher eosinophil concentrations than individuals in temporary camps. Severe eosinophilia $\left(>5 \times 10^{9} / \mathrm{L}\right)$ is indicative of extreme helminth infestations and only found in sedentarized camps. In contrast, people residing in sedentarized camps with some form of infrastructure (i.e. church or water pump) had lower odds (by $80 \%$ ) of neutrophilia. One major bacterial disease among the Agta is Mycobacterium tuberculosis (TB), demonstrated by the fact that $41.7 \%$ of the 24 individuals suffering from neutrophilia had clinically diagnosed or suspected TB. In contrast, percentage of time spent foraging had little effect on health. Individuals in households who foraged more had a $76 \%$ reduction in odds of presenting with lymphocytosis than households who foraged less. However, indicators of helminthic or bacterial infections were not affected by percentage of time spent foraging (SI appendix tables S11-13).
205 
Table 1.

\begin{tabular}{|c|c|c|c|c|}
\hline Measure & Abnormal values & $\%$ affected & Explanatory variables & Indication of \\
\hline
\end{tabular}

Lymphocytosis $\quad$ Lymphocytes $>3 \times 10^{9} / \mathrm{L} \quad 67 \% \quad$ Out-of-camp mobility $\mathrm{OR}=2.8, \mathrm{p}=0.03 ;>75 \%$ foraging: Viral infection 344

Out-of-camp mobility $\mathrm{OR}=2.8, \mathrm{p}=0.03,>75 \%$ foraging: Viral infection $\quad 345$
$\mathrm{OR}=0.24, \mathrm{p}=0.04$ (baseline $<75 \%$ foraging)

Neutrophilia Neutrophils $>7 \times 109 / \mathrm{L} \quad 6.5 \% \quad$ Settled camp: $\mathrm{OR}=0.2 \mathrm{p}=0.01$ baseline: mobile camp Bacterial infection 346

Eosinophilia $\quad$ Eosinophils $>0.5 \times 10^{9} / \mathrm{L} \quad 71.3 \% \quad$ Semi-permanent housing: $\beta=0.53+0.2, p=0.004 ; \quad$ Helminthic infection 348

$\begin{array}{ll}\text { Semi-permanent housing: } \beta=0.53+0.2, p=0.004, & 348 \\ \text { Permanent housing: } \beta=0.45+0.2 p=0.007 \text { baseline: } & 349\end{array}$

temporary

: White blood cell types, medically abnormal thresholds, frequency, predictors and health implications. Eosinophilia is a continuous analysis due so to 351 differentiate between individuals with mild eosinophilia, medium and extreme eosinophilia, as these reflect significant variance in helminth load (see SI appendix). All other analysis are logistic, $n=293$ due to missing household wealth data and inconclusive test results.

A

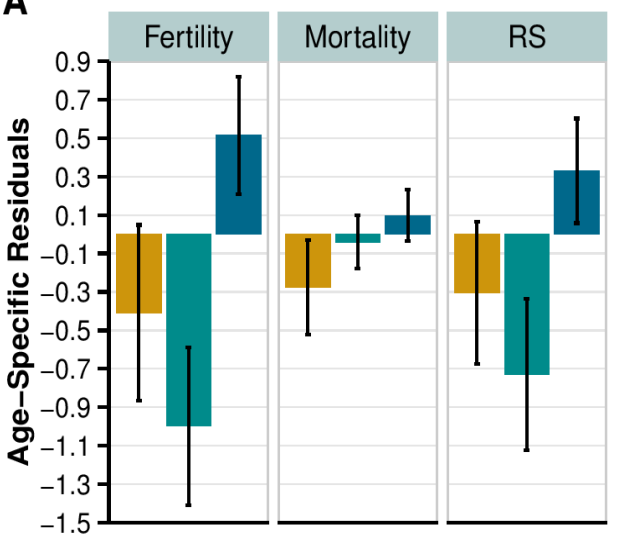

Settlement Status
B

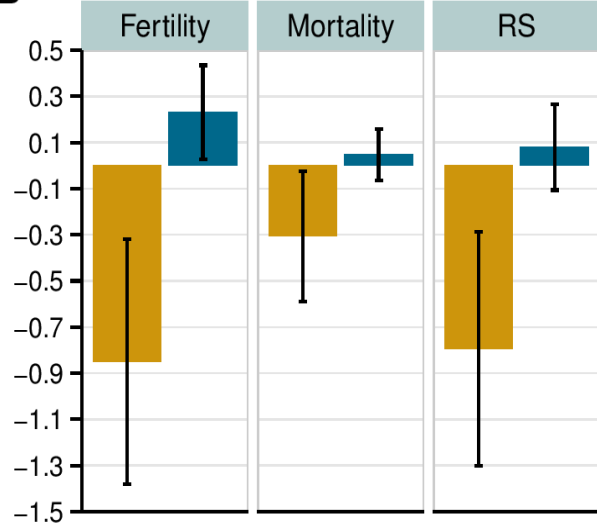

Amount of foraging

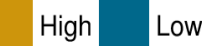

Fig. 2. Relationship between measures of transition and age-specific fertility, mortality and reproductive success (RS) separated by a) individual settlement status and $b$ ) degree of foraging. Error bars represent standard errors of the mean and $n=90$.

To explore helminth burden further we collected 30 fecal samples from Agta who had presented with eosinophilia. All 30 samples tested positive for helminth ova. The mean number of species was $1.6+0.7$ and included: roundworm (Ascaris Lumbricoides, $83.3 \%$ ); hookworm (Ancylostoma duodenale or Necator americanus, 46.7\%); and whipworm (Trichuris trichiura, 30\%). Due to the small sample size non-parametric Kruskal-Wallis tests were run on three measures of transition. Living in a sedentarized camp was associated with a 0.65 mean increase in parasite species number compared to living in mobile camps $\left(\mathrm{X}^{2}=5.9, \mathrm{df}=1, p=\right.$ 0.02 ). Household participation in cash labor decreased helminth loads by $37.4 \%\left(\mathrm{X}^{2}=8.9, \mathrm{df}=1, p=0.003\right)$ indicating that families who interact with the wider economy may be more able, financially and/or behaviorally, to acquire anthelmintic medicines. While cash labor is a positive predictor, the proportion of time spent foraging is not $\left(X^{2}=3.3\right.$, df $\left.=1, p=0.2\right)$. Our results indicate that although increased helminthic load is likely to be a consequence of transition to agriculture, it is settlement, rather than cultivation, which predicts helminth infestation.

Child mortality is increased in settled camps. We examined whether the negative effects of settlement on health also extended to child mortality rates (including all post-natal deaths until age 16). Out of 520 reported live births from 124 mothers, $19 \%$ of children died before age one, $13.9 \%$ from one to five and $6 \%$ from five to fifteen years. Those rates are lower than the $28-29 \%$ previ ously reported for neighboring Agta $(65,69)$. Mothers residing in camps with permanent housing experienced significantly highe childhood mortality rates, compared to mothers living in camp with temporary housing $(\beta=0.7+0.3, p=0.005)$. The relationship for semi-permanent camps was in the same direction but of marginal significance $(\beta=0.5+0.3, p=0.08)$. This relationship results in a $63.2 \%$ increase in mortality rates for settled mother living in sedentarized camps (0.93) when compared to nomadic mothers in temporary camps (0.57). Thus, the sedentarized camp with the highest helminth and viral disease rates also experience the highest childhood mortality rates. Following the morbidity results, proportion of time spent in cultivation had no significan relationship with child mortality $(\beta=0.01+0.04, p=0.8)$.

A quantity-quality trade-off explains higher reproductive succes in transitioning Agta. Our results show that women who settle down trade child survival for fertility, increasing their overall reproductive success (Figure 2a). Settled women $(\beta=0.8+$ $0.4, p=0.03$ ) had significantly more children surviving to age 16 (our measure of reproductive fitness) than mobile women. Although these settled women faced higher child mortality rates, they also experienced extremely high fertility. As a result of the negative morbidity effect of sedentarized camps compared to the positive fertility effect of settled households, women residing in 
permanent camps suffered lower child survival $(\beta=-1.2+0.5, p$ $=0.01$ ) compared to temporary camps, once household mobility has been controlled for. Figure $2 \mathrm{a}$ demonstrates that mobile women living in sedentarized camps faced the worst situation as they experienced both higher mortality rates associated with permanent camps and the lower fertility rates associated with high mobility. Thus, they experienced 0.73 lower fitness, given their age. Overall, our results show that settled mothers, in spite of the higher mortality rates, had an extra 0.59 offspring (4.4) surviving to age 16 compared to mobile women (3.8), a relative increase of $15.8 \%$ in reproductive fitness.

Our other indicator of 'transition', the percentage of time involved in foraging, also predicted reproductive success $(\beta=$ $1.2+0.6, p=0.05$ ). We find that mothers who foraged more than $75 \%$ of the time had 0.74 less children surviving to age 16 given their age, while mothers who spent less time foraging experienced 0.04 more children surviving to age 16 (figure $2 b$ ). Thus, while abandoning foraging does not significantly affect child mortality rates, it does increase fertility, and consequently mother's reproductive success. Consequently, while cultivation is associated with increased fertility, sedentarization is associated with both increased fertility and increased morbidity and mortality. The overall consequence of this combined process is a reproductive trade-off where settled mothers involved in cultivation have the highest reproductive fitness, despite increased morbidity and mortality.

\section{Discussion}

The variability of lifestyles currently observed within the Agta allows the testing of hypothesis proposed by archaeologists to explain the demographic processes taking place during the $\mathrm{Ne}-$ olithic transition $(8,29)$. Current evidence from archaeology and other sources suggests a Neolithic paradox as fertility increased alongside morbidity (58). Here we have tried to shed light on this debate by offering an adaptive explanation for the Neolithic transition. We propose that a life history trade-off between offspring quantity and quality is able to explain a suite of traits including higher fertility, mortality, morbidity and overall reproductive success observed in sedentarized Agta practicing cultivation. In so far that variability observed within the Agta is reflective of Neolithic trends, our results illuminate the adaptive mechanisms involved in transition to sedentarized food production. In doing so we provide empirical evidence that a quantity-quality trade-off can explain the demographic changes associated with cultivation and sedentism.

Our data indicates that sedentarization was associated with increased risk of infectious disease. Viral infections were higher in the larger, less mobile camps, with increased cultivation. Camp size and population density (measured here through out-of-camp mobility) were significantly correlated with viral infections in our data. This follows expectations of transmission of viruses such as Caliciviruses and Rotoviruses, causing gastroenteritis an influenza (38), both common among settled Agta and dependen on higher population densities (70). This mirrors the finding of increased risk of high C-reactive protein concentrations (indicative of an inflammatory response) in children among more acculturated Tsimane forager-horticulturalists (71). In particular, cultivation may independently predict increased viral load (indicated by the association between lymphocytosis and foraging due to poorer nutritional quality of agricultural staples. Increase consumption of rice with cultivation entails a loss of dietary breadth and macro- and micro-nutritional quality $(72,73)$ leading to malnutrition, which is a significant predictor of disease and mortality (74).

As with viral infections, Agta living in more permanent camps (as predicted by camp housing type) were also worse off in term of helminthic infections, matching theoretical predictions $(29$,
$33,43,44,75)$. Contrary to this, some studies have argued the high helminth burden was a major selective pressure before the Neolithic $(37,39,76)$ due to extreme helminth rates in many semi-sedentarized, farmer-foragers $(31,37,77)$. However, these groups are no longer fully mobile. Supporting our interpretation in an isolated Amazonian group (78), Hadza children (79) and lower helminth loads among isolated and mobile Saluma and Yanomama groups (80). While some parasitic species were hosted by our hominid ancestors $(29,81,82)$, there is little archaeological evidence for an extreme intestinal parasite burden predating agriculture $(33,41-43,45,81)$. Thus, increased viral and helminth burdens are costs associated with sedentarization. It should be noted that settled Agta did not, however show an increased incidence of neutrophilia, which in our sample is often caused by TB. Chronic bacterial infections, such as TB, have evolved to survive at low population densities, thus likely predate agriculture (83, 84). The lower incidence of neutrophilia in settled Agta camps is likely due to the limited access to medical care, which might also explain the finding that cash labor decreased helminth loads by $37.4 \%$. Since westernized medicine is a recent occurrence, the overall increased helminthic load in sedentarized camps may be underestimating the effect that settlement had on human populations during the Neolithic transition.

We found that more permanent camps had significantly higher childhood mortality rates, matching archaeological evidence from the Neolithic (6). Our results also revealed significantly higher fertility rates in settled women, particularly those transitioning from foraging to cultivation. Agriculture has been associated with fertility increases $(85,86)$ as the reduction in energy expenditure with settlement (87) and increased carbohydrate consumption $(10,88)$ are associated with increased BMI, which correlates with shorter inter-birth intervals and higher fertility (89). This suggests a pathway through which increased cultivation could lead to increased fertility. An additional pathway, as suggested by Kramer and Boone (90), considers the increased economic productivity of children in agriculture which reduces maternal constraints and thus, increases her fertility. Our study remains correlative, which leaves unanswered the question of the causal direction between fertility, sedentarization and food production. Since sedentarization correlates with food production in the Agta, with more sedentarized households abandoning foraging, it seems that these two process are interdependent, and thus are treated here as part of a process that involved a cluster of 'transition' traits. This is likely to be the case for the Neolithic transition as well, as many have reported the interplay between sedentarization and food production leading to the demographic changes associated in the origins of agriculture $(4,7)$. Further limitations stem from using a cross-sectional study design to explore longer-term trends, thus, it is unclear to what degree sedentism is reflective of mobility trends beyond our two-year sample period. However, a cross-sectional study design does permit the exploration of intra-population variability without requiring this variability to be recent or unilineal. Furthermore, foraging and mobility behaviors are likely altered during the heavy rainy and typhoon season not captured here as data collection occurred during the dry period. However, this does mean there is no systemic temporal bias in the data as camps were visited within the same season. Finally, more detailed dietary data would be required to explore these questions in more depth, allowing us to better separate the effects of 'transition'.

In summary, our study of the Agta provides evidence supporting the hypothesis that while the transition to agriculture in the Neolithic significantly depressed health, the overall fitness payoff was greater. Therefore, our proposed quality-quantity trade-off provides an adaptive mechanism that reconciles deteriorating health, increased mortality and demographic expansion following 
the spread of agriculture in the Neolithic. Finally, since high fertility rates were accompanied by relatively high mortality rates, the trade-off also explains why population numbers did not explode during the Neolithic but instead increased relatively slowly (8), perhaps as large increases in fertility were matched by increases in mortality (17)

\section{Methods}

Data collection occurred over two field seasons from April to June 2013 and February to October 2014 during the Isabela dry season. There are around 1,000 Agta living in Palanan municipality, Isabela Province, located in northeastern Luzon. The Agta are primarily fisher-foragers, residing either at the coast or inland watersheds. Hunting does occur, however fishing is far more common: out of 2168 work activities $54 \%$ were spent fishing, $20 \%$ gathering and $3 \%$ hunting. The remaining 'non-foraging' activities consist of cultivation $(13.5 \%)$ and wage labor $(9.5 \%)$. There is, however significant variance in diet (Figure S1) and activities between households: mobile households spent only $3.5 \%$ of their work activities in cultivation and $0 \%$ of time in wage activities in cultivation and wage labor, respectively. We found $26 \%$ Agta activities in cultivation and wage labor, respectively. We found $26 \%$ Agta move of once every 10 days. Similarly, the degree of camp sedentarization varies drastically as some camps change daily and consists of temporary shelters while others consist of permanent wooden structures built around churches and cultivation plots. Some Agta living in permanent camps closer to town have limited access to medical care and government provisioning However, the effects of these sporadic interventions on the Agta health are limited (see SI appendix for further ethnographic description). We stayed around 14 days in each of the 13 camps and conducted reproductive histories to quantify demographics and interviews to produce an index of household belongings. After creating an emic-sourced list of 'important' belongings we asked each household whether they had each of the 14 objects, and if so how many. This was then weighted based on how many times each item were staggered time intervals based on spot observation techniques ( $S$ ) appendix staggered time intervals based on spot observation techniques (SI appendix figure $\$ 4)$. We categorized each individual's activity at the allocated time, separating hunting, fishing, foraging, wage labor and cultivation. Thus, we
can quantify the proportion of time individuals spent in foraging activities can quantify the proportion of time individuals spent in foraging activities camp and its changing composition as we visited each camp two to three camp and its changing composition as we visited each camp
times (depending on whether it had dissolved into new camps).

These data created the 'transition' variables which were broken into: a) camp sedentarization measured by i) camp housing type (temporary, semi-permanent or permanent), ii) a binary sedentarized camp variable denoted by the presence of church and/or water pump and iii) out-of-

Bar-Yosef O, Meadow RH (1995) The origins of argiculture in the near east. Last Huntersinst Farmers, eds Price D, Gebauer AB (school ol American Research Press, Santa Fe), pp

2. Bollongino R, et al. (2013) Stone Age Central Europe. Science (80) 342:479-481.

Piperno D, Pearsall DM (1998) The origins of agriculture in the Lowland Neotropics (Academic Press, London). st Farmers, eds Price DT, Gebauer AB (School of American Research Press, Santa Fe), pp

Richerson PJ, Boyd R, Bettinger RL (2001) Was Agriculture Impossible during the Pleisocene but Mandatory during the Holocene ? A Climate Change Hypothesis. Am Antiq

Cohen MN, Crane-Kramer GMM (2007) Ancient Health: Skeletal indicators of agriculturel and economic intensification (University Press of Florida, Gainesville).

Hayden B (1995) A new overview of domestication. Last Hunters-First Farmers, eds Price D, Gebauer AB (School of American Research Press, Santa Fe), pp 215-242.

8. Bocquet-Appel J, Naji S (2006) Testing the Hypothesis of a Worldwide Neolithic Demographic Transition: Corroboration from American Cemeteries. Curr Anthropd 47(2):341-365.

9. Starling AP, Stock JT (2007) Dental indicators of health and stress in early Egyptian and Nubian agriculturalists: A difficult transition and gradual recovery. Am J Phys Anthropol 134(4):520-528.

10. Herrera E (2000) Metabolic adaptations in pregnancy and their implications for the availability of substrates to the fetus. Eur J Clin Nutr 54 Suppl 1:S47-S5

JCL, Soch $J$ (2007) The biology of the colonizing ape. Yearb Phys Anthropd 50:191-222.

Bandy MS (2005) New World settlement evidence for a two-stage Neolithic Demographic Transition. Curr Anthropol 46(S):S109-S115,

13. Bellwood P, Oxenham M (2008) The expansions of farming societies and the role of the Neolithic Demographic Transition. The Neolithic Demographic Transition and Is Consequences, eds Bocquet-Appel J, Bar-Yosef O (Springer, London), pp 13-34.

14. Downey SS, Bocaege E, Kerig T, Edinborough K, Shennan S (2014) The Neolithic Demographic Transition in Europe: Correlation with Juvenility Index Supports Interpretation of the Summed Calibrated Radiocarbon Date Probability Distribution (SCDPD) as a Valid Demographic Proxy. PLoS One 9(8): 105730. camp mobility between three visits, 0 representing camp abandonment 0.5 when $50 \%$ of individuals remained and 1 when no one leaves; b not during two years of fieldwork; c) house hold belongings a camp or list of common objects; and d) household foraging, a binary variabse in which 1 equals when mothers spent more than 75\% of food production which 1 equals when mothers spent more than $75 \%$ of food production and wage labor. Due to multicollinearity, only one of the variables in the amp sedentarization to multicollinearity, only one of the variables in the lowest AIC in univariate regressions. White blood cell composition analysis as conducted on HemoCue ${ }^{\circledR}$ WBC DIFF and we collected fecal samples from 30 individuals with intestinal parasites symptoms. Further information and descriptive statistics for these variables can be found in SI appendix tables \$2 and S3. Logistic and linear multilevel models (MLM) were conducted in version 3.1.2 (92) using the Ime4 package (93). All models were originally un with level 1 as the individual, level 2 as the household and level 3 the camp. Levels that failed to improve the model were removed to produce the most parsimonious model. Only theoretically informed variables wer ntered into the model; however, as many variables co-vary, the maxima model suffered from multicollinearity (variance inflation factor scores of more than 2.5 (94)). Therefore, two models were produced, separating mousehold mobility from household foraging, to correct for any remaining cex mea re (ext mean relatedness and household dependents and belongings) and the ukocyte is dependent on the circulating levels of other leukocytes, whether individual had abnormally high levels of any white blood cell types was controlled for as well as total white blood cell count in each analysis. Due the smaller sample size non-parametric Kruskal-Wallis tests were used to examine the data on polyparasitic infections. All model betas are reported in text alongside standard errors. This research and fieldwork was approved by UCL Ethics Committee (UCL Ethics code 3086/003) and carried out with permission from local government and tribal leaders in Palanan. Informed consent was obtained from all participants, and parents signed the informed consents for their children (after group and individual consultation and explanation of the research objectives in the Agta language). As part of the process, people identified as having any disease were provided with medical care in partnership with the local government.

\section{Acknowledgements.}

This project was funded by the Leverhulme Trust grant RP2011-R-045 to A.B.M., R.M. also received funding from European Research Council Advanced Grant AdG 249347. We thank the Human Evolutionary Ecology and Hunter-Gatherer groups at UCL and Matt Thomas for their comments on earlier drafts, the three reviewers for their constructive critique, ou assistants in the Philippines, and most importantly, the Agta.

5. Hershkovitz I, Gopher A (2008) Demographic, Biological and Cultural Aspects of the

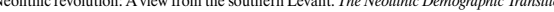
and Its Consequences, eds Bocquet-Appel J, Bar-Yosef O (Springer, London), pp 441-482. 16. Willis A, Oxenham MF (2013) The neolithic demographic transitin and pe be

17. Zahid HJ, Robinson E, Kelly RL (2015) Agriculture, population growth, and statistica

8. Armelagos GJ, Goodman AH, Harper KN, Blakey ML (2009) Enamel hypoplasia and early Cohen MN, Armelogs GJ (1984) Paloopatholog the Origins of Agriculture (Acodenc Press, Gainesville

20. Cohen MN (1989) Health and the rise of civilisation (Yale University Press, London).

21. Kohler TA, Varien MD (2010) A Scale Model of Seven Hundred Years of Farming Settlements in Southwestern Colorado. Becoming Villagers, eds Bandy MS, Fox JR (University of Arizona Press, Tucson), pp 37-61.

22. Richards MP (2002) A brief review of the archaeological evidence for Palaeolithic and Neolithic subsistence. Eur J Clin Nutr 56(12):16

23. Hershkovitz I, et al. (2008) Detection and molecular characterization of 9000-year-old Mycobacterium tuberculosis from a neolithic settlement in the Eastern mediterranean. PLoS One 3(10): $1-6$

24. Greenblatt CL, Spigelman M (2003) Emerging pathogens: archaeology, ecology and evolution of infectious disease eds Greenblatt CL, Spigelman M (Oxford University Press, Oxford). . Rasmussen S, et al. (2015) Early Divergent Strains of Yersinia pestis in Eurasia 5,000 Years
Ago. Cell 163(3):571-582.

26. Mathieson I, et al. (2015) Genome-wide patterns of selection in 230 ancient Eurasians. Nature advance on.

the Chinese Neolithenfer R a., Zhijun W (2002) Diet and health changes at the end of Anthropol 117(1):15-36.

Danford ME, Jacobi KP, Wrobel GD, Glassman S (2007) Health and the transition to Horticulture in the south-central United States. Ancient Health. Skeletal Indicators of Agriculture and Economic Intensification., eds Cohen MN, Crane-Kramer GMM (University Press of Florida, Gainesville), pp 65-79.

29. Barrett R, Kuzawa CW, McDade T, Armelagos GJ (1998) Emerging and re-emergin infectious diseases: The Third Epidemiologic Transition. Annu Rev Anthropol 27(1):247-271.

PNAS | Issue Date | Volume | Issue Number | 5

613 
. Cockburn TA (1977) Infectious Diseases in Ancient Populations. Culture, Disease and Healing. Studies in Medical Anthropology, ed Landy D (Macmillan Publishing, New York), pp 83-96.

Froment A (2014) Human Biology and Health of African Rainforest Inhabitants. HunterGatherers of the Congo Basin: Cultures, Histories and Biology of African Pygmies, ed Hewle BS (Transaction Publishers, London), pp 117-164.

Jackson J a., Friberg IM, Little S, Bradley JE (2009) Review series on helminths, immun modulation and the hygiene hypothesis. Immunology 126(1):18-27.

Leles D, et al. (2010) A parasitological paradox: Why is ascarid infection so rare in the Bethony J, et al. (2006) Soil-transmitted helminth infections: ascariasis, trichuriasis, an hookworm. Lancet 367(9521):1521-1532.

35. Dounias E, Froment A (2006) When forest-based hunter-gatherers become sedentary Consequences for diet and health. Unasylva 57:26-33,

6. Hotez PJ, Bethony J, Bottazzi ME, Brooker S, Buss P (2005) Hookworm: “The great infectio of mankind." PLoS Med 2:0187-0191

37. Hurtado a. M, Frey MA, Hurtado I, Hill KR, Baker J (2008) The role of helminthes in human evolution. Medicine and Evolution: Current Applications, Future Prospects, eds Elto

S, O'Higgins P(CRC Press, London), pp 153-180.
Van Blerkom LM (2003) Role of Viruses in Human Evolution. Am J Phys Anthropol 122:14-46.

Rook GAW (2009) Review series on helminths, immune modulation and the hygiene S Ser $S C$ (2012) El Sci 279(1746):4305-4321.

Hugot JP, Reinhard KJ, Gardner SL, Morand S (1999) Human enterobiasis in evolution: origin, specificity and transmission. Parasite 6 (3):201-8.

2. Reinhard K, Ambler JR, Mcguffie M(1985) Diet and Parasitism at Dust Devil cave. Am Anti 50(4):819-824

43. Reinhard KJ,Araujo A (2012) Synthesining parasitology with archaeology in paleopathology. The Global History of Paleapathology, eds Buikstra JE, Roberts C (Oxford University Press, Oxford), pp 751-764.

44. Reinhard KJ (1988) Cultural ecology of prehistoric parasitism on the Colorado Plateau as evidenced by coprology. Am J Phys Anthropol 77(3):355-366.

Santoro C, Dorsey Vinton S, Reinhard KJ (2003) Inca Expansion and Parasitism in the Luu Valley: Preliminary Data. Mem Inst Oswaldo Cruz 98:161-163.

46. Cohen MN (2009) Introduction: Rethinking the Origins of Agriculture. Curr Anthropol 50(5):591-59:

47. Hutchinson DL, Norr L, Teaford MF (2007) Outer Coast Foragers and Inner Coast Farmer in Late Prehistoric North Carolina. Ancient Healith: Skeletal Indicators of Agricutural an Gainesville), pp 52-6

48. Pinhai R, Stock JT (2011) Human Bioarchaeology of the Transition to Agriculture. northeast Thailand. Bioarchaeology of Southenst Asia, eds Oxenham MF, Tayles N (Cambride University Press, Cambridge), pp 191-219.

50. Humphrey LT, et al. (2014) Earliest evidence for caries and exploitation of starchy plant food in Pleistocene hunter-gatherers from Morocco. Proc Natl Acad Sci 111(3):954-959.

Lukacs JR (1992) Dental paleopathology and agricultural intensification in south Asia: ne evidence from Bronze Age Harappa. Am J Phys Anthropol 87(2):133-150.

52. Oxenham MF, Tayles N (2006) Synthesising Southeast Asian population history and palaehealth. Bioarchaeology of Southeast Asia, eds Oxenham MF, Tayles N (Cambridge University Press, Cambridge), pp 335-349.

53. Oxenham MF, Nguyen LC, Nguyen KD (2008) Oral health in northern Vietnam. Bull IndoPacific Prehist Assoc 22:121-134.

54. Pietrusewsky M, Ikehara-Quebral R (2007) The bioarchaeology of the Vat Kommou cemetry Angkor Borei, Cambodia. Bull Indo-Pacific Prehist Assoc 26:86-97.

Tayles N, Domett KM, Halcrow S (2009) Can dental caries be interpreted as evidence of farming? The Asian experience. Com

56. Waker P, Hewlett B (1990) Dental health diet and social status among Central Africen foragers and farmers. Am Anthropol 92(2):383-398.

foragers and farmers. Am Anthropol 92(2):383-398. Nature 418(6898):700-7.

. Lambert PM (2009) Health versus fitness: competing themes in the origins and spread of agriculture? Curr Anthropol 50(5):603-608.

Borgerhoff Mulder M (2000) Optimizing Offspring: The Quantity-Quality Trade-off in Agropastoral Kipsigis. Evol Hum Behav 21(6):391-410

Draper P, Hames R (2000) Birth order, sibling investment, and fertility among Ju/Hoan

('Kung). Hum Nat 11(2):117-156.
Gillespie DO., Russell AF, Lummaa V (2008) When fecundity does not equal fitness evidence of an offspring quantity versus quality trade-off in pre-industrial humans. Proc Soc B Biol Sci 275(1635):713-722

62. Hagen $\mathrm{EH}$, Barrett HC, Price $\mathrm{ME}$ (2006) Do human parents face a quantity-quality tradeoff? Evidence from a shuar community. Am J Phys Anthropol 130:405-418.

Hill K, Hurtado AM (1996) Aché Life History: The Ecology and Demography of a Foragin People (Aldine de Gruyter, New Haven).

Lawson DW, Alvergne A., Gibson M. (2012) The life-history trade-off between fertility an child sil Sci 279(1748):4755-4764

66. Rai NK (1990) Living in a lean-to: Philippine Negito forroger in (Leiden University) Michigan, Ann Arbor)

67. Peterson JT (1978) The Ecology of Social Boundaries: Agta Foragers of the Philippines (University of Illinois Press, London)

6 | www.pnas.org --- --
8. Kelly RL (2013) The Lifeways of Hunter-Gatherers: The Foraging Specturm (Cambridge University Press, Cambridge)

(1998) Population Dynamics of a Philippine Rain Forest People: The San Ildefonso Agta (University Press of Florida, Gainesville).

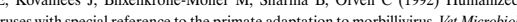
33:275-286.

1. McDade TW, et al. (2005) Predictors of C-reactive protein in Tsimane' 2 to 15 year-olds in lowland Bolivia. Am J Phys Anthropol 128(4):906-913.

bioavailability. J Nutr 133(9):2973S-7S.

73. Mcdonell A, Oxenham MF (2014) Localised Primary Canine Hypoplasia: Implications for 758 Maternal and Infant Health at Man Bac, Vietnam, 4000-3500years BP. Int J Osteoarchaeol 759 24(4):531-539.

74. Who (2012) WHO Children reducing mortality. WHO Media Cent:1. Mascie-laylor CGN (1993) The Biological Anthropology of Disease. The Anthropology of 76

Con, PJ (200) Taylor CGN (Oxford University Press, Oxford), pp 1-72. 762

Cooper PJ (2004) Intestinal worms and human allergy. Parasite Immunol 26(11-12):455-467. 763

Tanner S, et al. (2009) Influence of helminth infections on childhood nutritional status in 764

London D, Hruschka D (2014) Helminths and human ancestral immune ecology: What is the 765

London D, Hruschka D (2014) Helminths and human ancestral immune ecology: What is the
evidence for high helminth loads among foragers? Am J Hum Biol 26:124-129.

$\begin{array}{ll}\text { evidence for high helminth loads among foragers? AmJ Hum Biol 26:124-129. } & 766 \\ \text { Jelliffe DB (1962) The Children of the Hadza Hunters. Trop Pediatr 60:907-913. } & 767\end{array}$

$\begin{array}{ll}\text { 80. Confalonieri U, Ferreira LF, Araujo A (1991) Intestinal Helminths in Lowland South } & 767 \\ \text { American Indians Some Evolutionary Interpretations. Hum Biol 63(6) } 863-873 & 768\end{array}$

Goncialves MLC, Araújo A, Ferreira LF (2003) Human Intestinal Parasites in the Past: New 769

Findings and a Review. Mem Inst Oswaldo Cruz 98(SUPPL. 1):103-118.

2. Sprent JFA (1969) Helminth "zoonoses": an analysis. Helminthol Abstr 38:333-351. 771

83. Armelagos GJ, Harper KN (2005) Genomics at the Origins of Agriculture. Evol Anthropol 771

84. Sreevatsan S, et al. (1997) Restricted structural gene polymorphism in the Mycobacterium 773

$\begin{array}{ll}\text { tuberculosis complex indicates evolutionarily recent global dissemination. Proc Natl Acad Sci } & 774 \\ \text { US A 94(18):9869-74. } & 775\end{array}$

85. Bentley GR, Jasienska G, Goldberg T (1993) Is the Fertility Higher Than Agriculturalists That of Nonagriculturalists? ?'. Curr Anthropol $34(5): 778-785$.

66. Sellen D, Mace R (1997) Fertility and mode of subsistence: a phylogenetic analysis. Curr Anthropol 3(5):878-889.

B7. Blurton Jones N (1987) Bushman birth spacing: Direct tests of some simple predictions. Ethol

Hardy K, Brand-Miller JC, Brown KD, Thomas MG, Copeland L (2015) The importance of etary carbohydrate in human evolution. $Q$ Reviol $90(3) \cdot 251-268$.

Tacer D (1991) Fertily related changes in maternel boly conpoir Papua New Guinea. Am J Phys Anthropol 85(4):393-406. Kramer KL, Boone JL (2002) Why Intensive Agriculturalists Have Higher Fertility: A 784 Gibson M, Mace R (2005) Helpful grandmothers in rural Ethiopia: A study of the effect of 785 kin on child survival and growth. Evol Hum Behav 26(6):469-482.

92. R Core Team (2012) R: A language and environment for statistical computing. 787

93. Bates D, Bolker B, Maechler M, Walker S (2013) Ime4 R Package.

94. Zuur AF, Ieno EN, Elphick CS (2010) A protocol for data exploration to avoid common 788

$\begin{array}{ll}\text { statistical problems. Methods Ecol Evol 1(1):3-14. } & 789 \\ \text { Griffin PB, Griffin MB (1992) Fathers and Childcare among the Cagayan Agta. Father-Child } & 790\end{array}$ Relations: Cultural and Biosocial Contexts, ed Hewlett BS (Transaction, New Brunswick), pp 791 $297-320$.

96. Bion GP, B.Griffin M (1997) Agta Foragers. Aust J Anthropol 8:259.

97. Headland TN (1989) Population Decline in a Philippine Negrito Hunter-Gatherer Society. Am J Hum Biol 72:59-72.

Dyble M, et al. (2015) Sex equality can explain the unique social structure of hunter-gatherer bands. Science 348(6236): 796-798

Griffin BM (2012) The Cultural Identity of Foragers and the Agta of Palanan, Isabela the Philippines. Anthropos 91(1996): 111-123.

. Estioko-Griffin AA, Griffin PB (1981) The beginning of cultivation among Agta hunterA Societies, ed Olofsen $\mathrm{H}$ (Forest Research Institute, Laguna), pp 55-72.

Griffin PB (1985) A contemporary view of the shift from hunting to horticulture. Recent pp 349-352.

12. Headland TN, et al. (1989) Hunter-Gatherers and Their Neighbors from to the Prehistory Present. Curr Anthropol 30:43-66.

103. Headland TN (1984) Agta Negritos of the Philippines. Cult Surv Q 8(3):29-31.

104. Dounias E, Froment a. (2011) From foraging to farming among present-day forest huntergatherers: consequences on diet and health. Int For Rev 13(3):294-304.

5. Morris SS, et al. (1999) Precision, accuracy, and reliability of hemoglobin assessment with use of capillary blood. Am J Clin Nutr 69(6):1243-1248.

6. Greer PJ, et al. (2013) Wintrobes Clinical Hemaology (Lippincott Williams \& Wilkins).

107. Beck N (2008) Diagnostic Hematology (Springer, London). van Assendelft OW (2002) Calibration, control of hematology analyzers. Adv Adm Lab 811

Mitaishvili R (2010) The Human Blood: Composition, Typing Lab Test Interpretation (RM Global Health).

Wahed A, Dasgupta A (2015) Hematology and Coagulations: A Comprehensive Review for Board Preparation, Certification and Clinical Practice. (Elsevier, London).

Footline Author 